\title{
6 Untersuchung: Die Macht des Definierens im Bereich psychischer Gesundheit und Krankheit am Beispiel des Burnout-Diskurses
}

\subsection{Vorlauf und Beginn des Burnout-Diskurses}

\subsubsection{Wann ,beginnt' ein Diskurs?}

Wenn man der Frage nachgehen möchte, wann ein Diskurs ,beginnt', so ist - ausgehend von der dieser Arbeit zugrunde liegenden Definition von Diskurs ${ }^{389}$ - der Zeitpunkt entscheidend, ab dem man als Analysandin so etwas wie ein „Textnetz" zu einem bestimmten Thema und intertextuelle semantische Beziehungen innerhalb dieses Netzes oder einen gemeinsamen Kommunikationszusammenhang erkennen und rekonstruieren kann (vgl. Kap. 2.3).

In diesem Kapitel soll gezeigt werden, dass es neben der ab einem bestimmten Zeitpunkt steigenden Trefferzahl von Texten, die das Suchwort Burnout enthalten, weitere Elemente auf der diskursiven sprachlichen Oberfläche gibt, die den Beginn dieses Diskurses thematisieren und ihn damit ,selbst ${ }^{\star}$ konstituieren, entwickeln lassen und verfestigen.

Durchsucht man Archive von Tages- bzw. Wochenzeitungen/-magazinen, beispielsweise der FAZ, des SPIEGELs und der ZEIT, die bis 1945 zurückgehen, so findet man für den aus dem englischen Sprachraum entlehnten Ausdruck Burnout/Burn-out vereinzelte Treffer Anfang und weitere Treffer Ende der 1980erund Anfang der 1990er-Jahre. ${ }^{390}$ Damit ist allerdings noch nicht geklärt, ob es

389 Diskurs wurde mit Rückgriff auf Felder (2012: 122) als „Text- und Gesprächsnetz zu einem Thema“ definiert, dessen Texte untereinander nachweisbare semantische Beziehungen aufweisen (vgl. Busse/Teubert 1994: 14). Siehe zur weiteren Begriffsbestimmung von >Diskurs in dieser Arbeit auch Kap. 2.3 dieser Arbeit.

390 Siehe im QV im Abschnitt 8.1.6 die Artikel „Tennis: Das Letzte abverlangt“ im SPIEGEL 49/1989 (= DER SPIEGEL 1989: 222-224), in dem Steffi Graf als drohendes Beispiel eines „Tennis-Burnouts“ angeführt wird; oder der Beitrag „Nichts als Ruhe“ im SPIEGEL 52/1988, in dem Burnout als eine „mysteriöse Seelenkrankheit“ beschrieben wird (= DER SPIEGEL 1988: 162-164); siehe auch DER SPIEGEL (1990). In der FAZ findet man einen längeren Übersichtsartikel zur Burnout-Thematik auf der Intensivstation schon 1981 (= Stein 1981: 8) und weitere Treffer, einerseits 1989 als Kommentar zum Beitrag im SPIEGEL „Nichts als Ruhe“ (siehe in der FAZ = möl. 1989: 33) und andererseits einen Leserbrief zur Belastung von Altenpflegern (= Seibertz 1989: 12). In der ZEIT findet man erste Artikel mit diesen Suchwörtern ebenfalls in den 1980er Jahren, z. B. in einem Artikel, der den Zustand auf Intensiv-Stationen beschreibt (= Plog 1984), oder 1988 im Themenfeld von Management und Führungsstil (= Mayer-List 1988).

D Open Access. (c) 2021 Theresa Schnedermann, publiziert von De Gruyter. (๕)BY-NC-ND Dieses Werk ist lizensiert unter einer Creative Commons Namensnennung - Nicht-kommerziell - Keine Bearbeitung 4.0 International Lizenz. https://doi.org/10.1515/9783110727838-006 
frühere Texte gibt, die mit Wortbildungsableitungen des Verbs ausbrennen auf ähnliche psychische Befindlichkeiten referieren. Dieser Frage widmet sich das folgende Kapitel (Kap. 6.2). Dennoch ist dieses vermehrte In-Gebrauch-Kommen des neuen entlehnten Ausdrucks, insbesondere in den hier untersuchten Medientexten, aber auch in allgemeinsprachlichen Wörterbüchern und Fachlexika, als Indiz für ein eigenständiges Diskursthema zu werten. Zudem steigt die Verwendungshäufigkeit im Vergleich zum vorherigen Zeitraum merklich an, wie auch die Zeitverlaufsgrafik im Online-Wortschatz-Informationssystems Deutsch (OWID) des Leibniz-Instituts für Deutsche Sprache zum Stichwort Burnout aufzeigt. ${ }^{391}$

Neben diesen quantitativen Befunden zur Frequenz der Verwendung des Ausdrucks Burnout gibt es weitere sich in Texten manifestierende Zeichenketten, die das ,Neue‘ dieses Begriffs/Themas anzeigen und seine Begriffsgeschichte mit konstituieren.

Zum einen findet man den Ausdruck Burnout in deutsch-, aber auch englischsprachigen Neologismen-Wörterbüchern und zum ersten Mal in Fachlexika ${ }^{392}$ und allgemeinsprachlichen Wörterbüchern ${ }^{393}$ der 1980er und 1990er Jahre. Dies zeigt, dass die Lesart 'psychisch-physische Erschöpfung in Verbindung mit einer Arbeitstätigkeit' des Verbs burn out und dessen substantivierte Form (burnout, $n$.) zunächst im englischen und nach der Entlehnung im deutschen Sprachraum

391 Siehe dazu die Zeitverlaufsgrafik im Online-Wortschatz-Informationssystems Deutsch (OWID) zum Neologismus Burn-out des Leibniz-Instituts für Deutsche Sprache (IDS) unter http:// www.ids-mannheim.de/kl/neoplots/owid/179379.html (zuletzt eingesehen am 04.12.2019). Diese basiert auf Daten des Deutschen Referenzkorpus und dokumentiert damit das Aufkommen und die Verbreitung von Burnout im größten deutschsprachigen maschinenlesbaren Textkorpus. Vgl. auch Kap. 5.1.3 dieser Arbeit, Abb.6.

392 Das Stichwort Burnout findet man das erste Mal im Jahr 1993 in der 11. Auflage des „Dorsch Psychologisches Wörterbuch“ im „Ergänzungsteil“ für neu hinzugekommene Stichwörter (= Dorsch ${ }^{11} 1993:$ 887). In der „Enzyklopädie der Psychologie“ findet man z. B. in Band 4 des Themenbereichs „Pädagogische Psychologie“ 1997 einen Eintrag dazu (=Dittman-Kohli/Sowarska/ Timmer 1997: 198). Im klinischen Wörterbuch Pschyrembel erscheint das Stichwort erst in der 259. Auflage im Jahr 2002 (= Pschyrembel ${ }^{259}$ 2002: 325). Siehe alle Belege im QV unter Abschnitt 8.1.2.

393 Das Stichwort Burnout findet man mit Referenz auf einen psychischen Zustand das erste Mal in der 2. Auflage (1993) des „DUDEN: Das große Wörterbuch der deutschen Sprache“ (Band 2, Bin-Far, = Duden ${ }^{2} 1993:$ 613). In der ersten Auflage dieses Wörterbuchs aus dem Jahr 1976 war Burnout ebenfalls schon enthalten, jedoch nur als Terminus der Raumfahrt (= DUDEN 1976, Band 1, A-Ci). Im Rechtschreibduden ist das Stichwort „Burn-out-Syndrom“ allerdings erst im Jahr 2000 verzeichnet (DUDEN: Rechtschreibung der deutschen Sprache ${ }^{22}$ 2000: 253). Beim Verb ausbrennen ist die psychische Lesart schon früher belegt. Darauf wird im folgenden Kap. 6.2.2 noch ausführlich eingegangen. Im „Deutschen Wörterbuch“ von Wahrig/Wahrig-Burfeind findet man das Stichwort das erste Mal in der Neubearbeitung von 1994 auf S. 369: „Burning out“ „andauernder Erschöpfungszustand aufgrund körperl., geistiger und seelischer Überanstrengung (durch Beruf, Familie u. a.) [engl., zu burn out ,verausgaben'].“ Siehe für alle angegebenen Quellen im QV unter Abschnitt 8.1.1. 
als neuwertig angesehen wurden: In mehreren (Neologismen-)Wörterbüchern sowohl aus Großbritannien als auch aus den USA findet man einen Eintrag zum Substantiv burnout/burn-out mit dieser psychosozialen Bedeutungsangabe. Die meisten dieser Wörterbücher sind Anfang der 1990er Jahre erschienen und dokumentieren neue Wörter im anglo-amerikanischen Wortschatz der 1970er und 1980er Jahre (vgl. New Collegiate Dictionary 1975; Merriam Websters' Collegiate Dictionary 1993; Green 1991; Tulloch 1991; Barnhart/Steinmetz 1990). ${ }^{394}$

Für das Deutsche findet man im Neologismenwörterbuch für die 1990er Jahre von Herberg/Kinne/Steffens (2004) u. a. folgenden Eintrag (siehe Abbildung 7):

\section{Aufkommen: seit Anfang der 90er Jahre des 20. Jahrhunderts in Gebrauch}

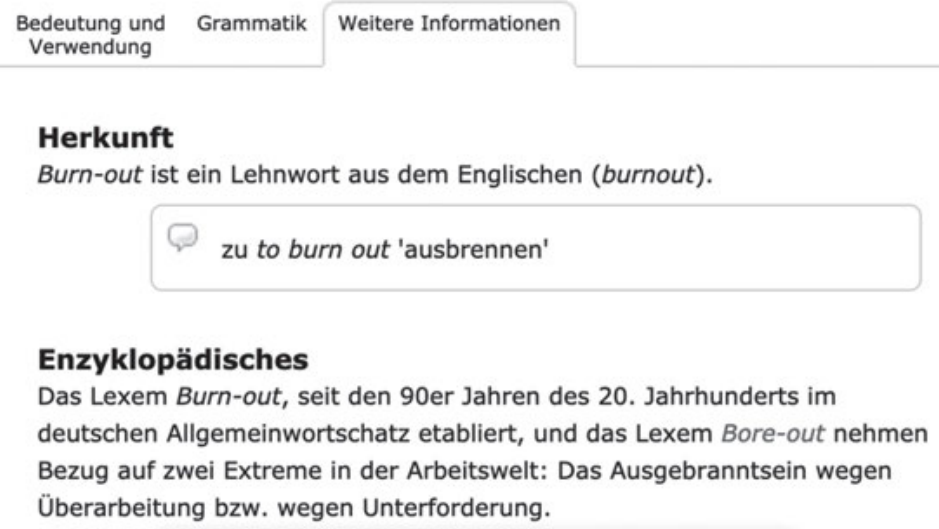

Abb. 7: Auszug aus der Onlineversion des Neologismen-Wörterbuchs von Herberg/Kinne/ Steffens (2004) zum Lemma Burn-out, (c) Leibniz-Institut für Deutsche Sprache. ${ }^{395}$

394 Im Merriam-Websters Collegiate Dictionary von 1975 erscheint das Substantiv burnout nur mit folgender Bedeutungsangabe: "the cessation of operation of a jet or rocket engine; also: the point at which burnout occurs”. In der 10. Auflage (1993) auf Seite 153 des Merriam Websters Collegiate Dictionary ist beim Substantiv dann die psychische Erschöpfung als Folge von anhaltendem Stress mitaufgenommen und um die Jahresangabe 1940 (wahrscheinlich für die technische Bedeutung) ergänzt. Im „Dictionary of Neologisms“ von Green aus dem Jahr 1991, das den britischen Wortschatz seit 1960 fokussiert, wird die spezielle psychische Lesart für das Verb to burn out und das Aufkommen des Substantivs burn-out mit einem Beleg von 1981 flankiert (Green 1991: 40). The „Oxford Dictionary of new words“ bezeichnet das Substantiv burn-out als "very fashionable” in den späten 1980er Jahren (= Tulloch 1991: 47). Für das in den USA erarbeitete „Barnhart Dictionary of New English“ (1990) setzt der figurative Gebrauch des Substantivs burnout im Jahr 1975 ein (= Barnhart/Steinmetz 1990: 71). Siehe für alle angegebenen Quellen im QV unter Abschnitt 8.1.1. 395 Online einsehbar unter: https://www.owid.de/artikel/179379 (zuletzt eingesehen am 4.12.2019). 
Man könnte einwenden, dass die Aufnahme von neuen Wörtern in Wörterbücher selbst von deren Belegfrequenz abhängt und dass dies daher nur ein weiterer Verweis auf die gestiegene Frequenz sei. Mit Rückgriff auf Foucault kann man die Aufnahme in ein Wörterbuch allerdings auch als „diskursives Ereignis“ (vgl. Foucault [1970] 1993: 22f.) deuten. Denn die Aufnahme eines Wortes in ein Wörterbuch geschieht zwar zunächst als Reaktion auf eine im Vergleich zu früheren Zeiträumen gestiegene Frequenz eines Wortes, sie kann aber ihrerseits auch maßgeblich zur Bekanntheit dieses Wortes beitragen: Das Wörterbuch als Produkt gesellschaftlich anerkannter lexikografischer Praxis setzt dieses Wort in Beziehung zum bestehenden Wortschatz, klassifiziert es als neu, etabliert und autorisiert damit zugleich seine Existenz. Das In-Gebrauch-Kommen der Zeichenkette Burn-out/Burnout im Deutschen wird durch die Aufnahme in (Neologismen-)Wörterbücher explizit für „Anfang der 90er Jahre des 20. Jahrhunderts“ aufgezeigt und bestätigt (siehe oben Herberg/ Kinne/Steffens 2004).

Es gibt weitere Textpassagen, die die Funktion erfüllen, die Existenz des Phänomens/Begriffs >BURNOUT $\mathrm{zu}$ bestätigen. Das sind solche, in denen die Begriffs- und Entdeckungsgeschichte bzw. „Ersteinführung“ des Begriffs thematisiert wird und ein Rückbezug auf Entdeckerfiguren stattfindet. ${ }^{396}$ So beispielsweise im Vorwort zu einem Sammelband von Farber 1983 (Beleg 1), im Kapitel „Begriffsgeschichte“ in einem Übersichtsartikel des deutschen Ärzteblatts (Beleg 2), in einem Beitrag in der FAZ aus dem Jahr 1992 (Beleg 3), im SPIEGEL aus dem Jahr 2011 (Beleg 4) oder in der Versionengeschichte von Wikipedia (Beleg 5):

1) [...] The concept of burnout was born in the early 1970s, its heritage embedded in the ideas and efforts of Herbert Freudenberger in New York and Christina Maslach and Ayala Pines in California. This volume presents a "state-of-the-art" look at burnout at the end of its first decade - a period in which this concept has made its way into the popular vocabulary and dramatically changed the ways in which human service professionals and the general public have thought about human service work. [...]

(Farber 1983: ix) (Unterstreichung T.S., siehe im QV unter 8.1.5)

396 Liebert beschreibt in seiner Monografie „Wissenstransformationen. Handlungssemantische Analysen von Wissenschafts- und Vermittlungstexten“ eine ähnliche Praktik: Die „Mitteilung der Chronologie einer Entdeckung als personalisierte Entdeckungsgeschichte“ sei ein „beliebtes Verfahren der Vermittlung“ (Liebert 2002: 13). Liebert bezieht sich dabei auch auf den Begriff der „Ersteinführung“, der von Wimmer 1979 im Kontext seiner Referenzsemantik aufgebracht wird (vgl. Wimmer 1979: 168), und schreibt dazu: „Die Grundidee liegt also darin, nicht die gesamte mit den Termini verbundene Theorie einzuführen, sondern die Situation der Referenzfixierung innerhalb einer Sprechergruppe zu rekonstruieren [...]“ (Liebert 2002: 86). 
2) [...] So, wie wir ihn heute verstehen, tauchte der Begriff Burn-out erstmals 1974 in den USA auf, wo er von dem Psychoanalytiker Herbert J. Freudenberger (5) und etwa gleichzeitig von Ginsburg popularisiert wurde (4). [...]

(Kaschka/Korczak/Broich 2011) (Unterstreichung T.S., siehe im QV unter 8.1.4)

3) [...] Der Begriff burn-out wurde 1974 in den Vereinigten Staaten geprägt, und schon Anfang der achtziger Jahre gab es in Deutschland einen ersten „Boom“ von Burn-out-Veröffentlichungen. Was damals noch als Skurrilität behandelt wurde, ist heute zur Lawine geworden. Mehr als tausend wissenschaftliche Bücher und Artikel wurden bisher darüber veröffentlicht. [...]

(FAZ, 29.02.1992 = Gross: 41) (Unterstreichung T.S., siehe im QV unter 8.1.6)

4) [...] Anfang der siebziger Jahre war es der deutsch-amerikanische Psychoanalytiker Herbert Freudenberger, der den Begriff Burnout populär machte. In New York hatte der Seelenklempner beobachtet, dass ehrenamtliche Mitarbeiter von Hilfsorganisationen nach Phasen der Überlastung nicht mehr konnten. [...]

(DER SPIEGEL 4/2011 = Dettmer/Shafy/Tietz 2011: 116) (Unterstreichung T.S., siehe im QV unter 8.1.6)

5) [...] Der Begriff Burnout (engl. Ausbrennen) bezeichnet einen besonderen Fall berufsbezogener, anhaltender Erschöpfung und wurde 1974 erstmalig von Freudenberger eingeführt. [...] $\quad$ (Wikipedia, 19.4.2004) ${ }^{397}$ (Unterstreichung T.S., siehe im QV unter 8.1.8)

Diese Rückverweise finden sich, wie die Beispiele zeigen, sowohl im englischsprachigen als auch im deutschsprachigen fachlichen und öffentlichen Diskurs seit den 1980er und 1990er Jahren bis in die Gegenwart. ${ }^{398}$ Neben dem Bezug auf den Entdeckungszeitraum ${ }^{399}$ wird häufig auch die Ausbreitung der Verwendung des Begriffs im alltagsweltlichen Kontext betont. Damit wird zudem ein zeitlicher Gegensatz aufgemacht zwischen einem Zeitraum vor und nach der Einführung und Verbreitung des Ausdrucks/Begriffs Burnout, was seine Neuigkeit und Erklärungsbedürftigkeit unterstreicht. Auch metasprachliche Zeichen oder Zusätze (typografische Hervorhebungen z.B. durch Anführungszeichen oder

397 Geht man in der „Versionengeschichte“ auf die ersten Einträge zurück, so wurde am 19. April 2004 (ca. einen Monat nach dem ersten Eintrag zum Thema „Burnout“) der Zusatz „1974 erstmalig von Freudenberger eingeführt“ hinzugefügt. Diese Version des Wikipedia-Artikels ist abrufbar unter: https://de.wikipedia.org/w/index.php?title=Burn-out\&oldid=1112383 (zuletzt eingesehen am 4.12.2019).

398 Weitere Beispiele aus dem fachlichen Diskurs sind u. a.: Schmidbauer 1982: 64; Fisher 1983: 40; Kleiber/Enzman 1990: 10ff.; Demerouti 1999: 1 und 3ff.; Sonneck/Fengler 22009; Barth 2001/2010: 83 (siehe für die angegebenen Quellen im QV unter Abschnitt 8.1.2 und 8.1.5); weitere Beispiele aus dem öffentlichen Diskurs sind u. a.: Die ZEIT Nr. 18/2001 (= Schenk 2001: 61); FAZ Nr. 68/2007 (= Lutterotti 2007: N2); sueddeutsche.de vom 17. Mai 2010 (= Beck 2010, siehe für die letzten angegebenen Belege im QV unter Abschnitt 8.1.6) und AU (2017b) und PH 10/2002 (= Ernst 2002: 3), siehe für die letzten Belege im QV unter 8.1.7).

399 Meist wird Bezug genommen auf einen Text von H. Freudenberger aus dem Jahr 1974, teilweise auch auf Ch. Maslach, A. Pines, S.G. Ginsburg oder H.B. Bradley. 
Kursivsetzung, ${ }^{400}$ fokussierende oder Distanz anzeigende Formen wie sogenannt, Ausspracheangaben ${ }^{401}$, Übersetzungen) sowie kritische Reflexionen über die Verwendung des Ausdrucks Burnout können diese Wirkung verstärken. Sowohl in englischsprachigen als auch in deutschsprachigen Texten des fachlichen und öffentlichen Diskursstrangs wird wiederholt die Frage aufgeworfen, ob man es bei diesem Gegenstand/Begriff mit einem 'neuen' Phänomen zu tun habe oder ob dies nur ein neuer Name für ein eigentlich bekanntes, vorher anders benanntes Phänomen sei.

If the relationship between burnout and constructs like depression and job satisfaction are too high, then the construct of burnout may be just a new name of an old idea.

(Meier 1984: 212, Unterstreichung T.S., siehe im QV unter 8.1.5)

Schon im Jahre 1911 beschreibt Siegbert Schneider in einem Artikel (des „Oberpfälzer Schulanzeigers“) die Symptome einer „modernen Lehrer-Krankheit“ mit dem Namen „Neurasthenie“, die den Burnout-Symptomen auffallend ähnelt. Burnout ist zweifellos schon ein älteres Phänomen, das durch die Benennung und Beachtung durch Wissenschaftler jedoch plötzlich in den Mittelpunkt des Interesses rückte [...]. Zuvor wurde das Phänomen mit Begriffen wie „Stress“, „Entfremdung“, „Depression“. „Erschöpfung“ oder „Arbeitsunzufriedenheit“ umschrieben.“ “402

(Barth ${ }^{2} 2001:$ 72, Unterstreichung T.S., siehe im QV unter 8.1.2)

Etwa 60 Patienten sind hier, Unternehmer, Manager, Lehrer, Selbstständige, die meisten um die 50. Viele leiden unter Symptomen, für die Ärzte keine physischen Ursachen finden können: Schlaflosigkeit, Herzrasen, Schweißausbrüchen, Magenkrämpfen, Tinnitus. Der Kern ihres

400 Siehe z. B. die Aufzählung in einer Studie von Bermejo/Muthny (1993: 110) in der Fachzeitschrift Psychotherapie Psychosomatik und medizinische Psychologie (PPmP), in der nur Burnout in einfachen Anführungszeichen erscheint: „Arbeitsbelastung, Überdruß, Streß, ,Burnout u.ä. sind Schlagwörter, die in den letzten Jahren immer häufiger im Zusammenhang mit helfenden oder Sozialberufen genannt werden“ (Im QV unter 8.1.4 bei PPmP).

401 So zum Beispiel in einem Beitrag aus der SZ aus dem Jahr 1994: „[...] Burnout-Syndrom (neudeutsch: börnaut)“ (= Rheinz 1994, SZ, 31.12.1994, im QV unter 8.1.6).

402 Ein weiteres Beispiel aus dem Fachtextekorpus findet man in der Zeitschrift „Der Internist 11/2012“ (= Kapfhammer 2012: 1276, im QV unter 8.1.5). Letzterer beschreibt bedeutungsähnliche fachliche Konzepte wie folgt: „In einer historischen Perspektive wird leicht erkennbar, dass Burnout keineswegs neuartige Phänomene erfasst. Vielmehr beschrieben schon konzeptuelle Vorläufer wie die Neurasthenie und Psychasthenie im ausgehenden 19. Jahrhundert oder die Erschöpfungsdepression und das chronische Müdigkeitssyndrom im 20. Jahrhundert sehr ähnliche, in den einzelnen historischen Epochen weit verbreitete Befindlichkeiten“ (Kapfhammer 2012: 1276). 
Leidens liegt woanders - in einer kranken Seele. Sie sind depressiv, haben Angstzustände oder Panikattacken. Neurasthenie hieß das früher, heute spricht man von Burn-out. ${ }^{403}$

(DIE ZEIT Nr. 46/2014, = Bund/Rudzio 2014, Unterstreichung T.S., siehe im QV unter 8.1.6)

Implizit geht damit teilweise eine Abwertung der neuen Bezeichnung einher und ihr Nutzen oder auch Existenzrecht wird in Zweifel gezogen. Paradoxerweise verstärkt diese metasprachliche Thematisierung jedoch gleichzeitig die Aufmerksamkeit für das Phänomen, wie folgendes Beispiel zeigt:

\begin{abstract}
Although burnout is a byword to many people, it is viewed as a buzzword by others. According to these critics, burnout is simply old wine in a new bottle - a trendy name for a problem that has been around for a long time. "They used to call it depression, but now it's known as "burnout,"” [sic]. Trumpets one newspaper headline; [...].
\end{abstract}

(Maslach 1982: 29, Unterstreichung T.S., siehe im QV unter 8.1.5)

Maslach beschreibt, dass kritische Stimmen Burnout als "trendy name" für "depression" beschreiben, sie weist aber gleichzeitig darauf hin, dass es die Vokabel Burnout auf diese Weise in die "headline" eines Zeitungsartikels geschafft hat und damit in die Welt hinaustrompetet wird.

Aus linguistischer Perspektive ist zunächst - unabhängig von der Frage, ob >BURNOUT< auf der Sachverhalts- und Konzeptebene etwas Neuwertiges gegenüber sachverwandten Phänomenen/Konzepten darstellt - das wiederholte Auftreten dieser metasprachlichen Kritik an verschiedenen Stellen im Diskurs interessant. Denn erstens wird `Burnout durch diese Reflexionen in ein mehr oder weniger festes Begriffsfeld um Begriffe wie `Depression`, >Neurasthenie, , Erschöpfung etc. und dessen semantische Beziehungen integriert. Zweitens schwingt in der Negierung, Burnout beschreibe kein neuwertiges Phänomen, eine deontische Ebene bzw. Praktik mit, dass bei einem neuen Begriff Besonderheiten desselben deutlich im semantischen Feld abgegrenzt und damit sichtbar gemacht werden sollten. Diese Forderung nach Trennschärfe und Deutlichkeit findet sich, wie gezeigt wurde, auch in den herausgearbeiteten Erwartungen an die Tätigkeit des Definierens wieder (vgl. Kap. 4.2.2).

Drittens scheint der ,neue' Ausdruck Burnout kommunikative Funktionen zu erfüllen, welche die älteren Ausdrücke wie zum Beispiel Neurasthenie nicht (mehr) erfüllen können. Denn vergleicht man verschiedene Ausgaben des „Dorsch Psychologischen Wörterbuchs“, so fällt zweierlei auf:

403 Weitere Belege: Der SPIEGEL „Zeit der Exzesse“, 7.12.2009: 152 (= Kurbjuweit/Steingart/ Theile 2009: 152); Süddeutsche Zeitung, ,Jahrmarkt der Suggestionen und Simulationen“ vom 9.11.1994 (= Köhler 1994: 914); im QV unter 8.1.6. 
- Erstens, dass der sowieso schon recht kurze Eintrag zur „Neurasthenie“ der 11. Auflage (1991, siehe Beleg 1) in der 13. Auflage (1998, siehe Beleg 2) nochmals deutlich gekürzt und im Zuge dessen nur noch als Konzept der Neurosenlehre Sigmund Freuds erscheint.

- Zweitens, dass am Ende des Eintrags zum Stichwort „Neurose“ in der 13. Auflage (1998, siehe Beleg 3) der Hinweis aufgenommen wird, dass dieser Terminus in aktuellen Klassifikationswerken „nicht mehr benutzt“ werde; in der 14. Auflage von 2004 (Beleg 4) wird dieser Hinweis dann sogar an den Anfang des Eintrags gestellt:

1) Neurasthenie [gr.], „Nervenschwäche“, nervöse Reizbarkeit. Geringe seelische Belastungsfähigkeit, leichte Erschöpfbarkeit. Der Begriff wurde 1879 von dem amerikanischen Arzt G.M. Beard eingeführt. $\rightarrow$ Neurose

(Dorsch ${ }^{11}$ 1991: 443)

2) Neurasthenie, psychovegetatives Syndrom; eine der Aktualneurosen bei Freud $\rightarrow$ Neurose

(Dorsch $\left.{ }^{13} 1998: 572\right)$

3) Neurose, [...] Im $\rightarrow$ ICD-10 und $\rightarrow$ DSM-IV wird der Oberbegriff Neurose nicht mehr benutzt. Allerdings wird im ICD-10 der Begriff „neurotisch“ gelegentlich verwendet und auch für Oberklassen wie „neurotische Belastungs- und somatoforme Störungen“ benutzt.

(Dorsch ${ }^{13} 1998: 577$ )

4) Neurose $(=\mathrm{N}$.$) , heute im \rightarrow$ DSM-IV nicht mehr verwendeter (da diskriminierender) Begriff, [...]

(Dorsch ${ }^{14}$ 2004: 643)

Mit der Neurosenlehre scheint also auch der Begriff der >Neurasthenie in gewisser Weise nicht mehr ganz zeitgemäß zu sein, und in eben diesem Zeitraum erscheint der Eintrag zu „Burnout“ (erstmals im Ergänzungsteil der erweiterten nachgedruckten 11. Auflage des Dorsch $\left.{ }^{11} 1991\right)$ und mit ihm weitere Lexikoneinträge beispielsweise zu „Streß am Arbeitsplatz“ (erstmals im Dorsch ${ }^{12} 1994$ ) oder „Arbeitszufriedenheit“ (erstmals im Dorsch ${ }^{13} 1998$ ). Aus der Perspektive „temporaler Modalität“ (Köller 1995: 40) wurde die Gültigkeit des Terminus Neurasthenie von Burnout zumindest teilweise abgelöst. ${ }^{404}$ Gleichzeitig wird gefordert, dass man den Terminus Burnout nur verwenden solle, wenn er auf etwas ,Neues` verweise, woraus dann wiederum eine Pflichtimplikation zur weiteren Differen-

404 Vgl. dazu auch die Einschätzung aus dem Lehrbuch zur „Psychotherapie und Psychosomatik“ von Ermann ('2016: 290): „Wenn an Stelle organbezogener Klagen (,Organneurose') Erschöpfung, rasche Ermüdung, Müdigkeit und Konzentrationsstörungen im Vordergrund der Beschwerden stehen, spricht man von Neurasthenie. Dieses typische Krankheitsbild des Beginns des 20. Jahrhunderts wird heute kaum mehr diagnostiziert. Es scheint in den modernen Diagnosen Fatigue-Syndrom und Burn-out-Syndrom aufgegangen zu sein.“ Auch für den Psychiater Allen

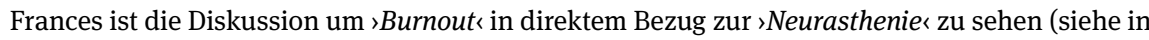
einem FAZ-Interview, Freund (2013)) und dieser Bezug wird auch bei Hank (2014a) auf FAZ.NET hergestellt; siehe die Zeitungsbelege im QV unter 8.1.6. 
zierung dieses Ausdrucks/Begriffs durchscheint (vgl. ebd.). Der neue Ausdruck Burnout teilt zwar Symptome wie 'schnelle Erschöpfbarkeit' mit der Neurasthenie, aber es scheint ein Bedürfnis gegeben zu haben, die mit dem ,alten` Begriff verbundenen Konnotationen und zugrunde liegenden Theorien und Forschungsmethoden $\mathrm{zu}$ verlassen, hier insbesondere die psychoanalytischen Grundannahmen wie zum Beispiel Triebkonflikte. Im Foucault'schen Sinne ist dies ein Indiz, dass sich „der Wille zum Wissen“ im Fach Psychologie/Psychiatrie verändert hat (vgl. Foucault [1970] 1993: 15 und Kap. 4.1.3 dieser Arbeit). Neurasthenie und Burnout sind damit „Lexeme[n], die einen bestimmten medizinischen [bzw. psychologischen, T.S.] Diskurs zu einer bestimmten Zeit geprägt haben“ bzw. prägen (vgl. Riecke 2016: 246).

Schließlich führen die Verweise auf die schnelle Verbreitung bzw. Popularisierung dieser neuen Bezeichnung für das psychische Phänomen $\mathrm{zu}$ weiteren sprachreflexiven Anschlussfragen: Gibt es diskursiv-semiotische oder sprachgeschichtliche Indizien für Faktoren, die die postulierte schnelle Akzeptanz der neuen Bezeichnung in der Sprachgemeinschaft und das Nebeneinander der bedeutungsähnlichen Begriffe beeinflusst haben könnten? Das folgende Kapitel nähert sich diesen Fragen, indem es anhand einer diachronen Durchsicht von allgemeinen deutschen und englischen Wörterbüchern, Neologismenwörterbüchern und Belegen des Deutschen Textarchivs ${ }^{405}$, des DWDS-Kernkorpus des 20. Jahrhunderts ${ }^{406}$ und Recherchen im Deutschen Referenzkorpus ${ }^{407}$ untersucht, in welchen Kontexten und in welcher Weise Ableitungen des Verbs ausbrennen/ to burn out vor dem sogenannten Beginn des Burnout-Diskurses zur Bezeichnung psychischer Zustände verwendet wurden.

\subsubsection{Metaphorische Verwendungsweisen der Ausdrücke (to) burn(out) und (aus)brennen im allgemeinen und fachlichen Sprachgebrauch vor Beginn des Burnout-Diskurses Mitte der 1970er Jahre}

Um herauszufinden, ob die Ausdrücke (to) burn out/burnt out und ausbrennen/ ausgebrannt schon vor dem Beginn des psychologisch-medizinischen BurnoutDiskurses Mitte der 1970er Jahre in ähnlich ,übertragener` Bedeutung im allgemei-

405 Die Beschreibung und Textauswahl des Deutschen Textarchivs ist einsehbar unter: https:// dwds.de/d/k-referenz\#dta (Stand: 4.12.2018).

406 Die Beschreibung des DWDS-Kernkorpus ist einsehbar unter: https://dwds.de/d/kreferenz\#kern (Stand: 4.12.2018).

407 Zur Beschreibung des Deutschen Referenz-Korpus siehe: http://www1.ids-mannheim.de/ $\mathrm{kl} /$ projekte/korpora (Stand: 4.12.2018). 
nen Sprachgebrauch und im fachlichen Kontext üblich waren, lohnt sich ein Blick in historische Textkorpora (DTA 1473-1927 und DWDS-Kernkorpus 1900-1999) ${ }^{408}$, Fachbücher und -zeitschriften ${ }^{409}$ und Wörterbücher, die den allgemeinen Wortschatz und Fachwortschatz vor dieser Zeit beschreiben. Die Durchsicht nimmt vor allem deutschsprachige Quellen in den Blick, da in dieser Arbeit der deutsche Burnout-Diskurs im Untersuchungsfokus steht. Für den englischen Sprachgebrauch wurden verschiedene Wörterbücher konsultiert. ${ }^{410}$

Für die englische Sprache dokumentiert beispielsweise das Oxford English Dictionary (OED) das Aufkommen von sprachlichen Verwendungsformen mit Belegen aus der jeweiligen sprachlichen Periode. Zum Verb to burn (out) findet man in der aktuellen Online-Version folgende Einträge:

„c. lit. and fig. With certain modifying adverbs. to burn out, forth: to burst out in flame (arch.). to burn out, also (quasi-refl. and pass.) to burn itself out, to be burnt out: to burn until extinguished by want of fuel; spec. ( $a$ ) of an electrical valve, fuse, etc.; $(b)$ of a space rocket. Also, to burn oneself out: to exhaust one's strength (by over-exertion) (1937 in Partridge Dict. Slang s.v.). [...] $]^{\star 411}$

(Unterstreichung T.S.)

408 Gesucht wurde 1.) im DTA = Deutsches Textarchiv (1473-1927), dessen Textauswahl unter folgender Adresse nachgelesen werden kann http://www.deutschestextarchiv.de/doku/textauswahl, und 2. im Kernkorpus des Digitalen Wörterbuchs der deutschen Sprache=DWDS-Kernkorpus 1900-1999. Dessen Textauswahl ist unter folgender Adresse dokumentiert: https://www.dwds. de/d/k-referenz\#kern (Stand: 23.2.2018).

409 Da der Fokus der vorliegenden Arbeit nicht auf Formulierungsvarianten von PSYCHISCHEN ERSCHÖPFUNGSZUSTÄNDEN im 19. und frühen 20. Jahrhundert liegt, sondern auf der Entwicklung des ,modernen‘ Burnout-Diskurses, kann die Durchsicht nach der Verwendung des Ausdrucks ausgebrannt und alternativer Formulierungen mit Bezug auf psychische Befindlichkeiten nur in ausgewählten Fachbüchern und -Zeitschriften des 19. Jahrhunderts erfolgen. Einige bedeutende psychiatrische Lehrwerke sind im Korpus des Deutschen Textarchivs enthalten (z. B. von Emil Kraepelin und Wilhelm Griesinger). Berücksichtigt wurden darüber hinaus Quellen, die bei einer Google-Buchsuche von 1800-1930 zum Suchwort ausgebrannt einsehbar sind und die eine übertragene psychische Lesart aufweisen. Die Belege z. B. aus der „Allgemeine[n] Zeitschrift für Psychiatrie und psychisch-gerichtliche Medizin“ oder Monografien wie der „Gesundheitslehre für Geistigbeschäftigte“ von Joseph-Henri Reveillé-Parise (1840) werden mit den Belegen aus dem DTA und dem DWDS-Kernkorpus im Folgenden zusammen aufgeführt. Sie sind über Fußnoten und im Quellenverzeichnis jedoch einzeln vermerkt.

410 Siehe dazu die in Kap. 5.1.1 aufgeführten englischsprachigen Wörterbücher.

411 Das Oxford English Dictionary Online ist über die Universitätsbibliothek Heidelberg einsehbar: https://www.oed.com/view/Entry/25028?rskey=sTN97b\&amp;result=1\&amp;isAdvanced=false (zuletzt eingesehen am 26.11.2019). 
Als Beleg für die „quasi-reflexive“ Verwendung des Verbs wird u. a. folgender Beleg aus Shakespeares Richard II. angegeben:

1597 [His rash fierce blaze of riot cannot last / For] violent fires soon burn out themselues. William Shakespeare, Richard II, ii. i. 34 [Unterstreichung T.S.]

In der Übersetzung von Christoph Martin Wieland lauten diese Verse folgendermaßen, wobei jeweils der Satz zuvor und danach hier mit zitiert seien, um die Stelle kontextuell besser einordnen zu können:

Mich däucht, ich bin ein neubegeisterter Prophet, und sterbend weissage ich so von ihm. Seine rasche, ausgelassene, unbezähmte Jugendhize, kan nicht von langer Dauer seyn; ein heftiges Feuer brennt sich bald selbst aus. Sanfte Regen dauren lange, plözliche Stürme gehen bald vorüber; der wird bald müde, der anfangs die Sporren zu stark gebraucht; und wer allzugierig ißt, hat am bäldesten genug. ${ }^{412}$

[Unterstreichung T.S.]

In dieser Szene bewertet John of Gaunt das Verhalten von Richard dem II. als „rasch“ bzw. übereilt (rash) und als „unbezähmt“ bzw. zu stark und gewaltsamaufrührerisch (fierze blaze of riot) und prophezeit, dass „heftiges Feuer“ (violent fieres) schnell „sich selbst“ ausbrennt. Der bei Shakespeare aufgerufene Kontext zeigt, dass die Verwendung von Wörtern aus dem Begriffsfeld rund um >Feuer (blaze; fires; to burn itself out) zur Beurteilung von menschlichen Charakter- und/ oder Verhaltensweisen im Sprachgebrauch dieser Zeit schon verankert war. Schlägt man bei den Verben to burn bzw. brennen im OED und Grimm'schen Wörterbuch ( $\left.{ }^{1} \mathrm{DWB}\right)$ nach und sieht auf die Bedeutungsbeschreibungen des Basisverbs ohne Präfix-Ableitungen (mit aus-, ver- oder durch-), so ist in beiden Sprachen der figurative Gebrauch für 'emotionale, leidenschaftliche Angelegenheiten' seit Jahrhunderten verbürgt:

Beispiele aus dem ${ }^{1} \mathrm{DWB}^{413}$ und $\mathrm{OED}^{414}$, [Unterstreichungen T.S.]:

\section{OED: c1385 Chaucer „Legend Good Women“ 1747}

Desyr That in his herte brende as any fer.

412 Die Übersetzung von Wieland aus der ersten Szene des zweiten Auszugs ist online einsehbar unter: https://gutenberg.spiegel.de/buch/leben-und-tod-konigs-richard-des-zweyten-2176/9 (zuletzt eingesehen am 10.12.2019).

413 Der Eintrag zu brennen ist abrufbar unter: http://woerterbuchnetz.de/cgi-bin/WBNetz/ wbgui_py?sigle=DWB\&mode=Vernetzung\&lemid=GB11198\#XGB11198 (zuletzt eingesehen am 10.12.2019).

414 Ebenfalls recherchiert über die Online-Version des OED, einsehbar über die Universitätsbibliothek Heidelberg: https://www.oed.com/view/Entry/25028?rskey=sTN97b\&amp;result=1\&amp;isAdvanced=false (zuletzt eingesehen am 14.7.2021). 
OED: 1611 „Bible (King James)“ Gen. xliv. 18

Let not thine anger burne against thy seruant.

OED: 1720 J. Ozell et al. tr. R. A. de Vertot „Hist. Revol. Rom. Republic“ I. v. 297: Virginius burnt with Impatience to revenge himself of Appius.

${ }^{1}$ DWB: Günther 509 (= Ch. Günther, zu Joh. Christ. Ernestis doctorwürde, 1716) kommt alle, deren fleisz zu guten künsten brennt.

1DWB: Gotter 2, 227 (= Friedrich Wilhelm Gotter, Merope, 2. Aufzug, 3. Auftritt, 1773) ich brannte, meine jugendkraft in waffen zu üben.

1DWB: Bürger 13' (= Gottfried August Bürger, Leonore, 1773)

o mutter, mutter, was mich brennt, das lindert mir kein sacrament.

Im letzten Beispiel (aus der Ballade „Lenore“ von Bürger, 1773) brennt der Verlust des Geliebten und das brennende Gefühl ist zugleich der Verlust des Glaubens und Lenores Untergang. Weitere Belege zeigen außerdem die enge Verbindung zwischen körperlichen und seelischen Symptomen im Verb brennen:

${ }^{1}$ DWB: fastn. $775,16^{415}$

die minn mich ser hat erzund

inniklich in meins herzen grund,

das mügt ir erkennen

bei der varb, die mich thut prennen.

[Unterstreichungen T.S.]

OED: 1697 Dryden tr. Virgil „Georgics“ iii, in tr. Virgil Wks. 106

With two fair Eyes his Mistress burns his Breast.

[Unterstreichungen T.S.]

In Belegen der seit Jahrhunderten nachweisbaren Kookkurrenz „brennender Schmerz" aus dem Deutschen Textarchiv (DTA) zeigen sich ebensolche semantischen Verbindungslinien zwischen körperlichen, seelischen und religiösen Krankheits-, Therapie- und Heilungsvorstellungen (Unterstreichungen T.S.):

DTA: Ideler, Karl Wilhelm: Der religiöse Wahnsinn, erläutert durch Krankengeschichten. Ein Beitrag zur Geschichte der religiösen Wirren der Gegenwart. Halle (Saale), 1847.

415 Das Zitat ist auch einsehbar unter: https://archive.org/stream/fastnachtspiele07schegoog/ fastnachtspiele07schegoog_djvu.txt (zuletzt eingesehen am 7.12.2018). 
Nicht nur empfand sie dabei einen brennenden Schmerz in den Geschwüren, sondern sie fühlte auch zugleich die ewige Höllenpein, durch welche Gott ihr ankündigte, sie solle die Menschen zur Buße und Besserung auffordern, damit sie nicht auf immer in den Feuerpfuhl geriethen.

Auch in den Belegen aus dem DTA und DWDS zu den Formen ausbrennen und ausgebrannt ${ }^{416}$ findet man viele Beispiele aus Gebrauchsliteratur, Belletristik, Zeitungen und Wissenschaft ${ }^{47}$, in denen durch Vergleiche und Metaphorik eine übertragene Bedeutungsebene ins Spiel kommt und Verbindungslinien zwischen natürlich-elementaren, körperlichen, seelischen und religiösen Erscheinungen gezogen werden. Britt-Marie Schuster merkt in Bezug auf die Textsorte Krankengeschichten in frühen psychiatrischen Fachzeitschriften des 19. Jahrhunderts an: „Alle mit Feuer assoziierbaren Begriffe wie lodern, entbrennen, aber auch glimmen und verlöschen sind für die Versprachlichung psychischer Abweichungen verwendbar“ (Schuster 2009: 379). Des Weiteren schreibt Schuster, dass ausgebrannt innerhalb der sich im 19. Jahrhundert herausbildenden psychiatrischen Schreibpraxis eine „lange tradierte Bezeichnung für das Endstadium einer Depression“ sei (Schuster 2010: 13; 2009: 380). ${ }^{418}$

416 Dies Suche nach ausbrennen ergab im DTA in allen „Textklassen“ 194 Treffer (https:// www.dwds.de/r?q=ausbrennen\&corpus=dta\&date-start=1473\&date-end=1927\&genre=Belletristik\&genre $=$ Wissenschaft\&genre $=$ Gebrauchsliteratur\&genre $=$ Zeitung\&format $=$ full\&sort $=$ da te_asc\&limit=100, Recherche-Stand: 7.12.2018) und nach ausgebrannt 541 Treffer (https://www. dwds.de/r?q=ausgebrannt\&corpus=dta\&date-start=1473\&date-end=1927\&genre=Belletristik\&genre=Wissenschaft\&genre $=$ Gebrauchsliteratur\&genre $=$ Zeitung\&format $=$ full\&sort $=$ date asc\&limit=100, Recherche-Stand: 7.12.2018). Die Suche nach ausbrennen im DWDS-Kernkorpus 1900-1999 ergab 50 Treffer, von denen aus urheberrechtlichen Gründen aber nur 38 Treffer angezeigt werden (https://www.dwds.de/r?q=ausbrennen\&corpus=kern\&date-start=1900\&date-end=1999\&genre=Belletristik\&genre $=$ Wissenschaft\&genre $=$ Gebrauchsliteratur\&genre $=-$ Zeitung\&format=full\&sort=date_asc\&limit=10, Recherche-Stand: 7.12.2018). Die Suche nach ausgebrannt im DWDS-Kernkorpus 1900-1999 ergab 266 Treffer, von denen 188 Treffer angezeigt werden (https://www.dwds.de/r?q=ausgebrannt\&corpus=kern\&date-start=1900\&date-en $\mathrm{d}=1999$ \&genre $=$ Belletristik\&genre $=$ Wissenschaft\&genre $=$ Gebrauchsliteratur\&genre $=$ Zeitung\&f ormat=full\&sort=date_asc\&limit=10, Recherche-Stand 7.12.2018). Siehe im QV unter 8.1.1.

417 Im Deutschen Textarchiv unter der Rubrik „Wissenschaft“ sind viele Werke naturwissenschaftlicher Fächer (z. B. Geologie, Chemie, Physiologie) enthalten, die den Ausdruck ausgebrannt in seiner ,wörtlichen' Bedeutung verwenden. Es finden sich jedoch einzelne Belege mit Verwendung von Formen des Verbstamms ausbrenn ${ }^{\star}$ in übertragener psychischer Bedeutung in Werken der Theologie, Philosophie und Volkswirtschaft. Obwohl das DTA auch frühe psychiatrische Lehrwerke enthält (z. B. von Wilhelm Griesinger und Emil Kraepelin) ergibt die Suche nach dem Verbstamm ausbrennen oder ausgebrannt aus dem psychiatrischen Fachbereich im DTA keine Treffer. 418 In folgenden Nachschlagewerken des ausgehenden 19. Jahrhunderts findet man keinen ,eigenen` Eintrag zu ausgebrannt, was dafür spricht, dass das Partizip ausgebrannt eher in Be- 
Innerhalb der Belege zeigen sich semantische Unterschiede, je nachdem, ob das Verb ausbrennen intransitiv oder transitiv verwendet wird. Mit dem intransitiven Verb wird ein Transformations- bzw. Reduktionsprozess beschrieben, ohne dass ein Auslöser für diesen Prozess explizit angegeben werden muss. Dieser durch das intransitive Verb beschriebene Vorgang, dass etwas vorher in einer bestimmten Qualität vorhanden war und dann durch das Feuer reduziert bzw. vernichtet wird, wird auf verschiedene lebensweltliche und psychische Umstände übertragen. Das Rahmenkonzept, das hinter den folgenden Beispielen steht, lässt sich als >Verlust, Reduzierung, Aufhören von x` formulieren:

A) Beispiele für 'x verliert Kräfte/Stoffe, die am Leben erhalten, bzw. hat diese verloren`(Unterstreichungen T.S.):

1) DTA: Steinbach, Georg: LeichPredigt Bey der Christlichen Begrebnüs. Liegnitz, 1603.

Aber ehe wir hinaus kamen/ war er mitlerzeit sanfft vnd seliglich eingeschlaffen/ vnd ohn alles zucken der Glieder wie ein ausgebrantes Licht ausgegangen/ Jm 50. Jahr seines Ehestandes/ im 52. Jahr seines Predigampts/ vnd im 77. Jahr seines alters.

2) DTA: Güntherus, Wolfgang: König Davids Sterbekunst Oder Christliche Leichpredigt. Görlitz, 1634.

Das Liecht seines lebens bald außgebrennet/ biß auff ein klein strümplein.

3) DTA: Lohenstein, Daniel Casper von: Großmüthiger Feldherr Arminius oder Herrmann. Bd. 1. Leipzig, 1689.

Wie eilends pflegt das Tacht des Lebens auszubrennen!

4) DTA: Arnim, Achim von; Brentano, Clemens: Des Knaben Wunderhorn. Bd. 2. Heidelberg, 1808.

schreibungen bestimmter Stadien anderer Krankheitsbilder (wie z. B. Depression) verwendet wurde und weniger eine eigene Krankheitsentität darstellte. Schuster beschreibt für psychiatrische Krankengeschichten, die im 19. Jahrhundert in damals entstehenden Fachzeitschriften veröffentlich wurden, die „terminologische Prägung ausgebrannter Defekt für das Endstadium einer Depression“ (Schuster 2009: 380; vgl. auch Schuster 2010: 13; 312; 414). Im „Deutschen Krankheits-Namenbuch“ (1899) von Max Höfler findet man einen Eintrag zu „Melancholie“, in dem allerdings nicht von ausgebrannten Zuständen gesprochen wird. Im Rahmen mittelalterlicher Vorstellungen von der „Melancholie“ wird nur Blut, das „erschwarzet [verbrannt]“ ist, erwähnt und aus mittelalterlichen Medizinschulen wird von „schwarzgallige[r] Bluthefe“, die „gleich als Asche aller vier Feuchten“ in den Neben-Nieren und der Milz aufzufinden sei, berichtet (Höfler 1899: 409). Schließlich gibt es bei Höfler noch einen Eintrag zu „verbrannte Melancholey“, mit der man sich damals allerdings auf Veränderungen der Hautpigmentierung durch oxidiertes „Bilirubin“ bei „Lebersyphilis“ bezog (Höfler 1899: 410). Im „Wörterbuch der klinischen Kunstausdrücke“ von Otto Dornblüth findet man Einträge zu den Stichwörtern Depression, Abspannung, Verstimmtheit, Melancholie, wobei jedoch ausgebrannt in diesen Einträgen ebenfalls nicht verwendet wird. 
Kein Fünkchen mehr in der Asche ist, Mein Kerzchen ist längst ausgebrannt, Adi, Adi mein Engelsschätzchen, Jezt reis' ich nach Engelland.

Zum dritten Beispiel findet man etwa hundert Jahre später bei Adelung sogar einen metasprachlichen Verweis, dass die Metapher des „Lebens Docht“ bzw. Tacht/Tocht (in oberdeutscher Mundart nach Adelung), „eine schon sehr abgenutzte Figur [sei], das Leben zu benennen“ ${ }^{\star 419}$ und mit ihr wohl auch die Metapher des ausbrennenden Dochts/der ausbrennenden Kerze für das Sterben bzw. den Verlust der Lebenskräfte. ${ }^{420}$

Schuster führt anhand von Beispielen aus Krankengeschichten des frühen Psychiatriediskurses in der ersten Hälfte des 19. Jahrhunderts aus, dass es in dieser Zeit die Vorstellung ,einer beeinflussbaren Lebenskraft [gab], die bspw. durch heftige Leidenschaften geschwächt werden kann und in der Folge Krankheiten ausbildet“ (Schuster 2010: 195). Bei dieser Vorstellung überkreuzten sich „medizinische und philosophische Denkmodelle (so die Schellingsche Naturphilosophie)“ und der „Magnetismus (der gerade in den ersten Jahrzehnten des 19. Jahrhunderts populäre Mesmerismus)“(ebd.).

B) ix verliert Empfindungen wie Mut, Spannkraft, Motivation, Leidenschaft, Glauben ..., weitere Qualitäten, Fähigkeiten ... bzw. hat diese verloren` (Unterstreichungen T.S.):

1) DTA: Abschatz, Hans Assmann von: Poetische Ubersetzungen und Gedichte. Leipzig, 1704.

Laß deiner Flammen heilge Glutt Manch ausgebrandtes Hertz entstecken/ Erhebe den entsuncknen Mutt/ Und lehre/ was die Tugend nüzt/ Die/ wenn die Laster todt/ im Tode lebend macht.

2) DTA: Brockes, Barthold Heinrich: Physikalische und moralische Gedanken über die drey Reiche der Natur. Bd. 9. Hamburg u. a., 1748.

Kaum aber war die Quelle weg, war alles plötzlich ausgebrannt, Und die Veränderung nicht glaublich, es schwand mit der erloschnen Glut Begierde, Flüchtigkeit und, Brunst, und, sammt den Kräften, Wut und Muth, Die Nerven, welche diese Brunst zu sehr, zu heftig ausgespannt, Die zogen sich im Augenblick, beraubt von ihren vor'gen Flammen, Geschwächt, erkältet, ganz erschöpft, und recht als wie gelähmt, zusammen.

3) DTA: Jean Paul: Dritte Abteilung Briefe. In: Jean Pauls Sämtliche Werke. Historisch-kritische Ausgabe. Abt. 3, Bd. 3. Berlin, 1959.

419 Siehe im Grammatisch-kritischen Wörterbuch der Hochdeutschen Mundart, Band $1{ }^{2} 1793$ von Johann Christoph Adelung, S. 1507, online einsehbar unter: http://woerterbuchnetz.de/cgibin/WBNetz/wbgui_py?sigle=Adelung\&mode=Vernetzung\&lemid=DD00708\#XDD00708 (zuletzt eingesehen am 12.12.2019, siehe im QV unter 8.1.1).

420 Siehe dazu auch folgenden Beleg im DTA: Gotter, Friedrich Wilhelm: Die Erbschleicher. Leipzig, 1789: „Der Alte ist ja so fertig, als ein ausgebranntes Docht.“ 
Solche Schmerzen wie du in Hof in mir sahest, finden keinen Weg mehr in die alte Seele, die ausgebrant und durchgearbeitet ist. ${ }^{421}$

4) DTA: Laube, Heinrich: Das junge Europa. Bd. 3. Mannheim, 1837.

Obwohl ich sonst stumpf und gleichgültig geworden, die Sonnenstrahlen, mein eigentlich Gottesleben, zündeten doch eine kleine Flacke in meiner ausgebrannten Seele.

5) Hohnbaum, Dr. (1845): Ueber den Wechsel zwischen Heiterkeit und Traurigkeit beim Irren. In: Allgemeine Zeitschrift für Psychiatrie und psychisch-gerichtliche Medizin, 2/1845, S. $260^{422}$

In diesem aufgeregten Zustand verblieb die Kranke 8 Monate, worauf er allmählig in ein vollkommenes stadium melancholicum überging. Eine solche vollkommen abgeschlossene Umkehrung des ganzen Charakter- und Gemüthszustandes ist mir nie vorgekommen. Ihr jetziger Gemüthszustand war einer ausgebrannten, verkohlten Brandstätte zu vergleichen. Mit freudenleerem, traurigem Blicke schlich sie im Haus umher, [...].

6) DTA: Falke, Jakob von: Die deutsche Trachten- und Modenwelt. Ein Beitrag zur deutschen Culturgeschichte. Bd. 1. Leipzig, 1858.

[...] wir müssen uns hineinversetzen in die Zeit der Galanterie und der irrenden Ritter, in die Zeit, da die Ritterschaft, arm an poetischen Großthaten, die ausgebrannte Phantasie und die erloschene Ehrbegierde an den Heldenbildern der Amadis aus Gallia und der Lanzelot vom See wieder zu erhitzen suchte, in die Zeit, da die Ritter Romane lasen, aber nicht mit ihren Thaten machten oder erlebten.

7) DTA: Frapan, Ilse [i. e. Ilse Akunian]: Flügel auf! Novellen. Berlin, 1895.

Lieber Gott, so kleine herrenlose Kohlen sind immer da, auch in dem allerausgebranntesten Herzen.

8) DWDS-Kernkorpus (1900-1999): Simons, Oliver (Hg.), Deutsche Autobiographien 1690-1930, Berlin: Directmedia Publ. 2004 [1927], S. 50398

Das Jugendfeuer, mit dem ich jede Art der Arbeit und Beanspruchung auf mich nahm, wenn sie nur im Dienst der vielgeliebten Wissenschaft stand, war ausgebrannt.

421 Jean Paul verwendet das Partizip ausgebrannt recht häufig in Briefen, um seine innere Verfassung oder die anderer Menschen zu beschreiben, wie mehrere Textstellen im DTA belegen. Siehe unter: https://www.dwds.de/r?q=ausgebrannt\&corpus=dta\&date-start=1473\&date-end=1927\&genre=Belletristik\&format=full\&sort=date_asc\&limit=100 (zuletzt eingesehen am 12.12.2019). Ähnlich in Bezug auf die „Melancholie“ in einem Werk von 1840, „Gesundheitslehre für Geistigbeschäftigte“ von Joseph-Henri Reveillé-Parise, das den „geistigen Zustand“ von Personen, die mit dem Kopf arbeiten, also z. B. von Gelehrten, Schriftstellern oder Staatsdienern, untersucht: „Das Feuer ihrer Seele ist ausgebrannt und es bleibt ihnen nichts als die kalte Wirklichkeit, [...], S. 220. Einsehbar unter: https://books.google.de/books?id=46o_ AAAAcAAJ\&pg=PA220\&dq=ausgebrannt + melancholie\&hl=de\&sa=X\&ved=0ahUKEwigqKHf 2Z3fAhUGUBUIHYRPCFYQ6AEIKDAA\#v=onepage \&q=ausgebrannt $\% 20$ melancholie \&f=false (zuletzt eingesehen am 12.12.2019), siehe im QV unter 8.1.7. Vgl. dazu auch den Topos der „sitzende[n] Lebensart“ im Zusammenhang mit psychischen Krankheiten in psychiatrischen Texten des 19. Jahrhunderts (Schuster 2010: 184ff.).

422 Einsehbar unter: https://books.google.de/books?id=9cUAAAAAYAAJ\&pg=PA260\&dq=ausgebrannt+psychiatr\&hl=de\&sa $=X \& e i=d T 5 q V I e B 04 j 10 M X-g e g E \& r e d i r \_e s c=y \# v=$ onepage\& $\mathrm{q}=$ ausgebrannt\%20psychiatr\&f=false, zuletzt eingesehen am 12.12.2019, siehe im QV unter 8.1.4. 
9) DWDS-Kernkorpus (1900-1999): Dwinger, Edwin Erich: Die letzten Reiter, Jena: Eugen Diederichs 1935, S. 55

Ich bin eben müde, bin eben völlig ausgebrannt ...

10) DWDS-Kernkorpus (1900-1999): Horster, Hans-Ulrich [d.i. Rhein, Eduard]: Ein Herz spielt falsch, Köln: Lingen 1991 [1950], S. 290

Ihm ist, als sei er völlig ausgebrannt, als säße hier nur noch eine leere Menschenhülle in dem tiefen Ledersessel.

11) DWDS-Kernkorpus (1900-1999): Adorno, Theodor W.: Minima Moralia, Frankfurt a. M.: Suhrkamp 1971 [1951], S. 319

Der Inhalt des Schocks wird gegenüber seinem Reizwert real gleichgültig, wie er es in der Beschwörung der Dichter ideell war; möglich sogar, daß das von Poe und Baudelaire ausgekostete Grauen von Diktatoren verwirklicht, seine Sensationsqualität verliert, ausbrennt.

12) DWDS-Kernkorpus (1900-1999): DER SPIEGEL, „Das ist ein dummes Gefühl“, 19.10.1981: 24 (siehe auch im QV unter 8.1.6, = Der SPIEGEL 1981)

Trotz aller Mißerfolge im Verteidigungsressort erscheint Apel den Freidemokraten keineswegs ausgebrannt. ${ }^{423}$

13) DWDS-Kernkorpus (1900-1999): DER SPIEGEL, „Es kann jede Frau treffen, zu jeder Zeit“, 17.11.1986: 109 (siehe auch im QV unter 8.1.6, = S., Sigrun 1986)

Nach den Minuten der Todesangst, Anspannung und Selbstbeherrschung fühle ich mich erschöpft und leer, ausgebrannt vor Haß und Ekel.

In den meisten Beispielen ist der Prozess des Ausbrennens schon abgeschlossen, was durch das Partizip Perfekt in prädikativer oder attributiver Verwendung (war ... ausgebrannt; bin/ist/fühle mich ... ausgebrannt, ausgebranntes Herz, ausgebrannte Phantasie) angezeigt wird. Gängige vom metaphorischen Ausbrennen affizierte Gegenstände sind schon in den Belegen des 18. Jahrhunderts Herz und Seele, in weiteren Belegen aber auch Begierde, Mut, Wut, Phantasie, Jugendfeuer, Gemütszustand usw. Auslösende Faktoren sind teilweise (siehe z. B. Beleg 13) zwar aus dem Kontext erschließbar, aber im Valenzrahmen keine notwendigen Ergänzungen. Die Kontexte vermitteln alle den Eindruck, dass das, was ausbrennt, eine positive Wertigkeit besitzt. Der Vorgang des Ausbrennens wird vor diesem Hintergrund als negative Zustandsveränderung konstituiert. Dies zeigen vor allem auch Beispiele, in denen angedeutet wird, dass man sich Kräften zuwendet, die die erloschenen Qualitäten wieder ,entfachen' könnten (z. B. Glauben (1, 4 und 7), Tugend (1), Orientierung an Vorbildern (6)).

Dass diese Verwendung im Partizip Perfekt und dem Verb sein zur Bezeichnung psychischer Erschöpfung Mitte des 20. Jahrhunderts fest im allgemeinen

423 Ähnlich auch eine Einschätzung im SPIEGEL zu Hans-Dietrich Genschers Entschluss, nicht mehr für den Parteivorsitz der FDP zu kandidieren. In diesem Artikel liest man: „Der Mann sei fertig, ausgebrannt nach zehn Jahren Doppelbelastung als Außenminister und Parteivorsitzender, heißt es im Kanzleramt.“ (Der Spiegel, 04.06.1984: 15, siehe im QV unter 8.1.6). 
Wortschatz verankert war, zeigen auch die Einträge in der ersten Auflage des WAHRIGs aus dem Jahr 1966 („Das große deutsche Wörterbuch“)424 oder des DUDENs aus dem Jahr 1976 („Das große Wörterbuch der deutschen Sprache“) ${ }^{425}$. Beide geben im Wörterbucheintrag zum Verb ausbrennen die Verwendung ,hat seine seelischen Kräfte völlig verbraucht“ (WAHRIG) bzw. „seelisch und körperlich völlig erschöpft“ (DUDEN) an, und der DUDEN ergänzt „«meist im 2. Part. ausgebrannt `“. In letzterem findet sich auch noch folgender Verweis auf den Sprachgebrauch im Sport: „(Sport) physisch nicht mehr in der Lage, seine sportliche Höchstleistung zu erbringen: dieser Läufer ist a.:“ (Unterstreichung T.S.).

Sieht man sich hingegen Belege mit dem transitiven Verb ausbrennen an, so fällt auf, dass die Sachverhalte, die im übertragenen Sinn ausgebrannt werden (sollen), teilweise eine negative Wertigkeit besitzen. Sehr verbreitet in moralischen und religiösen Kontexten ist das Konzept, dass durch das Ausbrennen der Gegenstand eine >Reinigung/Läuterung، erfährt. Hier scheinen verschiedene Praktiken und Vorstellungswelten mit hineinzuspielen: zum einen beispielsweise die schon in ahd. Zeit belegte chirurgische Praxis, eine Wunde auszubrennen ${ }^{426}$ und dadurch zu reinigen, zum anderen die Feuerprobe, mit der man die Reinheit von Gold überprüfte (Röhrich 1991, Bd. 1, S. 441), und die auch in christliche Vorstellungen vom „Feuer der Anfechtung“ als Prüfung der Echtheit des Glaubens Eingang fand (Unterstreichungen T.S.): ${ }^{427}$

1) DTA. Neuer Lust- und Lehrreicher Schau-Platz. Nürnberg, 1685.

Wofern man aber heutiges Tages allen Dieben Nasen/ Ohren/ und Hände abschneiden/ oder ihnen gewisse Merck-Mahle ausbrennen sollte/ so würden ihrer in einem gemeinen Wesen offters mehr als Tugendhaffte Leute gefunden werden.

2) DTA: Spener, Philipp Jakob: Theologische Bedencken. Bd. 2. Halle (Saale), 1701. Ach laß dieses feuer der anfechtung dazu gesegnet werden/ daß dadurch meines glaubens gold rechtschaffen geläutert/ von den schlacken und aller unreinigkeit gesäubert/ und insgesamt alles/ was von liebe dieser welt bey mir übrig seyn möchte/ ausgebrandt werde.

3) DTA: Lutz, Samuel: Ein Wohlriechender Straus Von schönen und gesunden Himmels-Blumen. Basel, 1736.

Wie will GOtt diesen köstlichen Balsam in sie giessen, wann das Geschirr nicht von erst wohl geschwenckt ist durch das Thränen-Wasser, der Unrath aus Leib und Seel ausge-

424 Wahrig (1966: Spalte 499).

425 DUDEN (1976: 241).

426 Vgl. den Eintrag zu „brennīsen“ im Wörterbuch (= Band 2) von „Die Frühgeschichte der mittelalterlichen medizinischen Fachsprache im Deutschen“ von Riecke (2004, Band 2, S. 545).

427 Röhrich (1991: 441) schreibt hierzu: „Den Anlaß zur Bildung gibt die Stelle in den Sprüchen Salomonis 17,3, die im Luthertext lautet: ,Wie das Feuer Silber und der Ofen Gold, also prüft der Herr die Herzen'; Röhrich gibt als ähnliche Stellen z. B. noch Sacharja 13,9 und 1. Petrus 1, 7 an. 
brannt durch den Feuer-Eifer der Heiligkeit GOttes gegen der Sünd; wie will man ihm aber thun, wann GOtt nicht will und seine Flamme das Hertz nicht durchwandlet. ${ }^{428}$

Das Konzept der >Reinigung` oder sogar >Vernichtung` wird über das Verb ausbrennen in weiteren Kontexten von Politik, Geschichte, Ästhetik bis hin zu radikalen, menschenverachtenden Ideologien aufgerufen. Beispiele aus dem nationalsozialistischen „Völkischen Beobachter“, der als Quelle im DWDS-Korpus (1900-1999) durchsuchbar ist, zeigen, dass ausbrennen in dieser Verwendungsweise gängiger Bestandteil nationalsozialistischer Vernichtungs-Metaphorik war (Unterstreichungen T.S.):

DWDS-Kernkorpus (1900-1999): Völkischer Beobachter (Ausgabe A / Norddeutsche Ausgabe), 15.06.1934

Ich habe den Befehl gegeben, die Hauptschuldigen an diesem Verrat zu erschießen, und ich gab weiter den Befehl, die Geschwüre unserer inneren Brunnenvergiftung und der Vergif-

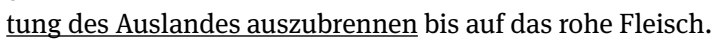

DWDS-Kernkorpus (1900-1999): Völkischer Beobachter (Berliner Ausgabe), 02.03.1935

Er hat strikten Befehl, die Ruhe und Ordnung in Tirol wiederherzustellen und mit drakonischen Mitteln jeden weiteren Widerstand auszubrennen.

Diese übertragene Redeweise vom auszubrennenden Geschwür bezogen auf gegnerische Weltanschauungen bzw. als negativ erkannte Sachverhalte findet man auch in folgender autobiografischer Notiz von 1914 (Unterstreichungen T.S.):

DWDS-Kernkorpus (1900-1999): Blos, Wilhelm: Denkwürdigkeiten eines Sozialdemokraten, Bd. 1. In: Simons, Oliver (Hg.) Deutsche Autobiographien 1690-1930, Berlin: Directmedia Publ. 2004 [1914], S. 9720

Als ich dort ankam, sah ich gleich, daß die Aussaat des Hasses aus der »Kölnischen Zeitung«, die Sozialdemokratie, diese »Eiterbeule am deutschen Volkskörper«, müsse ausgebrannt werden, auf fruchtbaren Boden gefallen war.

428 Weitere Beispiele bei Dannhauer, Johann Conrad: Catechismus-Milch. Bd. 9. Straßburg, 1672, S. 279; Arnold, Gottfried: Unpartheyische Kirchen- und Ketzer-Historie. Bd. 2 (T. 3/4). Frankfurt (Main), 1700, S. 291. Online einsehbar unter: https://dwds.de/r?q=ausbrennen\&corpus=dta\&date-start=1473\&date-end=1927\&genre=Belletristik\&genre=Wissenschaft\&genre=Gebrauchsliteratur\&genre=Zeitung\&format=full\&sort=date_asc\&limit=100 (zuletzt eingesehen am 04.12.2018). 
Man findet ähnliche Formulierungen eines ,läuternden' Ausbrennens aber auch in ästhetischen und weiteren lebensweltlichen Zusammenhängen (Unterstreichungen T.S.):

\section{DWDS-Kernkorpus (1900-1999): Vossische Zeitung (Sonntags-Ausgabe), 06.03.1921}

Den Epilog macht der Künstler, der seinen Untergang ekstatisch genießt, der sein Zeitliches im wilden Leben ausbrennt, um die innere Form zu verewigen. ${ }^{429}$

DWDS-Kernkorpus (1900-1999): Tucholsky, Kurt: Sechzig Fotografien. In: Kurt Tucholsky, Werke - Briefe - Materialien, Berlin: Directmedia Publ. 2000 [1924]

Aber es ist Pflicht jedes einzelnen, diesen Wahnsinn bei sich auszubrennen, im eignen Hause und nicht bei den andern.

Die transitive Verbform wird neben den gerade beschriebenen Beispielen aber auch in Zusammenhängen verwendet, in denen positive Kräfte ausgebrannt und damit 'zu einem Ende gebracht werden'. Hierbei ist besonders interessant, welche Sachverhalte die semantisch aktive Rolle in den folgenden Belegen aus Belletristik und philosophischer Literatur einnehmen. Hier findet man neben dem KRIEG, der um die Weltkriegsjahre in einigen Beispielen erscheint und dort insbesondere auf das ausgebrannte Hab und Gut referiert, nun auch die ARBEIT als möglichen Auslöser des figurativen Ausbrennens (Unterstreichungen T.S.):

DWDS-Kernkorpus (1900-1999): Neutsch, Erik: Spur der Steine, Halle: Mitteldeutscher Verl. 1964 [1964], S. 267

Unsere Familien hat der Krieg ausgebrannt ...

DWDS-Kernkorpus (1900-1999): Bloch, Ernst: Das Prinzip Hoffnung Bd. 2, Berlin: Aufbau-Verl. 1955, S. 493

Weil einen die Arbeit ausgebrannt hat und man weder für die Anmut noch den Frieden des Glücks Zeit und Beispiel hat.

Des Weiteren finden sich Belege, in denen Erinnerungsstücke oder geistesgeschichtliche Bewegungen als auslösende Faktoren erscheinen (Unterstreichungen T.S.):

DWDS-Kernkorpus (1900-1999): Langgässer, Elisabeth: Das unauslöschliche Siegel, Hamburg: Claassen 1959 [1946], S. 570

Dieses Bildchen, ganz winzig, aber unendlich scharf, schien sich wie mit photographischen Säuren seiner Erinnerung eingeätzt und ihn, ohne daß er es wußte, beständig begleitet zu haben; es hatte den Hintergrund seines Daseins ganz und gar ausgebrannt, ihn zerstört und

429 Weiterer Beleg siehe DWDS-Kernkorpus (1900-1999): Rubiner, Ludwig: Die Anonymen. In: Deutsche Literatur von Lessing bis Kafka, Berlin: Directmedia Publ. 2000 [1912], S. 143/351. 
war wie ein flüssiges Feuer den Rändern entlanggelaufen ...

\section{DWDS-Kernkorpus (1900-1999): DER SPIEGEL, 10.03.1980}

Heute ist dieser Typ verschwunden; der Bolschewismus hat ihn ausgebrannt.

Interessant ist schließlich folgender Beleg von 1911, der die Beziehung zwischen zwei Menschen als ausgebrannt bezeichnet und mit einem elektrischen „Kurzschluss“ vergleicht. Die Elektrizitätslehre hat nach Schuster seit ,ihrer Konstitution als Disziplin [...] großen Einfluss auf andere Disziplinen, so auf die Theologie und Philosophie" und auf die sich etablierende Psychiatrie im 19. Jahrhundert: „Energie gehört damit zu den Initialmetaphern, wobei die Elektrizität (auch Energieerhaltungs- und Entropiesatz werden verwendet) die damit verbundenen metaphorischen Modelle vorgibt“ (Schuster 2010: 203). Des Weiteren lässt sich schon für das 19. Jahrhundert nach Schuster nachweisen, „dass Kraft und Energie austauschbar gebraucht werden“ (ebd.). Dieser Vergleich steht in Verbindung $\mathrm{zu}$ Belegen des jüngeren Burnout-Diskurses der letzten Jahrzehnte, in denen >Burnout`häufig mit einer leeren Batterie verglichen wird, wobei das folgende Beispiel durch den „Kurzschluss“ ein 'plötzliches’ Ausbrennen inszeniert. ${ }^{430}$

\section{DWDS-Kernkorpus (1900-1999): Meisel-Hess, Grete: Die Intellektuellen. In: Deutsche Literatur von Frauen, Berlin: Directmedia Publ. 2001 [1911], S. 48560}

Evas Ehe bestand in äußerer Ordnung, aber Olga merkte bald, daß es hier so war, wie bei einer elektrischen Anlage, in der der Strom fehlt, - tot, ausgebrannt, durch irgendeinen schlimmen $\underline{\text { Kurzschluß }}$ vernichtet, - ein komplizierter Apparat ohne die treibende Kraft, um deretwillen er errichtet wurde.

Die reflexive Form „sich ausbrennen“ schließlich wird in den untersuchten deutschen Wörterbüchern (siehe Kap. 5.1.1 zum Wörterbuchkorpus) nicht aufgeführt und auch in den Belegen des DTA und DWDS finden sich kaum Beispiele. Englische Wörterbücher (z. B. in den aktuellen Online-Ausgaben des OED und Cambridge Dictionarys ${ }^{431}$ sowie des Oxford Dictionary of new words von $1991^{432}$ )

430 Obgleich man anmerken muss, dass im Beispiel von 1911 von einem „Kurzschluss“ gesprochen wird, was eher einem plötzlichen Durchbrennen als einer langsamen Entleerung einer Batterie entspricht. Doch auch das Konzept des Ausbrennen als >plötzliches Ereignis` bzw. Durchbrennen findet sich im jüngeren Burnout-Diskurs wieder: „Ihr altes Leben zerbrach, als sie in der Mittagspause eine Glühbirne kaufen wollte“ (SPIEGEL 4/2011: Das Volk der Erschöpften = Dettmer/Shafy/Tietz 2011: 115, im QV unter 8.1.6).

$431 \mathrm{https}$ //dictionary.cambridge.org/dictionary/english/burn-yourself-out (zuletzt eingesehen am 12.12.2019, siehe im QV unter 8.1.1).

432 Die Verwendung als Substantiv burn-out wird in diesem Neologismenwörterbuch verbucht und zwar als Substantiv, das auf die reflexive Verbphrase zurückgehe: "A noun formed on the 
belegen hingegen eine reflexive Verwendung, wie auch das Beispiel von Shakespeare zu Beginn dieses Kapitels zeigt. Dennoch wird der Prozess des Ausbrennens auch in deutschsprachigen Beispielen auf die Person oder Sache, die im übertragenen Sinn ausbrennt, durch sinnverwandte Verben im Kotext oder das Pronomen selbst rückbezogen (Unterstreichungen T.S.):

\section{DTA: Klingemann, Ernst August Friedrich: Nachtwachen. Penig, 1805.}

In solchen und dergleichen Fragmenten habe ich mich abgearbeitet, und mich ordentlich methodisch auszuschreiben gesucht, wie mancher Dichter, der seine Gefühle so lange auf dem Papiere von sich giebt, bis sie zuletzt alle abgegangen sind, und der Kerl selbst ganz ausgebrannt und nüchtern dasteht.

DWDS-Kernkorpus (1900-1999): Tucholsky, Kurt: Sechzig Fotografien. In: Kurt Tucholsky, Werke - Briefe - Materialien, Berlin: Directmedia Publ. 2000 [1924]

Aber es ist Pflicht jedes einzelnen, diesen Wahnsinn bei sich auszubrennen, im eignen Hause und nicht bei den andern.

DWDS-Kernkorpus (1900-1999): Horkheimer, Max u. Adorno, Theodor W.: Dialektik der Aufklärung, Amsterdam: Querido 1947 [1944], S. 14

Rücksichtslos gegen sich selbst hat die Aufklärung noch den letzten Rest ihres eigenen Selbstbewußtseins ausgebrannt.

Sucht man im Deutschen Referenzkorpus des Leibniz-Instituts für Deutsche Sprache im Archiv W im Korpus der ZEIT (1953-2018), so findet man auch einige wenige Belege für die reflexive Verbverwendung sich ausbrennen (Unterstreichungen T.S.). ${ }^{433}$

\section{DIE ZEIT, 24.11.1961, S. 49; Der Totengräber des Amateursports (= Natan 1961)}

Gordon Pirie war ein großer Läufer, der sich zehn Jahre lang „ausgebrannt“ hatte. Wie alle Sportsleute, die Schlagzeilen in der Presse gemacht haben, nahm er sich selbst zu ernst und

verbal phrase burn oneself out, meaning 'to use up all one's physical or emotional resources'; the noun burn-out already existed in the more literal sense of the complete destruction of something by fire, as well as in two technical senses" (Tulloch 1991: 47, siehe im QV unter 8.1.1).

433 Gesucht wurde im ZEIT-Korpus mit folgender Suchsyntax: sich /sO \&ausbrennen, das heißt sich und verschiedene Flexionsformen des Verbs ausbrennen sollen in einem Satz zusammen vorkommen. Die Suche ergab 89 Treffer, abzüglich doppelter Belege haben 56 Belege einen semantischen Bezug zu psychischem Erleben. Doch nur drei Belege (siehe oben) entsprechen wirklich der reflexiven Verbverwendung sich ausbrennen. Die anderen Belege verwenden das Verb ausbrennen in einem Satz mit einem weiteren reflexiven Verbgefüge wie z. B. die meisten halten sich für ausgebrannt, fühlen sich ausgebrannt oder sich wird zwar innerhalb des Satzes, aber in einem anderen Nebensatz verwendet, z. B. putscht sich auf [...], findet sich aber nur zu oft leer, ausgebrannt (Recherche-Stand: 7.12.2018). 
den Sport zu wichtig und begriff nicht, daß es heute viel wichtigere Dinge gibt als einen Meilenlauf.

DIE ZEIT, 3.6.1966, S. 23; Die Anatomie eines Mordes (= Federspiel 1966)

Capotes langes Schweigen nach „Breakfast at Tiffany’s“ (er schrieb Reportagen, Interviews und Filmdrehbücher) überbrückt passiv-schöpferisch jene Krise, die er bei Kollegen beobachtet hatte: „Die Sache ist die, daß die ungefähr gleichaltrigen amerikanischen Kollegen sich mit dem ausbrennen, mit dem sie einst begonnen haben. Sie haben keine zweite Chance. Aber ich gab mir selber eine zweite Chance. [...].“

Die Zeit, 17.12.1993, S. 60; Na? Warum lacht ihr wieder? (= Fitzel 1993)

Als entschiedener Antimilitarist schrieb er eine begeisternd-satirische Flugschrift über den kommenden Luftmilitarismus in dem Glauben, daß man den durch die Vermehrung seiner Schrecken abschaffen könnte. Mit Leidenschaft kümmerte er sich um sein letztes Projekt, die Erfindung des Perpetuum mobiles, das „perpeh“, wie er es zärtlich nannte. Wenn man den Briefen an Ernst Rowohlt glaubt (in dessen Verlag 1910 darüber ein schmales Büchlein erschien), betrieb er diese Verrücktheit, diese technische Unmöglichkeit wie immer mit aller Ernsthaftigkeit und Überzeugung. Man erkennt die Idee, die sich dahinter verbirgt; ein Objekt, eine Maschine zu schaffen, die vollkommen, rein und in sich geschlossen ist - die sich niemals verausgabt. Und gleichzeitig erhält man den Eindruck, daß Scheerbart sich mehr und mehr verausgabt - ausbrennt. Seine Briefe werden kürzer, hastiger, immer neue Projekte werden in Angriff genommen.

Bei der Durchsicht dieser Belege in der ZEIT fällt des Weiteren auf, dass die Mehrworteinheit sich ausgebrannt fühlen in dieser Zeitung zwar seit den 1980er Jahren verwendet wird, aber seit den 1990ern ansteigt. ${ }^{434}$ In den Belegen der DTA und DWDS-Korpus findet man nur einen solchen Beleg. Bemerkenswerter als die Anzahl dieser Belege ist dabei, dass diese Mehrworteinheit überwiegend in Berufskontexten benutzt wird, die auch in Texten zum Burnout-Syndrom aufgerufen werden: kreative (Belege 1 und 2), medizinisch-soziale (Belege 3 und 4), pädagogische (Belege 5 und 6) gesellschaftlich-politische (7) und generell Berufe, in denen es um Hochleistung und/oder ein Leben in der Öffentlichkeit geht, wie beispielsweise im Sport bei Fußballtrainern (8) oder der Musik-/Schauspielbranche (9) (zur weiteren Auswertung typischer ,Betroffenen-Gruppen‘ siehe auch Kap. 6.2.4.1). Es gibt zuletzt auch

434 Die Belege aus dem definierten ZEIT-Korpus in DeREKo zeigen folgende Verwendungshäufigkeit der Mehrworteinheit sich ausgebrannt fühlen nach Jahrzehnt in absoluten Häufigkeiten (diese wurde im Korpus mit folgender Suchsyntax sich /sO \&ausbrennen /sO \&fühlen recherchiert: 1980er: 4 Treffer (eine Dublette), 1990er: 3 Treffer, 2000er: 11 Treffer, 2010-2018: 13 (eine Dublette) Treffer und in relativen Häufigkeiten (normiert an der jeweiligen Korpusgröße je Vergleichszeitraum) in den 1980er Jahren 0.0728 pMW, in den 1990er Jahren 0.0608 pMW, in den 2000er Jahren 0.1660 pMW und von 2010-2018 0.2059 pMW (Recherche-Stand 07.12.2018). Zur Methode der Berechnung der relativen Häufigkeiten vgl. Perkuhn/Keibel/Kupietz (2012: 79). 
Belege, die das Gefühl des Ausgebranntseins als gesamtgesellschaftliches Phänomen beschreiben $(10,11)$ (Unterstreichungen T.S., alle Belege im QV unter 8.1.6): ${ }^{435}$

1) DIE ZEIT, 4.7.1980, Graham Greenes Fluchtwege (= Leonhardt 1980)

Er glaubte nicht, daß er danach noch einen Roman schreiben werde. Dies war wohl der letzte. Er hieß „Ein ausgebrannter Fall“ („A Burnt-Out Case“, 1961), und ausgebrannt war eben nicht nur der Leprakranke im dereinst belgischen Kongo, sondern auch sein Autor fühlte sich so, am Ende.

2) DIE ZEIT, 10.4.1987, Mann in der Revolte (= Rossum 1987)

Noch nicht dreißig Jahre alt, veröffentlicht er 1942 seinen Roman „Der Fremde“. Einige Monate später folgt sein philosophischer Essay über den „Mythos vom Sisyphos“, in dem er seine Theorie des Absurden formuliert. Fünf Jahre danach erscheint der unter großen Mühen geschriebene Roman „Die Pest“. Und nachdem 1951 sein umfangreicherer Essay „Der Mensch in der Revolte“ vollendet ist, fühlt sich Camus literarisch völlig ausgebrannt. ${ }^{436}$

3) DIE ZEIT (Online-Ausgabe), 27.6.2002; Raus aus dem Altersgefängnis! (= Tenbrock/ Uchatius 2002)

Natürlich nicht alle. Natürlich schmerzen manchem Maurer mit 40 die Knochen, fühlt sich mancher Krankenpfleger mit 50 ausgebrannt, ist ein Müllwerker froh, wenn er keine Tonnen mehr heben muss. Aber mit jeder Maschine, die noch einen stupiden Arbeitsschritt ersetzt, werden sie weniger. In den USA macht körperlich schwere Arbeit nur noch fünf bis zehn Prozent aller Jobs aus.

4) DIE ZEIT (Online-Ausgabe), 1.2.2007, Guter Arzt, kranker Arzt (= Viciano 2007)

Seine persönlichen Grenzen überschritt er, als er im Laufe seiner Facharztausbildung auf die Intensivstation kam. Dort musste er mal früh, mal spät, mal nachts arbeiten. Hatte er Spätdienst, verbrachte er den Vormittag im Labor. Zwei Mitarbeiter hatten seine Forschergruppe verlassen, und die Ergebnisse ließen auf sich warten. »Da wurde der Druck immer größer«, sagt der junge Arzt. Er habe nicht mehr schlafen, nicht mehr strukturiert denken, nicht mehr Sachen nacheinander erledigen können. Und er fühlte sich körperlich und psychisch ausgebrannt.

435 Belege aus dem SPIEGEL-Korpus zur Suchsyntax sich /sO \&ausbrennen /sO \&fühlen im Deutschen Referenzkorpus (DEREKo) zeigen ebenfalls einen leichten Anstieg dieser Mehrworteinheit über die Jahrzehnte sowohl in absoluten als auch in relativen Häufigkeiten (in DEREKo wurden die Jahrgänge 1947 bis 2018 durchsucht): 1980er Jahre: 3 Treffer (plus ein Treffer in anderer Verwendung) 0.0911 pMW; 1990er Jahre: 5 Treffer (plus ein Treffer in anderer Verwendung) 0.1201 pMW; 2000er Jahre: 20 Treffer (plus 2 Dubletten) 0.1270 pMW; 2010-2018: 31 Treffer (plus eine Dublette), in diesem Zeitraum fallen aber 7 Treffer auf verschiedene Besprechungen des Films „Fühlen Sie sich manchmal ausgebrannt und leer?“, 0.1660 pMW. Bemerkenswert ist allerdings auch in den SPIEGEL-Treffern, dass diese Mehrworteinheit wieder insbesondere im Kontext von Pflege- und pädagogischen Berufen erscheint, als Zeitphänomen u. a. der New Economy bzw. IT-Wirtschaft beschrieben wird und auch Beispiele aus dem Sport oder kreativen Medien- und Kunstbereich enthält. 436 Weitere Belege: DIE ZEIT, 29.9.1989, „Der alte Mann, die Frauen und der Gott“ (= Leonhardt 1989: 76) ebenfalls über Graham Greene; Die ZEIT, 7.1.2016, „Alma spielt“ über Erschöpfung bei Musikwunderkindern (= Heuser 2016: 13). 
5) DIE ZEIT (Online-Ausgabe), 01.5.1992, Lehrer: Der Ruin eines Berufs (= Etzold 1992) Kollektive Erschöpfung als Folge andauernder Überforderung angesichts der Widersprüche, mit denen sie fertig werden müssen - ein derart kompliziertes Krankheitsbild kann in der Öffentlichkeit kaum auf Verständnis stoßen. Daß Lehrer sich ausgelaugt und ausgebrannt fühlen, daß sie öfter krank feiern, häufiger „kuren“ und sich früher pensionieren lassen, ja daß bei Lehrerinnen angeblich gar die Schwangerschaften länger dauern als bei anderen Frauen, liefert nur - im Verein mit den vielen Ferien und der vermeintlich kürzeren Arbeitszeit - weitere Indizien für die Unterstellungen von Wehleidigkeit.

6) DIE ZEIT (Online-Ausgabe), 10.4.2001; Pädagogisch spät berufen (= Schmidt 2001) Nicht nur die schlechte Bezahlung dürfte manchem Lehrerkandidaten wehtun, der Beruf hat noch andere Kehrseiten: Das öffentliche Ansehen von Lehrern ist mies, sie werden häufig krank, und jeder Dritte in diesem Job fühlt sich überfordert oder ausgebrannt. $^{437}$

7) DIE ZEIT (Online-Ausgabe), 13.7.2006; Löws Aufstieg (= Dehne 2006)

Jürgen Klinsmann indessen sagte, er werde längere Zeit benötigen, um sich zu erholen. Auch das sei ein Grund gewesen, das Bundestraineramt nicht fortzuführen. Er fühle sich ausgebrannt und habe keine Energie mehr, um die Arbeit in dieser Intensität fortzusetzen. Im nächsten halben Jahr würde er Urlaub machen und kein Angebot aus einem sportlichen Bereich annehmen. ${ }^{438}$

8) DIE ZEIT (Online-Ausgabe), 9.6.2005; »Es ist gefährlich aufzuwachen« (= Lebert/ Mohaupt 2005)

Schneider: Ich kann Ihnen erzählen, warum ich Schauspielerin geworden bin. Es war Mitte der achtziger Jahre: Ich war ausgebrannt von der Zeit in Las Vegas, und auch das Musikmachen mit meiner Band fühlte sich nicht mehr so gut an. [...].

9) DIE ZEIT, 26.3.1993, S. 13; Die graue Revolution (= Borgeest/Perina 1993)

Auch die Westdeutschen gehen, mehr oder weniger freiwillig, immer früher in Rente derzeit mit durchschnittlich 59,4 Jahren die Arbeiter, mit 61,4 Jahren die Angestellten. Viele sind gesundheitlich ruiniert, fühlen sich verschlissen, ausgebrannt, für diese Art von Berufsleben nicht mehr geschaffen.

437 Weitere Belege zu sich ausgebrannt fühlen im Kontext pädagogischer Berufe: DIE ZEIT (Online-Ausgabe), 18.10.2007: Von wegen freie Nachmittage (= Hasse 2007); DIE ZEIT (OnlineAusgabe), 6.3. 2008, Lehrer: Der Beruf im Überblick (= Ebitsch 2008: 76); DIE ZEIT, 9.7.2015, Nachmittags kommt Tante Sonja (= Fokken 2015: 59); Weitere Belege zur Thematisierung von $>$ Burnout im Kontext pädagogischer Berufe: DIE ZEIT 23/1996 (= Gsteiger 1996); DIE ZEIT Nr. 51/1998 (= Etzold 1998); DIE ZEIT 25/1999 und 49/1999 (= Etzold 1999a/b); DIE ZEIT 45/2011 (= Spiewak 2011); in DIE ZEIT 12/1996 (= Kanders) wird eine Umfrage zitiert, dass sich die Belastung bei der Mehrheit der Lehrer/innen in Grenzen halte.

438 Weiterer Belege für sich ausgebrannt fühlen bei Fußballtrainern: DIE ZEIT (Online-Ausgabe), 28.2.2008, Ausgebrannte Helden (= Willmann 2008: 39). In diesem Artikel der ZEIT geht es auch um „Fußballtrainer“; DIE ZEIT (Online-Ausgabe), 25.7.2013: „Ich mag diese totale Zuspitzung“ (= Gilbert/Kammertöns 2013). 
10) DIE ZEIT (Online-Ausgabe), 6.1.2011; Im Namen des Herrn (= Berbner 2011) Glaubt man Manfred Dahm, war die Zeit für diesen Beratungsansatz überfällig: "Viele Leute fühlen sich ausgebrannt und leer. Daran ändert auch das dritte Handy und der vierte Urlaub nichts.« Die Wirtschaftskrise war für ihn nur ein Symptom für das mangelnde Miteinander im westlichen Wirtschaftssystem, allerdings eines, das die Notwendigkeit neuer Umgangsformen im Arbeitsalltag offenlege. [...]. ${ }^{439}$

Aufgrund der gesichteten und beschriebenen Belege scheint es, als ob die Redeweise sowohl vom reflexiven Handeln (sich ausbrennen; etwas bei sich selbst ausbrennen) als auch der direkte Rückbezug auf die Person (ich bin/er bzw. sie ist ausgebrannt/sich ausgebrannt fühlen) ohne Zwischenentitäten (wie Seele oder Herz, die gewissermaßen pars pro toto ausbrennen) Mitte/Ende des 20. Jahrhunderts verstärkt in Gebrauch kommt. Auch Formulierungen mit dem Substantiv Burnout/Burnout-Syndrom beziehen sich auf die ganze Person und nicht nur auf einen Teil (sie leidet an Burnout, er hat ein Burnout-Syndrom, * seine Seele hat ein Burnout-Syndrom). Um diesen Eindruck zu erhärten, müsste ein noch weitaus umfangreicheres historisches Korpus aufgebaut werden. Das vorliegende Kapitel kann vor dem Hintergrund der Kernfragestellungen dieser Arbeit jedoch nur einen Überblick über den Sprachgebrauch vor dem Beginn des ,eigentlichen Burnout-Diskurses geben. Dennoch kann man an diese beschriebene Tendenz anknüpfen, denn die Auffächerung der Gefühlslage und die Verantwortung der Person für den eigenen Prozess des Ausbrennens sind dann auch konstante Subthemen im Burnout-Diskurs. Des Weiteren würde dies auch zu dem „Trend“ passen, den Herberg/Kinne/Steffens et al. (2004) für einige Neologismen der 1990er Jahre festgestellt haben: Mit neuen Wörtern wie Wellness oder Work-Life-Balance würde der „eigenen Befindlichkeit erhöhte Aufmerksamkeit“ geschenkt werden. ${ }^{440}$

Überaus deutlich zeigt die untersuchte Beleglage des Weiteren, dass die substantivierten Formen das Ausbrennen und Ausgebranntsein erst Ende des 20. Jahrhunderts in Gebrauch kommen. Sie dienen mitunter als Übersetzung für die in den 1980er und 1990er Jahren noch neuen Bezeichnungen Burn(-)out(-Syndrom) (Unterstreichungen T.S.):

439 Weitere Belege für sich ausgebrannt fühlen bezogen auf größere Bevölkerungsgruppen (mit kritischem Unterton): DIE ZEIT (Online-Ausgabe), 8.12.2011: Gefühlte Epidemie (= Pawelzik 2011); DIE ZEIT (Online-Ausgabe), 12.7.2012: Coach oder Couch (= Grefe 2012).

440 Vergleiche den Eintrag unter „Weitere Informationen“/„Sprachreflexives“ in der OnlineVersion des Wörterbuchs „Neuer Wortschatz: Neologismen der 90er Jahre im Deutschen“ unter: http://www.owid.de/artikel/401042 (zuletzt eingesehen am 12.12.2019). 


\section{DIE ZEIT, 13.12.1991, S. 11; Das Gymnasium; Hauptschule der Nation (= Scholz 1991)}

[...] Lehrerkonferenzen, in denen es um pädagogische Diskussionen gehen müßte, werden zu frustrierenden Ritualen, die in den meisten Fällen von den Direktoren „im Stile eines Frontalunterrichts“ geleitet werden, die sie „zur Selbstdarstellung mißbrauchen“, wie die Erziehungswissenschaftler Georg E. Becker und Gernot Gonschorek herausgefunden haben, die über das „Burnout-Syndrom“ (Ausgebranntsein) bei Lehrern geforscht haben. ${ }^{441}$

Der SPIEGEL, 8/2016, Alles ist dunkelgrau, S. 126 (= Schmitter 2016)

Auf einen Schlag bekannt wurde er 2010 mit einem Essay über die „Müdigkeitsgesellschaft“, in dem er das weitverbreitete Gefühl der Überforderung und des Ausgebranntseins mit dem politischen Paradigmenwechsel seit 1989 zusammenbrachte: [...]. ${ }^{442}$

Im Deutschen Referenzkorpus im Archiv $\mathrm{W}^{443}$ zeigt sich folgende Verteilung für das Suchwort Ausgebranntsein*: 1980er: 2 Treffer (0.0181 pMW), 1990er: 87 Treffer (0.0648 pMW), 2000er: 126 Treffer (0.0349 pMW) und 2010-heute: 228 Treffer (0.0511 pMW). ${ }^{444}$ Für die substantivierte Form das Ausbrennen/des Ausbrennens/ dem Ausbrennen ${ }^{445}$ findet man im Archiv W bis in die 1990er Jahre keine Treffer im übertragenen Sinn. In den 1990er Jahren 26 Treffer, in den 2000er Jahren 59 Treffer und 2010-2018 71 Treffer. Vergleicht man diese Zahlen mit Zahlen zum Neologismus Burn(-)out(-Syndrom $)^{446}$ im Archiv W vom DeReKo, so sieht man allerdings, dass jene Bezeichnungen sich im Vergleich zu letzterer weniger stark durchgesetzt haben: 1980er: 16 Treffer; 1990er: 521 Treffer; 2000er: 5.350 Treffer, 2010-heute: 15.736 Treffer (Stand: 26.3.2018).

441 Die hier zitierte Fachpublikation ist Becker/Gonschorek (1991), im QV unter 8.1.5.

442 Weitere Belege siehe zum Beispiel: Der SPIEGEL, 26.12.1988, Nichts als Ruhe, S. 162; Der SPIEGEL, 29.12.1998, Wie ein Samurai, S. 87; Die ZEIT (Online-Ausgabe), 6.10.2011, Ausgebrannt (= Heinrich 2011); siehe alle Belege im QV unter 8.1.6.

443 Gesucht wurde im Archiv „W-öffentlich - alle öffentlichen Korpora des Archivs W“. Dieses umfasste zum Recherche-Zeitpunkt 80.499.090 Wörter (Stand: 25.03.2018). Einsehbar im eingeloggten Zustand unter: https://cosmas2.ids-mannheim.de/cosmas2-web/faces/investigation/ queryString.xhtml, (Stand: 25.3.2018). Das Archiv setzt sich zusammen aus verschiedenen Tagesund Wochenzeitungen, Magazinen, Wikipediaseiten usw.

444 Letzter Recherche-Stand für die Trefferzahlen und relativen Häufigkeiten 12.12.2019.

445 Gesucht wurde nach das / +w2 Ausbrennen*, des / +w2 Ausbrennens ${ }^{*}$, dem / +w2 Ausbren$n e n^{*}$, also mit dem Artikel und im Abstand von maximal zwei Wörtern Ausbrennen mit Beachtung des Großbuchstabens am Anfang (Letzter Recherche-Stand: 30.9.2018).

446 Als Suchausdruck wurde Burn +out* eingegeben mit Beachtung der Großschreibung am Anfang. So sind die verschiedenen Schreibweisen Burnout und Burn-out und verschiedene Kompositabildungen, bei denen Burnout/Burn-out Erstglied ist, abgedeckt. Wenn man auch Kleinschreibung und Komposita, bei denen Burnout das Zweitglied bildet zulässt, ergeben sich weitere Treffer (Letzter Recherche-Stand: 30.9.2018). 
Unabhängig von den Gebrauchshäufigkeiten fällt auf, dass die substantivierten Formen alle etwa zur gleichen Zeit im Sprachgebrauch aufkommen und dass auch im englischsprachigen Raum das Nomen als neuwertig in diesem Zusammenhang empfunden wurde, wie die Neologismenwörterbücher belegen. ${ }^{447}$ Betrachtet man mit Köller (2004) diesen Befund aus pragmatisch-instruktionssemantischer Sicht, so liegt die Vermutung nahe, dass dieser metaphorische Erschöpfungsprozess bzw. -zustand mit den substantivierten Formen als abgrenzbare Entität objektiviert werden sollte. Es gab wohl das Bedürfnis, dieses psychische subjektive Gefühl als etwas zu versprachlichen, das ,eigenständig existiert und was sich im physischen und geistigen Raum als eigenständige Größe auch isolieren“ (Köller 2004: 349) und von anderen vergleichbaren Entitäten bzw. negativ konnotierten Diagnosen wie z. B. der Depression oder älteren Termini wie der Neurasthenie differenzieren lässt. Das Oxford Dictionary of New Words vermutet nämlich, dass die Bezeichnung Burnout ein Ersatzausdruck für Depression oder Nervenzusammenbruch sei: Burnout habe „the more old fashioned terms depression (imprecise except as a clinical term) and nervous breakdown (for cases of complete burn-out)“ abgelöst (Tulloch 1992: 47). Auch in folgendem Beleg im SPIEGEL (1) wird Burnout dem Ausdruck Depression gegenübergestellt, und zwar als `Selbstzuschreibung` gegenüber einer weniger beliebten `Fremdzuschreibung , die man durch die substantivische Form nunmehr haben kann (im Vergleich zu ich bin ausgebrannt) und dadurch auch behandeln und überstehen kann, wie Beleg 2 verdeutlicht (Unterstreichungen T.S.):

\section{(1) DER SPIEGEL, 06.02.2012: 122; Schwermut ohne Scham}

Solche Sätze haben sich im deutschsprachigen Raum zur Auffassung verdichtet, Burnout sei eine eigene Krankheit, und zwar der Leistungsträger; jener, die sich für ihre Firma aufopfern. Der Münchner Psychiater Werner Kissling hat Hunderte Manager und Führungskräfte gesprochen, die sich erschöpft fühlen. „Dass er Depressionen habe, sagt keiner von ihnen“, so Kissling. „Aber Burnout haben sie gern. Das tragen viele von ihnen wie ein stolzes Abzeichen vor sich her.“

(2) Süddeutsche Zeitung, 02.04.2012: 23; Schunkeln zum Abschied

[...] Nach SZ-Informationen hat es Anbahnungsgespräche mit Ralf Rangnick gegeben, der nach überstandenem Burnout-Syndrom ab Juli wieder arbeiten wird. Rangnick, 53, könnte das sein, was Leverkusen bisher vergebens suchte, ein sogenannter Konzepttrainer mit Autorität. Beide Seiten, so ist zu hören, können sich eine Zusammenarbeit vorstellen.

447 Vgl. Green 1991: 40; Tulloch 1991: 47; (Barnhart/Steinmetz 1990: 71). 
Die Substantivierung bietet zusätzlich die Möglichkeit für komprimierende Ausdrucksweisen (vgl. von Polenz ${ }^{3} 2008$ : 24ff.). Über Komposita wie Burnout-Syndrom, Burnout-Falle, Burnout-Beauftragte(r), Burnout-Prävention wird eine Menge an Inhalten komprimiert transportiert, die das Phänomen in Bereichen wie der Arbeitsmedizin oder betrieblichen Gesundheitsvorsorge und deren Praktiken verorten.

Ein Indiz dafür, dass sich die psychische Lesart des Substantivs Burnout mit der Zeit im allgemeinen Wortschatz etabliert hat, sind schließlich Belege, in denen die psychische Lesart der Metapher >Burnout « auf weitere Zielbereiche übertragen wird: z. B. in den Titeln „WWF warnt: „Erde steht vor Burn-Out“ (dpa 2016 auf FAZ.NET) oder „Nach der Bürgerschaftswahl. Burnout in Bremen“ (Altenbockum 2015, FAZ, im QV unter 8.1.6). ${ }^{448}$

\subsubsection{Zusammenfassung}

Auf der Grundlage der dargelegten metaphorischen Verwendungsformen ausgehend von den Verben ausbrennen/to burn out kann man vermuten, dass das entlehnte Wort Burnout/Burn-out/Burnout-Syndrom deshalb eine recht schnelle Verbreitung im deutschen Sprachgebrauch nehmen konnte, da die dahinter stehende Metaphorik einerseits nicht neu war (siehe Kap. 6.1.2), durch weitere Konzepte aus dem Bereich der noch jüngeren Arbeits- und Organisationspsychologie und durch politische Bemühungen zum Schutz der psychischen Gesundheit möglicherweise gestützt wurde (vgl. 5.2) und darüber hinaus der entlehnte Ausdruck dennoch Möglichkeiten für neue Konnotationen gegenüber bestehenden Begriffen wie der >Neurasthenie oder >Depression $`$ eröffnete (vgl. Kap. 6.1.1).

Des Weiteren zeigen sich bei der Entlehnung von Burnout einige sprachgeschichtliche Tendenzen der „deutschen Gegenwartssprache“ (Riecke 2016: 245f.) bzw. des „jüngsten Neuhochdeutschen“ (Bär 2000: 10), die in Kap. 5.3.1. schon angesprochen wurden: In der zweiten Hälfte des 20. Jahrhunderts kommt es zu einer „Verbreitung englischer Wörter“ in den Fachsprachen durch „,die Vormachtstellung der USA in den Bereichen Wissenschaft und Technik“ (Riecke 2014: 88), die sich insbesondere auch im Fach Psychologie bemerkbar macht, wie in Kap. 5.2 und 5.3.1 beschrieben wurde. Zudem erhalten Ausdrücke aus der Fachsprache generell zunehmend größeren Einfluss auf die Alltagssprache, diese wirkt aber auch in die Fächer zurück. Burnout ist ein Paradebeispiel für einen solchen wechselseitigen Transfer: Eigentlich war das Wort zuvor in der angloamerikanischen Alltagssprache gebräuchlich, dann ver-

448 Ähnliche Belege: FAZ (2007b); Meiritz (2011, SPIEGEL ONLINE); Boecker (1993, DIE ZEIT), alle Belege im QV unter 8.1.6. 
wendeten Fachleute wie Freudenberger den Ausdruck zur Beschreibung der Folgen ehrenamtlicher Arbeit in den sogenannten „free clinics“ in den USA und schrieben darüber Fachartikeln, und von dort wirkte er auf den alltagssprachlichen Diskurs zurück. ${ }^{449}$ Im deutschen Sprachraum ist die Vorstellung des metaphorischen Ausbrennens, wie dargelegt wurde, ebenfalls schon lange Zeit im alltäglichen Sprachgebrauch angelegt. Entlehnt wird dann schließlich mit Burn(-)out/Burnout-Syndrom ein im Bereich der psychotherapeutisch-klinischen Praxis und Arbeits- und Organisationspsychologie verwendetes Konzept, ${ }^{450}$ das sich jedoch ebenfalls schnell im alltäglichen deutschen Sprachgebrauch weiterverbreitet. Schließlich zeigt sich bei der Entlehnung des Wortes Burnout auch die für das jüngste Neuhochdeutsche postulierte „kommunikative Globalisierung“ (Bär 2000:12), wobei einige aus dem Englischen stammende Wörter zur Beschäftigung mit Konzepten wie >(gesunder) Lebensstik oder ein `(gesundes) Verhältnis zur Arbeit ‘ in den 1990er Jahren entlehnt wurden wie zum Beispiel Work-Life-Balance ${ }^{451}$, Workaholiker ${ }^{452}$, Coaching ${ }^{453}, \mathrm{TCM}^{454}$ oder Bezeichnungen rund um den Bereich (Medical) Wellness ${ }^{455}$ und Fitness.

Wie in Kapitel 6.1.1 dargelegt, thematisiert der Diskurs an vielen verschiedenen Stellen die Einführung, Verwendung und Begriffsgeschichte von `Burnout wodurch er den Beginn der Beschäftigung mit diesem Thema auf Mitte der 1970er Jahre verlegt. Im Diskurs wird zudem die Frage aufgeworfen, was neu an diesem

449 Vgl. dazu die Analyse in Kap. 6.2.2.2 dieser Arbeit, wie Freudenberger (= Freudenberger 1974, im QV unter 8.1.5) über Fragen >BuRNouT` als Thema auffächert und die Praktik Freudenbergers, den Gegenstand BuRnout durch die Angabe der lexikalischen Bedeutung des Verbs to burn out zu bestimmen.

450 Die Autoren der ersten Fachpublikationen im englischsprachigen Raum stammen aus diesen Fachbereichen. Diese Fächer-Zuordnung erhärtet sich dann auch im deutschsprachigen Diskurs, wenn man vergleicht, aus welchen Fachbereichen die Autorinnen und Autoren, die über das Thema „Burnout“ schreiben, stammen oder in welchen Übersichts- und Lehrwerken welcher Unterfächer innerhalb des medizinisch-psychologischen Spektrums der Begriff verwendet wird (vgl. Kap. 6.2.4.1. dieser Arbeit).

451 Vgl. den Wörterbucheintrag in OWID: http://www.owid.de/artikel/401042 (zul. eingesehen am 12.12.2019).

452 Vgl. den Wörterbucheintrag in OWID: http://www.owid.de/artikel/404196 (zul. eingesehen am 12.12.2019).

453 Die Trefferzahlen im Archiv W des Deutschen Referenzkorpus zeigen einen sprunghaften Anstieg der Verwendung des Wortes Coaching in den 1990er Jahren. Bei den deutlich geringeren Treffern in den Jahrzehnten zuvor dominiert zudem die Bedeutung der 'sportlichen Betreuung', wohingegen in den 1990er Jahren das Coaching in Unternehmens- und beruflichen Kontexten aufkommt. 454 Vgl. den Wörterbucheintrag in OWID: http://www.owid.de/artikel/404057 (zul. eingesehen am 12.12.2019).

455 Vgl. den Wörterbucheintrag in OWID: http://www.owid.de/artikel/116622 (zuletzt eingesehen am 12.12.2019). 
Begriff/Phänomen sei und wodurch er sich von sachverwandten Begriffen unterscheide. Es wird damit im Diskurs ein Spezifizierungs- und Abgrenzungsbedürfnis formuliert, das mit dem Wunsch einhergeht, die Begriffsbildung in diesem Bereich $\mathrm{zu}$ vereinheitlichen und zu kontrollieren ${ }^{456}$. Ähnlich wie ein Gesprächsthema dadurch aufkommen kann, dass die am Gespräch beteiligten Personen einen Gesprächsinhalt als „spezifikationsbedürftig“ ansehen ${ }^{457}$, so kann ein im Diskurs verbalisiertes Präzisierungsbedürfnis den Anfangspunkt für Definitionsbemühungen darstellen. Diese diskursiven Bemühungen sollen in den folgenden Kapiteln einer genauen Analyse unterzogen werden.

\subsection{Definieren als zentrale Diskurspraxis im Burnout-Diskurs}

\subsubsection{Beschreibung des Analysevorgehens und die Analyseperspektive der >Unifizierung،}

Im ersten Schritt geht die Analyse je Diskursstrang (fachintern und fachextern) und textuellen Erscheinungsformen (vgl. Kap. 5.3) von Textstellen aus, in denen entweder von den Autoren/Autorinnen explizit definiert wird (z. B. dadurch, dass der Abschnitt mit Definition überschrieben ist) oder die den Ausdruck Burnout auf dargelegte Sachverhalte und Begriffsinhalte bewusst gleichsetzend beziehen, $d . h$. den Begriff und Sachverhalt >BURNOUT« punktuell ${ }^{458}$ im Rahmen eines Abschnitts reflektierend erklären, „bestimmen, festlegen; angeben oder beschreiben, worum es sich [dabei, T.S.] handelt“459. Der kommunikative Zweck einer Definitionshandlung besteht im strengen Sinn und insbesondere in Fachtexten darin, dass der Definierende das in Frage stehende (neue) Zeichen benennt (= Definiendum) und (intendiert) in eine Entsprechungs-, bzw. Äquivalenzbeziehung (= Definitor) zu anderen bereits bekannten Zeichen oder Zeichenketten stellt (= Definiens), wodurch er das Definiendum für sich selbst und etwaige Rezipienten (hinreichend genau, wesentlich bzw. in seiner Typik erfassend und mit dem Anspruch der Adäquatheit

456 Vgl. Köller 2004: 250, der Definitionen im Kontext seiner Ausführungen zur Entstehung von „Gegenstandswissen“ die Funktion des Kontrollierens zuspricht.

457 Vgl. zum Thema-Begriff dieser Arbeit Kap. 2.3. Dieses Verständnis von `Thema lehnt sich an Konerding (2007: 110) an, welcher sich wiederum auf Ochs-Keenan/Schieffelin (1976) beruft. 458 Das Adjektivpaar punktuell - flächig, das ich im Laufe der nächsten Kapitel zur Analyse der definitorischen Praktiken heranziehe, geht zurück auf Gardt, der im Rahmen seines Entwurfs eines Textsemantischen Analyserasters „punktuelle“ und „flächige Formen der Bedeutungskonstitution“ in einem Text unterscheidet (Gardt 2012: 64); siehe auch Kap. 2.3 dieser Arbeit.

459 Siehe die Bedeutungsangabe zum Verb definieren auf Duden online unter: https://www. duden.de/rechtschreibung/definieren (Recherche-Stand: 4.6.2018). 
und intersubjektiven Gültigkeit) feststellt oder festsetzt (und von anderen Sachverhalten/Begriffen/Termini differenziert und in bestehendes Wissen einordnet) ${ }^{460}$ (vgl. Kap. 4.3.4). Diese Abschnitte können im Diskurs typo- und topografisch herausgehobenen erscheinen. An diesen Punkten definitorischer Verdichtung wird analysiert, welche sprachlichen Mittel sich auf der Wort-, Satz- und Textabschnittsebene zeigen, die den Gegenstand BuRNOUT definieren. Es wird ferner darum gehen, inwiefern sich diese Mittel auf die in Kapitel 4 herausgearbeiteten Ansprüche an Definitionsprozesse und damit verbundene Verfahrensformen bzw. definitorische Teilpraktiken (wie z.B. klassifizieren, ein- und zuordnen, spezifizieren, differenzieren) beziehen lassen (Kap. 6.2.2.4 und 6.2.3.3). Der sprachliche Zeichengebrauch an diesen Stellen definitorischer Verdichtung wird aus sprachsystematischer und pragmatisch-praxeologischer Perspektive (vgl. Kap. 4.3.2 und 4.3.3) analysiert.

Im zweiten Schritt werden die gefundenen sprachlichen Mittel im Hinblick auf ihr indexikalisch-definitorisches Potenzial auch in der Fläche der Diskursstränge über verschiedene Einzeltexte hinweg mit besonderem Fokus auf ihre fach- und sozio-kulturellen Einbettungsbedingungen (vgl. Felder/Gardt 2015: 15) über die untersuchten Jahrzehnte gesichtet. Es wird dabei mit dem Beschreibungsinstrumentarium der Praktiken geprüft, vor welchen fach- und bereichskulturellen sowie sozial-kulturellen Rahmenbedingungen diese expliziten Definitionsbemühungen stattfinden und in welcher Weise diese Handlungs- und Verhaltensroutinen definitionsähnliche Effekte stimulieren können (Kap. 6.2.4.0 bis 6.2.4.3). Es geht zum einen darum herauszuarbeiten, welche Spuren (unwillkürlicher) sozio-kultureller Praktiken und Normen der Diskurs punktuell und in der Fläche aufweist, die auf den Prozess des Definierens und die Wissenskonstituierung und -strukturierung einwirken. Zum anderen soll aus einer praxeologischen Perspektive gefragt werden, welche „funktionsähnlichen sprachlichen Mittel“461 bzw. sprachlichen Stimuli Aufgaben des Definierens ebenfalls erfüllen können und welche Bandbreite sich im Diskurs diesbezüglich aufzeigen lässt. Definieren wird so als diskursive Prozedur, die sich sowohl punktuell als auch flächig über die Diskursstränge und die Zeit hinweg in reflektierten und nicht-reflektierten Teilpraktiken vollzieht,

460 Die in Klammern stehenden Kriterien, die auf Stimmen im Burnout-Diskurs zum Definitionsbegriff beruhen (vgl. Kap. 4.2.4), werden bei der Auswahl der zu exzerpierenden Textstellen nicht absolut gewichtet. In einem zweiten Schritt wird jedoch untersucht, ob diese ,strengeren“ DefinitionsKriterien sich in diskursiv-semiotischen Strukturen manifestieren, die nicht punktuell im Rahmen einer expliziten Definitionshandlung, sondern eher verdeckt in der Fläche des Diskurses auftreten. 461 Online veröffentlichtes Manuskript von Felder: https://www.gs.uni-heidelberg.de/md/neuphil/gs/sprache02/projekte/felder_form-funktion-perspektivenwechsel.pdf, S. 4 (zuletzt eingesehen am 12.12.2019, im Literaturverzeichnis unter Felder (ohne Jahresangabe)). 
analysiert. ${ }^{462}$ Dabei geht die Arbeit davon aus, dass der Übergang von reflektierter zu nicht vollständig reflektierter Sprachverwendung fließend ist.

Die Analyse erfolgt demnach in folgenden Schritten:

1) Es werden Textstellen definitorischer Verdichtung (siehe oben) in den verschiedenen textuellen Erscheinungsformen des Diskurses sondiert und analysiert, welche sprachlichen Zeichen ${ }^{463}$ (lexikalische und grammatische) ${ }^{464}$ in diesen Passagen definitorische Aufgaben (Kap. 4.2.2) erfüllen.

2) Auf dieser Basis werden Hypothesen für diskursive Teilpraktiken ${ }^{465}$ formuliert, die als prozedural-performative Handlungs- und Verhaltensroutinen zur diskursiven Entstehung einer Definition beitragen.

3) Ausgehend von diesen Hypothesen wird schließlich der Diskurs in seiner Breite nach weiteren Spuren dieser definitorischen Praktiken untersucht. Die Analyse ergibt folgende Teilpraktiken, die in den Kapiteln 6.2.4.1 und 6.2.4.2 dargelegt werden: Praktiken der Zuweisung, Einordnung sowie Einund Abgrenzung als Mittel definitorischer Sachverhaltsfestsetzung/-stellung (Kap. 6.2.4.1); Praktiken der Verknappung, Gewichtung und Geltungsvali-

462 Hier wird, wie zu Beginn des Kapitels bereits erwähnt, das Konzept der punktuellen und flächigen „Bedeutung in Texten“ von Gardt auf den Diskurs übertragen (Gardt 2009: 1202 und Gardt 2012: 64). Vgl. auch Kap. 2.3 dieser Arbeit.

463 Die vorliegende Arbeit legt einen Fokus auf die Analyse von Spuren definitorischer Praxis im Medium vertexteter Sprache. Dies geschieht zum einen vor dem Hintergrund, dass die interaktive wissenschaftliche Wissensgenerierung in heutigen Gesellschaften zu großen Teilen auf schriftlichen Bezugnahmeformen basiert. Bei Texten des öffentlichen Diskurses, sofern diese multimodal, z. B. mit Bildern erscheinen, werden diese Zeichenbeziehungen anhand von Einzelbeispielen in die Analyse mit einbezogen (vgl. Felder/Mattfeldt 2015). Zum anderen konzentriert sich die Arbeit auf das Medium Sprache und damit einhergehende intramediale Relationen vor der Analyse intermedialer Verhältnisse. Damit schließe ich mich L. Jäger an, der empfiehlt, zunächst die „Medialität des Mediums Sprache selbst stärker in den Fokus der Aufmerksamkeit zu rücken“ [...], ,,weil sich vielleicht erst von hier aus Aufklärung darüber gewinnen läßt, was die Medialität von Medien ausmacht‘ (Jäger 2000, S. 10)“ (Jäger 2010b: 302).

464 Zur Unterscheidung von lexikalischen und grammatischen Zeichen siehe Köller (2004: 313ff.). 465 Vgl. die Arbeitsdefinition diskursiver Praktik, die in Kap. 4.3.4 dargelegt wurde. Der Kern des Begriffs der `Praktik ist prozedural-performativ. Denn er führt eine Beschreibungsebene ein, die Prozesse benennt, von denen praktische Effekte ausgehen. In diesen Prozessen verbindet sich die Zweckoder Aufgabenebene mit den semiotischen Ressourcen. Der Begriff `Praxis` bezeichnet im Vergleich zu •Praktikı die Gesamtheit aller Praktiken (Konerding 2009: 83; vgl. Kap. 4.1.1 dieser Arbeit). Wenn in diesem und den folgenden Kapiteln von definitorischen Teilpraktiken die Rede ist, so soll damit des Weiteren verdeutlicht werden, dass mit diesen Praktiken verschiedene Teilaufgaben erfüllt werden können, die mit den Erwartungen an die Tätigkeit des Definieren korrespondieren (vgl. dazu Kap. 4.2.2 dieser Arbeit, z. B. die Erwartung und die daraus erwachsenden Teilaufgaben, dass ein Gegenstand möglichst eindeutig, trennscharf und allgemeinverbindlich festgesetzt werden soll). 
dierung als Mittel definitorischer Wissensgenerierung (Kap. 6.2.4.2); ${ }^{466}$ In diesem Analyseschritt wird außerdem die Frage fokussiert, in welcher Hinsicht (fach)kulturelle und soziale Rahmenbedingungen und Praktiken, also z. B. disziplineigene Methoden und Vorgehensweisen und gewohnheitsmäßige gesellschaftliche Umgangsformen mit Phänomenen aus dem Bereich zwischen `Gesundheit ` und `Krankheit`, in die diskursive Praxis des Definierens hineinwirken. Sobald sich Spuren solcher sozialen oder fachkulturellen Überzeugungen an den Textoberflächen im Diskurs auffinden lassen, werden sie hier nicht als außerhalb des Diskurses liegende Praktiken, sondern als integraler Bestandteil diskursiver Praktiken verstanden. ${ }^{467}$

4) Zuletzt werden die definitorischen Teilpraktiken, die sich punktuell und in der Fläche der jeweiligen Diskursstränge in unterschiedlicher Ausformung manifestieren, in ein Modell diskursiver Definierenspraxis integriert und bezogen auf die jeweils gesondert untersuchten Diskursstränge zu Typen diskursiver Definitionspraxis zum Phänomen bzw. Begriff des >BURNOUTSYNDROMS verdichtet (siehe Kap. 7.2). Am Diskursthema orientiert schließt sich hieran die Frage an, welche Aspekte des Phänomens durch die unterschiedlichen Definitionstypen akzentuiert werden und welche (Krankheits/ Gesundheits)-Konzepte und -Attribute dadurch dominant erscheinen.

Die Blickrichtung der Analyse und der damit verbundenen Schritte kann man auch mit dem Konzept der >Unifizierung` umschreiben. Damit ist gemeint, dass sich die Analyse in den folgenden Kapiteln auf sprachliche Mittel und Praktiken im Diskurs konzentriert, die statt einer agonalen, eine vereinheitlichende, sprich unifizierende Dimension transportieren und damit den Definitionsprozess unterstützen. Das heißt, es geht darum, sprachliche Mittel und Praktiken herauszuarbeiten, die dazu führen, dass bestimmte Konzepte in einem Diskurs Dominanz erhalten und als ,konsensuale‘ Zentren erscheinen. ${ }^{468}$ Es sind Mittel und Prakti-

466 Zum Konzept der `Verknappung`vgl. Foucault ([1970] 1993: 17ff.) und Kap. 4.1.3 dieser Arbeit. 467 Siehe die Argumentation dieser Arbeit in Kap. 4.1.3 zum Verhältnis von Sprache, Diskurs und außersprachlicher Wirklichkeit und zur binären Begrifflichkeit diskursiver und nichtdiskursiver Praktiken ausgehend von Foucault ([1977b] 2003), Schriften, Bd. 3: 396.

468 Die Bezeichnung „konsensuale Zentren“, die Felder (2015: 115) den „agonale[n] Zentren“, d.h. den „konfligierende[n] Geltungsansprüche[n] von Wahrheitsaussagen“, gegenüberstellt passt hier insofern, dass die untersuchten Praktiken dazu führen, dass die konfligierenden Konzepte im Diskurs weniger sichtbar werden und dadurch der Eindruck von Übereinstimmung entsteht. Es ist damit jedoch nicht gemeint, dass dieser Konsens von den beteiligten Sprecherinnen und Sprechern immer intendiert erzeugt wird. Es geht darum, zu zeigen, dass sich fachlicher und gesellschaftlicher ,Konsens ‘ im Diskurs auch durch nicht (gänzlich) intendierte Verhaltensroutinen abzeichnen kann. 
ken, die die Verfestigung bzw. einheitliche Fassung eines Begriffs im Diskurs mit bewirken, sei es durch Verknappungspraktiken und die Reduzierung konfligierender Konzepte (vgl. Kap. 6.2.4.2) oder durch konstant wiederkehrende Einordnungspraktiken (vgl. Kap. 6.2.4.1). Im Ko- und Kontext der Definitionen findet man jedoch auch sprachliche Mittel der Agonalität (vgl. zum Konzept der >Agonalität` Felder 2012; 136; 2015: 108ff.; Mattfeldt 2018: 52ff.). Auf das Verhältnis zwischen unifizierenden und agonalen Dimensionen im Burnout-Diskurs wird in Kap. 7.3 eingegangen.

\subsubsection{Sprachliche Mittel des Definierens im fachinternen Diskursstrang als sich punktuell zeigende Praktiken mit definitorischer Funktion}

\subsubsection{In Fachlexika, Hand- und Lehrbüchern}

In Kap. 5.3.3.1 wurden die Texte der Fachlexika und Hand- und Lehrbücher im Hinblick auf ihre kommunikativen Entstehungsbedingungen und grundlegenden variationslinguistischen Charakteristika ausführlich beschrieben. Die Kurzfassung dazu lautet: Diese Texte wenden sich an (werdende) Fachexpertinnen und -experten verschiedener Unterdisziplinen (z. B. Arbeits- und Organisationspsychologie) einer Dachdisziplin (z. B. der Psychologie). Es sind medial und konzeptionell schriftliche Texte, verfasst in der Standardvarietät des Neuhochdeutschen und sie haben einen mittleren Fachlichkeitsgrad. ${ }^{469}$ Die hauptsächliche Textfunktion ist informativ, das in Frage stehende Lemma soll beschrieben bzw. definiert werden. Mit diesen Voraussetzungen hängt zusammen, dass man in diesem Diskursstrang viele Textstellen findet, in denen explizit definiert wird; explizit deshalb, weil Definitionen genuiner Bestandteil des Erscheinungsformats sind, wie zum Beispiel in Fachlexika und teilweise in Hand- und Lehrbüchern, oder der Textabschnitt mit Definition überschrieben ist. Die folgende Analyse der sprachlichen Mittel, die in diesen Definitionen erscheinen, geht von sieben Beispielen aus, auf die sich die Analyse rückbezieht. Darüber hinaus werden weitere Beispiele an den jeweiligen Stellen der Untersuchung direkt (für den besseren Lesefluss teilweise in Fußnoten) zitiert, um die Analyseschritte transparent $\mathrm{zu}$ machen und die interpretativen Schlüsse zu plausibilisieren. In den folgenden Beispielen werden Stellen definitorischer Verdichtung aus den Texten exzerpiert.

469 Für einen mittleren Fachlichkeitsgrad spricht, dass einerseits, wie für ein Fachlexikon zu erwarten, Termini in den Einträgen vorkommen, dass diese aber andererseits in demselben Eintrag oder in der derselben Publikation expliziert werden. Vgl. dazu auch Kap. 5.3.3.1. 
Betrachtet man die folgenden Beispiele, insbesondere 2-6, so fällt auf, dass sie sich dahingehend ähneln, dass sie >Burnout` als einen dreidimensionalen Begriff beschreiben und sich dabei jeweils paraphrasierend auf die gleiche Quelle (die Definition von Maslach/Jackson) beziehen. Das dominante Vertextungsmuster dieser Beispiele ist die Zustandsbeschreibung, teilweise kombiniert mit explikativen Passagen, die Ursache-Folge-Beziehungen (hypothetisch) angeben: ${ }^{470}$ Die (dreidimensionale) Symptomkonstellation wird in komprimierter Ausdrucksweise im Nominalstil dargestellt, die transformativen Prozesse, die zu diesem Zustand geführt haben, werden als Erklärung für die Zustände mehr oder weniger explizit angeführt. Die Beispiel 1 und 7 stellen im Vergleich zu 2-6 eigenständigere Definitionsversionen dar. Alle Belegbeispiele werden nun zunächst in einem Block mit kurzen einführenden Kommentaren dargestellt, um im Anschluss die sprachlichen Mittel des Definierens vergleichend zu analysieren.

Das erste Beispiel stammt von Schmidbauer (1991: 41). Es ist ein frühes Beispiel für einen Eintrag in einem „Lexikon der Grundbegriffe“ für das Fach Psychologie, das Schmidbauer herausgegeben hat. Es erläutert den zu definierenden Gegenstand „Burnout/,Ausgebrannt““ anhand einer generischen Aussage über „Berufstätige“, denen dieses Attribut unter denen im Konditionalsatz folgenden Bedingungen zugeschrieben wird. Durch die Apposition (,vor allem in den sozialen Arbeitsbereichen“) wird die Gruppe von Berufstätigen noch weiter eingeschränkt. Der Vorgang wird unter den beschriebenen Bedingungen als sich wiederholend habitueller und generalisierbarer Prozess beschrieben:

1) Beispiel 1 Schmidbauer, Psychologie - Lexikon der Grundbegriffe (1991): 41 (im QV unter 8.1.2)

Burnout ,Ausgebrannt' sind Berufstätige, vor allem in den sozialen Arbeitsbereichen $(\rightarrow$ Helfersyndrom), wenn sie in dauernden Gefühlen der Überforderung und einem unbefriedigten Verhältnis von Leistung und Erfolg leben. Je unrealistischer die Erwartungen an die Tätigkeit und je ungünstiger die institutionellen Bedingungen, desto schneller macht sich der Burnout bemerkbar. Die subjektive Anstrengung steigt, aber die Qualität der Leistung sinkt. Zumindest teilweise Entlastung ermöglicht Supervision.““711

470 Die analytische Zuordnung zu Mustern der definitorischen Entfaltung orientiert sich an linguistischen Publikationen zur „thematischen Entfaltung“ und Vertextungsmustern, die in Kapitel 6.2.2.3 ausführlich dargelegt werden.

471 Ähnliche Beispiele, die `Burnout` in sozialen Berufen verorten und mit DiskrepanzErfahrungen beschrieben werden, findet man z.B. auch bei Grulke/Larbig (2001: 562) und Dittmann-Kohli et.al. (1997: 198); beide Belege im QV unter 8.1.2. 
Das zweite Beispiel ist der erste Eintrag zum Begriff >Burnout im „Dorsch Lexikon der Psychologie“, ein Fachwörterbuch, das seit den 1920er Jahren regelmäßig in Neuauflage erscheint: ${ }^{472}$

\section{2) Dorsch, Psychologisches Wörterbuch ( $\left.{ }^{11} 1991\right): 887$ (im QV unter 8.1.2)}

Burnout [engl.], Ausbrennen. Syndrom, das bei professionellen Helfern als Folge von Überlastung auftritt, u. a. gekennzeichnet durch emotionale Erschöpfung, Dehumanisierung (zynisch abwertende Haltung gegenüber dem Hilfesuchenden) und das Gefühl, der beruflichen Aufgabe nicht mehr gewachsen zu sein. Es gibt einen standardisierten Fragebogen zur Erfassung des Syndroms: Maslach Burnout Inventory (MBI). Maslach $1982 .{ }^{473}$

Beispiel 3 entstammt einem Fachwörterbuch eines Spezialfachs der angewandten Psychologie. Der Text in der 2. Auflage ( $\left.{ }^{2} 2001\right)$ und 4. Auflage $\left({ }^{4} 2010\right)$ ist unverändert. Die Autorin Annemarie Barth bespricht im Artikel „Burnout bei Lehrern“ im „Handwörterbuch Pädagogische Psychologie“ mehrere Burnout-Konzepte im Unterkapitel „Begriff und Konzeptionen“, aber das folgende Zitat ist im Lehrbuch grau hinterlegt, und durch fette Schriftstärke sind einzelne Phrasen vom restlichen Text abgesetzt. Sie präsentiert die Definition nach Maslach (1982) in deskriptiv-explikativer Themenentfaltung:

3) Barth (2001/2010): Handwörterbuch Pädagogische Psychologie: 70 (Die Definition ist im Original grau hinterlegt, Hervorhebungen im Original, im QV unter 8.1.2)

\section{Das Burnout-Syndrom: drei Dimensionen}

Maslach (1982) versteht Burnout als ein Syndrom, das aus emotionaler Erschöpfung, Dehumanisierung und verminderter Leistungsfähigkeit bei der Arbeit zusammengesetzt ist.

Emotionale Erschöpfung. Damit ist gemeint, dass man sich emotional überfordert und von den Kontakten mit den anderen Menschen ganz ausgelaugt fühlt. Wenn die emotionalen Reserven einer Person erschöpft sind, kann sie anderen nichts mehr geben.

472 Zur Geschichte des Wörterbuchs siehe: https://dorsch.hogrefe.com/ueber-den-dorsch (zuletzt eingesehen: 14.7.2021).

473 Diese Definition ist im Dorsch bis zum Jahr 2013 über vier Auflagen hinweg kaum verändert worden. Im Jahr 1998 erfolgte ein weiterer bibliografischer Verweis (neben Maslach 1982 auf Burisch 1989). Im Jahr 2004 wurde das Burnout-Phänomen innerhalb des Lexikons mit dem Chronic-Fatigue-Syndrom verknüpft: (,Zuweilen wird das B. mit $\rightarrow$ CFS (Chronic-FatigueSyndrom) in Zusammenhang gebracht. Burisch 1989, Maslach 1982“). In der Ausgabe des Jahrs 2009 wird erstmals „Dr. Peter Day, Univ. Tübingen“ als Autor des Eintrags genannt. Im Jahr 2013, in der 16. Auflage des Wörterbuchs, wurde der Eintrag zu „Burnout“ schließlich von Matthias Burisch grundlegend erneuert und erweitert. Die Zeichenzahl des neuen Eintrags hat sich im Vergleich zum Eintrag von 2004 etwa um den Faktor Sieben erhöht. 


\begin{abstract}
Dehumanisierung. Sie bezieht sich auf nicht-mit-fühlende und herzlose Reaktionen gegenüber den Personen, mit denen man zusammenarbeitet und um die man sich kümmern soll. Die Menschen werden immer weniger als Personen ( $\rightarrow$ Personzentrierte Unterrichtung und Erziehung), sondern vielmehr als Objekte gesehen. Es entsteht eine negative, zynische Einstellung gegenüber den Hilfesuchenden, die oft dazu führt, dass man der Meinung ist, diese seien selber schuld an ihren Problemen und Schwierigkeiten und würden sie sogar verdienen.
\end{abstract}

Gefühl verminderter Leistungsfähigkeit bei der Arbeit. Dieses Gefühl bezieht sich darauf, dass man sich immer weniger kompetent und erfolgreich fühlt. Die Betroffenen beginnen, sich selber negativ zu bewerten, sie sind unzufrieden mit ihren beruflichen Leistungen, ihr Selbstwertgefühl ( $\rightarrow$ Selbstkonzept) insgesamt leidet. Nur wenn alle drei Dimensionen betroffen sind, wird von „Burnout“ gesprochen.

Weitere Beispiele aus diesem Diskursstrang, die zwar mehrere Definitionen bzw. Beschreibungskonzepte erwähnen und/oder Kritik an der zur „Diagnose“ gewordenen Definition >Burnout üben, dennoch aber wie im 3. Beleg von Barth die Definition von Maslach (und Jackson) deutlich hervorheben (z. B. typografisch oder topografisch zu Beginn des Kapitels oder durch eine vergleichsweise ausführliche Zitierweise) findet man z. B. auch bei Krapp/Weideman ( $\left.{ }^{4} 2001: 326\right)$; Weber ( ${ }^{3} 2011$ : 426), Groth (2013); Kunter/Pohlmann (2015: 274); Gerrig/Zimbardo $\left.\left({ }^{20} 2015\right)\right)^{474}$ (alle im QV unter 8.1.2) und siehe auch Beleg 5 und 6. In Kap. 6.2.4.2 wird der verknappenden Wirkung dieser typografischen Gestaltungspraktiken noch weiter nachgegangen. Es deutet sich hier schon ein Konflikt zwischen einer als repräsentativ vs. deklarativ verstandenen >Definition` an, der im folgenden Kapitel 6.2.2.2 bei der Analyse der Texte in Fachzeitschriften und Monografien noch deutlicher zu Tage tritt und dort an vergleichenden Beispielen erläutert wird (zum Zusammenhang repräsentativer und deklarativer Merkmale von Definitionshandlungen mit Bezug auf Searle (1980: 84; 88ff.) vgl. Kap. 4.3.2 dieser Arbeit).

Das vierte Beispiel zeigt den Eintrag zu >Burnout`im Klinischen Wörterbuch Pschyrembel. Den diskursiven Stellenwert dieses Wörterbuchs, der über das Fach der Medizin hinausgeht, kann man daran ablesen, dass etwas für die breitere Öffentlichkeit als anerkanntes medizinisches Phänomen gilt, wenn es in diesem Wörterbuch enthalten ist ${ }^{475}$, und dass diese Definition im Wikipedia-Beitrag zu >Burnout`sieben Jahre lang als Grundlage für den ersten Definitionsabschnitt dient (vgl. Kap. 6.2.3.2 dieser Arbeit). Wie in den Ausgaben des psychologischen

474 Die gerade genannten Quellen befinden sich im QV unter 8.1.2.

475 Vgl. einen Verweis auf das klinische Wörterbuch Pschyrembel in einem Artikel der ZEIT vom 2. Dezember 1999 (= Blech 1999, im QV unter 8.1.6), in welchem dem Konzept `Burnout mit Misstrauen begegnet wird, denn in „Nachschlagewerken wie dem Pschyrembel taucht der Begriff erst gar nicht auf.“ Burnout wurde erst in der 259. Auflage (2002) in den Pschyrembel als Stichwort aufgenommen. 
Wörterbuchs Dorsch bis zum Jahr 2013 (siehe Bsp. 2) erscheint hier eine Definition aus drei Symptomen, die auf die Autorinnen Ch. Maslach und S. E. Jackson (1981) zurückgeht. ${ }^{476}$ Die Quelle wird hier allerdings nicht genannt.

4) Pschyrembel, Klinisches Wörterbuch ( $\left.{ }^{261} 2007\right): 295$ (Unterstreichung T.S., sonstige Hervorhebungen im Original, im QV unter 8.1.2):

Burn|out-Syn|drom ( $\uparrow$; engl. out aus; Syndrom $\left.{ }^{\star}\right)$ n;

Burned-out-Syndrom, auch Ausbrennen; Zustand emotionaler Erschöpfung, reduzierter Leistungsfähigkeit u. evtl. Depersonalisierung inf. Diskrepanz zw. Erwartung u. Realität; Endzustand eines Prozesses von idealist. Begeisterung über Desillusionierung, Frustration u. Apathie; Häufigkeit: geschätzte Prävalenz ca. 10 \% der Arbeitnehmer mit entspr. Berufen (20-30\% aller Arbeitnehmer sind gefährdet); Sympt.: psychosomati. Erkr., Depression od. Aggressivität, erhöhte Suchtgefahr; Ther.: im fortgeschrittenen Stadium Psychotherapie; bei gleichzeitiger Depression Antidepressiva

Beispiel 5 ist ein Auszug aus dem Teilband „Organisationspsychologie - Grundlagen und Personalpsychologie der „Enzyklopädie der Psychologie“. In diesem Beispiel hinterfragen die Autoren, ob >Burnout` ein eigenständiges Konzept gegenüber anderen „Maßen beeinträchtigten Befindens“ und „Stresssymptomen“ (Zapf/Semmer 2004: 1072) darstellt und führen die folgende Begriffsbestimmung auf Maslach zurück:

5) Zapf/Semmer (2004: 1072) (Fettdruck im Original, Unterstreichung T.S., im QV unter 8.1.2):

\subsubsection{Burnout: Ein spezielles Konstrukt?}

Auf ein Konzept soll hier noch kurz eingegangen werden, weil es als ein sehr spezifisches Konstrukt diskutiert wird, das sich a) von anderen Maßen beeinträchtigten Befindens unterscheide und b) auf spezifische Aspekte der Arbeit zurückzuführen sei - das Konzept des ,Burnout‘, des ,Ausgebrannt-Seins“ (Büssing \& Perrar 1992; Büssing \& Schmitt, 1998, Maslach, 2000; Schaufeli \& Buunk, 1996, Schaufeli \& Enzmann, 1998, Schaufeli, Maslach \& Marek, 1993). Nach dem besonders verbreiteten Konzept von Maslach besteht Burnout aus drei Komponenten: Emotionale Erschöpfung bezieht sich auf das Gefühl, ausgelaugt und erschöpft zu sein, Depersonalisation beinhaltet, dass man sich von den Menschen, mit denen man berufsmäßig zu tun hat - Patienten, Klienten, Schüler etc. - innerlich distanziert und sie wie Objekte behandelt, und schließlich drückt sich das Gefühl, den gestellten Ansprüchen nicht mehr zu genügen, in einem Gefühl reduzierten Leistungsvermögens aus. [Kursivsetzungen im Original]

476 Im Korpus der Fachlexika, Hand- und Lehrbücher gibt es weitere Beispiele, die `Burnout fast ausschließlich nur mit Bezug auf Quellen, an denen Ch. Maslach beteiligt ist, beschreiben und diese Dreifaktoren-Symptomstruktur repetieren, so Zimbardo (51992: 477); Nowak (2010: 69); Kirchler (32011: 293ff.); Gerrig ( ${ }^{20} 2015$ : 500). Alle Quellen im QV unter 8.1.2. Siehe zu diesen Verknappungspraktiken auch Kap. 6.2.4.2 dieser Arbeit. 
In Beispiel 6 behandelt der Autor Niclas Schaper >Burnout ‘ im Rahmen der Kapitel „Wirkungen der Arbeit“ und „Spezifische Auswirkungen von Stress“ im Lehrbuch zur Arbeits- und Organisationspsychologie (Nerdinger/Blickle/Schaper ${ }^{3} 2014$ ). Schaper nennt zwar nicht nur eine Definition, aber folgende in Beleg 6 wird farblich abgesetzt, mit „Definition“ überschrieben in einem Kasten abgedruckt. Eine Seite später (Schaper ${ }^{3}$ 2014: 532) werden die drei Hauptsymptome nach Maslach/ Jackson (1984) in einem Schaubild in ein „Modell der Burn-out-Entstehung“ nach Cordes/Dougherty (1993) eingeordnet und über Pfeile mit verschiedenen Stressorenquellen und weiteren „Einstellungs- und Verhaltenssymptomen von Burn-out“ verbunden und dadurch in eine Bedingungs-Reihenfolge gebracht („Persönliche Stressoren“ und „Arbeits- und Organisationsstressoren“ führen $\mathrm{zu} \rightarrow$ „Emotionaler Erschöpfung“, auf diese folgt $\rightarrow$ „Depersonalisation“ und auf diese $\rightarrow$ das „Gefühl, persönlich nichts mehr zu erreichen bzw. zu leisten“, alle drei Hauptsymptome führen $\mathrm{zu} \rightarrow$ den weiteren Symptomen):

6) Schaper, Lehrbuch (32014: 531f.) (im QV unter 8.1.2):

Eine der bekanntesten Definitionen nach Maslach und Jackson (1984) ist die folgende:

Definition [Die Definition ist im Lehrbuch eingerahmt und durch die Schriftfarbe vom restlichen Text abgesetzt, Kommentar T.S.]

Burn-out bezeichnet einen besonderen Zustand berufsbezogener chronischer Erschöpfung insbesondere in Sozial- und Pflegeberufen. Es wird meist als ein Syndrom aus emotionaler Erschöpfung, Depersonalisierung und reduzierter Leistungsfähigkeit beschrieben. Emotionale Erschöpfung ist durch hohe interpersonelle Anforderungen und die Beanspruchung emotionaler Ressourcen gekennzeichnet. Die Betroffenen fühlen sich durch den Kontakt mit anderen Menschen emotional überanstrengt und ausgelaugt. Depersonalisation beinhaltet negative, gefühlslose und zynische Einstellungen gegenüber Klienten, Kunden oder Patienten; ein Zustand, in dem die Betroffenen gefühlslose und abgestumpfte Reaktionen gegenüber ihren Klienten zeigen. Persönliche Leistungseinbußen beschreibt die Tendenz, die eigene Arbeit negativ zu bewerten und ein Gefühl mangelnden bzw. schwachen beruflichen Selbstwertes zu entwickeln. [...]

Beispiel 7 schließlich zeigt in Auszügen den deutlich überarbeiteten Eintrag im Psychologischen Wörterbuch Dorsch aus dem Jahr 2013 (16. Auflage), der von M. Burisch verfasst wurde. Hier ist bemerkenswert, dass als Definiensausdruck das seltener verwendete Wort Krise $(n)^{477}$ statt Syndrom, Zustand oder Begriff/Konzept

477 Dittmann-Kohli/Sowarska/Timmer (1997: 198, im QV unter 8.1.2) verwenden allerdings ebenfalls schon sechzehn Jahre zuvor das Hyperonym Krise. Sie definieren >Burnout in Band 4 der Serie „Pädagogische Psychologie“ der „Enzyklopädie der Psychologie“ wie folgt: „Burnout ist eine in neuerer Zeit oft beschriebene Motivationskrise, die zunächst vor allem bei Pflegepersonal beobachtet wurde und auch schon im jüngeren Erwachsenenalter auftreten kann. Burnout 
wie in den anderen Beispielen verwendet wird, dass für einen Lexikoneintrag viele Distanzierungsmarker gesetzt werden und dass die Definition im zweiten Abschnitt argumentativ weitergeführt wird:

7) Dorsch/Burisch ( ${ }^{16}$ 2013: 312f.) (Unterstreichungen T.S., im QV unter 8.1.2):

(= B.) [engl. burn brennen, out aus], [AO, GES, KLI, PÄD], ist der Oberbegriff für bestimmte Typen persönlicher Krisen (Krise), die mit eher unauffälligen Frühsymptomen beginnen und in völliger Arbeitsunfähigkeit oder im Suizid enden können. Diverse Phasenmodelle unterscheiden rein intuitiv zw. drei und zwölf Entwicklungsstadien. Wegen des Prozesscharakters von B. sind Prävalenzraten (Prävalenzrate) nicht sinnvoll anzugeben. In großen repräsentativen Bevölkerungsstichproben Deutschlands bezeichneten sich 10,5\% (2007) bzw. 7,7\% (2011) als «ausgebrannt». [...]

Die Symptomatik wird nicht einheitlich beschrieben - Burisch (2010) trug mehr als 130 Symptome zus. - und überlappt mit der diverser anderer Störungsbilder (z. B. Depression). Als Kernsymptome gelten emotionale Erschöpfung, Unzufriedenheit mit der eigenen Leistung, Depersonalisation (Depersonalisations-Syndrom), (treffender: Dehumanisierung) und Überdruss. Dies aber wohl v.a., weil so die Skalen des Maslach Burnout Inventory (MBI) (Maslach et al. 1996; 22 Items) bzw. des Tedium Measure (Aronson et al. 1983; 21 Items) benannt sind, zweier amerikanischer Fragebögen, für deren dt. Übersetzungen u. a. Normen fehlen. Als Alternativen kommen v.a. die Burnout-Screening-Skalen I und II (BOSS) (Hagemann \& Geuenich 2009; je 30 Items) und das Hamburger Burnout-Inventar (HBI; zugänglich über www.burnout-institut.eu/Burnout-Test.8.0.html; 40 Items) infrage. Die unscharfe Abgrenzung trägt dazu bei, dass B. in der ICD-10 (International Classification of Diseases (ICD)) nur als (nicht kassenfähige) Zusatzdiagnose Z73.0 auftaucht, was sich in der ICD-11 kaum ändern wird. U. a. aus diesem Grunde wird dem Begriff $B$. von manchen Psychiatern neuerdings die Existenzberechtigung abgesprochen. Eine Definition dreier niederländischer Spitzenverbände von Medizinern und Psychologen (zugänglich z. B. über www.burnout-institut.eu/Burnout-Service.33.0.html; niederländische Def. von B.), deren Trennschärfe die der üblichen ICD-Def. mind. erreicht, ist in Deutschland noch weitgehend unbekannt. Immerhin empfahl die Dt. Gesellschaft für Psychiatrie, Psychotherapie und Nervenheilkunde im März 2012, von Z73.0 vermehrt Gebrauch zu machen.

[...] [Es folgen noch zwei Absätze zu Ansätzen der Ätiologie und Therapie sowie die Autorenangabe Matthias Burisch und Literaturempfehlungen, T.S.]

Wie oben schon angeführt, ist das dominante Vertextungsmuster der meisten Beispiele die Zustandsbeschreibung ergänzt durch explikative Passagen, die die Entstehung von Burnout beispielsweise über konditionale Satzgefüge erläutern. Innerhalb dieser Beschreibungen werden die Definitionen mit syntaktischen Mustern wie

ist mit Zuständen wie Depression, Unempfindlichkeit gegenüber den Patienten, Apathie und Arbeitsunlust verbunden und scheint eine Reaktion auf die erlebte Diskrepanz zwischen tatsächlichen Arbeitsbedingungen und den ursprünglichen Idealen zu sein. Gerade bei sehr ,menschenfreundlichen“ Leuten tritt Burnout auf (Schaufeli, 1990).“ 
$x$ bezeichnet $y, x$ versteht $y$ als $z, x$ besteht aus $y, x$ ist der Oberbegriff für $y$ lexikalisch eingeleitet. Dem Bezugsgegenstand (BuRnout) und Ausdruck Burnout ${ }^{478}$ werden in der Beziehung der Äquivalenz Merkmalsbeschreibungen zugeordnet. ${ }^{479}$ In den darauf folgenden Sätzen oder Absätzen folgen ähnliche zuordnend-prädizierende Satzmuster, die BuRnouT sowie SYMPTOMEN von BuRnOuT (emotionale Erschöpfung, Depersonalisierung, reduzierte Leistungsfähigkeit) Eigenschaften zuschreiben: $x$ beschreibt $y$; $x$ wird beschrieben als; $x$ bezieht sich auf, $x$ ist gekennzeichnet durch; $x$ beinhaltet/beschreibt $y$. Diese Zuordnungspraktik entspricht formal dem klassischen Definitionsschema nach dem Muster genus proximum und differentia specifica: Es werden Hyperonyme bzw. Definiens-Nomina höherer Abstraktion angegeben (Zustand, Syndrom, Tendenz, Konzept, Krise), die über mehrgliedrige Attribut-Phrasen und/oder Relativsätze spezifiziert bzw. charakterisiert werden: Zustand berufsbezogener chronischer Erschöpfung, Syndrom aus emotionaler Erschöpfung, ...; ein Zustand, in dem ..., Syndrom, das aus ... zusammengesetzt ist. Inwiefern es sich bei diesen Angaben jedoch wirklich um nächsthöhere Gattungsbegriffe (mit Bezug auf ein festes Begriffssystem) handelt und inwiefern sich die in den Belegen angeführten spezifizierenden Merkmale für >Burnout` klar und deutlich von anderen sachverwandten Begriffen derselben Hierarchiestufe innerhalb eines Begriffssystems unterscheiden, wird in den Belegen nicht expliziert. Denn es erfolgt keine genaue Zuordnung zu einem Klassifikationssystem wie der ICD oder dem DSM: Es wird darauf verwiesen, dass das Konzept `Burnout ‘ in der ICD-10 nur als Zusatzdiagnose ohne scharfe Abgrenzung zu anderen ICD-Konzepten auftauche (vgl. Beleg 7 oben). Die Einordnung in eine organisationspsychologische Systematisierung von „Stresssymptomen“ wird kritisch diskutiert (vgl. Beleg 5 oben, Zapf/ Semmer (2004: 1072f.).

Betrachtet man die Definiensausdrücke höherer Abstraktion genauer, so scheint insbesondere das Wort Syndrom in den Definitionen 2, 3, 4 und 6 eine für die weitere Definition gliedernde Funktion zu übernehmen und eine Aussage über die Eigenart des Gegenstands zu treffen. Denn `Syndrome ‘ beruhen in der Medizin auf der Zusammenstellung ,von Krankheitssymptomen, deren Kombination für ein Krankheitsbild typisch ist““480 (vgl. Kap. 3.1 dieser Arbeit zur medizinischen Semio-

478 Das entlehnte Wort Burnout/Burn-out wird in die bestehende Terminologie eingeordnet. 479 In den Fachlexika wird Burnout zunächst übersetzt und dann durch typografische Konvention innerhalb der Textsorte Wörterbuchartikel (Doppelpunkt, Punkt, direkter Anschluss der Erläuterung ohne Verb) mit den erläuternden Folgezeichen in Beziehung gesetzt. Durch die vorherrschende Textfunktion von Wörterbuchartikeln, Begriffen/Sachverhalten eine Bedeutung/ Erklärung zuzuordnen, kann hier ebenfalls eine Äquivalenzbeziehung unterstellt werden.

480 Vgl. die Bedeutungsangabe für Syndrom auf der Webseite von „Das Wortauskunftssystem zur deutschen Sprache in Geschichte und Gegenwart“(DWDS), abrufbar unter: https://dwds.de/ 
tik). Dass die Eigenschaft der 'Typizität' von großer Bedeutung ist, zeigt Beispiel sieben. Burisch vollzieht zwar eine Abkehr vom Begriff Syndrom, aber die 'Typizität', die dem Begriff Syndrom inhärent ist, wird auch für den neuen Oberbegriff Krise wieder mit hineingeholt (bestimmte Typen persönlicher Krisen). Wie solch ein Typus einer persönlichen Krise aussehen soll, wird allerdings nicht erläutert.

Die Spezifizierung beginnt im 6. Beispiel oben mit dem bewertenden Adjektiv eines besonderen Zustands, womit direkt eine Abgrenzung zu normalen alltäglichen Erschöpfungszuständen markiert wird, in Beispiel 3 wird das Syndrom über einen anschließenden Relativsatz näher bestimmt. In allen Beispielen werden in äußerst sprachökonomischer komprimierter ${ }^{481}$, bei den Lexikoneinträgen sogar teilweise in elliptischer Form, weitere extensionale Bestimmungsmerkmale (berufsbezogen, chronisch, in Sozial- und Pflegeberufen, bei professionellen Helfern, bei der Arbeit, in völliger Arbeitsunfähigkeit ... enden) angeführt, die den Anwendungsbereich des Begriffs auf (psychosoziale) Berufe oder beanspruchende Tätigkeiten festlegen. Folgt man weiter zunächst einmal der Analyse von Beispiel 6, so fallen in den folgenden Sätzen sprachliche Mittel auf, die das Syndrom durch Angaben zu seiner Dauer bzw. Frequenz und Intensität weiter eingrenzen sowie durch (implizite) gradierende Zusatzinformationen $\mathrm{zu}$ anderen Zuständen und (implizit aufgerufenen) Erwartungsnormen in Beziehung setzen: Ein Adjektiv wie chronisch verweist darauf, dass der Zustand andauert bzw. immer wiederkehrt und sich dadurch von 'normaler' 'reversibler' Erschöpfung unterscheidet. Auch in Beispiel 1 werden die Gefühle der Überforderung durch das Adjektiv dauernde temporal gedehnt. ${ }^{482}$ Es sind keine unmarkiert normalen, sondern hohe interpersonelle

wb/Syndrom, (zuletzt eingesehen am 12.12.2019). Zur Geschichte des Syndrom-Begriffs bzw. der Syndromenlehre vgl. Schuster (2010: 308, Fußnote 14).

481 Die Textstellen sind im Sinne von P. von Polenz’ Satzsemantik „,komprimiert“, da die Zahl der zu verstehenden Inhaltsteile größer als die Zahl der Ausdruckseinheiten ist (vgl. von Polenz ${ }^{3} 2008: 25 \mathrm{ff}$.). Dies wird insbesondere ersichtlich, wenn man die hypotaktischen Strukturen der Attribut- und Nominalkomplexe auflöst. Für die Nominalphrase Syndrom aus emotionaler Erschöpfung, Dehumanisierung ... könnte man folgende explizite Umformulierungen vorschlagen: 'Man kann das Syndrom [= Zeichen, die typischerweise zusammen auftreten/sich körperlich, im Fühlen oder im Verhalten zusammen zeigen] daran erkennen, dass die Emotionen [der Personen, die in Sozial- und Pflegeberufen arbeiten] erschöpft sind und die Personen [die in Sozialund Pflegeberufen arbeiten] depersonalisiert wurden/sich depersonalisiert haben, d. h. dass sie sich von den Personen, die sie versorgen [sollen], und ihrem eigenen Fühlen entfremdet haben.' Man erkennt beim Umformulieren außerdem, dass einige semantische Informationen darüber, wer oder was durch wen oder was beeinflusst wird, unbestimmt bleiben.

482 Vgl. dazu auch Dorsch ( ${ }^{14} 2004:$ 157) und ( ${ }^{15} 2009$ : 163): Burnout wird dort mit dem „CFS (Chronic-Fatigue-Syndrom) in Zusammenhang gebracht“; vgl. Dorsch ( ${ }^{17} 2014$ : 312): „durch chronische Fehlbeanspruchung in Dauerstress-Situationen“; Krapp/Weidemann ( $\left.{ }^{4} 2001: 326\right)$ : „durch lang anhaltende berufliche Belastung“; Sonneck/Fengler (22009: 104): „Emotionale Erschöp- 
Anforderungen, denen die ,Betroffenen' ausgesetzt sind; die Leistungsfähigkeit, die für sich genommen kein Symptom wäre, erscheint als symptomwürdig, weil sie reduziert, vermindert ist (Bsp. 3; 4; 5; 6) bzw. die Personen sich immer weniger kompetent und erfolgreich fühlen und Leistungseinbußen verbunden mit mangeln$\underline{\text { dem }}$ bzw. schwachem Selbstwertgefühl zeigen (Bsp. 3; 6). ${ }^{483} \mathrm{Im}$ von Burisch überarbeiteten Lexikoneintrag im Dorsch in Beispiel 7 wird davon gesprochen, dass Burnout zu völliger Arbeitsunfähigkeit führen könne, bei Gusy/Kleiber (1998: 315, im QV unter 8.1.2) wird das Symptom extreme Müdigkeit angeführt. Durch diese sprachlichen Mittel der Graduierung werden die beschriebenen körperlichen, psychischen Anzeichen und Verhaltensweisen zu „Signalen“ für etwas, „die nach einer Erklärung schreien“ (Sebeok 1984: 42; vgl. von Uexküll 1984b: 32). ${ }^{484}$ Ein ähnliches Beziehungsgefüge erzeugen Wörter wie überanstrengt, überfordert, Überlastung, Überforderung (Bsp. 1, 2, 3, 6). ${ }^{485}$ Denn sie zeigen an, dass die Anstrengung/ Belastung in irgendeiner Art und Weise über Standardwerte von Anstrengung/ Belastung, die man bei der Arbeit mit Menschen haben sollte, hinausgeht. An dieser Stelle kommen verschiedene sozial-kulturelle Praktiken als Referenzwerte ins Spiel (z. B.: Unter welchen Bedingungen eine Handlungs- und Verhaltensweise bei speziellen Anforderungen als 'normal' angesehen wird), die durch wiederkehrende Zeichenkombinationen über verschiedenen Texte des Diskurses hinweg aufgerufen werden und den Gegenstand bzw. den Begriff >BURNOUT` zwischen den Polen sgesund ‘ und `krankı verorten. Sie werden in Kapitel 6.2.4.1 im Rahmen der Spurensuche von Praktiken der Ein- und Abgrenzung sowie Ein- und Zuordnung in der Breite des Diskurses näher erläutert werden.

Es stechen zudem Negationsmittel ins Auge. Diese dienen interessanterweise in den angeführten Beispielen kaum dazu, den Begriff `Burnout ` als Gesamtkonzept ex negativo zu definieren, dadurch das aufgezählt wird, was >Burnout ‘nicht ist (nur in Beispiel 7 wird z. B. davon gesprochen, dass Burnout eine „nicht kassenfähige“

fung bezieht sich auf chronische Müdigkeit von früh bis spät, Tag und Nacht“; siehe alle angeführten Belege im QV unter 8.1.2.

483 Weitere Beispiele: „verminderter Leistungsfähigkeit“ (Krapp/Weidemann ${ }^{4} 2001$ : 326); „reduziertem Leistungsvermögen, hohe emotionale Anforderungen“ (Zapf/Semmer 2004: 1072); „reduzierte persönliche Leistungszufriedenheit und Leistungseinbuße, Reduzierung der Arbeit auf das Allernötigste, Leistungsunzufriedenheit“ (Sonneck/Fengler 22009: 104); „dem Gefühl, immer weniger zu leisten“ (Kirchler ${ }^{3} 2011: 294$ ), „Konzentrationsprobleme, verlangsamtes Denken“ (Deister ${ }^{4}$ 2009: 271) etc.; im QV unter 8.1.2.

484 Vgl. dazu die Termini Zeichen, Signal, Symptom in der medizinischen Semiotik Kap. 3.1 dieser Arbeit.

485 Weitere Beispiele: „Überbeanspruchung“ (Semmer 1992: 749); „Übermüdung“(Richter/Hacker 1998: 144); „,durch berufliche Überforderung, gelegentlich auch Unterforderung, entstandene[n] Streßreaktion“ (Sonneck/Fengler ${ }^{2} 2009$ : 104), etc.; alle Belege im QV unter 8.1.2. 
Diagnose sei, oder man findet einzelne Belege des Satzmusters Burnout ist nicht/ kein(e) $x$, sondern ...) in den Lexika, Hand- und Lehrbüchern. ${ }^{486}$ Die Negationsmittel dienen in der Regel vielmehr dazu, einzelne Symptome des Sachverhalts/Begriffs $>$ BURNOUT`zu spezifizieren. Dadurch erfüllen die Negationsmittel eine ähnliche Funktion wie die sprachlichen Mittel der Graduierung. Sie grenzen das Verhalten bzw. Erleben, das Personen mit >Burnout ‘ zeigen, von 'normalem' >Verhalten/ Erleben` ab und umgrenzen den Begriff `Burnout dadurch weiter. So beispielsweise, wenn das Symptom >Depersonalisierung، wiederholt über privative, mit Negationsaffixen/-suffixenversehene Adjektivebeschrieben wird (gefühllos, herzlos, nicht-mitfühlende, Bsp. 3, 6, unmenschlich (Gerrig/Zimbardo ${ }^{20} 2015$ : 500)), und zwar im Zusammenhang mit dem Verhalten, das die ,Betroffenen“ ihren „Klienten, Kunden oder Patienten“ gegenüber zeigen. ${ }^{487}$ Dieses Symptom lässt sich nur vor dem Hintergrund einer anthropologischen und/oder berufskulturellen Norm verstehen, dass Personen, die in Sozial- und Pflegeberufen arbeiten, Mitgefühl zeigen sollten. Es steht dem Konzept der eigentlich 'üblichen' >teilnehmenden Fürsorge، für eine auf Hilfe angewiesene Person gegenüber. ${ }^{488}$ Die ,Betroffenen' haben sich oder wurden von den Personen, um die sie sich kümmern sollen, wegbewegt, wie das Wort De-personalisierung/De-humanisierung durch das Affix $d e^{-489}$ und die Markierung mit der Endung -ierung ${ }^{490}$ andeutet. Diese berufskulturellen und anthropo-

486 So schreibt Barth ( $\left.{ }^{4} 2010\right)$ im Handwörterbuch Pädagogische Psychologie: „Burnout tritt nicht als Folge vereinzelter traumatischer Erlebnisse auf, sondern als schleichende seelische Auszehrung“ (Barth ${ }^{4} 2010$ : 83, Unterstreichung T.S.). Ein weiterer Beleg, in dem ein Negationselement mit dem substitutiven mehrteiligen Konnektor nicht nur, sondern auch verbunden wird, definiert >Burnout als Gesamtkonzept zwar nicht negativ, grenzt es über diese Konstruktion aber vom sachverwandten Begriff `Stress ab: „Neben der Gemeinsamkeit zwischen Burnout und Streß bestehen also auch deutliche Unterschiede insofern, als mit dem Grad der persönlichen Erfüllung und der Depersonalisation nicht nur unmittelbare Folgen von Arbeitsbeanpsruchungen benannt sind, sondern auch Beziehungen zwischen den Motiven und Wertbereichen der Arbeitenden und ihrer Arbeitstätigkeit (Enzmann 1994)“ (Richter/Hacker 1998: 144f., Unterstreichungen T.S.).

487 Weitere Beispiele: „gefühlsarme ... Reaktion“ (Richter/Hacker 1998: 144), „,negative Haltungen sowie zynische und dehumanisierende Reaktionen“ (Gusy/Kleiber 1998: 317); „Gefühllosigkeit“ (Wegge 2004: 729); Dieses Wortbildungsmuster findet sich außerdem in der Beschreibung weiterer Merkmale des Burnout-Zustands: „Erfolg- und Machtlosigkeit“ (Sonneck/Fengler ${ }^{2}$ 2009: 104); alle Belege im QV unter 8.1.2.

488 Nach Leisi (1961: 43) zeichnen sich privative Adjektive dadurch aus, dass sie die „Abwesenheit von Dingen oder Eigenschaften im Gegensatz zum Normalen“ bezeichnen.

489 Lat. 'weg - von'.

490 Substantive auf -ierung sind aus Verben auf -ieren gebildet. Vgl. den Eintrag auf Duden online zu -ieren: „drückt in Bildungen mit Adjektiven - seltener mit Substantiven - aus, dass eine Person oder Sache in einen bestimmten Zustand gebracht, zu etwas gemacht wird“. Online einsehbar unter: https://www.duden.de/rechtschreibung/_ieren (zuletzt eingesehen am 12.12.2019). 
logischen Normen, die als Referenzwerte hinter den Definitionen stehen, werden ebenfalls in Kap. 6.2.4.1 eingehender analysiert.

Betrachtet man Tempus und Modus der finiten Verben in den vorliegenden Definitionen, so stehen diese überwiegend im Präsens Indikativ. Das Präsens ist nach Weinrich zum „besprechenden Tempus-Register“ zu zählen (Weinrich 32005: 198f.). Im Indikativ wird „ein fester Geltungsanspruch erhoben“ (ebd.: 199), die Grundinformation und im Fall der Definitionen damit auch die aufgestellten Äquivalenzbeziehungen werden dadurch hinsichtlich ihres Geltungsanspruchs nicht metainformativ eingeschränkt (Köller 2004: 448f.). Dieser Modus stützt den Anspruch allgemeiner Gültigkeit und Validität, der von Definitionen erwartet wird (vgl. Kap. 4.2.2).

In der Definition von Schaper ( ${ }^{3} 2014$ ) (siehe Beleg 6 oben) findet sich im zweiten Satz der Definition allerdings ein konkreter Hinweis, dass die dargelegten Aussagen auf einer Mehrheitsmeinung beruhen (Es wird meist als ein Syndrom ... ). Damit wird die Geltung des Folgenden einerseits eingeschränkt, indem gesagt wird, dies sei die Ansicht der meisten Fachleute, aber nicht aller. Andererseits wird die Geltung der Definition durch diesen gewissermaßen intertextuellen Bezug auf mehrheitliche konsensuale Stimmen ${ }^{491}$ im fachlichen Diskurs aber auch gestützt. ${ }^{492}$ In der Definition im Dorsch aus dem Jahr 1993 findet sich kein Hinweis auf der Sprachoberfläche, der die Gültigkeit der Definition einschränkt, im Gegenteil, sie wird durch den Verweis es gibt einen standardisierten Fragebogen als Teil statistisch-validierter Forschung ausgegeben; im Pschyrembel hingegen wird das Symptom Depersonalisierung über das abgekürzte Adverb evtl. als genuiner Bestandteil der Definition in Frage gestellt. Deutlich abgesetzt von den anderen Belegen ist Beispiel 7, der Wörterbucheintrag von Burisch in der 16. Auflage im Dorsch aus dem Jahr 2013. Die Allgemeingültigkeit bisheriger Burnout-Definitionen wird über verschiedene Mittel in Frage gestellt: So werden verschiedene Ansprüche, die von (natur)wissenschaftlicher Warte aus an Definitionen gestellt werden, verneint: Die Phaseneinteilung sei „rein intuitiv“, die Beschreibung der Symptomatik „nicht einheitlich“, „Normen fehlen“ bei den Skalen, die Burnout messen und „die unscharfe Abgrenzung“ trage dazu bei, „dass B. in der ICD-10 (International Classification of Diseases (ICD)) nur als (nicht kassenfähige) Zusatzdiagnose Z73.0“ auftauche. Burisch verwendet hier ein Sprachhandlungsmuster, das Busch (2006: 61) als gängiges Muster des semantischen Kampfs im Wissensbereich Medizin herausgearbeitet hat: Mit Bezug auf die im Diskurs formulierten wissenschaftlichen Kriterien des Definierens (vgl. 4.2.3) wirft

491 Vgl. dazu die Analyse polyphoner Strukturen bei Angermüller/Scholz (2013: 306.f.) sowie Kap. 4.1.2 dieser Arbeit.

492 Vgl. das Konzept der „transkriptive[n] Bezugnahmeformen“ bei Jäger (2012b: 16) sowie Kap. 4.1.2 dieser Arbeit. 
Burisch den Autoren/Autorinnen dieser Definitionen „[M]ethodologische Fehler“ vor uns spricht ihren Definitionen damit die „Wissenschaftlichkeit“ ab (vgl. Busch 2006: 61). Auf weitere sprachliche Mittel, die definitorische Ansprüche konterkarieren und dadurch den Geltungsanspruch einschränken und auf semantische Kämpfe hinweisen, wird in Kap. 7.3 eingegangen.

Weitere Sprachmittel, welche die Geltung der vermittelten Grundinformation verallgemeinern und damit stützen, sind die Gattungs- und Sammelbezeichnungen (professionelle Helfer, Hilfesuchende, Erwerbstätige mit Burnout-Syndrom, Betroffene, Klienten, Kunden, Patienten, ...) oder auch das unpersönliche Pronomen man (in Beispiel 2 und 4). In diesen Definitionen agieren nicht Individuen miteinander, sondern Personen- bzw. Risikogruppen und bei diesen Personengruppen werden bei gleichen äußeren Rahmenbedingungen (bei der Arbeit, in Sozial- und Pflegeberufen, Arbeitnehmer mit entsprechenden Berufen, ...) die besagten Symptome als 'typisch' konstatiert. Der beschriebene Zustand/Prozess wird so als verallgemeinerbares individuelles Erlebnis und damit als medizinischpsychologisches „Legizeichen“ konstituiert (vgl. Kap. 2.2.2 und Peirce CP 8.335 ${ }^{493}$ ). Durch die Angabe von typischen ,Betroffenen'-Gruppen und deren charakteristischen Merkmalen wird das Phänomen/Konzept zudem weiter kategorisiert und spezifiziert. In den Lexika-, Hand- und Lehrbüchern werden beispielsweise folgende Personengruppen auch durch sprachliche Marker (wie besonders, oft, vor allem ...) als besonders ,betroffen“ hervorgehoben: „Es [Burn-out] wird oft von Menschen erlebt, die in Berufen arbeiten, die ein hohes Maß an sehr intensivem persönlichen Kontakt mit Patienten/-innen, Klienten/-innen oder der Öffentlichkeit erfordern“ (Gerrig/Zimbardo ${ }^{20} 2015$ : 500); „Der Begriff Burnout [...] bezeichnet ein Syndrom, von dem vor allem Angehörige von Sozialberufen betroffen sind“ (Schüpbach/Kause: 2009: 505); „Burnout-Effekte werden häufig bei Personen beobachtet, die besonders engagiert ihre Arbeit verrichten“ (Kirchler ${ }^{3} 2011$ : 294).

Weitere Validierungsverfahren, welche die Definition dadurch stützen, dass sie auf impliziten Regeln beruhen, die im medizinisch-gesundheitlichen Bereich die Geltung eines Sachverhalts stützen können, werden in den Kap. 6.2.4.2 behandelt.

Zusammenfassend kann man daher folgern, dass die Definitionen in den Fachlexika, Hand- und Lehrbüchern „Realdefinitionen“ darstellen (vgl. Kap. 4.2.3). Damit ist gemeint, dass sich diese Definitionen nicht darauf beschränken, eine Bedeutungserklärung innerhalb eines terminologischen Systems für den Begriff/das Konzept `Burnout` zu geben, sondern die Definitionen sollen dazu dienen, repräsentatives

493 Vgl. dazu folgendes Zitat von Peirce: „I define an Index as a sign determined by its dynamic object by virtue of being in a real relation to it. Such is a Proper Name (a legisign); such is the occurrence of a symptom of a disease. (The symptom itself is a legisign, a general type of a definite character. The occurrence in a particular case is a sinsign.)" (CP 8.335, Unterstreichung T.S.). 
Wissen über den außersprachlichen Sachverhalt BuRNOuT unter der Bezeichnung Burnout zu verbreiten. ${ }^{494}$ Diese Definitionen werden vor einem ontologischen Denkhorizont formuliert, der von den Definitionen eine Aussage über einen Sachverhalt im „Reich der Wirklichkeit“ erwartet (vgl. Felder 2006a: 167). Das bestätigen auch die in den obigen Beispielen beschriebenen Skepsis-Signale gerade dadurch, dass dort im Hinblick auf eine unterstellte unmittelbar gegebene Beobachtungsebene in Zweifel gezogen wird, ob das Symptom Depersonalisierung ,wirklich“ oder nur „evtl.“ dazugehört (Pschyrembel ${ }^{261} 2007=$ Bsp. 4) und ob man mit dem neuen Begriff/ Konzept >Burnoutı auf Sachverhalte referiert, die in anderen „Maßen beeinträchtigten Befindens“ noch nicht konzeptualisiert sind (Zapf/Semmer $2004=$ Bsp. 5). Die ausdrucksseitigen Objektivierungsformen und deren etwaige Einflüsse auf die Konstituierung des Begriffs werden hingegen nicht reflektiert. ${ }^{495}$

Fachkulturell vertreten diese Textsorten das Ideal größtmöglicher Neutralität und Unabhängigkeit von der Subjektivität des Verfassers/der Verfasserin, was auch im unpersönlichen und komprimiert gehaltenen Formulierungsstil zum Ausdruck kommt.

Man findet in diesen untersuchten Stellen demnach von der Morphem- bis hin zur Textebene zahlreiche sprachliche Mittel, die das Ziel von Definitionen, einen Gegenstand allgemeingültig bzw. kollektiv akzeptiert einzugrenzen und festzusetzen, in der Zusammenschau bewirken. Einige dieser Mittel, wie z. B. die sprachlichen Mittel der Graduierung, entfalten diese eingrenzende Wirkung insbesondere bei Gegenständen, die nicht absolut, sondern nur graduell definiert werden können. Sachverhalte der Medizin fallen genuin in diesen Bereich (vgl. Kap. 3.2 dieser Arbeit). Alle, die mit klinischen Phänomenen zu tun haben, treffen nach Fleck ([1927] 1983) ,auf einen gewaltigen Reichtum und Individualität dieser Phänomene, die die Vielheit ohne klare, abgegrenzte Einheiten begleiten, voller Übergangs- und Grenzzustände.“

Im Folgenden werden nun im Vergleich die sprachlichen Mittel des Definierens, die sich in den Textsorten der Fachzeitschriften und Sammelbände zeigen, analysiert (Kap. 6.2.2.2). Auf dieser Basis erfolgt eine tabellarische Zusammenführung der sprachlichen Mittel des Definierens im fachlichen Diskursstrang in Kapitel 6.2.2.4.

494 Zum Konzept der `Wissensqualitätı und dem Aspekt des repräsentativen Wissens vgl. Zifonun/Hoffmann/Strecker (1997: 618f.) sowie Felder (2006a: 169).

495 So wird in keiner dieser Definitionen die Metapher des Brennens/Ausbrennens und damit einhergehende Bedeutungsaspekte (z. B. dass diese Prozessverben kein Agens benötigen) reflektiert. Vgl. Bär (2015: 178; 183) und Kap. 4.2.0 dieser Arbeit zur Unterscheidung von Begriff und Konzept. 


\subsubsection{In Fachzeitschriften, Fachbüchern und Sammelbänden}

In Kap. 5.3.3.2 wurden die Texte in Fachzeitschriften, Fachbüchern und Sammelbänden varietätenlinguistisch eingeordnet. Zusammenfassend sind dies schriftliche Texte, die sich vorwiegend von Fachleuten an Fachleute derselben Unter- oder Dachdisziplin und teilweise angrenzender Fächer richten und dadurch einen (mittel-)hohen Fachlichkeitsgrad aufweisen. Die Textfunktion von Übersichts- und Einleitungstexten ist informativ-orientierend. Originalarbeiten und Kasuistiken sind spezifischer ausgerichtet und bezwecken hauptsächlich einen Erkenntniszuwachs in Bezug auf konkrete Fragestellungen. Berichte über Umfragen ${ }^{496}$ und „ratgebende Aufklärungstexte“497 in Fachzeitschriften richten sich ebenfalls vorwiegend an Fachkolleginnen und -kollegen, ebenso wie Buchrezensionen.

Ähnlich wie bei den Handbuch- und Lehrbuchtexten gibt es auch in Fachzeitschriftenartikeln explizites Reflektieren über Definitionen und Hinweise auf definierende Passagen dadurch, dass das Kapitel mit Definition überschrieben ist oder im Fließtext selbst die Ausdrücke definieren/Definition oder syntaktische Muster verwendet werden, die den Ausdruck Burnout inhaltlich bestimmen. Die definierenden Kapitel und Passagen befinden sich überwiegend am Anfang der Fachartikel (z. B. in den Abstracts, in den einleitenden Kapiteln). ${ }^{498}$ Die Anfangsposition ist vor dem Hintergrund, dass Definitionen zur Festsetzung von Begriffen dienen, nicht verwunderlich. Fachartikel erläutern in der Regel zu Beginn die Begriffe/Konzepte, mit denen sie im weiteren Verlauf arbeiten.

Im Folgenden werden zehn Beispiele verschiedener Textsorten aus den Fachzeitschriften/Monografien nacheinander dargestellt, auf die sich die folgende Analyse der sprachlichen Mittel des Definierens in diesem Diskursstrang bezieht. Die Beispiele wurden so ausgewählt, dass sie die Bandbreite verschiedener Textsorten und Spezialfächer, die sich mit BuRnout befassen, widerspiegeln. Des Weiteren werden zu Beginn zwei Beispiele aus dem frühen englischen Fachdiskurs zitiert, auf die sich die Texte im fachinternen Diskursstrang und auch die hier aufgeführten Beispiele häufig rückbeziehen. Weitere Belege aus dem Korpus werden an den jeweiligen Stellen in Fußnoten angeführt.

496 Zum Beispiel Dieke/Schmidt/Katzer (2002) oder Will (2014) im Dt. Ärztebl. oder Weiß (2013) in der PPmP im QV unter 8.1.4.

497 Die Textsorte „ratgebender Aufklärungstext“ wird in Kapitel 5.3.3.4 eingehend beschrieben. Beispiele aus dem fachlichen Korpus sind: Freudenberger (1974, im QV unter 8.1.5), Madel (2003) im Dt. Ärztebl. oder Bergner (2016) in der DMW (beide im QV unter 8.1.4).

498 Die Anfangskapitel, in denen sich die Autoren/Autorinnen mit definitorischen Fragen auseinandersetzen, sind mit „Fragestellung“, „Einleitung“ überschrieben oder haben keine Überschrift. In folgenden Texten aus dem Korpus findet man dies beispielhaft: Reime/Steiner (2001: 304) in der PPmP; Schmitz (2001) und Neuenschwander (2003: 210) in PiEU (alle Belege im QV unter 8.1.4); Schuster (2010) in der Zeitschrift Verhaltenstherapie, im QV unter 8.1.5. 
Beispiel 1 ist ein Auszug aus dem häufig als Gründungstext titulierten Beitrag von Freudenberger aus dem Jahr $1974 .^{499}$ Der Text beginnt mit einer Frage, die eine Definition herausfordert. Freudenberger beantwortet diese Frage, indem er die Bedeutungsangaben eines Wörterbuchs zum Verb burn-out auf Erlebnisse im Arbeitsalltag der ehrenamtlichen Einrichtung überträgt. Daraufhin zählt er Einzelsymptome auf, wiederum als Beantwortung spezifizierter Fragen (What are the behavioral signs?). Im weiteren Verlauf des Textes gibt Freudenberger Ratschläge, wie man als betroffene Einrichtung mit dem Problem >BURN-OUT< umgehen oder ihm vorbeugen kann.

1a) Freudenberger (1974: 159) (Unterstreichungen T.S., im QV unter 8.1.5):

\section{WHAT IS BURN-OUT?}

The dictionary defines the verb "burn-out" as "to fail, wear out, or become exhausted by making excessive demands on energy, strength, or resources." And that is exactly what happens when a staff member in an alternative institution burns out for whatever reasons and becomes inoperative to all intents and purposes. [...]

[...]

\section{THE PHYSICAL SignS}

The physical signs are easy to spot. For one, there is a feeling of exhaustion and fatigue, being unable to shake a lingering cold, suffering from frequent headaches and gastrointestinal disturbances, sleeplessness and shortness of breath. In short, one becomes too somatically involved with one's bodily functions.

\section{WHAT ARE THE BEHAVIORAL SIGNS?}

A staff member's quickness to anger and his instantaneous irritation and frustration responses are the signs. The burn-out candidate finds it just too difficult to hold in feelings. He cries too easily, the slightest pressure makes him feel overburdened and he yells and screams. With the ease of anger may come a suspicious attitude, [...]. He may resort to an excessive use of tranquilizers and barbiturates. [...]

He becomes excessively rigid, stubborn, and inflexible. [...] He becomes the "house cynic." [sic] Anything that is suggested is bad rapped or bad mouthed. [...]

The person looks, acts and seems depressed. [... $]^{500}$

Dass dieses Frage-Antwort-Muster, das Freudenberger verwendet, auch von späteren Autoren übernommen und explizit mit dem Akt des Definierens verbunden wird, zeigt folgendes Beispiel (siehe den Hinweis „Definition“ in Abbildung 8 am Rand):

$499 \mathrm{Zu}$ den Rückverweisen auf H. Freudenberger als `Entdeckerfigur` des Burnout-Phänomens vgl. Kap. 6.1.1.

500 Ähnlich Freudenberger (1975: 73, im QV unter 8.1.5). 
1b) Seibold (Litzcke)/Schuh (52010: 157) (im QV unter 8.1.5):

\subsection{Was ist Burn-out?}

In der englischen Umgangssprache bedeutet Burn-out so viel wie: Man Definition ist so erschöpft, dass man nichts mehr tun oder geben kann (engl.: to burn out $=$ ausbrennen). Der amerikanische Psychoanalytiker Freudenberger (1974) führte den Begriff »Burn-out« in die Psychologie ein. Er stellte bei hochmotivierten Sozialarbeitern in alternativen Organisationen fest, dass sie auffallig oft etwa ein Jahr nach Arbeitsaufnahme psychisch geradezu zusammenbrachen.

Abb. 8: Auszug aus der Monografie von Seibold (Litzcke)/Schuh (52010: 157). Reprinted/ adapted by permission from Springer Nature: Springer eBook, Das Burn-out-Syndrom by Sven Max Litzcke [Seibold], Horst Schuh, ( 2010.

Das zweite Beispiel ist ein Auszug aus einer Übersichtsarbeit von Maslach/Jackson (1984) in der Buchreihe „Applied Social Psychology Annual“ zu ihrer eigenen bisherigen Burnoutforschung in den USA Mitte der 1980er Jahre. Der Beitrag ist deshalb für die vorliegende Arbeit bedeutsam, da die Autorinnen darin eine Definition des Begriffs `Burnout geben, deren triadische Symptomstruktur sowohl in den Fachlexika, Hand- und Lehrbüchern (siehe Kap. 6.2.2.1) als auch in vielen Fachartikeln und Veröffentlichungen im fachexternen Diskurs aufgegriffen wird:

2) Maslach/Jackson (1984: 134) (Unterstreichungen T.S., im QV unter 8.1.5):

We have defined burnout as a syndrome of emotional exhaustion, depersonalization, and reduced personal accomplishment that can occur among individuals who work with people in some capacity. Emotional exhaustion refers to feelings of being emotionally overextended and drained by one's contact with other people. Depersonalization refers to an unfeeling and callous response toward these people, who are usually the recipients of one's service or care. Reduced personal accomplishment refers to a decline in one's feelings of competence and successful achievement in one's work with people. This definition of burnout, which is now being used widely in ongoing research, was not based on a theoretical model but was derived empirically. Several years of earlier exploratory research provided the groundwork for the development of both our conceptual definition and our standardized measure..$^{501}$

Das dritte Beispiel entstammt dem Beginn der Einleitung zur ersten umfassenden deutschsprachigen internationalen Bibliografie zum Thema „Burnout“ von Kleiber/Enzmann (1990: 11). Der Beitrag nimmt teilweise Elemente von Freudenberger (1974) (Beleg 1, siehe oben) auf. Manche Symptome von Freudenberger (1974) überschneiden sich auch mit den Symptomen von Maslach/Jackson (1984)

501 Diese Definition in ähnlicher Form findet man z. B. auch bei Maslach/Jackson (1981); Maslach (1993); Maslach/Jackson/Leiter ('1996), siehe im QV unter 8.1.5. 
(Beleg 2, siehe oben), so werden im folgenden Beispiel die Symptome ERschöPFUNG und ZYNISMUS genannt, die auch in den obigen Belegen beschrieben werden:

3) Kleiber/Enzmann (1990: 11) (Unterstreichungen T.S., im QV unter 8.1.5):

Mit dem Begriff Burnout werden in der psychologischen Literatur vorrangig negative Folgen von Arbeitsbeanspruchungen psychosozialer Berufsgruppen thematisiert. Der Begriff hat Ende der 70er und Anfang der 80er Jahre zuerst im Bereich der helfenden Berufe in den USA in kurzer Zeit eine große Verbreitung gefunden. [...] Als eigentlicher Beginn der Burnoutdiskussion in der Psychologie gilt jedoch ein Artikel von Freudenberger (1974), in dem Burnout als ein Phänomen beschrieben wird, bei dem aufopferungsvolle, pflichtbewußte und ehemals besonders engagierte Mitarbeiter - vorwiegend aus alternativen Selbsthilfe- oder Kriseninterventionsstationen - beginnen, körperliche Symptome von Erschöpfung und Müdigkeit zu zeigen, sie zu reizbaren, mißtrauischen, halsstrarrigen Mitarbeitern werden und eine negative und zynische Einstellung zu ihrer Arbeit und den Klienten entwickeln, was zugleich mit einer depressiven Symptomatik einhergeht. ${ }^{502}$

Beispiel 4 ist eine Definition aus dem englischsprachigen Überblickswerk „The burnout compendium to study and praxis: a critical analysis“ von W. Schaufeli und D. Enzmann. Dieses Kompendium ist im Jahr 1998 erschienen mit dem Anspruch, den Status quo der Burnout-Forschung umfassend zusammenzustellen. Die Autoren führen in ihrem Werk zunächst an, dass die Gefahr besteht, dass über $\mathrm{zu}$ weite Symptomlisten die eigentliche Bedeutung des Burnoutkonzepts verlorengeht. $^{503}$ Sie präsentieren ein Fallbeispiel eines Lehrers (ebd.: 20), ordnen Symptome verschiedenen Symptomlisten (,affective“, „cognitive“, „physical“, „behavioral“, ...) zu (ebd.: 21ff.), diskutieren verschiedene Definitionen anderer Autoren/Autorinnen, die sie in Zustands- und Prozessdefinitionen einteilen (ebd.: 31ff.), und fassen deren Gemeinsamkeiten zusammen. ${ }^{504}$ Bei den Zustandsdefini-

502 Ähnliche Übersichtsartikel aus den späten 1980er und 1990er Jahren sind Enzmann/Kleiber (1989); Büssing (1992) und Richter/Hacker (1998) aus dem Bereich Arbeits- und Organisationspsychologie, Dittmann-Kohli/Sowarska/Timmer (1997) aus dem Bereich Pädagogische Psychologie; ausführliche deutschsprachige Monografien zum Stand der Burnout-Forschung sind Burisch (1989; $\left.{ }^{2} 1994\right)$ und Rook (1998). In diesen Monografien wird in den Unterkapiteln zur Definition des Burnout-Konzepts eine etwas breitere Übersicht über verschiedene Definitionen als in den Zeitschriftenübersichtsartikeln angegeben. Siehe auch das nächste Beispiel von Schaufeli/ Enzmann (1998, Beleg 4); siehe die Belege im QV unter 8.1.2 und 8.1.5.

503 Schaufeli/Enzmann (1998: 19): “Myriad possible burnout symptoms and definitions exist. The more or less exhaustive list of burnout symptoms that is presented in the first part of this chapter shows that the concept can easily be expanded to mean anything, so that there is the danger that in the end it does not mean anything at all."

504 Ein ähnliches Vorgehen der Darstellung verschiedener Definitionen und einer abschließenden Zusammenfassung findet man z. B. bei Rösing (2003: 58ff.); Seibold/Schuh (2010: 161); 
tionen präsentieren sie Maslach/Jackson ( $\left.{ }^{2} 1986\right)$ als „probably most cited definition of burnout“ (ebd.: 31), Pines/Aronson (1988) als „slightly broader definition“ (ebd.: 32). Bei den Prozessdefinitionen heben sie zum Beispiel die auch in anderen Überblickswerken häufig erwähnte Definition von Cherniss (1980a/1980b) und eine Definition nach Edelwich/Brodsky hervor. ${ }^{505}$ Auf dieser Basis weisen sie auf die Schwierigkeit hin, eine „consensus definition“ $\mathrm{zu}$ formulieren (ebd.: 36). Dennoch finden sie es wichtig, eine eigene Arbeitsdefinition, die auf den dargelegten Symptomen und Definitionsvorschlägen basiert, aufzustellen (ebd.).:

\section{4) Schaufeli/Enzmann (1998: 36) (Unterstreichungen T.S.):}

Our definition is based on the review of burnout symptoms, as well as on the previous state and process definitions:

Burnout is a persistent, negative, work-related state of mind in 'normal' individuals that is primarily characterised by exhaustion, which is accompanied by distress, a sense of reduced effectiveness, decreased motivation, and the development of dysfunctional attitudes and behaviours at work. This psychological condition develops gradually but may remain unnoticed for a long time by the individuals involved. It results from a misfit between intentions and reality in the job. Often burnout is self-perpetuation because of inadequate coping strategies that are associated with the syndrome.

Diese aus Vorgängerdefinitionen hervorgegangene Arbeitsdefinition von Schaufeli/Enzmann (1998) wird im Anschluss an ihre Veröffentlichung von einigen Autorinnen und Autoren aufgenommen, so z. B. in späteren Übersichtswerken/-artikeln (von Rösing in eigener Übersetzung (2003: 65, im QV unter 8.1.5); in deutscher Übersetzung von Burisch (52014: 22, im QV unter 8.1.5) ${ }^{506}$; Weimer/Kraus (2011: 240,

Rook (1998: 109); Weimer/Kraus (2011: 240); siehe alle Belege im QV unter 8.1.5; Beschoner/ Schönfeld-Lecuona/Braun et al. (2009) in PiD; Bauer/Häfner/Kächele et al. (2003: 214) in PPmP 53 (5), im QV unter 8.1.4.

505 Die Publikationen von Cherniss werden bspw. im Rahmen der Forschungsliteratur erwähnt bei Künzel/Schulte (1986: 303); Enzmann/Kleiber (1989: 41ff.); Büssing (1992: 43); Burisch (1989, $\left.{ }^{2} 1994,{ }^{3} 2006,{ }^{5} 2014\right)$; Burke/Greenglass/Schwarzer (1996: 262); Rook (1998: 44ff.; 109); Seibold/ Schuh (52010: 160f.); alle Belege im QV unter 8.1.5; Bauer/Häfner/Kächele et al. (2003: 214) und Söllner/Behringer/Böhme et al. (2016) in PPmP; Beschoner/Schönfeldt-Lecuona/Braun et al. (2009) in PiD, im QV unter 8.1.4. Edelwich \& Brodsky werden z. B. bei Demerouti/Nachreiner/ Bakker (2001, im QV unter 8.1.5) und Bergknapp (2009, PiD, im QV unter 8.1.4) referiert.

506 Die Übersetzung von Burisch (52014: 22) lautet: „Burnout ist ein dauerhafter, negativer, arbeitsbezogener Seelenzustand ,normaler` Individuen. Er ist in erster Linie von Erschöpfung gekennzeichnet, begleitet von Unruhe und Anspannung (Distress), einem Gefühl verringerter Effektivität, gesunkener Motivation und der Entwicklung dysfunktionaler Einstellungen und Verhaltensweisen bei der Arbeit. Diese psychische Verfassung entwickelt sich nach und nach, kann den betroffenen Menschen aber lange unbemerkt bleiben. Sie resultiert aus einer Fehlanpassung 
im QV unter 8.1.5)), im HTA-Bericht zur „Differentialdiagnostik des BurnoutSyndroms“ (Korczak/Kister/Huber 2010: 14, ebenfalls in der Übersetzung von Burisch ${ }^{5}$ 2014: 22, im QV unter 8.1.5) sowie von den Autoren des Messinstruments „Copenhagen Burnout Inventory“ (siehe Beispiel 6b).

Beispiel 5 ist einer Originalarbeit von Büssing/Schmitt entnommen, die 1998 in der Zeitschrift für Arbeits- und Organisationspsychologie (ZfAO) erschienen ist. Die Autoren befassen sich im ersten Kapitel mit verschiedenen wissenschaftlichen Burnout-Konzepten, stellen heraus, in welchen Punkten sich diese überschneiden, und geben an, dass sie sich bei ihrer folgenden Analyse zu Arbeitsbelastungen im Burnoutprozess auf die Definition von Maslach/Jackson (siehe oben Beispiel 2) stützen:

5) Büssing/Schmitt (1998: 76/77) (Unterstreichungen T.S., im QV unter 8.1.4, ZfAO):

Einleitung [fett gedruckt als Kapitelüberschrift, T.S.]

Der Begriff Burnout wurde 1974 von Freudenberger eingeführt. Er bezeichnete damit ein Syndrom, das besonders engagierte und pflichtbewußte Helfer in Sozialberufen zeigen, und das zum einen durch körperliche Symptome von Müdigkeit, Gereiztheit, Unausgeglichenheit und zum anderen durch eine mißtrauische und negativ zynische Grundstimmung gegenüber Kollegen, Klienten und der eigenen Arbeit gekennzeichnet ist, die oftmals mit depressiver Verstimmtheit einhergeht. Vor allem Maslach und Jackson (1981) sowie Cherniss (1990) entwickelten im weiteren [sic] wissenschaftliche Konzepte für das Phänomen, die in der Sozialpsychologie und in der Klinischen Psychologie verwurzelt sind und die Eingang in weitere psychologische Teildisziplinen, wie z. B. in die Arbeits- und Organisationspsychologie gefunden haben.

Den verschiedenen Modellen von Burnout liegen unterschiedliche Definitionen des Phänomens zugrunde. Konsens besteht darin, daß Burnout als eine spezifische Beanspruchungsfolge bei helfenden Berufen (bzw. allgemeiner bei personenbezogenen Dienstleistungsberufen) betrachtet werden kann, für die eine Auseinandersetzung mit Belastungen im Beruf und besondere Erwartungen sowie eine besondere Identifikation mit dem Beruf charakteristisch sind (Büssing \& May, 1997; Cherniss, 1993). [...]

Die Definition, die in dieser Arbeit Anwendung findet, stammt von Maslach und Jackson 1982, 1984). [...].

Ähnliche Originalarbeiten und Studien, die sich mit verschiedenen BurnoutKonzepten befassen oder die Güte der Burnout-Definition (kritisch) reflektieren und sich dann für die Durchführung der eigenen Studie für ein Messinstrument entscheiden, in diesen Fällen für die Definition nach Maslach/Jackson und das MBI (teilweise neben anderen damit kombinierten Messinstrumenten), sind z.B.: das

von Intentionen und Berufsrealität. Burnout erhält sich wegen ungünstiger Bewältigungsstrategien, die mit dem Syndrom zusammenhängen, oft selbst aufrecht.“ 
Phasen-Modell von Golembiewski/Munzenrider (1996, im QV unter 8.1.5), dem das MBI zugrunde liegt, Gamsjäger/Sauer (1996), Stöckli (1999), Schmitz/Leidl (1999), Schmitz (2001), Neuenschwander (2003) - alle in PiEU (im QV unter 8.1.4); Dormann/Zapf/Isic (2002), Neubach/Schmidt (2004), Freund/Diestel/Schmidt (2012) - alle in ZfAO (im QV unter 8.1.4); Schuster (2010) in „Verhaltenstherapie“ (im QV unter 8.1.5); Stoeber/Rennert (2008) in Anxiety, Stress \& Coping (im QV unter 8.1.5); Gumz/Brähler/Erices (2012); Gumz/Erices/Brähler et al. (2013); Bugaj/ Müksch/Ehrental et al. (2016) in PPmP (im QV unter 8.1.4); Stier-Jarmer/Frisch/ Oberhauser et al. (2016) und Schneider/Hilbert/Hamann et al. (2017) im Dt. Ärzteblatt (im QV unter 8.1.4); Kinzl/Traweger/Biebl et al. (2006) in der Deutschen Medizinischen Wochenschrift; Burke/Greenglass/Schwarzer (1996) und Schwarzer/Schmitz/Tang (2000) in „Anxiety, stress \& coping“ (im QV unter 8.1.5).

Die folgenden zwei Beispiele werden unter der Rubrik „operationale Definition“ zusammengefasst, da es in den folgenden Beispielen entweder um eine Überprüfung bestehender Messinstrumente oder um Weiterentwicklungen bzw. neue Messinstrumente zum Konzept bzw. Begriff >Burnout geht. Messinstrumente in Form von Fragebogen müssen das, was sie messen möchten, vorher definiert haben. Aus diesem Grund stellen die verschiedenen Messinstrumente an sich schon operationale Definitionen des Konzepts `Burnout « dar.

Beispiel 6a) ist ein Auszug aus der deutschen Übersetzung des Maslach Burnout Inventory ${ }^{507}$ von Büssing/Perrar (1992) in der Zeitschrift „Diagnostica“:

\section{6a) Büssing/Perrar (1992: 336f.), (im QV unter 8.1.5):}

Die deutsche Fassung lehnt sich eng an die von Maslach und Jackson (1981) vorgegebenen Item-Inhalte sowie an ihre Skalierung an und erfaßt entsprechend der BurnoutKonzeptualisierung des MBI anhand von 25 Items die vier hypothetischen Konstrukte: (1) Emotionale Erschöpfung, (2) Persönliche Erfüllung und Leistung, (3) Depersonalisation und (4) Betroffenheit, [...]. [S. 332] [...]

1) Ich fühle mich durch meine Arbeit ausgebrannt.

2) Der direkte Kontakt mit Menschen bei meiner Arbeit belastet mich zu stark.

3) Den ganzen Tag mit Menschen zu arbeiten, ist für mich wirklich anstrengend.

507 Einige Jahre zuvor hatten Kleiber/Enzmann (1986: 66-67) in einem Artikel zum Thema „Helfer-Leiden: Überlegungen zum BURNOUT in helfenden Berufen“ ebenfalls schon eine deutsche Übersetzung vorgelegt. Die Übersetzung ist ähnlich, unterscheidet sich aber in der Wahl einzelner Ausdrücke, z. B. verwenden Kleiber/Enzmann das Wort Klienten statt Patienten und Item 4 lautet bei Kleiber/Enzmann (1986) z. B.: „Von den Problemen meiner Klienten bin ich persönlich berührt.“ Maslach/Jackson verwenden in einer späteren Fragebogen-Version ihres Messinstruments ebenfalls einen Ausdruck, der mehrere Dienstleistungsberufe umfasst, indem sie dort nicht mehr von patients, sondern von recipients sprechen (Maslach/Jackson/Leiter ${ }^{3} 1996$ ). 
4) Ich fühle mich von den Problemen meiner Patienten persönlich betroffen.

5) Ich glaube, daß ich manche Personen so behandle, als wären sie unpersönliche „Objekte“.

6) Ich fühle mich durch meine Arbeit emotional erschöpft.

7) Ich habe das Gefühl, daß ich durch meine Arbeit das Leben anderer Menschen positiv beeinflusse. [...]

[weiter bis Item 25]

(Büssing/Perrar 1992: 336f.)

Die Autoren übernehmen von Maslach/Jackson, dass die Items anhand einer „Häufigkeits- wie auch eine[r] Intensitätsantwortskala“ (Büssing/Perrar 1992: 332) beantwortet und jeweils verschiedenen Konzeptdimensionen (semotionale Erschöpfungs, 'persönliche Leistungsfähigkeit‘ etc.) zugeordnet werden. Sie kommen bei ihrer Überprüfung der Itemkennwerte, Faktorenstruktur, Reliabilität und Konstruktvalidität zu dem Ergebnis, dass das MBI Defizite im Bereich der Faktorenstruktur und internen Konsistenz einzelner Skalen aufweise (Büssing/Perrar: 347).

Stellvertretend für weitere Messinstrumente, die etwa zeitgleich zum MBI oder später entstanden oder das MBI (auf der Grundlage der Kritik an diesem) weiterentwickelten und als eigenständige Messinstrumente hervorgetreten sind, ${ }^{508}$ sei

508 Das „Tedium-Measure“, später „Burnout-Measure“, von Pines/Aronson/Kafry (1983, im QV unter 8.1.5) ist nach Kaschka/Korczak/Broich (2012: 783, Dt. Ärztebl.) nach dem MBI das am häufigsten verwendete Messinstrument. Ein aus dem MBI weiterentwickeltes Inventar ist das Oldenburg-Burn-out-Inventar (OLBI), siehe bei Demerouti (1999, im QV unter 8.1.5); Demerouti/ Bakker (2008, im QV unter 8.1.5). Toker/Shirom/Shapira et al. (2005: 350, im QV unter 8.1.5) stellen eine Parallele zwischen den Erschöpfungskomponenten des OLBI und den Items des Messinstruments „Shirom Melamed Burnout Measure (SMBM)“ her. Das Hamburg Burnout Inventar (HBI) geht auf Burisch zurück. Es enthält die drei Dimensionen des MBI und erweitert das Konzept um weitere sieben Dimensionen. Die Entwicklung wurde nach Burischs eigener Aussage seit Anfang der 1990er Jahre „vor allem in Form von unveröffentlichten Diplomarbeiten“ durchgeführt (Burisch ${ }^{5} 2014:$ 38). Ein technischer Bericht dazu, den Burisch dort zitiert, ist ebenfalls „unpublished“. Eine Beschreibung der Dimensionen findet man in der Dissertation von Weber (2014: 68f., im QV unter 8.1.5) und über eine Internetsuche zum Stichwort „Hamburg Burnout Inventar“ unter https://burnout-institut.eu/fileadmin/user_upload/HBI-Report_V1.pdf auf den Internetseiten des „Burnout Instituts Norddeutschland (BIND)“, das Burisch 2008 gründete und das auch kostenpflichtige Beratung nach Ausfüllen des hauseigenen Fragebogens anbietet: https://www.burnout-institut.eu/Burnout-Test.8.0.html (zuletzt eingesehen am: 13.12.2019). Ein im deutschsprachigen Raum neueres Fragebogenwerkzeug stellen die BOSS-Screening-Skalen von Hagemann/Geuenich (2009) dar (im QV unter 8.1.5), z. B. verwendet bei Scholz/Neumann/ Steinmann et al. (2015, PPmP, im QV unter 8.1.4). Des Weiteren gibt es das „Arbeitsbezogene Verhaltens- und Erlebnismuster (AVEM)“ von Schaarschmidt/Fischer (1997, im QV unter 8.1.5), das Überschneidungen zum Burnout-Konzept aufweist (z. B. verwendet oder ausführlich zitiert bei Bauer (2009, PiD); Klusmann/Kunter/Trauwein (2009, PiEU); Scholz/Neumann/Steinmann et al. (2015, PPmP), alle im QV unter 8.1.4. Ein auf den Sport zugeschnittenes Messinstrument ist 
hier das Copenhagen Burnout Inventory (CBI) von Kristensen/Borritz/Villadsen et al. (2005) aus der Zeitschrift „Work \& Stress“ zitiert, da man an diesem Beispiel gut erkennen kann, wie eng die Konstruktion eines Messinstruments und die Definition des zugrunde liegenden Konstrukts zusammenhängen. Kristensen/Borritz/Villadsen et al. entwickeln von der Kritik der Monopolstellung des MBI ausgehend das CBI. ${ }^{509}$ Am MBI kritisieren sie vor allem, dass das Messinstrument drei eigenständige Faktoren erfassen würde ("three distinct and different dimensions"), Maslach und Jackson in ihrer Burnout-Definition aber von einem gleichzeitigen Auftreten dieser drei Faktoren ausgehen würden ("simultaneous occurrence of all three dimensions”). Dieser Auszug aus dem CBI zeigt, dass bei operationalen Definitionen der Anspruch auf Adäquatheit zwischen Definiens und Definiendum (vgl. Kap. 4.2.2) deutlich zum Vorschein kommt. Im Anschluss an ihre Kritik erläutern Kristensen/ Borritz/Villadsen et al. mit Bezug auf und in Übereinstimmung mit anderen ebenfalls häufig zitierten Forscherinnen und Forschern des Burnout-Diskurses (sie zitieren z. B. Schaufeli/Enzmann 1998, siehe Bsp. 4 oben), welche Hauptmerkmale dem Burnout-Konzept für sie zukommen ("the core of burnout is fatigue and exhaustion”), welche Rolle die Metapher der „leeren Batterie“ (“the flat battery”) dabei für sie spielt und was den Unterschied zwischen ,einfacher‘ >Müdigkeit` ("fatigue”) bzw. `Erschöpfung` ("exhaustion”) und `Burnout` ausmacht.

6b) Kristensen/Borritz/Villadsen et al. (2005: 194; 196f.) (Unterstreichungen T.S., Kursivsetzungen im Original, im QV unter 8.1.5)

Unclear relationship between the MBI and the concept of burnout? At first glance the MBI and the Maslach definition of burnout seem to match each other perfectly. The definition (above) includes three dimensions - emotional exhaustion, depersonalisation, and reduced personal accomplishment - and so does the MBI. The operationalisation of burnout is, however, still unclear to us. According to the definition, burnout is characterized by the simultaneous occurrence of all three dimensions, but according to the MBI manual the three dimensions should be measured independently, and they have been confirmed by factor analyses as three distinct and different dimensions. [...]

der „Athlete Burnout Questionnaire (ABQ)“ von T.D. Raedeke, der in der Studie von Gustafsson/ Skoog (2012, im QV unter 8.1.5) verwendet wird und auf dem MBI basiert (vgl. ebd: 184). Eine Übersicht zu Messinstrumenten zum Konzept >Burnout ‘ findet man bei Burisch ( $52014: 35 \mathrm{ff}$.) und Korczak/Kister/Huber (2010: 20ff.); beide im QV unter 8.1.5.

509 "As a consequence of the dominant position of the MBI, this questionnaire and the Maslach definition of burnout have become two sides of the same coin: Burnout is what the MBI measures, and the MBI measures what burnout is” Kristensen/Borritz/Villadsen et al. (2005: 193). Studien, die das CBI verwenden, sind z. B.: Avanzi/Zaniboni/Balducci et al. (2014, im QV unter 8.1.5); Götze/Brähler/Gansera et al. (2015, PPmP, im QV unter 8.1.4); Stöbel-Richter/Daig/Brähler et al. (2013, im QV unter 8.1.4); . 
The Copenhagen Burnout Inventory

[...]

In the CBI the core of burnout is fatigue and exhaustion. This is in accordance with the historical development of the burnout concept, and also with a recent definition by one of the leading researchers in the field, Schaufeli. In 2001, Schaufeli and Greenglass defined burnout as "a state of physical, emotional and mental exhaustion that results from long-term involvement in work situations that are emotionally demanding" (Schaufeli \& Greenglass, 2001, p. 501). Interestingly, this definition is almost identical to the definition by Pines and Aronson from 1988: "A state of physical and emotional exhaustion caused by long-term involvement in situations that are emotionally demanding” (Pines \& Aronson, 1988, p. 9). [...] While "the flat battery" remains the main metaphor for burnout, it is important to emphasize that burnout is not just fatigue or exhaustion. If this were the case we would not need the concept at all. In our understanding of the concept the additional key feature is the attribution of fatigue and exhaustion to specific domains or spheres in the person's life. One such domain is work and a more specific domain is client work. In the following we elaborate further on the three parts of the CBI.

(S. 196f.)

Kristensen/Borritz/Villadsen et al. (2005) unterscheiden zwischen einem „personal burnout“, einem „work-related Burnout“ und einem „client-related burnout“, je nachdem, worauf die Befragten selbst ihre über einen längeren Zeitraum anhaltende Erschöpfung zurückführen. Sie präsentieren die einzelnen Unterfragebögen und deren Items in Tabellen, auf die sie im Fließtext mit folgendem Satz verweisen: „The questions and the basic characteristics of the three CBI scales are shown in Table II“ (ebd.: 199). Item-Beispiele für das „work-related burnout“ sind (ebd.: 200):

Do you feel worn out at the end of the working day?

Are you exhausted in the morning at the thought of another day at work?

Do you feel that every working hour is tiring for you?

Do you have enough energy for family and friends during leisure time? (inverse scoring)

Is your work emotionally exhausting?

Does your work frustrate you?

Do you feel burnt out because of your work?

In Beispiel 7 ist die Textsorte Abstract, hier eines Übersichtsartikels von Bauer/ Häfner/Kächele et al. (2003) aus der Zeitschrift „Psychotherapie, Psychosomatik, medizinische Psychologie“ (PPmP), aufgenommen. Interessant an diesem Beispiel ist, dass Freudenberger wieder als `Begründer « genannt wird und im folgenden Satz die Symptomtrias nach Maslach/Jackson folgt. Die drei Symptome werden jedoch ohne intertextuellen Verweis auf diese Quelle zitiert, wodurch 
es so erscheint, als ginge diese Definition auf Freudenberger zurück. Die Definition erscheint an dieser Stelle ohne Distanzierungsmarker. Im weiteren Textverlauf, an topografisch unauffälligerer Stelle als im Abstract, wird jedoch davon gesprochen, dass „keine allgemein gültige Definition von Burn-out“ existieren würde, und es werden neben Freudenberger und Maslach/Jackson noch weitere „Burnout-Konzepte“ dargelegt (vgl. Bauer/Häfner/Kächele et al. 2003: 214). ${ }^{510}$ Inwiefern auch die Platzierung und typografische Gestaltung bestimmte Definitionen hervorheben und damit den Diskurs verknappen bzw. eine Definition im Diskurs dominant setzen kann, wird in Kap. 6.2.4.2 ausführlicher beschrieben.

7) Bauer/Häfner/Kächele et al. (2003: 213), PPmP (im QV unter 8.1.4):

Zusammenfassung [fett gedruckt als Überschrift, T.S.] (Unterstreichungen T.S.):

Vorgelegt wird eine Übersicht über wissenschaftliche Konzepte und psychologische sowie klinisch-psychosomatische Aspekte des Burn-out-Syndroms. Nach neueren Untersuchungen befinden sich in Deutschland bis zu 25\% der insgesamt 36 Millionen Erwerbstätigen in einer gesundheitlichen Situation, die der New Yorker Arzt und Psychoanalytiker Herbert J. Freudenberger 1974 erstmals als Burn-out-Syndrom bezeichnet hat. Burn-out-Dimensionen sind: 1. emotionale Erschöpfung (emotional exhaustion), 2. eine gefühllose, gleichgültige oder zynische Einstellung gegenüber Klienten, Kunden oder Kollegen (depersonalisation) sowie 3. eine negative Einschätzung der persönlichen Leistungskompetenz (low personal accomplishment). Erwerbstätige mit Burn-out-Syndrom leiden an einer breiten Palette psychosomatischer Beschwerden: Schlafstörungen, chronische Schmerzen ohne Befund, funktionelle Herz-Kreislauf-Beschwerden sowie unspezifische Beschwerden des Magens und des Darmes. [...]

Beispiel 8a-1 zeigt einen Übersichtsartikel in der Deutschen Medizinischen Wochenschrift, der mit einem Fallbeispiel einsteigt, was in Fachbeiträgen zum Phänomen BURNOUT in den Korpustexten vergleichsweise selten geschieht. Es wird der Fall einer alleinerziehenden Mutter beschrieben. Am Ende wird von einem „Psychiater“ ein „Burnout-Syndrom“ diagnostiziert: ${ }^{511}$

510 Ähnliche Beispiele für Artikel aus dem Korpus der Fachzeitschriften, Sammelbände und Monografien, die die Definition von Maslach/Jackson teilweise ohne explizite Nennung der Quelle an exponierter Stelle platzieren (im Abstract oder Einleitungskapitel des Artikels) und/oder mit Phrasen verbinden, welche die Geltung dieser Definition hervorheben (teils auch trotz vorheriger oder folgender Kritik am Fehlen einer allgemeingültigen Definition), sind: Nil/Jacobshagen/ Schächinger et al. (2010) im „Schweizer Archiv für Neurologie und Psychiatrie“, im QV unter 8.1.5; Schmitz (2001) in PiEU, im QV unter 8.1.4; Bergner (2016) und Koehler/Koehler (2014) in der DMW; Bugaj/Müksch/Ehrenthal et al. (2016) in der PPmP. Ein ähnliches Vorgehen wurde auch in einigen Lehrwerken beobachtet (vgl. Kap. 6.2.2.1, z. B. bei Groth 2013; Schaper ${ }^{3} 2014$; Barth ${ }^{4} 2010$ ).

511 Weitere ausführliche Fallbeispiele, aber nicht immer mit den Gliederungs-Kategorien „Anamnese“, „Befund“, „Therapie“ findet man im Korpus z. B. bei Schaufeli/Enzmann (1998: 
8a-1) Kissling/Mendel/Förstl (2014: 2587), DMW (Unterstreichungen T.S., im QV unter 8.1.4): Der konkrete Fall [farblich als Überschrift abgesetzt, T.S.]

Anamnese [farblich als Überschrift abgesetzt, T.S.]

Die 45-jährige Patientin fühlte sich erschöpft, niedergeschlagen, klagte über Tinnitus, immer wieder auftretendes Herzrasen, Magenschmerzen, häufige Infektionen der Atemwege und eine Gewichtsabnahme von $6 \mathrm{~kg}$ in den letzten 6 Monaten.

Vor 2 Jahren war sie von ihrem Ehemann überraschend verlassen worden und war seither alleinerziehende Mutter von 2 Söhnen (6 und 13 Jahre). Der jüngere Sohn war seit einem Jahr schwer erkrankt. Um die Wohnung alleine weiter finanzieren zu können, arbeitete sie zusätzlich zu ihrer Anstellung als Stationsleiterin in einer chirurgischen Klinik oft bis spätabends als Bedienung in einem Restaurant. Die Patientin hatte sich vor 18 Monaten als Stationsleitung auf eine große Intensivstation versetzen lassen, wurde aber von dem dortigen Team noch nicht in vollem Umfang als Leitung anerkannt. Sie stand wegen ihrer Kinder jeden Morgen um 5.30 Uhr auf, kam häufig abends erst um 24 Uhr ins Bett und litt seit längerem unter Einund Durchschlafstörungen. Der Freundeskreis habe sich nach der Scheidung komplett von ihr zurückgezogen, für Hobbys und Erholung bleibe keine Zeit mehr. Die Patientin leide seit mehr als einem Jahr unter den oben beschriebenen Symptomen und sei in letzter Zeit zunehmend zynisch und gereizt den Kollegen und häufig auch ihren Kindern gegenüber. Immer häufiger fand sie nur noch nach dem Genuss von 1-2 Flaschen Bier abends in den Schlaf.

Untersuchungsbefund [farblich als Überschrift abgesetzt, T.S.]

Eine ausführliche internistische Untersuchung erbrachte keine pathologischen Befunde, insbesondere keinen Hinweis auf Hypothyreose, Diabetes, Malignome oder eine kardiologische Erkrankung. Ebenso zeigte eine HNO-ärztliche Untersuchung keine behandlungsbedürftigen Erkrankungen.

Therapie und Verlauf [farblich als Überschrift abgesetzt, T.S.]

Die Patientin wurde zur weiteren diagnostischen Abklärung dem Psychiater vorgestellt, der ein Burnout-Syndrom diagnostizierte und die Patientin in eine auf Depression und Burnout spezialisierte psychiatrische Abteilung überwies.

Betrachtet man nur die zitierten Passagen, die die erste Seite dieses Beitrags von Kissling/Mendel/Förstl (2014) füllen, so könnte es so erscheinen, als würde der Artikel die psychiatrische Diagnose >Burnout`, die am Ende wie ein Kommentar für die zuvor geschilderte Fallbeschreibung vergeben wird, als gesichert erachten.

20), bei Weimer/Kraus (2011: 242) in der Zeitschrift „Der Psychotherapeut“ oder bei Wilms (2009) und Kopka/Ast/Hügel/Köllner et al. in PiD, in den verschiedenen Auflagen der Monografie von Burisch zum „Burnout-Syndrom“ (1989, $\left.{ }^{3} 2006,{ }^{5} 2014\right)$, im Vermittlungs- und Überblickswerk der Autoren Hillert/Marwitz (2006: 17ff.) und bei Cherniss (1980a: 14f.) (1980b: 5f.). In einigen Beiträgen des Fachkorpus werden einzelne Aussagen in der Ich-Perspektive aus Interviews mit Personen mit Burnout-Symptomatik oder Fallbeispiele eingestreut (vgl. z. B. Fisher 1983 im Sammelband von Farber (Hg.) (1983); Kratzer 2012 im Dt. Ärztebl.; Geyerhofer/Unterholzer 2009 in PiD). 
Ähnlich wie bei Beispiel 7 erfolgt allerdings auch in diesem Beitrag erst im weiteren Verlauf des Textes eine Distanzierung und das Definiensnomen Krankheit wird abgelehnt und durch ein anderes Definiensnomen substituiert (,sondern ein Erschöpfungs- und Überforderungssyndrom, [...] Risikozustand“):

\section{8a-2) Kissling/Mendel/Förstl (2014: 2588) (Unterstreichungen T.S.):}

Laut der Deutschen Gesellschaft für Psychiatrie und Psychotherapie, Psychosomatik und Nervenheilkunde (DGPPN) ist Burnout keine nach ICD-10 diagnostizierbare Krankheit, sondern ein Erschöpfungs- und Überforderungssyndrom, das einen Risikozustand für eine spätere nach ICD-10 diagnostizierbare - psychiatrische oder körperliche Krankheit darstellt (wie z. B. eine Depression oder eine Hypertonie) [3]. Diese Einschätzung wird auch von der Mehrheit der Psychiater und Psychotherapeuten geteilt [6]. Die DGPPN empfiehlt deshalb, immer zuerst die jeweils zutreffende ICD-10-Diagnose (z. B. Depression oder Hypertonie) zu verschlüsseln. Wenn darüber hinaus angenommen wird, dass bei der Entstehung und Aufrechterhaltung dieser als Hauptdiagnose festgestellten Erkrankungen ein Erschöpfungszustand im Sinne eines Burnouts eine entscheidende Rolle gespielt hat, sollte zusätzlich die ICD-10-Anhangsziffer Z

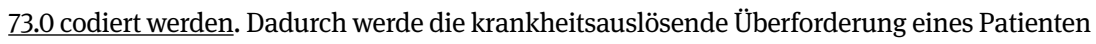
gekennzeichnet und entsprechende spezifische Therapiemaßnahmen nahegelegt. ${ }^{512}$

Auch Weimer/Kraus (2011) klassifizieren im Anschluss an ihr Fallbeispiel in der Zeitschrift „Der Psychotherapeut“ >Burnout als `Zusatzdiagnose im Rahmen der ICD-10 ${ }^{513}$ und begründen, warum sie diese Zusatzdiagnose wählen:

8b) Weimer/Kraus (2011: 242), Der Psychotherapeut (Unterstreichungen T.S., im QV unter 8.1.5):

Bezogen auf die bedrückte Stimmung, die Antriebsstörung, den Interessenverlust, Schuldund Versagensgefühle wird eine mittelgradige depressive Episode (F32.1) diagnostiziert und die Diagnose um den Zusatz Z73.0, Ausgebranntsein oder Burn-out als Zustand der totalen Erschöpfung ergänzt. Diese Unterkategorie aus dem Bereich Z73 Probleme mit Bezug auf Schwierigkeiten bei der Lebensbewältigung wird gewählt, da Herr L. eine ausgeprägte Erschöpfungssymptomatik bietet, die er ursächlich im Arbeitsbereich festmacht.

512 Die Autoren schreiben dazu: „Burnout ist keine anerkannte medizinische Diagnose. Subjektiv haben ca. 8 \% der Erwerbstätigen das Gefühl, an einem Burnout zu leiden.“ [Dieser Satz steht im Original am linken Rand optisch abgesetzt und fett gedruckt mit der Überschrift „Kurz gefasst“, Anm. T.S.]. Auf derselben Seite erfolgt dann noch folgende definitionsartige „kurzgefasste“ Aussage am Rand: „Burnout ist ein Erschöpfungszustand, der in eine psychiatrische oder körperliche Krankheit übergehen kann.“ [Im Original ebenfalls am linken Rand optisch abgesetzt und fett gedruckt, Anm. T.S.]. (Kissling/Mendel/Förstl 2014: 2588).

513 In der ICD-9 (1979) gab es noch keinen Eintrag zu „Ausgebranntsein“ bzw. „Burnout“. Die Ziffer Z73.0 wurde mit Einführung der ICD-10 in das Regelwerk aufgenommen. Die deutsche Version von 2004 ist einsehbar unter: https://www.dimdi.de/static/de/klassifikationen/icd/icd-10gm/kode-suche/htmlgm2004/index.htm?gz70.htm+ (zuletzt eingesehen am 18.2.2019). 
Die Zuschreibung zur Arbeitswelt geschieht durch den Patienten selbst („die er ursächlich ... “). Damit ist für Weimer/Kraus in diesem Beispiel, wie in Beispiel 6b für Kristensen/Borritz/Villadsen et al. (2005) bei der Konzipierung des Copenhagen Burnout Inventory, der Rückbezug der ,betroffenen' Person auf die Arbeit als Quelle der Erschöpfung das „additional key feature“ (Kristensen/Borritz/Villadsen et al. 2005: 193).

Als neuntes Beispiel sei noch auszugsweise ein kritischer Übersichtsbeitrag von Kaschka/Korczak/Broich aus dem „Deutschen Ärzteblatt“ von 2011 zitiert, der mit dem Titel „Modediagnose Burn-out“ für Aufmerksamkeit sorgte und auf einem HTA-Bericht ${ }^{514}$ basiert (Korczak/Kister/Huber 2010). Dieser Beitrag ist interessant, da er das Ziel hat, die Fachmeinungen zum Burnout-Phänomen in einer kritischen Übersicht zu bündeln und Empfehlungen für den klinischen Bereich und weitere Forschungsrichtungen zu formulieren. Des Weiteren kommt diesem Text Schlüsseltextcharakter zu, da er bzw. der HTA-Bericht, auf dem er basiert, in der Folgezeit häufig in den Medien und von Fachleuten aufgegriffen wurde (vgl. Kap. 1.2). ${ }^{515}$

9) Kaschka/Korczak/Broich (2011: 781f., Dt. Ärztebl.) (Unterstreichungen T.S., im QV unter 8.1.4):

ZUSAMMENFASSUNG [farblich und durch Fettdruck als Überschrift markiert, T.S.]

514 Zum Thema „Burnout“ sind zwei HTA-Berichte erschienen: Zur „Differentialdiagnostik des Burnout-Syndroms“ (Korczak/Kister/Huber 2010) und zur „Therapie des Burnout-Syndroms“ (Korczak/Wastian/ Schneider 2012). Die Abkürzung HTA geht zurück auf „Health Technology Assessment“. Unter HTA wird nach der Bundesärztekammer die „systematische, evidenzbasierte Bewertung medizinischer Verfahren und Technologien im Hinblick auf deren Effekte auf die Gesundheitsversorgung verstanden“. Siehe unter: https://www.bundesaerztekammer.de/aerzte/ qualitaetssicherung/health-technology-assessment/ (zuletzt eingesehen am 12.12.2019).

515 Artikel verschiedener Fachrichtungen nehmen auf diesen Beitrag bzw. den HTA-Bericht Bezug: z. B. Berger/Falkai/Maier 2012; Gumz/Brähler/Erices 2012; Dörr/Nater 2013; Riedel-Heller/ Luppa/Seidler et al. 2013; Oepen/Gruber 2014; Kissling/Mendel/Förstl 2014; Gahntz/Graefe 2016. Die Publikation im Dt. Ärztebl. wurde des Weiteren schon ein paar Tage nach ihrem Erscheinen in den Wikipedia-Eintrag zu „Burnout“ aufgenommen (siehe Versionengeschichte am 23.11.2011 zwischen 00:06 und 12:40 Uhr, die Versionengeschichte mit diesen Einträgen ist abrufbar unter: https://de.wikipedia.org/w/index.php?title=Burn-out\&action=history\&year=2011\&month=-1\&tagfilter= (zuletzt eingesehen am 12.12.2019)); Der Beitrag im Dt. Ärztebl. wird auch in der überregionalen Presse aufgenommen: in der FAZ am 6.10.2010 (= Kaulen 2010); in der ZEIT, 49/2011 (= Albrecht 2011a) und in SPIEGEL WISSEN 1/2012 (= Gatterburg/Großbongardt 2012). Dass das Thema „Modediagnose“ in Bezug auf Burnout in den Jahren 2010/2011und auch noch nach 2011 im Diskurs verhandelt wurde zeigen Belege in der FAZ und im SPIEGEL: In der FAZ vom 24.3.2011 wird „Chefarzt Hans-Peter Unger“ mit dem Vortrag „Burn-out - Modediagnose oder stille Epidemie?“ angekündigt (= FAZ 2011a), und im SPIEGEL wird der Psychiater Ulrich Hegerl zitiert, der „vor dem Modewort“ warnt (Kramer 2011), vgl. auch im SPIEGEL Glaubitz (2011). 
[...] Ergebnisse: Bisher existieren weder eine verbindliche Definition noch ein valides, allgemeingültiges differenzialdiagnostisches Instrument für das Burn-out-Syndrom. Die Symptomatik lässt sich den Dimensionen emotionale Erschöpfung, Depersonalisation und reduzierte Leistungsbereitschaft beziehungsweise -fähigkeit zuordnen. Vorliegende Studien zur Epidemiologie und Differenzialdiagnostik sind überwiegend von geringer Evidenz. Kontrollierte Therapiestudien fehlen.

$[\ldots]$

Definition [farblich und durch Fettdruck als Überschrift markiert, T.S.]

Eine allgemeingültige, international konsentierte Definition von Burn-out gibt es derzeit nicht. Die Übersicht stellt deshalb kausale Faktoren und Entstehungsmodelle vor, die veranschaulichen sollen, was der Begriff Burn-out umfasst.

[Die Symptome und typischen Phasen werden in Abbildungskästen dargestellt, T.S., im Folgenden wird der Inhalt zweier Informationskästen zitiert]

\section{Kasten 1}

\section{Symptom-Cluster bei Burn-out (modifiziert nach [4]; Langfassung im Internet)}

- Warnsymptome der Anfangsphase

- vermehrtes Engagement für Ziele

- Erschöpfung

- reduziertes Engagement

- für Patienten und Klienten

- für andere allgemein

- für die Arbeit

- erhöhte Ansprüche

- emotionale Reaktionen; Schuldzuweisung

- Depression

- Aggression

- Abbau

- der kognitiven Leistungsfähigkeit

- der Motivation

- der Kreativität

- Entdifferenzierung

- Verflachung

- des emotionalen Lebens

- des sozialen Lebens

- des geistigen Lebens

- psychosomatische Reaktionen

- Verzweiflung

\section{Kasten 2}

Burn-out-Phasenmodell (nach [8])

- Zwang, sich zu beweisen (übertriebener Ehrgeiz) 
- Verstärkter Einsatz

- Vernachlässigung eigener Bedürfnisse

- Verdrängung von Konflikten und Bedürfnissen

- Keine Zeit mehr für nicht-berufliche Bedürfnisse

- Zunehmende Verleugnung des Problems, abnehmende Flexibilität im Denken/Verhalten

- Rückzug, Orientierungslosigkeit, Zynismus

- Verhaltensänderung/psychische Reaktionen

- Verlust des Gefühls für eigene Person/Bedürfnisse

- innere Leere, Angstgefühle, Suchtverhalten

- zunehmende Sinnlosigkeit und Desinteresse

- körperliche, gegebenenfalls lebensbedrohliche Erschöpfung

(Kaschka et al. 2011: 782)

Das 10. Beispiel ist einem Beitrag von Berger/Falkai/Maier (2012) aus dem Deutschen Ärzteblatt entnommen, der mit „Burnout ist keine Krankheit“ überschrieben ist. Der Beitrag stellt Kernpunkte eines Positionspapiers der „Deutschen Gesellschaft für Psychiatrie und Psychotherapie, Psychosomatik und Nervenheilkunde“ (DGPPN) zusammen. Er argumentiert einerseits dafür, dass `Burnout $\iota$ von anderen in der ICD-10 klassifizierten Krankheiten und alltagssprachlichen Gebrauchsweisen genauer abgegrenzt werden müsse und weist ihm andererseits einen Platz im Klassifikationssystem, wenn auch nur als „Risikozustand“ (siehe im Schaubild, Abb. 9), zu. Dabei formulieren die Autoren im Fließtext und im Schaubild das Ziel, die „,on Freudenberger oder Maslach als Burn-out bezeichneten Beschwerden in Übereinstimmung mit der ICD-10“ differenzierend zu bestimmen:

10) Berger/Falkai/Maier (2012): 213, Dt. Ärztebl. (Unterstreichungen T.S., im QV unter 8.1.4, Beleg 10 entspricht Abbildung 9 auf der nächsten Seite)

Interessant an diesem Beispiel ist, dass die Autoren Sprachrohr einer Fachgesellschaft (der DGPPN) sind und dass sie eine Verbindung der ICD-10 und einschlägiger Burnout-Beschwerden von „Freudenberger oder Maslach“ anstreben. Des Weiteren verwenden sie konsekutive Satzgefüge, die sie mit einer Aufforderung bzw. Instruktion verbinden, wie man in der sprachlichen Festsetzung und Diagnostizierung nicht verfahren solle (,wenn diese absehbar zeitlich begrenzt sind [...], sollte nicht von Burnout gesprochen werden“), um >Burnout nicht krankheitswertigen Zuständen abzugrenzen. Die Fälle, in denen von >Burnout bei der Diagnosestellung gesprochen werden soll, werden im nächsten Satz durch das adversative Adverb jedoch und negierende Sprachmittel den 'gesunden' Zuständen gegenübergestellt. 


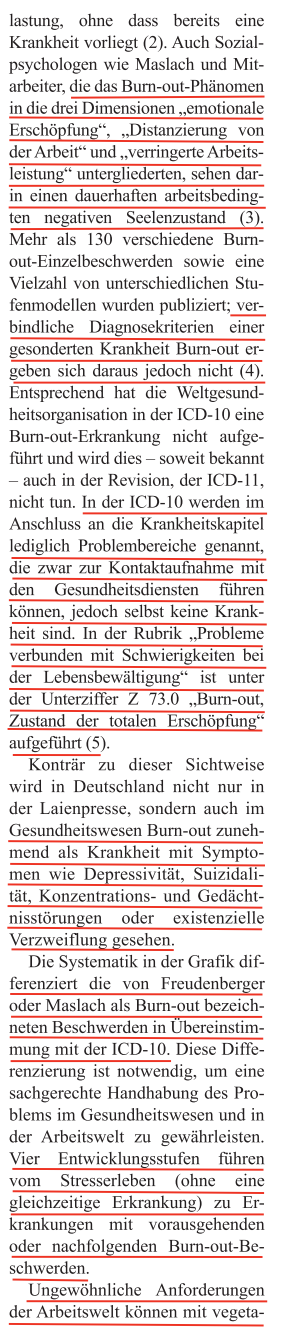

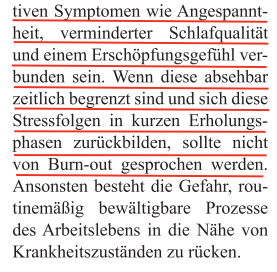

Viele Ursachen für andauernde Arbeitsüberforderung

Hält der Erschöpfungszustand jedoch mehrere Wochen bis Monate an, ist ein Ende nicht absehbar, und führen kurze Erholungsphasen nicht zu einer Rückbildung, sollte von einem Burn-out gesprochen werden. Erlebte, andauernde $\mathrm{Ar}$ beitsüberforderung kann ein breites Spektrum von Ursachen umfassen, wie arbeitsplatzbezogene Faktoren, das heißt real unbewältigbarer $\mathrm{Ar}$ beitsanfall, mangelnde Anerkennung durch Vorgesetzte, fehlende Abgrenzung zum Privatleben. Auch individuelle Faktoren wie reduzierte Stresstoleranz, überhöhte Zielsetzungen, Perfektionismus, Selbstüberschätzung oder unzureichende Qualifikation können ursächlich sein. Bedeutsam ist das individuelle Zusammenspiel beider Aspekt
Allgemein gültige Schwellenwerte gibt es folglich nicht.

Burn-out-Beschwerden sollten jedenfalls mit der Ziffer Z 73.0 von den konsultierten Ärzten oder Psychologischen Psychotherapeuten kodiert werden. Denn auch bei Abwesenheit definierter ICD-Krankheiten stellt Burn-out einen Risikozustand für nachfolgende psychische oder körperliche Erkrankungen dar. Besonders gefährdet sind Menschen mit entsprechenden früheren Erkrankungsphasen von Depressionen, Angst- oder Abhängigkeitserkrankungen. Die klinische Erfahrung zeigt, dass Burn-out auch körperliche Krankheiten wie Tinnitus, Hypertonie oder Infektionskrankheiten begünstigen kann. Wenn bei einem Patienten infolge einer längerfristigen Arbeitsüberforderung eine Erkrankung nach den ICD-10-Kriterien wie Depression, Angststörung, chronisches Schmerzsyndrom, Tinnitus oder Bluthochdruck aufgetreten ist, sollte die Krankheitsverschlüsselung nach ICD-10 erfolgen, da sie das weitere Vorgehen bestimmt. Wenn angenommen wird, dass die Arbeitsüberforderung zu Entstehung und Aufrechterhaltung der Krankheit beiträgt, sollte regelhaft die zusätzliche Kodierung mit der Anhangsziffer Z 73.0 vorge nommen werden. Diese Zusatzko-

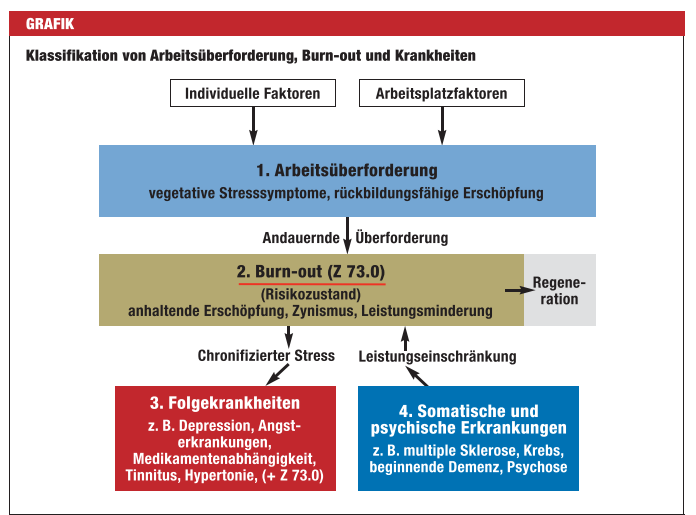

Abb. 9: Auszug und Schaubild aus Berger/Falkai/Maier (2012: A-701) im Deutschen Ärzteblatt (Unterstreichungen T.S.), () Berger/Falkai/Maier, Dt. Ärztebl., 2012.

Im Anschluss daran sei eine Definition aus einer Originalarbeit der Zeitschrift „Psychotherapie, Psychosomatik und Medizinische Psychologie“ zitiert, die es sich zum Ziel setzt, die Konstruktvalidität von `Burnout ` in Abgrenzung zur `Depression` zu erforschen: 
11) Reime/Steiner (2001: 304) in PPmP (Unterstreichungen T.S., im QV unter 8.1.4):

Obwohl mittlerweile tausende Studien zum Thema Burnout vorliegen, wurde seine Konstruktvalidität - insbesondere gegenüber der Depression - äußerst selten empirisch überprüft [1]. Burnout gilt als ein Syndrom von emotionaler Erschöpfung, Depersonalisation und subjektiver Leistungsverringerung, das vor allem in helfenden Berufen beobachtet wurde [2]. Emotionale Erschöpfung (EE) wird als Gefühl der Überbeanspruchung und des Ausgelaugtseins beschrieben. Depersonalisation (DP) bezieht sich auf die desinteressierte und abgestumpfte Haltung gegenüber Klienten. Subjektive Leistungsverringerung (SL) umschreibt das Gefühl, bei der Arbeit zu versagen.

Die Gemeinsamkeiten zwischen Burnout und einer Depression bzw. „dysthymen Störung“ ([3], S. 407) bestehen in „... Interessens-, Motivationsverlust und Apathie, Rückzug, negativem Selbstwertgefühl und dem Gefühl mangelnder Kompetenz“ ([4], S. 87). Die Differenzen betreffen z. B. die Intensität der Symptome. Unter einer Depression sollen die Betroffenen stärker leiden als unter Burnout, so dass erstere sich mehr auf den Alltag und das gesundheitliche Wohlbefinden auswirken soll [1,5-7]. Burnout soll sich im Gegensatz zur Depres-

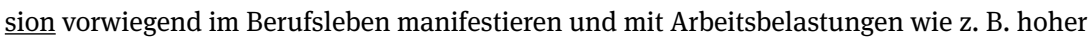
Fallzahl, Zeitdruck und fehlender Anerkennung durch Vorgesetzte zusammenhängen [1, 4-7]. Empirische Studien fanden mittelhohe statistische Zusammenhänge zwischen Depression und EE und niedrigere zwischen Depression und DP bzw. SL [4-7].

Zunächst wird die Definition, die auf Maslach/Jackson zurückgeht, im ersten Absatz zitiert. Die Symptome dieser Konstruktdefinition werden von den Autorinnen der Studie mit dem Maslach Burnout Inventory bei einer Stichprobe gemessen und mit Messwerten der „Allgemeinen Depressionsskala (ADS)“ derselben Studienteilnehmer/innen korreliert. Interessant sind zum einen die inhaltlichen Unterschiede zwischen $>$ Burnout und $>$ Depression , die die Autorinnen aus der Forschungsliteratur extrahieren und als Thesen ihrer Studie zugrunde legen (z. B. Differenzen in Bezug auf die Intensität und Manifestationsorte der Symptome). Zum anderen sind die sprachlichen Formen aufschlussreich, mit denen sie $>$ Burnout $<$ und $>$ Depression voneinander abgrenzen (stärker ... als, mehr auf ..., im Gegensatz zur, Zusammenhänge/Gemeinsamkeiten zwischen ....), ${ }^{516}$ und schließlich ist die Verwendung des

516 Es gibt noch weitere Beispiele im Fachdiskurs, die \Burnout $<$ mitunter dadurch zu definieren suchen, dass sie es von sachverwandten Konzepten abgrenzen. So z. B. Dörr/Nater (2013) in der PPmP, die es sich zur Aufgabe gemacht haben, ,verschiedene in der Literatur beschriebene Syndrome, die Erschöpfung als ein Kernsymptom beinhalten, vorzustellen und die Abgrenzung der Begrifflichkeiten zu diskutieren“ (ebd.: 69; Im QV unter 8.1.4). >Burnout « wird bei Dörr/Nater von den Begriffen >Neurasthenie`, >Chronic Fatigue Syndrome`(CFS) und >Depression` abgegrenzt. Vgl. zur Gegenüberstellung von `Burnout und `Depression` auch Nil/Jacobshagen/ Schächinger et al. (2010) im „Schweizer Archiv für Neurologie und Psychiatrie“ (im QV unter 8.1.5). Eine Gegenüberstellung von >Burnout $\mathrm{zu}$ anderen in der ICD-10 klassifizierten Diagnosen findet man auch bei Hamann/Parchmann/Mendel et al. (2013) in „Der Nervenarzt“ (im QV unter 8.1.4) und Beschoner/Schönfeldt-Lecuona/Braun et al. (2009) in PiD (im QV unter 8.1.4). Bakker/Schaufeli/ 
Modalverbs sollen hervorzuheben: Dieses zeigt an, dass sich die Begriffskonzeption und -abgrenzung noch in der empirischen Überprüfungsphase befindet.

Abschließend sei eine Meldung der Weltgesundheitsorganisation (WHO) vom 29. Mai 2019 zitiert. In dieser wird gemeldet, dass die Definition von >Burnout für die ICD-11, die ab Januar 2022 in Kraft tritt, überarbeitet wurde. Burnout wird darin wie folgt definiert:

12) Burn-out an "occupational phenomenon": International Classification of Diseases (Unterstreichungen T.S., im QV unter 8.1.3, WHO 5/2019)

28 May 2019 - Burn-out is included in the 11th Revision of the International Classification of Diseases (ICD-11) as an occupational phenomenon. It is not classified as a medical condition.

It is described in the chapter: 'Factors influencing health status or contact with health services' - which includes reasons for which people contact health services but that are not classed as illnesses or health conditions.

Burn-out is defined in ICD-11 as follows:

"Burn-out is a syndrome conceptualized as resulting from chronic workplace stress that has not been successfully managed. It is characterized by three dimensions:

feelings of energy depletion or exhaustion;

increased mental distance from one's job, or feelings of negativism or cynicism related to one's job; and reduced professional efficacy.

Burn-out refers specifically to phenomena in the occupational context and should not be applied to describe experiences in other areas of life."

Burn-out was also included in ICD-10, in the same category as in ICD-11, but the definition is now more detailed.

The World Health Organization is about to embark on the development of evidence-based guidelines on mental well-being in the workplace. ${ }^{517}$

Die Überarbeitung ist in zweifacher Hinsicht interessant. Die Überkategorie als Zusatzdiagnose wird beibehalten, d. h. Burnout wird nicht in den Status einer Hauptdiagnose („not classed as illnesses“) erhoben. Die Symptombeschreibung wird allerdings gegenüber der Fassung in der ICD-10 maßgeblich erweitert. In der ICD-10 wurde Burnout nur in der Kategorie „Z73 Probleme mit Bezug auf Schwierigkeiten bei der Lebensbewältigung“ erwähnt und mit „Ausgebranntsein“ übersetzt. $^{518}$ In

Demerouti et al. (2000) erforschen die Eigenständigkeit des Konzepts `Burnout` gegenüber der >Depression` unter Bezug auf die sozialpsychologische „equity theory“ (siehe im QV unter 8.1.5). 517 Diese Definition der WHO ist einsehbar unter: https://www.who.int/mental_health/evidence/burn-out/en/ (zuletzt eingesehen am 13.12.2019, siehe auch im QV unter 8.1.3).

518 Die Kategorie Z73 in der ICD-10 ist unter folgendem Link einsehbar: https://www.icd-code.de/icd/ code/Z73.html (zuletzt eingesehen am 13.12.2019, siehe auch im QV unter DIMDI (2019b) und 8.1.3). 
dieser neuen Fassung sind drei Symptome aufgenommen, die Ähnlichkeiten zu den Symptomen aufweisen, die in diesem Kapitel zurückgehend auf Maslach/Jackson (1984) beschrieben wurden (siehe oben Beleg 2). Des Weiteren wird Burnout eindeutig als `berufliches Phänomen` (,occupational phenomenon“) klassifiziert.

Bei den Definitionen in Fachlexika, Hand- und Lehrbüchern findet man, wie in Kap. 6.2.2.1 beschrieben wurde, überwiegend realdefinitorische Zustandsbeschreibungen, die mit dem Schema genus proximum (über die Nennung von Hyperonymen wie Syndrom, Zustand) - differentia specifica (über direkt angeschlossene spezifizierende Attributphrasen und Relativsätze) dem Ausdruck Burnout meist über Verbalphrasen, die ein Äquivalenzverhältnis realisieren, Merkmale zuweisen. Nach dieser Form funktioniert auch die Definition in Beleg 2. Das ist die im englischen Original zitierte Definition von `Burnout von Maslach/Jackson, welche das Vorbild für die meisten Lexika, Hand- und Lehrbücher darstellt (siehe Kap. 6.2.2.1) und die neben anderen Definitionen auch im weiteren fachlichen Korpus sehr häufig thematisiert oder paraphrasierend erwähnt wird (siehe die Belege 5, 6a, 6b, 7, 9, 10, 11). Möglicherweise stellt die Definition von Maslach/Jackson schon aufgrund dieser ,klassischen' komprimierten Form ein gutes Muster für alle Fälle bereit, bei denen auf engem Raum definiert werden soll: so einerseits für Fachlexika und Handbücher und andererseits für Abstracts, Einleitungskapitel von Fachzeitschriftenartikeln und Monografien und Klassifizierungsmanuale (vgl. Belege 7, 9, 11). Die Definition mit der Symptom-Trias von Maslach/Jackson wird erstaunlicherweise selbst dann reproduziert, wenn die Definition und Messung von Maslach/Jackson im Fachartikel im weiteren Verlauf kritisiert wird, zusammen mit der Aussage, es gäbe keine allgemein gültige Definition (vgl. die Belege 7 und 9 und Fußnote 510 oben).

Die weiteren in diesem Kapitel angeführten Beispiele zeigen jedoch deutlich, dass es in den fachlichen Zeitschriftenartikeln und Monografien eine größere sprachlich-formale und inhaltliche Varianz des Definierens als in den Lexika, Handund Lehrbüchern gibt und dass eine Definition häufiger einzelnen Autorinnen/ Autoren explizit und teilweise kritisch zugewiesen wird. Ein Abgleich mit Definitionsarten, wie sie in Definitionslehren der fachkommunikativen, terminologischen sowie wissenschaftsgeschichtlichen und -theoretischen Literatur aufgestellt und in Kap. 4.2.3-4.2.4 im Überblick dargestellt wurden, macht die größere Varianz sichtbar: - So führt Freudenberger in Beleg 1 beispielsweise zunächst als Beantwortung der Frage „What is burn-out?“ eine Nominaldefinition aus einem allgemeinsprachigen Wörterbuch an („The dictionary defines the verb ,burn-out“ as ... ") und überträgt diese Bedeutung, die durch das Verb den Fokus auf den Prozess des Ausbrennens rückt, dann durch die Formulierung ,and that is exactly what happens when a staff member [...] burns out“" auf die real beobachteten Zustände, wodurch er die Nominal- in eine Realdefinition überführt (vgl. Belege 
1a und 1b oben). ${ }^{519}$ Nach Köller (2004: 677) mühten sich schon die Gesprächspartner des Sokrates mit diesen „Was-ist-Fragen“, denen sie mit „Wesensdefinitionen in Form von Begriffsdefinitionen“ beizukommen suchten. Sie wichen aufgrund der Unabschließbarkeit solcher Fragen auf Beispielerzählungen aus (ebd.). Ein ähnliches Vorgehen praktiziert auch Freudenberger: In den folgenden Abschnitten spezifiziert er das Phänomen bzw. Konzept über die Präsentation einer eher lockeren Reihung von Beispielen und Entwicklungsstufen aus dem Klinikalltag, die das Symptom-Tableau in seiner Entstehungsgeschichte darstellt. ${ }^{520}$ Dieses Vertextungsmuster, über das die Symptome dem Ausdruck Burnout meist über mehrere Unterkapitel zugeordnet werden, findet man in Fachlexika und Handbüchern schon aus Platzgründen in der Regel nicht, häufiger hingegen in Monografien zum Thema „Burnout“ (Cherniss 1980a; Veninga/ Spradley 1981; Burisch 1989 und folgende Auflagen; Schaufeli/Enzmann 1998; Seibold/Schuh ${ }^{5}$ 2010). Einige dieser Überblickswerke, die dieses FrageAntwort-Format mit anschließenden Beispielerzählungen und Symptomlisten aufweisen, wenden sich neben Fachleuten des engeren Kollegenkreises auch an Fachleute angrenzender Fächer und an interessierte Laien bzw. Personen, die mit BuRnout in ihrem Alltag zu tun haben. Das Frage-Antwort-Schema könnte man daher auch als Vermittlungsstrategie deuten.

Indirekt befriedigt Freudenberger allein dadurch, dass er die Was-istFrage stellt, den Anspruch, der an Definitionen häufig gestellt wird, ${ }^{521}$ durch sie etwas über das Wesen oder den Kern eines Phänomens zu erfahren (vgl. Kap. 4.2.2; 4.2.3). Köller (2004: 677) schreibt hierzu:

Was-ist-Fragen korrelieren wohl mit dem menschlichen Bedürfnis, sich geistige oder materielle Vorstellungsobjekte als Wesenheiten vorzustellen, die substanzhaft für sich bestehen und nicht Produkte subjektiver Objektivierungsprozesse sind. Mit Was-ist-Fragen konstituieren wir uns eine eigenständige Objektwelt, zu der die Subjekte in eine dialogische Beziehung treten können.

$519 \mathrm{Zu}$ Nominal- und Realdefinition siehe Kap. 4.2.3 dieser Arbeit. Metasprachliche Reflexionen zu burn out/ausbrennen oder Überlegungen zur Metapher Burnout im Rahmen der Begriffsbestimmung findet man auch in Burischs Monografie zum Burnout-Syndrom ( 2014 : 9-10, im QV unter 8.1.5) und bei Bergknapp (2009) in PiD (im QV unter 8.1.4). Seibold/Schuh (52010: 157) rekapitulieren in ihrer Monografie „Stress, Mobbing und Burn-out am Arbeitsplatz“ die Überleitung einer Nominal- in eine Realdefinition von Freudenberger (1974; 1975), siehe Beleg 1b.

520 In philosophisch-terminologisch geprägter Literatur wird diese Art des Definierens, eine Aneinanderreihung von typischen Beispielen, als ostensive Definition bezeichnet. Vgl. Kap. 4.2.3 dieser Arbeit und Prechtl (52016: 131).

521 Dass Was-ist-Fragen eng mit der Tätigkeit des Definierens verbunden werden, zeigt auch die Zulassungsarbeit von Anna-Lena Schwarzhof: Sie erprobt Was-ist-Fragen als ,intuitiven“ Zugang zu einer Definitionsanalyse im Mikroplastikdiskurs (Schwarzhof 2019: 24ff.). 
In Beleg 3 fassen Kleiber/Enzmann mit Bezug auf den Text von Freudenberger (1974) diese Art des Definierens (zunächst nominale, danach ostensive und genetische Definition) $)^{522}$ in Kurzform zusammen.

- Die Belege 6a und 6b präsentieren Formen und Überlegungen zum operationalen Definieren (vgl. Kap. 4.2.3), indem Fragebogen-Items, die `Burnout` messen und definieren sollen, angeführt werden und das Beziehungsverhältnis zwischen Konzeptvariablen und beobachteten Merkmalen reflektieren. Später in diesem Kapitel wird auf diese Beispiele noch ausführlicher eingegangen.

- Beleg 8a-1 zeigt eine Definitionsform, die eng mit der Textsorte der Kasuistik oder Fallbeschreibung verknüpft ist und die Ähnlichkeiten zu Definitionen „durch eine Skizze (per quandam imaginationem)“ bzw. „durch ein Beispiel (velut)“(vgl. Cassiodorus/Pronay 2014: 132) aufweist. Diese Formen findet man schon in Definitionslehren der Philosophie- und Wissenschaftsgeschichte (vgl. Kap. 4.2.3). Darüber hinaus lassen sich Fallbeispiele mit „genetischen“ Definitionen vergleichen, die etwas dadurch definieren, dass sie seine Entstehung beschreiben. Dass dieser Text als Definitionsvorschlag gewertet wird, hängt zudem damit zusammen, dass auf die Schilderung des Fallbeispiels mit Aufzählung verschiedener Symptome und Belastungsfaktoren im Abschnitt „Therapie und Verlauf“ eine Zuordnung zum Ausdruck Burnout-Syndrom erfolgt: Erstens wird die Patientin schon einmal in den Bereich der Psychiatrie überstellt und dort wird ein „Burnout-Syndrom diagnostiziert[e]“ (siehe Beleg 8). Auf diese Weise werden die zuvor präsentierten Symptome im Diagnoseausdruck Burnout-Syndrom gebündelt und mit diesem identifiziert und der Einzelfall (Sinzeichen) dieser Patientin wird in einen intersubjektiven fachkulturellen Zusammenhang eingeordnet (Legizeichen).

- Beleg 10 und 11 führen in einem kurzen Ausschnitt vor Augen, wie durch Gegenüberstellung einzelner Kriterien ('zeitliche Ausdehnung', 'reversibel') bei >Burnout und sachverwandten Konzepten bzw. Begriffen das für einen Begriff charakteristische definitorische Merkmal gesucht wird und in bestehende Klassifikationssysteme integriert wird, grafisch unterstützt durch ein Flussdiagramm.

Des Weiteren ist auffällig, dass häufiger als dies bei den Lexika und Handbüchern der Fall ist, mehrere, ,konkurrierende‘ Definitionen aufgeführt und/oder Distanzmarker gesetzt werden. ${ }^{523}$ In einigen Texten erscheint zudem ein Hinweis, dass es noch

522 Zur Erläuterung dieser Definitionsarten siehe auch Kapitel 4.2.3 dieser Arbeit.

523 Zum Beispiel über Anführungszeichen: „Schutz vor ,Burn-out““ (Berger/Schneller/Maier (2012: 1364) in Der Nervenarzt im QV unter 8.1.4); Im Beitrag von Hamann/Parchmann/Mendel et al. (2013: 838) werden die Bezeichnungen Burnout-Störungen und Diagnose in Anführungszeichen gesetzt (ebenfalls in Der Nervenarzt). Kapfhammer (2012: 1277 in „Der Internist“ im QV 
keine ,allgemeingültige“ Definition bzw. einheitliche und im Klassifikationssystem gültige Diagnose gebe, womit aber auch ausgedrückt wird, dass eine Assimilierung der verschiedenen Definitionsvorschläge hin zu einer Konsens-Definition angestrebt wird (vgl. dazu v. a. die Belege 7, 8a-2, 9, 10). Es zeigt sich hier, dass im Forschungsprozess durchaus verschiedene Definitionsvorschläge nebeneinander existieren können. Beleg 6a spricht von „hypothetischen Konstrukte[n]“ der MBI-Skalen. In den Artikeln der Fachzeitschriften und Monografien handelt es sich demnach um Definitionsvorschläge mit repräsentativ-festsetzender Funktion ${ }^{524}$, die aufgezählt oder einander gegenübergestellt und diskutiert werden (siehe Beleg 4, 5 und 6b). In manchen Publikationen werden sie aber auch zusammengeführt, oder eine Definition wird als Definition, die von einem Kollektiv geteilt wird, hervorgehoben:

So z. B. in Beleg 5 über die Formulierung: Konsens besteht darin, dass [...] (Büssing/Schmitt 1998) oder in Beleg 6b: this definition is almost identical to the definition by [...] (Kristensen/ Borritz/Villadsen et al. 2005). Weitere Belege aus dem Korpus: Erschöpfung und der Depersonalisation als Kerndimensionen des Burnout (Freund/Diestel/Schmidt 2012: 144, ZfAO); vorherrschende Resignation, Motivationseinschränkungen, herabgesetzte Widerstandsfähigkeit gegenüber Belastungen und negative Emotionen zählen [...] zum Kern des Burnout-Syndroms (Schaarschmidt/Kieschke/Fischer 1999: 250, PiEU); Burnout wird ziemlich übereinstimmend als langandauernde Streßreaktion aufgefaßt und meist [...] als ein psychisches Drei-KomponentenSyndrom [...] definiert (Schmitz 1998: 129, PiEU); Einigkeit besteht über folgende 3 Hauptkriterien: 1.) Emotionale Erschöpfung [...], 2.) Depersonalisation [...], 3.) Leistungsabnahme [...] (Bergner 2016: 976, DMW); In der Befragung [...] wurde nach Symptomen emotionaler Erschöpfung gefragt, die als erste Phase und Kerndimension eines Burn-out-Syndroms gilt (Ansmann/ Nitzsche/Neumann 2014: A264, Dt. Ärztebl.); [...] verbinden verschiedene Ansätze und entwickeln daraus folgende Arbeitsdefinition (Weimer/Kraus 2011, im QV unter 8.1.5); [...] präzisiert eine Reihe von Merkmalen, die er als zentral ansieht: [...]. (Rösing 2003, im QV unter 8.1.5); [...] Risikozustand [...]. Diese Einschätzung wird auch von der Mehrheit der Psychiater und Psychotherapeuten geteilt (Kissling/Mendel/Förstl 2014: 2588, DMW); Sehr häufig wird unter Burnout ein arbeitsbezogenes Syndrom verstanden, das nach Maslach et al. [7, 8] hauptsächlich gekennzeichnet ist durch die 3 Kernsymptome [...] (Oepen/Gruber 2014: 269, PPmP); Unter B. wird im Allgemeinen ein Konglomerat einer schleichenden seelischen Auszehrung verstanden. Ein drohender Burnout kündigt sich mit den Warnsignalen einer chronischen Erschöpfung und Ermüdung an (Schenk/Vogt/Wippert 2006: 14, in der Zeitschrift „Leistungssport“, im QV unter 8.1.5); Leitsymptom des Burn-out-Zustands ist ein andauerndes Erschöpfungserleben (Berger/Schneller/ Maier 2012, Der Nervenarzt, im QV unter 8.1.4); Die hier vorgestellten Definitionen haben einen gemeinsamen Nenner. Demzufolge bezeichnet das BOS das Bild eines beruflich und/oder familiär überforderten Menschen, [...] (Beschoner/Schönfeldt-Lecuona/Braun et al. 2009: 216, PiD, im QV unter 8.1.4); den Kardinalsymptomen emotionale Erschöpfung, Gleichgültigkeit und Zynismus

unter 8.1.5) spricht im Zusammenhang mit der Prävalenz in der Bevölkerung über „Burnoutetikettierte Symptome“.

524 Zum Zusammenhang repräsentativer und deklarativer Merkmale von Definitionshandlungen vgl. auch Kap. 4.3.2 dieser Arbeit. 
Kunden gegenüber bzw. verminderter Lebenszufriedenheit im Sinne der Definition von Maslach und Shirom (Kopka/Ast/Hügel et al. 2009: 230, PiD, im QV unter 8.1.4); Als Hauptmerkmal von Burnout gilt emotionale Erschöpfung (Dicke/Holzberger/Kunina-Habe nicht 2016: 246, in PiEU, im QV unter 8.1.4); Folgende 3 Dimensionen von Beschwerden haben sich als gemeinsamer Nenner verschiedener Burnout-Konzeptionen etabliert [...] tiefgreifende Erschöpfung, [...]. (grosse Holtforth/Keller/Hochstrasser 2016: 26, PiD, im QV unter 8.1.4); Zum typischen Erscheinungsbild gehören Erschöpfung, Depersonalisation und berufliche Überforderung. Hinzu kommt ein Leistungsabfall (Zeidler/Musalek 2017: 78, PiD).

Solche Formulierungen, die sich zahlreich in den Texten finden, bewirken eine Zusammenführung unterschiedlicher Diskursstimmen und deuten auf Konsensfindungsprozesse hin mit dem Ziel, sich innerhalb eines Fachs auf eine allgemein anerkannte Festlegung zu verständigen.

Diese repräsentativen Formen des Definierens, bei denen sich diejenige Definition vorläufig durchsetzt, die sich in Bezug auf die beobachteten Verhältnisse oder durch ihre Verwendung in der Forschung zu bewähren scheint, ${ }^{525}$ passen zur Grundidee der „Logik der Falsifikation“ nach Popper (vgl. Küttner 1992: 80; Prechtl ${ }^{2}$ 1999: 175) und zum theoretisch unabschließbaren Sinnbildungsprozess nach Peirce (vgl. Kap. 2.2.2 und 4.3.2 dieser Arbeit; CP 8.148). Eine einheitliche Definition mit deklarativer Kraft wird im fachlichen Kontext allerdings dann gefordert, wenn diese Definition Eingang in die internationale Klassifikation der Krankheiten finden soll und wenn therapeutische Behandlungen auf diese Diagnose abgerechnet werden können sollen (vgl. Kap. 4.3.2). Diese beiden Pole repräsentativ-falsifizierbarer bzw. deklariert-konsentierter Gültigkeit einer Definition stehen sich gegenüber, bedingen sich aber auch gegenseitig. Denn eine Definition, die deklariert wird, ist einerseits Ergebnis eines Einigungsprozesses unter Fachleuten, der wiederum auf Überschneidungen oder Übereinkünften verschiedener Definitionen beruht und in regelmäßigen Abständen einer Überprüfung unterzogen wird. ${ }^{526}$ Andererseits wirkt sich der Anspruch kollektiver Akzeptanz und die damit verbundene Wirkmächtigkeit einer Definition mit deklarativer Kraft innerhalb der klinischen Verschreibungs- und Forschungspraxis schon auf den ersten Akt aus, in dem eine repräsentative Definitionsbehauptung neu aufgestellt wird. Denn auch schon bei dieser ersten definitorischen Behauptung soll ein gewisser Grad an überindividueller Geltung erfüllt werden, sei dies durch den Bezug auf „regelhafte Verweise“ zum Verwendungszusammenhang

525 Wobei damit nicht gesagt sein soll, dass, nur weil ein Messinstrument oder eine Definition von verschiedenen Studien weiterverwendet wird, dies ihren Bewährungsgrad eindeutig belegt. Denn dies können auch diskursive Übernahmepraktiken sein, die die Probleme einer Definition/ eines Messinstruments im Rahmen der Studie nicht thematisieren.

526 Das Verfahren der Krankheitsnormierung für die ICD und das DSM laufen nach diesem Schema ab. Vgl. zur Darstellung dieser Klassifikationsprozesse Saß/Saß-Houben (2005: $137 \mathrm{ff}$.). 
dieses Zeichens in einer Fach- oder Sprachgesellschaft (vgl. Bär 2015: 34) oder über die Adäquatheit zwischen Definiens und Definiendum.

Die Autorinnen und Autoren der Fachzeitschriftenartikel und Monografien thematisieren damit weitaus stärker explizit die Gütekriterien von Definitionen, deren Geltungsbedingungen und das Verhältnis zwischen Definiens und Definiendum, als dies in den Fachlexika, Hand- und Lehrbüchern der Fall ist. Dies wird besonders deutlich an den operationalen Definitionen (vgl. Beleg 2, 6b), welche die Relation zwischen Repräsentiertem und Repräsentierendem meist ontologisch verankern. So betonen Maslach und Jackson beispielsweise ausdrücklich, dass sie ihre „conceptual definition“ und „standardized measure“ von der Empirie abgeleitet haben (,derived empirically“):

This definition of burnout, which is now being used widely in ongoing research, was not based on a theoretical model but was derived empirically. Several years of earlier exploratory research provided the groundwork for the development of both our conceptual definition and our standardized measure.

(Maslach/Jackson 1984: 134, im QV unter 8.1.5)

Und die Autorinnen und Autoren des Copenhagen Burnout Inventory (Kristensen/Borritz/Villadsen et al. 2005) legen dar, welche Schwachstellen sie im Beziehungsverhältnis von Messinstrument, Definition und Operationalisierung (der ,Übersetzung، von abstrakten Konzepten in beobachtbare bzw. messbare Merkmale) beim MBI von Maslach/Jackson sehen (vgl. Beleg 6b).

Die Beispiele im Belegblock 6 zeigen dadurch, wie entscheidend das Messinstrument und mit diesem seine Operationalisierung über Fragebogen-Items für das Konzept und damit auch für die Definition von `Burnout ‘ ist. Im Fragebogen von Maslach/Jackson, der in Beleg 6a in der deutschen Übersetzung und Fassung von Büssing/Perrar zitiert ist, wirkt das erste Item (Ich fühle mich durch meine Arbeit ausgebrannt) wie ein Hinweis darauf, unter welcher Überschrift die folgenden Fragen zu lesen sind, und die suggestive Kraft dieser ersten Perspektive zur Bewertung des 〉Gefühls am Ende eines Arbeitstages` ist nicht zu unterschätzen. Im englischen Original steht diese Fragebogen-Aussage allerdings zumindest in späteren Fassungen nicht auf Platz 1, sondern wird als 8. Aussage in den Fragebogen integriert (Maslach/Jackson/Leiter ${ }^{3}$ 1996). Durch das erste Fragebogen-Item in der Version von Büssing/Perrar (1992, Beleg 6a oben) werden die inhaltlichen Grundkomponenten des Burnout-Konzepts direkt abgesteckt: Es handelt sich um einen sindividuell als negativ erlebten egressiven Gefühlszustand, der mit der Arbeit zusammenhängtく. Wenn in einem Fragebogen das Wort ausgebrannt selbst in den ersten Aussagen des Fragebogens verwendet wird, deutet dies darauf hin, dass die Erstellerinnen und Übersetzer des Fragebogens davon ausgingen, dass das Wort im alläglichen Sprachgebrauch hinlänglich bekannt ist. Es stellt sich in diesem Zusammenhang 
außerdem die Frage, ob Testpersonen den ersten Fragebogen-Items generell eine das Thema der Erhebung bestimmende Funktion zusprechen und welche Eigenhypothesen sie daran anknüpfen, die ihr Antwortverhalten beispielsweise im Sinne sozial erwünschter Eigenschaften ${ }^{527}$ beeinflussen können. In der empirischen psychologischen Forschung spricht man in diesem Zusammenhang von „Antworttendenzen“ sowie von „Kontext-, Einbettungs- und Reihenfolgeeffekten“ (Bierhoff/ Petermann 2014: 114). Gut erforscht sind zum Beispiel der Primacy-Effekt und der Recency-Effekt (vgl. ebd. 199), die bewirken, dass die ersten oder die letzten gegebenen Informationen bei der Beurteilung eines Sachverhalts übergewichtet werden, weshalb Handreichungen zur Entwicklung psychologischer Befragungen die Darbietung des Fragebogens in unterschiedlichen Reihenfolgen an die Testpersonen empfehlen. Ein Blick in zwei jüngere Publikationen mit Hinweisen für empirische Fragebogen-Forschungsdesigns (Bierhoff/Petermann 2014; Porst 2014), in denen auch die Entwicklung von Skalen mit „Fragebogen-Fragen“ (Porst 2014: 99) bzw. „Vorformulierten Aussagen (Items)“ (Bierhoff/Petermann 2014: 256) beschrieben wird, ergibt, dass die perspektivierende und definierende Kraft von sprachlichen Formulierungen dort als möglicher Einflussfaktor prinzipiell einbezogen wird. Die beispielsweise bei Porst aufgelisteten „Gebote“ sind allerdings ziemlich allgemein gehalten, ohne Hinweise auf die Formenvielfalt sprachlicher Zeichen, mit denen beispielsweise etwas „unterstellt“ werden kann: „2. Gebot: Du sollst lange und komplexe Fragen vermeiden!“ (Porst 2014: 103ff.) oder „4. Gebot: Du sollst doppelte Stimuli und Vermeinungen vermeiden!“ (ebd.: 106ff.) oder „5. Gebot: Du sollst Unterstellungen und suggestive Fragen vermeiden!“ (ebd.: $107 \mathrm{ff}.){ }^{528}$ In welchem Maß einzelne sprachliche Benennungsalternativen Wirk- oder Störfaktoren in der empirischen Forschung über Fragebogendesigns darstellen, könnten linguistisch-psychologische Anschlussstudien erforschen.

Betrachtet man die einzelnen Items des „Maslach Burnout Inventory“ und „Copenhagen Burnout Inventory“, so enthalten sie ähnlich wie die beschriebenen Definitionen in den Fachlexika, Hand- und Lehrbüchern sprachliche Mittel der Graduierung. Schließlich basiert die Gesamtkonzeption des Fragebogens auf

527 Vgl. z. B. das Konzept der „sozialen Erwünschtheit“: „Darunter versteht man die verständliche Tendenz von Teilnehmern, auf die Items des Fragebogens so zu antworten, dass sie einen möglichst günstigen Eindruck hervorrufen.“ (Bierhoff/Petermann (2014: 114).

528 Bierhoff/Petermann (2014: 140) verweisen auf eine Publikation von Hannelore Weber (2006), in der sie auf ein methodisches Problem innerhalb der differenziellen Psychologie hinweist, das auftritt, wenn „Persönlichkeitsmerkmale mit Folgen in Verbindung gebracht werden, die in der begrifflichen Bedeutung des Persönlichkeitsmerkmals schon enthalten sind“ (Bierhoff/Petermann (2014: 140) über Weber 2006). 
gradueller Konzeption, denn die Versuchspersonen sind dazu aufgerufen, je nach Häufigkeit und Intensität ihre Zustimmung zu den Items kenntlich zu machen.

So unterschiedlich demnach die angeführten Definitionsbeispiele der Fachzeitschriftenartikel und Monografien auf der Ebene der Definitionsentfaltung auf Satzund Textabschnittsebene sind, so weisen die meisten in Bezug auf die sprachlichen Mittel, die auf der Ebene der Morpheme und (Mehr-)Worteinheiten eine Abgrenzung zu normalen alltäglichen Erschöpfungszuständen bzw. zu normalem Verhalten, Erleben und normalen Arbeitsbedingungen spezifizieren, viele Ähnlichkeiten untereinander und mit den Definitionen der Fachlexika, Hand- und Lehrbücher auf. Denn die beschriebenen physischen und psychischen Merkmale werden auch in diesen Belegen erst dadurch zu erklärungsbedürftigen Symptomen,

1.) dass sie im Vergleich zu ähnlichen, implizit mitgemeinten 'gesunden' bzw. 'normalen' physischen und psychischen Referenz-Zuständen ein reduziertes oder gesteigertes Maß präsentieren, ${ }^{529}$ oft verbunden mit einer Bewertung der Intensität, Dauer oder Häufigkeit der beschriebenen PHYSISCHEN und PSYCHISCHEN MERKMALE bzW. SYMPTOME: ${ }^{530}$

- Fokus auf somatischer Ebene: suffering from frequent headaches and gastrointestinal disturbances, too somatically involved with one's bodily functions (Freudenberger 1974, im QV unter 8.1.5); Er klagt häufig über andauernde Müdigkeit, neigt vermehrt zu psychosomatischen Krankheiten wie Magengeschwüren, Migräneanfällen, Ekzemen und Asthma (Schmidbauer 1982, im QV unter 8.1.5); chronische Schmerzen ohne Befund (Bauer/Häfner/Kächele et al. 2003, PPmP, im QV unter 8.1.4); besonderer Fall schwerer Erschöpfung (Eckart 2008, DMW, im QV unter 8.1.4); andauerndes Erschöpfungserleben (Berger/Schneller/Maier 2012, Der Nervenarzt, im QV unter 8.1.4); immer wieder auftretendes Herzrasen, häufige Infektionen der Atemwege und eine Gewichtsabnahme (Kissling/Mendel/Förstl 2014, DMW, im QV unter 8.1.4), ausgeprägte Erschöpfungssymptomatik (Weimer/Kraus 2011, Der Psychotherapeut, im QV unter 8.1.5); verminderte Schlafqualität (Berger/Falkai/Maier 2012, Dt. Ärztebl., im QV unter 8.1.4); Anstieg in körperlichen Beschwerden (Zimmer/Klausmann 2016, PiEU, im QV unter 8.1.4); häufig, aber auch durch somatoforme Beschwerden (u.a. Fatigue, Schwindel, gastrointestinale Symptome, chronische Schmerzen) und Suchtverhalten (schädlicher Gebrauch von Alkohol, Stimulantien, Schlaf- und Beruhigungsmitteln) gekennzeichnet (Söllner/Behringer/Böhme 2016, PPmP, im QV unter 8.1.4);

- Fokus auf psychosozialer Ebene: langandauernde Stressreaktion (Schmitz 1998, in PiEU, im QV unter 8.1.4); staff member's quickness to anger and his instantaneous irritation and frustration responses, finds it just too difficult to hold in feelings. He cries too easily, the slightest pressure makes him feel overburdened, excessive use of tranquilizers, excessively

529 Auch Schuster verweist in ihrer Arbeit zur Entwicklung der psychiatrischen Schreibpraxis von 1800-1939 darauf, dass Graduierungen eine wichtige Rolle spielen, um ,die Stärke oder Ausprägung eines Symptoms oder einer Krankheit anzugeben“ (vgl. Schuster 2010: 238f.).

530 Die hier zitierten Belege wurden orthografisch und flexionsmorphologisch angeglichen und daher kursiv gesetzt, sonst sind sie aber im Vergleich zu den Originalen unverändert. 
rigid (Freudenberger 1974, im QV unter 8.1.5); reduced personal accomplishment, being emotionally overextended, decline in one's feelings of competence (Maslach/Jackson 1984, im QV unter 8.1.5); persistent, negative, work-related state of mind, reduced effectiveness, decreased motivation, (Schaufeli/Enzmann 1998, im QV unter 8.1.5); Every working hour is tiring you? (Kristensen/Borritz/Villadsen et al. 2005, im QV unter 8.1.5); Sympathie für Klienten [...] schwindet und macht mißtrauischer Distanz Platz. [...] (Schmidbauer 1982, im QV unter 8.1.5); belastet mich zu stark, ist für mich wirklich anstrengend (Büssing/Perrar 1992, im QV unter 8.1.5); Leistungseinbuße, reduzierte Leistungsbereitschaft bzw. -fähigkeit, reduziertes Engagement für Patienten und Klienten, Abbau, Verflachung, zunehmende Verleugnung des Problems, abnehmende Flexibilität im Denken/Verhalten, zunehmende Sinnlosigkeit (Kaschka/Korczak/Broich 2011, im Dt. Ärztebl., im QV unter 8.1.4); vermindertes Engagement [...], mit eingeschränkter Distanzierungsfähigkeit, hoher Resignationstendenz, geringem Wohlbefinden sowie herabgesetzter Widerstandsfähigkeit (Stück/Rigotti/Balzer 2005, in PiEU, im QV unter 8.1.4); [...] ständige Frustration, [...] seelische Überlastungen (Eckart 2008, in DMW, im QV unter 8.1.4); geringe Arbeitszufriedenheit und geringes Wohlbefinden bzw. Lebensqualität, d.h. intensiveres Burnout-Erleben (Hillert/Koch/Lehr 2013, Der Nervenarzt, im QV unter 8.1.4); typische Erschöpfungsbilder infolge emotionaler Überforderung (Kettler 2012: A-1189f., Dt. Ärztebl.); durch das permanente Gefühl, der Arbeit nicht gerecht zu werden und an den Grenzen der Leistungsfähigkeit zu kämpfen (Madel 2015, Dt. Ärztebl., im QV unter 8.1.4); Dazu kommt ein starker Interessenverlust an dem zuvor übertrieben ausgeführten Vorgang (Schneider/Bühler 1998, Dt. Ärztebl., im QV unter 8.1.4).

2.) dass sie ähnliche mitgemeinte Referenzzustände bzw. Normen, die ein 'gesundes', 'normales', 'funktionales' oder 'sozial erwünschtes' VerHALTEN und ERLEBEN darstellen, negieren oder zu diesen in Opposition stehen:

- Fokus auf somatischer Ebene: being unable to shake a lingering cold; sleeplessness, chronische Schmerzen ohne Befund, unspezifische Beschwerden des Magens und des Darmes (Bauer/Häfner/Kächele et al. 2003, in PPmP, im QV unter 8.1.4); Appetitlosigkeit, Libidoverlust, Gewichtsverlust, Unruhe, überhaupt nicht mehr abschalten können, nachhaltig unkonzentriert (Wilms 2009, in PiD, im QV unter 8.1.4); Unvermögen, sich zu entspannen (Geyerhofer/Unterholzer 2009, in Psychotherapie im Dialog, im QV unter 8.1.4); Ruhelosigkeit (Rösing 2003 nach Schaufeli/Enzmann 1998, im QV unter 8.1.5); Schlaflosigkeit (von Känel 2008, im QV unter 8.1.5); unspezifische [...] somatische Beschwerden (Stöbel-Richter/Daig/Brähler et al. 2012, in PPmP, im QV unter 8.1.4);

Fokus auf psychosozialer Ebene: inflexible (Freudenberger 1974, im QV unter 8.1.5); depersonalization, unfeeling and callous response toward these people, who are usually the recipients of one's service or care (Maslach/Jackson 1984, im QV unter 8.1.5); Ferien lassen nicht neue Kräfte schöpfen, sondern den Zwang zum Wiederantreten der Arbeit nur düsterer scheinen (Schmidbauer 1982, im QV unter 8.1.5); negative und zynische Einstellung zu ihrer Arbeit und den Klienten (Kleiber/Enzmann 1990, im QV unter 8.1.5); negative, work-related state of mind; distress, dysfunctional attitudes and behaviours at work; a misfit between intentions and reality in the job; inadequate coping strategies that are associated with the syndrome (Schaufeli/Enzmann 1998, im QV unter 8.1.5); Entpersönlichung führt zu einer indifferenten Haltung gegenüber den Klienten (Ulich 1991, in PiEU, im

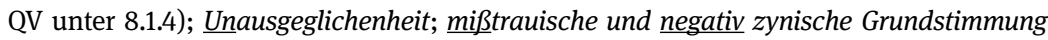


gegenüber Kollegen (Büssing/Schmitt 1998, ZfAO, im QV unter 8.1.4); daß ich manche Personen so behandle, als wären sie unpersönliche „Objekte“ (Büssing/Perrar 1992, im QV unter 8.1.5); gefühllose, gleichgültige Einstellung; negative Einschätzung der persönlichen Leistungskompetenz (Bauer/Häfner/Kächele et al. 2003, in PPmP, im QV unter 8.1.4), Depersonalisation, keine Zeit $\underline{\text { mehr }}$ für nicht-berufliche Bedürfnisse; Orientierungslosigkeit, Zynismus; innere Leere; zunehmende Sinnlosigkeit und Desinteresse (Kaschka/ Korczak/Broich 2011, im Dt. Ärztebl., im QV unter 8.1.4); führen kurze Erholungsphasen nicht zu einer Rückbildung (Berger/Falkai/Maier 2012, Dt. Ärztebl.; Es fällt mir schwer, mich nach einem Arbeitstag zu entspannen. Ich bin nicht davon überzeugt, dass ich meine Arbeit gut mache. (Zimmermann/Kaiser/Bernholt et al. 2016, in PiEU, im QV unter 8.1.4); Ich fühle mich (nicht) voller Tatkraft (Buschmann/Gamsjäger 1999, in PiEU, im QV unter 8.1.4); So wird Kompensation [...] gesucht, die aber misslingt (Will 2014, in Dt. Ärztebl., im QV unter 8.1.4); ( ... ich kann nicht mehr ... alles lastet auf mir"), habe sich andererseits aber immer wieder angetrieben (Wilms 2009, PID, im QV unter 8.1.4); Das Ziel wird erreicht, die Anerkennung, Dankbarkeit, das Lob, der Lohn fallen jedoch nicht den Erwartungen gemäß aus (Beschoner/Schönfeldt-Lecuona/Braun et al. 2009, PiD, im QV unter 8.1.4); ... ist in der Pubertät, aber ihre Symptome reichen weit über normale pubertäre Gefühlsschwankungen hinaus (Schulte-Markwort 2015, im QV unter 8.1.5); Typische Symptome des manifesten Burnout-Syndroms sind Frustration, Hilflosigkeit, Unzufriedenheit, Erschöpfung und Ineffizienz (Schneider/Bühler 1998, Dt. Arztebl., im QV unter 8.1.4).

3.) dass sie Eigenschaften von Personen mit Burnout benennen, die in der Gesellschaft zwar ein hohes sozial-ethisches Ansehen besitzen, mit denen diese Personen jedoch teilweise über einem implizit mitgemeinten sozial-ethischen Normalmaß liegen (`Aufopferung` liegt über dem ,Normalmaß`auf einer Skala des `Einsatzes im Beruf $\triangleleft$ ). Durch den Burnout-Prozess werden diese zugeschriebenen Eigenschaften jedoch reduziert oder auch (negativ) transformiert:

- aufopferungsvolle, pflichtbewußte und ehemals besonders engagierte Mitarbeiter; sie zu reizbaren, mißtrauischen, halsstarrigen Mitarbeitern werden (Kleiber/Enzmann 1990, im QV unter 8.1.5); besondere Identifikation mit dem Beruf (Büssing/Glaser 1993, in ZfAO, im QV unter 8.1.4); besonders engagierte und pflichtbewußte Helfer in Sozialberufen, besondere Erwartungen sowie eine besondere Identifikation mit dem Beruf (Büssing/Schmitt 1998, in ZfAO, im QV unter 8.1.4); vermehrtes Engagement für Ziele, Zwang, sich zu beweisen (übertriebener Ehrgeiz); verstärkter Einsatz (Kaschka/Korczak/Broich 2011, im Dt. Ärztebl., im QV unter 8.1.4); überhöhte Zielsetzungen, Perfektionismus, Selbstüberschätzung (Berger/ Falkai/Maier 2012, im Dt. Ärztebl., im QV unter 8.1.4); zu hohe persönliche Erwartungen an die eigene Leistung (Eckart 2008: 34, DMW, im QV unter 8.1.4); extrem stark ausgeprägtes Perfektionismusstreben (Wilms 2009, in PiD, im QV unter 8.1.4); Burnout-gefährdet scheinen allerdings Persönlichkeiten, welche sich bei der Arbeit übermässig verausgaben (sog. overcommitment), [...], gesteigerter Einsatz für Ziele, Zunahme der Überstunden [...] reduzierte soziale Interaktion, negative Einstellung zur Arbeit ... (von Känel 2008 im QV unter 8.1.5).

In vielen dieser Beispiele wird eine Vorher-Nachher-Konstellation erzeugt, wobei die Personen vorher gesellschaftlich eher positiv bewertete Eigenschaften zeigen, 
die im Zuge des Burnout-Prozesses verschwinden oder sich in ihr Gegenteil verkehrt haben.

Und in einigen Definitionen findet über die Referenz auf die URSACHEN oder Umgebungsfaktoren, die den Prozess des Ausbrennens beeinflussen, eine weitere Eingrenzung des Phänomens/Konzepts statt. Dabei findet man wieder quantifizierende, gradierende und negierende sprachliche Mittel, die diese Umgebungsfaktoren dadurch von 'normalen', 'nicht-krankmachenden' UMGEBUNGSFAKTOREN DES ARBEITS- UND PRIVATLEBENS abgrenzen:

from long-term involvement in work situations that are emotionally demanding (Kristensen/ Borritz/Villadsen et al. 2005, im QV unter 8.1.5); outcome of chronic depletion of the individual's coping resources resulting from prolonged exposure to stress (Toker/Shirom/Shapira et al. 2005: 344); arbeitete sie zusätzlich zu ihrer Anstellung als Stationsleiterin in einer chirurgischen Klinik oft bis spätabends als Bedienung in einem Restaurant (Kissling/Mendel/Förstl 2014, in DMW, im QV unter 8.1.4) ; $^{531}$ misfit between intentions and reality in the job (Schaufeli/Enzmann 1998, im QV unter 8.1.5); high job demands and low resources (Demerouti/Bakker 2008, im QV unter 8.1.5); Burnout is a process in which the psychological resources of an employee are gradually depleted as a consequence of prolonged stress at work (Ahola/Hakanen/Perhoniemi et al. 2014: 29, im QV unter 8.1.5); Konstrukt einer chronischen Anpassungsreaktion infolge dauernden Stresses (Dech 2009: 210, PiD, im QV unter 8.1.4); andauernde Arbeitsüberforderung, real unbewältigbarer Arbeitsanfall, mangelnde Anerkennung durch Vorgesetzte, fehlende Abgrenzung zum Privatleben (Berger/Falkai/Maier 2012, im Dt. Ärztebl., im QV unter 8.1.4); Im Verlauf eines Arbeitslebens kann es üblicherweise immer mal wieder zu vorübergehenden Überlastungen kommen, [...]. Bei länger anhaltender Überforderung kann der Begriff Burnout [...] genutzt werden (Schramm/Berger 2013, in Der Nervenarzt, im QV unter 8.1.4).

Die Abgrenzung zu sachverwandten Konzepten wie der >Depression`, >Belastungsreaktion`, >Anpassungsstörung` oder >Angststörung` wird über ähnliche graduierende, vergleichende, steigernde, negierende und adversative sprachliche Mittel vollzogen, allerdings im Vergleich zur Abgrenzung vom sog. ,Normalzustand' nicht ganz in derselben Vorkommensdichte:

- lack of reciprocity at work is predictive of both burnout and depression, and that a lack of reciprocity in private life is predictive of depression, but not of burnout (Bakker/Schaufeli/ Demerouti et al. 2000: 261); in a Dutch study among teachers a reduced sense of superiority, i.e., the inability to rate oneself favourably in comparison to others, was related to depressive symptoms but not to burnout (Ahola/Hakanen/Perhoniemi et al. 2014: 30, im QV unter 8.1.5); Suizidgedanken und Suizidalität bestanden im gesamten Zeitraum nicht; seien jedoch Gedanken an den Tod aufgetreten, die aber nicht persistierten. Die vegetativen Beschwer-

531 Im Fallbeispiel von Kissling/Mendel/Förstl (2014) (= Beleg 8) erfolgt die ,krankmachende‘ Steigerung durch die Menge der für sich schon belastenden Einzelfaktoren (alleinerziehend, krankes Kind, finanzielle Probleme, Doppelbelastung durch Kinder und zwei Arbeitsstellen etc.). 
den (Herzklopfen, Hitzewallungen, Übelkeit) sind weiterhin mittelgradig ausgeprägt; eine phobische Symptomatik bzw. Vermeidungsverhalten besteht jedoch nicht. (Wilms 2009, PiD, im QV unter 8.1.4); Während beim BOS Symptome wie depressive Verstimmung, Verlust von Freude und Interessen sowie rasche Ermüdbarkeit und reduzierter Antrieb durchaus vorkommen können, finden sich Schuldgefühle, reduziertes Selbstwertgefühl, Appetit- und Libidoverlust eher nicht. Le ausgeprägter ein BOS ist, umso höher ist die Korrelation mit einer depressiven Episode (Beschoner/Schönfeldt-Lecuona/Braun et al. 2009, PiD, im QV unter 8.1.4); Eine Studie zur Konstruktvalidität von Burnout in Abgrenzung zur Depression von Reime und Steiner [86] zeigt eine moderate Korrelation von $r=0,44$ zwischen der allgemeinen Depressionsskala (ADS [87]) und der Subskala ,emotionale Erschöpfung“ aus dem MBI, jedoch lediglich eine geringe Korrelation (von $r=0,22)$ mit der Skala „Depersonalisation/Zynismus“" des MBI und eine nicht signifikante Korrelation mit abnehmender subjektiver Leistungsfähigkeit $(r=-0,18)$ (Dörr/Nater 2013 PPmP).

Wie gezeigt wurde, weisen die Definitionen in Lexika- Hand- und Lehrbüchern einige Ähnlichkeiten, aber auch Unterschiede zu den sich in Texten der Fachzeitschriften und Monografien/Sammelbände punktuell manifestierenden Definitionen auf. Diese Gemeinsamkeiten und Unterschiede werden im folgenden Kapitel nochmals zusammengefasst, die sprachlichen Mittel des Definierens im fachlichen Diskursstrang tabellarisch zusammengeführt und im Rahmen des praxeologischen Analysevorgehens dieser Arbeit heuristisch mit den Teilpraktiken und sprachlichen Stimuli des Definierens, die sich in der Fläche der Diskursstränge zeigen und in den Kapiteln 6.2.4.1 und 6.2.4.2 beschrieben werden, verknüpft.

\subsubsection{Exkurs: Bemerkung zur Verwendung des Analysebegriffs >Definitionsentfaltungs}

Mit dem Analysebegriff $>$ Definitionsentfaltung ` bezieht sich die Arbeit auf Literatur zur thematischen Entfaltung bzw. zu Vertextungsmustern (nach Konerding 2005: 9ff.; Brinker/Cölfen/Pappert ${ }^{8}$ 2014: 60ff.; Müller 2007: 77 ff.; Gansel/Jürgens ${ }^{3} 2009$ : 148ff. mit Bezug auf Heinemann/Viehweger 1991: $237 \mathrm{ff}$. und Sandig 1986: 182f.). Analysekategorien dieser Theorien werden auf Textpassagen, in denen definiert wird, angewendet.

Die Arbeit stützt sich mit Konerding (2005: 10) zunächst auf den Thema-Begriff nach Ochs-Keenan/Schieffelin (1976), nach dem „Diskurstopiks bzw. -themen [...] spezifikationsbedürftige Wissensbestände bei Interaktionspartnern“ sind und die „in einer Frage explizit Ausdruck erlangen“ (Konerding 2005: 11). Vom Gespräch auf den Diskurs übertragen heißt dies, dass ein Diskursthema ,intertextuell“ in einzelnen Texten als ,Gesprächsbeiträgen' verhandelt wird (ebd.: 9). Wissensbestände, „die hinsichtlich ihrer Detailliertheit bestimmter Komponenten unterspezifiziert sind“ und die in „sogenannten Ergänzungsfragen (bzw. „w-Fragen“)“ Ausdruck erlangen, werden nach Konerding in primär deskriptiven Texten entfaltet (ebd.: 11). „, [V]orgebliche Wissensbestände, für die eine akzeptable präferenz- 
bezogene Einbettung übergeordneter Wissenszusammenhänge gefordert ist und für die eine rezipientenbezogene Möglichkeit der Akzeptanz durch diese Einbettung geleistet werden soll“, erlangen in „,sogenannten Entscheidungsfragen (ja/ nein-Fragen) Ausdruck“. Letztere werden in primär argumentativen Texten verhandelt (ebd.). Diese grundlegende Dichotomie wird für die vorliegende Arbeit zur Beurteilung definierender Textabschnitte übernommen, die beispielsweise einerseits auf die Frage „Was ist Burnout?“ und andererseits auf Fragen des Typs „Ist Burnout eine >Krankheitı?“ definitorisch antworten.

Die Arbeit unterscheidet des Weiteren in Anlehnung an Brinker/Cölfen/ Pappert ( ${ }^{8} 2014:$ 60ff.) und Müller (2007) zwischen deskriptiven, narrativen und explikativen Mustern der thematischen bzw. definitorischen Entfaltung. Deskriptive und narrative Texte werden mit Rückgriff auf die Argumentation Müllers (2007: 82) zunächst durch das Kriterium des entfalteten Redegegenstandes unterschieden: „Erzählen soll die Vertextung von Ereignissen und Prozessen heißen; diese sind als auf der Zeitachse sich vollziehende Entwicklungen gedacht“(ebd.), die „prozessual-aktional[e]“ repräsentiert werden (Brinker/Cölfen/Pappert ${ }^{8} 2014$ : 66). „Beschreiben wird die Vertextung von Gegenständen und synchronisch gedachten Konstellationen genannt“ (Müller 2007: 82, Kursivsetzung im Orig.).

Die erzählende Themenentfaltung in diesem weiten Sinn wird in dieser Arbeit allerdings nochmals graduell nach stilistischen und semantischen Kriterien unterteilt, und zwar in die Schemata `Referieren` und `Narration im engeren Sinn ২. Mit >Referieren` werden Textpassagen beschrieben, die „ergebnisorientiert“ eine eher „sachlich-registrierend[e], stets um Objektivität [...] bemühte“ Darstellung bei der Repräsentation des Ereignisses/Vorgangs wählen (vgl. Heinemann/Viehweger 1991: 239; Gansel/Jürgens 332009: 150). Bei Vorgängen kommt zudem das von Brinker/Cölfen/Pappert ( ${ }^{8} 2014:$ 62) genannte semantische Kriterium des 'Regelhaften' hinzu. >Narrationen im engeren Sinn` sind „ereignisorientiert“ (Gansel/ Jürgens ${ }^{3}$ 2009: 150) und elaborieren die Erzählung der Ereigniskette dramatisch. ${ }^{532}$

532 Marcus Müller kritisiert dieses stilistische Unterscheidungskriterium in Bezug auf Brinkers Unterscheidung zwischen >Beschreiben` und `Erzählen` (Müller bezieht sich dabei auf Brinker ( ${ }^{6} 2005$ : 65ff.)). Für Brinker zähle auch die Entfaltung eines einmaligen Vorgangs zur deskriptiven Themenentfaltung, wenn diese „weder sich noch den Rezipienten selbst in die Darstellung“ involviere, „sondern das Ereignis vielmehr in einer neutralen und distanzierenden Diktion“ präsentiere (Müller 2007: 81). Müller argumentiert, dass jedoch auch in „synchronisch gedachten Konstellationen“, also Texten, die beispielsweise einen Gegenstand beschreiben, „dramatische Elaboration“ zum Einsatz kommen kann. In dieser Arbeit wird diese stilistische Unterscheidung als graduelles Unterscheidungskriterium dennoch übernommen, da sie nützlich ist, um Narrationen in fachlichen Kontexten von Narrationen in fachexternen Kontexten zu unterscheiden. Letztere zeichnen sich nämlich in der Tat durch eine dramatische Elaboration im Vergleich zu den in fachlichen Kontexten referierten klinischen Einzelfällen ab. Diese stilistische Unterscheidung wird in dieser Arbeit jedoch nicht 
Die explikative Themenentfaltung bzw. Definitionsentfaltung wird nach Heinemann/Heinemann (2002: 188, zitiert bei Müller 2007: 80) „,als ,Mischkonzept““ beschreibender und argumentativer Formen gesehen, da „,Erklären ja heißt, Sachverhalte zu beschreiben und ggf. argumentativ zu stützen“ (Müller 2007: 80). Es wird dennoch teilweise mit dem Terminus explikativ gearbeitet, wenn in Definitionen Passagen erscheinen, die einen „Abhängigkeitszusammenhang“ (vgl. Jahr 2000: 386) oder eine Folgebeziehung konstatieren, bei der die „rezipientenbezogene Möglichkeit der Akzeptanz“ (Konerding 2005: 11) von Seiten der Emittenten vorausgesetzt wird.

\subsubsection{Zusammenfassung sprachlicher Mittel des Definierens im fachinternen Diskursstrang aus punktueller Perspektive und Ausblick auf sprachliche Stimuli des Definierens in der Fläche der Diskursstränge}

In den vorigen Kapiteln (6.2.2.1 und 6.2.2.2) wurden von der Morphem- bis zur Textabschnittsebene sprachliche Mittel extrahiert, mit denen definitorische Aufgaben in den Texten sowohl der Fachlexika, Hand- und Lehrbücher als auch der Fachzeitschriften, Monografien und Sammelbände erfüllt werden. In ersteren dominieren Formulierungen wie: Ausbrennen. Syndrom, das bei ... auftritt, u. a. gekennzeichnet durch; B. wird als Syndrom aus ... beschrieben; B. bezeichnet einen besonderen Zustand berufsbezogener Erschöpfung; Burnout wird definiert als Syndrom aus .... Diese syntaktischen Muster ordnen Burnout zuerst einem Definiens-Ausdruck höherer Abstraktion $\mathrm{zu}$ und weisen ihm damit einen Platz in einem mehr oder weniger festen Begriffssystem zu (genus proximum) und spezifizieren es daraufhin durch qualitätsattributierende Nominalphrasen oder Relativsatzgefüge (differentia specifica). ${ }^{53}$ Die Richtung der Festlegung erfolgt vom Allgemeinen zum Speziellen. Die Definitionen werden in den Hand- und Lehrbüchern unter Berufung auf die Autoren/Autorinnen der jeweiligen Definition aufgeführt, in einigen Fachlexika auch ohne Nennung derselben. Distanzmarker werden in den Fachlexika kaum und in den Hand- und Lehrbüchern teilweise gesetzt (siehe Kap. 6.2.2.1). Bei diesen in der Regel im Indikativ Präsens verfassten Definitionen geht es nicht nur um Festlegungen der Bedeutung des Worts Burnout, sondern um Realdefinitio-

genutzt, um zwischen >Beschreiben` und `Erzählen` zu unterscheiden, sondern um verschiedene narrative Vertextungsmuster analysieren zu können: `Referieren` und `Erzählen im engeren Sinn. 533 Vgl. die an die Diskussion über die Termini genus proximum und differentia specifica in der Terminologielehre und Philosophiegeschichte anschließende Frage, ob in Definitionen dieses Formats wirklich nur ein möglicher nächsthöherer Gattungsbegriff in einem festen Begriffssystem angegeben wird oder ein hierarchisch weniger genau festgelegter Überbegriff in einem Begriffssystem mit unscharfen, auch graduellen Kategoriengrenzen, gewählt wird. Diese Diskussion wird in Kapitel 4.2.4 rekapituliert und in Kapitel 6.2.2.1 auf die Analyse der Belege bezogen. 
nen, deren Korrektheit sich an der adäquaten Beschreibung der außersprachlichen Wirklichkeit bzw. anderen zeichengebundenen kollektiven Erfahrungen und Perspektivierungsanstrengungen bemisst (vgl. Kap. 6.2.2.1 und Köller 2004: 219). Die Art der Definitionsentfaltung ${ }^{534}$, die mit diesen syntaktischen Mustern korrespondiert, ist in der Regel eine beschreibende. Meist wird über die Wahl eines nominalen Definiens-Ausdrucks höherer Abstraktion in diesen Definitionen (Syndrom, Zustand, Folge, Krise, Konzept etc.) eine synchrone Sicht auf den Sachverhalt/das Konzept eingenommen. Zugleich wird durch den Definiens-Ausdruck eine erste Einordnung bzw. Kategorisierung zu Definiens-Metakonzepten, z. B. ıZustand/Eigenschaft‘, >Vorgang/Prozess`, `Gattung` oder `Ereignis`, vorgenommen (vgl. zu diesen Kategorisierungen von Polenz ${ }^{3} 2008$ : 159 ff.; ${ }^{535}$ Konerding 2005: 16; Konerding 1993; Adamzik 2004: 122ff.). Daran anschließend werden zur Spezifizierung und/oder Unterscheidung von anderen sachverwandten Konzepten charakteristische Merkmale meist über Nominalphrasen aufgezählt, die wiederum auf telische oder egressive Verben zurückgehen (erschöpfen, reduzieren, depersonalisieren) und damit charakteristische transformative Teilvorgänge bezeichnen, die zum definierten Endzustand geführt haben. Verallgemeinernde Pronomen (man) oder Gattungsnamen (bei professionellen Helfern) in generischer Referen $z^{536}$ weisen den beschriebenen Endzustand eines transformativen Prozesses als etwas aus, das nicht nur einem Subjekt, sondern unter denselben Bedingungen verschiedenen Subjekten, die dies meist in der semantischen Rolle der Experiencer ${ }^{537}$ erleben, zugeschrieben wird.

Auch wenn dieses Muster und die mit ihm einhergehenden syntaktischen qualitätsattribuierenden Muster (Relativsatzgefüge, präpositionale, attributive

534 Siehe hierzu die Ausführungen im letzten Kapitel 6.2.2.3.

535 Vgl. hierzu die Prädikatsklassen, die Peter von Polenz in seiner Satzsemantik in Kap. 2.1.4.2 ausführt (von Polenz ${ }^{3}$ 2008: $159-167$ ) und die hier in äußerst verkürzter Form wiedergegeben werden: HANDLUNG (mit dem Merkmal der 'Absichtlichkeit' verbunden), VORGANG (mit dem Merkmal der 'Unabsichtlichkeit' verbunden), ZUSTAND (mit dem Merkmal 'grundsätzlich veränderlich' und mit Orts- oder Zeitangaben verbunden), EIGENSCHAFT (mit dem Merkmal 'grundsätzlich unveränderlich' verbunden), GATTUNG (mit dem Merkmal 'essentielle (nicht akzidentielle) Eigenschaft eines Objekts’ verbunden). Bei Definitionen ist die Prädikatsklasse „Gattungsprädikate“ besonders einschlägig. So kann man in folgendem Beispiel Burnout ist ein besonderer Zustand berufsbezogener chronischer Erschöpfung das Definiendum Burnout der definierenden Gattung ১berufsbezogene chronische Erschöpfungszustände` zuordnen. Man könnte diese definitorische Aussage aber zugleich auch der Kategorie „Zustandsprädikate“ zuordnen, die Peter von Polenz als „Aussagen über grundsätzlich veränderliche, also irgendwann eingetretene und irgendwann endende physische oder psychische Zustände von Lebewesen, Sachen oder Abstraktbegriffen“ beschreibt (von Polenz ${ }^{3} 2008$ : 162f.).

536 Zur generischen Referenz vgl. Schmidt-Brücken (2015: 29ff.) und Blühdorn (2001).

537 Zustandsträger/Experiencer ist eine „Person oder Figur, die etw. wahrnimmt, fühlt oder denkt“ (Müller 2007: 94; vgl. auch Primus 2012: 25f.). 
Nominalphrasen) in den Texten der Fachzeitschriften und Monografien ebenfalls sehr häufig erscheinen, so zeigen diese im Vergleich zu den Lexika und Handbüchern dennoch eine größere Formenvielfalt, um den Ausdruck Burnout auf die ihn bestimmenden Sachverhalte und Begriffsinhalte identifizierend-gleichsetzend zu beziehen:

1. durch die gerade beschriebene deskriptive Definitionsentfaltung, die sich am Muster genus proximum - differentia specifica orientiert (vgl. z. B. Beleg 2, 4, 5 und 12 in Kap. 6.2.2.2);

2. durch die Beschreibung, welche charakteristischen Eigenschaften und generalisierbaren Vorgänge bzw. „Phasen“ sich bei den ,Merkmalsträgern“, also >PERSONEN MIT BuRNOUT<, zeigen (vgl. Beleg 1, 4, 9 in Kap. 6.2.2.2);

3. durch die Frage „Was ist Burnout?“ und daran anschließende Antwortmuster, die den Prozess oder Zustand 〉Burnout` beschreibend/referierend charakterisieren und durch Aufzählungen von Symptomen und Phasen in Einzelmerkmale bzw. -vorgänge aufgliedern (vgl. Beleg 1 in Kap. 6.2.2.2);

4. durch die Zuordnung einer kurzen beschreibenden Definition des Konzepts >Burnout und seiner Unterfaktoren $\mathrm{zu}$ einer Zusammenschau von Items eines Fragebogens, die die Burnout-Dimensionen durch beobachtbare Unterfaktoren konkretisieren (vgl. Beleg 6a und 6b in Kap. 6.2.2.2);

5. durch die Einordnung des Definiensausdrucks in eine feste Oberklasse in einem deklarierten Klassifikationssystem wie der ICD-10 oder ICD-11 und einer anschließenden Kurzbeschreibung (vgl. Beleg 12 in Kap. 6.2.2.2);

6. durch die Zuordnung des Diagnosebegriffs `Burnout zu einem zuvor sachlichreferierenden Fallbericht, in dem die in der Untersuchung in Erfahrung gebrachten Merkmale/Symptome in zeitlich geordneter Abfolge, aber mit Blick auf die zugeordnete Diagnose ergebnisorientiert präsentiert werden (vgl. Beleg 8 in Kap. 6.2.2.2);

7. durch den Vergleich des Begriffkonzepts >Burnout mit sachverwandten 'nicht-krankheitswertigen' Zuständen (siehe Beleg 10 in Kap. 6.2.2.2) und sachverwandten klinischen Diagnosekonzepten wie der >Depression ‘ (siehe Beleg 11 in Kap. 6.2.2.2);

8. durch die argumentative Zurückweisung eines Definiens-Nomens höherer Abstraktion (z. B. Diagnose, Krankheit) in bisherigen im Diskurs etablierten Definitionsvarianten und die explizite Abgrenzung von anderen taxonomisch festgelegten sachverwandten Konzepten/Sachverhalten (vgl. Beleg $8 a-2$ und 10 in Kap. 6.2.2.2);

9. durch die Reformulierung einer anderen (meist deskriptiven) Definition und anschließender argumentativer Zurückweisung bestimmter Attribute als 'wesentlich' bzw. 'konstitutiv' (vgl. z. B. eine Studie, in der das Attribut 'über- 
mäßiges Engagement' zurückgewiesen wird (Schmitz/Leidl 1999 in PiEU, im QV unter 8.1.4));

10. durch die argumentativ-operationale Zurückweisung bestimmter Attribute/ Attributskonstellationen als 'wesentlich' bzw. 'konstitutiv' (z. B. Zurückweisung des Merkmals Depersonalisierung) für das operationalisierte Konzept >Burnout` und die anschließende Substitution durch eine eigene deskriptivoperationale Definitionsentfaltung (vgl. z. B. Beleg 6b in Kap. 6.2.2.2).

Bei den Fallberichten (siehe in der gerade präsentierten Aufzählung Punkt 6) erfolgt die Richtung der definitorischen Festlegung im Gegensatz zum Muster „genus proximum - differentia specifica“ vom singulären Erleben (Sinzeichen) hin zur verallgemeinernden Diagnose (Legizeichen). Die Fallberichte unterscheiden sich von Fallnarrationen in Medientexten, da die geschilderten Ereignisse ohne Spannungsbogen „ergebnisorientiert“ und ohne explizite oder nur wenige emotionalisierende Bewertungselemente „institutionsgebunden“ präsentiert werden (vgl. Sandig 1986: 184; Gansel/Jürgens ${ }^{3} 2009$ : 149ff.). Die öffentlich-medialen Fallnarrationen werden im folgenden Kapitel 6.2.3.1 eingehender beschrieben.

Die Fragebogen-Items im Rahmen psychologischer Messinstrumente (siehe in der gerade präsentierten Aufzählung Punkt 4) wurden ebenfalls dafür entwickelt, das individuelle Erleben der Testpersonen anzusprechen. Sie werden damit einerseits im Gewand singulären Erlebens dargeboten, stellen aber andererseits verallgemeinerte Eigenschaften und Verhaltensweisen dar, die als typische Faktoren bzw. Symptome des Konzepts >Burnout` bei der Testerstellung entworfen wurden.

Die Originalarbeiten verwenden meist eine knappe Zustandsbeschreibung mit Andeutung des Transformationsprozesses als Vertextungsmuster oder referieren direkt auf das verwendete Fragebogen-Messinstrument, teilweise mit Nennung von Beispiel-Items der Messinstrumente (z. B. Dormann/Zapf/Isic 2002: 206, ZfAO; Freund/Diestel/Schmidt 2012: 146, ZfAO; Zimmermann/Kaiser/Bernholt et al. 2016: 262f., PiEU, im QV unter 8.1.4). Manche diskutieren auch unterschiedliche Burnout-Definitionen (siehe Beleg 4, 5 und 6b in Kap. 6.2.2.2).

Bei Übersichtsartikeln, die in der Regel verschiedene Definitionen präsentieren und gewichten, sind die meisten Definitionen klassische Zustandsbeschreibungen einer aus egressiven Prozessen hervorgegangenen Symptomatik, wie in den Fachlexika, Hand- und Lehrbüchern (siehe die Belege 2, 3, 4, 5 und 7 in Kap. 6.2.2.2). Manche der zitierten Definitionen beziehen jedoch auch den Prozess und/oder ursächliche Faktoren der Genese des Phänomens BuRNout explizierend mit ein (siehe die Belege 4, 9 und 10 in Kap. 6.2.2.2). Des Weiteren können die Grenzen zwischen den Unterkapiteln nicht immer eindeutig ausgemacht werden, d. h. das Kapitel „Definition“ ist gerahmt von Kapiteln zur „Begriffsgeschichte“, 
„Symptomatik“ oder zu „Erklärungsmodellen“. Da die im Kapitel „Definition“ zusammengestellten Sätze aber ebenfalls häufig auf typische Symptome und Bedingungsfaktoren referieren, wirken die in diesen Rahmenkapiteln ausgeführten Passagen - Symptomlisten, Modelle typischer aufeinander folgender Phasen, kurze charakteristische Beispiele oder Ätiologiemodelle - ebenfalls in den Definitionsprozess mit hinein (siehe Beleg 9 in Kap. 6.2.2.2 und Korczak/Kister/Huber 2010: 14ff., im QV unter 8.1.5).

Die Formenvielfalt korrespondiert damit, dass in Artikeln der Fachzeitschriften und Monografien durchaus verschiedene Definitionen nebeneinander präsentiert, diskutiert und in manchen Publikationen zusammengeführt werden. In Kap. 6.2.2.2 wurde dieses Vorgehen als Definieren mit repräsentativ-festsetzender Funktion beschrieben (siehe insbes. Beleg 4, 5, 6b und 11 in Kap. 6.2.2.2) und auf das Falsifizierungsprinzip moderner naturwissenschaftlicher Forschung bezogen (vgl. Prechtl ${ }^{2}$ 1999: 175). Definitionen in Fachlexika und Handbüchern sowie Abstracts von Übersichtsartikeln in Fachzeitschriften, die eine knappe Definition teilweise ohne Angabe der Quelle geben (z. B. Schmidbauer 1991; Pschyrembel 2007, im QV unter 8.1.2) und dadurch wie eine monolithische Stimme im Diskurs erscheinen, unterstützen hingegen die Vorstellung einer allgemeingültigen Definition mit deklarativer Kraft. ${ }^{538}$ Eine solche Definition wird in Fachkreisen aus praktischen Gründen gefordert, wenn die Definition Eingang in internationale Klassifikationssysteme für Krankheiten finden soll und wenn therapeutische Behandlungen darüber abgerechnet bzw. Personen dadurch krankgeschrieben werden können sollen (vgl. Searle 1980: 99 und Kap. 4.3.2). Dies beweisen die vielen Verweise in Fachartikeln, vor allem in Zeitschriften klinischer Fächer (Der Nervenarzt, Deutsches Ärzteblatt, Der Internist), die das Fehlen einer ,international konsentierte[n] Definition“ anmerken (vgl. Beleg 9 und 10 in Kap. 6.2.2.2 und z. B. in der Zeitschrift Der Internist bei Kapfhammer 2012: 1277; in Der Nervenarzt bei Hamann/Parchmann/Mendel et al. 2013; in der DMW bei Kissling/Mendel/ Förstl 2014, im QV unter 8.1.4). Doch die letzten Kapitel haben gezeigt, dass trotz dieser kritischen Anmerkungen gegenüber einer in klinischen Fachkreisen kollektiv akzeptierten Definition einige typische Berufsfelder (z. B. psychosoziale Berufsgruppen), kennzeichnende Symptome (z. B. Erschöpfung, Zynismus, Leistungsreduktion, auf den Arbeitsbereich bezogen/ursächlich im Arbeitsbereich, Schlafprobleme etc.) und Namen (Freudenberger, Maslach/Jackson) auch in den Texten der Fachzeitschriften und Monografien immer wieder an exponierter Stelle (z. B. im Abstract, in typografisch hervorgehobenen Passagen, in Schaubildern,

538 Zum Zusammenhang repräsentativer und deklarativer Merkmale von Definitionshandlungen vgl. auch Kap. 4.3.2 dieser Arbeit. 
Zusammenfassungen, zuvorderst in Aufzählungen) erscheinen. Diese diskursiven Auffälligkeiten können eine Reduktion von Definitionsvarianten bewirken, die möglicherweise eine Konsensfindung und Einordnung in momentan gültige Klassifikationssysteme vorbereiten können (vgl. dazu besonders Beleg 10 und Beleg 8a-2, der die Einordnung von Beleg 10 ebenfalls aufnimmt, in Kap. 6.2.2.2). Wie weitreichend die unifizierende Wirkung dieser diskursiven Praktiken der Bezugnahme bzw. Verknappung ist, wird in Kap. 6.2.4.2 noch eingehender unter Berücksichtigung der fach- und sozio-kulturellen Einbettungsbedingungen der Texte untersucht.

In der Zusammenschau vermitteln die analysierten Definitionen der Fachzeitschriften, Sammelbände und Monografien zwar ein weniger homogenes bzw. vielstimmigeres Bild als die Definitionen in den Fachlexika, Hand- und Lehrbüchern, und sie variieren formal stärker auf der Ebene der Definitionsentfaltung (siehe Tabelle 1 unten). Trotz der verschiedenen oben erläuterten Definitionsentfaltungstypen entsteht aber dennoch der Eindruck von Überschneidung zwischen den Definitionen der beiden fachinternen Diskursstränge. Denn erstens ähneln sich alle Definitionen auf der Ebene der Morpheme und (Mehr-) Worteinheiten, wenn es um die sprachliche Abgrenzung zu normalen alltäglichen Erschöpfungszuständen bzw. normalem Verhalten, Erleben und normalen Arbeitsbedingungen geht (vgl. Kap. 6.2.2.1 und 6.2.2.2 und siehe Tab. 1 unten). Zweitens werden Konzept-Komponenten wie >übermäßige Erschöpfung`, ১Überforderung، sowie ,Verlust von vormaligen Möglichkeiten oder Fähigkeiten in nahezu allen Definitionen versprachlicht. Drittens wird in beinahe allen Definitionen ein Bezug zur Arbeitswelt meist über die Verwendung von Gattungsbezeichnungen (staff member, Mitarbeiter, psychosoziale Berufsgruppen, helfende Berufe, ...) hergestellt.

In den folgenden drei Tabellen werden zunächst in Tabelle 1 die sprachlichen Mittel nochmals zusammengefasst, die in exponierten Passagen an der Textoberfläche der fachinternen Diskursstränge punktuell definitorische Aufgaben und Ansprüche erfüllen (Kap. 6.2.2.1 und 6.2.2.2). Zusätzlich wird in Tabelle 1, Spalte 3 rekapituliert, welche abstrakt-idealen Erwartungen an eine (gute) Definition und damit an den Akt des Definierens als regelgeleiteten sozialen Prozess im fachlichen, aber auch öffentlichen Burnout-Diskurs selbst gestellt werden und welche Teil-Funktionen des Definierens daraus erwachsen (vgl. Kap. 4.2.2). In den darauf folgenden Tabellen (Tab. 2.1-2.2) werden dann zwei Gruppen definitorischer Teil-Praktiken mittlerer Abstraktion vorgestellt, die im Zuge der Analyse entwickelt wurden und die zwischen den sprachlichen Zeichenressourcen und den abstrakt-idealen Ansprüchen an die Tätigkeit des Definierens vermitteln. 
Tab. 1: Sprachliche Mittel, die definitorische Aufgaben erfüllen (punktuelle Perspektive), und Ansprüche, die von Stimmen im Diskurs an die Tätigkeit des Definierens gestellt werden (im fachlichen/fachinternen Diskursstrang).

\begin{tabular}{lll}
\hline $\begin{array}{l}\text { Sprachliche } \\
\text { Ebenen }\end{array}$ & Textoberfläche: & Abstrakt-ideale \\
& (Sprachliche) Zeichenressourcen, die definitorische & Ansprüche an \\
& Aufgaben erfüllen (punktuelle Perspektive, Kap. 6.2.2.1 & die Tätigkeit des \\
& Definierens im \\
& und 6.2.2.2) & Burnout-Diskurs \\
& (Kap. 4.2.2) \\
& (Die Positionen \\
& der einzelnen \\
& "Ansprüche“ in der \\
& Tabelle beziehen \\
& sich nicht exakt auf \\
& die (sprachlichen) \\
& Zeichenressourcen \\
& bzw. könnten an \\
& mehreren Stellen \\
& platziert werden.) \\
\hline
\end{tabular}

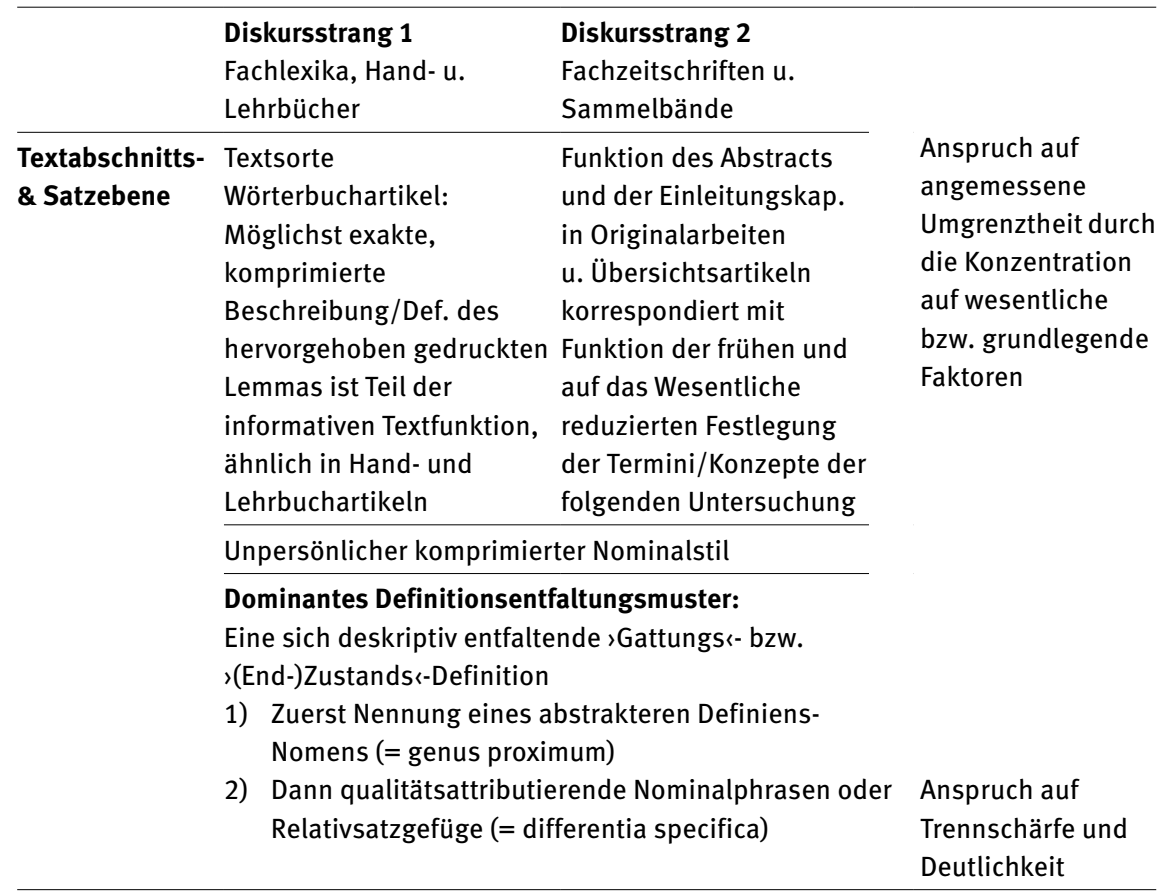


Tab. 1 (fortgesetzt)

zuweisend-prädizierende Satzmuster mit Verbgefügen
oder Satzzeichen, die eine Äquivalenz- oder
Entsprechungs-Relation herstellen (= Definitor):
Burnout wird definiert als Syndrom aus [...]; $x$
versteht Burnout als ein Syndrom, das aus [...]
zusammengesetzt ist; $x$ ist gekennzeichnet durch, $x$
wird beschrieben als; Burnout-Merkmale sind: [...];
according to the definition burnout is characterized
by...

Zusätzliche relationale syntaktische Muster in Fachaufsätzen/-büchern, mit denen das zu Definierende auf die definitorische Proposition (Definiens) bezogen wird:

- X ist $y$, wenn (kond. Satzgefüge in attributiver Funktion)

- Was-ist-Frage-Antwort-Muster (Frage enthält das Definiendum - Antwort das Definiens)

- Nominaldefinition eines Ausdrucks (aus dem Wörterbuch, der Umgangssprache) wird auf den beobachteten Sachverhalt übertragen

- Nach Nennung des Definiendums (als Überschrift oder vor dem Doppelpunkt) Aufzählungen typ. Symptome und Bsp. (vgl. stipulat. Def.) ${ }^{539}$

- Referieren eines Fallbeispiels mit anschließ. Diagnosekommentar, der das Definiendum nennt (vgl. genet. Def.)

- Nennung des Namens des Messinstruments und anschließende Präsentation der operationalisierenden Fragebogen-Items (vgl. operationale Def.)

- Negierte Äußerungseinheiten in Verbindung mit substitutiven Konnektoren zur Zurückweisung und anschließenden Ersetzung von Definiens-Nomina bzw. deren Rahmenkonzepten (keine Krankheit, sondern Risikozustand)

- Äußerungseinheiten mit adversativen und negierenden Sprachmitteln, um Komponenten von `Burnoutı von anderen `Zuständen`, ১Merkmalen`, `Bedingungen` zu unterscheiden.

\author{
Anspruch auf \\ exakte und \\ eindeutige \\ Bestimmung oder \\ Zuordnung
}

Anspruch auf Adäquatheit zwischen Definiens und Definiendum

539 Zur Begriffsbestimmung der „stipulativen Definition“, „,genetischen Definition“ und „operationalen Definition“ vgl. Kap. 4.2.3 dieser Arbeit. 
Tab. 1 (fortgesetzt)

(Mehr-)Wort- u. Kategorisierung durch Definiens-Nomina mittlerer Morphemebene und hoher Abstraktion (Bezug auf Metakonzepte, Grundkonzepte des jeweiligen Fachs)

Syndrom, Oberbegriff für bestimmte Ivpen persönlicher Krisen, Maß beeinträchtigten Befindens, Zustand berufsbezogener chronischer Erschöpfunq, Indikator (nachhaltiger psychischer) Beanspruchung

Hervorgehobene Gattungs- und Sammelbezeichnungen, die teilweise durch ,typische' Zusatzangaben spezifiziert werden Besonders betroffen sind Helfer mit geringem Selbstwertgefühl [...]; Syndrom, von dem vor allem Angehörige von Sozialberufen betroffen sind; es wird oft von Menschen erlebt, die [...]; so scheinen gerade die allerbesten Leute bestimmter Berufsgruppen [...]; offenbar bevorzugt besonders engagierte Mitarbeiter trifft [...]

(Abgrenzende) Spezifizierung durch:

- Adjektive, Partizipien

- Abgrenzung einzelner Symptome und Entstehungsbedingungen von 'normalen' Zuständen/Bedingungen/ Erwartungsnormen und sachverwandten Konzepten durch sprachliche Mittel zum Anzeigen von Dauer, Frequenz, Intensität, Graduierung, Negierung, Gegensätzen z. B. chronische Erschöpfung, völlige Arbeitsunfähigkeit, hohe interpersonelle Anforderungen, mangelndem Selbstwertgefühl, überanstrengt, kaum noch Freizeitaktivitäten, hält der Erschöpfungszustand jedoch mehrere Wochen [...] an; die Menschen werden immer weniger als Personen [...], sondern als Objekte gesehen; Vermeidungsverhalten besteht jedoch nicht

Gewichtung einzelner Merkmale/Definitionen durch Lexeme und Mehrworteinheiten wie: Kernsymptome, Hauptkriterien, the core of burnout ..., the main metaphor for burnout [...], in erster Linie gekennzeichnet von [...], Leitsymptom des Burn-outZustands 
Tab. 1 (fortgesetzt)

\begin{tabular}{|c|c|}
\hline $\begin{array}{l}\text { Intersubjektivierung bzw. Konsensstiftung durch: } \\
\text { Formulierungen, die zwischen verschiedenen } \\
\text { Definitionen die Gemeinsamkeiten hervorheben, z. B. } \\
\text { Konsens besteht darin, dass; this definition is } \\
\text { almost identical by [...]; findet sich auch wieder in } \\
\text { [...]; Einigkeit besteht über folgende } 3 \text { Hauptkriterien [...]; } \\
\text { Studien [...] führten zu dem auch noch heute weitgehend } \\
\text { anerkannten Ergebnis, dass Burnout als Syndrom von [...] } \\
\text { zu verstehen ist; Die am häufigsten zitierte Beschreibung } \\
\text { von Burnout geht auf [...] zurück; Beschwerden, die häufig } \\
\text { drei Dimensionen zugeschrieben werden [...]; } \\
\text { Sprachliche Mittel des Geltungsanspruchs } \\
\text { - Präsens, Präteritum in Fallberichten oder in } \\
\text { Definitionen, die auf Zitaten beruhen } \\
\text { - Indikativ } \\
\text { - Nomen, Adjektive, Pronomen, die die } \\
\text { empirische Forschungspraxis und intersubjektive } \\
\quad \text { Gültigkeit unterstreichen: empirisch, die } \\
\quad \text { meisten Fachleute, man, standardisierter } \\
\text { Fragebogen, ... }\end{array}$ & $\begin{array}{l}\text { Anspruch auf } \\
\text { eine neutrale, } \\
\text { objektorientierte } \\
\text { Sachverhaltskon- } \\
\text { stitution / } \\
\text { Begriffsbildung }\end{array}$ \\
\hline
\end{tabular}

Die in Tabelle 1 zusammengefassten sprachlichen Mittel (Spalte 2, Tab. 1) wurden an Textstellen des fachlichen Diskursstrangs herausgearbeitet, in denen der Begriff $>$ Burnout entweder von den Autoren/Autorinnen explizit als Definitionsgegenstand behandelt wurde (z. B. dadurch, dass der Abschnitt mit Definition überschrieben ist) oder in denen der Ausdruck Burnout auf dargelegte Sachverhalte und Begriffsinhalte bewusst identifizierend-gleichsetzend bezogen und damit punktuell im Rahmen eines Abschnitts reflektierend erklärt, bestimmt oder festgelegt wurde. ${ }^{540}$ Betrachtet man nun genauer, welche Effekte von diesen sprachlichen Mitteln ausgehen, und bezieht diese auf die im Diskurs selbst formulierten abstrakt-idealen Ansprüche an die Tätigkeit des Definierens ${ }^{541}$ (Spalte 3, Tab. 1), so erhält man |Praktiken mittlerer Abstraktion|, die zwischen den konkreten sprachlichen Mitteln und abstrakten Definitions-Ansprüchen vermitteln: Diese Praktiken verbinden einzelne Zwecke oder Aufgaben, die aus den Definitions-Ansprüchen

540 Siehe die Bedeutungsangabe zum Verb definieren in Duden online unter: https://www. duden.de/rechtschreibung/definieren (zuletzt eingesehen am 17.12.2019).

541 Vgl. Kap. 4.2.2 dieser Arbeit: In den Texten der fachinternen und fachexternen Diskursstränge zum Thema „Burnout“ wurden anhand metasprachlicher Äußerungen die Ansprüche, die im Diskurs selbst an die Tätigkeit des Definierens gestellt werden, herausgearbeitet. 
erwachsen, mit verfügbaren sprachlichen Ressourcen. Ihr praktisch-prozesshafter Charakter lässt sich am besten dadurch kennzeichnen, dass sie durch Verben benannt werden (siehe unten Spalte 2, Tab. 2.1). In diesen durch die Verben benannten Handlungen oder Prozessen verbindet sich die Zweck- oder Aufgabenebene mit den sprachlichen Ressourcen. Gruppiert man diese Verben wiederum zu Überkategorien, so ergeben sich |definitorische Praktiken der Zuweisung, Einordnung sowie Ein- und Abgrenzung| (siehe Tab. 2.1) und |definitorische Praktiken der Verknappung, Gewichtung und Geltungsqualifizierung| (siehe Tab. 2.2). Die Herleitung dieser Praktiken wird anhand der folgenden zwei Tabellen erläutert:

Tab. 2.1: Exemplarische Herleitung der |definitorischen Teilpraktiken der Zuweisung, Einordnung sowie Ein- und Abgrenzung| (im fachlichen/fachinternen Diskursstrang).

\begin{tabular}{|c|c|c|}
\hline $\begin{array}{l}\text { Textoberfläche (punktuelle } \\
\text { Perspektive, Kap. 6.2.2.1 und } \\
6.2 .2 .2 \text { ) }\end{array}$ & Praktiken mittlerer Abstraktion & $\begin{array}{l}\text { Einzelne Ansprüche } \\
\text { an die Tätigkeit des } \\
\text { Definierens im Burnout- } \\
\text { Diskurs (Kap. 4.2.2) }\end{array}$ \\
\hline $\begin{array}{l}\text { zuordnend-prädizierende } \\
\text { Satzmuster wie z. B. Burnout wird } \\
\text { definiert als Syndrom aus [...]; X } \\
\text { versteht Burnout als ein Syndrom, } \\
\text { das aus [...] zusammengesetzt ist; } \\
\text { x ist gekennzeichnet durch, } x \text { wird } \\
\text { beschrieben als; }\end{array}$ & $\begin{array}{l}\text { |Definiens u. Definiendum } \\
\text { einander zuweisen/doppelt } \\
\text { referieren|, }{ }^{542} \\
\text { |gleichsetzen|, } \\
\text { |sortal kategorisieren } \\
\text { /einordnen|, }{ }^{543}\end{array}$ & $\begin{array}{l}\text { Anspruch auf } \\
\text { Adäquatheit zwischen } \\
\text { Definiens und } \\
\text { Definiendum }\end{array}$ \\
\hline
\end{tabular}

542 Stanaitytė (2005: 97 f.) beschreibt diese Praktik des Zuweisens in Alltagsdefinitionen unter Rückgriff auf die erste Auflage der Satzsemantik von P. v. Polenz (1985) folgendermaßen: „Wenn man davon ausgeht, dass Alltagsdefinitionen eine lexikalische Einheit in ihrer Bedeutung erläutern (aus der satzsemantischen Sicht betrachtet sind es Referenzstellen bzw. Bezugsobjekte), so hieße dies, dass das Explizieren einer Bedeutung ein Akt des doppelten Referierens ist: zunächst wird ein Bezug zu einer Referenzstelle hergestellt, worauf dann der zweite Akt des Referierens folgt, indem auf die gleiche Referenzstelle ein weiterer Bezug genommen wird, der sich von dem ersten dadurch unterscheidet, dass er andere Referenzausdrücke zur gleichen Bezugsstelle verwendet.“ 543 Die Praktik des |Kategorisierens| macht darauf aufmerksam, dass bei der Verwendung eines Definiens-Ausdrucks (z. B. Burnout ist ein Syndrom) verschiedene semantische Kategorisierungen vorgenommen werden: durch die Wortart Substantiv beispielsweise wird eine „kategoriale Bedeutung “ transportiert: Denn diese Formulierung fordert uns dazu auf, das zu Definierende als etwas „das eigenständig existiert und was sich im physischen Raum als eigenständige Größe auch isolieren lässt“ oder als „eigenständig betrachtete Größe im geistigen Raum“ zu verstehen (Köller 2004: 349 f.); Wenn man Hyperonyme dieser Definiens-Substantive bildet, so erhält man eine übergeordnete Interpretationsperspektive. Konerding entwickelte dieses Verfahren der Hyperonymenreduktion und Benennung von Matrixframes im Bereich der Framesemantik (vgl. Konerding 1993; 2005). In dieser Arbeit wird im Folgenden von `Metakonzepten in diesem Zusammenhang gesprochen. 
Tab. 2.1 (fortgesetzt)

\begin{tabular}{lll}
\hline Textoberfläche (punktuelle & Praktiken mittlerer Abstraktion & $\begin{array}{l}\text { Einzelne Ansprüche } \\
\text { an die Tätigkeit des }\end{array}$ \\
$\begin{array}{l}\text { Perspektive, Kap. 6.2.2.1 und } \\
\text { 6.2.2) }\end{array}$ & Definierens im Burnout- \\
& Diskurs (Kap. 4.2.2) \\
\hline
\end{tabular}

1) Zuerst Nennung eines Hyperonyms, Verwendung von Fachtermini (Syndrom, Depersonalisation ...)

2) Dann qualitäts-attributierende Nominalphrasen oder Relativsatzgefüge zur Spezifizierung

Spezifizierende Adjektive, Partizipien

Gattungs- und Sammelbezeichnungen sprachliche Mittel zum Anzeigen von Dauer, Frequenz, Intensität, Graduierung, Negierung, z. B. chronische Erschöpfung, völlige Arbeitsunfähigkeit, hohe interpersonelle Anforderungen, mangelndem Selbstwertgefühl, überanstrengt

|klassifizieren/in eine Taxonomie einordnen|, ${ }^{544}$ |aufgliedern|, |spezifizieren|, |charakterisieren/ kennzeichnen|, |ein typ. Bsp. referieren/typisieren|, |Ursache-Folge-Beziehungen explizieren, implizieren|, |durch Angabe von weiteren Kriterien von sachverw. Konzepten abgrenzen und damit eingrenzen|, leine Kategorisierung oder Klassifizierung negieren/ zurückweisen und ersetzen|, leine (einzelne) Kennzeichnung negieren/zurückweisen und ersetzen| (etc. s. Tab. 1)

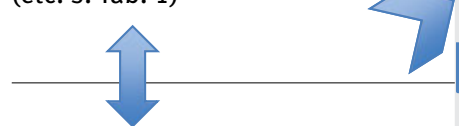

Ausblick auf Kap. 6.2.4.1

Textoberfläche (flächige Perspektive)

Sprachliche Stimuli definitoischer Praktiken der |Zuweisung, Einordnung sowie Ein- und Abgrenzung| in der Fläche der Diskursstränge
Definitorische Praktiken der Zuweisung, Einordnung sowie Ein- und Abgrenzung|
Anspruch auf exakte und eindeutige Bestimmung oder Zuordnung

Anspruch auf Trennschärfe und Deutlichkeit

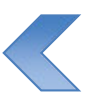

Anhand der in Tabelle 2.1 (Spalte 1) aufgeführten Satzmuster wird das zu Definierende mit einem zumeist |sortal-kategorisierenden| oder auch |taxonomisch-

544 Die Praktik des |Klassifizierens| bezieht sich in diesem Rahmen stärker als das |Kategorisieren| auf die Zuordnung zu festgelegten Klassen in terminologischen Systemen oder gesellschaftlich-fachlichen Taxonomien wie der Internationalen Klassifikation der Krankheiten (ICD). 
einordnenden $\left.\right|^{545}$ Definiensausdruck höherer Abstraktion (Syndrom, Krise, Risikozustand) |gleichgesetzt|, welcher dann seinerseits noch mit weiteren, das Phänomen |kennzeichnenden| und von anderen Phänomenen |unterscheidenden| Angaben versehen und dadurch |spezifiziert| wird. Wenn diese semantische Relation ohne Distanzmarker konstituiert wird, dann wird eine Adäquatheitsbeziehung zwischen Definiens und Definiendum (Tab. 2.1, Spalte 3) unterstellt. Die weiterhin angeführten sprachlichen Mittel können zur Befriedigung der Ansprüche auf Exaktheit und Eindeutigkeit bzw. Trennschärfe und Deutlichkeit verwendet werden, um den zu definierenden Zustand/Prozess von ähnlichen Zuständen/Prozessen |abzugrenzen| und das Phänomen weiter zu |spezifizieren| und |einzugrenzen|.

In Kap. 6.2.4.1 wird der Analyseblick ausgehend von den in Tabelle 2.1 dargestellten sprachlichen Mitteln und Teilpraktiken des Definierens, die punktuell-lokal an einschlägigen Textstellen herausgearbeitet wurden, nochmals geweitet und gefragt, vor welchen fach-, bereichs- und sozial-kulturellen Rahmenbedingungen diese expliziten Definitionsbemühungen stattfinden und in welcher Weise diese Handlungs- und Verhaltensroutinen, die teilweise im retrospektiven Bezug auf generisches Vorwissen ihren Ausdruck finden (vgl. Schmidt-Brücken 2015: 86), definitionsähnliche Effekte stimulieren können. Um analytisch zwischen den sprachlichen Zeichenressourcen $\mathrm{zu}$ unterscheiden, die sich einerseits punktuell-konzentriert in einem Abschnitt und andererseits verstreut an verschiedenen Stellen in den Texten der Diskursstränge zeigen, wird im ersten Fall von sprachlichen Mitteln und im zweiten Fall von sprachlichen Stimuli gesprochen. Dieser Aufgliederung liegen keine Unterschiede der analytischen Grundperspektive zugrunde. In beiden Fällen werden Zeichenressourcen mit definitorischer Formungskapazität durch Spurenlese an den Textoberflächen des Diskurses gesichtet. Bei der flächigen Spurensuche wird jedoch stärker als bei der auf einen Abschnitt konzentrierten Analyse davon ausgegangen, dass die Autorinnen und Autoren der analysierten Texte an diesen Stellen nicht bewusst intendiert bzw. kontrolliert definieren, sondern unwillkürliche Sprachverwendungskonstellationen routinemäßig wiederholen, die zur diskursiven Entstehung einer Definition beitragen können. ${ }^{546}$ Das heißt, sie verwenden bestimmte Zeichen nicht bewusst kombiniert, um definitorische Teil-Funktionen zu erfüllen, sondern bestimmte,

545 Die |so| ausgezeichneten auf Verben zurückgehenden Formen verweisen im Folgenden auf die Praktiken mittlerer Abstraktion (siehe Spalte 2, Tab. 2.1).

$546 \mathrm{Zu}$ den Analysekonzepten `Spur`, ১Spurensuche`, ১Praktik`, ১Praxis` und damit einhergehenden Analyseaspekten, wie z. B. der „Unwillkürlichkeitsgedanke[n]“ (Müller 2015: 18/19), die Einbettung jeder (Sprach-)handlung in (fach-)kulturelle, soziale Bedingungsgefüge (vgl. Deppermann/Feilke/Linke 2016: 9ff.; Konerding 2015b) oder „Medialität“ (Jäger 2015; Spitzmüller/ Warnke 2011: 183f.) und „Materialität“ (Deppermann/Feilke/Linke 2016.: 5ff.; Spitzmüller/Warn- 
wiederholt auftretende Zeichenkombinationen, seien sie bewusst gesetzt oder nicht, können wie Reize in einem Reiz-Reaktions-Schema bestimmte definitionsähnliche Effekte stimulieren. Diese Unterscheidung rekurriert auf die Grundthese der vorliegenden Arbeit, dass Definieren in diesem Diskurs weit mehr ist als ein Satz oder Textabschnitt, der eingeführt von der sprachlichen Formel $x$ ist/bedeutet/bezeichnet $y$ punktuell, intentional und explizit in terminologisch genau gefassten fachsprachlichen Kontexten geäußert wird.

Der Anspruch auf allgemeine Gültigkeit, Validität und Intersubjektivität und die Konzentration auf wesentliche Faktoren beim Definieren wird in der folgenden Tabelle 2.2 durch sprachliche Mittel gewährleistet, von denen zum einen eine unifizierende ${ }^{547}$ und zum anderen eine die vorliegende Definition verstärkende Wirkung ausgeht. Das heißt, dass diese sprachlichen Mittel einerseits Einzelmerkmale |zusammenfassen und -führen| und dadurch bewirken, dass der Diskurs sich an diesen Stellen |verdichtet| und um manche |Ausdrucksvarianten reduziert| wird. Andererseits werden dadurch aber auch Merkmale oder Definitionsversionen |gewichtet| bzw. |hervorgehoben|. Weitere Sprachzeichen mit metainformativem Status (wie z. B. Präsens und Indikativ oder Zeichen, die auf die empirischobjektive Entstehung der Definition referieren) können schließlich die Geltung der arrivierten Definitionsvarianten und deren Einordnungs- und Abgrenzungspraktiken |validieren|, dadurch dass sie die zugrunde liegende definierende Proposition als |allgemein zeitlos bzw. generisch| (vgl. Schmidt-Brücken 2015: 41) und |als faktisch-sichere Gegebenheit qualifizieren|, |verallgemeinern| und |objektivieren| (siehe Tab. 2.2 auf der folgenden Seite).

Praktiken der Verknappung zeigen sich allerdings auch über Textgrenzen hinweg, wenn manche Quellen besonders häufig in intertextuellen Bezugnahmen zitiert oder reformuliert werden oder wenn ähnliche Schlüsselwörter zu Beginn einer Original- oder Übersichtsarbeit von den Autoren/Autorinnen der Texte hinzugefügt werden. Diese Verknappungsprozeduren, die über Textgrenzen hinweg ihre Wirkung entfalten, werden in Kap. 6.2.4.2 beschrieben. Verknappende Praktiken können dadurch, dass sie den theoretisch unbegrenzten polyphonen Möglichkeitsraum des Diskurses einschränken, indirekt zur Konsensbildung und zum Eindruck überindividueller Geltung bezogen auf einen Wissensgegenstand beitragen.

Auch die in Tabelle 2.2 angeführten sprachlichen Mittel der Geltungsvalidierung erscheinen nicht nur punktuell in den Textpassagen, in denen explizit definiert wird, sondern Spuren dieser Geltungsvalidierung findet man auch im wei-

ke 2011: 166ff.) bzw. „Formungskapazität der Diskurse“ (Felder/Gardt 2015: 19) vgl. im Überblick die Kap. 4.1.2-4.1.4 dieser Arbeit.

547 Zum Begriff der `Unifizierung`vgl. Kap. 6.2.1 dieser Arbeit. 
Tab. 2.2: Exemplarische Herleitung der |definitorischen Teilpraktiken der Verknappung, Gewichtung und Geltungsvalidierung| (im fachlichen/fachinternen Diskursstrang).

Textoberfläche (punktuelle

Perspektive, Kap. 6.2.2.1 und

6.2.2.2)

- Formulierungen wie:

Konsens besteht darin, dass [...];

findet sich auch wieder in [...].; $x / y$

verbinden verschiedene Ansätze

und entwickeln [...] folgende

Arbeitsdefinition; [...] Definition

[...], die sich in der Literatur mit

Abstand am häufigsten findet;

recent definition by one of the

leading researchers in the field,

die meisten Fachleute ..., Einigkeit

besteht über ...

- typografische Hervorhebungen:

Symptomaufzählungen

eingerahmt in einem Kasten

- topografische Hervorhebungen

dadurch, dass sie in Textsorten

wie Wörterbuchartikeln oder

Abstracts oder zu Beginn einer

Aufzählung genannt werden

- Stil: Unpersönlicher komprimierter Nominalstil; Pronomen man, Passivkonstruktionen

- Textsorten: Wörterbuchartikel, fachl. Textsorten

- Modus: Präsens, Indikativ

- Aussagesätze

- Nomen, Adjektive, die die empirische Forschungspraxis unterstreichen: empirisch, standardisierter Fragebogen, ...

\section{Teilpraktiken mittlerer \\ Abstraktion}

|verallgemeinern/generalisieren|,

|als faktisch-sichere

Aussagen qualifizieren|, lals im Fach und/oder in geltenden Taxonomien als mehrheitlich oder allgemein anerkannte Aussage qualifizieren|,

Igenerisch referieren generisch prädizieren (auf Eigenschaften, Vorgänge/Habitus)|,

|unifizieren|
Einzelne Ansprüche an die Tätigkeit des Definierens im Burnout-

Diskurs (Kap. 4.2.2)

Anspruch auf angemessene Umgrenztheit durch die Konzentration auf wesentliche bzw. grundlegende Faktoren

Anspruch auf allgemeine Gültigkeit, Validität, Intersubjektivität

Anspruch auf eine neutrale, objektorientierte Sachverhaltskonstitution/ Begriffsbildung 
Tab. 2.2 (fortgesetzt)

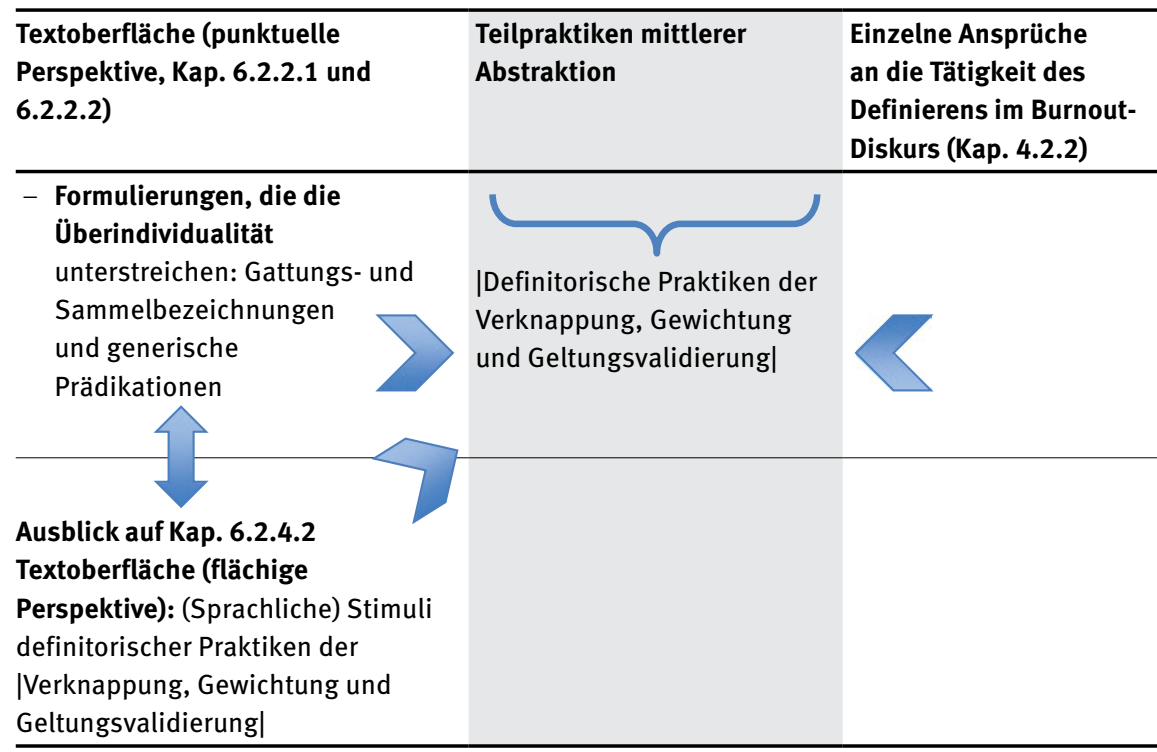

teren Kontext und auf der transphrastischen und intertextuellen Ebene: Durch Textsorten wie den „Wörterbuchartikel“ kann der Eindruck einer angemessen umgrenzten, monolithischen Definition mit deklarativer Kraft gestützt werden, dadurch, dass diese Textsorte mit gesichertem, von fachlichen Diskursautoritäten verfasstem Wissen in Verbindung gebracht wird; ein wiederkehrender konventionalisierter Textaufbau bei Fachtexten ${ }^{548}$ kann zudem Zuordnungspraktiken zum medizinischen Bereich im Allgemeinen und damit den ,Krankheitsstatus“ eines Sachverhalts im Konkreten verstärken, wie in Kap. 6.2.4.2 noch dargelegt wird. In diesem Zuge gilt es schließlich auch das besondere geltungsqualifizierende Potenzial im Bereich medizinischer Semiotik zu berücksichtigen, z. B. durch welche Zeichendimensionen und Teilbedeutungen etwas einerseits als individuell erlebtes, aber dennoch 'objektives' Symptom erscheinen kann und

548 Vgl. dazu z.B. die von der Deutschen Medizinischen Wochenschrift empfohlene KapitelReihenfolge zum Abfassen von „Kasuistiken“: Titel, englisches und deutsches Abstract (ohne „Literaturverweise“ und „keine Verweise auf Abbildungen, Tabellen“), das in sich gegliedert sein soll (,Gliedern Sie [...] das Abstract bitte in Anamnese und klinischer Befund, Untersuchungen, Diagnose, Therapie und Verlauf, Folgerung“), dann 3-5 Keywords, Einleitung und danach die Kasuistik, die sich aus Unterkapiteln zur Anamnese, Diagnostik, Therapie und Verlauf und Diskussion zusammensetzen soll. https://www.thieme.de/statics/dokumente/thieme/final/de/dokumente/zw_dmw/ DMW_Autorenrichtlinien_Kasuistik.pdf,_darin Seite 1-3 (zuletzt eingesehen am 20.12.2019). 
welche medizinischen Termini im Rahmen fachkultureller Praktiken solche Geltungsmechanismen in besonderer Weise transportieren (z. B. Syndrom).

Im folgenden Kapitel werden zunächst ebenfalls aus punktueller Perspektive die sprachlichen Mittel herausgearbeitet, die in den Texten des fachexternen Diskursstrangs definitorische Aufgaben erfüllen. Daran anschließend soll der Blick in die Fläche der Diskursstränge geweitet werden und der Frage nachgegangen werden, welche Spuren (unwillkürlicher) sprachlicher und sozio-kultureller Praktiken der Diskurs fachintern und -extern aufweist, die einzelne Ansprüche an die Tätigkeit des Definierens erfüllen und dadurch in ihrer Gesamtheit über einen gewissen Zeitraum hinweg auf die diskursive Entstehung einer Definition einwirken. Beschreibungssprachlich werden diese Befunde in den Kapiteln 6.2.4.1-6.2.4.3 als „,sprachliche Stimuli definitorischer Praktiken der Zuweisung, Einordnung sowie Ein- und Abgrenzung“ sowie „sprachliche Stimuli definitorischer Praktiken der Verknappung, Gewichtung und Geltungsvalidierung“ zusammengefasst und analysiert.

\subsubsection{Sprachliche Mittel des Definierens im fachexternen Diskursstrang als sich punktuell zeigende Praktiken mit definitorischer Funktion}

\subsubsection{In Pressetexten und populärwissenschaftlichen Zeitschriften}

Varietätenlinguistische Charakteristika von Pressetexten und Vermittlungstexten in populärwissenschaftlichen Zeitschriften wurden in den Kapiteln 5.3.3.3 und 5.3.3.4 ausführlich dargelegt. Diese Texte werden von (Wissenschafts-)Journalisten/Journalistinnen, aber auch von Fachleuten verfasst und wenden sich meist an eine Vielzahl möglicher Rezipientinnen und Rezipienten. Der Adressatenkreis ist anonym, heterogen und dispers (vgl. Burger/Luginbühl 42014: 8). Populärwissenschaftliche Zeitschriften wie „Psychologie Heute“ oder „Gehirn\&Geist“ können im Vergleich zu tagesaktuellen Presseerzeugnissen jedoch in stärkerem Maße eine Rezipienten-Zielgruppe anvisieren, die an Erkenntnissen aus den Fächern Psychologie/Medizin und benachbarten Wissenschaften interessiert ist, und diese Themen im Rahmen von Themenheften auch ausführlicher behandeln. Dadurch ist der Umfang der Artikel und der Fachlichkeitsgrad in einigen populärwissenschaftlichen Zeitschriften etwas höher als in der Tagespresse. In den Kapiteln 5.3.3.3 und 5.3.3.4 wurden auch die gängigen Presse- und Vermittlungstextsorten beschrieben, die >BURNOUT< als Haupt- oder zumindest ausführlicheres Subthema behandeln.

In Folgenden werden anhand von Beispielen, die die Spannbreite der Textsorten abdecken, sprachliche Mittel des Definierens beschrieben. Wie in den vergangenen Kapiteln erfolgt die Beschreibung der definitorischen Sprachmittel anhand von Beispieltexten, in denen der Ausdruck Burnout auf dargelegte Sachverhalte und Begriffsinhalte bewusst gleichsetzend als Entsprechung bezogen wird (vgl. Kap. 6.2.1). 
Die Mehrheit der Texte, die als Hauptthema >BURNOUT` behandeln, lassen sich den Textsorten Magazinbericht und Problemdarstellung zuordnen. Magazinberichte enthalten in der Regel mehr personalisierte Fallschilderungen als Problemdarstellungen, sie schließen häufig auch mit einer persönlichen Szene den Artikel ab, setzen mehr auf Spannung und detaillierte Fallschilderungen und arbeiten in stärkerer Weise mit visuellen und typografischen Blickfängern (z. B. durch Abbildungen, Grafiken, Tabellen). In Problemdarstellungen liegt der Fokus stärker auf einer „systematischen Entfaltung des Textinhalts“, wodurch das Thema „in mehrere gleichrangige Teile oder Aspekte“ aufgliedert wird (Lüger 21995: 118). Doch die Grenzen zwischen diesen Artikelformen sind fließend. Diese Artikelformen finden sich in allen untersuchten Dekaden, weshalb im Folgenden zunächst Beispiele dieser Textsorten aufsteigend aus jeder Dekade und verschiedenen Publikationsorganen vorgestellt werden (siehe die Belegbeispiele 1-6).

Beispiel 1 ist ein Magazinbericht des SPIEGELS vom 26.12.1988. Es ist einer der ersten Beiträge im deutschsprachigen Mediendiskurs, der ausführlich auf „eine mysteriöse Seelenkrankheit“ eingeht, die „immer mehr Ärzte und Therapeuten“ beschäftigt (DER SPIEGEL, Nr. 52 (1988): 162-164, im QV unter 8.1.6). Typisch für die Textsorte Magazinbericht ist die formulierte These im hervorgehoben gedruckten Vorspann des Artikels und der Wechsel von personalisierten Szenen (siehe die Abschnitte 1-4 unten) und Zitaten von Expertinnen und Experten (siehe die Abschnitte 5, 6, 8, 9). Der Text beginnt mit drei szenischen Beispielen, die jeweils das Verhalten dreier Personen im jungen und mittleren Berufsalter schildern: Dem „begeisterten“ Start ins Berufsleben wird eine spätere unmotiviertere Haltung gegenübergestellt, die auch mit psychosomatischen Symptomen einhergeht. Es folgt in Absatz 5 eine erste Experteneinschätzung zum „neuen Patiententyp“ und in Absatz 7 wird die Reaktion dieses neuen Patiententyps von anderen möglichen Reaktionen („Kündigung“, „Rebellion am Arbeitsplatz“) abgegrenzt. In Absatz 8 wird der ,Entdecker“ Herbert Freudenberger als Person eingeführt, die für „krisenhafte Entwicklungen dieser Art“ eine „Diagnose“ mit den Symptomen „Dauermüdigkeit, Gleichgültigkeit und Zynismus“ prägte. Die zuvor geschilderten Szenen und Expertenstimmen werden dadurch mit dem Diagnosewort Burnout verknüpft. Interessant ist des Weiteren, dass berichtet wird, Freudenberger habe herausgefunden, dass diese Entwicklungen und Symptome „mit traumatischen Kindheitserlebnissen wenig zu tun hatten.“ Erklärungskonzepte der psychoanalytischen Theorie werden von ihm für diesen Patiententyp demnach zurückgewiesen. ${ }^{549}$

549 Dieser Abschnitt im SPIEGEL-Artikel nimmt wohl Bezug auf folgende Aussage Freudenbergers in seinem Buch „The high cost of high achievment“, das er zusammen mit der Journalistin Geraldine Richelson 1980 herausgegeben hat: „Many oft he people who come to my office describe similar feelings, and yet they are neither neurotic nor psychotic in the historical sense of those 
1) DER SPIEGEL Nr. 52 (1988: 162-164) (Unterstreichung und Nummerierung der Absätze, T.S., sonstige Hervorhebungen im Original, im QV unter 8.1.6)

26.12.1988

PSYCHOLOGIE

Nichts als Ruhe

Vor allem Menschen in helfenden Berufen, die mit besonderem Elan ins Berufsleben gestartet sind, verfallen zuweilen in eine mysteriöse Seelenkrankheit.

[1] Zu Beginn seiner Karriere war der Berliner Professor für seine Studenten jederzeit zu sprechen. Neugierig und umtriebig engagierte sich der Wissenschaftler für Studienreform und Selbstverwaltung und war stets dabei, wenn ein „autoritärer Zopf“ abzuschneiden war.

[2] Heute beschränkt der inzwischen 53jährige Professor seine Begegnungen mit Studenten, „diesen narzißtischen Kretins“, auf ein kühles Minimum, sitzt Konferenzen seufzend ab und lebt müde und resigniert zwischen seinen Bücherwänden. Neuerdings ist der Akademiker regelmäßig beim Arzt: Er schläft miserabel, und sein Herz setzt zu unvermittelten Sprüngen an.

[3] Die junge Lehrerin begann ihre Lehrzeit an einer nordrhein-westfälischen Gesamtschule voller Elan. Doch bereits nach einem Jahr im Schuldienst ist sie „ein Nervenbündel“, fällt nach dem Unterricht in einen Erschöpfungsschlaf und brütet nachts über ihren Stundenvorbereitungen: „Ich hätte nie gedacht, daß ich mal so werden könnte wie die säuerlichen alten Kollegen, die die Kinder bloß anschreien und Strafarbeiten aufgeben“, seufzt sie und erzählt, inzwischen haue sie auch dazwischen, „anders kommt man einfach nicht durch“.

[4] Eine Münchner Psychologin, 34, arbeitet als Projektleiterin im Strafvollzugsbereich. Anfangs voller Begeisterung über den „neuen Job“, ertappt sie sich jetzt dabei, daß sie im Büro die Stunden bis zum Dienstschluß zählt. Sie leidet unter einem „quälenden Gefühl der Hilflosigkeit und des Ausgeliefertseins“, begleitet von Schlaflosigkeit, Kopf- und Rückenschmerzen.

[5] Die rätselhafte Krankheit, bei der Idealismus, Arbeitseifer und Begeisterung schwinden und zeitgleich körperliche Beschwerden auftreten, beschäftigt immer mehr Ärzte und Therapeuten. Der Hamburger Professor und Psychotherapeut Gerhard Portele spricht von einem „neuen Patiententyp“, der, getrieben von diffusem Unbehagen, psychologische Hilfe sucht.

words. That they are suffering is undeniable. [...] Soon I recognized a phenomenon at work that had less to do with a childhood trauma than with a relatively recent malaise“ (Freudenberger/Richelson 1980: xv-xvi). Diese Zurückweisung psychoanalytischer Theorievokabeln für die „,neuen Patiententypen“ passt zu der Beobachtung in Kap. 6.1.1, dass die Neurosenlehre Freuds im Zuge der 1980er- und 90er-Jahre zunehmend kritischer gesehen wurde (vgl. dazu den folgenden Hinweis in der 13. Auflage des psychologischen Lexikons DORSCH ${ }^{13} 1998:$, Im $\rightarrow$ ICD-10 und $\rightarrow$ DSM-IV wird der Oberbegriff Neurose nicht mehr benutzt.“). In diesem Zuge wird auch der Begriff der `Neurasthenie`, der auf der Neurosenlehre beruht, als nicht mehr ganz zeitgemäß eingeordnet. Auch Freudenberger geht es um die Beschreibung von Erschöpfung bei Personen, die er entgegen bisheriger psychoanalytischer Theoriebildung als „weder neurotisch noch psychotisch“ beschreibt. 
[6] Während noch vor einigen Jahren Beziehungs- und Eheprobleme im Mittelpunkt therapeutischer Bemühungen standen, seien es heute Karriere- und Leistungsstreß, Frustrationen am Arbeitsplatz. Die selbstzweiflerische Frage „Was soll das eigentlich, was ich da mache?" ist inzwischen, so Portele, das beherrschende Thema, Arbeitsstreß werde in zunehmendem Maße als Lebensverhinderung empfunden.

[7] Ein Kennzeichen der Büroneurotiker ist, daß ihre Unzufriedenheit weder Kündigung noch Rebellion am Arbeitsplatz auslöst, sondern sich nach innen richtet, Körper und Seele malträtiert und zu dramatischen Persönlichkeitsveränderungen führen kann.

[8] „Burnout“ heißt die knappe Diagnose, die der amerikanische Psychoanalytiker Herbert J. Freudenberger für krisenhafte Entwicklungen dieser Art prägte, nachdem Patienten in Verbindung mit ihrer Arbeit über Dauermüdigkeit, Gleichgültigkeit und Zynismus klagten, Zustände, die, wie Freudenberger herausfand, mit traumatischen Kindheitserlebnissen wenig zu tun hatten.

[9] Unter seinen ausgebrannten Patienten fand Freudenberger auffällig viele Menschen, die ursprünglich mit idealistischem Schwung einen dienenden Beruf ergriffen hatten: etwa Rechtsanwälte, Krankenschwestern, Ärzte, Sozialarbeiter, Richter und Politiker. „Nur wer einmal gebrannt hat“, folgerte Burnout-Entdecker Freudenberger, „kann ausbrennen.“ [...]

Das zweite Beispiel ist eine Problemdarstellung aus der Süddeutschen Zeitung vom 31.12.1994. Auch dieser Artikel steigt mit einem Fallbeispiel ein, an das zum Ende des ersten Abschnitts eine Aussage aus der Psychologie zum „Burnout-Syndrom“ im Sinne eines einordnenden Fachkommentars direkt angeschlossen wird („Hohe Erwartungen, heißt es in der Psychologie, [...] ergeben ein Burnout-Syndrom“). Der Artikel fasst im weiteren Verlauf ohne weitere Personalisierungen psychologische Theorien und Untersuchungen zum Phänomen BuRNOUT zusammen und bezieht sich dabei explizit auf das Buch „Das Burnout-Syndrom. Theorie der inneren Erschöpfung“ von M. Burisch. Dabei verwendet der Beitrag verschiedene sprachliche Mittel, um zwischen den Bezeichnungen Burnout, Burnout-Syndrom bzw. Ausbrennen und weiteren Satz- und Textabschnitten eine Entsprechungsrelation herzustellen, z. B. über einen Doppelpunkt im Übertitel des Beitrags, durch ein konsekutives Verb wie ergeben am Ende des ersten Abschnitts, das Sachverhalte zu Bedingungen für den Folgezustand Burnout erklärt; durch das syntaktische Muster „erscheint Burnout [...] als [...]“ am Ende des dritten Abschnitts oder durch eine Aufzählung verschiedener beruflicher und gesellschaftlicher Gruppen in Abschnitt 4 („Krankenschwester auf einer Intensivstation [...]“), die von den zuvor genannten Entstehungsbedingungen des Burnout betroffen sind.

2) Süddeutsche Zeitung, 31.12.1994 (= Rheinz 1994) (Unterstreichung und Nummerierung der Absätze, T.S., sonstige Hervorhebungen im Original, im QV unter 8.1.6)

Burnout: ,Die phantasievolle Schwungkraft, der muntere Idealismus' sind dahin [hier Unterstreichung im Original] 


\section{Wenn berufliches Feuer zur Asche wird}

Sich leer und ausgebrannt fühlen - die Gründe für ein weit verbreitetes Phänomen in unserer Gesellschaft

Von Hanna Rheinz

[1] Unmerklich veränderte sich sein Berufsalltag. Eine ausgebliebene Beförderung; bei der Jahresprämie war er schlicht übergangen worden. Wenig später kam der neue Kollege. Er setzte sich dem Altgedienten vor die Nase und nahm ihm, ohne mit der Wimper zu zucken, die angestammte Aussicht. Aus dem schleichenden Zerfall war unterdessen galoppierende Unzufriedenheit geworden. Er murrte des öfteren, aber ohne Ergebnis. Sein Verhandlungsgeschick sank gleichermaßen. Der einstige Überflieger galt inzwischen kaum mehr als unterer Durchschnitt. Angewidert blickte er morgens in die Runde. Das war nicht mehr der Ort, an dem man ihm einst die Goldene Verkaufsnadel verlieh. Innerlich hatte er längst gekündigt. Er fühlte sich ausgebrannt. Hohe Erwartungen, heißt es in der Psychologie, gepaart mit dem Wunsch, helfen zu wollen oder anderen wenigstens moralisch ein Vorbild zu sein, ergeben ein Burnout-Syndrom (neudeutsch: börnaut).

$[\ldots]$

[3] Doch warum verwandelt sich ein hoffnungsvoll entfachtes Berufsfeuer überhaupt in kalte, ausgebrannte Asche? Der Hamburger Psychologe Mathias Burisch hat die Vielzahl der im Umfeld des Burnout-Syndroms entwickelten psychologischen Theorien und betrieblichen Untersuchungen zusammengetragen (Das Burnout-Syndrom - Theorie der inneren Erschöpfung, Springer-Verlag, Heidelberg 1994, 38 Mark). Bezeichnenderweise wurde das BurnoutPhänomen zuerst bei ehrenamtlichen Mitarbeitern diverser Hilfsorganisationen in den USA beobachtet. Hier stand der Ehrgeiz, helfen zu wollen oder etwa bei der Betreuung von Drogenabhängigen, sogar gesellschaftlich etwas zu bewegen, in krassem Gegensatz zu den geringen Erfolgen dieses Unterfangens. [...] Daher erscheint Burnout zunächst als Streß durch ,langdauernd zu hohe Energieabgabe für zu geringe Wirkung bei ungenügendem Energienachschubs.

\section{Die Flinte ins Korn geworfen}

[4] ,Ausbrennen“ ist jedoch nicht nur das Schicksal jener Legionen hilfloser und unterbezahlter Helfer, die über die Undankbarkeit der Welt (und der ihrer Klienten im besonderen) lamentieren. Ausbrennender Energieverbrauch bei fehlendem Nachschub trifft inzwischen auch jene, die in großen Konzernen, Krankenhäusern und staatlicher Institutionen arbeiten. Die Krankenschwester auf einer Intensivstation leidet darunter, der Verkaufsrepräsentant, Eltern, die ein behindertes Kind, Kinder, die ihre greisen Eltern pflegen, der Schalterbeamte, der Lehrer angesichts der lärmenden, gewaltbereiten Heranwachsenden oder der Drogentherapeut, der nach Jahren die Flinte ins Korn wirft und resigniert und verbittert erkennt, daß all sein Bemühen vergeblich war. Und je höher die Erwartungen und die persönliche Anteilnah$\underline{\text { me, dies zeigen empirische Untersuchungen an ,Ausbrennern' in verschiedenen Projekten im }}$ sozialen Bereich, desto bitterer ist das Scheitern.

Das dritte Beispiel zeigt eine Problemdarstellung aus der Zeitschrift „Psychologie Heute“ aus dem Jahr 2000 von Dieter Smolka (= Smolka 2000, PH, im QV unter 8.1.7), in der „Burnout“ im Zusammenhang mit Lehrerbelastung behandelt 
wird. Zu Beginn des Artikels wird die aktuelle Lage beschrieben, die von schlechten Arbeitsbedingungen im Lehrerberuf berichtet („die fehlenden Junglehrer“, „Mehrarbeit bei gleichzeitigem Abbau der Entlastungen“ etc., ebd.: 39). In der dritten Spalte (daraus stammt der unten zitierte Abschnitt) wird >Burnout als ein Zustand eingeführt, für den „Menschen in Berufen“ besonders anfällig seien, „bei denen hohes persönliches Engagement im täglichen Umgang mit anderen Menschen gefordert wird“ (ebd.). >Burnout ‘ wird weiter wie folgt definiert:

\section{3) Psychologie Heute, Heft 4/2000 (= Smolka 2000: 39f.)}

(Unterstreichung, T.S., Kursivsetzung im Original, im QV unter 8.1.7)

$[\ldots]$

Burnout ist eine körperliche und emotionale Erschöpfung aufgrund dauernder Anspannung, ständiger sozialer Begegnungen, täglichen Stresses. Die Wirkungen ist dann besonders tiefgreifend, wenn aufreibende Arbeit und dauernde Belastung von wenig Anerkennung und mitmenschlicher Unterstützung begleitet sind. Burnoutgefährdete Menschen fühlen sich isoliert. schämen sich ihres Zustandes - und verschlimmern ihn damit. Ideen und Projekte, die früher die Arbeit auszeichneten, gibt es nicht mehr; vielmehr findet Dienst nach Vorschrift statt. Das Gefühl von „innerer Kündigung“ und Hilflosigkeit, kann sich zu chronischer Verzweiflung und Sinnlosigkeit verdichten, wie die Schulpsychologen Christine Enders und Roland Storath an einem Beispiel verdeutlichen:

Eine Lehrerin begann vor etwa 15 Jahren ihren Dienst. Voller Elan widmete sie sich der Arbeit. Sie plante Projekte hatte guten Kontakt zu ihren Schülern. Häufig unternahm sie Wanderungen und Klassenfahrten. Der Unterricht machte ihr und den Schülern Spaß, die Erfolge waren groß. Im Kollegium hatte sie viele Kontakte und war durch ihr kollegiales Verhalten sehr beliebt. In einem schleichenden Prozess verwandelte sich die fröhliche, engagierte Lehrerin. Der Unterricht wurde immer weniger vorbereitet: „Da hab ich doch Routine; wer nach zehn Jahren noch was vorbereitet, ist selbst schuld!“ Die Pausen im Lehrerzimmer dehnten sich über den Gong hinaus aus. Die Äußerungen über die Klasse wurden verächtlicher, destruktiver: „Die sind so doof, das ist nicht auszuhalten. "Der Ton den Schülern gegenüber wurde zunehmend aggressiver. Gelegentlich tauchte die etwas wehmütige Erinnerung an frühere Klassen auf, verknüpft mit der Annahme, diese Schüler seien eben einfach anders gewesen, mit ihnen habe man sehr viel mehr anfangen können, mit der jetzigen Klasse lohne sich das alles nicht. Weniger Kontakt zu Kolleginnen und Kollegen, auch hier eher Nörgeln als Gedankenaustausch. Die Aussicht, den Beruf noch lange Jahre ausüben zu müssen ohne erkennbare Perspektiven auf Veränderung, lähmte die Lehrerin mehr und mehr.

In diesem Beispiel folgt das Fallbeispiel auf einen Absatz, in dem `BURNOUT` mit dem Kardinalsymptom 'körperlicher' und 'emotionaler' >ERSCHÖPFUNGく identifiziert wird und anhand verschiedener Entstehungsbedingungen (,,aufgrund dauernder Anspannung“, „wenig Anerkennung“ etc.) und durch Verknüpfung zu Konzepten wie der sinneren Kündigung` weiter eingegrenzt wird. Das Fallbeispiel gestaltet den qualitativen und temporalen Kontrast (markiert durch „nach zehn Jahren“) 
zwischen dem Verhalten der Lehrerin vor dem Beginn des Ausbrennens und dem Zustand danach in narrativer Breite aus. Dieses Muster des qualitativen und temporalen Kontrasts kann man in vielen Texten des medialen Vermittlungsdiskurses beobachten, teilweise wird dieser Kontrast auch durch Tempuswechsel in den narrativen Szenen (vom Präteritum zum Präsens, siehe Beleg 1 oben) unterstützt. $^{550}$

Das vierte Beispiel zeigt eine Problemdarstellung, die in der FAZ am 03.04.2004 erschienen ist. Die Autorin Ursula Kals benennt im ersten Abschnitt zunächst Eigenschaften, die für Personen, die ,an Burn-out-Symptomen“ erkranken, typisch sind. Im zweiten Abschnitt „charakterisiert“ sie >Burnout lichen Diskursstrang sehr häufig zitierten Symptome „emotionale Erschöpfung, Dehumanisierung und Zynismus“, die auf Maslach/Jackson 1981 zurückgehen. Unter Berufung auf Expertenmeinungen wird Burnout zudem als Folgeerscheinung von „Disstress“ bestimmt. Etwas später erläutert der zitierte Arbeitsmediziner am konkreten Einzelfall noch eine „typische Burn-out-Patientin“.

4) Frankfurter Allgemeine Zeitung, 3.4.2004: 55 (= Kals 2004) (Unterstreichung T.S., sonstige Hervorhebungen im Original, im QV unter 8.1.6)

\section{Ausgebrannte Mitarbeiter können das Ergebnis schlechter Führung sein / Von Ursula Kals}

Krank werden die Engagierten. Diejenigen, die sich für ihren Beruf begeistern, die sich hoch mit ihrer Tätigkeit identifizieren, darin Sinn und Selbstverwirklichung finden und ein ausgeprägtes Verantwortungsgefühl besitzen. Also all jene Führungskräfte, Ärzte, Lehrer, die sich hundertzwanzigprozentig für das Wohl ihrer Mitarbeiter, Patienten und Schüler einsetzen. Gefährdet sind auch Menschen in sozialen und pflegerischen Berufen, die sich für andere aufopfern und am „Helfersyndrom“ leiden. Wer sich hingegen entspannt durchhangelt, bis er um 16.29 Uhr seinen Computer herunterfahren kann, der läuft nicht Gefahr, an Burn-out-Symptomen zu erkranken.

Neben komplexen Wechselwirkungen läßt sich ein Burn-out-Syndrom durch drei Elemente charakterisieren: emotionale Erschöpfung, Dehumanisierung und Zynismus und schließlich eine verminderte Leistungszufriedenheit. „Weitgehende Einigkeit besteht heute darin, daß Distress, also negativer Streß, ein Schlüsselphänomen in der Entstehung des Burn-out darstellt“, sagt der Arbeitsmediziner Andreas Weber.

[...]

Eine typische Burn-out-Patientin, die Weber während eines Forschungsprojekts kennengelernt hat, ist Eva Berger, die anders heißt. Den Schulalltag empfindet die Gymnasiallehrerin inzwischen „wie eine Bleiweste“, ihre fünf Stunden Unterricht montags erlebt sie „wie eine 38-Stunden-Schicht“. Die einfachsten Dinge bewältigt sie nicht mehr, die Alltagsroutine

550 Weiteres Beispiel mit Tempuswechsel in der narrativen Szene: „Früher ging sie gerne zur Arbeit. [...] Heute schleppt sie sich morgens lustlos an den Schreibtisch [...]“ (PH 1/2017, = Langosch 2017); siehe auch PH 9/2011 (= Leggewie 2011), Belege im QV unter 8.1.7. 
überfordert sie. Läßt ein Schüler seinen Füller fallen, schreit sie ihn an. Die Pädagogin in Fürth hat mit Englisch und Deutsch korrekturintensive Fächer. Sie möchte alles gut, gründlich und gerecht machen und scheitert an diesen Ansprüchen. [...]

Das fünfte Beispiel zeigt einen Beitrag aus der populärwissenschaftlichen Zeitschrift „Gehirn\&Geist“ aus dem Jahr 2016 (= Falkai 2016, G\&G, im QV unter 8.1.7). Der Autor Peter Falkai ist Direktor der Klinik für Psychiatrie und Psychotherapie an der Universität München. Er war von 2010-2012 Präsident der Deutschen Gesellschaft für Psychiatrie und Psychotherapie, Psychosomatik und Nervenheilkunde (DGPPN) und ist Mitherausgeber der deutschen Fassung der Neuauflage des Klassifikationssystem für psychische Störungen DSM-5 (Falkai/Wittchen et al. 2015, im QV unter 8.1.3). ${ }^{551}$ Der Artikel ist der Rubrik „Diagnose und biologische Grundlagen“ in einem Dossier-Heft zum Thema „Burnout“ zugeordnet (siehe Abschnitt 1, Beleg 5 unten). Im Zentrum des Beitrags steht die Frage, ob „Burnout“ nicht häufig eigentlich eine „Depression“ oder „Angststörung“ meine (siehe Abschnitt 2, Beleg 5 unten). Die Symptome von >Burnout< und `Depression , werden in einem Informationsabschnitt, der typografisch vom restlichen Fließtext abgesetzt ist, direkt einander gegenübergestellt (siehe die Abschnitte 7-9 in Beleg 5 unten). Falkai vergleicht dabei den Symptomkatalog des Diagnose-Konzepts >Depression` nach der ICD-10 $0^{552} \mathrm{mit}$ den drei Hauptsymptomen des Burnout-Konzepts nach Maslach/Jackson (1981, im QV unter 8.1.5). Eine Seite zuvor sind zudem in leicht modifizierter Form die Fragen des Maslach Burnout Inventory (MBI) der Fragebogen-Version „Human Services Survey“ für Berufe im Sozial- und Gesundheitssektor der dritten Auflage aus dem Jahr 1996 als Selbsttest für die Leser/innen in deutscher Übersetzung abgedruckt (siehe unten Beleg 5, G\&G 2016: 23). Dies ist erstaunlich, da man auch die Fragebogenversion des MBI-General-Survey (Maslach/Jackson/

551 Die deutsche Fassung des DSM-5, das Diagnosemanual für psychische Störungen der American Psychiatric Association, erschien in erster Auflage 2015. In den Medien wurde damals darüber berichtet, dass „Burnout“ nicht als eigenständige Störung in die neue Fassung des DSM-5 aufgenommen wurde (siehe SPIEGEL ONLINE vom 12.07.2012 (= Hauschild 2012, im QV unter 8.1.6). In Kapitel 6.2.2.2 wurde ein Beitrag aus dem Dt. Ärztebl. analysiert, an dem Peter Falkai ebenfalls als Autor beteiligt war (vgl. Beispiel 10 in Kapitel 6.2.2.2.; = Berger/Falkai/Maier 2012, im QV unter 8.1.4).

552 Die Leitlinien zur Anwendung der ICD-10 geben vor, dass zwei von den folgenden drei Hauptsymptomen über einen Zeitraum von mehr als zwei Wochen zutreffen müssen: ICD-10, Kap. F32 [231] (siehe dort Kapitel F32): „depressive, gedrückte Stimmung; Interessenverlust und Freudlosigkeit; Verminderung des Antriebs mit erhöhter Ermüdbarkeit (oft selbst nach kleinen Anstrengungen) und Aktivitätseinschränkung.“ (https://www.leitlinien.de/nvl/html/depression/kapitel-2, zuletzt eingesehen am 16.6.2019). 
Leiter ${ }^{3}$ 1996: 19ff., im QV unter 8.1.5) hätte abdrucken können. Denn dieser bezieht alle Berufe in die formulierten Aussagen mit ein. ${ }^{553}$ Mit dem MBI-General-Survey lägen allerdings bisher „erst wenige Erfahrungen vor“, wie Burisch 2014 konstatierte (Burisch ${ }^{5} 2014$ : 36, im QV unter 8.1.5). In jedem Fall können diese im Magazin Gehirn\&Geist abgedruckten 22 Items (in Abb. 10 werden die ersten 15 Items zitiert) und ihre Auswertung als eine operationale Definition des Phänomens BuRnout innerhalb dieses Artikels gelesen werden (vgl. dazu auch Kap. 6.2.2.2 die Belege 6a und 6b).

Der Einstieg in den Artikel ist ein ausführliches Einzelfallbeispiel eines Werbetexters, dessen Arbeitsleistung sich immer weiter verschlechtert und dessen Hausarzt bei ihm schließlich die Diagnose Burnout stellt (siehe die Abschnitt 3-5 in Beleg 5 unten). Im Anschluss erhält das Beispiel dieses Werbetexters durch den Vergleich mit Prominenten, die öffentlich zugegeben haben, dass sie an Burnout ,litten‘, über den Einzelfall hinaus Geltung (siehe Abschnitt 6 unten). Am Ende des Beitrags wird Burnout schließlich als „Beschreibung eines Risikostadiums für die Entwicklung körperlicher und psychischer Probleme“ in die psychiatrische Terminologie eingeordnet (siehe Abschnitt 11 unten). Die Bezeichnung Risikostadium kann als bewusster terminologischer Fixierungsversuch gelesen werden, da Falkai diese Wortwahl auch in fachlichen Texten und dortigen Abbildungen verwendet (vgl. dazu Beleg 10 in Kap. 6.2.2.2, = Berger/Falkai/Maier (2012: 213), im Dt. Ärztebl. im QV unter 8.1.4).

5) Gehirn\&Geist Dossier 1/2016: 20-25 (= Falkai 2016) (Unterstreichung und Abschnittsmarkierung, T.S., sonstige Hervorhebungen im Original, im QV unter 8.1.7)

[1] DIAGNOSE UND BIOLOGISCHE GRUNDLAGEN [als Übertitel in Versalien und mit Unterstreichung gesetzt, T.S.]

[2] DIAGNOSTIK hinter den meisten Fällen von Burnout stecken noch schwerere psychische Erkrankungen: Depressionen oder Angststörungen etwa. Werden diese dank vermeintlichem Burnout-Befund frühzeitig erkannt, steigen die Heilungschancen. [als Vorspann etwas größer als folgender Fließtext gedruckt, die folgende Überschrift ist deutlich größer gedruckt als die sie umgebenden Texte, T.S.]

553 In der ersten Auflage des Malsach Burnout Inventory (MBI) werden die Fragebogen-Items nicht mit Ja/Nein, sondern mit einer Häufigkeits- und Intensitätsskala beantwortet (vgl. Maslach/Jackson 1981; Büssing/Perrar 1992: 332, im QV unter 8.1.5). In der 2. Fassung des MBI (1986) wird die Intensitätsskala „zugunsten eines einzigen Häufigkeitsformats fallengelassen“ (Burisch 52014: 36, im QV unter 8.1.5). In der dritten Fassung von 1996 haben Maslach/Jackson/Leiter die Häufigkeitsskala („0-6 frequency ratings“) ebenfalls beibehalten und neben dem Fragebogen, der sich auf die „Human Services“ konzentriert, noch eine Fassung für den Bereich „Educators“ und für andere Berufe (MBI - Generaly survey) hinzugefügt. Vgl. Maslach/Jackson/Leiter ( ${ }^{3} 1996$ : 2; 22, im QV unter 8.1.5). 


\author{
Eine scheinbare \\ Pandemie \\ VON PETER FALKAI
}

[3] Das Aus für Bernd Hinze* kam schleichend. Der 38-Jährige liebte seinen Beruf als Werbetexter - eigentlich. Zuletzt nahmen die Anforderungen in der Agentur jedoch ständig zu. Immer mehr Projekte wanderten auf seinen Schreibtisch. Die Tage füllten sich sukzessive mit Besprechungen, Kundengesprächen und Präsentationen. Anfangs gab ihm dieses Mehr an Aufgaben, das schnell getaktete Arbeitsleben einen gewissen Kick. Aber mit der Zeit nagte es zunehmend an seinen Ressourcen. Hinze opferte mehr und mehr Freizeit. Bereits morgens um sieben saß er am Schreibtisch, um ungestört wichtige Arbeiten zu erledigen. Gegen 19 Uhr war er erschöpft, musste jedoch von zu Hause aus noch E-Mails beantworten oder Telefonkonferenzen abhalten. Der Kontakt zu Freunden schlief nach und nach ein, seine Frau zog sich zurück, und seinen kleinen Sohn sah er praktisch gar nicht.

[4] Zunehmend wandelte sich die Freude am Job in Abscheu - insbesondere jüngeren Kollegen gegenüber, die vermeintlich leistungsfähiger waren. Hinze konnte sich immer schlechter konzentrieren und fühlte sich schon morgens erledigt. Dazu gesellten sich körperliche Beschwerden: Kopfweh, Magendrücken, Rückenschmerzen. Urlaub - sofern er sich denn welchen gönnte - vermochte die Lage nicht mehr zu verbessern.

[5] Irgendwann ging gar nichts mehr. Hinze verließ die Agentur früher als gewohnt und ging direkt ins Bett. Trotzdem fühlte er sich morgens wie gerädert. In der Firma war er unfähig, auch nur den Inhalt seiner E-Mails zu erfassen. Eine Kollegin sprach ihn an, sie erlebe ihn als abwesend, fast schon verwirrt. Das war der Punkt, an dem Bernd Hinze einsah, dass er Hilfe brauchte. Noch am selben Tag ging er zum Hausarzt. Der tippte auf Burnout - als Folge chronischer Arbeitsüberlastung.

[6] Werbetexter Hinze befindet sich in bester Gesellschaft. Die Fußballer Ralf Rangnick und Sebastian Deisler [...] sind nur einige aus der langen Liste Prominenter, die zugaben, irgendwann in ihrem Leben unter einem Burnout gelitten zu haben. Es scheint heute viele $\mathrm{zu}$ treffen. [...] [S. 20 des Beitrags, Unterstreichung, T.S.]

* Name von der Redaktion geändert [...]

[Zusammenfassung der S. 21-23, T.S.: In diesen Abschnitten wird eine Erklärung für den Anstieg der Zahl der Arbeitsunfähigkeitstage aufgrund psychischer Erkrankungen gesucht, dabei wird auch auf das Krankheitsbild der „Neurasthenie“ und seine Verbreitung zu Beginn des 20. Jahrhunderts eingegangen. Des Weiteren ist eine grafische Übersicht zu den „häufigsten psychischen Störungen in Deutschland“ abgedruckt.] 
[Zitierter Auszug aus dem Selbsttest aus Gehirn\&Geist, Dossier 1/2016 (= Gehirn\&Geist 2016, im QV unter 8.1.7). Der gesamte Selbsttest ist im Original beige und hier grau hinterlegt, T.S.]

\section{DIAGNOSE UND BIOLOGISCHE GRUNDLAGEN / DIAGNOSTIK}

Sind Sie ausgebrannt? [Überschrift ist außerdem rosafarben, T.S.]

Ein Team um die Psychologin Christina Maslach von der University of California in Berkeley entwickelte schon in den 1980er Jahren einen Fragenkatalog zur Erfassung des Burnout-Syndroms. Das Maslach Burnout Inventory (MBI), auf dem auch der folgende Selbsttest beruht, gilt heute als wichtiges Hilfsmittel, um das Burnout-Risiko abzuschätzen.

1. Ich fühle mich durch meine Arbeit emotional erschöpft.

$($ ) $=\mathbf{J A}$

\」- NEIN

[im Orig. zwei Kästchen, bei „JA" rosafarben, bei ,NEIN" schwarz, T.S.]

2. Ich fühle mich am Ende eines Arbeitstags verbraucht.

3. Ich fühle mich bereits ermüdet, wenn ich morgens aufstehe und einen neuen Arbeitstag vor mir liegen sehe.

4. Ich kann es leicht verstehen, wie andere Menschen über bestimmte Themen denken.

5. Ich habe das Gefühl, einige andere Menschen so zu behandeln, als wären sie Objekte.

6. Den ganzen Iag mit Menschen zu arbeiten, strengt mich an.

7. Ich gehe erfolgreich mit den Problemen anderer Menschen um.

8. Ich fühle mich durch meine Arbeit ausgebrannt.

9. Ich habe das Gefühl, durch meine Arbeit das Leben anderer Menschen zu beeinflussen.

10. Ich bin Menschen gegenüber abgestumpfter geworden, seit ich diese Arbeit ausübe.

11. Ich befürchte, dass mich meine Arbeit weniger mitfühlend macht.

12. Ich fühle mich sehr energiegeladen.

13. Ich fühle mich durch meine Arbeit frustriert.

14. Ich habe das Gefühl, in meinem Beruf hart zu arbeiten.

15. Es interessiert mich nicht wirklich, was mit anderen Menschen geschieht.

[Es folgen noch die Items 16-22, T.S.]

Auswertung:

Zählen Sie die Antworten auf der linken Seite zusammen. Wenn Sie mehr als zehn Punkte erreichen, sind sie burnoutgefährdet. Der Test liefert jedoch nur eine grobe Orientierung und kann keine ärztliche Diagnose ersetzen. Im Zweifelsfall sollten Sie sich professionelle Hilfe suchen.

Gehirn\&Geist, nach: Maslach, C. et al.: The Maslach Burnout Inventory Manual. 3rd Edition. Consulting Psychologists Press, Palo Alto 1996

Abb. 10: Items 1-15 des Maslach Burnout Inventory, zitierter und im Layout nachempfundener Auszug aus dem Selbsttest in Gehirn\&Geist (2016: 23), Textauszug @ Maslach et al. 1996, Gehirn\&Geist 2016. 
[Eine Seite später (S. 24) gibt es folgende hervorgehoben gedruckte Informationseinheit zur Abgrenzung bzw. Überschneidung von `Burnout` und `Depression`:]

Depression und Burnout: Fließende Grenzen [im Orig. fett und farbig als Überschrift markiert, T.S.]

[7] Eine 37-jährige Juristin schildert in meiner Sprechstunde, sie sei bereits seit einem halben Jahr weniger belastbar als zuvor und ständig müde. Außerdem fange sie wegen kleinster Probleme an zu weinen und sei überhaupt schon seit Wochen traurig - ohne dass sie dafür einen Grund benennen könne. Zudem beschreibt sie sich als unkonzentriert: Um einfache Schreiben zu erfassen, brauche sie ewig, Meetings zögen oft einfach so an ihr vorbei. Das sei aber vielleicht auch kein Wunder, schließlich schlafe sie nachts schlecht. Meist, so erzählt sie, wache sie gegen 2 Uhr auf und grüble dann stundenlang. Abgenommen habe sie auch. Und überhaupt fühle sie sich wertlos.

[8] Diese Schilderung führt zur Diagnose einer mittelgradigen Depression. Denn um eine Depression zu diagnostizieren, müssen über einen Zeitraum von mehr als zwei Wochen mindesten zwei von drei Hauptsymptomen zutreffen: gedrückte Stimmung, Interessenverlust und Antriebsmangel. Je nachdem, über wie viele der sieben Zusatzsymptome - etwa Schlafstörungen, verminderter Appetit oder Suizidgedanken - ein Patient zudem berichtet, unterscheiden Ärzte zwischen leichter, mittelgradiger oder schwerer Depression (siehe Grafik unten).

[9] Allerdings: Betrachtet man den Symptomkatalog, so trifft einiges auch auf das BurnoutSyndrom zu. Dessen zentrales Merkmal, »anhaltende Erschöpfung und Leistungsminderung«, meint letztlich nichts anderes als das Hauptsymptom »Antriebsmangel und erhöhte Ermüdbarkeit« im Diagnosekatalog für Depressionen. Und hinter dem für Burnout typischen Zynismus verbergen sich möglicherweise »Gefühle von Schuld und Wertlosigkeit sowie negative und pessimistische Zukunftsperspektiven«, also Zusatzsymptome der Depression. pf

[Unter diesem Informationseinheit ist noch eine Info-Grafik abgebildet, T.S.]

[10] [...] es scheint, als ob der Begriff Burnout heute etwas inflationär eingesetzt wird. So bezog sich die ursprüngliche Definition des "Staff Burnout«, die 1974 der deutschamerikanische Psychotherapeut Herbert Freudenberger (1926-1999) prägte, auf eine Überforderung im beruflichen Leben. Aktuell verwenden ihn viele im Zusammenhang mit verschiedensten Erschöpfungssyndromen - gleich ob diese nun auf Grund einer privaten oder beruflichen Überforderung entstanden sind. [Unterstreichungen, T.S.]

\section{Vorbote einer Depression}

[11] Hinzu kommt: Burnout - das ist derzeit fachlicher Konsens - gilt als Vorbote einer psychischen Erkrankung. Statt einer eigenständigen Diagnose handelt es sich hier vielmehr um die Beschreibung eines Risikostadiums für die Entwicklung körperlicher und psychischer Probleme. [...]

[Es folgen weitere Abschnitte zur Verwendung des Begriffs Burnout in der heutigen Praxis, T.S.]

Bernd Hinze ließ sich vier Wochen lang stationär behandeln. Die Ärzte in der Klinik stellten die Diagnose einer mittelgradigen Depression. Der Hausarzt hatte jedoch Burnout diagnos-

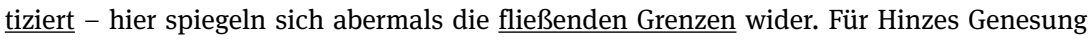
spielt das nur eine untergeordnete Rolle, doch die Krankenkassen erkennen Risikostadien wie Burnout nicht an, sondern zahlen nur für die Begleiterkrankungen - ein Unding in einer Zeit, in der wir Erkrankungen früher erkennen und besser behandeln können. [...] 
Ähnlich wie die bisher präsentierten Texte, die „Burnout“ vor allem über Fallbeispiele und Expertenmeinungen vorgestellt haben, sind auch ratgebende Aufklärungstexte aufgebaut. Diese erscheinen insbesondere in Zeitschriften wie der „Apotheken Umschau“ und „Psychologie Heute“, aber auch in der Tagespresse. ${ }^{554}$ Sie unterscheiden sich von den Magazinberichten und Problemdarstellung vor allem dadurch, dass sie die Leser/innen stärker einbeziehen (z. B. indem sie die Leser/innen direkt ansprechen) und dass sie ihnen konkrete Vorschläge bieten, welche Handlungen, Verhaltensänderungen oder Handlungsabfolgen ausgeführt werden müssen/können/soll(t)en, wenn in einer bestimmten Situation ein spezifischer Zustand (wieder) herbeigeführt, beseitigt, bewahrt oder verhindert werden soll (vgl. Franke 1996: 252; zur weiteren Charakterisierung ratgebender Aufklärungstexte siehe ausführlich Kap. 5.3.3.3 und 5.3.3.4).

Das sechste Beispiel zeigt einen ratgebenden Aufklärungstext mit „appellativinstruierender Funktion“ (vgl. Stanaitytė 2005: 58) aus der „Apotheken Umschau“ aus dem Jahr 1997. Dieser Text wählt direkt zu Beginn ein syntaktisches Muster im Vorspann, das den Einzelnen vor dem Hintergrund einer generischen Identifizierungsfolie anspricht: „Wer an seiner Arbeit keinen Spaß mehr findet, sich immer schlechter konzentrieren kann [...], der braucht vielleicht Hilfe von außen.“ Der Leser wird ermutigt, seine Gefühlslage mit den geschilderten symptomatischen Erlebensweisen, die über den Einzelfall hinaus Gültigkeit beanspruchen, zu vergleichen. Der Haupttext beginnt daran anknüpfend wie in den bisher präsentierten Beispielen mit einem personalisierten Fallbeispiel, das durch einen Diagnosekommentar einer Therapeutin und eine Übersetzung der Bezeichnung Burnout ins Deutsche abgeschlossen wird (siehe Abschnitt 1 in Beleg 6 unten). Doch auch schon Übertitel, Titel und Untertitel bilden zusammen ein semantisches Gefüge, durch das Burnout als ein spezifikationsbedürftiger Begriff präsentiert, als Syndrom klassifiziert und über vier aufgezählte Symptome im Untertitel und den Zusatz der 'Hilfsbedürftigkeit' schon vorab bestimmt wird. Schließlich wird in diesem Beitrag eine Art Checkliste typografisch abgesetzt präsentiert (siehe Abschnitt 3 unten), anhand derer die Leser/innen selbst entscheiden können, ob sie von „Burnout“ betroffen sind. Im Vergleich zum MBI-Selbsttest in Beispiel 5 sind diese aufgelisteten Symptome aber nicht mit „Ja“ oder „Nein“ zu beantworten, sondern sie werden direkt als „Alarmzeichen“ präsentiert, was zur direktiven Grundfunktion der Warnung dieses ratgebenden Aufklärungstextes passt.

554 Z. B. in der Frankfurter Allgemeinen Sonntagszeitung, FAS, 20.04.2014, Nr. 16, S. 31 mit dem Übertitel und Titel: „WAS TUN BEI BURNOUT? Tipps für Erschöpfte und Therapien für Ausgebrannte. Erschöpfung und Burnout verhindern“ (= Weiguny/Nienhaus 2014c, im QV unter 8.1.6). 
6) Apotheken Umschau 12/1997-A: 20-23 (= AU 1997, Unterstreichung und Abschnittsmarkierung, T.S., sonstige Hervorhebungen im Original, im QV unter 8.1.7)

\section{Burnout-Syndrom}

Total ausgebrannt - und jetzt?

Wer an seiner Arbeit keinen Spaß mehr findet, sich immer schlechter konzentrieren kann, sich ständig müde und erschöpft fühlt, der braucht vielleicht Hilfe von außen.

[1] Der erste Pinselstrich fiel ihr schon immer schwer. Seit ein paar Tagen war es aber irgendwie anders. Sie starrte wieder und wieder auf die weiße Leinwand. Ihr fiel einfach nichts mehr ein. Absolut nichts. Die Malerin Frauke B. fühlte sich nur noch leer. Ihre Therapeutin hat es später beim Namen genannt, was die Malerin selbst vage Schaffenskrise nannte. Sie leiden unter einem „Burnout-Syndrom, aus dem Englischen von ausgebrannt.“

[2] Seit Anfang der 70er Jahre fassen Arbeitsmediziner und Psychologen das Phänomen der inneren Erschöpfung unter diesem Begriff zusammen. Ehemals hochmotivierte Mitarbeiter kehren scheinbar plötzlich ins Gegenteil um. [...]

[Zweites Fallbeispiel, Arzt in eigener Praxis, T.S.]

[3] Sind Sie reif für die Insel?

Diese Alarmzeichen sollten Sie beachten:

- Sie sind ständig müde

- Sie haben das Gefühl, nie Zeit zu haben

- Das Aufstehen morgens fällt Ihnen schwer

- Sie reagieren oft grundlos gereizt

- Sie beschränken Ihre sozialen Kontakte auf Klienten, Patienten und Kunden

- Sie haben das Gefühl unentbehrlich zu sein

- Sie vernachlässigen seit längerem Ihre Hobbys und Interessen

$-[\ldots]$

- Sie leiden häufig unter Kopf-, Magen oder Rückenschmerzen

Weitere Fallbeispiele, die in Print- und Onlinemedien präsentiert werden, basieren auf Erzählungen aus der Ich-Perspektive von Betroffenen. Diese schildern, ähnlich wie die anderen Fallbeispiele, den Weg von (besonderem) Engagement und übermäßigen Arbeitseinsätzen hin zu psychosomatischen Symptomen wie Schlaf- und Magenproblemen, extremer Müdigkeit, Gereiztheit oder Ängsten. Das Folgende Beispiel ist eines von dreien, das auf SPIEGEL ONLINE am 08.08.2012 veröffentlicht wurde: ${ }^{555}$

555 Weitere Fallbeispiele aus der Ich-Perspektive findet man zum Beispiel: auf ZEIT Campus 3/2010 das Beispiel eines Jungprofessors (= Schwabe 2010, im QV unter 8.1.6) oder in der FAS das Beispiel eines freiberuflichen Architekten vom 13.09.2015, Nr. 37: 35 (= Oberhuber 2015, im QV unter 8.1.6); in der Zeitschrift Stern Nr. 50/2015: 52-58 (= Schmitz 2015, im QV unter 8.1.7). Des 
Beleg 7: SPIEGEL ONLINE, 08.08.2012 (= Abé 2012) (Unterstreichung T.S., sonstige Hervorhebungen im Original, im QV unter 8.1.6)

Nach dem Burnout

"Es könnte jederzeit wieder passieren"

Es gibt ein Leben nach dem Zusammenbruch - und das soll anders sein als vorher, entspannter, gelassener. Aber wie setzt man die guten Vorsätze um? Drei Betroffene erzählen, wie sie sich und ihren Alltag nach der Diagnose Burnout verändert haben.

[...] [1. Teil: Chef eines Ingenieurbüros plus Trennung von Partnerin, 2. Teil: Fachkrankenschwester, T.S.]

3. Teil: Ein Berater in einem großen Unternehmen: "Ich muss höllisch aufpassen”

„Ich würde mich nicht als geheilt bezeichnen, eher als stabil. So wie ein trockener Alkoholiker. Ich muss höllisch aufpassen. Ich bin stets ein ehrgeiziger Typ gewesen.

In der Schule hatte ich Bestnoten, für meine Doktorarbeit bekam ich einen Preis. Ich fühlte mich nur dann anerkannt, wenn ich Leistung brachte. Entsprechend verhielt ich mich auch im Berufsleben. Ich wollte meine Aufgaben immer perfekt erledigen. So stieg ich schnell weiter auf. Schließlich bekam ich eine Führungsposition, übernahm sehr viel Verantwortung.

In meiner Branche kann man sich keine Fehler leisten. In den schlimmsten Phasen arbeitete ich etwa 80 Stunden in der Woche, 140 Überstunden im Monat. Wenn ich nach Hause kam, konnte ich nicht mehr abschalten. In dieser Zeit litt auch die Beziehung zu meiner Frau, für meine Kinder hatte ich kaum mehr Zeit. Mein Blick verengte sich. Ich war gefangen in einem Tunnel, es gab nur noch die Karriere.

So fühlte ich mich einsamer. Nachts wachte ich auf, schwitzte. Morgens musste ich mich übergeben. Dann trank ich etwas Wasser, schluckte ein paar Stück Traubenzucker und ging in mein erstes Meeting.

\section{Es tut mir gut, Zeit mit meinen Kindern und meiner Frau zu verbringen}

Ich fing eine Gesprächstherapie an. Der Arzt wollte mich krankschreiben. Aber ich weigerte mich. Ich musste erst ganz ausbrennen, um zu erkennen, dass es so nicht weiterging mit mir. Im Herbst 2010 war es schließlich so weit, drei Jahre nachdem ich diese Führungsposition übernommen hatte: Ich hatte einen Burnout. [...]

Berichte über statistische Erhebungen, Umfragen, Studien, Forschungsergebnisse und (falsche) Überzeugungen zum Thema „Burnout“ in der Bevölkerung stellen die zweite größere Gruppe von Texten dar, in denen sich die Autorinnen und Autoren mit dem Gegenstand bzw. Begriff >BURNOUT` auseinandersetzen.

Weiteren gibt es Berichte von ,Betroffenen“ im Interview: So zum Beispiel von Miriam Meckel im ZEITmagazin Nr. 39/2014, 6.10.2014 (= Koelbl 2014, im QV unter 8.1.6). Miriam Meckel hat in einem Buch „Brief an mein Leben“ (2010) das „persönliche Protokoll einer Burnout-Krise“ veröffentlicht (= Meckel ${ }^{4}$ 2013, im QV unter 8.1.7). 
„Burnout“ wird in diesen Texten nicht an sich thematisiert, sondern jeweils im Zusammenhang mit Nachrichten, wie z. B. mit ansteigenden Fehlzeiten (siehe Beispiel 8) oder mit prominenten Personen (aus dem Leistungssport) ${ }^{556}$, die mit >BURNOUT< in Verbindung gebracht werden (siehe Beispiel 9). In den folgenden beiden Meldungen wird >BURNOUT< in knapper Form definiert: Im achten Beispiel, indem Verhaltensweisen und Eigenschaften aufgezählt werden, die „immer mehr Arbeitnehmer“ zeigen und die mit der „Diagnose: Burnout“ anschließend verknüpft werden (Bohsem 2012a: 17, SZ, im QV unter 8.1.6); und im neunten Beispiel, indem angegeben wird, was unter dem „sogenannten Burn-out-Syndrom“, unter dem Sven Hannwald leide, zu verstehen sei und was die „Betroffenen“ im Gegensatz zum vorherigen gesunden Zustand „verloren“ hätten.

8) Süddeutsche Zeitung: 17.08.2012 (= Bohsem 2012a: 17) [Unterstreichung T.S., sonstige Hervorhebungen im Original, im QV unter 8.1.6]

\section{Fleißig, flexibel - und krank}

Auch im vorigen Jahr gab es mehr Fälle von Burn-out in den Betrieben. Das treibt die Kosten der Krankenkassen in die Höhe. Experten machen die Anforderungen der Berufswelt verantwortlich.

\section{VON GUIDO BOHSEM [fett gedruckt im Original]}

Berlin - Das ist für immer mehr Arbeitnehmer das Leben: Sie sind rund um die Uhr erreich-

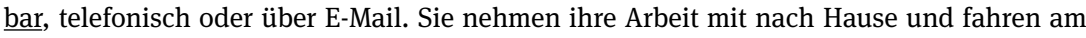
Wochenende für ein paar Stunden ins Büro. Sie arbeiten rund um die Uhr, um ein Projekt fertigzustellen - und wenn der Chef am folgenden Tag das nächste bestellt, sind sie wieder voll dabei. Flexibilität ist das große Stichwort, und für den Beschäftigten heißt das, seine Freizeit flexibel für die Arbeit zu verplanen.

Diagnose: Burn-out. Immer mehr Menschen können diese Art von Flexibilität nicht mehr ertragen. Sie scheitern an permanenter Überforderung und Dauerbelastung. Die Arbeit

556 Weitere Beispiele aus dem Korpus, in denen über Burnout im Spitzensport berichtet wird: über Burnout im Tennis „Selbst Steffi Graf, die eherne, ist ausgebrannt“ (SPIEGEL 49/1989: 222f., im QV unter 8.1.6); In der SZ wird Dagmar Ruhwandl, Fachärztin für Psychiatrie und Psychotherapie, zitiert: Bei „Spitzenkräften aus der Wirtschaft als auch bei Sportlern wie [Sebastian] Deisler“ träten ähnliche Burnout-Symptome auf. „,Erstens beinhaltet Burn-Out die Symptome einer Depression, wie etwa Überforderung, Erschöpfung oder Leistungsabfall‘, sagt Ruhwandl. ,Zweitens handelt es sich sowohl bei Spitzenkräften aus der Wirtschaft als auch bei Sportlern wie Deisler um so genannte High Potentials, also ehrgeizige und leistungsstarke Persönlichkeiten, häufig noch dazu im Rampenlicht der Öffentlichkeit stehend““ (= Steinkohl/Hölper 2003: 35 in der SZ, im QV unter 8.1.6); SPIEGEL ONLINE berichtet am 26.10.2011: „Michael Sternkopf nimmt sich eine Pause vom Fußball. Der ehemalige Bayern-Profi leidet am Burnout-Syndrom.“ (= Aha/ sid 2011b/SPIEGEL ONLINE, im QV unter 8.1.6) sowie FAZ.NET, 02.03.2012 mit dem Titel „Fußballerin mit Burnout“ (= Wenzel 2012/FAZ.NET, im QV unter 8.1.6). 
macht sie krank. Das jedenfalls legt der neue Fehlzeiten-Report des Wissenschaftlichen Instituts der Allgemeinen Ortskrankenkassen AOK nahe. Rund 130000 Menschen waren im vergangenen Jahr insgesamt 2,7 Millionen Tage wegen Burn-outs krankgeschrieben. Die Fehlzeiten sind somit elfmal so hoch wie noch im Jahr 2004. Von 1994 bis 2011 hat sich die Anzahl der psychischen Erkrankungen verdoppelt. Alleine die AOK-Einheiten gaben im vergangenen Jahr 9,5 Milliarden Euro für ihre Behandlung aus.

9) FAZ, 03.05.2004 (= dpa 2004a: 38) [Unterstreichung T.S., sonstige Hervorhebungen im Original, im QV unter 8.1.6]

Mit Burn-out-Syndrom in der Klinik [hier Unterstreichung im Original]

\section{„Dem Menschen Hannawald helfen“}

FRANKFURT (dpa). Der Zusammenbruch von Skispringer Sven Hannawald hat beim Deutschen Skiverband (DSV) große Sorge ausgelöst. Der am sogenannten Burn-out-Syndrom leidende 29 Jahre alte Mannschafts-Olympiasieger aus Hinterzarten wird in einer Rehaklinik stationär behandelt und damit für längere Zeit ausfallen. Mit Burn-out-Syndrom wird ein „Ausgebranntsein“ beschrieben. Den Betroffenen geht die psychische und physische Leistungsfähigkeit sowie die Kraft zum Regenerieren verloren, ihre Energiereserven sind erschöpft. „Wir können nur hoffen, daß Sven wieder ganz gesund wird. Wir werden ihn auf gar keinen Fall unter Druck setzen“, sagte Bundestrainer Wolfgang Steiert. „Jetzt ist das Wichtigste, zuerst dem Menschen, dann dem Sportler Sven Hannawald zu helfen“, erklärte Sportdirektor Thomas Pfüller. Bereits während der vergangenen Saison hatte Hannawald drei Wettkampfpausen eingelegt und sich im Februar vorzeitig von den Schanzen verabschiedet. Der begnadete Skiflieger, der 2000 und 2002 jeweils Weltmeister wurde, klagte über fehlende Frische und Explosivität.

Nach den Fußballprofis Sebastian Deisler (Bayern München) und Jan Simak (Hannover 96) ist Hannawald bereits der dritte prominente Sportler, der den auf ihm lastenden Druck nicht mehr verkraftete. $[. .$.

Blickt man aus diachroner Sicht auf die Medientexte, so erscheinen ca. ab dem Jahr 2010 Zeitungsmeldungen dieser Art vermehrt mit einem kritischen Unterton gegenüber der Verwendung des Begriffs > Burnout ` als Diagnosekonzept (vgl. oben auch Beispiel 5 in der Zeitschrift Gehirn\&Geist, Falkai 2016). ${ }^{557}$ Die Kritik wird meist begleitet von der Feststellung, dass es eine ,inflationäre Verwendung des

557 In den untersuchten Texten von DIE ZEIT und ZEIT ONLINE zwischen 1984 und 2015, in denen das Stichwort bzw. Wortbildungen mit dem Stichwort Burnout vorkommen (die Belege der ZEIT wurden über das Deutsche Referenzkorpus des IDS mit der Suchsyntax ${ }^{*}$ Burn-out ${ }^{*}$ oder ${ }^{*}$ Burnout ${ }^{*}$ recherchiert), erscheint eine explizite Kritik an der Verwendung des Ausdrucks Burnout statt Depression das erste Mal im Artikel „Die versteckte Krankheit“ (= Bahnsen 2009, DIE ZEIT (Online-Ausgabe), 19.11.2009, im QV unter 8.1.6). Im Jahr 2007 findet man allerdings schon einen Beitrag über Angststörungen in der ZEIT, der darauf aufmerksam macht, dass , lieber über Burn-outs oder Urlaubsreife“ berichtet werde und dass Angststörungen (auch dadurch) häufig nicht erkannt würden (= Schüle 2007, DIE ZEIT (Online-Ausgabe), 19.04.2007, im QV unter 8.1.6). 
Begriffs Burnout“ (vgl. Kaulen 2010: S. N1 in der FAZ) gebe, dass das Phänomen nicht ausreichend erforscht sei und dass es keine „einheitliche, international gültige wissenschaftliche Definition“ gebe, mit der man es von anderen Krankheiten wie der „Depression“ abgrenzen könne (ebd.). Es wird dennoch häufig die Definition bzw. die Symptomtrias nach Maslach/Jackson als diejenige angeführt, die die Mehrheitsmeinung der „Ärzte“ wiedergebe: ${ }^{558}$

10) FAZ, 06.10.2010, Nr. 232, S. N1 (= Kaulen 2010) (Unterstreichung T.S., sonstige Hervorhebungen im Original, im QV unter 8.1.6)

\section{„Burnout“: Hat die Diagnose Methode, oder ist es Mode?}

Die inflationäre Verwendung des Begriffs Burnout suggeriert, dass eine ganze Gesellschaft psychisch erschöpft ist. Das legen auch die Schätzungen der Betriebskrankenkassen nahe, die von neun Millionen Betroffenen ausgehen. Bezieht man diese Zahl auf die Gruppe der Erwerbstätigen, muss jeder vierte Arbeitnehmer ausgebrannt sein. Burnout ist demnach eine Epidemie - aber eine, für die es keine einheitliche, international gültige wissenschaftliche Definition gibt.

In den untersuchten Texten von FAZ und FAZ.NET zwischen 1992 und Januar 2017 (die Belege von FAZ und FAZ.NET wurden über das Online-Archiv www.faz-biblionet.de mit der Suchsyntax * burnout ODER * burn-out ODER burnout ${ }^{*}$ ODER burn-out* im Titelbereich zuletzt am 18.2.2017 recherchiert) wird zwar schon in einem Artikel aus dem Jahr 2004 ein Experte mit den Worten zitiert, dass Burnout „eine arbeitsbezogene Form depressiver Störungen“ sei (= Kals 2004, FAZ: 55, im QV unter 8.1.6) und in einem Beitrag aus dem Jahr 2004 wird Burnout schon als „Zusatzdiagnose“ (= Obermeier 2004, FAZ.NET, 20.08.2004, im QV unter 8.1.6) bezeichnet, aber es wird keine gleichzeitige Kritik an der Verwendung des Ausdrucks Burnout oder seiner Definition geübt. Diese Kritik findet man zuerst in einem Artikel vom 26.05.2010, in dem berichtet wird, dass die Forscher sich noch uneins seien, „ob es sich um eine Modediagnose handelt“ (= Stock 2010, FAZ, im QV unter 8.1.6) und in einem Artikel vom 06.10.2010 in der FAZ mit dem Titel „,Burnout': Hat die Diagnose Methode, oder ist es Mode?“ (= Kaulen 2010, FAZ, im QV unter 8.1.6). In der „Apotheken Umschau“ wird in der Ausgabe 10/2011-A: 34 darauf hingewiesen, dass das „Syndrom [...] nicht als offizielle Diagnose“ gelte und in der Ausgabe 6/2012-B auf Seite 10 (im QV unter 8.1.7) wird die positivere Konnotation von Burnout gegenüber der Depression betont. In der Zeitschrift DER SPIEGEL wird der Begriff Burnout als Diagnosevokabel das erste Mal im Artikel „Volk der Erschöpften“ explizit mit Bezug auf Fachleute aus der Psychiatrie als ein „,beschönigendes Synonym für die stigmatisierte Depression“ kritisiert (= Dettmer/Shafy/Tietz 2011: 116, DER SPIEGEL, im QV unter 8.1.6).

558 In folgenden Beiträgen wird zum Beispiel auf die Symptomtrias nach Maslach/Jackson (1981) verwiesen, Maslach und Jackson werden jedoch meistens nicht explizit genannt: SZ 03.03.2001, S. V1-1 (= Brunner 2001, im QV unter 8.1.6); FAZ 03.04.2004, Nr. 80: 55 (= Kals 2004, im QV unter 8.1.6); DIE ZEIT 27.07.2006 Nr. 30 (Online-Ausgabe) in einem Interview mit dem Medizinsoziologen Johannes Siegrist (= Kuhrt 2006, im QV unter 8.1.6); Süddeutsche Zeitung, 29.11.2008: S. V2/9 mit explizitem Bezug auf Maslach und Jackson (= SZ 2008a, im QV unter 8.1.6); FAZ 26.05.2010, Nr. 119, S. B4 mit explizitem Bezug auf Maslach (= Stock 2010); Karriere-SPIEGEL 31.10.2013 (= Burisch 2013); FAZ.NET, 28.01.2016 (= Dunsch 2016). 
Jeder hat ein intuitives Verständnis davon, aber weder die internationale Klassifikation der Krankheiten noch das „Diagnostische und Statistische Handbuch psychischer Störungen“ listen Burnout als eigenständige Erkrankung. Es fehlt auch ein allgemein anerkanntes Instrument, um die Störung von anderen Krankheiten, etwa der Depression und den Angsterkrankungen, abzugrenzen. Trotzdem wird sie laufend diagnostiziert.

$[\ldots]$

Der Begriff Burnout leitet sich aus der Arbeitswelt her. Die meisten Ärzte verstehen darunter einen Zustand aus arbeitsbedingter Erschöpfung, Selbstentfremdung, Zynismus und verminderter Leistungsfähigkeit [14]. Im praktischen Alltag zählen noch Dutzende weiterer Symptome dazu, etwa verstärkte Anspannung, Schlafstörungen, Unruhe, Konzentrationsschwäche, mangelnde Motivation und reduzierte Arbeitsleistung. Für Korczak sind diese Zuschreibungen ein beliebiger Katalog negativer Befindlichkeitsstörungen, die keine Rückschlüsse auf den Beginn der Symptome zulassen. In der wissenschaftlichen Literatur werde die Störung deshalb auch als „randunscharfe Menge“ bezeichnet, so der Medizinsoziologe im Gespräch. [...].

Seit Beginn der 2010er Jahre gibt es zudem vermehrt Texte, die man den Textsorten einordnender Kommentar oder auch berichtartiger „Faktencheck“ zuordnen könnte. Im Folgenden sei aus einem Kommentar aus DER ZEIT als elftem Textbeispiel zitiert (Albrecht 2011a, im QV unter 8.1.6). ${ }^{559}$ Dieser Artikel zeigt, dass durch die Kritik an der Definition, die gleichzeitig auch verdeutlicht, welche Ansprüche an eine Definition gestellt werden (vgl. Kap. 4.2.2), neue Definitionsbemühungen ausgelöst werden. Aus diesem Grund wird dieser Kommentar im Folgenden etwas ausführlicher analysiert mit Bezug auf konkrete Textabschnitte (siehe unten Beispiel 11): Im Vorspann wird eine Was-ist-Frage gestellt, gefolgt von der Feststellung, dass Fachleute um eine „klare Definition“ ringen würden (zur Verbindung von Was-ist-Fragen und Wesensdefinitionen siehe Kap. 6.2.2.2, S. 224-225). Im zweiten Absatz des Haupttextes wird die Weite des Begriffs kritisiert, wenn gesagt wird, die Vokabel stehe „mittlerweile für fast alle Arten psychischer Beschwerden, die in Verbindung mit hoher Arbeitsbelastung “ aufträten. Diese Kritik wird im dritten Absatz durch Bezug auf einen Artikel im „Deutschen Ärzteblatt“ zur „Modediagnose Burnout“ noch ausgeweitet (vgl. dazu auch Beleg 9 in Kap. 6.2.2.2). Danach wird die Begriffsgeschichte im vierten Absatz rekapi-

559 Ähnliche Beispiele aus dem Korpus: Der mit „Faktencheck“ übertitelte Beitrag „Elf Mythen über Burnout“ in G\&G 2016: 13-18 (= Melchers/Plieger 2016, im QV unter 8.1.7); oder in einem Interview in PH, in dem Isabella Heuser, Leiterin der Klinik für Psychiatrie und Psychotherapie der Charité, zu Laienkonzepten über Burnout interviewt wird (Psychologie Heute Dez. 2011: 30-33, (= Tenzer 2011b) im QV unter 8.1.7); oder ein medienkritischer Artikel über die Magazinberichte zu BuRnout, der auch einzelne Aspekte des Phänomens Burnout kritisch beleuchtet in der FAZ vom 10.02.2012, Nr. 35: 35 (= Geyer 2012, im QV unter 8.1.6). 
tuliert. Dabei wird das Hyperonym „psychische Erkrankung“ mit Bezug auf H. Freudenbergers ,ursprüngliche‘ Festsetzung des Begriffs `Burnout` zurückgewiesen und durch „Folge einer Arbeitsüberlastung in Gesundheitsberufen“ ersetzt (vgl. dazu auch Falkai 2016: 25 in G\&G, Beleg 5 oben). Im fünften Absatz wird die fehlende Einheitlichkeit in der Forschung zur Definition von >Burnout k konstatiert. Die auf Maslach/Jackson (1981) zurückgehende Symptomtrias, die in den Kapiteln 6.2.2.1 und 6.2.2.2 schon ausführlich beschrieben wurde, wird als einzige „dürre Erkenntnis“ ausgewiesen und in modifizierter Form reformuliert (,irgendwie unter emotionaler Erschöpfung, dem Gefühl der Leblosigkeit und verminderter Leistungsfähigkeit leiden“). Im sechsten Abschnitt wird mit Bezug auf Expertenmeinungen aus der Medizin und Psychotherapie gefragt, ob Burnout nur eine andere, jedoch weniger abschreckende Bezeichnung für Depression sei (vgl. dazu auch Falkai 2016 in G\&G, Beleg 5 oben). Im siebten bis zehnten Abschnitt wird betont, dass die mangelnde Trennschärfe zwischen `Burnout gefährlich sein könne. ${ }^{560}$ In den letzten beiden hier zitierten Absätzen (11 und 12) werden dann beobachtete Unterscheidungskriterien von >Burnout und >Depression genannt. Nach der Meinung eines dazu zitierten Experten, müsste es weitere fachliche typologisierende Anstrengungen für eine „differenzierte Betrachtung der Erschöpfungszustände“ geben, die dann als Definitionen in Leitlinien übernommen werden könnten.

11) DIE ZEIT, Nr. 49/2011, 01.12.2011 (= Albrecht 2011a) (Gefettete Überschriften wie im Original, Unterstreichung und Nummerierung der Absätze T.S., im QV unter 8.1.6)

\section{Erschöpfungsdepression: Burn-out}

Was ist das eigentlich? Mediziner und Psychologen ringen um eine klare Definition. Erst wenn sie gefunden ist, können Depressive die angemessene Therapie erhalten und Überforderte Unterstützung im Alltag erhalten.

Von Harro Albrecht

1. Dezember 2011, 7:00 Uhr DIE ZEIT Nr. 49/2011 38 Kommentare

[1] Das Wort für extreme Erschöpfung ist inzwischen auch Pop: »Ich hab Burn-out. I feel so outgeburnt. I have my Burn-outing", sang der Comedian Johann König auf dem Satiregipfel der ARD . »Das ist wirklich toll. Ich lass mich bedienen und krümel alles voll.»

[2] Man könnte empört einwenden, hier mache sich jemand lustig auf Kosten leidender Mitbürger. Doch König hat nur satirisch verarbeitet, was viele spüren: Nach der Burn-out-Erkenntniswelle folgt nun eine Art Burn-out-Übertreibung. Die Vokabel steht mittlerweile für

560 Die Gefahr, die „für psychisch Schwerkranke[n]“ bestehe, dadurch dass Therapieplätze an „weniger belastete[r] Menschen wie Burnout-Patienten“ vergeben würden, wird auch in SPIEGEL ONLINE am 26.06.2013 aufgegriffen (= Hauschild 2013, im QV unter 8.1.6). 
fast alle Arten psychischer Beschwerden, die in Verbindung mit hoher Arbeitsbelastung auftreten. Das fordert nicht nur Comedians zum Spott heraus, es reizt auch Ärzte und Psychotherapeuten zum Widerspruch.

[3] Modediagnose Burn-out, titelt Mitte November das Deutsche Ärzteblatt. Der inflationäre Gebrauch des schwammigen Begriffs Burn-out sei »Verwirrung stiftend, irreführend und längerfristig stigmaverstärkend«, klagt Ulrich Hegerl, Vorsitzender der Stiftung Deutsche Depressionshilfe. Und der Münsteraner Psychotherapeut Markus Pawelzik befürchtet, dass das Wort "als sozial akzeptierte Entschuldigung herhalten muss für ein schlechtes Einteilen der eigenen Kräfte«. Der Zweifel am Massenphänomen Burn-out ist der vorläufige Höhepunkt einer sehr wechselhaften 40-jährigen Wortkarriere.

[4] Mit dem Begriff Burn-out hatte Herbert Freudenberger 1974 die verbreitete chronische Erschöpfung von Menschen in helfenden Berufen, von Krankenschwestern oder Altenpflegern charakterisiert. Der amerikanische Psychoanalytiker verstand den Zustand explizit nicht als psychische Erkrankung, sondern als Folge einer Arbeitsüberlastung in Gesundheitsberufen. Bald eroberte der Begriff Deutschland und fand Anwendung in immer neuen Arbeitsfeldern.

[5] Dann verbreitete sich der Terminus von der Arbeitswelt hinüber in die Medizin. [...]. Bis heute ist Burn-out nicht einheitlich definiert. Es existieren lediglich ein Dutzend unspezifischer Tests und die dürre Erkenntnis, dass die Betroffenen irgendwie unter emotionaler Erschöpfung, dem Gefühl der Leblosigkeit und verminderter Leistungsfähigkeit leiden.

[6] Lange Zeit hat diese Definitionslücke niemanden gestört. Viele Ärzte und Psychotherapeuten waren zwar der festen Überzeugung, Burn-out sei nur ein neuer Name für Depressionen, benutzten das Wort aber dennoch gern. Es schreckte die Patienten weniger ab, weil jeder sich in den sprechenden Begriff hineindenken konnte. Wer fühle sich denn nicht mal am Morgen erschöpft und würde gerne im Bett bleiben, sagt der Leipziger Psychiater Ulrich Hegerl, das kenne jeder Mensch, und darin liege auch der Erfolg dieses Modeworts. Medien griffen das Thema begierig auf, reihenweise spezialisierten sich Psychosomatikkliniken auf die Behandlung der Ausgebrannten, die Bekenntnis- und Ratgeberliteratur füllt Regalmeter.

\section{"Eine Verharmlosung der Depression"}

[7] Doch jetzt macht sich Unbehagen unter den Experten breit. Das Wort, das zunächst den Dialog über psychische Erkrankungen erleichtert hat, wird zum Problem. Der Begriff Burnout werde völlig unterschiedslos sowohl für lebensbedrohliche Depressionen als auch für einfache Erschöpfungszustände nach anstrengenden Arbeitsphasen benutzt, sagt Ulrich Hegerl. Das sei falsch.

[8] [...] [Zusammenfassung folgender Abschnitt, T.S.: Überforderung und harte Arbeitsbedingungen als Ursache für Depression werden als selten bezeichnet gegenüber genetischer Veranlagung in Verbindung mit weiteren Faktoren wie Verlusterlebnissen.]

[9] Aber der Mythos von der »stressigen« Arbeit als Depressionspromotor ist in der Welt - und das hat Nebenwirkungen. "Eine Vermengung von Stress, Burn-out und Depression führt zu einer Verharmlosung der Depression«, sagt Ulrich Hegerl. Beiläufig raten Kollegen den Betroffenen, sie sollten einfach mal Urlaub machen. 
[10] Wenn man eine Depression habe, sagt Hegerl, dann sei davon dringend abzuraten. Urlaub helfe depressiven Menschen genauso wenig wie viel Schlaf. Angebracht seien Psychotherapie oder Antidepressiva. Diese Hilfe gebe es oft nicht, sagt Hegerl, wenn man das unter Burn-out laufen lasse. »Der beste Weg zu einem optimalen Umgang mit der Erkrankung Depression ist es, eine Depression auch Depression zu nennen«, sagt der Psychiater. Denjenigen, die nicht das klar definierte Bild einer Depression erfüllen, rät er, ihre Einstellung zur Arbeit zu verändern: Gelegentliche Überforderungen seien ein ganz normaler Teil des Lebens.

[11] Aber gibt es wirklich nur die beiden großen Gruppen? Auf der einen Seite die Depressiven und auf der anderen klagende Arbeitnehmer? Hegerls Stellvertreter in der Deutschen Depressionshilfe, Nico Niedermeier, unterscheidet weniger radikal. »Ich glaube schon, dass der Begriff Burn-out für manche Menschen nicht so falsch ist«, sagt der Münchner Verhaltenstherapeut. Einer seiner Klienten zum Beispiel zeige, solange er arbeite, alle Anzeichen einer schweren Depression. »Der schläft nicht, hat schwere Suizidgedanken, kann sich auf kaum etwas konzentrieren und hat seine Beziehungen vollkommen vernachlässigt«, sagt Niedermeier. Sobald dieser Klient aber in den Urlaub fahre, sei er binnen zwei Wochen wie ausgewechselt. Das sei vollkommen untypisch, ergänzt Hegerl. Die Depression nehme normalerweise im Urlaub eher zu.

[12] Nico Niedermeier empfiehlt eine differenzierte Betrachtung der Erschöpfungszustände. Ihm schwebt eine Typologie mit vier verschiedenen Charakteren vor. Erstens die Depressiven. Zweitens Menschen mit einem echten Burn-out. Drittens Arbeitnehmer, die eine Persönlichkeitsstörung haben und dadurch bei hoher Arbeitsbelastung durchs Raster fallen. Schließlich Menschen, die durch zu hohes Anspruchsdenken auffallen, sogenannte Hyperindividuierte. Irgendjemand, fordert der Verhaltenstherapeut, müsse Unterscheidungskriterien definieren und entsprechende Leitlinien entwickeln.

Als zwölftes Beispiel sei abschließend noch ein Interview der Zeitschrift DER SPIEGEL aus dem Jahr 2011 zitiert, in dem es um „den Rücktritt des am BurnoutSyndrom erkrankten Trainers Ralf Rangnick“ geht. ${ }^{561}$ Interviewt wird Thorsten Rarreck, der als Mannschaftsarzt des Fußball-Bundesligisten Schalke 04 in die Rücktrittsüberlegungen Rangnicks 2011 mit eingebunden war. Rarreck grenzt im Gespräch den Zustand, in dem man „einfach nur platt“ ist vom „Burnout“ ab. Aber auch er verwendet zur Benennung der Diagnose nicht Burnout, sondern

561 Weitere Beispiele aus dem Korpus zur Textsorte Interview: 1.) Ein Interview in der ZEIT (Online-Ausgabe), vom 27.07.2006: 50 mit dem Titel „Der öffentliche Patient“. Interviewt wird der Medizinsoziologe Johannes Siegrist. Ausgangspunkt ist der Rücktritt von Jürgen Klinsmann von seinem Amt als Fußballbundestrainer mit der Begründung, dass er „ausgebrannt“ sei (= Kuhrt 2006, im QV unter 8.1.6); 2.) Ein Interview in DER ZEIT vom 21.02.2008 mit dem Titel „Ausgebrannte Helden“. Interviewt wird ein Sportpsychologe. Es geht um Fußballtrainer, die ausbrennen. (= Willmann 2008, im QV unter 8.1.6); 3.) Ein Interview in der Apotheken Umschau mit Matthias Burisch aus dem Jahr 2011 mit dem Titel „Mit leerem Rucksack nach Hause gehen“ (= Rotherbl 2011, in Apotheken Umschau 10/2011 A: 34-37, im QV unter 8.1.7); Ein Interview mit dem Betriebsarzt Wolfgang Panter im Heft Focus Gesundheit (= Wagner Dez./Jan. 2012/2013: 49, im QV unter 8.1.7). 
vegetatives Erschöpfungssyndrom, wodurch die somatische Seite stärker betont wird. Er beruft sich auf zwölf Burnout-Stadien, welche den „Stadien innerhalb des Burn-out-Zyklus“ bei Freudenberger/North ('2002: 38f.; 121ff.) ähneln, und nennt Symptome (Appetitlosigkeit, Schlafstörungen), die wie in Beispiel 5 mit Bezug auf die ICD-10 ausgeführt wurde, auch auf eine depressive Episode hinweisen könnten. Im weiteren Verlauf des Gesprächs werden noch typische Persönlichkeitseigenschaften besprochen, durch die Menschen ,in ein Erschöpfungssyndrom hineinrennen“ könnten.

12) DER SPIEGEL 39/2011: 118 (Unterstreichung T.S., sonstige Hervorhebungen im Original, = Der SPIEGEL 2011d, im QV unter 8.1.6)

FUSSBALL: „Hart am Wind“ [Unterstreichung im Original]

DER SPIEGEL - Interview Szene Sport - 26.09.2011

Thorsten Rarreck, 47, Mannschaftsarzt des Fußball-Bundesligisten Schalke 04, über den Rücktritt des am Burnout-Syndrom erkrankten Trainers Ralf Rangnick

SPIEGEL: Sie haben bei Ralf Rangnick ein vegetatives Erschöpfungssyndrom diagnostiziert. Mussten Sie ihn zu einer Auszeit überreden?

Rarreck: Am Ende war er erleichtert, dass wir Ärzte ihn in dem bestätigten, was er selbst am Dienstag mit seiner Familie beschlossen hatte. Wir hatten ein Expertengremium gebildet, neben mir waren zwei Fachleute im persönlichen Kontakt mit ihm, drei weitere telefonisch eingebunden. Zwei Wochen zuvor jedoch hatte Überzeugungsarbeit dazugehört, dass Ralf Rangnick ein solches Syndrom bei sich überhaupt akzeptierte. Ich kenne ihn schon lange, und mir fiel auf, dass er nicht einfach nur platt war.

SPIEGEL: Sondern?

Rarreck: Beim Burnout unterscheidet man zwölf Stadien. Bei Stadium eins bis drei landen viele ehrgeizige Menschen, die einen Zwang sehen, sich zu beweisen, und eigene Bedürfnisse verdrängen. Ralf Rangnick war zuletzt bei acht bis neun, es musste die Reißleine gezogen werden.

\section{SPIEGEL: Wie äußerte sich das?}

Rarreck: Etwa durch Appetitlosigkeit, eine deutlich verminderte Nahrungsaufnahme sowie erhebliche Schlafstörungen. Stellen Sie sich einen angespannten Körper in einem unausgeruhten Zustand vor, nicht ausreichend mit Nährstoffen versorgt, quasi unterernährt. Das kann gefährlich werden. Vorher hatte er, im Stadium sechs ungefähr, die Probleme über mehrere Wochen verdrängt. Weil er sehr diszipliniert ist, ist es nicht aufgefallen. Er ist ja ein Mensch, der sehr viel Power hat, gerade deswegen ist er gefährdet.

[...]

SPIEGEL: Rangnick ist dafür bekannt, dass er von Mitarbeitern viel fordert, viel Energie verbraucht für scheinbare Banalitäten, kaum abschaltet. Wurde diese Stärke, die Leidenschaft und das Engagement im Job, nun zur Schwäche? 
Rarreck: $\underline{\text { So etwas ist typisch bei Menschen, die in ein Erschöpfungssyndrom hineinrennen, }}$ wenn sie es nicht gleichzeitig schaffen, die Gehirnfunktion herunterzufahren. Die Stressresistenz muss gecoacht werden. Eine gewisse Gelassenheit entwickeln, delegieren, abschalten können - das ist jetzt auch ein Hauptteil der Arbeit, die für Ralf Rangnick anliegt.

Im Folgenden werden die Beispiele 1-12 miteinander verglichen, um das Spektrum der sprachlichen Mittel des Definierens in fachexternen Medien- und Vermittlungstexten aus punktueller Perspektive aufzuzeigen. Als weitere Vergleichsfolie dienen die in den Kapiteln 6.2.2.1 und 6.2.2.2 herausgearbeiteten sprachlichen Mittel, durch die in den Fachlexika, Hand- und Lehrbüchern sowie Fachtexten und Monografien definitorische Aufgaben erfüllt werden. Denn durch diese Gegenüberstellung können Sprachgebrauchsformen mit definitorischer Funktion ${ }^{562}$, die entweder für den fachlichen oder fachexternen Diskursstrang spezifisch sind, direkt identifiziert werden.

Blickt man zunächst auf die Textabschnitts-, Satz- und Mehrwortebene, so fällt auf, dass sieben von zwölf Beispielen mit narrativen Vertextungsmustern arbeiten, sechs davon beginnen den Text nach dem Vorspann mit einer solchen Szene und steigen damit narrativ ins Thema ein.

Eine der ausführlichsten narrativ entfalteten Szenen findet man in Beispiel 5: Es wird dort die berufliche Geschichte einer Einzelperson erzählt, wie das Pseudonym „Bernd Hinze“ unterstreicht. Die Anonymisierung des Klarnamens macht darauf aufmerksam, dass diese Geschichte für die Öffentlichkeit eigentlich zu privat ist, jedoch dennoch erzählt wird, weil sie exemplarischen Charakter besitzt. Dasselbe Verfahren findet man auch in Beispiel 4 oben:

Eine typische Burn-out-Patientin, die Weber während eines Forschungsprojekts kennengelernt hat, ist Eva Berger, die anders heißt. Ihren Schulalltag empfindet die Gymnasiallehrerin inzwischen [...]. (= Kals 2004/FAZ: 55, Unterstreichung, T.S.)

Die anonymisierten Eigennamen werden mit Gattungsbezeichnungen („Werbetexter“ oder „Gymnasiallehrerin“) und weiteren charakterisierenden Merkmalen und Details aus dem Berufsalltag dieser Personen verknüpft („der 38-Jährige“, „Freude am Job“, „immer mehr Projekte“) und dadurch zu Figuren, die einen typischen Vorgang durchleben (vgl. dazu auch Beispiel 6 oben). Auch in anderen Texten findet man häufig das Muster ein(e) oder der/die typische(r) Burnout-Patient(in). ${ }^{563}$

562 Die Teilfunktionen einer diskursiven definitorischen Praxis und dabei zum Beispiel der Anspruch auf Trennschärfe und Deutlichkeit wurden in Kapitel 4.2.2 herausgearbeitet und sind in die Arbeitsdefinition einer diskursiven Praxis des Definierens eingegangen (siehe Kapitel 4.3.4). 563 Weitere Beispiele aus dem fachexternen Korpus sind z.B.: „Der typische Burnout-Patient neige zur Selbstüberschätzung [...]“ (Frömel/Recht 2011 in GEO WISSEN, Nr. 48/2011: 53); „Ein typi- 
In den anderen Beispielen mit narrativen Szenen (siehe Bsp. 1, 2, 3) erscheinen die Personen nur als Vertreter bestimmter Berufs- bzw. Personenklassen (,der Berliner Professor“, „die junge Lehrerin“, „eine Münchner Psychologin“, „der einstige Überflieger“). In Bsp. 9 hingegen wird über den „Zusammenbruch von Skispringer Sven Hannawald“ berichtet. Doch auch diesem prominenten Einzelfall aus dem Bereich des Profisports wird schon eine gewisse Musterhaftigkeit zugesprochen, wodurch das Phänomen BuRNouT als ein intersubjektiver Zustand ausgewiesen wird:

Nach den Fußballprofis Sebastian Deisler (Bayern München) und Jan Simak (Hannover 96) ist Hannawald bereits der dritte prominente Sportler, der den auf ihm lastenden Druck nicht mehr verkraftete.

(FAZ, 03.05.2004: 38, Unterstreichung T.S., siehe oben Bsp. 9)

Die Übertragung von prominenten Beispielen auf eine größere Menge von Personen, findet man auch in folgendem Beleg der Apotheken Umschau:

„Irgendwann kam ich an den Punkt, an dem ich all diese Anforderungen nicht mehr bewältigen konnte“, schilderte Sven Hannawald, der wohl bekannteste „Ausgebrannte“, seine Situation. So wie es dem erfolgreichen und beliebten Skispringer erging, fühlen sich Hunderttausende Deutsche: am Ende, völlig ausgelaugt, geistig, körperlich und emotional so erschöpft, dass sie ihren Alltagsanforderungen kaum noch nachkommen können.

(Apotheken Umschau 5/2005 B: 40, Unterstreichung T.S.)

Am Ende dieses Kapitels wird auf die sprachlichen Mittel des Geltungsanspruchs noch näher eingegangen.

Beispiel 5 zeigt einige Merkmale narrativer Themenentfaltung besonders deutlich (vgl. Kap. 6.2.2.3): Es wird aus der Rückschau eine Ereigniskette im epischen Präteritum präsentiert und die „Ereignisse verbinden sich in ihrer logischen, kausalen, zeitlichen Aufeinanderfolge“ (Heinemann/Viehweger 1991: 237). Der Endzustand wird zwar zu Beginn des Beitrags vorweggenommen (,Das Aus für Bernd Hinze` kam schleichend“), aber die Entwicklung hin zum kritischen Höhepunkt der Geschichte wird akkumulierend aufgebaut (,immer mehr Projekte wanderten auf seinen Schreibtisch“, „Hinze opferte mehr und mehr Freizeit“, „fühlte sich schon morgens erledigt“, „Irgendwann ging gar nichts mehr“). Dabei kommt es zu einer gegenläufigen Steigerung: Potenziell belastende Dinge (z. B. Kundengespräche, Aufgaben) nehmen zu und entlastende Dinge ab (z. B. Freizeit, Kontakt zu Freunden). Es kommt zu einer emotionalen Umkehr (,Zunehmend wandelte sich die Freude am Job in Abscheu“), die auch auf temporaler

scher Burnout-Patient hat meistens auch das Gefühl ... “ (Stock 2010, FAZ, 25.05.2010: B4); „Frau P. hat eine typische Burnout-Persönlichkeit, [...]“ SPIEGEL WISSEN 22.02.2011 (= Bruhns 2011). 
Ebene („anfangs“ (= positive Gefühle zur Arbeit) und „irgendwann“ (= negative Gefühle zur Arbeit)) ausgestaltet wird. Die erzählte Zeit, also der Zeitraum, über den sich die Transformation hin zum Burnout-Zustand vollzieht, bleibt vage. Am Ende der Szene erfolgt eine „zusammenfassende Einschätzung“564 durch den „Hausarzt“, der die Geschichte mit der Diagnose „Burnout - als Folge chronischer Arbeitsüberlastung“ kommentiert. Durch dieses Resümee von fachlicher Seite wird die zuvor dargebotene individuelle Geschichte mit einem Prozess, der bei „chronischer Arbeitsüberlastung“ zum „Burnout“ führt, gleichgesetzt. Man kann die Geschichte zudem auch als Explanans für die im letzten Absatz genannte Diagnosebezeichnung Burnout (= Explanandum und Definiendum) lesen. Das implizit aufgerufene Gesetz, das diese erzählte Entwicklung erklärt bzw. argumentativ stützt, könnte z. B. folgendermaßen lauten: Berufliche Belastungen, die quantitativ über einen längeren Zeitraum über das 'normale' Maß hinausgehen/sich immer weiter steigern, führen zu körperlichen Beschwerden, Erschöpfung und Leistungsverlust, insbesondere dann, wenn die Faktoren, die zur Regeneration wichtig sind, zugleich weniger werden. Diese Szene zeigt eine individuell-exemplarische, sich zu einer Wende/Krise hin kontrastiv ${ }^{565}$ steigernde $^{2}$ narrativ-explikative Ereignisdefinition mit auf die Ereigniskette folgendem Deutungskommentar. Das obige Beispiel 2 in der Süddeutschen Zeitung (Rheinz 1994: A2) funktioniert ebenfalls nach diesem Muster. ${ }^{566}$

Die Fallbeschreibungen in Beispiel 1 (SPIEGEL Nr. 52/1988: 162) stellen eine verkürzte Form dieser ausführlichen narrativen Kontrastdefinition dar. Denn sie konzentrieren sich ganz auf den temporalen und damit einhergehenden qualitativen sowie quantitativen Kontrast eines Ausgangszustands A, in welchem sich

564 Vgl. die Ausführungen Müllers zum Aufbau narrativer Themenentfaltung (Müller 2007: 79/80).

565 Kontrastiv, da die Anforderungen innerhalb der Erzählung zunehmen, wohingegen die Elemente, die für Pause und Erholung in der Erzählung angeführt werden, abnehmen. Dadurch wird narrativ das Kontrastverhältnis auf dem Weg zum kritischen Höhepunkt der Geschichte stilistisch verstärkt.

566 Weitere Beispiele aus dem Korpus, die >Burnout Wendepunkt steigernde Erzählung plus Deutungskommentar definieren sind z. B.: PH 1/2016: 18-21 (= Schönberger 2016a, im QV unter 8.1.7); G\&G 11/2005: 12-19 (= Kraft 2005, im QV unter 8.1.7). Andere narrative Erzählungen setzen bei der Krise ein und schildern von dort ausgehend den Kontrast, den der Burnout-Zustand im Vergleich zum vorigen besonders engagierten Leben darstellt. Vgl. zu diesem Muster z. B. auch faz.net 08.03.2010 (= Meck 2010, im QV unter 8.1.6); AU 11-2012-A: 22-26 (= Schweiger 2012); in der AU (05/14-A: 11) wird die Geschichte von Herbert Freudenbergers Burnoutentwicklung (= Wolfrum/Steinmüller 2014) und auf SPIEGEL ONLINE die Geschichte einer Krankenschwester (= Zeltner 2011) nacherzählt; alle Belege im QV unter 8.1.6 und 8.1.7. 
die Personen in ihrem Beruf in besonderem Maße eingebracht haben, und eines Zustands B, in welchem sich ihre Gefühle und Einstellung zur Arbeit negativ gewandelt haben, sie bestimmte Leistungen von früher nicht mehr erbringen und die Häufigkeit engagierter Aktionen abgenommen hat. Der Prozess, der diese Transformation herbeigeführt hat, wird nicht explizit erzählt, aber durch Tempuswechsel deutlich gemacht:

Zu Beginn seiner Karriere war der Berliner Professor für seine Studenten jederzeit zu sprechen. [...] Heute beschränkt der inzwischen 53jährige Professor seine Begegnungen mit Studenten [...] auf ein kühles Minimum, [...].

(SPIEGEL Nr. 52/1988: 162, Unterstreichungen T.S.)

Und auch in diesem Text folgt nach zwei weiteren narrativen Vignetten dieser Art ein diagnostischer Deutungskommentar (,Burnout heißt die knappe Diagnose, [...]“). Diese Beispiele könnte man daher als verkürzte Variation des in Beispiel 5 analysierten Definitionstyps ansehen. Ähnlich funktioniert auch Beispiel 6 in der „Apotheken Umschau“ (12/1997-A: 20). Beispiel 3 in der Zeitschrift „Psychologie Heute“ (Smolka 2000: 39f.) stellt ebenfalls diesen Kontrast ins Zentrum seines narrativen Fallberichts. Letzterem folgt nicht ein Deutungskommentar, sondern bevor die narrative Passage entfaltet wird, werden im Absatz zuvor Kernsymptome und Entstehungsbedingungen des Begriffs >Burnout ‘ genannt: „Burnout ist eine körperliche und emotionale Erschöpfung aufgrund dauernder Anspannung [...]“ (ebd.). In ähnlicher Weise ist auch in Beleg 4 das Fallbeispiel in den Gesamttext integriert (siehe oben Kals 2004: 55) und auch die Erzählung des Belegs 7 aus der Ich-Perspektive ist in dieser Weise aufgebaut.

Vergleicht man diese Beispielerzählungen in fachexternen Texten mit Fallberichten im fachinternen Diskursstrang, beispielsweise mit Kissling/Mendel/Förstl (2014: 2387, DMW, im QV unter 8.1.4), Weimer/Kraus (2011: 242ff.) oder dem in Beispiel 5 oben zitierten Fallbericht zur „Depression“, in dem der Autor Peter Falkai einen Fall aus seiner Sicht als Psychiater mit Bezug auf die ICD-10 schildert (Falkai 2016: 24 in G\&G, im QV unter 8.1.7), so kann man folgende Unterschiede erkennen: ${ }^{567}$ - Die erzählenden Passagen zu einem Fall sind in den fachlichen Texten meist in ein klinisches Setting eingebettet. Es wird deutlich, dass diese Geschichten aus einem Anamnese- oder Therapiegespräch stammen, da bestimmte Symptome eindeutig als Patientenrede ausgewiesen werden (Unterstreichungen T.S.): „Die 45-jährige Patientin [...] klagte über [...]““; „Die Patientin leide seit [...]“ (Kissling/ Mendel/Förstl 2014: 2587); „ Herr L. berichtete über kontinuierlich gestiegene

567 Zur Analyse von Fallbeispielen im fachinternen Diskursstrang vergleiche Kap. 6.2.2.2 und darin Beleg 8a-1. 
Versagensängste [...]“ (Weimer/Kraus 2011: 242) ${ }^{568}$; „Klar, sagt Frau M., darüber denke sie pausenlos nach“ (Wilms 2009: 237) ${ }^{569}$; „Um einfache Schreiben zu erfassen, brauche sie ewig“ (Falkai 2014: 24). In den fachexternen Texten berichtet bzw. erzählt ein Erzählmedium von den Gefühlen und veränderten Handlungen der betroffenen Personen, in manchen Texten wird die Erzählung durch Aussagen der ,Betroffenen“ in wörtlicher Rede ergänzt:

Am liebsten wäre Mareike nur noch im Bett geblieben. Schon am frühen Morgen fühlte sie sich müde, erschöpft, ausgebrannt. „Ich hatte keinen Antrieb zu gar nichts, alles war mir zu viel“", sagt die junge Frau. Gerade 30 war sie geworden, als sie spürte, ihr High-PotentialLeben gerät aus den Fugen: „Ich war im Paradies, und in mir fühlte ich die Hölle.“ [...] Schließlich wird Mareike in eine Klinik eingewiesen. Diagnose: „Schwerer Burnout“.

(Meck 2010, FAZ.NET, 08.03.2010, Unterstreichungen T.S., im QV unter 8.1.6)

- Fallbeispiele der Textsorte Kasuistik trennen zwischen „Anamnese“ bzw. „Aufnahmesituation“ und „Untersuchungsbefund“ oder „diagnostischer Beurteilung“ sowie „Therapie“ und „Verlauf“ (vgl. Kissling/Mendel/Förstl 2014 und Weimer/Kraus 2011). In fachexternen Texten beginnt die Erzählung des Fallbeispiels meist im beruflichen Kontext der Personen. Danach wird entweder der Weg zum Zusammenbruch geschildert oder die problematische Lage der Person und deren Entstehung. Die Erzähleinheit schließt oder wieder gerahmt von einem Diagnosekommentar von ärztlicher oder psychotherapeutischer Seite. Reflexionen $\mathrm{zu}$ verschiedenen diagnostischen Möglichkeiten („Die geschilderten Symptome bieten mehrere Möglichkeiten einer diagnostischen Einschätzung“, Weimer/Kraus 2011: 242) und differenzialdiagnostische Überlegungen („Eine ausführliche internistische Untersuchung erbrachte keine pathologischen Befunde“) sind in den fachexternen Texten in der Regel nicht in das erzählte Fallbeispiel integriert. Man findet jedoch insbesondere seit den 2010er Jahren Hinweise, dass die „Seelenpein [...] viele Namen“ habe: „Burnout, Erschöpfungssyndrom, Anpassungsstörung, Depression“ (Dettmer/Shafy/ Tietz 2011: 116 in DER SPIEGEL, im QV unter 8.1.6), dass es sich im „strikten Sinn [...] beim Burnout-Syndrom“ nicht ,um eine Krankheit“ handle (Meck 2010, faz. net) oder dass hinter einem ,,vermeintlichen Burnout-Befund“ meist „schwerere psychische Erkrankungen“" steckten (Falkai 2016: 20 in G\&G, im QV unter 8.1.7).

- Fallbeispiele der Textsorte Kasuistik berichten, wie oben beschrieben, aus dem Anamnesegespräch und sie rekapitulieren vom „Endzustand“ ausge-

568 In der Fachzeitschrift „Der Psychotherapeut“, im QV unter 8.1.4. 569 In der Zeitschrift PiD, im QV unter 8.1.4. 
hend den Fall (vgl. Kissling/Mendel/Förstl 2014 und Weimer/Kraus 2011). ${ }^{570}$ Dadurch ist die fachliche Fallschilderung ergebnisorientiert und spannungsarm. Das heißt, es wird nicht bewusst Spannung erzeugt, dadurch dass bestimmte Folgen zu Beginn zunächst nur angedeutet werden („Das Aus für Bernd Hinze kam schleichend.“ Falkai 2016: 20) oder Kontrastlinien (z. B. zwischen dem Rückgang der schützenden und dem Anstieg der belastenden Faktoren) erzählerisch ausgestaltet und dadurch betont werden.

- Die Wortwahl in den fachlichen Fallbeispielen erfolgt vor der Folie der Diagnosemanuale, durch die bestimmte Ausdrücke Schlüsselwortcharakter erhalten. Dies wird deutlich, wenn beispielsweise zeitliche Angaben gemacht werden, wie lange die Symptome schon bestehen („Sie fühle sich seit etwa sechs Monaten weniger belastbar, [...]“, siehe Beispiel 5 oben: 24). Denn für eine Beurteilung nach den Diagnosemanualen müssen bestimmte Kombinationen von Symptomen über einen bestimmten Zeitraum bestehen. Im ärztlichen Fallbericht wird diese Information eingeflochten (und im Gespräch erfragt), da sie diagnostische Relevanz besitzt. Ähnlichen Schlüsselwortcharakter für das Diagnosekonzept `Depression` haben Symptome wie „Gewichtsverlust“ oder „Schlafstörungen“ (ebd.).

In fachexternen Texten wird allerdings nicht nur narrativ definiert, wie die Beispiele 8-12 oben zeigen. In diesen Texten finden sich weitere zuordnendprädizierende Muster auf Satz- und Textabschnittsebene, die den Ausdruck Burnout in ein Äquivalenzverhältnis zu anderen Satz- oder Textstellen setzen. Teilweise weisen diese sprachlichen Mittel des Definierens Ähnlichkeiten zu den sprachlichen Mitteln mit definitorischer Funktion in Fachlexika und Handbüchern sowie fachlichen Zeitschriftenartikeln und Monografien auf, es gibt aber auch Besonderheiten:

1) Zunächst findet man ähnliche Verben, die den Ausdruck Burnout/Burn-out auf dargelegte Sachverhalte bzw. Begriffsinhalte identifizierend-gleichsetzend beziehen: zum Beispiel $x$ ist $y /$ ist gekennzeichnet durch $y,{ }^{571}$ definiert wird $x$

570 Dies beschreibt Schuster auch für Krankengeschichten psychiatrischer Schreibpraxis im 19. Jahrhundert: „Grundsätzlich gilt für die narrative Organisation, dass die Simultanität, Partikularität und Fragmentarizität des Erlebten in eine geordnete Sukzession gebracht wird, wobei das Schreiben insofern integrativ ist, dass es vornehmlich vom Resultat her gedacht wird““ (Schuster 2010: 163, Unterstreichung T.S.). Zu den Mitteln narrativer Inszenierung und Dramatisierung dieser Krankheitsgeschichten aus dem 19. Jahrhundert siehe Schuster (2010: 163ff.).

571 Beispiele aus dem Korpus: „Burn-out ist gekennzeichnet durch körperliche und seelische Erschöpfungsgefühle [...]“ (FAZ, 29.02.1992: 41,=Gross 1992); „Burnout ist keine Krankheit, sagen Mediziner. Burnout ist ein Risikozustand. Das wichtigste Erkennungszeichen: Man fühlt sich überwältigend erschöpft. [...]“ (FAZ, 19.04.2014,=Weiguny/Nienhaus 2014a, Unterstrei- 
$\underline{\text { als }} y,{ }^{572} x$ beschreibt $y,{ }^{573} x$ wird als $y$ bezeichnet,${ }^{574} x$ mit $y$ charakterisieren ${ }^{575}$ oder $y$ versteht unter $y z^{576}$. Sie ordnen den Ausdruck wie in den fachlichen Texten meist einem nominalen (hyperonymen) Abstractum zu, (z. B. Zustand, Endzustand, Erschöpfungsdepression, Symptomkomplex, Vorbote (einer psychischen Erkrankung), Vorläufersyndrom, (nicht) Krankheit, Zusatzdiagnose, Zusammenbruch (eines Menschen), Risikozustand, das Aus, Phase, Ausweichdiagnose, etc.), ${ }^{577}$ wodurch eine erste Kategorisierung stattfindet. Über attributive Adjektive und Nominalphrasen, Relativsätze, nominale Aufzählungen oder Informationen, die durch Adverbien wie typischerweise als charakteristische Zusatzkriterien für den beschriebenen Sachverhalt ausgewiesen werden, wird der Zustand oder Endzustand eines Prozesses weiter spezifiziert. Der Doppelpunkt als Satzzeichen, das in Verbindung mit diesen Verben

chung T.S.); „nahezu jeder Burnout ist eine Erschöpfungsdepression“ (Der SPIEGEL, 4-2011: 116, = Dettmer/Shafy/Tietz 2011); Unterstreichungen T.S., alle Belege im QV unter 8.1.6.

572 Beispiele dazu aus dem Korpus: „Definiert wird ein Burnout-Syndrom oder Ausgebranntsein als ein ,Zustand körperlicher, emotionaler und mentaler Erschöpfung““ (FAZ, 26.05.2010, Nr. 119: B4, = Stock 2010, Unterstreichung T.S.); „Der Arbeitspsychologe Arnold B. Bakker [...] stellte fest, dass es Lebenspartnern selbst bei großer Anstrengung nicht gelingt, ihre Gefühle und Probleme aus dem Arbeitsleben von zu Hause fernzuhalten. Dies betrifft beispielsweise Erschöpfung und Zynismus, die als Burnout-Bestandteile definiert sind [7]“ (SPIEGEL ONLINE, 30.12.2013, = Bürgel 2013, Unterstreichungen T.S.); beide Belege im QV unter 8.1.6.

573 „Burn-out beschreibt einen Risikozustand [...]“ (FAZ, 21.11.2013, Nr. 271: V1, = Berger 2013); „Totale Erschöpfung und die Gewissheit, am Ende seiner Kräfte zu sein: Was oft mit dem Begriff Burnout beschrieben wird, scheint der Endpunkt eines Weges zu sein“ (KarriereSPIEGEL 30.12.2013, = Bürgel 2013, Unterstreichungen T.S.); beide Belege im QV unter 8.1.6.

574 „Das klinische Wörterbuch Pschyrembel (via Wikipedia) erklärt, dass Burnout ,als Endzustand einer Entwicklungslinie bezeichnet werden kann [...]““ (SPIEGEL ONLINE, 12.02.2013 (= Lobo 2013)); „,Hohe berufliche Motivation, die in totale Abneigung gegen die Arbeit umschlägt dieses Phänomen wird mittlerweile mit einem eigenen Krankheitsbild bezeichnet, dem BurnoutSyndrom“ (DIE ZEIT (Online-Ausgabe), 20.09.2007 (= Werdes 2007, Unterstreichungen T.S.); beide Belege im QV unter 8.1.6.

575 „Mit dem Begriff Burn-out hatte Herbert Freudenberger 1974 die verbreitete chronische Erschöpfung von Menschen in helfenden Berufen, von Krankenschwestern oder Altenpflegern charakterisiert.“ (DIE ZEIT (Online-Ausgabe), 08.12.2011, = Albrecht 2011, im QV unter 8.1.6).

576 „Der Begriff Burnout leitet sich aus der Arbeitswelt her. Die meisten Ärzte verstehen darunter einen Zustand aus arbeitsbedingter Erschöpfung, Selbstentfremdung, Zynismus und verminderter Leistungsfähigkeit“ (FAZ, 06.10.2010, Nr. 232, S. N1, = Kaulen 2010, Unterstreichung T.S, im QV unter 8.1.6).

577 Siehe zu den Hyperonymen die Belege oben 1-12 und manager-magazin.de vom 19.12.2008 (= Heimann 2008); KarriereSPIEGEL 26.09.2011 (= Stehr 2011); SPIEGEL ONLINE, 12.02.2013 (= Lobo 2013); FAZ, 29.02.1992: 41 (= Gross 1992); faz.net, 20.08.2004 (= Obermeier 2004); alle Belege im QV unter 8.1.6. 
den Bezug zweier Satzbestandteile aufeinander bewirkt, findet ebenfalls häufig Verwendung (Unterstreichungen T.S.):

\section{A) DIE ZEIT (Online-Ausgabe), 25.04.2001: 61 (= Schenk 2001)}

Den Begriff prägte der New Yorker Psychoanalytiker Herbert Freudenberger schon in den siebziger Jahren. Seitdem beschäftigen sich Psychologen wie Mediziner intensiv mit diesem Phänomen. Burn-out beschreibt einen Zustand, in dem nichts mehr geht, die Betroffenen sich „ausgebrannt“ fühlen. Sie leiden unter körperlicher und seelischer Erschöpfung. Ein Zustand, der sich nicht schlagartig, sondern schleichend einstellt, meist Folge einer längeren Überforderung. Viele Symptome werden darunter zusammengefasst: psychosomatische Beschwerden wie Schlafstörungen, Herzprobleme oder Tinnitus. Im schlimmsten Fall kommt es zu schweren Depressionen, zum völligen Zusammenbruch, zu Selbstmordgedanken.

B) Gehirn\&Geist Dossier 1/2016: 25 (= Falkai 2016, aus Beispiel 5 (siehe oben))

Neben komplexen Wechselwirkungen läßt sich ein Burn-out-Syndrom durch drei Elemente charakterisieren: emotionale Erschöpfung, Dehumanisierung und Zynismus und schließlich eine verminderte Leistungszufriedenheit.

2) In Kap. 6.2.2.2 wurde schon beschrieben, dass auf Was-ist-Fragen häufig Definitionsbemühungen folgen. In den fachexternen Texten findet man einerseits die klassischen Was-ist-Fragen (siehe Bsp. 11 oben „Burnout. Was ist das eigentlich?" und die Bsp. C und D unten), andererseits beziehen die Fragen die Leser/innen häufig auch direkt mit in das Geschehen ein, meist durch die Verwendung des Pronomens man oder durch ein inkludierendes Wir oder durch eine vom Interviewer stellvertretend eingenommene Ich-Perspektive (siehe die Beispiele C, D und E): ${ }^{578}$

578 Weitere Beispiele aus dem Korpus: „Die gestresste Seele: Was ist noch Erschöpfung? Was ist schon Krankheit?“ FAZ, Nr. 35, 10.02.2012: 35 (= Geyer 2012); „Kennen Sie auch jemanden, der sich eine längere Auszeit nimmt - wegen Burnout? Haben Sie sich selbst auch schon einmal gefragt, wie lange Sie den ganzen Stress noch ertragen können? [...] Was ist noch normal? Wo fängt die Krankheit an? Und wer hat Schuld?“ FAS, Nr. 16, 20.04.2014: 27 (= Hank 2014b). „Unter Lehrern grassiert das Burnout-Syndrom. Doch was ist das?“, DIE ZEIT, 2. Dezember 1999 (= Blech 1999); ,[...] wann kann man von einem Burnout sprechen?““(Obermeier 2004, FAZ.NET), alle Belege im QV unter 8.1.6. Weitere Beispiele für inkludierendes Wir: „Wir sind die Ausgebrannten“ (PH 1/2016, = Nuber 2016a, im QV unter 8.1.7); „Wir Unausgeschlafenen“ (DIE ZEIT Nr. 44/2010, = Spork 2010 im QV unter 8.1.6). 
C) apotheken-umschau.de (Textfassung vom 18.10 .2011$)^{579}$ (Unterstreichungen T.S., im QV unter 8.1.7)

\section{Burn-out-Syndrom}

Erschöpft, müde, ausgebrannt - habe ich zu viel Stress oder schon ein Burn-out? Unser kleiner Test liefert Ihnen erste Hinweise. Dazu: Informationen zu Ursachen des Ausgebranntseins und Therapiemöglichkeiten bei totaler Erschöpfung

Was ist ein Burn-out-Syndrom?

Fußballtrainer Ralf Rangnick, Skispringer Sven Hannawald, Fernsehkoch Tim Mälzer - sie alle haben eines gemeinsam: Sie erlebten eine Phase der totalen Erschöpfung, mussten pausieren und wurden in den Medien als prominente Burn-Out-Betroffene bekannt. Das Gefühl ausgebrannt zu sein[,] haben aber nicht nur Prominente. [...]

D) SPIEGEL WISSEN 1/2012 (= Gatterburg/Großbongardt 2012) (Unterstreichung T.S., im QV unter 8.1.6)

[...] SPIEGEL: Was ist Burnout Ihrer Meinung nach?

Unger: Ein chronischer Erschöpfungsprozess, der durch eine emotionale Verstrickung mit Arbeit gekennzeichnet ist und der schließlich zu klar definierten Krankheiten führen kann wie etwa Depressionen, Angststörungen, Herzinfarkten oder auch chronischen Schmerzsyndromen..$^{580}[\ldots]$

E) Psychologie Heute 27/2011: 40-43 (= Schulze 2011) (Unterstreichung T.S., im QV unter 8.1.7)

\section{Burnout: Was uns gefährdet. Was uns schützt}

Wie erkennt man, ob eine Erschöpfung ganz normal ist oder ob man bereits am Burnoutsyndrom erkrankt ist? Und liegt es nur an der vielen Arbeit und den hohen Alltagsanforderungen, wenn man ausbrennt?

Das Gefühl kennen viele: Man ist müde schon beim Gedanken an die Arbeit, es freut einen nicht, zur Arbeit zu gehen, man hat den Eindruck, man drehte sich im Kreis wie ein Hamster im Laufrad, die Erkältung kommt und geht, und selbst nachts lässt einen der Gedanke an die Arbeit nicht mehr los. Ist das bereits ein Burnout?

\section{F) ZEIT ONLINE 29. Juli 2010 (= Heyn 2010)}

[...] ZEIT ONLINE: Wie kann ich feststellen, ob ich unter Burn-out leide?

Bergner: Erstes Symptom ist die emotionale Erschöpfung. Man kann sie selbst diagnostizieren. Sätze wie "Ich kann nicht mehr” oder "Es macht alles keinen Sinn” sind klassisch für emotional erschöpfte Menschen. Zweites Symptom ist die sogenannte Depersonalisation. Die Betroffenen ziehen sich zurück. Das dritte Symptom ist die Leistungsabnahme. Erst wenn alle drei Symptome erfüllt sind, lässt sich die Diagnose Burn-out stellen. [...]

579 Abgerufen von apotheken-umschau.de am 24.11.2012, siehe im QV im Abschnitt 8.1.7 unter AU (2011d).

580 Auch in diesem Beispiel sieht man die Zwischenposition zwischen noch 'gesunder' >Erschöpfung` und „klar definierten Krankheiten“, die dem Burnout-Konzept seit den 2010er Jahren vermehrt explizit zugeschrieben wird (vgl. Beispiel 9 oben). 
Dass diesen Fragen, häufig an Expertinnen und Experten gerichtet, große Bedeutung zukommt, kann man auch daran erkennen, dass sie im Kontext von Selbstfragebogen oder in ratgebenden Aufklärungstexten typografisch hervorgehoben als eigene Texteinheit und Rubrik (,Nachgefragt“) in einem größeren Text präsentiert werden, wie in folgendem Beispiel der Apotheken Umschau (Ausgabe 5/2005-B: 41, im QV unter 8.1.7, AU 2005b, siehe Abbildung 11) ${ }^{581}$ :

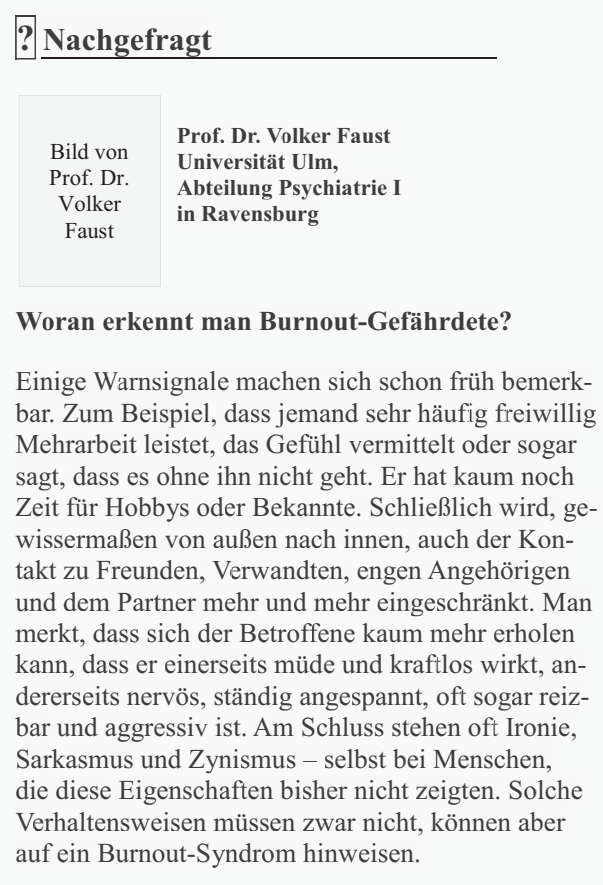

Abb. 11: Typografische Hervorhebung einer definitionseinleitenden Frage in der Apotheken Umschau 5/2005-B, S. 41 (Layout des Zitats nachempfunden, T.S.), Text @ Wort \& Bild Verlag/ Apotheken Umschau.

3) Auch die „Selbsttests“, die in einigen Übersichtsartikeln abgedruckt und mit denen das eigene „Burnout-Risiko“ abgeschätzt werden kann, werden meist mit Fragen eingeleitet. Meistens über geschlossene Entscheidungs- oder Alternativ-

581 Ähnlich auch in AU 12/1997-A: 20; PH 5/2009: 26; Und die Selbstfragebogen sind mit ähnlichen Fragen überschrieben: „Sind Sie Burnout-gefährdet?“ (PH 1/2016: 25); siehe den folgenden Spiegelstrich 3. 
fragen, mit denen die Leser/innen in der Regel direkt angesprochen werden: ${ }^{582}$ „Sind Sie ausgebrannt? [...]“ (siehe Bsp. 5 oben, G\&G Falkai 2016: 23); „Sind Sie reif für die Insel? [...]“ (siehe Bsp. 6 oben, AU 12/1997-A: 20); „Droht Ihnen der Burnout? Sind Sie noch völlig entspannt [...] oder schon chronisch überlastet? [...]“ (faz.net 25.04.2014) ${ }^{583}$; „Fühlen Sie sich ausgebrannt? Einen anerkannten Selbsttest finden Sie hier. [...]“ (Burisch, auf SPIEGEL ONLINE 25.05.2011). ${ }^{584}$ Die Selbsttests arbeiten dann entweder mit Aussagesätzen, denen die Leser/innen zustimmen oder die sie verneinen können (vgl. G\&G Falkai 2016: 23), oder mit verschiedenen Auswahlsets an Aussagesätzen, von denen die Personen dann jeweils eine Aussage wählen können (vgl. Burisch auf SPIEGEL ONLINE 2011). Man findet auch Tests mit Aussagen, die die Testpersonen anhand einer mehrstufigen Skala (dieser Aussage stimme ich nicht, bedingt bis voll zu) beantworten

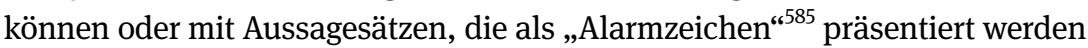
wie z. B. „Sie sind ständig müde; Sie haben das Gefühl, nie Zeit zu haben [...]“ (AU 1997: 20). Selbsttests oder Fragebogen operationalisieren den Begriff >Burnout unter dem Anspruch einer Realdefinition. Sie erfüllen definitorische Funktion, weil sie das Phänomen möglichst adäquat identifizierbar machen und den Begriff mit verschiedenen Aussagen zu Gefühlen und Symptomen differenzieren sollen (vgl. Kap. 6.2.2.2). Die einzelnen Aussagesätze bzw. Items eines Fragebogens und teilweise die Antwortmodalitäten arbeiten zudem mit sprachlichen Mitteln der Graduierung, Quantifizierung und Negation. In welcher Weise diese Sprachmittel in fachexternen Texten eingesetzt werden und dadurch definitorische Kraft entfalten, wird weiter unten noch ausführlich dargelegt.

582 Die Entscheidungs- oder Alternativfragen weisen darauf hin, dass die Selbsttests in populärwissenschaftlichen Magazinen und Medienartikeln den Gegenstand im Rahmen deskriptivargumentativer bzw. explikativer Themenentfaltung definieren. Vgl. Kap. 6.2.2.3 dieser Arbeit zum Analysebegriff des Definitionsentfaltungsmusters.

583 Abrufbar unter: https://www.faz.net/aktuell/wirtschaft/stress-burnout-ueberlastungt-burnouttest-12909273.html (zuletzt eingesehen am 12.12.2019). Dieser Test ist auch eingebettet in folgenden FAZ-Beitrag: Weiguny/Nienhaus (2014c), im QV unter 8.1.6.

584 Für die Fotostrecke, die auf den Test verweist siehe im QV im Abschnitt 8.1.6 unter SPIEGEL ONLINE (2011e). Für den Test, der von Matthias Burisch verfasst und auf SPIEGEL ONLINE veröffentlicht wurde, siehe im QV im Abschnitt 8.1.6 unter SPIEGEL-ONLINE, Burisch (2013).

585 Im fachexternen Diskursstrang wird auf die Hauptmerkmale von `Burnout häufig durch Ausdrücke referiert, die eine 'Gefahr' implizieren: „eines der wichtigsten Warnsymptome für Burn-out [...]“ (AU 10/2011-A: 35 = Rotherbl (2011) im QV unter 8.1.7); „Was sind die ersten Warnzeichen? Alarmierend ist [...] (Focus-Gesundheit Dez./Jan. 2012/2013: 49 = Wagner (2012/2013) im QV unter 8.1.7); „Warnzeichen für den heraufziehenden Zusammenbruch hatte es zuvor gegeben, aber sie waren ihm nicht klar“ (FAZ.NET, 28.01.2016, = Dunsch 2016 im QV unter 8.1.6). 
4) $\mathrm{Zu}$ den soeben beschriebenen Fragetypen, welche die Leser/innen einbeziehen, passen weitere Formulierungen im Umfeld von Definitionsbemühungen in fachexternen Texten, von denen sich die Leser/innen direkt angesprochen fühlen sollen. Es sind generalisierende Formulierungen, in denen sich das Individuum jedoch wiederfinden bzw. als Mitglied einer Gruppe erkennen kann: „Wer an seiner Arbeit keinen Spaß mehr findet, [...], der braucht vielleicht Hilfe von außen“ (siehe Bsp. 6 oben); „Krank werden die Engagierten. Diejenigen, die sich für ihren Beruf begeistern [...]“ (siehe Bsp. 4 oben). ${ }^{586}$ "Man ist müde schon beim Gedanken an die Arbeit“ (siehe oben Bsp. E, Schulze 2011: 40) oder durch die Verwendung des inkludierenden Wirs („spüren, wann wir uns zu viel zumuten“ (AU 2/2017-A: 13, (= Engelmann 2017), im QV unter 8.1.7). Diese Formulierungen, die zwischen Verallgemeinerung und Singularität und generischen und individuellen Bedeutungskomponenten changieren, findet man besonders in Magazinberichten oder Problemdarstellungen, die neben theoretischem Wissen über >BURNOUT< auch „unverbindliches Handlungswissen“ vermitteln (vgl. Kapitel 5.3.3.3 und 5.3.3.4, vgl. Franke 1997: 227). Sie fordern die Leser/innen auf, die beschriebenen Symptome mit ihrem eigenen Erleben abzugleichen, wodurch eine potenzielle intersubjektive Gültigkeit dieser Symptome und Umstände impliziert wird.

5) Auffallend ist zudem, dass in den fachexternen Texten Burnoutsymptome und Personeneigenschaften, die mit $>B U R N O U T<$ in Zusammenhang gebracht werden, fast durchgehend personalisiert (durch ein individuelles Beispiel oder durch die Charakterisierung von Personengruppen) und selten wie in Fachlexika und Handbüchern unpersönlich dargestellt werden. ${ }^{587}$ Vgl. dazu

586 Ähnlicher Beleg in der FAZ: „Wer sich diese Pausen versagt, fühlt sich über kurz oder lang gestresst“ (Weiguny/Nienhaus 2014b, FAZ); „Wer sein Pensum nicht schafft, der fliegt“ (Weigand 2006, FAZ), Belege im QV unter 8.1.6; „Wer nicht abschalten kann, wird krank“ (AU 1995c); „Wer seine Grenzen nicht mehr spürt, ist auf dem besten Weg in die totale Erschöpfung: das Burn-out“ (AU 2/2017: 3, = Haltmeier 2017), Belege im QV unter 8.1.7).

587 Eine im Vergleich dazu im unpersönlich-neutralen Lexikon-Stil gehaltene Definition findet man beispielsweise im Klinischen Wörterbuch Pschyrembel ( ${ }^{261} 2007$ : 295 , vgl. dazu auch Kap. 6.2.2.1 dieser Arbeit, Unterstreichung T.S.): „Burn|out-Syn|drom ( $\uparrow$; engl. out aus; Syndrom) n;

Burned-out-Syndrom, auch Ausbrennen; Zustand emotionaler Erschöpfung, reduzierter Leistungsfähigkeit u. evtl. Depersonalisierung inf. Diskrepanz zw. Erwartung u. Realität; Endzustand eines Prozesses von idealist. Begeisterung über Desillusionierung, Frustration u. Apathie; Häufigkeit: geschätzte Prävalenz ca. $10 \%$ der Arbeitnehmer mit entspr. Berufen (20-30\% aller Arbeitnehmer sind gefährdet); Sympt.: psychosomati. Erkr., Depression od. Aggressivität, erhöhte Suchtgefahr; Ther.: im fortgeschrittenen Stadium Psychotherapie; bei gleichzeitiger Depression Antidepressiva.“ In Lehrbüchern und Handbüchern, die sich an ein breiteres Fachpub- 
zum Beispiel Beleg 3 oben: Burnoutgefährdete Menschen fühlen sich isoliert [...]“; oder Beleg 8 oben: „Das ist für immer mehr Arbeitnehmer das Leben: Sie sind rund um die Uhr erreichbar, [...]“. Des Weiteren werden typische Eigenschaften von Personen mit 〉Burnout` benannt: Siehe aus Beleg 12 oben: „So etwas ist typisch bei Menschen, die in ein Erschöpfungssyndrom hineinrennen“; oder „Frau P. hat eine typische Burnout-Persönlichkeit.“ (SPIEGEL WISSEN 1/2011, = Bruhns 2011, im QV unter 8.1.6).

6) Es werden seltener als in Fachzeitschriftenartikeln, verschiedene Definitionsvorschläge bzw. definitorische Sachverhaltsfestsetzungen nebeneinander präsentiert, einander gegenübergestellt und diskutiert (vgl. dazu in den Fachartikeln Kap. 6.2.2.2). Diese Perspektivengegenüberstellung findet man nur in ausführlichen Magazinberichten (z. B. in einem zweiseitigen Informationskasten, der in einen Magazinbericht eingeschoben wurde, vgl. Simon (2011), in GEO WISSEN Nr. 48/2011: 56-57, im QV unter 8.1.7) oder in Übersichtsartikeln, die als „Faktencheck“ angelegt sind (vgl. Bsp. 9 oben; vgl. auch Melchers/Plieger 2016: 12-18, in G\&G Dossier, 1/2016). Die Präsentation von verschiedenen Definitionen zu >Burnout`, die man in fachlichen Übersichtsartikeln und Originalarbeiten findet, deutet darauf hin, dass die Definitionen in diesen Kontexten mit einer repräsentativ-falsifizierbaren Illokution einhergehen. ${ }^{588}$ Wenn im fachexternen Diskurs auf die unzureichende fachliche Einigkeit zu „eindeutigen Kriterien“ oder auf die bisher nicht erreichte Allgemeingültigkeit und Klarheit der Definition bzw. Diagnose Bezug genommen wird, so geschieht dies häufig ohne Bezug auf verschiedene Fachmeinungen. Das Fach erscheint in seiner Kritik als eine Stimme. ${ }^{59}$ In manchen Texten kommen allerdings auch konfligierende fachliche Meinungen in direkter Gegenüberstellung zu Wort (siehe dazu beispielsweise Beleg 11 oben, DIE ZEIT, Nr. 49/2011, 1.12.2011 (= Albrecht 2011a)).

7) Schließlich findet man in den fachexternen Texten auch wiederholt Formulierungen, die >Burnout von sachverwandten Begriffen, vor allem der >Depression $\iota$ abgrenzen möchten. An diesem Punkt zeigen sich vor allem seit den

likum richten, findet man allerdings personalisierende Formulierungen (vgl. z. B. Barth ${ }^{4} 2010$ : 83f. oder Schaper ${ }^{3}$ 2014: 531), alle Belege dieser Fußnote im QV unter 8.1.2.

588 Zum Zusammenhang repräsentativer und deklarativer Merkmale von Definitionshandlungen vgl. auch Kap. 4.3.2 dieser Arbeit.

589 Beispiele, in denen diese Kritik als eine allgemein fachliche bezeichnet wird: faz.net vom 08.03.2010 (= Meck 2010); DIE ZEIT (Online-Ausgabe), 12.08.2010 (= Elsing 2010); SPIEGEL ONLINE - Unispiegel - 26.02.2012, (= jjc/dpa 2012). Dieses Phänomen findet man auch in Interviews, in denen Experten befragt werden, so. z. B. in einem Interview der FAZ vom 20.08.2004 mit Dagmar Ruhwandl (= Obermeier 2004); alle Belege dieser Fußnote im QV unter 8.1.6. 
2010er Jahren konfligierende Einschätzungen im Diskurs. Manche fachlichen Akteure werden zitiert, die davon sprechen, dass der Begriff 〉Burnout « sich nur dadurch von der >Depression ‘ unterscheide, dass er weniger mit „Schwäche“ verbunden sei (siehe Beleg 5 oben, Falkai 2016: 22 in G\&G; siehe auch die Position Ulrich Hegerls, seit 2008 Vorsitzender der „Stiftung Deutsche Depressionshilfe“, in Beleg 11 oben). Die Symptome, die hinter diesen beiden Begriffen stünden, seien dieselben (ebd.). Andere Fachleute, die zitiert werden, erachten eine Differenzierung zwischen `Burnout ‘ und `Depression ` für sinnvoll, wie z. B. Nico Niedermeier in Beleg 11 oben, zur damaligen Zeit und bis heute ebenfalls Vorstandsmitglied der „Stiftung Deutsche Depressionshilfe“. Er plädiert für eine Differenzierung zwischen >Burnout $<$ und 〉Depression $<$ :

»Ich glaube schon, dass der Begriff Burn-out für manche Menschen nicht so falsch ist «, sagt der Münchner Verhaltenstherapeut. Einer seiner Klienten zum Beispiel zeige, solange er arbeite, alle Anzeichen einer schweren Depression. »Der schläft nicht, hat schwere Suizidgedanken, kann sich auf kaum etwas konzentrieren und hat seine Beziehungen vollkommen vernachlässigt«, sagt Niedermeier. Sobald dieser Klient aber in den Urlaub fahre, sei er binnen zwei Wochen wie ausgewechselt. Das sei vollkommen untypisch, ergänzt Hegerl. Die Depression nehme normalerweise im Urlaub eher zu.

(Unterstreichungen T.S., DIE ZEIT, Nr. 49/2011, Albrecht 2011a, im QV unter 8.1.6)

Bei diesem Beispiel eines Patienten, für den der Begriff `Burnout` besser passe als `Depression`, wird das Kriterium 'Symptome NUR während der Arbeitsphase', aber 'KEINE Symptome während der Urlaubszeit' als Merkmal eingeführt, das >Burnout ` von der >Depression ` abgrenzt. Durch den adversativen Konnektor wird die ARBEITSPHASE mit der URLAUbSzEIT kontrastiert. Zugleich wird ein Gegensatz zum Konzept `Depression` konstatiert: Denn der Rückgang der Symptome 'während der Urlaubszeit' wird als untypisch für die >Depression ‘ bezeichnet, in der sich die Symptome 'immer' und 'global' zeigen. Eine ähnliche Abgrenzung, findet man auch im folgenden Interview aus der Zeitschrift „Psychologie Heute“. In diesem Interview fragt Dörthe Binkert für „Psychologie Heute“ schon vorab unterscheidend (= PH, Binkert 2009: 30, im QV unter 8.1.7):

Burnout und Depression sind nicht dasselbe. An welchem Punkt wird aus dem Burnout eine Depression?

Ruth Enzler-Denzler, Juristin und Psychologin, die über „Burnout aus ressourcenorientierter Sicht“ promoviert hat, versprachlicht dieselbe Unterscheidung, dadurch dass sie die Extension des Begriffs `Burnout als geringer ansieht als die der >Depression`. Das >Burnout`-Merkmal, dass sich nur auf die Arbeit erstreckt, wird durch die Substitutiv-Konstruktion mit sondern für den Depressionsbegriff 
als zu begrenzt zurückgewiesen und im anschließenden Nebensatz durch „das private Umfeld“ erweitert und korrigiert (ebd.):

Burnout ist ein schleichender Prozess, der meiner Meinung nach, dann in eine Depression ,kippt‘, wenn nicht mehr nur die Arbeit, sondern auch das private Umfeld betroffen ist und sich ganz allgemein - nicht nur berufsbezogen - Gefühle der Leere und Sinnlosigkeit ausbreiten. [...]“".

(Unterstreichung T.S.)

In einem Beitrag in der ZEIT (= Scholter 2010, im QV unter 8.1.6) wird das „Burnout-Syndrom“ in einem typografisch vom restlichen Text abgesetzten Kasten ebenfalls abgegrenzt von einer „echten Depression“, da „Menschen mit Burnout emotional ansprechbar“ seien. Darum sei „ein an Burnout erkrankter Mensch auch gut behandelbar - zumeist auch ohne den Einsatz von Medikamenten“ (ebd.). Gehe „das Erschöpfungssyndrom jedoch in eine Depression über“, müsse „häufig eine Medikamentengabe erfolgen“ (ebd., Unterstreichung T.S.). Wie diese Beispiele zeigen, findet man in definierenden Textabschnitten im fachexternen Diskursstrang substitutive und adversative Konnektoren, mit denen >Burnout von anderen sachverwandten Konzepten wie der `Depression abgegrenzt wird, wodurch die definitorische Teilfunktion genauer Abgrenzung von bedeutungsnahen Konzepten erfüllt wird. Des Weiteren wird über diese Konnektoren angezeigt, dass die Extension des Burnoutbegriffs auch in Bezug auf die Personen, die von Burnout ,betroffen‘ sein können, größer sei, als zu Beginn angenommen wurde:

Aus Beleg 2, = Süddeutsche Zeitung, 31.12.1994 (= Rheinz 1994, im QV unter 8.1.6, Unterstreichung T.S.)

,Ausbrennen' ist jedoch nicht nur das Schicksal jener Legionen hilfloser und unterbezahlter Helfer, die über die Undankbarkeit der Welt (und der ihrer Klienten im besonderen) lamentieren. Ausbrennender Energieverbrauch bei fehlendem Nachschub trifft inzwischen auch jene, die in großen Konzernen, Krankenhäusern und staatlicher Institutionen arbeiten.

Das Gefühl ausgebrannt zu sein[,] haben aber nicht nur Prominente. (apotheken-umschau. de 2011, im QV unter 8.1.7, Unterstreichung T.S.)

Apotheken Umschau 10/1999 (= AU 1999b, im QV unter 8.1.6, Unterstreichung T.S.)

Nicht nur Topmanager und Börsianer trifft es: In Deutschland fühlt sich jeder vierte Arbeitnehmer in seinem Job unter Streß.

Über die substitutiven Konnektoren wird zudem deutlich, dass in Beiträgen etwa ab dem Jahr 2010 semantische Kämpfe um klassifizierende Ausdrücke für `Burnout` geführt werden, die aus dem fachlichen Diskurs stammen und über 
die im fachexternen Diskurs berichtet wird. ${ }^{590}$ Diese unterstreichen den weiter oben schon angesprochenen kritischen Unterton gegenüber der Verwendung des Begriffs `Burnout ‘ als Diagnosekonzept, da es ab diesem Zeitpunkt verstärkt

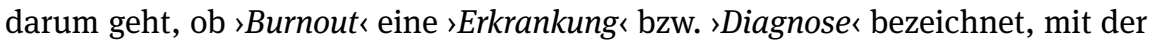
man Gelder des Gesundheitssystems in Anspruch nehmen kann, oder ,nur` eine noch nicht krankheitswertige `Erschöpfung`oder einen 〉Risikozustand`.

Beleg 11 oben, = DIE ZEIT, Nr. 49/2011, 1.12.2011 (= Albrecht 2011a, im QV unter 8.1.6, Unterstreichung T.S.)

Mit dem Begriff Burn-out hatte Herbert Freudenberger 1974 die verbreitete chronische Erschöpfung von Menschen in helfenden Berufen, von Krankenschwestern oder Altenpflegern charakterisiert. Der amerikanische Psychoanalytiker verstand den Zustand explizit nicht als psychische Erkrankung, sondern als Folge einer Arbeitsüberlastung in Gesundheitsberufen. Bald eroberte der Begriff Deutschland und fand Anwendung in immer neuen Arbeitsfeldern.

Aus Beleg 5 oben (= Falkai 2016, Unterstreichung T.S.)

Hinzu kommt: Burnout - das ist derzeit fachlicher Konsens - gilt als Vorbote einer psychischen Erkrankung. Statt einer eigenständigen Diagnose handelt es sich hier vielmehr um die Beschreibung eines Risikostadiums für die Entwicklung körperlicher und psychischer Probleme.

Und auch im Umfeld von konzessiven Konnektoren kann man unterschiedliche Sichtweisen zur Verwendung des Begriffs `Burnout beobachten, in diesem Fall zwischen „Fachleuten“ und „Beratern“ mit Blick auf Studierende, die „zunehmend an Burnout-Symptomen“ leiden sollen:

SPIEGEL ONLINE Unispiegel - 26.02.2012, (= jjc/dpa 2012) (Unterstreichung T.S., im QV unter 8.1.6)

Obwohl die meisten Fachleute den Begriff Burnout (die Verlinkung bei Burnout mit Unterstreichung im Orig., T.S.) angesichts der unterschiedlichen Erschöpfungserscheinungen mit Skepsis betrachteten, sehen 61 Prozent der Berater vor allem in den vergangenen fünf Jahren „einen deutlichen Anstieg von Burnout im engeren Sinne“, erklärte die Autorin der Diplomarbeit im Fach Soziologie an der TU Chemnitz, Doreen Liebold.

Im fachlichen Diskursstrang in den Texten der Fachlexika, Hand- und Lehrbücher findet man im Umfeld adversativer und substitutiver Konnektoren wenig semantische Kämpfe um das Hyperonym Diagnose oder Krankheit (vgl. Kap. 6.2.2.1). In

590 Zum korpuslinguistischen Auffinden agonaler Zentren, u. a. über adversative und substitutive Konnektoren, siehe Schedl (2017). 
Fachzeitschriften medizinisch-psychiatrischer Unterfachrichtungen hingegen manifestiert sich diese Kritik verstärkt seit den 2010er Jahren (vgl. Kap. 6.2.2.2). Es ist anzunehmen, dass diese kritischen Texte mitunter die Referenzquellen für die gerade dargelegten fachexternen Belege darstellen:

Korczak/Kister/Huber (2010: 13), HTA-Bericht (Unterstreichungen T.S., im QV unter 8.1.5)

Im Klassifikationssystem der American Psychiatric Association, dem diagnostischen und statistischen Handbuch psychischer Störungen (DSM-IV), wird Burnout nicht als eigenständige Diagnose aufgeführt”. Lediglich unter der Ziffer 68.20: „Probleme im Beruf“ " kann Burnout eingeordnet werden. Nach DSM-IV handelt es sich um ein Phänomen, das zu beobachten, aber nicht zu behandeln ist.“ “...]

Hamann/Parchmann/Mendel et al. (2013: 841) in der Zeitschrift Der Nervenarzt (Unterstreichung T.S., im QV unter 8.1.4)

Die Teilnehmer unserer Befragung hielten das Burnout-Syndrom überwiegend nicht für eine eigene diagnostische Entität, sondern eher für einen Überlastungszustand, der auch ein Risiko für eine spätere psychische Erkrankung darstellen kann.

Kissling/Mendel/Förstl (2014: 2588) in der Zeitschrift „Dt. med. Wochenschrift“ (Unterstreichungen T.S., im QV unter 8.1.4):

Laut der Deutschen Gesellschaft für Psychiatrie und Psychotherapie, Psychosomatik und Nervenheilkunde (DGPPN) ist Burnout keine nach ICD-10 diagnostizierbare Krankheit, sondern ein Erschöpfungs- und Überforderungssyndrom, das einen Risikozustand für eine spätere - nach ICD-10 diagnostizierbare - psychiatrische oder körperliche Krankheit darstellt (wie z. B. eine Depression oder eine Hypertonie) [3].

Interessant ist, dass der Dissens einen neuen Konsens hervorbringt, nämlich >Burnout`als >Risikozustand zu klassifizieren. Das Wechselverhältnis zwischen Konflikt und Konsens im Diskurs wird in Kapitel 7.3 noch eingehend behandelt.

Die substitutiven, adversativen und konzessiven Konnektoren werden außerdem dazu verwendet, den Burnout-Zustand von einer 'normalen' >Erschöpfung‘, 'normalen, zu erwartenden' >Arbeitsumständen`, >Persönlichkeitsmerkmalen` oder anderen möglicherweise 'gesünderen' >Umgangsformen mit beruflichen Problemen zu unterschieden. So zum Beispiel in Beleg 1, 2, 5, 6 und 11 oben (Unterstreichungen T.S.):

Substitutive Konnektoren:

Aus Beleg 1 oben = SPIEGEL Nr. 52 (1988: 162-164) (im QV unter 8.1.6)

Ein Kennzeichen der Büroneurotiker ist, daß ihre Unzufriedenheit weder Kündigung noch Rebellion am Arbeitsplatz auslöst, sondern sich nach innen richtet, Körper und Seele malträtiert und zu dramatischen Persönlichkeitsveränderungen führen kann. 


\section{Aus Beleg 12 oben = DER SPIEGEL (39/2011: 118) (Der SPIEGEL 2011d, im QV unter 8.1.6)}

Rarreck: [...] Ich kenne ihn schon lange, und mir fiel auf, dass er nicht einfach nur platt war.

SPIEGEL: $\underline{\text { Sondern? }}$

Rarreck: Beim Burnout unterscheidet man zwölf Stadien. [...]

\section{Adversative Konnektoren:}

Aus Beleg 2 oben = (Rheinz 1994, in SZ) $($ im QV unter 8.1.6)

,Ausbrennen ist jedoch nicht nur das Schicksal jener Legionen hilfloser und unterbezahlter Helfer, die über die Undankbarkeit der Welt (und der ihrer Klienten im besonderen) lamentieren. Ausbrennender Energieverbrauch bei fehlendem Nachschub trifft inzwischen auch jene, die in großen Konzernen, Krankenhäusern und staatlicher Institutionen arbeiten.

Aus Beleg 4 oben (= Kals 2004, in FAZ) (im QV unter 8.1.6)

Gefährdet sind Menschen in sozialen und pflegerischen Berufen, die sich für andere aufopfern und am „Helfersyndrom“ leiden. Wer sich hingegen entspannt durchhangelt, bis er um 16.29 Uhr seinen Computer herunterfahren kann, der läuft nicht Gefahr, an Burn-out-Syndromen zu erkranken.

\section{Aus Beleg 5 oben = (Falkai 2016, in G\&G) (im QV unter 8.1.7)}

Der 38-Jährige liebte seinen Beruf als Werbetexter - eigentlich. Die Tage füllten sich sukzessive mit Besprechungen, Kundengesprächen und Präsentationen. Anfangs gab ihm dieses Mehr an Aufgaben, das schnell getaktete Arbeitsleben einen gewissen Kick. Aber mit der Zeit nagte es zunehmend an seinen Ressourcen.

Bereits morgens um sieben saß er am Schreibtisch, um ungestört wichtige Arbeiten zu erledigen. Gegen 19 Uhr war er erschöpft, musste jedoch von zu Hause noch E-Mails beantworten oder Telefonkonferenzen abhalten. (ebd.)

Aus Beleg 6 = (Apotheken Umschau 12/1997-A (= AU 1997)) (im QV unter 8.1.7)

Der erste Pinselstrich fiel ihr schon immer schwer. Seit ein paar Tagen war es aber irgendwie anders.

\section{Konzessive Konnektoren}

SPIEGEL ONLINE, 08.08.2012 (= Abé 2012) (im QV unter 8.1.6)

Ich war nur noch müde. Einmal hatte ich sogar in meinem Auto auf einem Parkplatz übernachtet. Die Müdigkeit hatte mich so plötzlich überfallen, dass ich nicht mehr weiterfahren konnte, obwohl es nur noch zehn Kilometer bis zu meiner Wohnung gewesen wären. 
DIE ZEIT (Online-Ausgabe), 12.09.2013 (= Moulin 2013) (im QV unter 8.1.6)

„Obwohl ich nur noch Teilzeit arbeitete, übertrug man mir ständig Aufgaben, mit denen ich bis abends um 20 Uhr im Büro saß. Ich musste einen Babysitter kommen lassen, der die Kinder aus der Betreuung abholte. “ Vor gut vier Monaten erlitt die 40-Jährige ein Burn-out. „Am Ende hab ich nur noch geheult.“

Süddeutsche Zeitung, 04.01.2001 (=Reicherzer 2001: 3) (im QV unter 8.1.6)

Eine Hamburger Richterin, die gerade in Kassel angekommen ist, mitten im Burnout, erzählt von ihrer langen seelischen Not: „Ich fühle mich tot, nein eigentlich bin ich schon seit Jahren tot“, sagt sie. „Mir ist alles zuviel, Beruf, Familie, Freunde, obwohl ich eigentlich sehr gut organisiert bin. Doch entspannen kann ich mich schon lange nicht mehr. [...]“

Abgrenzungsfragen kommt, wie gerade dargelegt, in Textabschnitten der fachexternen Texte, die definitorische Funktionen erfüllen, große Bedeutung zu. Es geht einerseits um die Abgrenzung von psychischen Krankheiten wie der >Depression` (vgl. Falkai 2016: 21) und andererseits um die Abgrenzung von 'normalen' oder 'gesunden' `Erschöpfungs- oder Leistungs-Zuständen`. Folgende Frage in einem Beitrag der ZEIT fasst dieses im fachexternen Diskursstrang sich deutlich zeigende Orientierungsbedürfnis nochmals zusammen: „Habe ich ein Burn-out, eine echte Depression, oder bin ich nur erschöpft?“ (DIE ZEIT, Online-Ausgabe, 08.12.2011, = Albrecht/Schnabel 2011, im QV unter 8.1.6).

Auch in fachexternen Belegen findet man, ähnlich wie in den untersuchten fachlichen Texten (vgl. Kap. 6.2.2.1 und 6.2.2.2), vielfach sprachliche Mittel auf der Ebene der Morpheme und (Mehr-)Worteinheiten, mit denen angezeigt wird, wie lange oder oft, wie eingeschränkt oder stark im Vergleich zu anderen (implizit mitgemeinten) Zuständen oder Zeitpunkten, wie positiv oder negativ und wie (standardmäßig) erwartet oder unerwartet die beschriebenen oder erzählten Sachverhalte und Vorkommnisse sind. Dies sei anhand der oben zitierten Belege und weiterer Beispiele aus dem Korpus erläutert: So wird in den Belegen 1, 2, 3, 4 und 5 erzählt, wie sich das Verhalten und Erleben der Personen der Fallbeispiele ausgehend von einer positiven Situation bzw. einem hohen Motivationsmaß („liebte seinen Beruf“ (5); „Überflieger“ (2); „voller Elan“ (2 und 3)) verschlechtert oder ins Gegenteil hin zu einem negativen Zustand verkehrt (,immer weniger vorbereitet“ (3); „Irgendwann ging gar nichts mehr“ (5)). SYMPTOME und URSACHEN oder UMgebUngSFAKTOREN, die in Fachtexten durch komplexe Nominalphrasen ausgedrückt werden, wie zum Beispiel „real unbewältigbarer Arbeitsanfall“, „fehlende Abgrenzung zum Privatleben““591, „vermehrtes Engagement

591 Berger/Falkai/Maier 2012 im Dt. Ärzteblatt, im QV unter 8.1.4. 
für Ziele“ ${ }^{592}$, „Dehumanisierung und verminderte[r] Leistungsfähigkeit““593 oder „pos. und neg. Befindensindikatoren im Arbeitskontext““594, werden in narrativen Passagen der fachexternen Texte auf die konkrete Handlungsebene übertragen und aus der Erlebnisperspektive sprachlich vielgestaltig ausgeformt:

So findet man in den fachexternen medialen Beiträgen Intensitäts-, Fokus- und Abtönungspartikeln sowie Adjektive, die in dieser Funktion verwendet werden. Das sind z. B. gar, völlig, bereits, noch, erst, ja. In den Texten des fachinternen Diskursstrangs findet man einen solchen Partikelgebrauch seltener. ${ }^{595}$ Die Intensitätspartikeln wirken z. B. „intensivierend-steigernd“ oder „,abschwächend-abstufend“596 (Unterstreichungen T.S.):

„Irgendwann ging gar nichts mehr.“ (Beleg 5, Falkai 2016: 20, in G\&G, im QV unter 8.1.7)

Es sei, als ob man einen Porsche auf einen holprigen Feldweg setze und ihn so lange fahren lasse, bis er völlig ramponiert sei, beschreibt Siebecke die Situation vieler Betroffener. (SPIEGEL ONLINE, 28.5.2011, = Marquart 2011, SPIEGEL ONLINE, im QV unter 8.1.6)

In dieser Zeit litt auch die Beziehung zu meiner Frau, für meine Kinder hatte ich kaum mehr Zeit. (SPIEGEL ONLINE 8.8.2012, = Abé 2012, im QV unter 8.1.6)

[...] „Ich hatte keinen Antrieb zu gar nichts, alles war mir zu viel“ [...]. (faz.net. 08.3.2010, = Meck 2010, im QV unter 8.1.6)

[...] am liebsten würde man morgens gar nicht mehr aus dem Bett aufstehen. (AU 10/1995-B: 12, = AU 1995d, im QV unter 8.1.7)

592 Kaschka/Korczak/Broich 2011 im Dt. Ärzteblatt, im QV unter 8.1.4.

593 Barth ${ }^{4} 2010$ : 70, im QV unter 8.1.2.

594 Faltermaier/Hübner 2019, im QV unter 8.1.2.

595 Man findet Partikeln an einigen Stellen, zum Beispiel zur Veranschaulichung von Symptomen: "The burn-out candidate finds it just too difficult to hold in feelings"; "he cries too easily” (Freudenberger 1974: 160, im QV unter 8.1.5); Oder durch den Partikelgebrauch wird die Bedeutung des Symptoms `Zynismus siederaufgenommen und verdeutlicht: ,Es entsteht eine negative, zynische Einstellung gegenüber den Hilfesuchenden, die oft dazu führt, dass man der Meinung ist, diese seien selber schuld an ihren Problemen und Schwierigkeiten und würden sie sogar verdienen“ (Barth ${ }^{4} 2010$ : 84, im QV unter 8.1.2); Außerdem findet man den Partikelgebrauch in den Fachtexten mitunter in Aussagen von Betroffenen, wenn diese direkt zitiert werden: „Sie kommen am Abend nach Hause, sind völlig fertig, der Puls rast, [...]“ (Dt. Ärzteblatt, Kratzer (2011: A-2246), im QV unter 8.1.6). (Unterstreichungen T.S.)

596 Vgl. den Artikel „Intensitätspartikel“. In: Leibniz-Institut für Deutsche Sprache: „Systematische Grammatik“. Grammatisches Informationssystem grammis. DOI: 10.14618/grammatiksystem; Permalink: https://grammis.ids-mannheim.de/systematische-grammatik/391 (zuletzt eingesehen am 19.12.2019). 
Mit Abtönungspartikeln wird beispielsweise die Einstellung der Personen zu diesem Verhalten und Erleben wiedergegeben:

\begin{abstract}
Als freiberuflicher Architekt kann man so etwas. Es gibt keine Gewerkschaft, die für einen zuständig ist und sagt: Halt, jetzt reicht's! Ich hatte keinen Chef, dem aufgefallen wäre, was mit mir passierte, sondern einen, der mir Auftrag um Auftrag zuschanzte, weil ihm gefiel, dass ich für ihn arbeitete wie ein Berserker. [...]. Eigentlich bestand mein Leben nur noch aus Arbeiten, Essen, Einkaufen und Schlafen. Häufiger habe ich auch mit einer der Kolleginnen geschlafen, um mir ein bisschen Nähe zu holen. Mehr braucht der Mensch zum Leben ja nicht.

(FAS, 13.09.2015, = Oberhuber 2015: 35, im QV unter 8.1.6)
\end{abstract}

Die Fokuspartikeln werden eingesetzt, um eine verbalisierte Handlung auf einer mitgemeinten „Skala des Erwarteten“597 als 'normal'/'abnormal' einzustufen (Unterstreichungen T.S.):

Das kann sogar umschlagen in dehumanisierendes Verhalten, wie Verachtung, Zynismus oder Aggressivität.

(Psychologie Heute 10/1983: 24, im QV unter 8.1.7)

Sie stehen bei ihrer Arbeit unter Druck und Terminhetze, finden sogar zu Hause keine Ruhe. (AU 1985)

„Hinze [...] fühlte sich schon morgens erledigt.“

(Beleg 5, Falkai 2016: 20, in G\&G, im QV unter 8.1.7)

„Bereits morgens um sieben saß er am Schreibtisch, um ungestört wichtige Arbeiten zu erledigen. Gegen 19 Uhr war er erschöpft, musste jedoch von zu Hause noch E-Mails beantworten oder Telefonkonferenzen abhalten.“

(ebd.)

„Ich fühle mich bereits ermüdet, wenn ich morgens aufstehe und einen neuen Arbeitstag vor mir habe“

(G\&G 2016: 23, Item 3 aus dem Selbstfragebogen)

Oft sitzt er morgens schon um sieben Uhr im Büro und ist erst spätnachts wieder zu Hause.

(SPIEGEL ONLINE, 10.07.2012, = Briseño 2012, im QV unter 8.1.6)

Gerade die Leistungsfähigen sind die Opfer.

(SPIEGEL ONLINE, 28.2.2011, Klepsch 2011, im QV unter 8.1.6)

Mit der Fokus-Partikel sogar wird im ersten Beleg oben ein Hinweis darauf gegeben, dass dieses „dehumanisierende“ Verhalten (in diesem Kontext von

597 Vgl. den Artikel „Fokuspartikel“ verfasst von Eva Breindl und bearbeitet von Elke Donalies. In: Leibniz-Institut für Deutsche Sprache: „Systematische Grammatik“. Grammatisches Informationssystem grammis. DOI: 10.14618/grammatiksystem Permalink: https://grammis.ids-mannheim.de/systematische-grammatik/408, zuletzt eingesehen am 19.12.2019. 
„Angehörigen in helfenden Berufen) gegen alle Erwartungen verstößt. Mit den Fokus-Partikeln schon, bereits und erst werden in den darauf folgenden Beispielsätzen oben „Erwartungen hinsichtlich zeitlicher Abläufe“ (vgl. grammis 2018) und den dazu passenden Handlungen, Gefühlen etc. verbalisiert. Es ist z. B. gegen die Erwartung, dass man sich schon morgens_erledigt fühlt. Die zeitlichen Abläufe, die z. B. mit bereits morgens um sieben eingeleitet werden, könnten auf eine Erwartung anspielen, die von einem Acht-Stundentag, d. h. einer in Deutschland gesetzlich festgeschriebenen sozialen Praktik, ausgeht. Denn gemessen an dieser Erwartungsfolie arbeiteten die Personen der Fallbeispiele deutlich länger und liegen damit über der politisch festgelegten Standardarbeitsdauer. Mit der Partikel noch wird in diesem Zusammenhang ebenfalls „ein Überhang über einen vermeintlichen Endpunkt vermerkt.“599

Auch die Negationspartikel nicht und weitere Negationswörter häufig auch in Kombination mit dem Adverb mehr markieren, dass Erwartungen an physiologischpsychologische Vorgänge und das soziale Verhalten, seien sie an dieser Stelle stärker individuell oder kollektiv motiviert, nicht fortgesetzt bzw. erfüllt werden, wie folgende Beispiele verdeutlichen (Unterstreichungen T.S.):

Wenn ich nach Hause kam, konnte ich nicht mehr abschalten.

(SPIEGEL ONLINE 8.8.2012, = Abé 2012, im QV unter 8.1.6, im QV unter 8.1.6)

„Ich hatte keinen Antrieb zu gar nichts, alles war mir zu viel“, sagt die junge Frau. [...] „Ich brauchte die Anerkennung, bekam mich nicht mehr runtergeregelt.“

(faz.net. 8.3.2010, = Meck 2010, im QV unter 8.1.6)

[...] und seinen kleinen Sohn sah er gar nicht.

(Beleg 5, Falkai 2016)

Der Druck im Job war groß, für Freizeit und Freunde blieb wenig Zeit, Ärger oder Unbehagen drückte er weg. Doch seine Gefühle brachen sich auf körperlicher Ebene Bahn. Richard O. erlebte Angstzustände und Panikattacken, litt unter Lähmungserscheinungen in den Beinen, fühlte sich lustlos, freudlos, unkonzentriert.

(SPIEGEL WISSEN 1/2011, = Gatterburg 2011, im QV unter 8.1.6)

598 Vgl. zu den Fokuspartikeln schon und bereits den Artikel „Fokuspartikel und fokusbezogene Ausdruckssequenzen als Mittel der Diktumsgraduierungen“ der online zugänglichen „Systematischen Grammatik“ des Grammatischen Informationssystems „grammis“ des Leibniz-Instituts für Deutsche Sprache, letzte Änderung am Artikel am 18.09.2018, abrufbar unter: https:// grammis.ids-mannheim.de/systematische-grammatik/2483\#besonders, zuletzt eingesehen am 19.12.2019).

599 Zitiert aus dem Artikel „Fokuspartikel und fokusbezogene Ausdruckssequenzen als Mittel der Diktumsgraduierungen“ der online zugänglichen „Systematischen Grammatik“ des Grammatischen Informationssystems „grammis“ des Leibniz-Instituts für Deutsche Sprache, letzte Änderung am Artikel am 18.09.2018, siehe Link in Fußnote 598. 
Die Betroffenen haben den Eindruck, dass sie ihre täglichen Aufgaben nicht mehr bewältigen können, fühlen sich überfordert und müde. ${ }^{600}$

(www.apotheken-umschau.de, = AU 2011d, 18.10.2011, im QV unter 8.1.7)

Nach dem Sommerurlaub im vergangenen Jahr merkte Thomas F. ${ }^{\star}, 48$, dass etwas mit ihm nicht stimmte. Der Vorstandsvorsitzende eines börsennotierten Technologie-Unternehmens fühlte sich kein bisschen erholt.

(SPIEGEL ONLINE, 31.05.2012, = Kröher/Werle 2012, im QV unter 8.1.6)

Aufstehen, Duschen, Kaffee kochen, alles, was zur Tagesroutine gehört, wird zur unzumutbaren Kraftanstrengung. Die eigene Arbeit wird als wirkungslos erlebt [...].

(FAZ, 19.4.2014, = Weiguny/Nienhaus 2014a, im QV unter 8.1.6)

In den zitierten Beispielen werden Zustände und Praktiken negiert, die unter 'normalen' bzw. 'gesunden` bzw. 'wünschenswerten' Umständen nicht negiert wären. Durch die Negation werden sie zu einem Symptom des Konzepts `Burnout‘, wie auch folgende positive Aussagen des Selbstfragebogens aus Beleg 5 (orientiert am Maslach Burnout Inventory) zeigen, wenn sie mit einem Nein beantwortet werden:

18) Ich fühle mich angeregt, wenn ich eng mit anderen Menschen zusammengearbeitet habe.

19) Ich habe viele lohnende Ziele bei meiner Arbeit erreicht. [...]

21) Bei meiner Arbeit gehe ich mit emotionalen Problemen gelassen um.

Weiterhin findet man im fachexternen Diskursstrang eine große Bandbreite sprachlicher Mittel (z. B. Adjektive, Adverbien), durch welche die physischen und psychischen Merkmale und Verhaltensweisen, die als Symptome von >Burnout beschrieben werden, sowie Persönlichkeitseigenschaften, die mit >Burnout in Bezug gebracht werden, sowie Ursachen und Umgebungsfaktoren, zeitlich gedehnt, intensiviert und gesteigert oder als wiederholte Vorgänge dargestellt werden. Dadurch werden die beschriebenen Merkmale von 'normalem' Verhalten und Empfinden und Durchschnittsnormen abgegrenzt. Diese sprachlichen Mittel wurden im fachinternen Diskursstrang schon ausführlich beschrieben (vgl. Kap. 6.2.2.1 und 6.2.2.2), weshalb es an dieser Stelle genügt, einige Beispiele aus den Medien- und Vermittlungstexte zu zitieren (Unterstreichungen T.S.):

Denn die Leistungsfähigkeit nimmt dramatisch ab. Mercedes-Manager Bredack beobachtete stundenlang eine Fliege in seinem Büro. Tagelang fuhr er mit dem Auto herum, statt zu arbeiten. Und noch etwas kommt hinzu: Die Distanz zur Umwelt wird größer. Die Erschöpf-

600 Online nicht mehr in dieser Fassung abrufbar, abgerufen am 24.11.2012. 
ten werden zynisch, apathisch. [...] „Der typische Burnout-Patient ist der engagierte, kompetente Mitarbeiter in Führungsposition oder der Selbständige Mitte 40, der über Monate bis Jahre chronisch seine persönlichen Grenzen der Arbeitsfähigkeit überschritten hat", sagt Götz Mundle, medizinischer Geschäftsführer der Oberbergkliniken, die Burnout-Patienten behandeln.

(FAZ, 19.4.2014, = Weiguny/Nienhaus 2014a, im QV unter 8.1.6)

80 Prozent der Deutschen empfinden ihr Leben als stressig, jeder Dritte klagt über Dauerstress in Job, Haushalt, Schule oder Studium. Leistungsdruck, Terminhetze, fehlende Wertschätzung im Beruf oder in der Partnerschaft, aber auch chronische Unterforderung erzeugen Stress, der zu einer echten Plage werden kann.

(SPIEGEL WISSEN 22.02.2011, = Gatterburg 2011, im QV unter 8.1.6)

Man fühlt sich richtig leer. Es fehlt jeglicher Antrieb, überhaupt irgend etwas anzufangen.

(Apotheken Umschau 10/1995-B: 12, = AU 1995d, im QV unter 8.1.7)

Fühlt man sich zum Beispiel einmal im Monat nach der Arbeit erledigt, so ist das noch lange kein Burn-out. Erlebt man dieses Gefühl aber mehrmals in der Woche oder täglich, geht das über eine normale Stressreaktion hinaus. [...]

(Psychologie Heute 5/2009: 27, = Schulze 2009, im QV unter 8.1.7)

aufgrund dauernder Anspannung, ständiger sozialer Begegnungen, täglichen Stresses. Die Wirkung ist dann besonders tiefgreifend, wenn aufreibende Arbeit und dauernde Belastung von wenig Anerkennung und mitmenschlicher Unterstützung begleitet sind

(PH 4/2000, = Smolka 2000: 39, im QV unter 8.1.7)

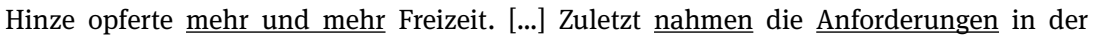
Agentur jedoch ständig zu. Immer mehr Projekte wanderten auf seinen Schreibtisch.

(Gehirn\&Geist 1/2016: 20, = Falkai 2016, in G\&G, im QV unter 8.1.7)

Die „Burnout-Syndrom“ genannte Überbeanspruchung von Geist und Körper ergreift Spitzensportler und Sachbearbeiter gleichermaßen

(FAZ, 14.7.2012, = FAZ 2012b, im QV unter 8.1.6)

Besonders häufig sind Personen betroffen, die hoch motiviert sind - mit hohen Perfektionsansprüchen, hohem beruflichen und/oder sozialen Engagement, hohem Kontrollbedürfnis und oftmals geringer Delegationsneigung.

(FAZ, 16.3.2012, = Siebecke 2012, im QV unter 8.1.6)

„Immer wieder befinde ich mich in Arbeitssituationen, die nur durch äußersten Arbeitseinsatz zu bewältigen sind“, schreibt ein erschöpfter Angestellter.

(PH 11/2000, = Kerber 2000, im QV unter 8.1.7)

Die auf den letzten Seiten beschrieben sprachlichen Mittel, die Burnout vom >Normalzustand chen dies dadurch, dass sie soziale und kulturelle Praktiken sowie biologische 
Normen als Referenzwerte evozieren. Ob Spuren solcher Praktiken über die Textgrenzen hinaus im fachexternen Diskursstrang stabil bleiben und inwiefern sie dadurch auf den diskursiven Prozess des Definierens einwirken können, wird in Kapitel 6.2.4.1 noch eingehender untersucht.

Zum Abschluss dieses Kapitels sei noch analysiert, ob sich in den Medienund Vermittlungstexten sprachliche Mittel manifestieren, mit denen die Autorinnen und Autoren einen Anspruch auf allgemeine Gültigkeit, Validität oder Intersubjektivität ihrer präsentierten Definitionen erheben, und ob bestimmte Merkmale des Konzepts sprachlich hervorgehoben präsentiert und dadurch zu >Kernmerkmalen` der Definition erhoben werden.

In den Medien- und Vermittlungstexten findet man neben dem Präsens auch das Präteritum und Perfekt in definierenden Passagen, die narrativ entfaltet oder zitierend auf eine andere Person als Urheber zurückgeführt werden. In den Fachlexika, Hand- und Lehrbüchern dominiert im Vergleich dazu in den definierenden Abschnitten das Präsens (vgl. Kap. 6.2.2.1), in Artikeln aus Fachzeitschriften, Sammelbänden und Monografien hingegen stößt man ebenfalls auf das Präteritum in referierten Fallbeispielen oder in Definitionen, die auf Zitaten beruhen (vgl. Kap. 6.2.2.2). ${ }^{601}$ Interessant ist, dass in einigen Texten des fachexternen Diskursstrangs gezielt Tempuswechsel eingesetzt werden, um den Kontrast zwischen 'gesunden'/'normalen' und 'ungesunden'/'abnormalen' Zuständen zu unterstreichen und um den Wechsel von erzählter und besprochener Welt zu markieren (vgl. Weinrich ${ }^{3} 2005$ : 198). So beispielsweise in Beleg 1 oben aus dem SPIEGEL (Nr. 52, 1988) und Beleg 2 oben aus der Süddeutschen Zeitung (31.12.1994, = Rheinz 1994) (Unterstreichungen T.S.):

\section{Aus Beleg 1 oben:}

Die junge Lehrerin begann ihre Lehrzeit [...] voller Elan. Doch bereits nach einem Jahr im Schuldienst ist sie ein Nervenbündel.

[...] Burnout heißt die knappe Diagnose, die der amerikanische Psychoanalytiker Herbert J. Freudenberger für krisenhafte Entwicklungen dieser Art prägte, nachdem Patienten in Verbindung mit ihrer Arbeit über Dauermüdigkeit, Gleichgültigkeit und Zynismus klagten, [...].

\footnotetext{
601 Vgl. z. B. Beleg 5 in Kap. 6.2.2.2 (= Büssing/Schmitt 1998: 76, in ZfAO): „Der Begriff Burnout wurde 1974 von Freudenberger eingeführt. Er bezeichnete damit ein Syndrom, das [...]“ (Unterstreichung T.S.) und Beleg 8a-1 in Kap. 6.2.2.2 (= Kissling/Mendel/Förstl 2014: 2587, in DMW): „Der konkrete Fall [als Überschrift hervorgehoben, T.S.] [...] Die 45-jährige Patientin fühlte sich erschöpft, [...] Die Patientin wurde [...] dem Psychiater vorgestellt, der ein Burnout-Syndrom diagnostizierte [...]“ (Unterstreichung T.S.). Belege im QV unter 8.1.4.
} 


\section{Aus Beleg 2 oben:}

Unmerklich veränderte sich sein Berufsalltag. [...] Der einstige Überflieger galt inzwischen kaum mehr als unterer Durchschnitt, [...] Er fühlte sich ausgebrannt. Hohe Erwartungen, heißt es in der Psychologie, gepaart mit [...] ergeben ein Burnout-Syndrom [...].

Durch die Tempuswechsel wird nicht nur die Abgrenzung zwischen einem erzählten Zustand 1 und erzählten Zustand 2 und dadurch eine binäre Sicht auf den 'gesunden, erwünschten, normalen' vorzeitigen und 'ungesunden, unerwünschten, abnormalen' Zustand erzeugt, sondern durch das Präsens kann das Erzählte nicht mehr „von einem distanzierten Sehpunkt aus“ als Beispiel eines Einzelnen betrachtet werden (vgl. Köller 2004: 436). Der kontrastive Einsatz des Präsens lenkt die Aufmerksamkeit auf die „Faktizität“ des thematisierten Tatbestands (vgl. ebd.: 433) und der Geltungsanspruch wird zudem durch den Gebrauch des Indikativs und durch den Verweis, dass diese Einschätzung von Fachleuten stammt, verstärkt. ${ }^{602}$ Einige Beispiele zitieren die Definition H. Freudenberger und repetieren damit eine schon im fachlichen Diskursstrang sich zeigende intertextuelle Bezugnahmepraktik, die der Erstdefinition besondere Geltung zuschreibt. ${ }^{603}$ Die Geltung dieser Erstdefinition zeigt sich auch darin, dass in der Diskussion um den ,richtigen' klassifizierenden Überbegriff Freudenbergers Erstcharakterisierung für die eigene Klassifizierung übernommen und mit dem Hinweis versehen wird, dass Stimmen, die sich auf ihn mit anderen Überbegriffen beriefen, einem Missverständnis auflägen (siehe oben Beleg 11, = Albrecht 2011a in der ZEIT, im QV unter 8.1.6).

Geltung wird den Definitionen zudem dadurch zugeschrieben, dass Fachleute aus dem Bereich der Medizin oder Psychotherapie zitiert werden, die sich teilweise selbst wiederum auf Mehrheitsmeinungen aus dem Fach berufen (,weitgehende Einigkeit besteht heute darin, dass [...]“, siehe oben Beleg 4), und dass referiertes Wissen diesen Bereichen zugeordnet wird: So in den von fachlicher Seite stammenden Kommentaren, die auf die narrativen Fallbeispiele folgen (siehe oben in den

602 Wenn in einem Artikel gegenüber der `Diagnose Burnout `eine kritische Grundhaltung besteht, dann wurde beobachtet, dass die auf die Erzählung oder den Fallbericht folgende ärztliche/psychiatrische Diagnosestellung als Teil der Geschichte weiter im Präteritum erzählt wird. Auf diese Art ist es im Folgenden leichter möglich, sich von dieser Form der Diagnosestellung im weiteren Verlauf des Textes zu distanzieren. Vgl. Beleg 5 oben (Falkai 2016 in G\&G, im QV unter 8.1.6) oder im fachlichen Diskursstrang Beleg 8a-1 (= Kissling/Mendel/Förstl 2014: 2587 in der DMW, im QV unter 8.1.4) in Kap. 6.2.2.2.

603 Vgl. das vermittlungssemantische Verfahren der „Mitteilung der Chronologie einer Entdeckung als personalisierte Entdeckungsgeschichte“ und das Moment der „Referenzfixierung“ und Ersteinführung bei Liebert (2002: 13) sowie Wimmer (1979: 168). Vgl. dazu auch Kap. 6.1.1 dieser Arbeit. 
Belegen 2, 3, 4, 5, 6), in Interviews mit Expertinnen und Experten (siehe z. B. Beleg 12 oben) oder durch Bezug auf Studien mit Formulierungen wie „,nach Untersuchungen von Wissenschaftlern“ (FAZ, Gross 1992) oder „Experten machen die Anforderungen der Berufswelt verantwortlich“ (siehe Beleg 8 oben, SZ, Bohsem 2012a).

Im Vergleich zum fachlichen Diskurs, in dem zur Geltungsvalidierung in einigen Texten auf die empirische Methodik hingewiesen wird, verweisen Zeitungsmeldungen und -berichte im Kotext der Burnoutdefinitionen häufig auf Prävalenzraten, die die Definition dadurch realsemantisch stützen. ${ }^{604}$

Wie weiter oben schon beschrieben wird fachlicher Disput seit den 2010er Jahren in den Medientexten zwar aufgegriffen, aber selten werden verschiedene fachlichen Positionen, die als gleichwertig ausgewiesen werden, in einem Text einander gegenübergestellt (eine Ausnahme wurde in Beleg 11 oben präsentiert). Häufiger wird eine leitende fachliche Kritik bzw. (Gegen)-Meinung (Burnout ist keine Krankheit, sondern ein Risikozustand) referiert, teilweise wird diese Fachmeinung der Meinung von Personen, die in der Praxis tätig sind, gegenübergestellt, ${ }^{605}$ oder an die Kritik wird der Bericht über einen fachlichen Minimalkonsens angeschlossen, der sich zumeist auf einzelne Kernsymptome bezieht: So zum Beispiel in Beleg 11, wenn von der „dürre[n] Erkenntnis“ gesprochen wird, „dass die Betroffenen irgendwie unter emotionaler Erschöpfung, dem Gefühl der Leblosigkeit und verminderter Leistungsfähigkeit leiden“; oder in folgendem Informationskasten aus der Zeitschrift GEOkompakt (08/2014 = Eberle 2014: 28, im QV unter 8.1.7, siehe Abbildung 12), in dem ebenfalls verneint wird, dass >Burnout als Krankheit anerkannt sei. Es werden aber dennoch drei Kernsymptome, die auf Maslach/Jackson (1981) zurückgehen, und eine besonders gefährdete Personengruppe benannt:

604 Vgl. zum Beispiel in der FAZ vom 24.09.2010: 62: „Steigende Belastungen im Beruf [...] führten immer häufiger zu psychosomatischen Erkrankungen wie Burnout, Depression [...].“ (= FAZ 2010); in der SZ vom 17.08.2012: 17 (= Bohsem 2012a): „Die Fehlzeiten durch Burnout waren 2011 elf mal so hoch wie noch 2004 [...]“ oder im SPIEGEL 29.05.2011 (= Zeltner 2011): „Fast jeder dritte Klinikarzt leidet Studien zufolge unter einem Burnout“; Belege im QV unter 8.1.6.

605 Im Text von Kaulen (2010, FAZ vom 6.10.2010: 232) wird der kritischen Betrachtung des zitierten Health-Technology-Assessment-Berichts (Burnout sei keine international anerkannte, allgemeingültig definierte Dia-gnose) die Diagnosetätigkeit der praktizierenden Ärzte gegenübergestellt. Letztere würden Burnout dennoch diagnostizieren. Ähnlich wird in SPIEGEL ONLINE Unispiegel vom 26.02.2012 berichtet, dass der Begriff `Burnout ` von den meisten Fachleuten angesichts der unterschiedlichen Erschöpfungserscheinungen mit Skepsis betrachtet würde, dass aber „62 Prozent der Berater vor allem in den vergangenen fünf Jahren ,einen deutlichen Anstieg von Burnout im engeren Sinne““ sehen würden (SPIEGEL ONLINE, Unispiegel (= jjc/dpa 2012), oder in einem Beitrag der ZEIT wird eine Psychiaterin, die sich auf Managerberatung spezialisiert hat, zitiert: Burnout sei ein „Modebegriff“, der „oft falsch verwendet werde“, aber es sei wichtig „Zustände von Erschöpfung und Ausbrennen“ zu erkennen und zu behandeln (= Schoener 2013, DIE ZEIT); Belege im QV unter 8.1.6. 


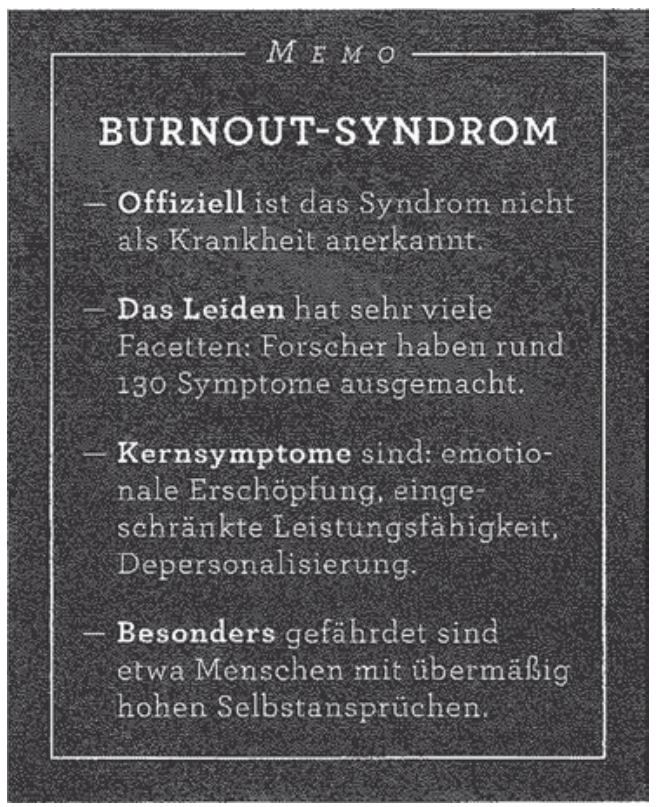

Abb. 12: Auszug aus GEOkompakt (08/2014=Eberle 2014: 28, Kopie in schwarz-weiß), (C) GEO 2014, Verlag Gruner + Jahr, Hamburg.

In vielen fachexternen Texten werden ähnlich wie im Fachdiskurs zudem einzelne Berufsgruppen - teilweise verbunden mit persönlichen Eigenschaften dieser Personen - hervorgehoben (insbesondere bei Führungskräften, vor allem ehrgeizige Leistungsträger). Einige Berufe werden als prototypisch markiert, dadurch dass man von ihnen ausgehend die Liste betroffener Gruppen adversativ/substitutiv erweitert (B. ist jedoch nicht nur das Schicksal jener Legionen hilfloser und unterbezahlter Helfer, sondern ...).

Des Weiteren werden einzelne Symptome sprachlich gewichtet, wie gerade schon im Kasten der Zeitschrift GEOkompakt (siehe Abb. 12) deutlich wurde. Im fachexternen Diskurs zeigt sich dabei im Vergleich zum fachinternen Diskurs wiederum stärker die Perspektive der potenziell betroffenen Personen. Denn es werden nicht nur wie in den Fachtexten Kernsymptome oder Hauptkriterien erwähnt, sondern die fachexternen Texte informieren oft über „einige Warnsignale“ oder „Alarmzeichen“, über „das wichtigste Erkennungszeichen“ oder darüber, welche „ersten Warnzeichen“ als „alarmierend“ anzusehen sind:

Das wichtigste Erkennungszeichen: Man fühlt sich überwältigend erschöpft. [...] (FAZ, 19.04.2014, = Weiguny/Nienhaus 2014a, Unterstreichung T.S.); nahezu jeder Burnout ist eine Erschöpfungsdepression (Der SPIEGEL, 4-2011: 116, = Dettmer/Shafy/Tietz 2011); Betrachtet man den Symptomkatalog, so trifft einiges auch auf das Burnout-Syndrom zu. Dessen zentrales 


\begin{abstract}
Merkmal, »anhaltende Erschöpfung und Leistungsminderung (Falkai 2016: 24, in G\&G, im QV unter 8.1.7); Was sind die ersten Warnzeichen? [im Orig. als Überschrift markiert] Alarmierend ist ein extrem ambitionierter Einsatz für das Unternehmen oder freiwillige unbezahlte Arbeit. Typisch für den Beginn der Erkrankung ist, dass Betroffene bei eingespielten Tätigkeiten plötzlich Fehler machen. Oder sie sind auffällig oft müde, unkonzentriert, klagen über Schlafstörungen, sind vergesslich, desinteressiert oder hyperaktiv. (Focus-Gesundheit, Wagner 2012/2013: 49); Diese Alarmzeichen sollten Sie beachten: Sie sind ständig müde; Sie haben das Gefühl, nie Zeit zu haben [...] (AU 1997: 20); Charakteristisch ist, dass sich die Betroffenen ständig müde und kraftlos fühlen, dennoch kaum Schlaf finden, innerlich unruhig und gereizt sind, nach der Arbeit kaum mehr abschalten und entspannen können (AU 2005b: 40); Gar nicht mehr abschalten können[,] gilt übrigens als eines der wichtigsten Warnsymptome für Burn-out. Welches sind die anderen? [als Frage hervorgehoben gedruckt] Erschöpfung, Leistungsabfall. Oft kommen dann körperliche Symptome wie Ohrgeräusche oder Magenprobleme hinzu (AU 10/2011-A: 35f., = Rotherbl 2011); Zu den wichtigen körperlichen Warnzeichen zählen Kopfschmerzen und Migräne, Rückenschmerzen, Magen-Darm-Störungen, erhöhter Blutdruck, Herzrasen und Schweißausbrüche, Tinnitus und Hörsturz. (DIE ZEIT (Onlineausgabe) 08.07.2010, = Bahnsen 2010, im QV unter 8.1.6); Drei Leitsymptome [...] kennzeichnen ein Burn-out-Syndrom. Typisch ist emotionale Erschöpfung: Die betroffene Person fühlt sich psychisch und körperlich ausgelaugt. Mit dem Energiemangel tauchen ständige Müdigkeit, Niedergeschlagenheit und Anspannungszustände auf. Zweitens zählen Zynismus, Distanzierung und Depersonalisierung dazu. [...]. Drittens nimmt die Arbeitsleistung ab. Betroffene haben das Gefühl (und oft es dies auch Fakt), dass sie nicht mehr so gute Arbeit leisten wie früher (DIE ZEIT (Onlineausgabe) 13.06. 2014, = Kleinschmidt 2014, im QV unter 8.1.6).
\end{abstract}

Sowohl Warnsymptome als auch die mit diesen Anzeichen verbunden Phasen oder Stufen der Erschöpfung werden in Magazinberichten teilweise typografisch hervorgehoben präsentiert. ${ }^{606}$

Für die Definitionen im fachlichen Diskursstrang wurde schon die generalisierende und damit geltungsqualifizierende Wirkung von Gattungsnamen beschrieben (Kap. 6.2.2.1), mit denen Personen- und Risikogruppen des Konzepts >Burnout benannt werden (z. B. professionelle Helfer). Denn dadurch, dass nicht Einzelpersonen, sondern Berufsgruppen als ,Betroffene‘ benannt werden, wird den beschriebenen Symptomen und Entstehungsbedingungen über den Einzelfall hinausgehend eine Regelmäßigkeit oder Typizität zugeschrieben. Doch der Beschreibung des Einzelfalls in den narrativen Belegen kommt ebenfalls eine validierende Funktion in Bezug auf den Hergang der Geschichte zu. Der Umstand, dass in Beleg 5 nicht einfach nur die Erlebnisse eines „Werbetexters“, sondern die Erlebnisse der konkreten Person „Bernd Hinze“ erzählt werden, führt dazu, dass die Geschichte in einem persönlich-unmittelbaren, subjektiv-singulären und dadurch authentischen Licht

606 So z. B. in der AU 2005b: 40 als Treppe mit zwölf Stufen; im SPIEGEL 2/2011: 117 als drei Stufen, die sich mit dem Stresskreislauf verbinden (= Dettmer/Shafy/Tietz 2011) oder im Stern vom 3.12.2015 als „Erschöpfungsspirale“ mit vierzehn Stufen (= Schmitz 2015: 53, im QV unter 8.1.7). 
erscheint (vgl. die Kategorie des Sinzeichens bei Peirce, Kap. 2.2.2). Bernd Hinze ist der >Eigendynamikı der auftretenden Symptome unmittelbar ausgesetzt, das zeigt sich auch dadurch, dass er häufig die semantische Rolle des Patiens, Rezipienten oder Wahrnehmenden einnimmt. ${ }^{607}$ Am Ende der Erzählung wird sein subjektives Erleben von außen durch die Bewertung des Hausarztes „Burnout - als Folge chronischer Arbeitsüberlastung“ von fachlicher Seite aus bestätigt. Es wird durch diesen fachlichen Kommentar des Weiteren impliziert, dass sich die Qualität der beschriebenen Gefühle und Beschwerden (vgl. die Kategorie der Qualizeichen bei Peirce, Kap. 2.2.2), die sich bei Bernd Hinze aktuell zeigen, mit anderen Fällen und dort beschriebenen Beschwerden vergleichen lassen. Mit anderen Worten: Der Arzt erkennt darin ein Muster bzw. einen Typus und identifiziert aufgrund seines erworbenen Vergleichswissens das aktuell geschilderte Beschwerdebild mit dem über die Aktualität hinausreichenden, verallgemeinerten Beschwerdebild Burnout (vgl. die Kategorie des Legizeichens bei Peirce, Kap. 2.2.2). `Burnout` wird dadurch sowohl singulär-subjektiv als auch kollektiv-intersubjektiv konstituiert.

Man könnte einwenden, dass die sprachlichen Mittel, die die gerade herausgearbeiteten semantischen Aspekte erzeugen (z. B. die 'Unmittelbarkeit des subjektiven Erlebens', 'Eigendynamik' des geschilderten Prozesses), zwar den Fortgang der Geschichte innerhalb der narrativen Einschübe plausibilisieren, aber dennoch nicht diskurs- bzw. fachübergreifend als sprachliche Mittel angesehen werden können, mit denen der definitorische Anspruch auf allgemeine Gültigkeit, Validität oder Intersubjektivität gestützt wird. Bei der Definition eines Gegenstands aus dem Fach Chemie, wie z. B. bei der Definition von `MOLEKÜL〈, dürfte die 'Unmittelbarkeit des subjektiven Erlebens' eine untergeordnete Rolle für die Geltung der Definition spielen. Der Aspekt der 'Eigendynamik' könnte hingegen ebenfalls zur Geltung einer Definition in diesem Bereich beitragen. Diese Gegenüberstellung geltungs-

607 Der Aspekt der 'Eigendynamik' bzw. 'Eigengesetzlichkeit' wird dadurch verstärkt, dass in der Erzählung die benannten UMGEBUNGSFAKTOREN personalisiert werden und als selbstinduzierte Bewegungen oder Prozesse erscheinen, wohingegen die Person Hinze überwiegend in der empfangenden, passiven oder wahrnehmenden Rolle verharrt: z. B. „Zuletzt nahmen die Anforderungen [...] ständig zu. Immer mehr Projekte wanderten auf seinen Schreibtisch. [...] Die Tage füllten sich sukzessive mit Besprechungen [...]. Anfangs gab ihm dieses Mehr an Aufgaben [...] einen gewissen Kick. Aber mit der Zeit nagte es zunehmend an seinen Ressourcen. [...] Dazu gesellten sich körperliche Beschwerden [...]“ (Beleg 5 oben, = Falkai 2016: 20, in G\&G, im QV unter 8.1.7). Friedemann Vogel beschreibt dieses Sprachgebrauchsmuster als „Autorität durch Anthropomorphisierung von Ausdrücken und Texten“ im Rahmen seiner Analyse zu „Sprachliche[n] Mittel[n] des Geltungsanspruchs im Kontext der Normgenese“ (vgl. Vogel 2012: 409f.). $\mathrm{Zu}$ semantischen Rollen und Verantwortungszuschreibungen in der Burnout-Ratgeberliteratur vgl. auch Schnedermann (i. Ersch.) und zu semantischen Rollen im Allgemeinen Primus (2012), von Polenz (32008: 169ff.) und Müller (2007: 94f.) sowie Kap. 6.2.4.2. 
qualifizierender sprachlicher Mittel für Akte des Definierens im Bereich Chemie und im Bereich psychischer Gesundheit und Krankheit führt vor Augen, dass in verschiedenen Fachgebieten und textuellen Erscheinungsformen unterschiedliche Funktions-Ansprüche an Definitionen gestellt werden können und dass diese auch mit unterschiedlichen sprachlichen Mitteln umgesetzt werden können. Die vorliegende Arbeit hat sich daher zum Ziel gesetzt, ein Analysemodell einer diskursiven Praxis des Definierens für den Bereich psychischer Gesundheit und Krankheit zu entwerfen (vgl. Kap. 4.3.4 und 7.1-7.2).

Im Anschluss an dieses Kapitel werden die Plattformen Wikipedia, NetDoktor und Onmeda einer Analyse unterzogen. Das Kapitel 6.2.3.3 dient schließlich der (tabellarischen) Zusammenfassung der analysierten sprachlichen Mittel des Definierens in den verschiedenen Texten des fachexternen Diskursstrangs (Medientexte, Vermittlungstexten in populärwissenschaftlichen Zeitschriften, Texte auf Onlineportalen).

\subsubsection{In Onlineplattformen: Wikipedia, NetDoktor und Onmeda}

Für die Untersuchung sprachlicher Mittel des Definierens auf der WikipediaPlattform lohnt sich zunächst ein Blick in einen Text, der dort unter der Überschrift „Wie schreibe ich gute Artikel“608 veröffentlicht ist und als Richtlinie für die „kollaborative Konstruktion von Wissensbeständen“ auf Wikipedia angesehen werden kann (Gredel 2016a). Dort findet man unter dem Gliederungspunkt „Begriffsdefinition und Einleitung“ die „Richtlinien“, dass jeder Artikel durch eine „Begriffsdefinition“ eröffnet werden soll, durch welche das „Lemma“ als Bezeichnung“ geklärt wird bzw. durch die der „Begriff in seiner Grundbedeutung“ erläutert werde. „Homonyme“ sollen unterschieden werden und der „Gegenstand des Artikels“ soll „möglichst präzise in seinen sachlichen Kontext“ eingeordnet werden. „Superlative“ sollen in der Definition vermieden werden, was an das „Grundprinzip“ für Wikipedianer erinnert, „persönliche Standpunkte aus Wikipedia-Artikeln herauszuhalten“ („neutraler Standpunkt“). ${ }^{609}$ In diesen Anleitungspunkten spiegeln sich Ansprüche an die Tätigkeit des Definierens wider, die in Kap. 4.2.2 herausgearbeitet wurden: so z. B. der Anspruch auf exakte und eindeutige Bestimmung bzw. Einordnung; der Anspruch auf angemessene

\footnotetext{
608 Siehe die folgende Anleitungsseite auf Wikipedia: https://de.wikipedia.org/w/index.php?title=Wikipedia:Wie_schreibe_ich_gute_Artikel\&oldid=188510203\#Begriffsdefinition_und_Einleitung (Artikelstand: 13.05.2019, zuletzt eingesehen am 03.12.2019).

609 Zum Grundprinzip des neutralen Standpunkts siehe: https://de.wikipedia.org/w/index. php?title=Wikipedia:Neutraler_Standpunkt\&oldid=187247426 (Artikelstand: 04.04.2019, zuletzt eingesehen am 03.12.2019).
} 
Umgrenztheit durch die Konzentration auf wesentliche bzw. grundlegende Faktoren und der Anspruch auf eine sachliche von emotionalen Konnotationen und Bewertungen freie Sachverhaltskonstitution/Begriffsbildung:

Aus dem Artikel „Wie schreibe ich gute Artikel“ in der Online-Enzyklopädie Wikipedia (Hinterlegung T.S., Unterstreichungen zur Kennzeichnung von Links im Orig., Links im Orig. blau, hier schwarz) ${ }^{610}$

Begriffsdefinition und Einleitung eröffnen den Artikel und leiten zum ersten Abschnitt über. Sie sollen das Lemma als Bezeichnung klären und den Begriff in seiner Grundbedeutung erläutern. Bei mehrdeutigen Benennungen ist eine Begriffsklärung sinnvoll und der Hinweis auf die Begriffsklärungsseite angebracht, damit Homonyme unterschieden werden. Der erste Satz ordnet den Gegenstand des Artikels möglichst präzise in seinen sachlichen Kontext ein. Hierbei werden der Titel des Artikels und eventuelle Synonyme in Fettschrift gesetzt. Beispiele:

- Aszites (von altgriechisch aбkiтns askítēs) ist die medizinische Benennung für eine übermäßige Ansammlung von Flüssigkeit in der Bauchhöhle, genauer im Peritoneal$\underline{\text { raum; }}$ andere Ausdrücke für diese krankhafte Flüssigkeitsansammlung sind Hydraskos, Bauchwassersucht oder Wasserbauch.

- Als Aszites, auch Hydraskos, Bauchwassersucht oder Wasserbauch, wird in der Medizin eine übermäßige Ansammlung von Flüssigkeit in der Bauchhöhle bezeichnet, genauer im Peritonealraum.

Unmittelbar darauf sollte eine kurze Einleitung mit einer Zusammenfassung der wichtigsten Aspekte des Artikelinhalts folgen. Die Einleitung soll einen kurzen Überblick über das Thema ermöglichen und das Lemma in Grundzügen erklären. [...]

- Der Leser sollte die Einleitung mit einem Blick erfassen können. Hier sind Hauptsätze besonders wichtig. Kurze, einfache und aussagekräftige Formulierungen sind besser als lange oder geschachtelte Erklärungen.

- Gib nach Möglichkeit Kurzinformationen zur Herkunft des Lemmas mit einer Übersetzung ins Deutsche an (wie zum Beispiel bei Virus, Software oder Exklave).

- Verweise auch kurz auf die historische Bedeutung des Begriffs, sofern es sich nicht schon um ein historisches Thema handelt.

- Erwähne Superlative in der Einleitung nur, falls sie zur Erklärung des Lemmas maßgeblich beitragen oder sich das Lemma ausdrücklich auf solche Besonderheiten (ältester, größter) stützt. Andere Superlative gehören an passende Stellen im Fließtext.

Umgangssprachlich wird das Wort Begriff oft undifferenziert verwendet. Im Rahmen einer Begriffsdefinition oder -klärung sollten jedoch Formulierungen wie „Der Begriff bezeichnet ..." vermieden werden, da sie erlauben, Bezeichnung und Bezeichnetes zu verwechseln und schon insofern missverständlich sind.

610 Siehe die folgende Anleitungsseite auf Wikipedia: https://de.wikipedia.org/w/index.php?title=Wikipedia:Wie_schreibe_ich_gute_Artikel\&oldid=188510203\#Begriffsdefinition_und_Einleitung (Artikelstand: 13.05.2019, zuletzt eingesehen am 03.12.2019). 
Der letzte Hinweis hängt mit dem Begriffsbegriff zusammen, der auf Wikipedia gepflegt wird und der im obigen Zitat direkt mit folgendem Wikipedia-Eintrag zu „Begriff“ verlinkt wurde:

Im weiteren Sinne bezeichnet das Wort Begriff in der Philosophie, wie ein Wort zu verstehen ist (im Sinne von „zu begreifen“). Es handelt sich somit um die Verbindung einer sprachlichen Bezeichnung mit einem Gedankeninhalt (einem Konzept im psychologischen Sinn). ${ }^{611}$

(Unterstreichungen zur Kennzeichnung von Links im Orig., Links im Orig. blau, hier schwarz)

Die frühen Einträge $\mathrm{zu}>$ Burnout auf Wikipedia verwenden aber das Muster, von dem oben abgeraten wird (der Begriff $x$ bezeichnet $y$, siehe unten den Eintrag vom 31.12.2004). Dies zeigt, dass der Begriffsbegriff auf Wikipedia bei Weitem nicht so einheitlich verwendet wird, wie angestrebt, bzw. in früheren Jahren noch nicht ausformuliert worden war.

Vor dem Hintergrund der oben genannten Ansprüche an die Tätigkeit des Definierens verwundert es nicht, dass man in den Begriffsdefinitionen der Wikipedia-Artikelversionen zu >Burnout einen unpersönlichen, sprachökonomisch komprimierten Formulierungsstil vorfindet (ähnlich wie in den Fachlexika und Handbüchern, siehe Kap. 6.2.2.1). Der erste Eintrag zu >Burnout auf Wikipedia stammt vom 12. März 2004 und besteht nur aus zwei Sätzen, die sehr allgemein gehalten sind, und auf Berufe im helfenden Bereich fokussieren:

$\mathbf{6 1 1}$ https://de.wikipedia.org/w/index.php?title=Begriff_(Philosophie)\&oldid=177395919 (Artikelstand vom 13.05.2019, zuletzt eingesehen am 03.12.2019). Auf einer anderen Wikipedia-Seite zum Eintrag Begriff, die der Kategorie „Alltagssprache und Etymologie“ zugewiesen wurde, werden noch weitere Begriffsbegriffe angeführt: „Mit dem Wort Begriff wird der Bedeutungsinhalt einer Bezeichnung oder Vorstellung angesprochen. Ein Begriff bildet dabei eine semantische Einheit, die Teil einer Proposition oder eines Gedankens ist. ${ }^{[1]}$ Die Abgrenzung zu Worten oder Ausdrücken als sprachlichen Einheiten und Vorstellungen als rein gedanklichen Einheiten ist jedoch im Alltagsgebrauch und in verschiedenen Fachsprachen - je nach Perspektive - oft unscharf: Teilweise wird Begriff als „mentale Informationseinheit“ verstanden, ${ }^{[2]}$ oder bedeutungsgleich zum ,Begriff‘ im Sinne der vormodernen philosophischen Tradition; unter Begriff kann aber auch ausdrücklich ein ,lexikalisiertes Konzept' verstanden werden, also eine Kombination von Bezeichnung und ,mentale[r] Repräsentation eines einzelnen Objekts oder einer kognitiven Kategorie‘.[3]“، (siehe: https://de.wikipedia.org/w/index.php?title=Begriff\&oldid=188998655 (Artikelstand vom 28.05.2019, zuletzt eingesehen am 03.12.2019, Unterstreichungen zur Kennzeichnung von Links im Orig. wurden entfernt). 


\section{Wikipedia, 12. März 2004, Eintrag zu „Burnout“ \\ Burnout \\ Unter Burnout/Ausbrennen versteht man verschiedene Formen beruflicher Überlastung durch Stress und andere Faktoren. Burnout tritt insbesondere in „helfenden Berufen“ auf Pflegeberufe, Lehrer, Sozialarbeiter. ${ }^{612}$}

(Unterstreichungen zur Kennzeichnung von Links im Orig., Links im Orig. blau, hier schwarz)

Ende des Jahres 2004 ist der Artikel deutlich überarbeitet und füllt ausgedruckt beinahe zwei Din-A-4-Seiten. Die neue Begriffsdefinition setzt dabei schon einige der in den Richtlinien genannten Kriterien um, wie z. B. die Angabe zur „Herkunft des Lemmas“ und „einer Übersetzung ins Deutsche“. Auch hier orientierten sich die Verfasser/innen des Wikipedia-Artikels wohl an Lexikon-Vorlagen, da in den Richtlinien der Wikipedia im Jahr 2004 die Angaben zum Definieren noch nicht so konkret wie im Jahr 2019 ausformuliert waren. ${ }^{613}$ Der Eintrag vom 31. Dezember 2004 ähnelt formal dem klassischen aristotelischen Muster von genus proximum - differentia specifica: Das hyperonyme Definiens-Nomen Erschöpfung wird attributiv spezifiziert durch die Adjektive berufsbezogen und chronisch. Die Ersteinführung wird auf H. Freudenberger und Ch. Maslach zurückgeführt und als Synonym wird Erschöpfungsdepression angegeben (die paradigmatische Bedeutungserläuterung durch Angabe von Synonymen wird jedoch in den Folgejahren nicht weitergeführt):

Wikipedia, 31. Dezember 2004, Eintrag zu „Burnout“ (Unterstreichungen zur Kennzeichnung von Links und Fettung im Orig., Links im Orig. blau, hier schwarz)

Der Begriff Burnout (engl. burn out: ausbrennen) bezeichnet einen besonderen Fall berufsbezogener, chronischer Erschöpfung und wurde 1974 erstmalig von Herbert Freudenberger und Christina Maslach eingeführt. Synonym wird der Begriff Erschöpfungssyndrom gebraucht.

Die folgende tabellarische Übersicht zeigt, dass das Grundmuster des „genus proximum - differentia specifica“ in den ersten Absätzen der Artikelversionen jeweils beibehalten wird. Als Definitorverben jeweils im ersten Satz der Beitragsversionen werden nur die folgenden drei Muster verwendet: $x$ bezeichnet $y$; mit $x$ wird $y$ bezeichnet und $x$ ist $y$. Der Definitionsabschnitt wird innerhalb dieses Rahmens von Jahr zu Jahr überarbeitet. Es wechseln die Definiens-Nomina höherer Abstraktion und vor allem die Einordnungspraktiken mit Bezug auf die Klassifikationssysteme ICD-10, ICD-11 und DSM-5. Darauf wird im Anschluss an die Präsentation der Tabelle noch

612 Diese Version ist abrufbar unter: https://de.wikipedia.org/w/index.php?title=Burn-out\&ol$\operatorname{did}=808670$ (zuletzt eingesehen am 03.12.2019).

613 „Wikipedia: Wie schreibe ich gute Artikel“ in der Fassung vom 21.12.2004, einsehbar unter: https://de.wikipedia.org/w/index.php?title=Wikipedia:Wie_schreibe_ich_gute_Artikel\&oldid=3945206\#Definition_des_Begriffs_und_\%C3\%9Cberschriften (zuletzt eingesehen am 23.6.2019). 
ausführlich eingegangen. Der einführende Definitionsabschnitt entwickelt sich in den Burnout-Artikelversionen der Wikipedia von einer knappen Definition, die aus ein bis zwei Sätzen mit einem Definiens-Nomen und zwei bis drei spezifizierenden Merkmalen besteht, zu einem Definitionsabschnitt, der diese Basisangaben um symptomatische und ursächliche Angaben, Betroffenen-Gruppen, Klassifizierungsfragen und Prävalenzangaben erweitert:

Tab. 3: Vergleich der einleitenden Abschnitte der Artikelversionen zu >Burnoutı in der OnlineEnzyklopädie Wikipedia von 2004-2019. ${ }^{614}$

\begin{tabular}{|c|c|c|c|}
\hline \multirow[t]{2}{*}{ Datum } & \multicolumn{2}{|l|}{ 1. Satz } & \multirow{2}{*}{$\begin{array}{l}\text { Weitere Merkmale und Angaben im } \\
\text { Abschnitt vor dem Inhaltsverzeichnis } \\
\text { (Definitor-Verb-Muster, Klassifizierung } \\
\text { ICD-10, Angaben zu Symptomen und } \\
\text { Entstehung) }\end{array}$} \\
\hline & $\begin{array}{l}\text { Definiendum } \\
\text { (schon mit } \\
\text { erläuternder } \\
\text { Übersetzung) }\end{array}$ & $\begin{array}{l}\text { Nominaler } \\
\text { Definiensausdruck } \\
\text { höherer Abstraktion } \\
\text { (grau hinterlegt } \\
\text { T.S.) - Spezifizierung } \\
\text { (Unterstreichung T.S.) }\end{array}$ & \\
\hline 31.12 .2004 & $\begin{array}{l}\text { "Der Begriff } \\
\text { Burnout }^{615} \\
\text { (engl. burn out: } \\
\text { ausbrennen)“ }\end{array}$ & $\begin{array}{l}\text { „einen besonderen } \\
\text { Fall berufsbezogener, } \\
\text { chronischer } \\
\text { Erschöpfung“ }\end{array}$ & $\begin{aligned}- & X \text { bezeichnet } y \\
- & \text { Nennung der Ersteinführung } \\
& \text { (FB, Masl.) }\end{aligned}$ \\
\hline 21.12 .2005 & $\begin{array}{l}\text { „Der Begriff } \\
\text { Burnout (engl. } \\
\text { burn out: } \\
\text { ausbrennen)“ }\end{array}$ & $\begin{array}{l}\text { „einen besonderen Fall } \\
\text { berufsbezogener, } \\
\text { chronischer } \\
\text { Erschöpfung“ }\end{array}$ & - wie 2004 \\
\hline 30.12 .2006 & $\begin{array}{l}\text { „Der Begriff } \\
\text { Ausgebranntsein } \\
\text { oder englisch } \\
\text { Burnout- } \\
\text { Syndrom } \\
\text { (engl. (to) burn } \\
\text { out - ausbrennen)“ }\end{array}$ & $\begin{array}{l}\text { „einen } \\
\text { besonderen Fall } \\
\text { berufsbezogener, } \\
\text { chronischer } \\
\text { Erschöpfung“ }\end{array}$ & $\begin{aligned} &- \text { X bezeichnet } y \\
&- \text { Nennung der Ersteinführung (FB) } \\
&- \text { Nennung Einflussfaktoren } \\
&- \text { Nennung (typischer) Symptome } \\
&- \text { Burnout in der ICD-10 als } \\
& \text { „Diagnoseschlüssel Z73.0 erfasst“ } \\
& \text { (ohne Distanzmarker) } \\
&- \text { Prominente mit Burnout in } \\
& \text { den Medien } \\
&- \text { („Skispinger Sven Hannawald“, } \\
& \text { „Rapper Eminem“) } \\
&\end{aligned}$ \\
\hline
\end{tabular}

614 In den Zitaten aus Wikipedia, die in der Tabelle zitiert werden, sind die Fußnoten und Verlinkungen, die auf Wikipedia gesetzt wurden, nicht enthalten.

615 Durch die Formulierung „,[d]er Begriff Burnout“ wird Burnout zudem der Gattung >Begriff prädikativ zugeordnet. Vgl. die Verwendung von Substantiven in „prädizierend-referierender [...] Funktion“ bei von Polenz (²008: $164 \mathrm{f})$.

616 In diesem Beispiel wird `Burnout durch das Kompositum Burnout-Syndrom der Gattung `Syndrom` prädikativ zugeordnet. 
Tab. 3 (fortgesetzt)

\begin{tabular}{|c|c|c|c|}
\hline \multirow[t]{2}{*}{ Datum } & \multicolumn{2}{|l|}{ 1. Satz } & \multirow{2}{*}{$\begin{array}{l}\text { Weitere Merkmale und Angaben im } \\
\text { Abschnitt vor dem Inhaltsverzeichnis } \\
\text { (Definitor-Verb-Muster, Klassifizierung } \\
\text { ICD-10, Angaben zu Symptomen und } \\
\text { Entstehung) }\end{array}$} \\
\hline & $\begin{array}{l}\text { Definiendum } \\
\text { (schon mit } \\
\text { erläuternder } \\
\text { Übersetzung) }\end{array}$ & $\begin{array}{l}\text { Nominaler } \\
\text { Definiensausdruck } \\
\text { höherer Abstraktion } \\
\text { (grau hinterlegt } \\
\text { T.S.) - Spezifizierung } \\
\text { (Unterstreichung T.S.) }\end{array}$ & \\
\hline 29.12 .2007 & $\begin{array}{l}\text { „Ausgebranntsein } \\
\text { oder englisch } \\
\text { Burnout- } \\
\text { Syndrom (engl. } \\
\text { (to) burn out: } \\
\text {,ausbrennen')“ }\end{array}$ & $\begin{array}{l}\text { „einen besonderen } \\
\text { Fall berufsbezogener } \\
\text { und/oder familiärer } \\
\text { chronischer } \\
\text { Erschöpfung“ }\end{array}$ & 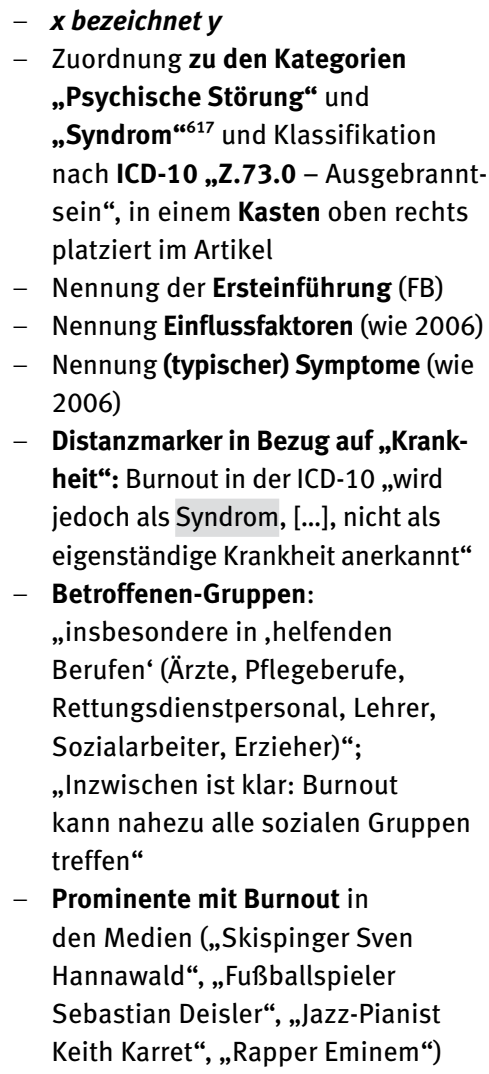 \\
\hline
\end{tabular}

617 Diese Zuordnung erscheint jeweils unten im Wikipedia-Artikel. Sie wird in dieser Tabelle aufgenommen, da sie eine globale Zuordnungspraktik für den gesamten Artikel darstellt. 
Tab. 3 (fortgesetzt)

\begin{tabular}{|c|c|c|c|}
\hline \multirow[t]{2}{*}{ Datum } & \multicolumn{2}{|l|}{ 1. Satz } & \multirow{2}{*}{$\begin{array}{l}\text { Weitere Merkmale und Angaben im } \\
\text { Abschnitt vor dem Inhaltsverzeichnis } \\
\text { (Definitor-Verb-Muster, Klassifizierung } \\
\text { ICD-10, Angaben zu Symptomen und } \\
\text { Entstehung) }\end{array}$} \\
\hline & $\begin{array}{l}\text { Definiendum } \\
\text { (schon mit } \\
\text { erläuternder } \\
\text { Übersetzung) }\end{array}$ & $\begin{array}{l}\text { Nominaler } \\
\text { Definiensausdruck } \\
\text { höherer Abstraktion } \\
\text { (grau hinterlegt } \\
\text { T.S.) - Spezifizierung } \\
\text { (Unterstreichung T.S.) }\end{array}$ & \\
\hline 30.12 .2008 & $\begin{array}{l}\text { „Ausgebranntsein } \\
\text { oder Burnout- } \\
\text { Syndrom (engl. } \\
\text { (to) burn out: } \\
\text {,ausbrennen')“ }\end{array}$ & $\begin{array}{l}\text { „eine besonders } \\
\text { ausgeprägte } \\
\text { berufliche und/ } \\
\text { oder familiäre } \\
\text { Erschöpfung“ }\end{array}$ & 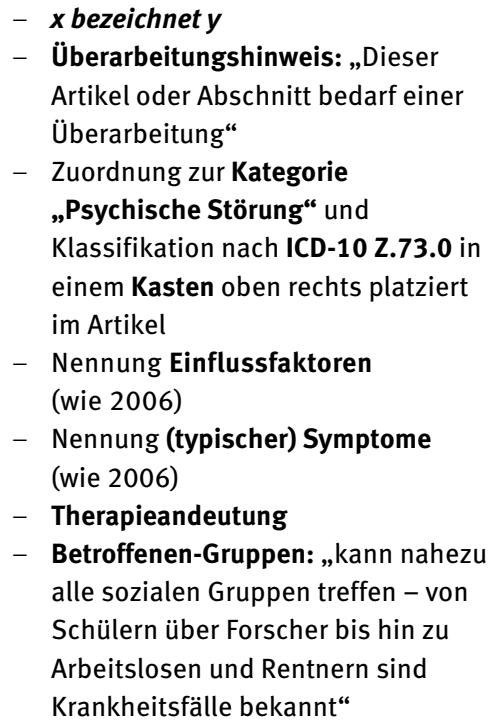 \\
\hline 26.12 .2009 & $\begin{array}{l}\text { „Ein Burnout- } \\
\text { Syndrom (engl. } \\
\text { (to) burn out: } \\
\text {,ausbrennen')“ }\end{array}$ & $\begin{array}{l}\text { „ein Zustand } \\
\text { ausgesprochener } \\
\text { emotionaler } \\
\text { Erschöpfung } \\
\text { mit reduzierter } \\
\text { Leistungsfähigkeit, } \\
\text { das als } \\
\text { Endzustand einer } \\
\text { Entwicklungslinie } \\
\text { bezeichnet werden } \\
\text { kann, die mit } \\
\underline{\text { idealistischer }} \\
\text { Begeisterung } \\
\underline{\text { beginnt und über }}\end{array}$ & $\begin{aligned}- & x \text { ist } y \\
- & \text { Überarbeitungshinweis: } \\
& \text { (siehe 2008) } \\
\text { - } & \text { Zuordnung zur Kategorie } \\
& \text { „Psychische Störung“ und } \\
& \text { Klassifikation nach ICD-10 Z.73.0 in } \\
& \text { einem Kasten oben rechts platziert } \\
& \text { im Artikel } \\
\text { - } & \text { Betroffenen-Gruppen: (siehe 2008) }\end{aligned}$ \\
\hline
\end{tabular}


Tab. 3 (fortgesetzt)

\begin{tabular}{|c|c|c|c|}
\hline \multirow[t]{3}{*}{ Datum } & \multicolumn{2}{|l|}{ 1. Satz } & \multirow[b]{2}{*}{$\begin{array}{l}\text { Weitere Merkmale und Angaben im } \\
\text { Abschnitt vor dem Inhaltsverzeichnis } \\
\text { (Definitor-Verb-Muster, Klassifizierung } \\
\text { ICD-10, Angaben zu Symptomen und } \\
\text { Entstehung) }\end{array}$} \\
\hline & $\begin{array}{l}\text { Definiendum } \\
\text { (schon mit } \\
\text { erläuternder } \\
\text { Übersetzung) }\end{array}$ & $\begin{array}{l}\text { Nominaler } \\
\text { Definiensausdruck } \\
\text { höherer Abstraktion } \\
\text { (grau hinterlegt } \\
\text { T.S.) - Spezifizierung } \\
\text { (Unterstreichung T.S.) }\end{array}$ & \\
\hline & & $\begin{array}{l}\frac{\text { frustrierende }}{\text { Ereignisse zur }} \\
\text { Desillusionierung } \\
\text { und Apathie, } \\
\text { psychosomatischen } \\
\text { Erkrankungen und } \\
\text { Depression oder } \\
\text { Aggressivität und } \\
\text { einer erhöhten } \\
\text { Suchtgefährdung } \\
\text { führt.“ }\end{array}$ & \\
\hline 30.12 .2010 & $\begin{array}{l}\text { „Ein Burnout- } \\
\text { Syndrom (engl. } \\
\text { (to) burn out: } \\
\text {,ausbrennen“) } \\
\text { bzw. } \\
\text { Ausgebranntsein“ }\end{array}$ & $\begin{array}{l}\text { „ein Zustand } \\
\text { ausgesprochener } \\
\text { emotionaler } \\
\text { Erschöpfung } \\
\text { mit reduzierter } \\
\text { Leistungsfähigkeit, } \\
\text { das als Endzustand } \\
{[\ldots . . \text { ““ (siehe wie in }} \\
\text { 2009) }\end{array}$ & $\begin{array}{l}-\quad x \text { ist } y \\
-\quad \text { Zuordnung zur Kategorie „Psychische } \\
\text { Störung“ und Klassifikation nach } \\
\text { ICD-10 Z.73.0 in einem Kasten oben } \\
\text { rechts platziert im Artikel } \\
\text { - } \text { Distanzmarker in Bezug auf } \\
\text { „Krankheit“ und Folge/Ursache: } \\
\text { „Burnout ist keine Krankheit mit } \\
\text { eindeutigen diagnostischen Kriterien } \\
\text { (siehe Abschnitt ICD), sondern eine } \\
\text { körperliche, emotionale und geistige } \\
\text { Erschöpfung aufgrund beruflicher } \\
\text { Überlastung und wird durch Stress } \\
\text { ausgelöst, der nicht bewältigt werden } \\
\text { kann.“ } \\
\text { Betroffenen-Gruppen: „Burnout } \\
\text { wurde zunächst in Helfenden } \\
\text { Berufen beschrieben und ist } \\
\text { auch in zahlreichen anderen } \\
\text { Berufsgruppen zu beobachten. } \\
\text { Dazu gehören Sportler, Politiker, } \\
\text { Forschungsmitarbeiter, } \\
\text { Langzeitpflegende kranker } \\
\text { Angehöriger bis hin zu Verkäufer.“ }\end{array}$ \\
\hline
\end{tabular}


Tab. 3 (fortgesetzt)

\begin{tabular}{|c|c|c|c|}
\hline \multirow[t]{2}{*}{ Datum } & \multicolumn{2}{|l|}{ 1. Satz } & \multirow{2}{*}{$\begin{array}{l}\text { Weitere Merkmale und Angaben im } \\
\text { Abschnitt vor dem Inhaltsverzeichnis } \\
\text { (Definitor-Verb-Muster, Klassifizierung } \\
\text { ICD-10, Angaben zu Symptomen und } \\
\text { Entstehung) }\end{array}$} \\
\hline & $\begin{array}{l}\text { Definiendum } \\
\text { (schon mit } \\
\text { erläuternder } \\
\text { Übersetzung) }\end{array}$ & $\begin{array}{l}\text { Nominaler } \\
\text { Definiensausdruck } \\
\text { höherer Abstraktion } \\
\text { (grau hinterlegt } \\
\text { T.S.) - Spezifizierung } \\
\text { (Unterstreichung T.S.) }\end{array}$ & \\
\hline 27.12 .2011 & $\begin{array}{l}\text { „Ein Burnout- } \\
\text { Syndrom (engl. } \\
\text { (to) burn out: } \\
\text {,ausbrennen`) } \\
\text { bzw. } \\
\text { Ausgebranntsein“ }\end{array}$ & $\begin{array}{l}\text { „ein Zustand } \\
\text { ausgesprochener } \\
\text { emotionaler } \\
\text { Erschöpfung } \\
\text { mit reduzierter } \\
\text { Leistungsfähigkeit, } \\
\text { das als Endzustand } \\
{[\ldots . . \text { “. (siehe wie in }} \\
2009 \text { und 2010) }\end{array}$ & $\begin{array}{l}-\quad x \text { ist } y \\
\text { - Zuordnung zur Kategorie „Psychische } \\
\text { Störung“ und Klassifikation nach } \\
\text { ICD-10 Z.73.0 in einem Kasten oben } \\
\text { rechts platziert im Artikel } \\
\text { - Distanzmarker in Bezug auf } \\
\text { „Krankheit“ und Folge/Ursache: } \\
\text { „Burnout ist keine Krankheit, sondern } \\
\text { ein Problem der Lebensbewältigung } \\
\text { (siehe Abschnitt ICD). Es handelt } \\
\text { sich um eine körperliche, emotionale } \\
\text { und geistige Erschöpfung aufgrund } \\
\text { beruflicher Überlastung. Diese } \\
\text { wird meist durch Stress ausgelöst, } \\
\text { der aufgrund verminderter } \\
\text { Belastbarkeit nicht bewältigt } \\
\text { werden kann.“ }\end{array}$ \\
\hline 30.12 .2012 & $\begin{array}{l}\text { „Ein Burnout- } \\
\text { Syndrom (engl. } \\
\text { (to) burn out: } \\
\text {,ausbrennen“) } \\
\text { bzw. } \\
\text { Ausgebranntsein“ }\end{array}$ & $\begin{array}{l}\text { „ein Zustand } \\
\text { ausgesprochener } \\
\text { emotionaler } \\
\text { Erschöpfung } \\
\text { mit reduzierter } \\
\text { Leistungsfähigkeit. } \\
\text { Es kann als } \\
\text { Endzustand [...]“ } \\
\text { (siehe wie in 2009, } \\
2010 \text { und 2011) }\end{array}$ & $\begin{array}{ll} & \text { x ist } y \\
\text { - } & \text { Zuordnung zur Kategorie } \\
\text { „Psychische Störung“ und } \\
\text { Klassifikation nach ICD-10 } \\
\text { Z.73.0 in einem Kasten oben } \\
\text { rechts platziert im Artikel } \\
\text { - } \text { „Das Burnout-Syndrom ist } \\
\text { international nicht als Krankheit } \\
\text { anerkannt, sondern gilt als Problem } \\
\text { bei der Lebensbewältigung (siehe } \\
\text { Abschnitt ICD). Es handelt sich } \\
\text { um [...] (Fortsetzung siehe wie } \\
\text { in 2011). }\end{array}$ \\
\hline
\end{tabular}


Tab. 3 (fortgesetzt)

\begin{tabular}{|c|c|c|c|}
\hline \multirow[t]{2}{*}{ Datum } & \multicolumn{2}{|l|}{ 1. Satz } & \multirow[b]{2}{*}{$\begin{array}{l}\text { Weitere Merkmale und Angaben im } \\
\text { Abschnitt vor dem Inhaltsverzeichnis } \\
\text { (Definitor-Verb-Muster, Klassifizierung } \\
\text { ICD-10, Angaben zu Symptomen und } \\
\text { Entstehung) }\end{array}$} \\
\hline & $\begin{array}{l}\text { Definiendum } \\
\text { (schon mit } \\
\text { erläuternder } \\
\text { Übersetzung) }\end{array}$ & $\begin{array}{l}\text { Nominaler } \\
\text { Definiensausdruck } \\
\text { höherer Abstraktion } \\
\text { (grau hinterlegt } \\
\text { T.S.) - Spezifizierung } \\
\text { (Unterstreichung T.S.) }\end{array}$ & \\
\hline .12 .2013 & $\begin{array}{l}\text { „Ein Burnout- } \\
\text { Syndrom (engl. } \\
\text { (to) burn out: } \\
\text {,ausbrennen“) } \\
\text { bzw. } \\
\text { Ausgebranntsein“ }\end{array}$ & $\begin{array}{l}\text { „ein Zustand } \\
\text { ausgesprochener } \\
\text { emotionaler } \\
\text { Erschöpfung } \\
\text { mit reduzierter } \\
\text { Leistungsfähigkeit. } \\
\text { Es kann als } \\
\text { Endzustand [...]“ } \\
\text { (siehe 2012) }\end{array}$ & $\begin{array}{l}-\quad x \text { ist } y \\
-\quad \text { Zuordnung zur Kategorie „Psychische } \\
\text { Störung“ und Klassifikation nach } \\
\text { ICD-10 Z.73.0 in einem Kasten oben } \\
\text { rechts platziert im Artikel } \\
-\quad \text { Distanzmarker in Bezug auf } \\
\text { „Krankheit“" und Folge/Ursache: } \\
\text { „Das Burnout-Syndrom ist } \\
\text { wissenschaftlich nicht als } \\
\text { Krankheit anerkannt, sondern } \\
\text { gilt im ICD-10 als Problem bei der } \\
\text { Lebensbewältigung. Es handelt sich } \\
\text { um [...] (Fortsetzung siehe wie in 2011). }\end{array}$ \\
\hline 014 & $\begin{array}{l}\text { „Ein Burnout- } \\
\text { Syndrom (engl. } \\
\text { burn out: } \\
\text {,ausbrennen') } \\
\text { bzw. } \\
\text { Ausgebranntsein“ }\end{array}$ & $\begin{array}{l}\text { „ein Zustand ausge- } \\
\text { sprochener emoti- } \\
\text { onaler Erschöpfung } \\
\text { mit reduzierter Leis- } \\
\text { tungsfähigkeit. Es } \\
\text { kann als Endzustand } \\
{[\ldots . . \text { ““ (siehe } 2012} \\
\text { und 2013) }\end{array}$ & $\begin{array}{ll}- & x \text { ist } y \\
- & \text { Zuordnung zur Kategorie „Psychische } \\
& \text { Störung“ und Klassifikation nach } \\
& \text { ICD-10 Z.73.0 in einem Kasten oben } \\
& \text { rechts platziert im Artikel } \\
- & \text { Distanzmarker in Bezug auf } \\
& \text { „Krankheit“ und Folge/Ursache: } \\
& \text { (siehe wie in 2013) }\end{array}$ \\
\hline 16.12 .2015 & $\begin{array}{l}\text { „Ein Burnout- } \\
\text { Syndrom (engl. } \\
\text { burn out: } \\
\text {,ausbrennen`) } \\
\text { bzw. } \\
\text { Ausgebranntsein“ }\end{array}$ & $\begin{array}{l}\text { „ein Zustand } \\
\text { ausgesprochener } \\
\text { emotionaler } \\
\text { Erschöpfung } \\
\text { mit reduzierter } \\
\text { Leistungsfähigkeit. } \\
\text { Es kann als } \\
\text { Endzustand [...]“ } \\
\text { (siehe 2012, 2013 } \\
\text { und 2014) }\end{array}$ & $\begin{array}{ll}- & x \text { ist } y \\
- & \text { Zuordnung zur Kategorie „Psychische } \\
& \text { Störung“ und Klassifikation nach } \\
\text { ICD-10 Z.73.0 in einem Kasten oben } \\
\text { rechts platziert im Artikel } \\
\text { - Distanzmarker in Bezug auf } \\
\text { „Krankheit“ und Folge/Ursache: } \\
\text { (siehe wie in 2013) }\end{array}$ \\
\hline 16.12 .2016 & $\begin{array}{l}\text { Mit Burnout- } \\
\text { Syndrom } \\
\text { oder Burn-out } \\
\text { (engl. burn out } \\
\text {,ausbrennen') }\end{array}$ & $\begin{array}{l}\text { „ein Zustand [...], } \\
\text { der von psychischer } \\
\text { und physischer } \\
\text { Erschöpfung } \\
\text { gekennzeichnet ist.“ }\end{array}$ & $\begin{array}{ll} & \text { mit } x \text { wird y bezeichnet } \\
\text { - } & \text { Zuordnung zur Kategorie „Psychische } \\
\text { Störung“ und Klassifikation nach } \\
\text { ICD-10 Z.73.0 in einem Kasten oben } \\
\text { rechts platziert im Artikel }\end{array}$ \\
\hline
\end{tabular}


Tab. 3 (fortgesetzt)

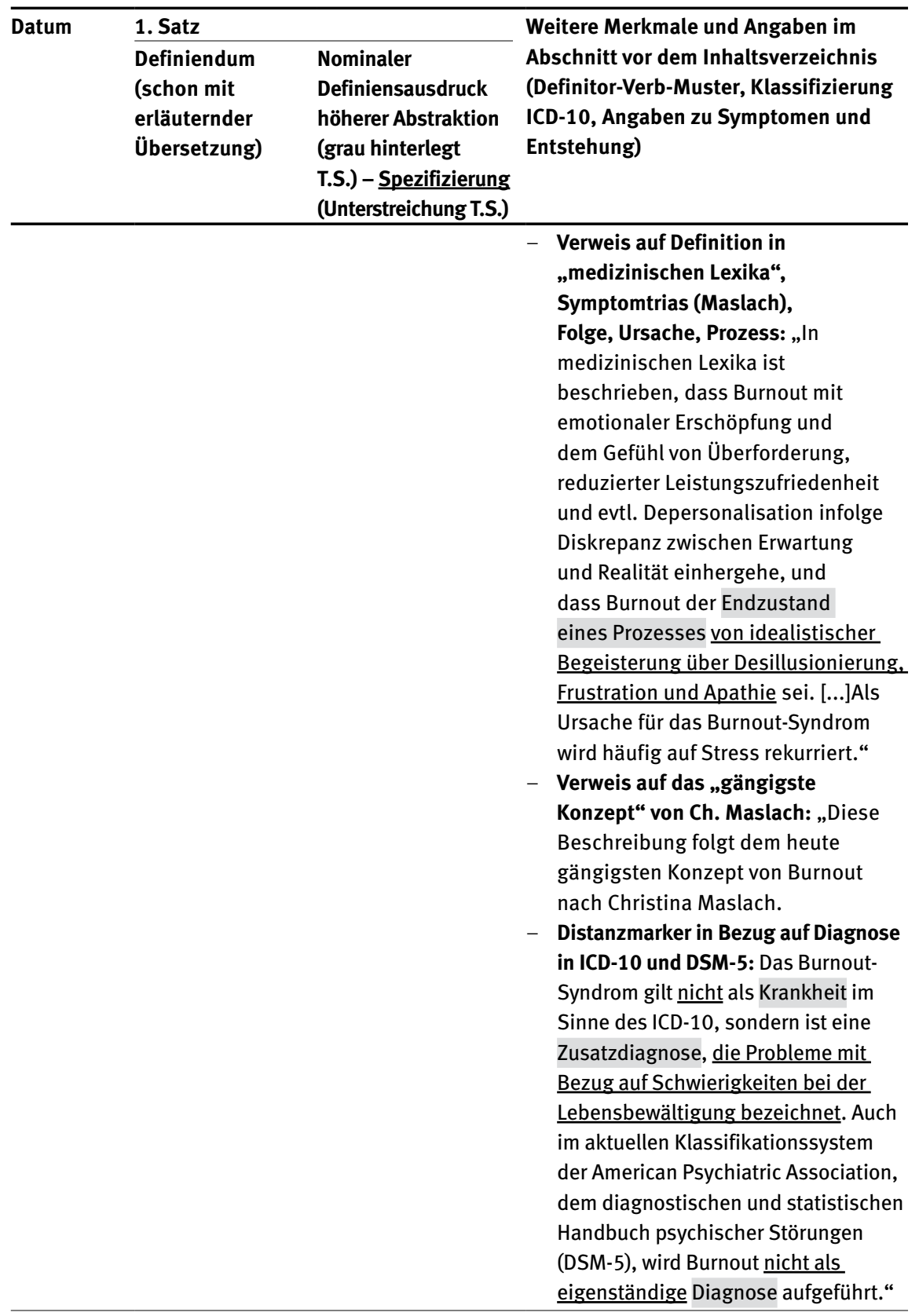


Tab. 3 (fortgesetzt)

\begin{tabular}{|c|c|c|c|}
\hline \multirow[t]{2}{*}{ Datum } & \multicolumn{2}{|l|}{ 1. Satz } & \multirow[b]{2}{*}{$\begin{array}{l}\text { Weitere Merkmale und Angaben im } \\
\text { Abschnitt vor dem Inhaltsverzeichnis } \\
\text { (Definitor-Verb-Muster, Klassifizierung } \\
\text { ICD-10, Angaben zu Symptomen und } \\
\text { Entstehung) }\end{array}$} \\
\hline & $\begin{array}{l}\text { Definiendum } \\
\text { (schon mit } \\
\text { erläuternder } \\
\text { Übersetzung) }\end{array}$ & $\begin{array}{l}\text { Nominaler } \\
\text { Definiensausdruck } \\
\text { höherer Abstraktion } \\
\text { (grau hinterlegt } \\
\text { T.S.) - Spezifizierung } \\
\text { (Unterstreichung T.S.) }\end{array}$ & \\
\hline 21.12. 2017 & $\begin{array}{l}\text { „Burn-out oder } \\
\text { Burnout, auch } \\
\text { Burnout-Syndrom } \\
\text { (englisch burn out } \\
\text {,ausbrennen')“ }\end{array}$ & $\begin{array}{l}\text { „ein Oberbegriff für } \\
\text { Typen persönlicher } \\
\text { Krisen, die mit } \\
\text { eher unauffälligen } \\
\text { Frühsymptomen } \\
\text { beginnen und } \\
\text { mit völliger } \\
\text { Arbeitsunfähigkeit } \\
\text { oder sogar Suizid } \\
\text { enden können. }\end{array}$ & 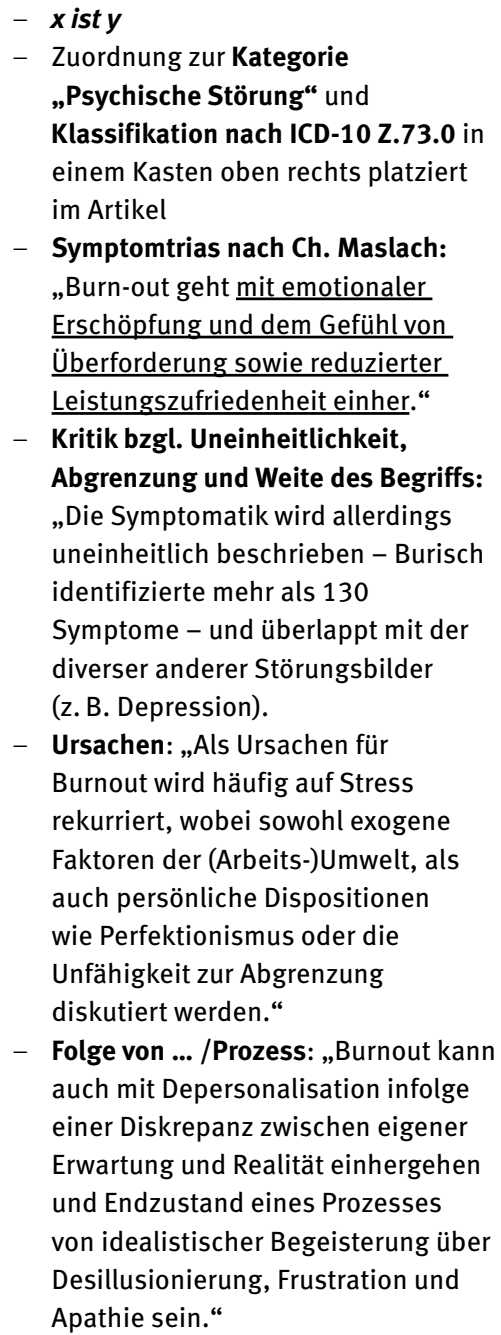 \\
\hline
\end{tabular}


Tab. 3 (fortgesetzt)

\begin{tabular}{|c|c|c|c|}
\hline \multirow[t]{3}{*}{ Datum } & \multicolumn{2}{|l|}{ 1. Satz } & \multirow{2}{*}{$\begin{array}{l}\text { Weitere Merkmale und Angaben im } \\
\text { Abschnitt vor dem Inhaltsverzeichnis } \\
\text { (Definitor-Verb-Muster, Klassifizierung } \\
\text { ICD-10, Angaben zu Symptomen und } \\
\text { Entstehung) }\end{array}$} \\
\hline & $\begin{array}{l}\text { Definiendum } \\
\text { (schon mit } \\
\text { erläuternder } \\
\text { Übersetzung) }\end{array}$ & $\begin{array}{l}\text { Nominaler } \\
\text { Definiensausdruck } \\
\text { höherer Abstraktion } \\
\text { (grau hinterlegt } \\
\text { T.S.) - Spezifizierung } \\
\text { (Unterstreichung T.S.) } \\
\end{array}$ & \\
\hline & & & $\begin{array}{l}\text { - Angabe Prävalenz: „Die Lebenszeit- } \\
\text { Prävalenz von Burnout in Deutschland } \\
\text { beträgt auf der Datengrundlage der } \\
\text { bevölkerungsrepräsentativen ,Studie } \\
\text { zur Gesundheit Erwachsener in } \\
\text { Deutschland (DEGS1)‘ } 4,2 \% \text { und die } \\
\text { 12-Monats-Prävalenz 1,5\%.“ }\end{array}$ \\
\hline 02.12 .2018 & wie 2017 & wie 2017 & - wie 2017 \\
\hline 24.06 .2019 & $\begin{array}{l}\text { „Burn-out } \\
\text { oder Burnout } \\
\text { (auch Burnout- } \\
\text { Syndrom, von } \\
\text { englisch burn out } \\
\text {,ausbrennen')“ }\end{array}$ & $\begin{array}{l}\text { „ein Oberbegriff für } \\
\text { bestimmte Arten von } \\
\text { persönlichen Krisen, } \\
\text { die als Reaktion auf } \\
\frac{\text { andauernden Stress }}{\text { und Überlastung }} \\
\frac{\text { am Arbeitsplatz }}{\text { auftreten.“ }}\end{array}$ & 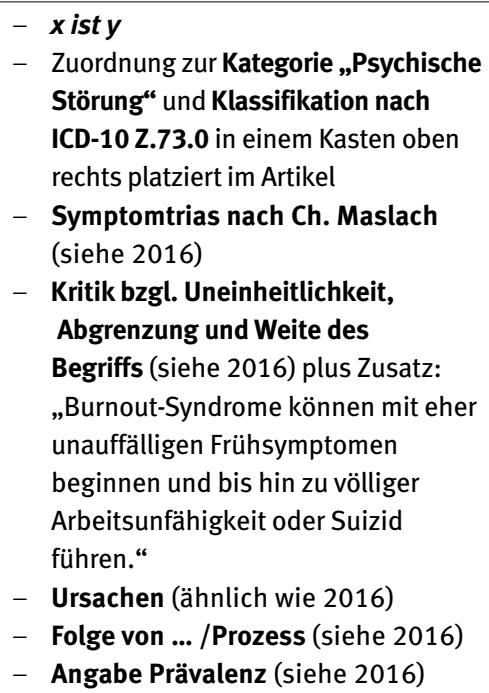 \\
\hline
\end{tabular}

Beim Definiensausdruck (siehe die 2. Spalte der Tabelle oben) zeigen sich einige Anpassungen über die Zeit. Bis Ende 2006 wird Burnout explizit als „Begriff“ definiert. 2007 fällt diese Angabe weg, was eine stärkere realdefinitorische Orientierung der Autorinnen und Autoren andeuten könnte. Interessant ist, dass im Jahr 2007 zudem der Ausdruck Syndrom an Burnout angehängt wird und der Definiensausdruck dadurch selbst ein Klassifizierunsgmerkmal bzw. eine Gattungszugehörigkeit erhält. Die Bezeichnung Burnout-Syndrom bleibt bis 
heute bestehen, rückt allerdings im Jahr 2017 an die dritte Stelle („Burn-out oder Burnout, auch Burnout-Syndrom“, Hervorhebung im Zitat entfernt, T.S.). Dass in den Jahren 2007 und 2008 der deutsche substantivierte Partizipialausdruck Ausgebranntsein verwendet wird, könnte damit zusammenhängen, dass in diesem Zeitraum der Informationskasten der ICD-10 oben im Artikel eingeblendet wird. Denn in der deutschen Version der ICD-10 lautet der Klassifizierungsschlüssel: „Z73 Ausgebranntsein [Burn-out]“. Auf den Einsatz dieses ICD-10-Kastens wird später in diesem Kapitel noch eingegangen.

Als Genera proxima dieser einleitenden Absätze (siehe die 3. und 4. Spalte der Tabelle oben) stellen sich heraus: Fall [...] Erschöpfung (2004-2007); Erschöpfung (2008); Zustand [...] Erschöpfung und Endzustand (2009-2010); Zustand [...] Erschöpfung, Endzustand und Problem (bei) der Lebensbewältigung (2011-2015); Zustand [...] Erschöpfung, Endzustand und Zusatzdiagnose (2016); Oberbegriff für Typen ... Krisen (2017-2019). Die Artikel setzen den Begriff `Burnout` damit von 2004 bis 2016 durchgängig aus der Perspektive eines Endzustands ins Licht. 2017-2019 wird aus der Perspektive des Endzustands die Perspektive des Wendepunkts durch das Hyperonym Krise.

Ab dem Jahr 2009 bis 2016 basiert der erste Definitions-Abschnitt auf der Definition des Klinischen Wörterbuchs Pschyrembel (2612007, siehe Beleg 4 in Kap. 6.2.2.1). Die Definition aus dem Pschyrembel wird nicht wörtlich zitiert, sondern es werden einzelne dort genannte Symptome und Phasen übernommen und in eine eigene weniger elliptische Satzstruktur gebracht. Zum direkten Vergleich sei im Folgenden nochmals die Definition aus der 261. Auflage des Pschyrembel und danach der Wikipedia-Eintrag aus dem Jahr 2009 zitiert. In diesem ist grau hinterlegt, welche Formulierungen von Wikipedia hinzugefügt wurden bzw. an welchen Stellen der Pschyrembel-Eintrag verändert wurde:

Pschyrembel, Klinisches Wörterbuch ( ${ }^{261}$ 2007): 295 (graue Hinterlegung T.S.):

Burn|out-Syn|drom ( $\uparrow$; engl. out aus; Syndrom) n;

Burned-out-Syndrom, auch Ausbrennen; Zustand emotionaler Erschöpfung, reduzierter Leistungsfähigkeit u. evtl. Depersonalisierung inf. Diskrepanz zw. Erwartung u. Realität; Endzustand eines Prozesses von idealist. Begeisterung über Desillusionierung, Frustration u. Apathie; Häufigkeit: geschätzte Prävalenz ca. 10 \% der Arbeitnehmer mit entspr. Berufen (20-30\% aller Arbeitnehmer sind gefährdet); Sympt.: psychosomati. Erkr., Depression od. Aggressivität, erhöhte Suchtgefahr; Ther.: im fortgeschrittenen Stadium Psychotherapie; bei gleichzeitiger Depression Antidepressiva

Wikipedia, 26. Dezember 2009, Eintrag zu „Burnout“ (graue Hinterlegung, T.S., Unterstreichungen zur Kennzeichnung von Links im Orig., Links im Original blau, hier schwarz)

Ein Burnout-Syndrom (engl. (to) burn out: ,ausbrennen“) ist ein Zustand ausgesprochener emotionaler Erschöpfung mit reduzierter Leistungsfähigkeit, das als Endzustand einer Ent- 
wicklungslinie bezeichnet werden kann, die mit idealistischer Begeisterung beginnt und über frustrierende Erlebnisse zu Desillusionierung und Apathie, psychosomatischen Erkrankungen und Depression oder Aggressivität und einer erhöhten Suchtgefährdung führt. ${ }^{[1]}{ }^{1]}$

Burnout kann nahezu alle sozialen Gruppen treffen - von Schülern über Forscher bis hin zu Arbeitslosen und Rentnern sind Krankheitsfälle bekannt. ${ }^{618}$

Der Wikipedia-Eintrag fügt auf der einen Seite zum Pschyrembel-Eintrag Verben und eine lexikalische Steigerungsform (ausgesprochener emotionaler Erschöpfung) hinzu, und er stellt semantische Beziehungsgefüge zwischen Symptomen und Prozessstufen her, die im Pschyrembel-Eintrag durch Aufzählung offen gehaltenen werden (im Wikipedia-Eintrag wird das zweite Symptom reduzierte Leistungsfähigkeit durch die Präposition mit zum Nebensymptom des Hauptsymptoms Erschöpfung und die frustrierenden Erlebnisse führen zur Desillusionierung). Auf der anderen Seite lässt der Wikipedia-Eintrag aber auch einige Angaben des Pschyrembel-Eintrags unerwähnt: So z.B. das dritte Symptom „Depersonalisierung“, das im Pschyrembel mit der Kennzeichnung „evtl.“ als nicht gänzlich gesichert angegeben wird, und die Angaben zur Prävalenz und Therapie. In den hier verglichenen Artikelfassungen findet man diesen Bezug auf den Pschyrembel per Fußnote, aber ohne genaue Kennzeichnung des Zitierten bis in Jahr 2015. Wikipedia präsentiert diese auf dem Pschyrembel basierenden Informationen in einer unpersönlichen Satzstruktur im Präsens Indikativ als intersubjektives und monolithisches fachliches Wissen. Der Verweis auf das klinische Wörterbuch in der Fußnote dient als Autoritätsanker. Im Jahr 2016 wird im Fließtext ergänzt, dass Burnout ,in medizinischen Lexika“ auf diese Weise beschrieben werde.

Ab dem Jahr 2017 wird der erste Absatz im Wikipedia-Artikel deutlich überarbeitet. Dies liegt jedoch vor allem daran, dass ab diesem Jahr nicht mehr die Definition in Anlehnung an den Pschyrembel zitiert wird, sondern ab diesem Jahr das Psychologische Wörterbuch DORSCH in der 16. Auflage als Grundlage herangezogen wird (Burisch im Dorsch ( ${ }^{16} 2013:$ 312f.); vgl. auch Beleg 7 in Kap. 6.2.2.1). Der Eintrag im DORSCH wurde von Matthias Burisch geschrieben, der im deutschen Diskurs viele Texte zum Thema „Burnout“ verantwortet und in verschiedenen Publikationsformen fachintern und fachextern zitiert wird. In Kap. 6.2.4.2 wird daran anknüpfend der Frage nachgegangen, inwiefern sich in solchen intertextuellen Bezugnahmepraktiken und intertextuellen Beziehungsgefügen Foucault'sche Prinzipien der Verknappung wie z. B. „,der Autor als Prinzip der Gruppierung von Diskursen“ (Foucault [1970] 1993: 20) oder der Disziplinen zeigt.

618 Artikelfassung vom 26.12.2009 auf Wikipedia zu >Burnout unter: https://de.wikipedia. org/w/index.php?title=Burn-out\&oldid=68443289\#cite_note-1 (zuletzt eingesehen am 3.12.2019). 
Des Weiteren wird es in Kap. 6.2.4.2 um die Frage gehen, ob das Prinzip „Autor“ und weitere Verknappungs- und Ausschließungsprozeduren zur Durchsetzung bestimmter definitorischer Konzeptualisierungen beitragen.

Die konstanten Verweise auf die ICD-10 ab dem Jahr 2004 zeigen, dass das internationale Klassifikationssystem der Krankheiten eine einflussreiche Autoritätsinstanz für die Definition auf Wikipedia darstellt. In den Jahren 2004-2006 wird im Fließtext ${ }^{619}$ der Artikelfassungen darauf verwiesen, dass „Burn-out“ in der „ICD-10 [...] als ,Ausgebranntsein“ und ,Zustand der totalen Erschöpfung“ mit dem Diagnoseschlüssel Z73.0 erfasst“ würde. In diesen Jahrgängen wird die ICD-10 eindeutig geltungsverstärkend angeführt. Ab dem Jahr 2007 wird der Burnout-Artikel innerhalb der Wikipedia-Plattform der Kategorie „Psychische Störung“ zugeteilt. ${ }^{620}$ Und ab dieser Zeit erscheint rechts oben im Artikel auch ein typografisch deutlich hervorgehobener Kasten, der die ICD-10-Kennung für Burnout angibt. Die Beschriftung dieses Kastens erfährt leichte Änderungen, die für die Klassifizierung und die deklarative Kraft der Definition von >Burnout als „Diagnose“ bedeutsam sind (siehe die Abbildungen 13-16):

$2007^{621}$

\section{Klassifikation nach ICD-10 \\ Z73.0 Ausgebranntsein}

Abb. 13: Kasten mit ICD-Kennung im Wikipedia-Beitrag zu >Burnout vom 29.12.2007 (zuletzt eingesehen am 3.12.2019), CC BY-SA 3.0 (https://creativecommons.org/licenses/by-sa/3.0/ legalcode).

619 In den Fassungen vom 31.12.2004 und 21.12.2005 zwei Absätze nach dem Inhaltsverzeichnis, in der Fassung vom 30.12.2006 im Absatz vor dem Inhaltsverzeichnis. Der Wikipediatext ist unter der Lizenz CC BY-SA 3.0 verfügbar.

620 Die Kategorie „Psychische Störung“ wurde auf Wikipedia am 02.05.2005 angelegt: https:// de.wikipedia.org/w/index.php?title=Kategorie:Psychische_St\%C3\%B6rung\&oldid=5616555 (zuletzt eingesehen am 24.6.2019).

621 In den Kästen gibt es jeweils noch eine Verlinkung auf die ICD-10, welche in den Abbildungen abgeschnitten wurde. Diese entspricht trotz alter Versionen des Wikipedia-Eintrags zu $>$ Burnout jeweils der neuesten ICD-10-Version zur Zeit des Abrufdatums. 
2008 (gleich 2009, ähnlich $2010^{622}$ )

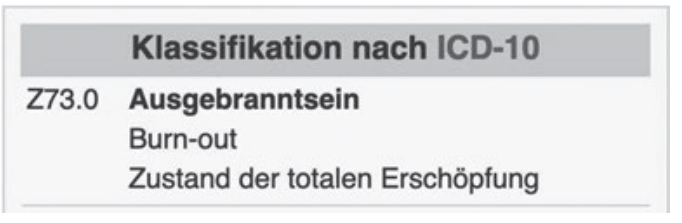

Abb. 14: Kasten mit ICD-Kennung im Wikipedia-Beitrag zu >BURNOUT` vom 30.12.2008 (zuletzt eingesehen am 3.12.2019), CC BY-SA 3.0 (https://creativecommons.org/licenses/by-sa/3.0/ legalcode).

2011 (gleich 2012, 2013, 2014, 2015)

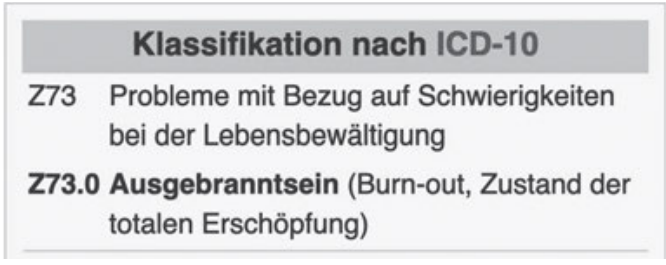

Abb. 15: Kasten mit ICD-Kennung im Wikipedia-Beitrag zu >BURNOUT vom 27.12.2011 (zuletzt eingesehen am 3.12.2019), CC BY-SA 3.0 (https://creativecommons.org/licenses/by-sa/3.0/ legalcode).

2016 (gleich 2017, 2018, 2019623)

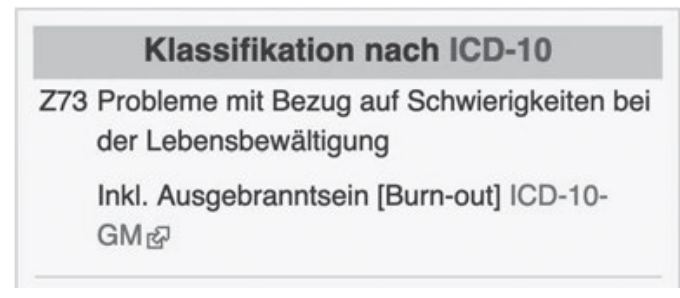

Abb. 16: Kasten mit ICD-Kennung im Wikipedia-Beitrag zu >BURNOUT` vom 28.12.2016 (zuletzt eingesehen am 3.12.2019), CC BY-SA 3.0 (https://creativecommons.org/licenses/by-sa/3.0/ legalcode).

622 Im Jahr 2010 wird allerdings noch hinzugefügt, dass es „in der englischen Originalfassung: 'state of vital exhaustion'“ heißen würde.

623 In der Artikelfassung vom 24.6.2019. 
Im Jahr 2007 wird im Kasten nur die deutsche Übersetzung „Ausgebranntsein“ zusammen mit der Kennung Z73.0 angegeben (siehe Abbildung 13). Im Fließtext im dritten Absatz wird allerdings schon darauf hingewiesen, dass Burnout trotz dieses Diagnoseschlüssels „nicht als eigenständige Erkrankung anerkannt würde“. ${ }^{624}$ Im Jahr 2008 und 2009 wird diese distanzierende Anmerkung in das letzte Kapitel „Burnout in der ICD“ des Wikipedia-Eintrags verschoben. ${ }^{625} \mathrm{Ab}$ dem Jahr 2010 werden die genannten Distanzmarker wieder in den ersten oder zweiten Absatz zu Beginn des Wikipedia-Artikels zu >Burnout $\iota$ und ab 2017 in eines der ersten Unterkapitel gestellt. ${ }^{626}$

Ab 2008 erfolgt in den ICD-Kästen zu Beginn der Artikel außerdem eine Expansion durch die Erläuterung „Zustand der totalen Erschöpfung“ (siehe Abbildung 14). Ab dem Jahr 2011 wird die Überkategorie von Z73 innerhalb der ICD-10 ebenfalls im Kasten angezeigt (siehe Abbildung 15): „Probleme mit Bezug auf Schwierigkeiten bei der Lebensbewältigung " und im Fließtext wird erläutert, dass Burnout demnach eine „Rahmen- oder Zusatzdiagnose und keine (!) Behandlungsdiagnose“ darstelle. Nur letztere könne „zum Beispiel die Einweisung in ein Krankenhaus ermöglichen.“627 Ein Jahr später werden im Abschnitt „Burn-out in der ICD-10“ noch weitere ICD-Diagnosen angeführt, die als „Behandlungsdiagnosen“ mit dem „Burnout-Syndrom“ in Bezug gebracht werden bzw. von diesem abgegrenzt werden könnten:

624 Vgl. die Artikelfassung vom 29.12.2007 auf Wikipedia zu >Burnout` unter: https://de.wikipedia.org/w/index.php?title=Burn-out\&oldid=40543491 (zuletzt eingesehen am 3.12.2019).

625 Burnout wird als „Einflussfaktor“, aber nicht als „Syndrom“ klassifiziert, da er dem Abschnitt $Z$ zugewiesen sei, der Faktoren beinhalte „, die den Gesundheitszustand beeinflussen und zur Inanspruchnahme des Gesundheitssystems führen““. Vgl. die Artikelfassung vom 30.12.2010 auf Wikipedia $\mathrm{zu}>$ Burnout $\iota$ unter: https://de.wikipedia.org/w/index.php?title=Burn-out\&oldid=83232737 (zuletzt eingesehen am 3.12.2019).

626 Die genauen Formulierungen in den Artikelversionen lauten: „keine Krankheit mit eindeutigen diagnostischen Kriterien, sondern eine körperliche, emotionale und geistige Erschöpfung aufgrund [...]“ (30.12.2010); „keine Krankheit, sondern ein Problem der Lebensbewältigung“ (27.12.2011); , ,ist international nicht als Krankheit anerkannt, sondern gilt als Problem der Lebensbewältigung“ (30.12.2012); , ist wissenschaftlich nicht als Krankheit anerkannt, sondern gilt im ICD-10 als ein Problem der Lebensbewältigung“(31.12.2013; 16.12.2014; 16.12.2015); „Das Burnout-Syndrom gilt nicht als Krankheit im Sinne des ICD-10, sondern ist eine Zusatzdiagnose, die Probleme mit Bezug auf Schwierigkeiten bei der Lebensbewältigung bezeichnet.“ (28.12.2016); ab dem Jahr 2017 erscheint der Hinweis im 4. Unterkapitel („Burnout als Diagnose“) des Burnout-Artikels: „Burnout ist im Unterschied zu Depression keine Behandlungs-, sondern eine Rahmen- oder Zusatzdiagnose.“ (21.12.2017); ähnlich in den Fassungen vom 2.12.2018 und 24.6.2019 (Unterstreichungen zur Kennzeichnung von Links in den Originalzitaten., Links im Orig. blau, hier schwarz).

627 Vgl. die Artikelfassung vom 27.12.2011 auf Wikipedia zu >Burnout $\iota$ unter: https://de.wikipedia.org/w/index.php?title=Burn-out\&oldid=97607282 (zuletzt eingesehen am 3.12.2019). 


\begin{abstract}
Dagegen ist die Feststellung einer Depression eine Behandlungsdiagnose. Ein reines Burnout-Syndrom ist ein Ausschlusskriterium für eine Neurasthenie (F48.0), ${ }^{[23]}$ die in der Diagnose aber als Burnout-basiert beschrieben wird und die Leistungspflicht eines Krankenversicherers begründen kann. Auch wird die Depersonalisierung (s. u. und F48.1) ${ }^{[23]}$ als ein mögliches Symptom des Burnouts betrachtet.

(Aus der Artikelversion zu „Burn-out“ vom 30.12.2012 der Online-Enzyklopädie Wikipedia, graue Hinterlegung T.S., Unterstreichungen zur Kennzeichnung von Links im Orig., Links im Orig. blau, hier schwarz)
\end{abstract}

Diese abgrenzende Klassifizierung wird mit leichten Veränderungen bis 2018 beibehalten. Ab 2016 bezieht der Kasten sich schon vorab auf die ICD-10-Onlineversion der WHO des Jahres 2019 (siehe Abbildung 16) und im Fließtext wird erwähnt, dass Burnout auch im DSM-5, dem diagnostischen und statistischen Handbuch psychischer Störungen der American Psychiatric Association, nicht „als eigenständige Diagnose aufgeführt“ sei. Im Jahr 2019 (Fassung vom 22.6.2019) ${ }^{629}$ gibt es schließlich wiederum eine Neuerung, die durch die ICD-11 entstanden ist, die die ICD-10 ab dem Jahr 2022 ablösen soll. Burnout bleibt ein Zusatzfaktor bzw. eine Zusatzdiagnose ${ }^{630}$, aber wird in der ICD-11 eindeutig als Syndrom der Arbeitswelt beschrieben:

\title{
Aus der Artikelversion zu Burnout vom 22.6.2019 der Online-Enzyklopädie Wikipedia
}

In der Version ICD-11, die ab Januar 2022 gelten soll, ist Burn-Out als Syndrom aufgrund von „Stress am Arbeitsplatz, der nicht erfolgreich verarbeitet werden kann“ definiert. ${ }^{[24]}$ Dabei heißt es ausdrücklich, das Syndrom solle nicht verwendet werden, um Erfahrungen in anderen Lebensbereichen zu erfassen, sondern auf den Arbeitsplatz beschränkt sein. ${ }^{[25]}$

Die vollständige Definition aus der ICD-11 reformuliert zudem die Symptomtrias, die auf Maslach/Jackson (1981) zurückgeht (siehe Beleg 12 in Kap. 6.2.2.2). In den Kapiteln 6.2.4.2 wird anhand von intertextuellen Bezugnahmepraktiken analysiert, wie sich diese drei Symptome im Burnoutdiskurs durchsetzen konnten, sodass sie nun in die ICD-11 aufgenommen wurden. Zuvor werden im Vergleich zu den Artikelfassungen der Wikipedia noch die sprachlichen Mittel des Definierens der Einträge zum Phänomen/Konzept >BURNouT` auf den Internetportalen „NetDoktor“ und „Onmeda“ analysiert.

628 Siehe die Artikelfassung vom 30.12.2012 auf Wikipedia zu `Burnout unter: https://de.wikipedia.org/w/index.php?title=Burn-out\&oldid=112270018 (zuletzt eingesehen am 3.12.2019).

629 Siehe die Artikelfassung vom 22.6.2019 auf Wikipedia zu >Burnout $<$ unter: https://de.wikipedia.org/w/index.php?title=Burn-out\&oldid=189780829 (zuletzt eingesehen am 3.12.2019).

630 Die ICD-11-Kategorie, in die Burnout eingeordnet ist, lautet: "Problems associated with employment or unemployment”, siehe: https://icd.who.int/browse11/l-m/en\#/http\%3a\%2f\%2fid.who. int\%2ficd\%2fentity\%2f129180281 (zuletzt eingesehen am 3.12.20, = WHO 2019b, im QV unter 8.1.3). 
Die zuletzt aktualisierten Fassungen der Einträge $\mathrm{zu}>$ Burnout auf diesen Plattformen (NetDoktor vom 28. Juni $2018^{631}$ und Onmeda vom 14.02.2019 ${ }^{632}$ ) weisen sowohl Unterschiede als auch Ähnlichkeiten zu den Wikipedia-Einträgen auf. Sowohl auf NetDoktor als auch auf Onmeda wird auf die ICD-10-Kennung verwiesen. Die Artikel ähneln den Wikipedia-Einträgen in der Kapitelstruktur. Sie weisen einen Einführungsabschnitt, Abschnitte zu Symptomen, Ursachen, Diagnosen, Phasen/Verlauf, Testung/Messung, Therapie, Prognose und Prävention auf. $\mathrm{Zu}$ Beginn der Artikel geben sie folgende zusammenfassende Kurzdefinitionen:

Onmeda, 14.02.2019, = Nagel (2019) (Unterstreichung, T.S., sonstige Hervorhebungen im Original, im QV unter 8.1.8)

\section{Was ist das Burnout-Syndrom?}

Unter dem Begriff Burnout-Syndrom versteht man einen Zustand totaler körperlicher, emotionaler und geistiger Erschöpfung sowie verringerter Leistungsfähigkeit. Übersetzt bedeutet der aus dem Englischen stammende Begriff Burnout so viel wie „Ausbrennen“. Menschen mit Burnout fühlen sich extrem erschöpft und innerlich leer.

NetDoktor, 28.06.2018, = Dobmeier/Fux 2018 (Unterstreichung, T.S., im QV unter 8.1.8)

Burnout beschreibt einen Zustand tiefer emotionaler, körperlicher und geistiger Erschöpfung. Die Betroffenen können sich nur schlecht konzentrieren, machen viele Fehler. Manche verlieren auch die Energie für ihr Privatleben. Burnout wird meist auf Überforderung und Stress im Beruf zurückgeführt. Die Ursachen sind aber vielfältig. Wie Sie Burnout erkennen, wie Sie sich schützen und Burnout überwinden, erfahren Sie hier.

Die Definition auf Onmeda beginnt mit einer Was-ist-Frage (siehe dazu Kap. 6.2.2.2, 224-225). Für den ersten Satz der Definition wählen sowohl die Autorin auf Onmeda als auch die Autorinnen auf NetDoktor das ,klassische‘ Definitionsschema. Sie ähneln darin den Definitionen in den Wikipedia-Artikeln, und sie definieren Burnout primär als einen Erschöpfungszustand, der durch attributive Ergänzungen noch weiter spezifiziert wird. Die Symptome werden in beiden Definitionen im Anschluss personalisiert, indem aufgezählt wird, wie sich „Menschen mit Burnout“ bzw. die „Betroffenen“ fühlen oder welche Verhaltens- und Erlebensweisen diese zeigen. NetDoktor gibt noch einen Hinweis auf die Entstehung von Burnout und spricht die Leser/innen im letzten Satz direkt an. Auch im Beitrag von Onmeda werden die Leser/innen im späteren Textverlauf direkt angesprochen (,Beleuchten Sie Ihren

631 Dieser Artikel ist abrufbar unter: https://www.netdoktor.de/krankheiten/burnout/ (zuletzt eingesehen am 24.11.2019).

632 Dieser Artikel ist abrufbar unter: https://www.onmeda.de/krankheiten/burnout_syndrom. html (zuletzt eingesehen am 24.11.2019). 
eigenen Perfektionsdrang und fahren Sie diesen falls notwendig runter." Hervorh. entfernt, T.S.). Durch diese zuletzt genannten Vermittlungsmethoden (Personalisierung und Leseransprache) unterscheiden sich die Artikel auf Onmeda und NetDoktor von den Wikipedia-Artikelfassungen, in denen die unpersönliche Schreibweise jeweils in den Artikelversionen beibehalten wird. Die Artikel auf Onmeda und NetDoktor stellen dadurch hybride Textformen zwischen Wikipedia und ratgebenden Aufklärungstexten dar. Denn sie kombinieren Darstellungsformen, die man in den Wikipedia-Artikeln findet, mit einigen Vermittlungsmethoden von medialen Vermittlungstextsorten (vgl. Kap. 6.2.3.1), wobei sie in der Personalisierung jedoch nicht so weit gehen, dass sie ihre Übersichtsbeiträge $\mathrm{zu}$ `Burnout mit einem Fallbeispiele oder verschiedenen narrativen Passagen einleiten.

Wie gezeigt wurde, weisen die sich punktuell manifestierenden Definitionen in den medialen Textsorten und auf Wikipedia, NetDoktor und Onmeda Gemeinsamkeiten, aber auch Unterschiede auf. Diese Gemeinsamkeiten und Unterschiede werden im folgenden Kapitel nochmals zusammengefasst und in diesem Zuge auch mit den sprachlichen Mitteln des fachinternen Diskursstrangs (vgl. Kap. 6.2.2.4) verglichen. Dazu werden die sprachlichen Mittel des Definierens für den gesamten fachexternen Diskursstrang tabellarisch zusammengeführt und im Rahmen des praxeologischen Analysevorgehens dieser Arbeit heuristisch mit den Teilpraktiken und sprachlichen Stimuli des Definierens, die sich in der Fläche der Diskursstränge zeigen und in den Kapiteln 6.2.4.1 und 6.2.4.2 beschrieben werden, verknüpft.

\subsubsection{Zusammenfassung sprachlicher Mittel des Definierens im fachexternen Diskursstrang aus punktueller Perspektive und Ausblick auf sprachliche Stimuli des Definierens in der Fläche der Diskursstränge}

In den letzten beiden Kapiteln wurden in Pressetexten, populärwissenschaftlichen Zeitschriften und Onlineportalen sprachliche Mittel von der Morphem- bis zur Textabschnittsebene beschrieben, die definitorische Aufgaben erfüllen.

Auf Wikipedia und den Online-Portalen dominieren ähnlich wie in den Fachlexika, Hand- und Lehrbüchern Formulierungen wie: der Begriff Burnout bezeichnet einen Zustand ... ; mit Burnout wird ein Zustand ... bezeichnet oder ein Burnout-Syndrom ist ein Zustand .... Diese syntaktischen Muster führen zu deskriptiven, kategorisierenden und taxonomisch-klassifizierenden Definitionen, da sie den zu definierenden Ausdruck bzw. Begriff (Burnout/Burn-out/Burnout-Syndrom) über das zugeordnete abstrahierende Nomen und dessen Spezifizierung (Zustand tiefer emotionaler, körperlicher und geistiger Erschöpfung) einem Bereich (PHYSISCHE(S) UND PSYCHISCHE(s) RESSOURCEN UND WOHLERGEHEN) zuordnen oder den Begriff in eine stabile taxonomische Ordnung wie zum Beispiel die ICD-10 eingliedern (z. B. dadurch, dass Burnout nach der ICD-10 keine Behandlungs-, sondern 
eine Zusatzdiagnose bezeichnet). Auf den Online-Portalen Onmeda und NetDoktor und in den Wikipedia-Artikelfassungen seit dem Jahr 2006 wird im Kotext der Definition auf die ICD-10 explizit Bezug genommen (s. Kap. 6.2.3.2). In einigen Fällen wird durch die Wahl des zu definierenden Ausdrucks bereits selbst eine Einordnung vorgenommen. Dies ist der Fall, wenn der Ausdruck Burnout-Syndrom definiert werden soll, denn das Determinativum Syndrom verortet die nachfolgende Definitionshandlung direkt im medizinischen Bereich.

Die Richtung der definierenden Bestimmung des Gegenstands erfolgt in den Onlineportalen vom Allgemeinen zum Speziellen, ähnlich wie in den Fachlexika, Hand- und Lehrbüchern und den meisten fachlichen Übersichtsartikeln und Originalarbeiten (vgl. Kap. 6.2.2.1-6.2.2.4). Die Definitionsentfaltung basiert meist auf einer Zustandsbeschreibung, die um typische Prozessstadien und ursächliche Faktoren auf dem ,Weg` zum Endzustand `Burnoutı ergänzt wird.

Interessant ist des Weiteren folgende Entwicklung, die man an den Definitionen der Wikipedia-Artikelversionen von 2004 bis 2019 beobachten kann: Zu Beginn werden die Definitionen ohne Quellverweis im unpersönlichen, wissenschaftlichen Stil als gesicherte Definitionen „der einen Wissenschaft“ (Liebert 2002: 11) präsentiert bzw. Distanzierungsmarker werden erst am Ende des Artikels und dadurch nicht im direkten Kotext der Definition platziert (so in den Artikelfassungen der Jahre 2008 und 2009). Seit dem Jahr 2010 wird diese Praxis dahingehend verändert, dass die Debatte um den Krankheits- bzw. Diagnosestatus von >Burnout « mit Bezug auf die ICD-10 und die Diskussion um einzelne Symptome und Ursachen in Teilen schon in die definierenden Abschnitte mit aufgenommen wird. Bei der Zurückweisung des Hyperonyms Krankheit bzw. Behandlungsdiagnose für Burnout und der Substitution durch Hyperonyme wie Problem der Lebensbewältigung oder Zusatzdiagnose wird auf die ICD-10 verwiesen, welche in diesem Fall jedoch wiederum als einzelne, allgemeingültige fachliche Stimme inszeniert wird (vgl. Kap. 6.2.3.2).

Das dominante Definitionsentfaltungsmuster in den meisten Magazinberichten und vielen ratgebenden Aufklärungstexten und Problemdarstellungen der untersuchten Zeitungen und Zeitschriften setzt sich deutlich vom Definitionsmuster der Onlineportale ab. In Kap. 6.2.3.1 wurde herausgearbeitet, dass in diesen Textsorten häufig narrativ über Fallbeispiele mit anschließendem Deutungskommentar definiert wird.

Diese Definitionen beginnen beim konkreten individuellen Beispiel. Meist wird die Geschichte eines Individuums, das zudem Vertreter/in einer beruflichen Rolle ist, erzählt. Die Erzählung läuft entweder auf einen Wendepunkt zu oder es wird eine Vorher-Nachher-Konstellation geschildert, wobei der vorige Zustand durchweg positiver ist als der Folgezustand. Abgeschlossen wird die Beispielgeschichte häufig mit einem Deutungskommentar, der von einer ärztlichen, therapeutischen Expertin/ einem Experten im Rahmen der Erzählung geäußert wird. Sie leiten von dieser Bei- 
spielerzählung zudem vielfach zum intersubjektiven, verallgemeinerbaren Zustand oder Prozess über, d.h., dass diese Beispielerzählung auch auf andere Personen bestimmter Berufsgruppen oder alle Personen übertragen wird. Von Fallbeispielen aus Fachzeitschriften unterscheiden sie sich unter anderem dadurch, dass sie erzählerisch Spannung erzeugen oder Kontrastlinien innerhalb der Erzählung durch gezielte Tempuswechsel oder sprachliche Mittel der Graduierung und Quantifizierung kreieren (zu den weiteren Unterscheidungsmerkmalen, siehe Kap. 6.2.3.1).

In Zeitungsmeldungen oder -berichten über Erhebungen, Umfragen, prominente Burnout-Fälle oder Studien und Interviews wird `Burnout «meist nur knapp in einem oder wenigen Sätzen definiert:

- In Interviews und im Titelbereich der Medientexte findet man häufig Frage-Antwort-Muster, wobei die Fragemuster stärker auf die Leser/innen bezogen sind als in Fachtexten und deren Orientierungsbedürfnis befriedigen sollen ${ }^{63}$;

- In Berichten über Sportler/innen oder Trainer/innen, die aufgrund von Burnout ausfallen, werden meist nur ein bis zwei Hauptsymptome angegeben. Dabei geht es in der Regel darum, dass die „Energiereserven“ oder die „Power“ erschöpft sind und dass diese für die Erfüllung der Anforderungen im Hochleistungssport nicht mehr ausreichen;

- Vergleicht man die Definitionen dieser Medientexte mit den Formen des Definierens, die aus wissenschaftsgeschichtlicher sowie linguistischer und terminologischer Literatur zusammengefasst wurden (vgl. Kap. 4.2.3 und 4.2.4), so wird deutlich, dass die fachexternen Definitionen in der Regel verschiedene Formen (also z. B. die klassische, genetische und ostensive Definition) verbinden. Man findet Kombinationen aus paradigmatischen Einzelbeispielen (Def. durch Beispiel), einer Übersetzung des Ausdrucks Burnout (Def. durch Übersetzung), durch Besetzung derselben Referenzstelle mit einem Hyperonym, einer Aufzählung typischer psychischer und somatischer Symptome (ostensive Def.) und einer knappen Angabe von Entstehungsbedingungen und persönlichen riskanten Eigenschaften (genetische Def.).

- Der Frage der 'korrekten' 'allgemeingültigen' taxonomisch-klassifizierenden Einordnung (in der Regel mit Bezug auf die ICD-10) wird in den Medientexten ab den 2010er Jahren neben anderen Formen des Definierens große Bedeutung beigemessen (siehe Kap. 6.3.1.1). Diese geht einher mit der Zurückweisung von Hyperonymen wie Diagnose oder Krankheit und der Angabe substitutiver Hyperonyme (z. B. Zusatzdiagnose, Risikozustand).

633 Insbesondere die folgenden Fragemuster findet man in den fachlichen Texten kaum: Wie-erkennen wir/erkenne ich $x$ ?; Was-ist-noch $x$ ? Was ist schon $y$ ? siehe Kap. 6.2.2.1 und 6.2.3.1. 
Bei den sprachlichen Mittel, die in den fachexternen Vermittlungstexten eingesetzt werden, um >Burnout v von 'normalen' 'alltäglichen' >ERScHöPFunGsZUSTÄNDEN` bzw. von 'normalem' >Verhalten und Erleben ‘ und 'normalen' >Arbeitsbedingungen dungen $\mathrm{zu}$ den sprachlichen Mitteln in Fachtexten aufzeigen, andererseits weisen einzelne Medientexte eine noch größere Varianz und Intensivierung der Symptome auf: Das heißt, dass die Charakterisierung von >Burnout in Medientexten häufig sowohl über Fokuspartikeln, Intensitätspartikeln und Abtönungspartikeln als auch über Adjektive und Adverbien, die die beschriebenen Sachverhalte quantifizieren oder steigern, sprachlich vielgestaltig ausgeformt wird. Mit dieser Fülle der sprachlichen Mittel geht einher, dass die SympтомE oder ENTSTEHUNGSBEDINGUNGEN zwar meist konkreter und häufig in höherer Steigerungsstufe als in den Fachtexten dargestellt werden, mit den dort genannten Symptomen und Entstehungsbedingungen aber große Ähnlichkeit aufweisen (siehe Kap. 6.2.3.1). ${ }^{634}$

In den Kapiteln 6.2.3.1 und 6.2.3.2 wurden schließlich sprachliche Mittel beschrieben, durch die einzelne Symptome des Konzepts >Burnout besonders hervorgehoben werden. Des Weiteren findet man Formulierungen, die explizit auf fachlichen Konsens verweisen oder sprachliche Strategien, um die fachliche Validität und intersubjektive Geltung der Definition zu unterstreichen. Diese sind in folgender Tabelle (siehe Tabelle 4) beschrieben zusammen mit den anderen sprachlichen Mitteln, die in exponierten Passagen an der Textoberfläche in den untersuchten fachexternen Texten definitorische Aufgaben übernehmen. Zusätzlich wird in der folgenden Tabelle, parallel zur zusammenfassenden Tabelle der definitorischen Sprachmittel im fachinterne Diskursstrang in Kap. 6.2.2.4, rekapituliert, welche abstrakt-idealen Erwartungen an eine (gute) Definition und damit an den Akt des Definierens als regelgeleiteten sozialen Prozess im öffentlichen Burnout-Diskurs selbst gestellt werden (Spalte 3) und welche Teil-Funktionen des Definierens daraus erwachsen (vgl. Kap. 4.2.2).

In Kapitel 6.2.2.4, in dem die sprachlichen Mittel des Definierens im fachinternen Diskursstrang zusammengefasst sind, wurde schon beschrieben, wie Spalte 2 und 3 analytisch aufeinander bezogen werden. Auch für den fachex-

634 Das Symptom „verminderte Leistungsfähigkeit“ (vgl. z. B. Barth 2001/2010: 70) wird beispielsweise konkret auf die Arbeit bezogen: „Die eigene Arbeit wird als wirkungslos erlebt.“ (FAZ, 19.4.2014, = Weiguny/Nienhaus 2014a.). Diese ,Übersetzungen` zwischen abstrakteren Symptombezeichnungen des fachlichen Diskursstrangs und medialen Ausformungen fußen auf Interpretationsakten und lassen sich in vielen Fällen nicht eindeutig zuordnen. 
Tab. 4: Sprachliche Mittel, die definitorische Aufgaben erfüllen (punktuelle Perspektive), und Ansprüche, die von Stimmen im Diskurs an die Tätigkeit des Definierens gestellt werden (im fachexternen-öffentlichen Diskursstrang).

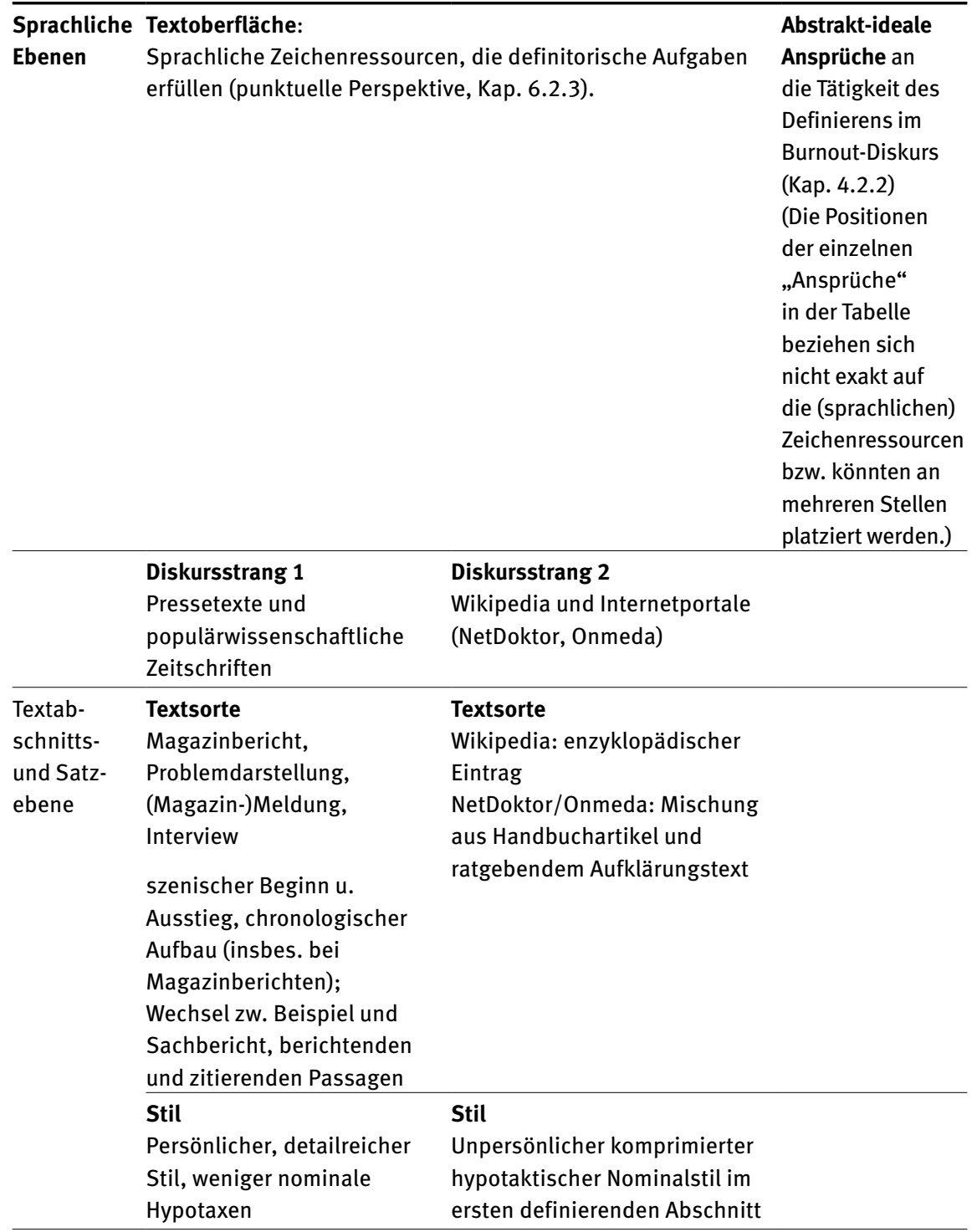


Tab. 4 (fortgesetzt)

\begin{tabular}{|c|c|c|}
\hline $\begin{array}{l}\text { Dominante } \\
\text { Definitionsentfaltungsmuster: } \\
\text { Eine sich exemplarisch } \\
\text { narrativ entfaltende Ereignis- } \\
\text { Definition plus } \\
\text { professionellem } \\
\text { verallgemeinerndem } \\
\text { (Diagnose-/Deutungs-) } \\
\text { Kommentar } \\
\text { zuordnend-prädizierende } \\
\text { Vertextungsmuster } \\
\text { durch anaphorische } \\
\text { oder kataphorische } \\
\text { verallgemeinernde } \\
\text { Deutungskommentare zur } \\
\text { erzählten bzw. folgenden } \\
\text { Ereigniskette: Irgendwann } \\
\text { ging gar nichts mehr [...]. } \\
\text { Der [Hausarzt] tippte auf } \\
\text { Burnout - als Folge von } \\
\text { Arbeitsüberlastung. }\end{array}$ & $\begin{array}{l}\text { Dominante } \\
\text { Definitionsentfaltungsmuster: } \\
\text { Eine sich deskriptiv entfaltende } \\
\text { (taxonomische) >Gattungs-- bzw. } \\
\text { (End-)Zustandsı-Definition } \\
\text { 1) Zuerst Nennung eines } \\
\text { abstrakteren Definiens- } \\
\text { Nomens: Zustand, Fall, } \\
\text { Erschöpfung } \\
\text { 2) Dann qualitätsattribuierende } \\
\text { Nominalphrasen oder } \\
\text { Relativsatzgefüge zur } \\
\text { Spezifizierung: totaler } \\
\text { körperlicher, emotionaler } \\
\text { und geistiger Erschöpfung } \\
\text { sowie verringerter } \\
\text { Leistungsfähigkeit } \\
\text { zuordnend-prädizierende } \\
\text { Satzmuster mit Verbgefügen } \\
\text { oder Satzzeichen, die } \\
\text { eine Entsprechungs- oder } \\
\text { Identifizierungs-Relation } \\
\text { herstellen (= Definitor): } \\
\text { Burnout, Burnout-Syndrom } \\
\text { bezeichnet y; mit B./B-S wird } \\
\text { y bezeichnet und B./B-S ist y; } \\
\text { unter B./B-S versteht man y; } \\
\text { B./B-S beschreibty }\end{array}$ & $\begin{array}{l}\text { Anspruch auf } \\
\text { exakte und } \\
\text { eindeutige Be- } \\
\text { stimmung und } \\
\text { Zuordnung }\end{array}$ \\
\hline
\end{tabular}

Weitere syntaktische Muster in den fachexternen Textsorten:

- zuordnend-prädizierende Satzmuster mit Verbgefügen oder Satzzeichen, die eine Äquivalenz- oder Identifizierungs-Relation herstellen

- Paradigmatische Bedeutungserläuterung über Synonyme und Übersetzung

- Aufzählung typischer Berufsgruppen, Gruppen von ,Betroffenen' (insbesondere Personen in ...)

- Aufzählung typischer, charakteristischer, häufiger Symptome und persönlicher und umweltbedingter Entstehungsbedingungen auch über konditionale Satzgefüge

- Was-ist-(schon/noch)-Frage-Antwort-Muster //

- Wie-erkennt-man-Frage-Antwort-Muster 
Tab. 4 (fortgesetzt)

- Negierte Äußerungseinheiten in Verbindung mit substitutiven Konnektoren zur Zurückweisung und anschließenden Ersetzung von Definiens-Nomina bzw. deren Rahmenkonzepten (keine Krankheit, sondern Risikozustand)

- Äußerungseinheiten mit adversativen, substitutiven und konzessiven Konnektoren, um Komponenten von >Burnouts von anderen ıZuständen ‘, >Merkmalen`, >Bedingungen ‘ zu unterscheiden.

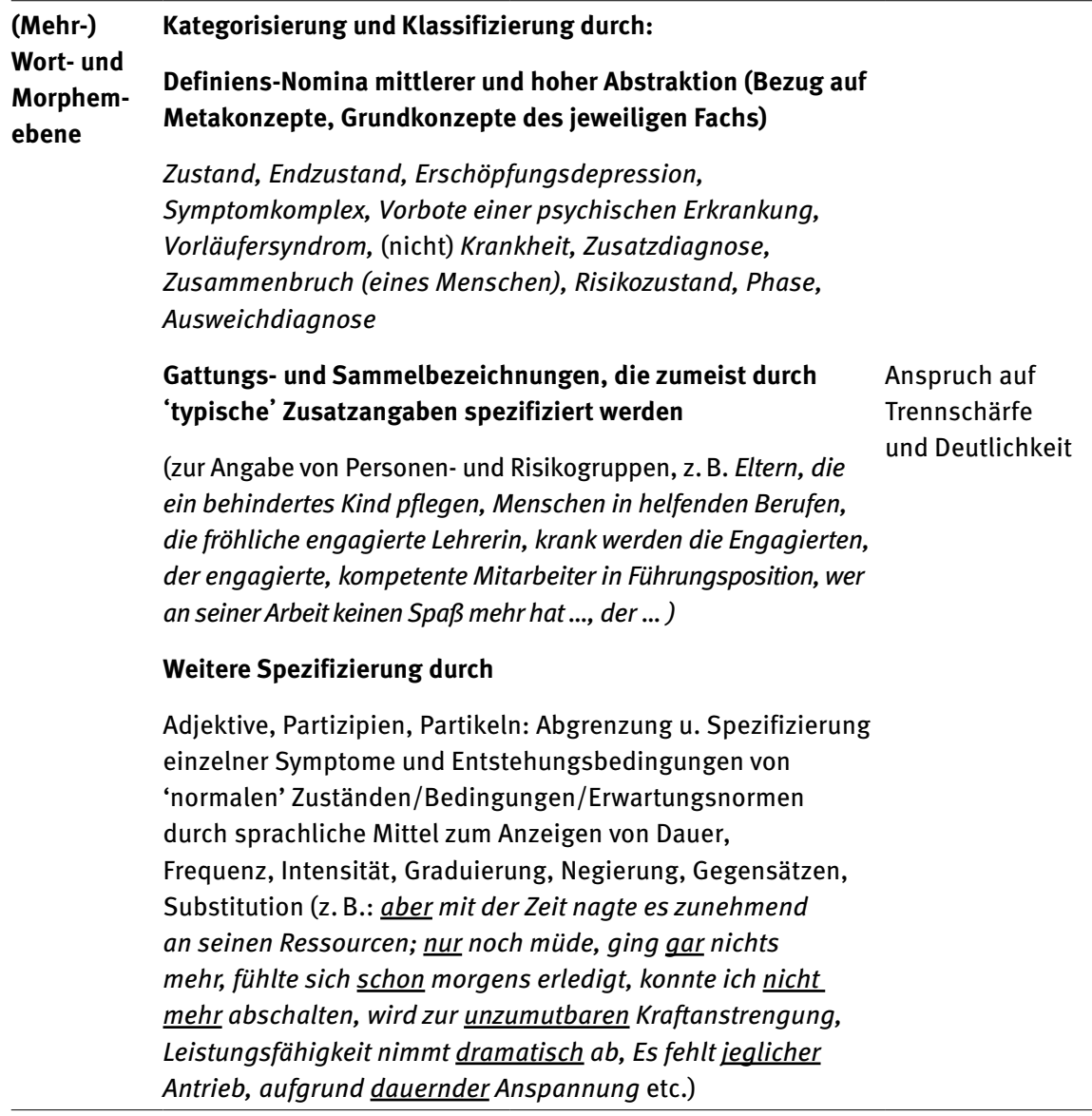


Tab. 4 (fortgesetzt)

\begin{tabular}{|c|c|}
\hline $\begin{array}{l}\text { Gewichtung einzelner Merkmale } \\
\text { das wichtigste Erkennungszeichen; ein deutliches Symptom } \\
\text { für Burnout; dessen zentrales Merkmal ..., dem für B. } \\
\text { typischen ... ; eines der wichtigsten Warnsymptome für Burn- } \\
\text { out; Was sind die ersten Warnzeichen? Alarmierend ist ... } \\
\text { Intersubjektivierung/Konsensstiftung durch } \\
\text { Weitgehende Einigkeit besteht heute darin, dass; Das ist } \\
\text { derzeit fachlicher Konsens }\end{array}$ & $\begin{array}{l}\text { Anspruch auf } \\
\text { angemessene } \\
\text { Umgrenzung und } \\
\text { Konzentration } \\
\text { auf wesentliche } \\
\text { Faktoren }\end{array}$ \\
\hline $\begin{array}{l}\text { Sprachliche Mittel des Geltungsanspruchs } \\
\text { - Außer in narrativen Passagen meist Präsens Indikativ } \\
\text { - Präteritum Indikativ in narrativen Passagen und in } \\
\text { zitierten Definitionen, in letzteren teilw. in Verbindung } \\
\text { mit Distanzmarkern, Wechsel vom Präteritum (erzählte } \\
\text { Welt, Vorzeitigkeit in Bezug auf den Zustand ıBurnoutı) ins } \\
\text { Präsens (besprochene Welt, Gleichzeitigkeit in Bezug auf } \\
\text { den Zustand ıBurnoutı) } \\
\text { - Rückbezug auf personalisierte Entdeckungsgeschichte } \\
\text { und den damit zusammenhängenden definierenden } \\
\text { Referenzfixierungsakt } \\
\text { - Rückbezug auf Expertentum bzw. die eine Wissenschaft } \\
\text { (vgl. Liebert 2002: 11) und (paraphrasierende) Zitate } \\
\text { von Expertendefinitionen (nach Untersuchungen von } \\
\text { Wissenschaftlern, } \\
\text { - Rückbezug auf Prävalenzangaben (Ansteigen von } \\
\text { psychosomatischen Erkrankungen wie Burnout ...) } \\
\text { Diagnosen wie „Depression“ oder „Burn-out“ führen zu } \\
\text { jeder zehnten Krankschreibung } \\
\text { - 'Unmittelbarkeit' und ‘Eigendynamik' der beschriebenen } \\
\text { Symptome und Bedingungen (Personen sind meist in } \\
\text { den semantischen Rollen des Patiens oder Experiencers); } \\
\text { Diagnose kommt von außen von Fachleuten }\end{array}$ & $\begin{array}{l}\text { Anspruch auf } \\
\text { eine neutrale, } \\
\text { objektorien- } \\
\text { tierte } \\
\text { Sachverhalts- } \\
\text { konstitution/ } \\
\text { Begriffsbildung }\end{array}$ \\
\hline
\end{tabular}

ternen Diskursstrang geht es darum, Praktiken zu eruieren, die zwischen den sprachlichen Zeichenressourcen und den abstrakt-idealen Ansprüchen an die Tätigkeit des Definierens vermitteln, dadurch dass sie einzelne Zwecke oder Aufgaben, die aus den Definitions-Ansprüchen erwachsen, mit verfügbaren sprachlichen Ressourcen verbinden. In Kap. 6.2.2.4 wurde deren praktisch-prozesshafter Charakter betont und erläutert, warum sich diese Praktiken am besten durch Verben benennen lassen (siehe unten Spalte 2 in Tabelle 5.1). Auch im fachexter- 
nen Diskursstrang ergeben sich zwei Gruppen definitorischer Praktiken, wenn man die verschiedenen Zwecke und Aufgaben, die aus den Ansprüchen an die Tätigkeit des Definierens erwachsen, mit den sprachlichen Ressourcen verbindet und bündelt: |definitorische Praktiken der Zuweisung, Einordnung sowie Ein- und Abgrenzung| (siehe Tab. 5.1) und |definitorische Praktiken der Verknappung, Gewichtung und Geltungsvalidierung| (siehe Tab. 5.2). In den folgenden zwei Tabellen werden diese Praktiken für den fachexternen Diskursstrang, d. h. ihre Bindegliedfunktion zwischen den punktuell herausgearbeiteten Zeichenressourcen (siehe Kapitel 6.2.3.1 und 6.2.3.2 und Tabelle 4) und Ansprüchen an die Tätigkeit des Definierens nochmals zusammenfassend dargestellt. Danach wird ein kurzer Ausblick auf die Kapitel 6.2.4.1 und 6.2.4.2 gegeben. In diesen Kapiteln wird es darum gehen, Zeichenkombinationen zu eruieren, die über Einzeltexte hinweg in der Fläche des Diskursstrangs wiederholt auftreten, definitionsähnliche Effekte stimulieren und die punktuellen Definitionsangebote dadurch verfestigen können.

Tab. 5.1: Exemplarische Herleitung der |definitorischen Teilpraktiken der Zuweisung, Einordnung sowie Ein- und Abgrenzung| (im fachexternen-öffentlichen Diskursstrang).

\begin{tabular}{lll}
\hline $\begin{array}{l}\text { Textoberfläche (punktuelle Perspektive, } \\
\text { Kap. 6.2.3.1 und 6.2.3.2) }\end{array}$ & Praktiken mittlerer Abstraktion & $\begin{array}{l}\text { Einzelne Ansprüche } \\
\text { an die Tätigkeit } \\
\text { des Definierens im } \\
\text { Burnout-Diskurs } \\
\text { (Kap. 4.2.2) }\end{array}$ \\
\hline
\end{tabular}

Onlineportale (bes. Wikipedia)

zuordnend-prädizierende Satzmuster wie

z. B. B./B.-S. bezeichnet y; unter B./B.-

S. versteht man $y ; \ldots$

1) Zuerst Nennung eines DefiniensNomens höherer Abstraktion (Zustand, Erschöpfung ...)

|Definiens u. Definiendum einander zuweisen/doppelt referieren|, ${ }^{635}$
Anspruch auf

Adäquatheit zwischen Definiens und Definiendum

635 Stanaitytè (2005: 97 f.) beschreibt diese Praktik des Zuweisens in Alltagsdefinitionen unter Rückgriff auf die Satzsemantik von P. v. Polenz (1985) folgendermaßen: „Wenn man davon ausgeht, dass Alltagsdefinitionen eine lexikalische Einheit in ihrer Bedeutung erläutern (aus der satzsemantischen Sicht betrachtet sind es Referenzstellen bzw. Bezugsobjekte), so hieße dies, dass das Explizieren einer Bedeutung ein Akt des doppelten Referierens ist: zunächst wird ein Bezug zu einer Referenzstelle hergestellt, worauf dann der zweite Akt des Referierens folgt, indem auf die gleiche Referenzstelle ein weiterer Bezug genommen wird, der sich von dem ersten dadurch unterscheidet, dass er andere Referenzausdrücke zur gleichen Bezugsstelle verwendet.“ 
Tab. 5.1 (fortgesetzt)

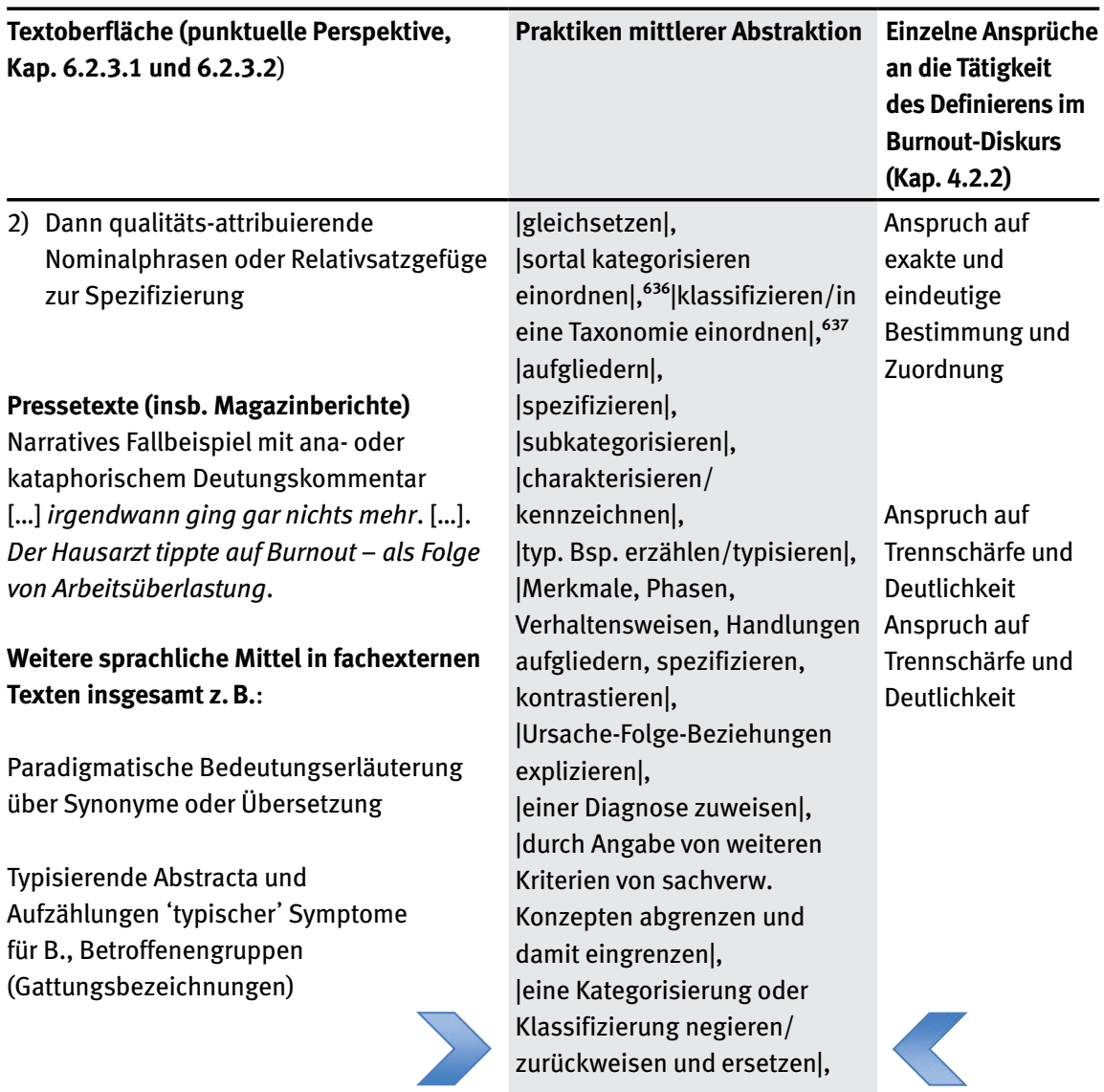

636 Die Praktik des |Kategorisierens| macht darauf aufmerksam, dass bei der Verwendung eines Definiens-Ausdrucks (z. B. Burnout ist ein Syndrom) verschiedene semantische Kategorisierungen vorgenommen werden: Durch die Wortart Substantiv beispielsweise wird eine „kategoriale Bedeutung“ transportiert: Denn diese Formulierung fordert uns dazu auf, das zu Definierende als etwas, „das eigenständig existiert und was sich im physischen Raum als eigenständige Größe auch isolieren lässt“, oder als „eigenständig betrachtete Größe im geistigen Raum“ zu verstehen (Köller 2004: 349 f.); Wenn man Hyperonyme dieser Definiens-Substantive bildet, so erhält man eine übergeordnete Interpretationsperspektive. Konerding entwickelte dieses Verfahren der Hyperonymenreduktion und Benennung von Matrixframes im Bereich der Framesemantik (vgl. Konerding 1993; 2005). Bei Syndrom käme man zur übergeordneten Interpretationsperspektive/zum Matrixframe `ZUSTANDヶ. In dieser Arbeit wird im Folgenden von `Metakonzepten in diesem Zusammenhang gesprochen.

637 DiePraktikdes|Klassifizierens| bezieht sich in diesem Rahmen stärker als das |Kategorisieren| auf die Zuordnung zu festgelegten Klassen in terminologischen Systemen oder gesellschaftlichfachlichen Taxonomien wie der Internationalen Klassifikation der Krankheiten (ICD). 
Tab. 5.1 (fortgesetzt)

\begin{tabular}{lll}
\hline $\begin{array}{l}\text { Textoberfläche (punktuelle Perspektive, } \\
\text { Kap. 6.2.3.1 und 6.2.3.2) }\end{array}$ & Praktiken mittlerer Abstraktion & $\begin{array}{l}\text { Einzelne Ansprüche } \\
\text { an die Tätigkeit } \\
\text { des Definierens im }\end{array}$ \\
& $\begin{array}{l}\text { Burnout-Diskurs } \\
\text { (Kap. 4.2.2) }\end{array}$ \\
\hline
\end{tabular}

Negierte Äußerungseinheiten in Verbindung mit substitutiven Konnektoren zur Zurückweisung und anschließenden leine (einzelne) Kennzeichnung negieren/zurückweisen und ersetzen|

Ersetzung von generalisierenden Abstracta, die an ein Klassifikationssystem rückgebunden sind

Tempuswechsel innerhalb der Erzählung sprachliche Mittel zum Anzeigen von Dauer, Frequenz, Intensität Graduierung, Negierung, z. B. chronische Erschöpfung, nur noch müde, fühlte sich schon morgens erledigt, Leistungsfähigkeit nimmt dramatisch $a b$,

etc. S. Tab. 4 in diesem Kapitel

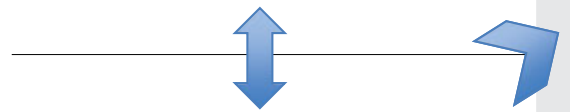

Ausblick auf Kap. 6.2.4.1 Textoberfläche (flächige Perspektive) Sprachliche Stimuli definitorischer Praktiken der |Zuweisung, Einordnung sowie Ein- und Abgrenzung| in der Fläche der Diskursstränge

Anhand der in Tab. 5.1 (Spalte 1) aufgeführten Satzmuster wird der zu definierende Ausdruck im Fall der Onlineportale meist schon selbst durch den Zusatz Syndrom dem medizinischen Bereich |zugeordnet $\left.\right|^{638}$ und danach, wie in den meisten fachinternen Textsorten (siehe Tab. 2.1 in Kap. 6.2.2.4), über ein generalisierendes Abstractum (Zustand ausgesprochener emotionaler Erschöpfung, Oberbegriff für bestimmte Arten von persönlichen Krisen) einem DefiniensMetakonzept |zugeordnet| und dadurch |kategorisiert|. Dieses Konzept wird dann

638 Die $\mid$ so| ausgezeichneten auf Verben zurückgehenden Formen verweisen im Folgenden auf die Praktiken mittlerer Abstraktion (siehe Spalte 2, Tab. 5.1). 
seinerseits noch attributiv oder prädikativ |spezifiziert| bzw. |subkategorisiert| (z. B. durch die Zusätze 'emotional' oder 'chronisch' zum Konzept `Erschöpfung`). In den Textversionen auf Wikipedia werden seit 2010 des Weiteren bestimmte Abstracta (Behandlungsdiagnose, Krankheit), die an das Klassifikationssystem ICD gesellschaftlich rückgebunden sind, direkt im Definitionsabschnitt zurückgewiesen und durch andere Abstracta dieses Klassifikationssystems (z. B. Problem der Lebensbewältigung, Zusatzdiagnose) ersetzt, wodurch >Burnout` innerhalb dieses Systems |eingeordnet| und |klassifiziert| wird.

Im Fall der analysierten Magazinberichte der im Korpus befindlichen Zeitungen und Zeitschriften wird der Erzählung eines Fallbeispiels eines Individuums ein fachlicher Deutungs- bzw. Diagnosekommentar |zugeordnet|, wodurch das Fallbeispiel |eingeordnet| und meist auch |klassifiziert| wird, und zwar mit Bezug auf das bestehende in einer Gesellschaft gültige Klassifikationssystem ICD. Je nach Erzählweise wird das im Fallbeispiel Thematisierte eher als >Transformationsprozess` oder als `Zustand, der im Kontrast zu einem früheren Zustand stehtı, |charakterisiert| und dadurch verschiedenen Metakonzepten (`Zustand «, `Ereignis $\triangleleft />P r o-$ zess ) |zugeordnet| und dadurch wiederum |kategorisiert|. Spezifiziert wird dieser >Prozess` oder `Zustand schließlich innerhalb der Erzählung durch die Aufzählung einzelner |charakterisierender| Begleitumstände oder Gefühle und Verhaltensweisen und deren |Gegenüberstellung| zu und |Abgrenzung| von 'normalen' Begleitumständen und Verhaltensweisen.

Erscheinen diese zuordnend-prädizierenden Vertextungs- und Satzmuster ohne Distanzmarker, so wird eine Adäquatheitsbeziehung zwischen Definiens und Definiendum unterstellt. Bei Definitionen, die ein zugeordnetes Definiensnomen höherer Abstraktion negieren, wird diese Adäquatheitsbeziehung zurückgewiesen. Daran schließt jedoch meistens der Vorschlag für ein anderes, als adäquater angesehenes Definiensnomen an (siehe oben die zitierten Beispiele der Textversionen auf Wikipedia seit dem Jahr 2010).

In der obigen Tabelle 4 wurden des Weiteren sprachliche Mittel zusammengestellt, durch die in den fachexternen Texten einzelne Merkmale innerhalb der Definition als Warnsymptome |hervorgehoben| werden oder durch die etwas als Mehrheitsmeinung, intersubjektiver Konsens oder objektive Tatsache markiert und dadurch |verdichtet| und |validiert| wird. Des Weiteren wurde gezeigt, dass sprachliche Zeichen, die beispielsweise die 'Eigendynamik' des in der Erzählung präsentierten Prozesses unterstützen, eine spätere Klassifizierung als Syndrom oder Krankheit |plausibilisieren| können. In welcher Weise sich diese sprachlichen Ressourcen mit den Ansprüchen, die im Burnout-Diskurs in Bezug auf die Geltung von Definition erhoben werden, verbinden, sei in der folgenden Tabelle (Tab. 5.2) nochmals zusammengefasst: 
Tab. 5.2: Exemplarische Herleitung der |definitorischen Teilpraktiken der Verknappung, Gewichtung und Geltungsvalidierung| (im fachexternen-öffentlichen Diskursstrang).

\begin{tabular}{|c|c|c|}
\hline $\begin{array}{l}\text { Textoberfläche (punktuelle Perspektive, } \\
\text { Kap. 6.2.3.1 und 6.2.3.2) }\end{array}$ & $\begin{array}{l}\text { Teilpraktiken mittlerer } \\
\text { Abstraktion }\end{array}$ & $\begin{array}{l}\text { Einzelne Ansprüche an die } \\
\text { Tätigkeit des Definierens } \\
\text { im Burnout-Diskurs } \\
\text { (Kap. 4.2.2) }\end{array}$ \\
\hline $\begin{array}{l}\text { - Gewichtung von Merkmalen und } \\
\text { Betroffenen-Gruppen durch Lexeme } \\
\text { und Mehrworteinheiten wie: } \\
\text { Kernsymptome, Hauptkriterien, eines } \\
\text { der wichtigsten Warnsymptome für } \\
\text { Burn-out; das wichtigste } \\
\text { Erkennungszeichen; insbesondere } \\
\text { Führungskräfte; besonders häufig } \\
\text { betroffen sind ... } \\
\text { - Verdichtung/Unifizierung durch } \\
\text { Formulierungen wie: Nach Meinung } \\
\text { der Fachleute ...; weitgehende Einigkeit } \\
\text { besteht heute darin, dass ...; der } \\
\text { allgemeine Konsens dahinter ...; } \\
\text { generell versteht man unter dem } \\
\text { Burnout-Syndrom ...; es beginnt meist } \\
\text { mit ...; } \\
\text { - typografische Hervorhebungen: von } \\
\text { Fragebogeneinheiten und } \\
\text { Phasenmodellen und Symptomen oder } \\
\text { Bedingungen im Titel und Vorspann des } \\
\text { Textes }\end{array}$ & $\begin{array}{l}\text { |assertieren|, } \\
\text { |Merkmale gewichten|, } \\
\text { |hervorheben|, } \\
\text { |bekräftigen|, } \\
\text { |plausibilisieren|, } \\
\text { |zusammenfassen|, } \\
\text { |verdichten|, } \\
\text { |Varianten reduzieren|, } \\
\text { |unifizieren|, } \\
\text { |Sachverhalte } \\
\text { objektorientiert (ohne } \\
\text { subjektive Bewertungen } \\
\text { seitens des Autors) } \\
\text { assertieren|, } \\
\text { |verallgemeinern|, } \\
\text { |auffordern, Merkmale } \\
\text { abzugleichen/auf } \\
\text { Übereinstimmung zu } \\
\text { prüfen|, } \\
\text { |deklarieren|639 }\end{array}$ & $\begin{array}{l}\text { Anspruch auf } \\
\text { angemessene Umgrenzung } \\
\text { und Konzentration } \\
\text { auf wesentliche bzw. } \\
\text { grundlegende Faktoren }\end{array}$ \\
\hline $\begin{array}{l}\text { Stil: Unpersönlicher komprimierter } \\
\text { Nominalstil, Pronomen man und } \\
\text { Passivkonstruktionen auf } \\
\text { Wikipedia/Onlineportalen; detailreicher } \\
\text { als in Fachlexika }\end{array}$ & & $\begin{array}{l}\text { Anspruch auf eine } \\
\text { neutrale, objektorientierte } \\
\text { Sachverhaltskonstitution/ } \\
\text { Begriffsbildung }\end{array}$ \\
\hline
\end{tabular}

639 Zum Zusammenhang repräsentativer und deklarativer Merkmale von Definitionshandlungen vgl. auch Kap. 4.3.2 dieser Arbeit. 


\begin{tabular}{lll}
\hline $\begin{array}{l}\text { Textoberfläche (punktuelle Perspektive, } \\
\text { Kap. 6.2.3.1 und 6.2.3.2) }\end{array}$ & $\begin{array}{l}\text { Teilpraktiken mittlerer } \\
\text { Abstraktion }\end{array}$ & $\begin{array}{l}\text { Einzelne Ansprüche an die } \\
\text { Tätigkeit des Definierens } \\
\text { im Burnout-Diskurs } \\
\text { (Kap. 4.2.2) }\end{array}$ \\
\hline
\end{tabular}

- Geltungsanspruch:

Text(abschnitts)-Ebene: Geltung der Textsorte: enzyklopädischer Artikel, Neutralitätsanspruch in den Statuten von Wikipedia;

Sprachliche Marker für Unmittelbarkeit und Eigendynamik der Ereignisse in den medialen Fallbeispielen als Garant für eine authentische bzw. alethische Vermittlung der Grundinformation Verweis auf wissenschaftliche Erforschung und intersubjektive Gültigkeit (einen anerkannten Selbsttest; in der Fachliteratur heißt es ...)

- Verallgemeinerung der Einzelfallbeispiele durch Gruppenbezeichnungen und statistische Prävalenzangaben

- Leseransprache: potenzielle Übereinstimmung der berichteten Ereignisse mit der Lebenswelt der Leser/innen; Aufforderung zur Prüfung der Geltung des Berichteten für die eigene Lebenswelt

- Modus: Präsens, Indikativ in Onlineportalen und bei Diagnosekommentaren in Magazinberichten

\section{Ausblick auf Kap. 6.2.4.2}

Textoberfläche (flächige Perspektive):

(Sprachliche) Stimuli definitorischer Praktiken der |Verknappung, Gewichtung und Geltungsvalidierung| 
Dem Anspruch auf angemessene Umgrenzung tragen Formulierungen Rechnung, die in der Vielzahl der Symptome einzelne lals besonders wichtig herausstellen| bzw. |gewichten|. Wie in Kap. 6.2.3.1 beschrieben, werden diese hervorgehobenen Symptome im fachexternen Diskursstrang häufig auch als Warnsymptome bezeichnet. Dadurch werden diese Symptome nicht nur |assertiert|, sondern sie implizieren auch die |Aufforderung|, die beschriebenen Symptome mit dem eigenen Gesundheitszustand |abzugleichen| und bei Übereinstimmung zu beachten. Denn „warnen ist ein Prädikat, das zwischen den Repräsentativen und den Direktiven steht: mit ihm ist sowohl ankündigen (repräsentativ) als auch auffordern (etwas nicht zu tun) (direktiv) impliziert“ (Harras/Winkler/Erb 2004) ${ }^{640}$. Maßstab für den Anspruch auf Adäquatheit zwischen Definiens und Definiendum ist bei einer direktiv-warnenden Definition demnach, dass möglichst alle 'gefährdeten’ Leser/innen bei diesem Abgleich Übereinstimmungen feststellen können. Die Verwendung des Ausdrucks Warnsymptome, den man mit 'Symptome, die als Warnung für die Entstehung einer Gesundheitsgefährdung gelten’ paraphrasieren kann, ist des Weiteren ein Indiz dafür, dass die Verfasser/innen der Texte auf eine teilweise schon gefestigte konvergente Bewertung des Phänomens zurückgreifen und dem Phänomen einen realen Gehalt zuschreiben. Denn es wäre kommunikativ inkonsistent, vor etwas zu warnen, dessen Realität man generell anzweifelt. Eine ähnliche faktizitätsbekräftigende Wirkung erzielen die in Medientexten angeführten Zahlenangaben, die unter Berufung auf Umfragestudien oder statistische Erhebungen über die Prävalenz von >Burnout` in der Bevölkerung berichten.

In Kapitel 6.2.3.1 wurde des Weiteren beschrieben, dass fachliche Kritik am Konzept `Burnout « seit den 2010er Jahren in den Medientexten zwar global aufgegriffen wird, dass aber selten verschiedene fachliche Positionen und Definitionen in einem Text einander als gleichwertige repräsentative Definitionen gegenübergestellt werden, wie beispielsweise in einigen Originalarbeiten aus dem Bereich der Arbeits- und Organisationspsychologie oder pädagogischen Psychologie zum Thema „Burnout“ (vgl. Kap. 6.2.2.2). Die fachexternen Texte lassen überwiegend fachliche Stimmen zu Wort kommen, die mit der Diagnostizierung und Behandlung psychischer Krankheiten befasst sind. Sie berichten deren Kritik, dass es für >Burnout im Vergleich zu anderen psychischen Krankheiten „keine einheitliche, international gültige wissenschaftliche Definition“ gebe (vgl. Beleg 10 in Kap. 6.2.3.1). In diesen Medientexten und auf Wikipedia wird damit nicht nur eine Definition, die die ,Realität‘ adäquat repräsentiert, sondern eine Definition mit deklarativer Kraft gefordert, auf deren Basis Krankschreibungen erfolgen

640 Vgl. den Eintrag zu warnen in der Onlineversion des „Handbuchs für deutsche Kommunikationsverben“ unter: https://www.owid.de/artikel/298934 (zuletzt eingesehen am 18.7.2019). 
können. Fachexterne Ratgebertexte schließlich beziehen sich einerseits ebenfalls auf die Forderung nach einer deklarativen Definition, andererseits präsentieren sie aber auch repräsentativ-direktive Definitionen, wenn sie, wie oben beschrieben, trotz des ,Defizits‘ einer als allgemeingültig deklarierten Definition Warnsymptome auflisten. In welcher Weise sich Definitionstypen mit repräsentativer, deklarativer und repräsentativ-direktiver (sprich warnender) Grundfunktion diskursiv beeinflussen können, wird in Kapitel 7.2 abschließend diskutiert werden.

Im folgenden Kapitel 6.2.4.1 wird der Frage nachgegangen, ob sich an den Textoberflächen der untersuchten Teilkorpora über die punktuellen Passagen des Definierens hinaus Aussage- und Zeichenformationen manifestieren, die das Phänomen BuRNout ein- bzw. abgrenzen und inwiefern sich diese flächigen Ein- und Abgrenzungspraktiken diskursiv stabil manifestieren und die expliziten (punktuellen) Definitionsbestrebungen beeinflussen.

\subsubsection{Sprachliche Stimuli definitorischer Praktiken im fachinternen und fachexternen Diskursstrang}

\subsubsection{Vorbemerkung}

Mit dem Wort Stimulus verwendet diese Arbeit im Folgenden einen Terminus, der auch im Fachbereich Psychologie/Medizin verwendet wird. Der Terminus soll darauf aufmerksam machen, dass bestimmte, wiederholt auftretende Zeichenkombinationen, seien sie bewusst gesetzt oder nicht, wie Reize in einem ReizReaktions-Schema, bestimmte Effekte mit großer Wahrscheinlichkeit hervorrufen können. Es geht im Folgenden darum, zu zeigen, dass bestimmte Verhaltensroutinen, deren Spuren im Diskurs ausfindig gemacht werden können, definitionsähnliche Effekte stimulieren können. Ferner geht es um die Frage, welchen Einfluss diskursive (flächige) Verweisstrukturen auf Definierensprozesse haben können und in welchem Maße sich flächige und punktuelle Praktiken des Definierens gegenseitig bedingen. In den vorausgehenden Kapiteln wurde an verschiedenen Stellen schon darauf verwiesen, dass sprachliche Mittel, die das Konzept und Phänomen >BURNOUT< ein- und abgrenzen, nicht selten sozial- und fachkulturelle Praktiken sowie biologische Normen als Referenzwerte ins Spiel bringen. Des Weiteren wurde punktuell schon herausgestellt, dass die Eingrenzung und Verortung des Phänomens dadurch verfestigt werden kann, dass es wiederholt in ähnlichen „Anordnungen der Äußerungsfolgen“ (Foucault [1969] 1981: 83f.) und unter vergleichbaren fach- oder bereichskulturellen Rahmenbedingungen erscheint. In den folgenden Kapiteln soll herausgearbeitet werden, ob sich diese Beobachtungen zu gewohnheitsmäßigen Verhaltens- und Handlungsweisen, sprich diskursiven Praktiken verdichten, welche die expliziten definitorischen Bemühungen begleiten, mit 
ihnen interagieren und auf diese Weise zur diskursiven Entstehung einer Definition des Phänomens `BuRNOUTく beitragen. In den folgenden Kapiteln liegt der Schwerpunkt auf der Analyse von Praktiken, welche die Eingrenzung von >Burnout $\triangleleft$ durch die wiederholte Zuordnung zu einem Bereich (Fach-, Forschungs- oder Praxisgebiet) und durch Formen der Abgrenzung von anderen sachverwandten Konzepten und Zuständen stützen oder verstärken. Die Analyse erfolgt direkt vergleichend im fachlichen und fachexternen Diskursstrang, da dadurch die Bedingungsverhältnisse zwischen diesen Diskurssträngen besser dargestellt werden können.

\subsubsection{Sprachliche Stimuli definitorischer Praktiken der Zuweisung, Einordnung sowie Ein- und Abgrenzung im fachinternen und fachexternen Diskursstrang}

Sowohl in den Texten der Fachlexika, Hand- und Lehrbücher und den Artikeln der Fachzeitschriften und Monografien als auch in den fachexternen medialen Texten und auf Onlineportalen konnten Stimuli für Praktiken extrahiert werden, die den definitorischen Anspruch auf angemessene Umgrenzung sowie eindeutige Trennschärfe und Deutlichkeit der Konstituierung des Gegenstands unterstützen. Zunächst werden verschiedene diskursive Relationsgefüge im fachinternen und fachexternen Bereich vorgestellt, die >BuRNOuT< dadurch eingrenzen, dass sie es stabil einem Bereich zuordnen. Daran anschließend wird analysiert, welche sprachlichen Stimuli den Bezug zu sozial-kulturellen Praktiken, Fach- und Normvorstellungen in den beiden Diskurssträngen auslösen, die >BURNOUT` von bedeutungsähnlichen Konzepten und dem sog. Normalzustand abgrenzen. Durch die parallele Beschreibung dieser Stimuli in fachlichen und fachexternen Texten wird direkt überprüft, ob sich diese Stimuli und die mit ihnen verbundenen Wissensbestände unterscheiden oder ob sie sich überschneiden und gegenseitig bedingen.

Zunächst ergibt der Blick in Überblickslehrwerke, die in das gesamte Forschungsgebiet der Psychologie einführen, thematisch folgende Verortung des Gegenstands >BURNOUT<: Zimbardo (51992, im QV unter 8.1.2) kommt auf >BURNOUT ‘ in Kap. 12 „Gesundheit, Streß und Bewältigungsstrategien“ in den Unterkapiteln „Gesundheitswesen und Gesundheitspolitik“ (S. 477) und „Dehumanisierung zur Selbstverteidigung“ (S. 590-591) zu sprechen; Myers (²014, im QV unter 8.1.2) behandelt >BURNOUT< in Kap. 19.2 „Arbeit und Gesundheit“ im Rahmen des Kap. 19, das einen Überblick über das psychologische Anwendungsfach der „Arbeits- und Organisationspsychologie“ gibt; in der Einführung von Gerrig/Zimbardo $\left({ }^{20} 2015\right.$, im QV unter 8.1.2) wird dem „Burnout“ in Kap. 12 „Emotionen, Stress, Gesundheit“ im Unterkapitel „Gesundheitspsychologie“ ein Abschnitt unter der Überschrift „Burnout im Beruf und das Gesundheitssystem“ gewidmet; im International Handbook of Psychology erscheint „,burnout“ im Kapitel „Developmental Psychology II: Adult- 
hood and Aging“ als mögliche Folge von „continuous occupational stress“. In diesem Kapitel werden Herausforderungen des Erwachsenenalters besprochen wie „work, marriage, childbearing“ etc. Es folgt eine Kurzdefinition, welche die dreigliedrige Symptomatik nach Maslach/Jackson ohne Angabe der Quelle wiedergibt (Pulkkinen 2000: 275, im QV unter 8.1.2). ${ }^{641}$ 'Burnout erscheint in diesen Kapitelüberschriften als ein Konzept, das zusammen mit den Themen „Gesundheit“, „Stress“ und „Arbeit“ verhandelt und in den Fachbereichen der Gesundheitspolitik, -psychologie sowie Arbeits- und Organisationspsychologie untersucht wird.

Betrachtet man daraufhin die Enzyklopädie der Psychologie, die seit den frühen 1980er Jahren erscheint und aktuell 88 verschiedene Bände umfasst, ${ }^{642}$ so erscheint `BURNOUT` über alle Handbücher hinweg als ein Phänomen, das in Arbeitskontexten verortet wird. ${ }^{643}$ Berufe, die aus der Perspektive verschiedener Fachrichtungen mit >BURNOUT< in Verbindung gebracht werden, sind vor allem klinische und pflegende Berufe ${ }^{644}$, Lehrberufe ${ }^{645}$, Berufe im Leis-

641 In einigen einführenden Überblickswerken zur Psychologie erscheint das Thema „Burnout“ aber auch nicht, z. B. Pawlik (2006) oder Schütz/Brand/Selg et al. (52015), im QV unter 8.1.2.

642 Man erhält 88 Treffer, recherchiert im Katalog der Deutschen Nationalbibliothek am 19.11.2018. Darunter sind jedoch auch Bände aus den frühen 1980er Jahren, die im Freihandbereich der Universitätsbibliothek Heidelberg nicht mehr vorgehalten werden, weil aktuellere Bände zu den jeweiligen Spezialfächern erschienen sind. Der Verlag Hogrefe stellt im Internet eine grafische Übersicht mit den schon veröffentlichten und geplanten Titeln der Enzyklopädie bereit. Es befindet sich allerdings keine Datumsangabe auf der Übersicht: https://www.hogrefe.de/fileadmin/user_ upload/hogrefe_de/Downloads/Enzyklopedie/EdP_Gesamtuebersicht.pdf (zuletzt eingesehen am: 27.12.2019). Die einzelnen Bände der Enzyklopädie, die in Tab. 6 im Anhang aufgeführt werden und in denen Burnout als Stichwort erscheint, sind im Quellenverzeichnis unter 8.1.2 aufgeführt.

643 Siehe im Anhang zu diesem Kapitel Tabelle 6 und dort die Zeilen 6, 13, 18, 19, 20, 22, 24 und 25. 644 Siehe im Anhang zu diesem Kapitel Tabelle 6 und dort die Zeilen 18, 19, 20 und 25; vgl. dazu auch weitere Fachzeitschriftenartikel z. B. in der Fachzeitschrift DMW: Eckart (2008); Bossenmayer (2012); Hamader/Nöhammer (2012); Bergner (2016); in der Fachzeitschrift PiD: Fengler (2001); in der Fachzeitschrift PPmP: Herschbach (1991), Bermejo/Muthny (1993), Seidler/Schreiber-Willnow/Hamacher-Erbguth et al. (2004), Zwack/Abel/Schweitzer (2011), Burger/Tektas/Paulsen et al. (2014) und Scholz/Neumann/Steinmann et al. (2015); im Dt. Ärztebl.: Stoschek (1996), Glöser (1996) und die (kritischen) Leserbriefe dazu von Deuser (1996), Hauck (1996) und Wächtler (1996); Schweizer (1997); Rottenfußer (1999) und die (kritischen) Leserbriefe dazu von Hien (1999), Dittrich (1999), Papenburg (1999) und Hammerström (1999); Abele (2001); Ruebsam-Simon (2002); Clade (2002); Madel (2003) und ein Leserbrief dazu von Drees (2003); Bergner (2004) und die (kritischen) Leserbriefe dazu von Schuster (2004), Drees (2004), Kolitzus (2004), Gebhardt (2004) und Wittmund (2004); Bühring (2005); Gothe/Köster/Storz et al. (2007); Knesebeck von dem/Klein/Frie et al. (2010), Unrath/Zeeb/Letzel et al. (2012), Voltmer/Kötter/ Westermann (2015), alle im QV unter 8.1.4).

645 Siehe im Anhang zu diesem Kapitel Tabelle 6 und dort die Zeilen 18, 22 und 24; vgl. dazu auch weitere Fachzeitschriftenartikel in PiEU: Stück/Rigotti/Mohr (2004); Stück/Rigotti/Balzer 
tungssport $^{646}$ und Berufe der Kundenbetreuung im Dienstleistungssektor ${ }^{647}$. >BURNOUT« wird insbesondere im Kotext der Themen „Stress“, „Belastung“, „Leistungsprobleme“ und psychische „Gesundheit“ verhandelt. Die meisten Einzelartikel, die das Stichwort Burnout enthalten, verzeichnen die Handbücher der Fachbereichs-Serie „Wirtschafts-, Organisations- und Arbeitspsychologie“ (5), „Medizinische Psychologie“ (4), „Sportpsychologie“ (4) und „Sozialpsychologie“ (3). ${ }^{648}$ In den enzyklopädischen Bänden der „klinischen Psychologie“ erscheint >BURNOUT « interessanterweise nur im Zusammenhang mit klinischem Pflegepersonal und Ärzten/Ärztinnen, die BuRnouT-SymPTome zeigen, nicht dagegen als Diagnose für Patienten/Patientinnen in der Klinik.

Betrachtet man im Vergleich zu dieser innerfachlichen Einordnung des Phänomens bzw. Begriffs 〉BURNOUT`, welchen Zeitungs-Ressorts Medientexte, die das Stichwort Burnout enthalten, zugeordnet werden (im Folgenden betrachtet am Beispiel der Süddeutschen Zeitung), so ergibt sich ein zunächst nicht allzu spezifisches Bild. Bei genauerer Betrachtung der Beleglage findet man jedoch Überschneidungen zu den Einordnungspraktiken im fachinternen Diskursstrang.

Bei den Texten der Süddeutschen Zeitung vom 1.1.1992 bis 31.12.2000 ${ }^{649}$ und 1.1.2011 bis 31.12.2012 ${ }^{650}$ findet man folgende zugeordnete Ressortverteilungen, die auf den ersten Blick wenig spezifisch erscheinen:

(2005); Wudy/Jerusalem (2011); Bodensteiner (2016); Dicke/Holzberger/Kunina-Habenicht et al. (2016); Keller-Schneider (2016); im Dt. Ärztebl.: Weber/Weltle/Lederer (2004) und Scheuch/ Haufe/Seibt (2015); sowie Becker/Gonschorek (1991) im QV unter 8.1.5.

646 Siehe im Anhang zu diesem Kapitel Tabelle 6 und dort die Zeile 22. Vgl. auch Schenk/Vogt/ Wippert (2006) in der Zeitschrift „Leistungssport“ und Altfeld/Kellmann (2013) in der Zeitschrift „Sportpsychologie“, beide im QV unter 8.1.5.

647 Siehe im Anhang zu diesem Kapitel Tabelle 6 und dort Zeile 20. Vgl. auch Dormann/Zapf/ Isic (2002) in der ZfAO, im QV unter 8.1.4.

648 Siehe im Anhang zu diesem Kapitel Tabelle 6.

649 Gesucht wurde mit folgender Suchsyntax: burn-out* ODER burnout* in den überregionalen und den Landkreisausgaben der Süddeutschen Zeitung. Vgl. auch Kap. 5.1.3 dieser Arbeit. Von dieser Recherche wurden nur einzelne Titel, die zitiert werden und das Thema „Burnout“ ausführlicher behandeln, in das Quellenverzeichnis im Anhang (siehe Süddeutsche Zeitung unter 8.1.6) übernommen. Kurze Meldungen zu einem Vortrag oder Seminar, die nur für diese Ressort-Recherche genutzt wurden, sind also nicht einzeln im Quellenverzeichnis aufgeführt. 650 Gesucht wurde mit folgender Suchsyntax: burn-out* ODER burnout ${ }^{*}$. Die Landkreisausgaben als Quelle wurden bei dieser Recherche allerdings aufgrund der Fülle der Ergebnisse nicht mehr berücksichtigt. Von dieser Recherche wurden nur einzelne Titel, die zitiert werden und das Thema „Burnout“ ausführlicher behandeln, in das Quellenverzeichnis im Anhang (unter Süddeutsche Zeitung, unter 8.1.6) übernommen. Kurze Meldungen zu einem Vortrag oder Seminar, die nur für diese Ressort-Recherche genutzt wurden, sind also nicht einzeln im Quellenverzeichnis aufgeführt. 


\section{2-2000 (167 Treffer)}

Nachrichten (43), Landkreis (17), Bildung und Beruf (15), München (9), Feuilleton (9), Beilage (9), Bayern (8), Service (7), Leserbriefe (6), Sport (5), Wirtschaft (4), Münchner Kultur (4), Die Seite Drei (3), Schule und Hochschule (3), Immobilien (2), Politik (2), Wissenschaft (2), Letzte Seite (2), Gesellschaft (2), Reise (1), Medien (1), Themen aus Deutschland (1), Themen (1), SZ am Wochenende (1), Sachbuch (1), Münchner Wirtschaft (1), Münchner Sport (1), Mode (1), Literatur (1), Computerseite (1), Berlin-Service (1), Berlin-Seite (1), Anzeige (1), SZ Extra (1)

\section{1-2013 (250 Treffer)}

Wirtschaft (39), Sport (34), Beruf und Karriere (22), Feuilleton (19), SZ Wochenende (18), Geld (17), Politik (15), Panorama (15), Medien (11), Forum (10), Beilage (8), Die Seite Drei (5), München/Bayern (5), Sport in Bayern (5), Reise (4), Forum \& Leserbriefe (3), Themen des Tages (3), Wissen (3), Literatur (3), Literaturbeilage (2), Mobiles Leben (2), Meinungsseite (2), JETZT.DE (2), Sportbeilage (1), Report (1), Filmseite (1)

Die größte Rubrik „Nachrichten“ zusammen mit der Rubrik „Landkreis“ im Abschnitt 1992-2003 enthält folgende Nachrichtenbereiche:

- über Burnout bei Lehrkräften im Kontext von Schule u. Kindergärten (24 Texte); ${ }^{651}$

- Seminar- oder Vortragsankündigungen zum Thema „Burnout“ (9 Texte);

- Burnout in Pfarrgemeinden/sozialen Berufen/im Bereich soziale Arbeit/bei der Nachbarschaftshilfe (6 Texte);

- Burnout bei Pflegekräften (in Altenheimen) (4 Texte); bei Krankenhauspersonal (2 Texte) und pflegenden Angehörigen (1 Text);

- Meldungen zu Burnout im Kontext der Themen Medikamenten-Abhängigkeit/Doppelbelastung/Wechseljahre bei Frauen (2 Texte);

- Meldungen zu Burnout bei Personen aus der Kommunalpolitik (1 Text);

- Burnout im Rahmen von Glossen (2 Texte);

- Bericht über Chronisches Erschöpfungssyndrom und Burnout (2 Texte) und über Burnout und Workaholismus (1 Text);

- Kabarettist, der einen Burnout-Lehrer imitiert (1 Text);

- eine Meldung über einen IT-Fachmann, der aussteigt und sich beruflich neu orientiert (1 Text).

651 Interessant ist, dass auch über Lehrer/innen mit vielen Berufsjahren oder über Pensionierungen von Schul-/Kindergarten-Rektoren/-Rektorinnen der Landkreise berichtet wird. In diesen wird meist lobend betont, dass sie kein `Burnout` hätten. Dies belegt wiederum, dass dieses Phänomen für den Lehrberuf (ab den 1990er Jahren) als typisch angesehen wird und die belobigten Personen sich von diesem eventuell als neu klassifizierten Typus absetzen: „Seit 31 Jahren, 30 davon an der Grund- und Hauptschule in Kirchseeon, ist Waltraud Müller Lehrerin. Aber von Frustration oder gar Anzeichen des ,Burn-out-Syndroms“ keine Spur“ (SZ, Landkreise, Ebersberg, 24.11.1998: 4). Ähnlich z. B. SZ, Landkreise, Starnberg, 29.6.1998: 2. 
Bei der zweiten Unterrubrik „Seminar- und Vortragsankündigungen“ ist wiederum interessant, an wen sich die Seminare richten, wenn sie nicht generell über den „Stand der Burnout-Forschung“ berichten oder sich an „alle“ wenden ${ }^{652}$. Denn auch hier finden sich die ‘typischen’ Betroffenengruppen wieder:

Er wies darauf hin, dass ein Grund für die zunehmende Überlastung der Lehrkräfte darin liegen könnte, dass das Durchschnittsalter der Lehrer steigt. [...]. Einmal im Monat werden Veranstaltungen von der GEW angeboten, die dem „Burnout“-Syndrom vorbeugen sollen.

(SZ, Landkreise, Erding,12.08.1999: 4).

Ein besonderer Baustein ist die Mitarbeiter-Fortbildung des Dachauer Forums: Lehrer, Erzieher, Eltern und alle Referenten des Forums bekommen Hilfestellung, um als Mensch und Berufstätiger zu überleben. So heißt es für einen ganzheitlichen Gesprächskreis: 'Den roten Faden finden' - gegen das Burn-out- Syndrom.

(SZ, Landkreise, Dachau, 18.01.1996: 3)

Ein Seminar für alle, die ehrenamtlich als Führungskräfte in Vereinen arbeiten, [...]. Neben Organisations- und Verwaltungskenntnissen stehen psychologische „Komponenten“ auf dem Lehrplan: Die Motivation der ehrenamtlichen Mitarbeiter, die Vermeidung und Beilegung von internen Streitigkeiten und die Verhinderung des „Burnout-Syndroms“ bei überarbeiteten Ehrenamtlichen nehmen einen großen Teil der Seminarzeit ein.

(SZ, Landkreise, München-Land, 03.06.1997: 7)

Mehrfachbelastungen bei Frauen führen oft zum „burn out Syndrom“. Um Beruf, Familie und Freizeit unter einen Hut zu bekommen, veranstaltet die Volkshochschule in Freising am Freitag, 18. Februar, von 18 bis 21 Uhr, und am Samstag, 19. Februar, von 10 bis 13 Uhr und von 15 bis $18 \mathrm{Uhr}$, ein Seminar. ${ }^{63}$

(SZ, Landkreise, Freising, 11.02.2000: 2)

In den Texten des Ressorts „Bildung und Beruf“ werden schließlich insbesondere die folgenden drei Betroffenen-Gruppen genannt: 1.) Personen, die in Unternehmen Führungspositionen bekleiden und/oder eine sehr hohe Wochenarbeitszeit aufweisen ab dem Alter von 30 Jahren; 2.) Personen, für die der Beruf im Mittel-

652 Vortragsankündigung allgemein zum Stand der Burnout-Forschung findet man z. B. in SZ, Landkreise, Fürstenfeldbruck, 27.04.1999: 12; oder eine Veranstaltung „Ausgebrannt und Ausgelaugt“, die sich an alle wendet, die etwas ,gegen die Feststellung ,Ich bin fix und fertig“ (Neudeutsch: Burnout)“ und gegen die danach aufgezählten Zustände machen möchten: „,Privat gebraucht, beruflich gefordert, gesundheitlich verbraucht, kräftemäßig ausgelaugt, menschlich überfordert und seelisch ausgebrannt' sind Zustände, über die viele klagen, die aber nicht dazu führen müssen, sich aufzugeben“ (ila/SZ, Landkreise, Fürstenfeldbruck, 11.01.1999: 4).

653 Weitere Seminarankündigungen, die sich an Frauen wenden: „Für alle Frauen, die sich ausgelaugt vom Alltag fühlen und sich Gutes tun wollen, bietet die Volkshochschule Dachau ein Anti-Streß-Seminar an.“ (SZ, 3.3.1997, Landkreise, Dachau: 3); vgl. auch SZ, Landkreise, Erding, 20.12.2000: R10. Das Thema „Überlastung“ und „Burnout“ speziell bzw. verstärkt bei Frauen wird z. B. auch in folgenden Beiträgen thematisiert FAZ (2001); Kals (2004, FAZ); Meck (2010, FAZ); mamk (2012, SPIEGEL ONLINE); DER SPIEGEL (1992); Brnada/Gasser/Kapeller (2010, DIE ZEIT), alle Belege im QV unter 8.1.6. 
punkt ihres Lebens steht; 3.) Personen, die Berufe und/oder Tätigkeiten im sozialen Bereich ausüben und eine Restgruppe:

„Berufstätige“, „,bereits 30-jährige“; ,vielen, deren Lebensmittelpunkt der Job ist“; ,[A]ngespannte Manager“; „Menschen, die mit ihrem Beruf verheiratet sind“, „Unternehmensberatungen sind prädestiniert für Workaholismus und Burnout“; „Führungskräfte scheinen auf Grund der Arbeitsstrukturen besonders gefährdet zu sein. Doch entgegen dem gängigen Klischee von der Managerkrankheit, wählen auch der kleine Angestellte oder die Hausfrau den Sprung in die Arbeitsflut als Strategie“; „Manager über 50“, „Menschen, die Hochleistung vollbringen und trotzdem ständig Angst um ihren Job haben müssen, können nicht motiviert sein“; ,der berufstätige Mensch“; „,ungmanagerin“, „der jungenhafte Geschäftsführer“; „Ausgelaugt von einer achtzig-Stunden-Woche in einer Unternehmensberatung, vernachlässigt der Enddreißiger auch seine Frau“; „sechs Risikotypen der sogenannten 'Workaholics'“; „Die Krankenschwester auf einer Intensivstation leidet darunter, der Verkaufsrepräsentant, Eltern, die ein behindertes Kind, Kinder, die ihre greisen Eltern pflegen, der Schalterbeamte, der Lehrer angesichts der lärmenden, gewaltbereiten Heranwachsenden oder der Drogentherapeut, der nach Jahren die Flinte ins Korn wirft und resigniert und verbittert erkennt, daß all sein Bemühen vergeblich war“;

Erstaunlich erscheint, dass es in diesem ersten Vergleichszeitraum der Süddeutschen Zeitung keine Rubrik zu „Gesundheit“ oder „Medizin“ gibt. Ausführliche Problem- oder Magazinberichte zum Thema „Burnout“ findet man vor allem im Ressort „Bildung und Beruf““54, in der Rubrik „Die Seite $3^{\text {“655 }}$ und vereinzelt im Ressort „Nachrichten“656 , „Sport““657 und „Wissenschaft“658.

Elf Jahre später im Belegzeitraum 2011-2012 findet man wie im ersten Zeitraum keine Rubrik „Gesundheit“ und ebenfalls nur wenige Beiträge im Ressort „Wissenschaft“. Der Bereich mit den meisten Beiträgen ist in diesem Zeitraum das Wirtschaftsressort. ${ }^{659}$ Dieses enthält, wie zuvor das Ressort „Bildung und Beruf“, neben Meldungen zu Fehlzeiten und politisch geforderten Aktionen auch die meisten ausführlichen (Problem-)Berichte ${ }^{660}$. Als ,Betroffenen`-Gruppen erschei-

654 SZ, 31.12.1994 (= Rheinz 1994: 801); SZ 19.4.1997 (= Mohr 1997: 801); SZ 16.01.1999 (= Dilk 1999: 53); SZ, 9.-10.12.2000 (= Gestmann 2000: V1/1); im QV unter 8.1.6.

655 SZ, 08.10.1994 (= Lebert 1994: 3); SZ, 10.4.1999 (= Emundts 1999: 3); im QV unter 8.1.6.

656 Z. B. SZ, Freising, 06.05 .1997 (= Weinfurtner 1997: 2).

657 So z. B. ein Problembericht über Erschöpfung und Burnout bei jungen Tennisprofis in SZ, 9.11.1994 (= Muz 1994: 64, im QV unter 8.1.6).

658 So z. B. zwei Beiträge über „Burnout“ in Verbindung mit Drogenkonsum im Silicon Valley (SZ, 24.10.2000, = Krempl 2000: V2/17) und Burnout in Verbindung mit Herz-Kreislauf-Erkrankungen (SZ, 20.1.1994, = tst 1994: 50); im QV unter 8.1.6.

659 Aufgrund der Fülle der Beiträge mit dem Stichwort Burnout ab den 2010er Jahren konnten in diesem Zeitraum die ländlichen Ausgaben nicht mehr berücksichtigt werden.

660 Z. B. SZ, 18.11.2011 (= Sogorski 2011: 19); SZ, 17.08.2012 (= Bohsem 2012a: 17); SZ, 18.8.2012 (= Brömmling 2012: 22). 
nen in diesem Ressort neben der nicht weiter spezifizierten Gruppe von Beschäftigten vor allem Personen mit Leitungsfunktion, hoher Verantwortung, in sozialen Berufen sowie fleißige, motivierte und mit der Arbeit identifizierte Personen ab dem Alter von 30 Jahren bis Ende 50:

„Gesundheit der Beschäftigten“; „Besonders betroffen sind die fleißigen und motivierten Mitarbeiter“; „Besonders betroffen sind laut Studie Arbeitnehmer mit Vorgesetzten-Funktion“; „Mitarbeiter“; „Menschen in Berufen mit hoher sozialer Verantwortung und geringer gesellschaftlicher Anerkennung werden öfter seelisch krank als andere“; Krankenschwestern, Sozialarbeiter, Erzieherinnen und Seelsorger sind hochgefährdet - Berufe, in denen auch der Fachkräftemangel viele zur Mehrarbeit zwingt“, „der moderne Mensch“; „Die Abteilungsleiterin ist krankgeschrieben“; „Der Rechtsanwalt gibt seine erfolgreiche Tätigkeit in der Anwaltskanzlei auf“; „Die Kollegin hat Depressionen, obwohl die doch viel erfolgreicher ist als man selbst.“; „warum hat sich der Mitarbeiter, der völlig in seiner Arbeit aufging, das Leben genommen?“; „Wer einen sozialen Beruf ausübt, ist deutlich häufiger von einem Burn-out betroffen.“; „So fehlten [...] vor allem Sozialpädagogen, Helfer in der Krankenpflege, Krankenschwestern und Kindergärtnerinnen bei der Arbeit. Häufig betroffen sind aber auch Werbefachleute sowie Wächter und Aufseher. Insgesamt leiden Frauen deutlich häufiger unter zu hoher Arbeitsbelastung. [...] Am häufigsten tritt das Phänomen im Alter zwischen 40 und 59 Jahren auf. “661

Das Ressort „Sport“ folgt von der Menge der Treffertexte, die das Stichwort Burnout/Burn-out enthalten, auf das Wirtschaftsressort. In diesen Texten wird vom (zeitweisen) Rücktritt von Sportlerinnen und Sportlern aufgrund von BurnoutSymptomen oder Burnout-Gerüchten, berichtet. ${ }^{662}$

Wie in den letzten Belegen deutlich wurde, werden nicht nur bestimmte Berufe häufiger genannt als andere, sondern man findet Sprachgebrauchsmus-

661 Weitere Belege: „Burn-out-Fälle in Dax-Konzernen“; „Entschleunigung der Burn-out-geplagten Gesellschaft“; „dass die Burn-out-Rate, die Anzahl derer mit Erschöpfungssyndrom, gerade in den sozialen Berufen besonders hoch ist“; „Mitarbeiter“; ,dass immer mehr Beschäftigte unter einem steigenden Arbeitsdruck und zunehmender Arbeitsverdichtung leiden“; „erinnert an Fußballtrainer oder Sportler mit Burn-Out-Syndrom und findet: ,Die Arbeitnehmer in den Betrieben verdienen die gleiche Aufmerksamkeit wie Prominente.“;; „Ein Seminar zur Prophylaxe von Burnout hilft nun den Mitarbeitern“; „im Umfeld des Bankers hieß es, er sei überarbeitet und leide an einem Burn-out“; „Viele Berufstätige sind betroffen“; „Der Konzern-Chef soll sich überarbeitet haben“; , ,dass Burn-out zu einem immer größeren Problem in der Bankenbranche werde“; ,,weil es oft die besonders Fleißigen, die besonders Fröhlichen trifft“; „Vom Burn-out-Syndrom und anderen psychischen Krankheiten sind [...] inzwischen 20 Prozent der Beschäftigten betroffen“; .

662 Zum Beispiel im Fußball über Ralf Rangnicks Rücktritt im Jahr 2011 und sein Wiedereinstieg im Jahr 2012 (SZ, 17.02.2012: 28 oder über den Ersatztorwart von Hannover 96 Markus Miller (SZ München, 23.11.2011: 28) oder im Tennissport über Florian Mayer (SZ, 22.12.2011: 39). Über Burnout im Leistungssport berichten auch einige Artikel im SPIEGEL/SPIEGEL ONLINE, im QV unter 8.1.6: Aha/sid (2011a); chp/sid (2011); Kraus (2012); DER SPIEGEL (1986); DER SPIEGEL (1989); DER SPIEGEL (1994); DER SPIEGEL (1995a); SPIEGEL ONLINE (2003a und b und 2004 und 2006); Weinzierl (2011); Ahrens (2011). 
ter, die auf diese Personengruppen, aufmerksam machen bzw. diese von anderen weniger betroffenen Gruppen dadurch abgrenzen:

Besonders betroffen sind; Menschen in Berufen mit ... werden öfter seelisch krank als andere; Wer einen ... ausübt, ist deutlich häufiger von einem Burn-out betroffen; gerade in den sozialen Berufen besonders hoch ist; oft die besonders Fleißigen; nicht nur ... leiden unter; $\underline{\text { insbe- }}$ sondere bei ... auftreten kann, die ...;

Durch diese und ähnliche Suchwörter findet man auch in den Texten der anderen Zeitschriftenkorpora und Onlineportale hervorgehobene ,Betroffenen'-Gruppen, die den Personen-Gruppen, die aus den Texten der untersuchten Ressorts der Süddeutschen Zeitung extrahiert wurden, ähneln (Unterstreichungen T.S.):

Doch nicht nur Manager oder Leistungssportler leiden unter Dauerstress und enormem Leistungsdruck. Seelische Erkrankungen haben in den letzten Jahren deutlich zugenommen.

(FAZ, 17.06.2007, = jur/FAZ 2007, im QV unter 8.1.6)

Das erklärt, warum nicht nur Spitzenmanager an Burn-out erkranken, sondern auch so mancher Hartz-IV-Empfänger. ～(ZEIT Wissen, Nr. 3/2014, = Willmann 2014, im QV unter 8.1.6)

Heute leiden insbesondere Führungskräfte und High-Potentials sowie Lehrer unter beruflicher Erschöpfung

(FAZ, 20.8.2004, = Obermeier 2004, im QV unter 8.1.6)

Am Burn-out erkranken primär die Leistungsträger einer Gesellschaft, also jene, die besonders leidenschaftlich für eine Sache brennen. （FAZ. 16.3.2012, S. B2, = Marlovitz 2012, im QV unter 8.1.6)

Experten zufolge trifft die Diagnose Burnout vor allem ehrgeizige Leistungsträger.

(SPIEGEL ONLINE 2011c, im QV unter 8.1.6)

Gerade die Leistungsträger fühlen sich bei der Prävention von Unternehmen und Vorgesetzten im Stich gelassen

(SPIEGEL ONLINE, 2012c, im QV unter 8.1.6)

Oft trifft es die, die alles geben.

(SPIEGEL ONLINE, 2012b, im QV unter 8.1.6)

Burn-out ist nicht nur im Kontext helfender Berufe zu finden, wie Christina Maslach, die bekannteste wissenschaftliche Vertreterin des Konzepts, ursprünglich dachte; auch Eltern, Sportler, Manager und Unterbeschäftigte beklagen Burn-out.

(Die Zeit, Online-Ausgabe, 08.12.2011, = Pawelzik 2011, im QV unter 8.1.6)

Vor allem Menschen, die stark in ihrem Beruf aufgehen und sich dabei für andere Menschen aufopfern, sind häufig vom Burnout-Syndrom betroffen. Meist geschieht dies in sozialen Berufen wie Lehrer, Arzt oder Krankenpfleger. Prinzipiell kann jedoch jeder, unabhängig vom Beruf, am Burnout-Syndrom erkranken, zum Beispiel auch durch familiäre Belastungen.

(Hervorhebungen durch Fettung im Orig., Onmeda.de, 14.2.2019 „Burnout-Syndrom: Ausgebrannt und erschöpft“, im QV unter 8.1.8) 
Dass Burnout insbesondere in Pflegeberufen auftritt, ist kein Wunder, weil es im zwischenmenschlichen Bereich immer Resonanzerwartungen gibt.

(PH, 01/2013, = Schönberer 2013, im QV unter 8.1.7)

Diese Befunde zu typischen Personengruppen in Texten, die >BURNOUT< thematisieren, passen des Weiteren zu den Berufskontexten, die in Kapitel 6.1.2 für die Mehrworteinheit sich ausgebrannt fühlen im ZEIT-Korpus extrahiert wurden (siehe Kap. 6.1.2). ${ }^{663}$

Weiterhin wird >Burnout dadurch stabil einem fachlichen Bereich zugeordnet, dass die Autorinnen und Autoren von Fachartikeln oder Vermittlungstexten sowie die Zitatgeber/innen in Medientexten über die Einzeltexte fachintern und fachextern hinweg aus ähnlichen Fachbereichen stammen.

Betrachtet man in einer auf kein einzelnes medizinisches Fach konzentrierten Zeitschrift wie dem „Deutschen Ärzteblatt“ die Fachrichtungen, aus denen die Autorinnen und Autoren der Original- und Übersichtsarbeiten sowie der Problem- und Kurzberichte zum Phänomen bzw. Konzept >Burnout`stammen, so erhält man folgende Übersicht: ${ }^{664}$

663 Und auch in den Unterkorpora von SPIEGEL/SPIEGEL ONLINE und FAZ/FAZ.NET findet man die Berufsgruppen Lehrer/innen, Sportler/innen und Trainer/innen, Prominente besonders häufig sowie die Gruppen Leistungsträger, Manager/innen, Führungskräfte, soziale Berufe (z. B. Polizisten/innen, Pflegepersonal, Ärztinnen/Ärzte, Sozialarbeiter/innen), Politiker/innen, Studierende und Callcenter-Mitarbeiter/innen: Belege im SPIEGEL/auf SPIEGEL ONLINE: DER SPIEGEL (1987; 1990; 1993; 1995b; 2001; 2010b); SPIEGEL ONLINE (2002a/b; 2003a/b; 2004; 2006; 2011a); Hinrichs/Koch/Meyer (2004); Brandt (2004); Hinrichs/Meyer (2004); Leffers (2007); Leffers (2008); Weinzierl (2011); Kraus (2012); Jjc/dpa (2012); dpa (2010); Hebel/Röbel (2013), Kröher (2012); Marquart (2011); Walter (2007); Meiritz (2011); Meiritz (2012); Wöhrle (2011); Maeck (2012); weitere Belege in PH 1/2013 (= Schönberger 2013); Belege in der FAZ/auf FAZ.NET: Stein (1981); Seibertz (1989); Gross (1992); dpa (2004 a/b); Hahn (2004); FAZ (2006); Lutterotti (2007); Nawrath (2007); Martin (2008); Reinsch (2010); Camp (2010); Daniels (2011); Otto (2011); Hetrodt (2013); Bender (2013); Palm (2013); dpa (2013); Zimmering (2014); Arens (2014); Astheimer (2014a); Breitbart/Schwenk (2015); Dieterle (2015); Dpa/svs/tine (2015); FAZ.NET (2015); FAZ (2016). Dass das Attribut 'Burnout' Führungskräften oder Personen im Spitzensport zugesprochen wird (in der Form einer Frage, ob sie sich je ausgebrannt gefühlt hätten/ausgebrannt sind), auch wenn es von diesen dann abgelehnt wird, zeigen Beeger (2015, FAZ.NET) und Eder (2016, FAZ.NET). In einer im Dt. Ärztebl. abgedruckten dapd-Meldung werden ebenfalls Berufsgruppen hervorgehoben: „Immer mehr Menschen fühlten sich ausgebrannt, vor allem Führungskräfte und engagierte Mitarbeiter“ (dapd 2011, Dt. Ärztebl, im QV unter 8.1.4). Einige Artikel gehen auch noch auf Jugendliche und Kinder als ,Betroffene‘ von >Burnout ‘ ein: Weiß (2016, FAZ); Sentker (2003, PH 2/2003); Otto (2016, PH 9/2016).

66431 Texte erfüllen diese Textsortenkriterien. Der älteste Fachartikel stammt aus dem Jahr 1995, der jüngste aus dem Jahr 2017. Bei Beiträgen, in denen (mehrere) Autoren/Autorinnen unterschiedlichen Fachrichtungen durch die Nennung ihrer Fachzugehörigkeit zugeordnet werden 
Tab. 7: Fachrichtungen der Autorinnen/Autoren, die über `Burnout` im Dt. Ärzteblatt schreiben (1995-2017).

Fachrichtung

Anzahl der Texte mit

Autoren/Autorinnen

dieser Fachrichtungen

Schnittstelle zwischen

- medizinischen und soziologischen Fächern und (arbeits-) soziologischen Fragestellungen

- psychologischen und (arbeits-)soziologischen Fächern/ Fragestellungen

Schnittstelle zwischen

Medizin und Psychologie:

Psychiatrie, Psychotherapie, Psychosomatik, medizinische

Psychologie

Gesundheitswissenschaften, Public-Health-Forschung,

Versorgungsforschung, Epidemiologie

\begin{tabular}{lr} 
Allgemeinmedizin & 3 \\
\hline Personalberatung, Supervision & 2 \\
\hline Pädagogik & 1 \\
\hline Medizinmanagement & 1 \\
\hline
\end{tabular}

BuRnout erscheint im „Deutschen Ärzteblatt“ ähnlich wie in der Handbuchreihe der Enzyklopädie der Psychologie als ein Phänomen, das von Fachkreisen aufgegriffen und erforscht wird, die an der Schnittstelle von psychologischen, medizinischen und soziologischen Fragestellungen forschen und weniger Grundlagen, sondern anwendungsbezogene Forschung betreiben.

Vergleicht man dies mit den in Medientexten zum Thema „Burnout“ zitierten Expertinnen und Experten und ihrer medialen Zuweisung, so erkennt man einige Ähnlichkeiten zur fachlichen Aufteilung im Deutschen Ärzteblatt, insbesondere auch in Bezug auf die Schnittstellenfächer. Fachleute, die in Beiträgen der FAZ zwischen $1992-2012^{665}$ zitiert werden, entstammen nach Häufigkeit und ähnlichen Überfächern gelistet, folgenden Fachbereichen:

können, wird jeder Autor/jede Autorin mit seinen/ihren Fachrichtungen auf die Fachgruppen in der nachfolgenden Tabelle verteilt, auch wenn dadurch ein Text zwei- oder mehrfach in der Zählung erscheint.

665 Dieser Auszählung liegen 57 Texte des FAZ-Teilkorpus zugrunde. Zur Korpuszusammenstellung siehe Kap. 5.1.3. 
Psychiatrie/Psychotherapie/psychosomatische Medizin (14); Medizin (allg.) (4); Arbeitsmedizin/Betriebsärzte (4); Psychoanalyse (3); Psychologie (allg.) (2); Psychokardiologie (1); Psychoanalytische Sozialpsychologie (1); Wirtschaftspsychologie (1); Sportpsychotherapie (1); Gesundheitspsychologie (1); Arbeitsökonomie (1); Sportmedizin (1); Neurologie (1); Medizinsoziologie (1); Managementforschung (1); Programmforschung (1),

Fachleute dieser Fachbereiche werden auch in anderen Magazinen und Zeitungen gerne zitiert, wie die folgenden Belege verdeutlichen (Unterstreichungen T.S.):

Eine Haltung, die Manfred Sigwart von vielen seiner Patienten kennt. Der Oberarzt der psychosomatischen Abteilung ist seit drei Jahren in der Habichtswald-Klinik in KasselWilhelmshöhe. （DIE ZEIT (Online-Ausgabe) 26.4.2001, = Schenk 2001, im QV unter 8.1.6)

Wie man aus dieser Stressspirale wieder herauskommt und was man tun kann, um Erschöpfung zu vermeiden, erläutert der Facharzt für Psychiatrie und Psychotherapie am nächsten Dienstag, 29. März, von 19 Uhr an in den Räumen der F.A.Z.-Redaktion. (FAZ, 24.03.2011, = Jff 2011, im QV unter 8.1.6)

Thorsten Rarreck, 47, Mannschaftsarzt des Fußball-Bundesligisten Schalke 04, über den Rücktritt des am Burnout-Syndrom erkrankten Trainers Ralf Rangnick

(DER SPIEGEL 39/2011: 118, = Der Spiegel 2011d, im QV unter 8.1.6)

„Hoher Druck produziert auf Dauer Ex-Leistungsträger, das kann keine Firma wollen“, sagt Kerstin Reviol, TÜV-Expertin für Arbeitspsychologie. [...]

Danach beginnt das, was der Psychologe die „Zuspitzung“ nennt. Er konfrontiert sie mit ihren persönlichen Prioritäten, fragt nach, wenn es um Freundschaften und das Verhältnis zum Partner geht. „Dann kommen wir meistens an den Punkt, an dem sie merken, dass sie auf dem Weg in den Burnout sind“, sagt Ahrens.

(SPIEGEL ONLINE, 23.5.2011 = Brenner 2011, im QV unter 8.1.6)

„Immer mehr Unternehmen investieren in das emotionale und psychische Wohlbefinden ihrer Mitarbeiter“, erklärt Tony Urwin, der leitende Arbeitspsychologe der Priory.

(DIE ZEIT, Nr. 5/2015, S. 28, = Jungclaussen 2015, im QV unter 8.1.6)

Zudem gibt es Personen, die in ihrem Beruf mit ,Burnout-Fällen` zu tun haben und die ebenfalls als Experten befragt werden:

Mit Burnout ist nicht zu spaßen: „In schweren Fällen sind die Betroffenen sogar suizidgefährdet“, sagt Frank Berndt. „Häufig geht an einer stationären Behandlung in einer Klinik dann kein Weg vorbei“, so der Führungskräftetrainer.

(manager-magazin.de, 19.12.2008, = Heimann 2008, unter SPIEGEL, im QV unter 8.1.6)

Millionen Deutsche leiden an Burnout - und immer mehr Arbeitnehmer zeigen Stresssymptome am Arbeitsplatz. Aber was lässt sich dagegen tun? Und wie erkennt man frühzeitig die Warnsignale des Körpers? Managementberater Rüdiger Klepsch gibt die wichtigsten Tipps.

(SPIEGEL ONLINE, 28.2.2011, = Klepsch 2011, im QV unter 8.1.6) 
Heiler ist Berater für betriebliche Gesundheitsförderung. So oder so ähnlich laufen viele seiner Untersuchungen ab. Wenn der Münchner mit seinem Team in ein Unternehmen geht, kann er anhand einer Reihe kleiner Tests das Burn-out-Risiko der Belegschaft grob einschätzen.

(SZ, 17.11.2012, S. V211, = Fiedler 2012, im QV unter 8.1.6)

Diese Belege haben exemplifiziert, dass die Erforschung von und der Umgang mit `BuRnout` primär den Fächern Medizin, Psychiatrie und Psychologie und Schnittstellenfächern wie zum Beispiel der Arbeitsmedizin oder der medizinischen Soziologie und medizinischen Psychologie zugewiesen wird. Wirtschaftsund Arbeitswissenschaftler/innen ohne klinischen Hintergrund werden in den Pressetexten ebenso, aber seltener befragt. Auf Wikipedia wird `BURNOUT seit dem Jahr 2007 der Kategorie „Psychische Störung“ und bis 2008 auch der Kategorie „Syndrom“ zugeordnet.

Dass medial in hohem Maße Personen des klinischen Bereichs (insbesondere Psychiater und Psychotherapeuten) befragt werden, ist ein interessanter Befund im Hinblick darauf, dass manche klinischen Fächer, insbesondere die Psychiatrie, dem Thema „Burnout“ am kritischsten gegenüberstehen und sich der Behandlung im Vergleich zu anderen Fächern erst spät angenommen haben: Vergleicht man durch eine Recherche in der psychologisch-bibliografischen Datenbank Psyndex und im Untersuchungskorpus, ab welchem Zeitpunkt Texte zum BuRNouT-Phänomen in der „Zeitschrift für Arbeits- und Organisationspsychologie“ im Vergleich zur psychiatrischen Zeitschrift „Der Nervenarzt“ auffindbar sind, so fällt Folgendes auf: In der Zeitschrift für Arbeits- und Organisationspsychologie erscheinen erste Beiträge, in denen das Konzept >Burnout in der Forschung Verwendung findet, bereits Anfang der 1990er Jahre (z. B. Büssing/Glaser 1991; 1993; Nijhuis/Smulders 1996; Büssing/ Schmitt 1998, ZfAO, im QV unter 8.1.4). In der klinisch-psychiatrischen Zeitschrift „Der Nervenarzt“ wird der Ausdruck Burnout hingegen erst in einem Übersichtsbeitrag im Jahr 2012 verwendet. Dies bedeutet natürlich nicht, dass in letzterer Zeitschrift in den Jahren zuvor keine Arbeiten zum Themenbereich `Depression<, 'Stress` und `Leistungsfähigkeit bei Arbeitnehmern/innen` erschienen sind, doch auch die Suche nach Beiträgen zu sachverwandten Themen ergab nur wenige Beitragstreffer in dieser Zeitschrift. ${ }^{66}$ Des Weiteren fällt auf, dass in der Zeitschrift „Der Nervenarzt" in allen sechs Texten seit dem Jahr 2012, die `Burnout` als Haupt- oder

666 Beiträge zu sachverwandten Themen wie `Berufsstress` (`occupational stress`), die in das Fachkorpus aufgenommen wurden, sind z. B. Christian (1981) zum ,allgemeinen psychosomatischen Syndrom“ bei Arbeitnehmern; Linden/Muschalla 2007; Siegrist (2013); Rau/Henkel (2013); Lederbogen/Ströhle (2012); alle Belege im QV unter „Der Nervenarzt“ in Abschnitt 8.1.4. 
Nebenthema behandeln, ein Bezug zur ICD-10 hergestellt wird, ${ }^{667}$ woran sich der Verweis anschließt, dass `Burnout in diesem Klassifikationssystem nicht als Krankheit/Diagnose, sondern nur unter der Rubrik Z als „Zusatzdiagnose (Z 73.0)“ gelistet sei. Es folgt in einigen Texten der Rat bzw. Vorschlag, >Burnout als „Risikozustand“ für eine „spätere psychische Erkrankung“ zu betrachten. Der Begriff `Burnout « wird in diesen Texten durch die Nennung der ICD-10 mit anderen Definitionen (z. B. >Depression` oder >Anpassungsstörung`), die in diesem Regelwerk deklarativ als Diagnosen definiert wurden, verglichen. Von deklarativen Definitionen kann in diesem Zusammenhang gesprochen werden, weil der propositionale Gehalt dieser Diagnosedefinitionen institutionell mit der Wirklichkeit dahingehend zur Deckung gebracht wird, dass jemand nur dann als >depressiv ‘ gilt und Leistungen der Krankenkasse in Anspruch nehmen kann, wenn er die dort definierten Symptome über einen bestimmten Zeitraum hinweg aufweist (vgl. Searle 1980: 98; Saß/Saß-Houben 2005: 137ff.; vgl. Kap. 4.3.2). In den Texten der „Zeitschrift für Arbeits- und Organisationspsychologie“ wird hingegen nur in einem von 18 Texten die ICD-10 zitiert. Die meisten Texte sind Originalarbeiten (15 Texte), 14 davon stützen sich zur Erhebung von (einzelnen) Burnout-Variablen auf das Maslach Burnout Inventory ${ }^{668}$, wobei sie teilweise aber auch andere Definitionen diskutieren (z. B. Büssing/Schmitt 1998) und mit anderen Messinstrumenten kombinieren (Michel/Stegmaier/Meiser et al. 2009) oder die Güte des Messinstruments überprüfen (z. B. Neubach/Schmidt 2000). Man sieht, dass in diesen Studien keine Notwendigkeit besteht, die Definition im Hinblick auf Kennwerte für Krankschreibungspraktiken zu deklarieren. Die Definitionen dienen als Arbeitsgrundlage zur Überprüfung von Hypothesen und die Definition kann oder soll durch den Forschungsprozess durchaus noch revidiert werden. Die „Repräsentativität“ bzw. Geltung der Definition wird jedoch dennoch daran bemessen, ob ihre Aussagen über den Bezugsgegenstand „der Welt entsprechen“ (Searle 1980: 84) bzw. an andere zeichengebundenen kollektiven Erfahrungen und an den Status quo des sich in Zeichen manifestierenden „Willen[s] zum Wissen“ (Foucault [1970] 1993: 15) anschließen (vgl. Kap. 4.3.2).

667 Siehe die Texte: Berger/Schneller/Maier (2012); Berger/Gravert/Schneller et al. (2013); Hamann/Parchmann/Mendel et al. (2013); Hillert/Koch/Lehr et al. (2013); Riedel-Heller/Luppa/ Seidler et al. (2013); Schramm/Berger (2013).

668 Büssing/Glaser 1991; Büssing/Glaser 1993; Büssing/Schmitt 1998; Dormann/Zapf/Isic 2002; Büssing/Glaser/Höge 2004; Neubach/Schmidt 2004; Lehr/Schmitz/Hillert 2008; Neubach/Schmidt 2008; Michel/Stegmaier/ Meiser 2009; Raeder/Mutz/Widmer et al. 2009; Freund/ Diestel/Schmidt 2012; Holstad/Rigotto/Otto 2013; Turgut/Michel/Sonntag 2014; Wassermann/ Hoppe/Reis et al. 2014.; alle Belege im QV bei ZfAO im Abschnitt 8.1.4. 
Betrachtet man in diesen beiden Zeitschriften, wie die Autorinnen und Autoren mit dem Ausdruck Syndrom umgehen, so zeigen sich ebenfalls deutliche Unterschiede: In der „Zeitschrift für Arbeits- und Organisationspsychologie“ wird der Ausdruck Syndrom nur in 5 von 18 Artikeln verwendet. ${ }^{669}$ In den zwölf untersuchten Texten der Zeitschrift „Der Nervenarzt“ gehen sechs Texte unter der Bezeichnung Burnout auf das dahinterliegende Phänomen ein. Von diesen sechs Texten erwähnt ein Text den Ausdruck Burnout-Syndrom ohne im direkten Kotext befindliche Distanzmarker (Jurkat/Richter/Cramer 2011). Der nächste Beitrag distanziert sich zu Beginn vom „medialen „Hype“ um das Thema Burn-out“ (Riedel-Heller/Luppa/ Seidler et al. (2013): 832) und ordnet das „Burnout-Syndrom“ in der Folge als nicht neue, aber „ernstzunehmende Risikokonstellation“ ein (ebd.: 834); in zwei weiteren Texten werden Burnout-Komposita wie „Burnout-Folgeerkrankungen“ verwendet, der Ausdruck Syndrom erscheint selbst nicht (Hillert/Koch/Lehr 2013; Rössler/ Hengartner/Ajdacic-Gross 2013). In den letzten drei dieser sechs Texte distanzieren sich die Autoren/Autorinnen ausdrücklich von der Bezeichnung Syndrom: Berger/ Schneller/Maier (2012) setzen es in Anführungszeichen, Schramm/Berger (2013) sprechen vom „so genannten Burnout-Syndrom[s]“ und Hamann/Parchmann/ Mendel et al. (2013: 840) sprechen im Abstract ihres Beitrags davon, dass das „Konzept des Burnouts und vor allem seine Verwendung als Diagnose [...] derzeit in der deutschen Psychiatrie sehr umstritten“ seien (Unterstreichung T.S.). Sie ergänzen dazu: „In den Medien und am Arbeitsplatz spielt das Burnout-Syndrom allerdings eine zentrale Rolle und die Fehltage aufgrund von Burnout steigen seit Jahren steil an“ (Unterstreichung T.S.).

Interessant ist, dass in Texten der „Zeitschrift für Arbeits- und Organisationspsychologie“, deren Definierensprozesse nicht auf das Klassifikationssystem bezogen sind, der Ausdruck Syndrom eher selten und im Falle seiner Verwendung neutral, d.h. ohne Distanzierungsmarker, verwendet wird. Der distanziertere Umgang mit dem Ausdruck Syndrom in Texten der Zeitschrift „Der Nervenarzt“ könnte hingegen damit zusammenhängen, dass mit der Verwendung des Aus-

669 In einem Text von Neubach/Schmidt (2000: 140) wird betont, dass die Autorinnen Maslach/Jackson (1981) >Burnout $<$ in ihrem Fragebogenmodell als mehrdimensionales Syndrom definiert hätten. In den vier anderen Texten wird der Ausdruck Syndrom ohne Quellenzuschreibung oder Distanzierung als Synonym neben Burnout verwendet (Büssing/Schmitt 1998; Kernen/ Meier 2002; Lehr/Schmitz/Hillert 2008; Neubach/Schmidt 2004); Siehe im QV unter ZfAO im Abschnitt 8.1.4. 
drucks Syndrom eine implizite Zuordnung zum klinischen Bereich einhergeht. ${ }^{670}$ Denn diese Distanzbekundung tritt häufig zusammen mit dem Verweis auf, Burnout sei keine Krankheit bzw. Diagnose, teilweise wird auch von Modediagnose gesprochen. ${ }^{671}$ Burnout wird stattdessen als Risikozustand für bestehende Diagnosen klassifiziert. Dass Fachleute aus der psychiatrischen Praxis eine solche bewusste Abwehrhaltung einnehmen, könnte ein Indiz dafür sein, dass sie einer im fachexternen Bereich schon konventionell gebrauchten Wortverbindung etwas entgegensetzen möchten. Denn das Kookkurrenzprofil zu Burnout in der Kookkurrenzdatenbank CCDB, die Medientexte auswertet, zeigt, dass Burnout und Syndrom bereits eine dominante Wortverbindungsstruktur eingegangen sind. ${ }^{672}$ In Kap. 7.3 wird anknüpfend an diese Beobachtung der Frage nachgegangen, inwiefern bestimmte diskursive Verfestigungen, die in dieser Arbeit im Rahmen diskursiver Praktiken des Definierens untersucht werden, einerseits den Eindruck von Konsens produzieren und andererseits Dissens provozieren.

Eine ebenfalls eingrenzende und durch Wiederholung in verschiedenen Texten des Diskurses sich verstetigende Rolle übernehmen die Schlüsselwörter oder Keywords, deren Angabe in vielen Fachzeitschriften zum festen Bestandteil der Textsorten Originalarbeit und Übersichtsarbeit/Review geworden ist. Vergleicht man die Schlüsselwörter aller Korpustexte der Zeitschriften „Deutsche medizinische Wochenschrift“, „Der Nervenarzt“, „Psychologie in Erziehung und Unterricht“, „Zeitschrift für Arbeits- und Organisationspsychologie“ und „Psychotherapie Psychosomatik und medizinische Psychologie“, dann ergibt sich folgende Übersicht: ${ }^{673}$

670 Siehe die Bedeutung auf Duden Online zum Ausdruck Syndrom: „Krankheitsbild, das sich aus dem Zusammentreffen verschiedener charakteristischer Symptome ergibt.“ https://www. duden.de/rechtschreibung/Syndrom (zuletzt eingesehen am 28.12.2019).

671 In den zitierten Texten der Zeitschrift „Der Nervenarzt“ wird bei Hamann/Parchmann/Mendel (2013: 839) referiert, dass Burnout als „eigenständiges Krankheitsbild“ von Teilnehmenden des Jahreskongresses der „Deutschen Gesellschaft für Psychiatrie, Psychotherapie und Nervenheilkunde (DGPPN)“ im Mittel „verneint“ wurde, und es wird von Burnout als Modediagnose (ebd.: 840) gesprochen.

672 Syndrom weist im Kookkurrenzprofil der Kookkurrenz-Datenbank „CCDB“ des LeibnizInstituts für Deutsche Sprache den höchsten Log-Likelihood-Ratio-Wert (LLR) auf. Gesucht wurde das Kookkurrenzprofil zu Burnout auf der Seite http://corpora.ids-mannheim.de/ccdb/ (zuletzt eingesehen am: 28.12.2019).

673 Das „Dt. Ärzteblatt“ weist in seinen Artikeln keine Schlüsselwörter/Keywords aus. 
Tab. 8: Schlüsselwörter/Keywords der Fachzeitschriften-Beiträge, die \BURNOUTく behandeln.

\begin{tabular}{|c|c|}
\hline Zeitschrift & Keywords/Schlüsselwörter \\
\hline $\begin{array}{l}\text { Deutsche med. } \\
\text { Wochenschrift } \\
\text { (DMW) }\end{array}$ & $\begin{array}{l}\text { Burn-out/Burnout-Syndrom (5) } \\
\text { ZUGEORDNETE, UNTERSUCHTE BEREICHE, PERSONENGRUPPEN: Ärzte/ } \\
\text { Ärztinnen (als Patienten) (3), MedizinstudentInnen; Arzt-Patient- } \\
\text { Interaktion; Suchttherapeuten/Suchthelfer; Krankenhaus; Schule; } \\
\text { Professionalisierung in der Altenpflege; Intensivmedizin } \\
\text { SYMPTOMATOLOGIE UND UNTERSUCHUNGSZUSAMMENHÄNGE ZU } \\
\text { ANDEREN (ÄHNLICHEN) FORSCHUNGSKONZEPTEN/-VARIABLEN, TLW. } \\
\text { HYPOTHETISCHE FAKTOREN FÜR ÄTIOLOGIE UND VERLAUF UND MÖGLICHE } \\
\text { ZIELE: Zu den Konzepten >Belastungı und >Beanspruchung` (4): } \\
\text { arbeitsbedingte Belastung, psychopathologische Symptombelastung, } \\
\text { Beanspruchungsfolgen, Belastungen am Arbeitsplatz; Stress/ } \\
\text { Stressoren/Stressreaktionen (3); Depression (2); psychische } \\
\text { Gesundheit (2); emotionale Erschöpfung; Depersonalisation; Angst; } \\
\text { CFS; Symptome; Ätiologie; Arbeitsbedingungen; Anforderungen; } \\
\text { Lärm; Schichtarbeit; Multitasking; Ressourcen; Unterbrechungen; } \\
\text { Behandlungsfehler; Krankheitsverlauf; } \\
\text { (MEDIZINISCHE, PSYcHOLOG.) VERFAHREN UND ZIELE: Prävention } \\
\text { (3), (Differential-)Diagnose (2), Therapie (2), (Betriebliche) } \\
\text { Gesundheitsförderung (2); Screening; Prävalenz; }\end{array}$ \\
\hline er Nervenarzt & 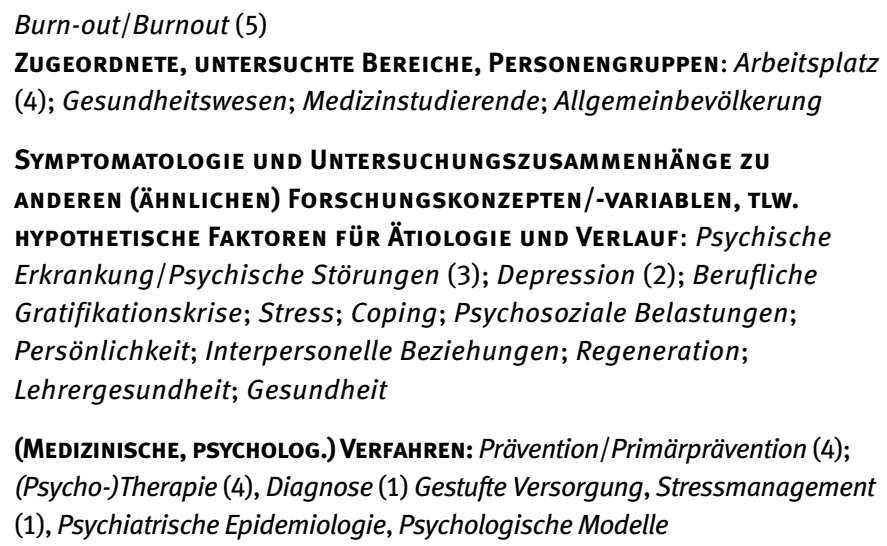 \\
\hline $\begin{array}{l}\text { Psychologie in } \\
\text { Erziehung und } \\
\text { Unterricht (PiEU) }\end{array}$ & $\begin{array}{l}\text { Ausbrennen (3) Burnout (9) „burnout“-Syndrom } \\
\text { ZUGEORDNETE ODER UNTERSUCHTE BEREICHE, PERSONENGRUPPEN: Burnout } \\
\text { im Studium (1) Lehrer-Burnout (1), Lehrkräfte (1) Lehrerbildung (1) Lehrer- } \\
\text { berufLehrerforschung (1), Lehrer (3) Grundschullehrkräfte (1) Vorberei- } \\
\text { tungsdienst (1), Referendariat (1), Sozialberufe }\end{array}$ \\
\hline
\end{tabular}


Tab. 8 (fortgesetzt)

\begin{tabular}{|c|c|}
\hline Zeitschrift & Keywords/Schlüsselwörter \\
\hline & 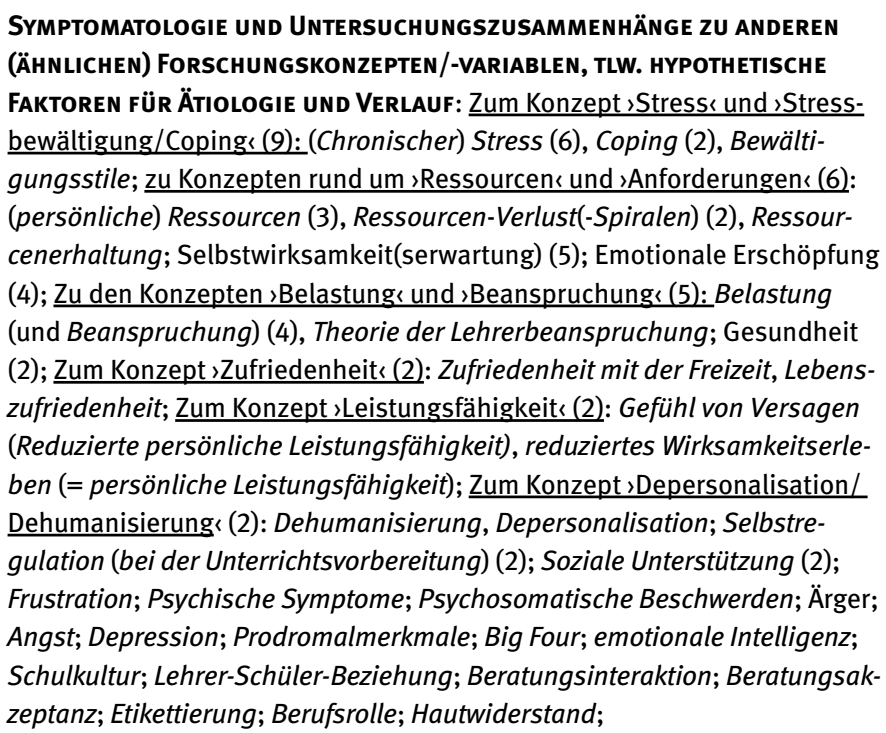 \\
\hline
\end{tabular}

FORSCHUNGSMETHOdEN: Veränderungsmessung; AVEM; LISREL (2); Trainingsevaluation; Intervention;

Zeitschrift für Arbeits- und Organisationspsychologie (ZfAO)
Burnout (5), Burnout-Dimensionen, Burnout-Modelle

Zugeordnete oder untersuchte Bereiche, Personengruppen: In den Bereichen >med. Versorgung und >Pfleges: Krankenhaus, Krankenpflegekräfte, Krankenpflege; Altenpflege/Altenpflegekräfte (3); Pflegepersonal, Ambulante Pflege; Call Center; Dienstleistung; Lehrer; Patchworker

SYMPTOMATOLOGIE UND UNTERSUCHUNGSZUSAMMENHÄNGE ZU ANDEREN (ÄHNLICHEN) FORSCHUNGSKONZEPTEN/-VARIABLEN, TLW. HYPOTHETISCHE FAKTOREN für ÄtIologie Und VerLauf: Zu den Konzepten >Belastungく und >Beanspruchung`(7): Arbeitsbelastungen (3), psychische Belastung, physische Belastung, Belastung, psychische Beanspruchung am Arbeitsplatz; zu Konzepten rund um >Ressourcen und >Anforderungen (4): (persönliche) Ressourcen (2), Job Demands-Resources Model, Arbeitsanforderungen; zum Konzept semotionale Erschöpfungs (4); Zum Konzept `Kontrolle ( (4): Demands-Control-Model, Kontrollspielräume, Kontrollmöglichkeiten, Selbstkontrolle; Zum Konzept >Emotion (3): Emotionsarbeit (2); emotionale Dissonanz; Depersonalisation; Tätigkeitsspielraum; Persönlichkeitsförderung; vollständige Tätigkeit; Qualifikationserfordernisse, Qualifikationsmöglichkeiten; Transformationale Führung; prozedurale Fairness; kognitive Irritation; Bewältigungsmuster; beruflicher Stress; psychische Störungen; Change- 
Tab. 8 (fortgesetzt)

\begin{tabular}{|c|c|}
\hline Zeitschrift & Keywords/Schlüsselwörter \\
\hline & $\begin{array}{l}\text { Charakteristika; Wahrnehmung des Arbeitskontextes; Reaktionen } \\
\text { während Veränderungen an Hochschulen; Mediatoreffekt; Ego-Depletion; } \\
\text { soziale Unterstützung; Gesundheitsbeschwerden; Fehlzeiten; flexible } \\
\text { Beschäftigung; Selbstwert; Arbeitszufriedenheit; Sinnstiftung; } \\
\text { FORSCHUNGSMETHODEN: Tätigkeits- und Arbeitsanalyse(verfahren) (3), } \\
\text { Clusteranalyse (2), Reliabilität, Validität; } \\
\text { ZIELE: Gesundheitsförderung; Persönlichkeitsförderung; Betriebliche } \\
\text { Gesundheitsförderung; Arbeits- und Gesundheitsschutz; }\end{array}$ \\
\hline $\begin{array}{l}\text { Psychotherapie, } \\
\text { Psychosomatik } \\
\text { und Medizinische } \\
\text { Psychologie (PPmP) }\end{array}$ & 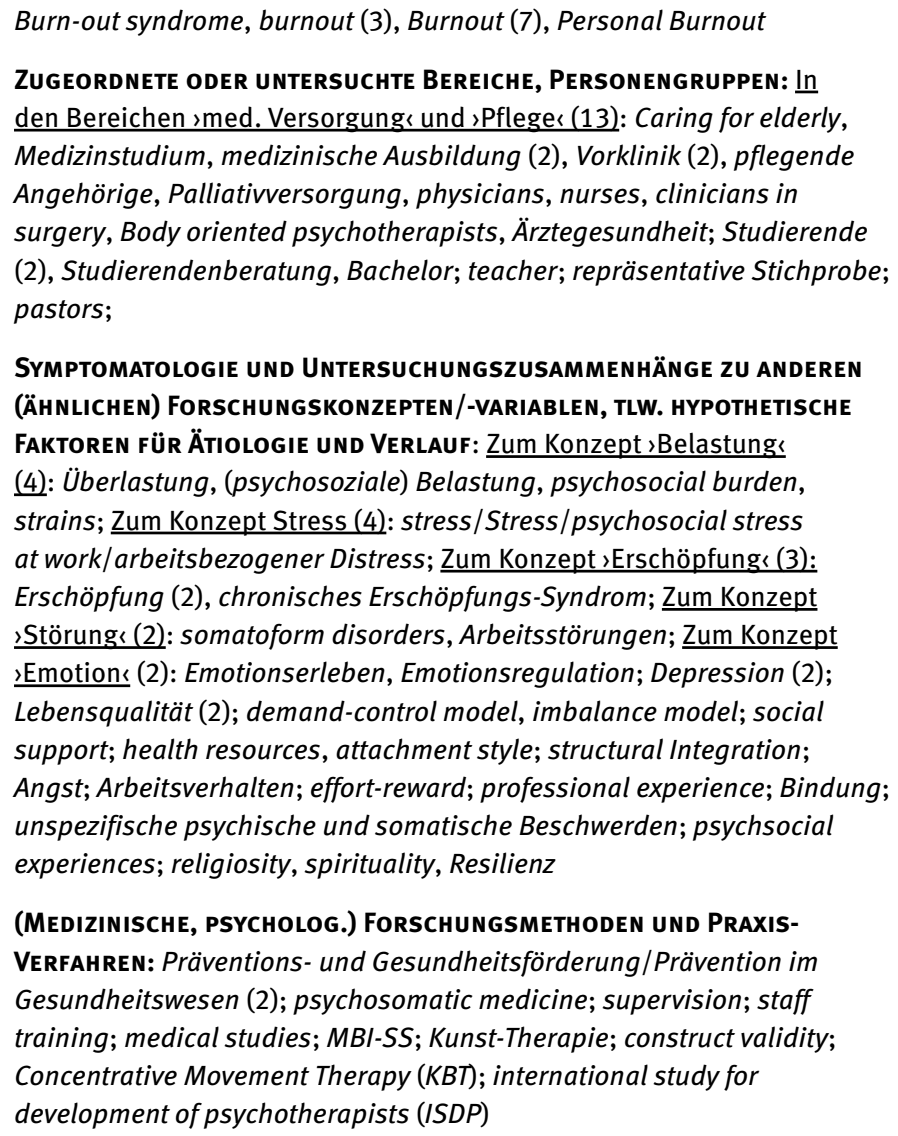 \\
\hline
\end{tabular}


Über alle Zeitschriften hinweg wird in den Keywords ein Bezug zur Arbeitswelt hergestellt, explizit über Ausdrücke wie Arbeitsbelastungen, Arbeitsstörungen oder dadurch, dass Berufsgruppen aufgezählt werden. Nicht verwunderlich ist, dass in den Zeitschriften, je nach Ausrichtung der Zeitschrift, bestimmte Berufsgruppen besonders im Fokus der Untersuchungen stehen (z. B. Lehrer in der Zeitschrift PiEU). Interessant ist jedoch, dass in den klinischen Zeitschriften die klinischen bzw. pflegenden Berufe selbst so intensiv beforscht werden und dass diese Personengruppe sich in fast allen Fachzeitschriften wiederfindet. ${ }^{674}$ Da Keywords in der Form der Aufzählung dargeboten werden, kann man, wenn man die Keywords ohne Vorwissen liest, teilweise nicht entscheiden, in welchem semantischen Verhältnis die dort genannten Wörter zum Begriff `Burnout s stehen. In den Zeitschriften „Der Nervenarzt“ und „PPmP“ werden beispielsweise in mehreren Artikeln die Schlüsselwörter psychische Erkrankung, psychische Störung oder auch somatoform disorder verwendet, die man in dieser Position als Hyperonyme zu >Burnout interpretieren könnte. >Burnout ' wird in einigen dieser Beiträge jedoch nicht selbst als >psychische Störung`, sondern als „Risikozustand“ klassifiziert, der zu einer >psychischen Störung، führen könne (z. B. Berger/Schneller/Maier 2012, Der Nervenarzt). ${ }^{675}$ Bei den Keywords werden meist verschiedene Kategorien zusammen dargeboten. Man muss aufmerksam sein, dass man bei ihrer Interpretation keine voreiligen Schlüsse über semantische Relationen zieht: So werden einerseits Symptome von >BuRNouT< gelistet, zwölfmal über vier Zeitschriften verteilt das Symptom (emotionale, chronische) Erschöpfung und viermal über drei Zeitschriften das Symptom Depersonalisation/Dehumanisierung. Dass dies Symptome sind, weiß man aber nur, wenn man zuvor einige Beiträge gelesen hat, in denen diese als Symptome von >Burnout benannt wurden. Depression erscheint siebenmal über die Zeitschriften verteilt, wobei es bei genauerer Textlektüre als Konzept, dessen Überschneidungen zu `Burnout` untersucht werden (z. B. Reime/Steiner 2001, PPmP), oder als das eigentliche Krankheitsbild hinter >Burnout behandelt wird (vgl. Hamann/Parchmann/Mendel 2013, Der Nervenarzt). Keywords, die ebenfalls über alle Fachzeitschriften hinweg auftreten, sind Wörter rund um die Begriffe $>$ Belastung $\triangleleft />B e$ anspruchung`(20 mal), >Stress` (16 mal), >Gesundheit` (14 mal), >Ressourcen und Anforderungen $(10 \mathrm{mal})$ sowie >Kontrolle/control` (5 mal), in denen auch Forschungsmodelle wie das „(Job) Demands-Resources Model“/“(Arbeitstätigkeit)-

674 Siehe in Tab. 8 in den Zeitschriften: DMW, Der Nervenarzt, ZfAO und PPmP.

675 Bei Bauer/Häfner/Kächele et al. 2003 (PPmP, im QV unter 8.1.4) wiederum dient ,somatoform disorder“ in den Keywords durchaus als Hyperonym, denn >Burnout ‘ wird im Artikel folgendermaßen beschrieben (ebd.: 216): „Das Burn-out-Syndrom ist also eine medizinisch relevante Gesundheitsstörung, [...].“ (Unterstreichung T.S.). 
Anforderungen-Ressourcen-Modell“676, das Demand-Control-(Support-)Modell/ Modell der Anforderung-Kontrolle-(Unterstützung) nach Karasek/Theorell 1990 (zitiert nach Rigotti 2019) ${ }^{677}$ und die „Norm zur psychischen Belastung und Beanspruchung “678 mitschwingen. Durch diese gerade aufgezählten Keywords wird `Burnout`stärker dem Bereich der `Salutogenese` und Prävention als der >Pathogenese` zugeordnet. Das wiederholte Auftreten gleicher oder ähnlicher Keywords kann daher die definitorische Einordnung eines Begriffs maßgeblich unterstützen. Die in den Keywords angesprochenen Konzepte und Modelle

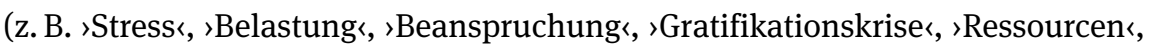
>Demands-Control-Model $)$ finden im öffentlichen Diskurs insbesondere Verbreitung über die Wikipedia-Artikelversionen zu „Burnout“ und Artikel in populärwissenschaftlichen Zeitschriften wie „Psychologie Heute“ oder „Gehirn\&Geist“ und in Metaphern, die diesen Erklärungsmodellen ähneln (siehe Kap. 6.2.4.2). ${ }^{679}$

676 Das „Anforderungs-Ressourcen-Modell“ ist „ein Rahmenmodell zur Erklärung von Gesundheit [...] aus systemischer bzw. ökologischer Perspektive. Es beruht auf stresstheoret. Modellen (Stress) wie der Salutogenese und wurde von dem Trierer Psychologen Peter Becker und Mitarbeitern 1994 entwickelt“ (= Faltermaier/Hübner 2019, Dorsch, Lexikon der Psychologie, Onlineausgabe: https://portal.hogrefe.com/dorsch/anforderungs-ressourcen-modell/, zuletzt abgerufen am 27.12.2019, im QV unter 8.1.2). Das „Job-demands-resources model“ wurde „als arbeitspsychol. Modell i. R. der Burn-out-Forschung entwickelt. Es dient der Erklärung von pos. und neg. Befindensindikatoren im Arbeitskontext. Faktoren der Arbeit können demnach eingeteilt werden in Anforderungen (demands) und Ressourcen (resources). Durch eine Häufung von Anforderungen (z. B. Zeitdruck, ungünstige Umgebungsbedingungen, Schichtarbeit) kommt es über den sog. health impairment process zu neg. Folgen wie Erschöpfung oder Burn-out.“ (Schladitz 2019, Dorsch, Lexikon der Psychologie, Onlineausgabe: https://portal-hogrefe-com. ubproxy.ub.uni-heidelberg.de/dorsch/job-demands-resources-modell/, zuletzt abgerufen über die Universitätsbibliothek Heidelberg am 27.12.2019, im QV unter 8.1.2, vgl. auch Demerouti/ Nachreiner/Bakker et al. 2001, im QV unter 8.1.5).

677 Rigotti (2019), Dorsch, Lexikon der Psychologie, Online-Ausgabe: https://portal-hogrefecom.ubproxy.ub.uni-heidelberg.de/dorsch/demand-control-support-modell/, zuletzt abgerufen über die Universitätsbibliothek Heidelberg am 27.12.2019, im QV unter 8.1.2).

678 Vgl. dazu im Überblick Nachreiner 2012: 8ff., im QV unter 8.1.5, sowie Kap. 5.2. dieser Arbeit. 679 „Gratifikationskrise nennen es Experten, wenn das Gefühl, der hohe berufliche Einsatz würde nicht ausreichend gewürdigt, sich zum zusätzlichen Stressfaktor entwickelt“ (G\&G 11/2005: 17. = Kraft 2005, im QV unter 8.1.7); „Auch Erschöpfung, vielleicht das zentrale Merkmal eines Burnout, entsteht aus einer übermäßigen Beanspruchung physischer und psychischer Ressourcen“ (G\&G, Dossier, 1/2016: 75, = Altstötter-Gleich 2016); „Schließlich haben sich verschiedene Studien mit dem Zusammenhang zwischen dem Fünf-Faktoren-Persönlichkeitsmodell (Big Five) und Burn-out beschäftigt. Hier scheint eine neurotische Persönlichkeit [...] besonders Gefahr [zu] laufen, einen Burn-out zu entwickeln.“ (PH 5/2009: 28, =Schulze 2009); Unterstreichungen in den Zitaten T.S. 
Wie in den Kapiteln 6.2.2.1 und 6.2.2.2 schon angedeutet wurde, wird der Gegenstand BuRNouT schließlich sowohl in den Fachtexten (in Lexika, Hand- und Lehrbuchtexten sowie Fachzeitschriften und Monografien) als auch in fachexternen Texten (Pressetexten und Onlineportalen) über sprachliche Mittel der Gradierung, Quantifizierung und Negation punktuell eingegrenzt, dadurch, dass bei den Symptomangaben verschiedene Bezugsnormen und Gegenpole impliziert werden. Diese geben Anhaltspunkte darüber, wie das Verhältnis zwischen Über-, Unter- und Normalmaß zu interpretieren ist. Treten diese punktuellen Eingrenzungspraktiken wiederholt an verschiedenen Stellen im Diskurs auf, so können sie den Gegenstand und das Konzept >BURNOUT< zwischen den Polen >normal//gesund ‘ und sabnormal//>krank verorten und dadurch weiter eingrenzen und festlegen. Sie entfalten insbesondere durch das wiederholte Auftreten in der Fläche des Diskurses ihre ab- und eingrenzende-definitorische Kraft. Die implizit aufgerufenen Referenznormen, Gegenpole und Vergleichsstandards, die im Folgenden anhand von Beispielen differenziert werden, sind Ausdruck diskursiver `Polyphonie` (vgl. Angermüller/Scholz 2013: 307), die hier als ein flächiges Diskursphänomen eingeordnet wird, da auf eine Partitur von Stimmen (kollektive Wissensbestände und Normvorstellungen) außerhalb des analysierten Textes/Textabschnitts (implizit) Bezug genommen wird: Wenn über eine Pflegeperson im Rahmen einer Symptombeschreibung gesagt wird, sie handle herzlos, dann schwingt in dieser Aussage eine ,generische Prädikation“ (vgl. SchmidtBrücken 2015: 38ff.) bzw. sozial-kulturelle Praktik mit, die unter 'normalen' Umständen für diesen Berufskontext `teilnehmende Fürsorge` vorsieht. ${ }^{680}$ Das für `Burnout ‘ oft als typisch genannte Symptom des ıZynismus « wird in solchen diskursiv-polyphonen Gegenüberstellungen ausgestaltet. Im Kontrast zu dieser implizit mitgemeinten sozial-kulturellen Praktik wird dieses Verhalten zum erklärungsbedürftigen Signal und damit zum Symptom (vgl. Sebeok 1984: 42; vgl. von Uexküll 1984b: 32). ${ }^{681}$

Sowohl über die verschiedenen Textsorten des fachlichen als auch über die Texte des fachexternen Diskursstrangs verteilt, findet man frequente, durative und steigernde Adjektive, Adverbien (vgl. Zifonun/Hoffmann/Strecker 1997: 1536ff., Band 2), Partikeln (diese vor allem fachextern) und Wortbildungselemente in Kombination mit negierenden Sprachmitteln, die die Bezugsausdrücke hinsichtlich ihrer Frequenz und Dauer sowie Intensität spezifizieren und damit antonyme oder auch komplementäre Gegensatzpole evozieren. ${ }^{682}$ Durch diese sprach-

680 Vgl. dazu Köller (2004): „Bei der Verwendung des Negationssuffixes -los gilt in der Regel die Annahme, dass die jeweilige Eigenschaft nicht erwünscht ist“ (Köller 2004: 365f.).

681 Vgl. dazu die Termini Zeichen, Signal, Symptom in der medizinischen Semiotik Kap. 2.2.1 dieser Arbeit.

682 Schippan (22002: 215) bestimmt „Antonymie“ wie folgt: „Die Negation eines Ausdrucks impliziert nicht notwendigerweise die Behauptung des anderen, sondern zwischen zwei antony- 
lichen Mittel wird beispielsweise angezeigt, dass der REFERENZZUSTAND bzw. das angeführte BEOBACHTUNGSMERKMAL (z. B. Überforderung) sich nur graduell von anderen Ausprägungen desselben MERKMALs (z. B. Unterforderung) unterscheidet bzw. oberhalb oder unterhalb eines GEMEINSAMEN DURCHSCHNITTSWERTS (z. B. weder zu hohe noch zu niedrige Anforderung an $x /$ sich/etwas) einer Skala eingeordnet werden kann. ${ }^{683}$ Es wird mit einem Wort wie Überforderung ein impliziter wertender Vergleich initiiert, denn Überforderung bedeutet, dass höhere Anforderungen an eine Person gestellt werden, als dies 'angemessen' erscheint. ${ }^{684} \mathrm{Um}$ die Bezugsnorm für diese einstufende Bewertung auszumachen, muss wiederum eine Vergleichsmenge mitgedacht werden. Ko- und kontextuell bieten sich in den untersuchten Belegen des fachlichen und fachexternen Burnoutdiskurses Normen an, die sich zum einen auf biologische Durchschnittswerte, zum anderen auf sozialkulturelle Praktiken und darüber hinaus auf Individualnormen beziehen:

Im Verlauf eines Arbeitslebens kann es üblicherweise immer mal wieder zu vorübergehenden Überlastungen kommen, die mit vegetativen Stresssymptomen wie Schweißausbrüchen, Schlafstörungen und einem allgemeinen Gefühl der Erschöpfung einhergehen können. Diese Arbeitsüberlastungsfolgen sollten sich jedoch im Rahmen von Wochenenderholung oder Urlauben rasch zurückbilden. Bei länger anhaltender Überforderung

mischen Polen gibt es Übergänge. [...] So treten Antonyme meistens dort auf, wo auf der Grundlage bestimmter Kriterien Qualitäten eingeschätzt und diese Qualitäten auf eine angenommene ,Null-Stufe“ bezogen werden.“ Bei der Komplementarität hingegen sind „Zwischenstufen nicht möglich. Handelt es sich um Adjektive, so sind sie oft nicht komparierbar, weil nicht graduierbar. Die Behauptung des einen impliziert die Verneinung des anderen“ (ebd.). Aus logischer Perspektive lassen sich >Antonymie und `Komplementarität eindeutig unterscheiden, im Sprachmaterial gibt es zwischen diesen aber fließende Grenzen (vgl. Gsell 1979: 107; Rachidi 1989: 95). Im Laufe des Kapitels wird noch auf die gradierbaren Komplementäre eingegangen (vgl. Rachidi 1989: 93). Nach Schuster wurde in der psychiatrischen Schreibpraxis auf die Erklärungskraft komplementärer Adjektivpaare (Schuster 2010: 236f.) und Graduierungen zur Angabe „der Stärke oder Ausprägung eines Symptoms oder einer Krankheit“ (ebd: 238) schon in der ersten Hälfte des 19. Jahrhunderts gesetzt. Später wurden mit dem Lehrbuch von Emil Kraepelin auch Affixe wie über- (z. B. in Überempfindlichkeit, Überanstrengung) produktiv (Schuster 2010: 290f.).

683 Vgl. Schippan 22002: 215; Rachidi (1989: 98) spricht vom „gemeinsamen Teilinhalt antonymer Adjektive“, der „im Bereich der Norm bzw. in der Indifferenzzone anzusiedeln ist.“ Sie bezieht sich dabei u. a. auch auf Gsell 1979.

684 Vgl. die Bedeutungsangabe auf ,duden.de“ zum Verb überfordern: „zu hohe Anforderungen an jemanden, sich, etwas stellen“ (Unterstreichung T.S.) und zum Intensitätspartikel $z u$ findet man folgenden Eintrag: „kennzeichnet ein (hohes oder geringes) Maß, das nicht mehr angemessen oder akzeptabel erscheint“ (Unterstreichung T.S.). Die Bedeutungsangaben sind einsehbar unter: https://www.duden.de/rechtschreibung/ueberfordern und https://www.duden.de/rechtschreibung/zu_Adverb (zuletzt eingesehen am 27.12.2019). 
kann der Begriff Burnout zur Charakterisierung eines Zustands chronischer emotionaler Erschöpfung, einer kritischen Distanz zu der als überfordernd erlebten Arbeit und einer Reduzierung der eigenen Leistungsfähigkeit bei vegetativen Stresssymptomen genutzt werden [8].

(Unterstreichung T.S.) (Schramm/Berger 2013: 816, Der Nervenarzt, im QV unter 8.1.4)

Wie das zitierte Beispiel zeigt, werden Überforderungserlebnisse im Arbeitskontext aufgerufen und mit somatischen („Schweißausbrüchen“) und emotionalpsychischen (,kritischen Distanz zu der [...] Arbeit“) Merkmalen sowie Verhaltensmerkmalen („Reduzierung der eigenen Leistungsfähigkeit“) verbunden. Auf den Kern der Bewertungsnorm wird im obigen Zitat durch das Verb sollen hingewiesen: Bei einem 'normalen', 'gesunden', ‘durchschnittlichen' Vertreter der Gattung sarbeitende Person`sollten sich diese Merkmale nach „,vorübergehenden Überlastungen“ am Wochenende oder im Urlaub rasch zurückbilden. Es kommen hier >biologische Belastungsgrenzen ‘ in Bezug auf die Länge und Häufigkeit der belastenden Faktoren (z. B. durchschnittliches Schlaf- und Ruhebedürfnis, durchschnittliche Konzentrationsspanne), aber auch >sozialkulturelle Idealnormen` in Bezug auf die Einstellung zur Arbeit (kritische Distanz zur Arbeit wird hier in Verbindung mit anderen Merkmalen als Syмртом für BuRNout angeführt) und sindividuell-durchschnittliche Erwartungsnormen « in Bezug auf die Leistungsfähigkeit (Reduzierung der eigenen Leistungsfähigkeit im Vergleich zu früher) ins Spiel.

In fachexternen Texten werden diese verschiedenen Ebenen und Normen in Bezug auf >BURNOUT< ebenfalls miteinander verbunden. Die Symptome werden dabei meist über eine noch konkretere Ebene vermittelt, wie der folgende „Selbsttest“" zeigt, der am Ende eines Magazinberichts auf faz.net präsentiert wird (Meck 2010, FAZ, im QV unter 8.1.6). Denn auch in diesem Test werden die in den Fragen präsentierten konkretisierten Merkmale erst dadurch zu „bedrohlichen“ Symptomen, dass die sich testende Person biologische Normalfunktionen (z. B.: Nach Schlaf/Urlaub fühlt man sich erholt) und sozial-kulturelle oder auch individuell-durchschnittliche Erwartungen (z. B.: Arbeit sollte Freude bereiten/ Bisher hat mir die Arbeit meistens Freude bereitet) als Vergleichswerte mitdenkt (faz.net 08.03.2010, = Meck 2010):

\section{Sind Sie von Burnout gefährdet? Ein Selbsttest}

Wenn Sie mehr als eine der folgenden acht Fragen mit „Ja“ beantworten, sollten sie überlegen, ob Sie vom Burn out-Syndrom bedroht sind:

- Gehen Sie lustlos zur Arbeit?

- Fühlen Sie sich morgens nach dem Schlafen wie zerschlagen?

- Belastet Sie der Umgang mit Kollegen, ziehen Sie sich zurück?

- Fühlen Sie sich von Ihren Mitmenschen genervt?

- Sind Sie öfter gereizt? 
- Fühlen Sie sich auch nach Urlaub oder Wochenende nicht richtig erholt?

- Sind Sie öfter erkältet, oder haben Sie Magen-, Kopf- oder Rückenschmerzen und Kreislaufprobleme?

- Haben Sie deutlich mehr Lust auf Zigaretten, Alkohol, Süßigkeiten oder Tabletten?

Die meisten im fachlichen und fachexternen Korpus angeführten SOMATISCHEN MERKMAle (z. B.: chronische Erschöpfung (durative Quantitätsantonymie), schwere Erschöpfung (gradierende Qualitätsantonymie), immer wieder auftretendes Herzrasen, öfter erkältet (frequente Quantitätsantonymie), verminderte Schlafqualität (gradierende Qualitätsantonymie)) werden auf den >durchschnittlich gesunden Menschen eines bestimmten Alters bezogen. Rachidi spricht in diesem Zusammenhang unter Rückbezug auf Leisi ( ${ }^{4} 1971:$ 101-105) von einer ISpeziesnorm` (Rachidi 1989: 99). Auch bei der Beschreibung PSYCHISCHER ZUSTÄNDE, PERSONELLER EIGENSCHAFTEN und VERHALTENSAUFFÄLLIGKEITEN kommen durchschnittliche menschliche und gruppenspezifische Vergleichswerte ins Spiel: So gibt es implizite Vorstellungen davon, wie eine normale Leistungsbereitschaft oder -fähigkeit einer (z. B. jugendlichen, erwachsenen) Person (z. B. mit bestimmter Ausbildung, Lebenssituation) aussieht, woran man eine durchschnittliche Motivation oder ein durchschnittliches Engagement in bestimmten Bereichen (z. B. Universität, Krankenhaus, Konzern) im Vergleich zu besonderem Engagement oder Idealismus erkennt und in welchen Situationen oder unter welchen Umständen (z. B. schlechtes Betriebsklima) es durchaus als ,normal' gilt, mit Misstrauen, Zynismus oder Frustration statt Zufriedenheit oder Selbstwirksamkeitserwartung zu reagieren. ${ }^{685}$ Anhand der Spezifizierungen, die im letzten Satz in Klammern hinzugefügt wurden, wird deutlich, dass es hier nicht nur um biologische, die menschliche Spezies betreffende graduelle Kennwerte geht, sondern dass je nach Kontext sozialgesellschaftliche und kulturelle Erwartungsnormen mit ins Spiel kommen. Betrachtet man diese Erwartungsnormen noch genauer, dann wird deutlich, dass es neben dem Bezug auf durchschnittliche Werte auch so etwas wie Ideal- und Tauglichkeitsnormen gibt, die in Konflikt zueinander geraten und dadurch zum SумРтом werden können, wie in den folgenden beiden fachlichen Kurzdefinitionen des Begriffs >Burnout nach Edelwich/Brodsky (1984) (zitiert in einer Tabelle bei Sonntag/Frieling/Stegmaier ${ }^{32012)}$ und Bergknapp (2009, PiD) und drei Fallbeispielen aus dem fachexternen Diskursstrang (SPIEGEL ONLINE, = Abé 2012 und FAZ.NET, $=$ Weiguny/Nienhaus 2014a) deutlich wird (Unterstreichungen T.S.):

685 Die kursivierten Wörter in diesem Satz und in den folgenden Absätzen gehen auf Belege im Korpus zurück. 
Edelwich/Brodsky (1984) (zitiert in einer Tabelle bei Sonntag/Frieling/Stegmaier ${ }^{32} 2012$ : 320, im QV unter 8.1.2)

Vierstufiger Prozess der Desillusionierung: 1. idealistische Begeisterung (unrealistische Erwartungen, übermäßiges Engagement) 2. Stagnation: Unzufriedenheit mit organisatorischen Rahmenbedingungen der Arbeit, weiterhin großes Engagement 3. Frustration der idealistischen Erwartungen 4. Apathie, Frustration, Zynismus, emotionaler Rückzug, Vermeidung von Klientenkontakten.

«... zunehmender Verlust an Idealismus und Energie ... » (S. 12) durch fortschreitende Desillusionierung, hervorgerufen durch Überidentifikation mit Klienten sowie durch Erfahrungen mit den Arbeitsbedingungen.

Edelwich/Brodsky (1984)

Bergknapp (2009: 240) (PiD, im QV unter 8.1.4)

Aus glühenden Idealisten wurden im Laufe der Zeit frustrierte müde und reizbare Zyniker, die am Ende gleichgültig, körperlich erschöpft und feindselig ihren Klienten gegenüber waren. ${ }^{686}$

SPIEGEL ONLINE, 08.08.2012 (= Abé 2012, im QV unter 8.1.6)

[...] Ich bin stets ein ehrgeiziger Typ gewesen.

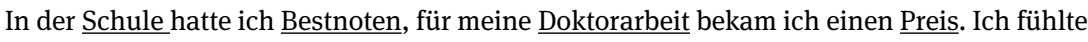
mich nur dann anerkannt, wenn ich Leistung brachte. Entsprechend verhielt ich mich auch im Berufsleben. Ich wollte meine Aufgaben immer perfekt erledigen. So stieg ich schnell weiter auf. Schließlich bekam ich eine Führungsposition, übernahm sehr viel Verantwortung.

In meiner Branche kann man sich keine Fehler leisten. In den schlimmsten Phasen arbeitete ich etwa 80 Stunden in der Woche, 140 Überstunden im Monat. Wenn ich nach Hause kam, konnte ich nicht mehr abschalten. In dieser Zeit litt auch die Beziehung zu meiner Frau, für meine Kinder hatte ich kaum mehr Zeit. Mein Blick verengte sich. Ich war gefangen in einem Tunnel, es gab nur noch die Karriere.

So fühlte ich mich einsamer. Nachts wachte ich auf, schwitzte. Morgens musste ich mich übergeben. Dann trank ich etwas Wasser, schluckte ein paar Stück Traubenzucker und ging in mein erstes Meeting.

$[\ldots]$

Im Herbst 2010 war es schließlich so weit, drei Jahre nachdem ich diese Führungsposition übernommen hatte: Ich hatte einen Burnout. [...]

686 Ähnliche Beispiele aus dem Korpus findet man auch im Pschyrembel, Klinisches Wörterbuch (2612007): 295: „Endzustand eines Prozesses von idealist. Begeisterung über Desillusionierung, Frustration u. Apathie“ (im QV unter 8.1.2); oder bei Barth 22001: 85: „Am Anfang des Burnouts steht fast regelhaft eine enthusiastische und idealistische Einstellung zum Beruf. Die Erfahrung, dass die idealistischen Ziele an der Realität scheitern, führt dazu, dass Zufriedenheit und Erfüllung im Beruf immer mehr in den Hintergrund geraten (Barth ${ }^{2} 2001 / 42010$ : 85, im QV unter 8.1.2). 
DIE ZEIT (Online-Ausgabe), 20.04.2011 (= Wagner 2011, im QV unter 8.1.6)

Helen Heinemann kennt viele solcher Geschichten, wie Marita sie erzählt, deren Name auf ihren Wunsch hin geändert wurde. Heinemann ist Psychotherapeutin und leitet das Institut für Burn-out-Prävention in Hamburg. Zynismus und Burn-out hängen eng zusammen Zynismus ist die erste Reaktion, wenn eine Situation im Beruf entsteht, aus der sich ein Burn-out entwickeln kann. Er entsteht unter anderem, wenn Ansprüche enttäuscht werden. wenn der eigene Idealismus und das eigene Engagement keinen Widerhall finden und dann die Resignation zum ersten Mal an die Bürotür klopft.

[Durch Unterstreichung hervorgehobene Links entfernt, T.S.]

FAZ.NET, 19.04.2014 (= Weiguny/Nienhaus 2014a, im QV unter 8.1.6)

Anfällig für den Zusammenbruch sind insbesondere zwei Typen von Menschen: Perfektionisten und Idealisten. Das sind perfiderweise häufig diejenigen, die in ihrer Arbeit besonders engagiert sind, voller Enthusiasmus für die Sache. Sie brennen für ihren Beruf - und dann sind sie irgendwann ausgebrannt. „Der typische Burnout-Patient ist der engagierte, kompetente Mitarbeiter in Führungsposition oder der Selbständige Mitte 40, der über Monate bis Jahre chronisch seine persönlichen Grenzen der Arbeitsfähigkeit überschritten hat“, sagt Götz Mundle, medizinischer Geschäftsführer der Oberbergkliniken, die Burnout-Patienten behandeln. Diese Grenzen überschreitet der Erschöpfte entweder, weil er sich moralisch verpflichtet fühlt - das sind die Idealisten, häufig unter Lehrern, Ärzten, Krankenschwestern zu finden - oder weil er selbst nach höchster Perfektion strebt, sich dabei unrealistische Ziele setzt, alles tut, um die eigene Grandiosität zu beweisen. „Narzisstische Persönlichkeiten sind anfällig“, sagt Mundle. Und Menschen, die schon in der Kindheit gelernt haben: Nur wenn ich etwas leiste, werde ich von meinen Eltern wahrgenommen.

Eigenschaften, die gesellschaftlich eigentlich ein eher hohes Ansehen genießen und in vielen Arbeitskontexten auch >Idealnormen darstellen, ${ }^{687}$ wie >Idealismus , ১Ehrgeiz«, ১Engagement « im Beruf und `Identifikation mit Personen/Dingen des eigenen Arbeitsumfeldes`, werden zu problematischen Eigenschaften, wenn sie in einem Übermaß vorkommen (übermäßiges Engagement, übermäßiger Ehrgeiz, nach höchster Perfektion, Überidentifikation, glühende Idealisten). Denn dieses Übermaß führt zu einer negativen Transformation der Gesundheit (Übelkeit, Schlafprobleme), negativen Emotionen gegenüber der Arbeit (Desillusionierung, Unzufriedenheit, Frustration, reizbare Zyniker) oder zur Beeinträchtigung der eigentlichen beruflichen Aufgabe (Apathie, gleichgültig, Vermeidung von Klientenkontakten, feindselig ihren Klienten gegenüber, Grenzen der Arbeitsfähigkeit überschritten, unrealistische Ziele). Dadurch werden die mit der jeweiligen Arbeit

687 So findet man beispielsweise Belege, die davon sprechen, dass „gerade die allerbesten Leute bestimmter Berufsgruppen am stärksten vom Ausbrennen bedroht zu sein“ scheinen (vgl. Barth 22001: 83, im QV unter 8.1.2). Hier spielen zweifelsohne auch Konnotationen der Metaphorik des „Brennens für etwas“ eine entscheidende Rolle. Vgl. Kap. 6.1.2 dieser Arbeit. 
verbundenen sTauglichkeitsnormen` verletzt. Zudem können andere soziale Erwartungen und Idealnormen (z. B. bezogen auf die Partner- und Elternrolle) mit der 'übererfüllten' Berufsrolle in Konflikt stehen. Das Engagement oder die Ideale „taugen“ nämlich nur, wenn sie zu den Realisierungsbedingungen passen, und Motivationsverlust wird als ,normal' bzw. ,gesund' angesehen, wenn die Zielerreichung über die Kräfte bzw. Möglichkeiten hinausgehen würde. Dies wird besonders deutlich, wenn von unrealistischen Erwartungen in Verbindung mit Idealismus gesprochen wird. Der vierstufige Prozess im oben zitierten Beispiel nach Edelwich/Brodsky evoziert eine Kausalkette, in der sich eine Aufspaltung in zwei sich gegenüberliegende Pole ergibt. Auf der einen Seite steht die 'grenzenlose, selbstlose' >Einsatzbereitschaft für positiv bewertete Ziele und Normen` und auf der anderen Seite der >Zynikerı, ein ,die Wertgefühle anderer herabset-

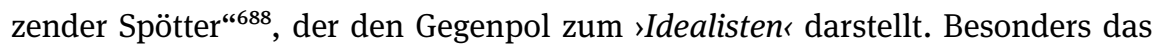
semantische Merkmal des Einsatzes oder Handelns aufgrund bestimmter `Werte wird hier in sein Gegenteil verkehrt. Die dahinterstehende Skala >wertbezogene(r) Einsatz-Zurückhaltung im Beruf (psychisch und physisch)< könnte man, wenn man jeden Zwischenzustand explizit formuliert, wie folgt darstellen:

Tab. 9: Skala ıwertbezogene(r) Einsatz-Zurückhaltung im Beruf (psychisch und physisch)<.

\begin{tabular}{|c|c|c|c|c|c|c|}
\hline $\begin{array}{l}\text { sübermä- } \\
\text { Biger } \\
\text { Einsatzı }\end{array}$ & $\begin{array}{l}\text { ssehr großer, } \\
\text { ständiger } \\
\text { Einsatzı/ } \\
\text { 'größter } \\
\text { Einsatz im } \\
\text { Vgl. zu xı - }\end{array}$ & $\begin{array}{l}\text { ıgrößerer } \\
\text { Einsatz als } \\
\text { xı/ıgroßer } \\
\text { Einsatz im } \\
\text { Vgl. zu xı }\end{array}$ & $\begin{array}{l}\text { sunmarkierter } \\
\text { durchschnitt- } \\
\text { licher } \\
\text { Einsatz`/ } \\
\text { sdurch- } \\
\text { schnittliche } \\
\text { Zurückhaltung, }\end{array}$ & $\begin{array}{l}\text { ıgrößere } \\
\text { Zurückhaltung } \\
\text { als xı/ıgroße } \\
\text { Zurückhaltung } \\
\text { im Vgl. zu xı }\end{array}$ & $\begin{array}{l}\text { ssehr große } \\
\text { Zurückhaltung } \\
\text { s/ıgrößte, } \\
\text { ständige } \\
\text { Zurückhaltung` }\end{array}$ & $\begin{array}{l}\text { sübermä- } \\
\text { ßige } \\
\text { Zurückhal- } \\
\text { tung، }\end{array}$ \\
\hline
\end{tabular}

Wenn man darüber nachdenkt, dass man zur Bezeichnung des rechts abgebildeten Pols statt von großer Zurückhaltung auch von geringem oder fehlendem Einsatz sprechen könnte, wohingegen fehlende Zurückhaltung als Benennungsalternative für den links abgebildeten Pol unpassend klingt, so stößt man auf ein Phänomen, dass Rachidi mit Bezug auf Gsell folgendermaßen umschreibt:

Unter Polarität soll in Anlehnung an Gsell (1979, 122f) die Tatsache verstanden werden, daß der ,naive Sprecher‘ eine deutliche ,positive“ oder ,negative“ Vorstellung mit je einem der Terme eines Gegensatzpaares verbindet, sowie die Übersetzung dieser Sprecherintuition in linguistische Begriffe.

(Rachidi 1989: 102)

688 Vgl. die Bedeutungsangabe auf „duden.de“, abrufbar unter: https://www.duden.de/rechtschreibung/Zyniker (zuletzt abgerufen am 18.01.2019). 
In diesem Zusammenhang ist des Weiteren interessant, dass in den untersuchten Texten PSYchISCHE ZuSTÄNDE und PERSONELLE EIGENSCHAFTEN häufig durch Gegensatzkonstellationen konstituiert werden, die in der linguistischen Literatur als „gradierbare Komplementäre“689 beschrieben werden. Gegensatzpaare dieses Typs seien jeweils auf eine Skala zu beziehen, „die eine unerwünschte Eigenschaft, wie z. B. Schmutz oder Ungenauigkeit, denotiert“ und deren positiver Endpunkt (z. B. Sauberkeit oder Genauigkeit) „das (völlige oder weitgehende) Fehlen der unerwünschten Eigenschaft markiert“ (Rachidi 1989: 93). Dieselbe Relation findet sich auch im Gegensatzpaar gesund - krank wieder und folgende ex- und implizit transportierten Gegensatzpaare in den Belegen des fachlichen Diskursstrangs lassen sich als gradierbar-komplementär klassifizieren: zufrieden - unzufrieden, frustriert, resigniert; (realistisch) - unrealistisch; (effektiv) - ineffektiv/verringerte Effektivität; (bewältigbar) - unbewältigbar, pflichtbewusst - (verantwortungslos), ehrgeizig (antriebslos oder gleichgültig); (flexibel) unflexibel, (funktional) - dysfunktional, (mitfühlend) - gefühllos, gleichgültig, herzlos, zynisch; (leistungsfähig) - reduzierte Leistungsfähigkeit/leistungsunfähig; distanzierungsfähig - (distanzierungsunfähig); (ausgeglichen) - unausgeglichen; (belastbar) - reizbar; (entspannt) - angespannt; (ruhig) - unruhig ... Die 'positiven' Pole dieser Gegensatzpaare lesen sich beinahe durchgehend wie eine Aufzählung erwünschter Eigenschaften in einer Stellenausschreibung. Sie sind teilweise in Klammern geschrieben, weil sie in den Texten zur Beschreibung von >Burnout` nicht immer explizit genannt werden. Ihre 'negativen' Gegenpole hingegen werden zur Beschreibung von >Burnout` häufig verwendet, wodurch dieses Konzept deutlich von den 'erwünschten' oder 'normalen' Leistungsprofilen abgegrenzt bzw. diesen sogar diametral gegenübergestellt wird.

Die genauere Betrachtung der sprachlichen Mittel der Gradierung, Quantifizierung, Polarisierung und Negation über die Einzeltexte hinweg hat gezeigt, dass `Burnout durch diese Mittel erstaunlich stabil sowohl fachintern als auch fachextern jenseits gesellschaftlich-kultureller Vorstellungen von 'normalen' bzw. 'erwünschten' oder 'gesunden' Erlebens- und Verhaltensweisen im berufli-

689 Gradierbare komplementäre Adjektivpaare (z. B. sauber - schmutzig) sind einerseits ähnlich wie antonyme Adjektivpaare (z. B. groß - klein) gradierbar (z. B. sauber (sauberer) - schmutzig (schmutziger)), andererseits „verhalten sich diese Adjektive logisch wie Komplementäre: Die Negation des einen Terms impliziert die Assertion des anderen“ (Rachidi 1989: 93). Rachidi verdeutlicht dies am Bsp. sauber - schmutzig: Wenn die Wäsche nicht sauber ist, dann ist sie „zumindest ein bißchen schmutzig“ (ebd.) und wenn eine Person nicht gesund ist, dann ist sie zumindest ein bisschen krank. Bei antonymen Gegensatzpaaren würde dieser „Test“ nicht funktionieren. Denn folgendem Satz würden Teilnehmer/innen eines Testrahmens nicht zustimmen: *Wenn Anja nicht groß ist, dann ist sie zumindest ein bisschen klein. 
chen Kontext verortet wird und durch verschiedene sprachliche Steigerungsmittel in Bezug auf die Dauer und Intensität der Merkmale auch deutlich von Konzepten wie `Stress` bzw. `Stressbelastung oder 'normaler' >Erschöpfung` unterschieden wird, die 'vorübergehend' sind und deren 'negative' Folgen innerhalb kurzer Zeiträume 'reversibel' sind.

Eine qualitative Abgrenzung zu sachverwandten Konzepten wie der $>$ Depression` oder >Neurasthenie findet in den untersuchten Texten des fachlichen und fachexternen Diskursstrangs allerdings nicht in gleich ausgeprägter Weise anhand dieser sprachlichen Mittel der Frequenz, Dauer, Intensität, Polarisierung und Negierung statt. Dies könnte damit zusammenhängen, dass bei allen Konzepten `Erschöpfung، eine Rolle spielt und die Merkmale der zuletzt genannten Konzepte mit eben diesen sprachlichen Mittel jenseits des durchschnittlichen gesunden Referenzpunkts verortet werden. Die Nähe zwischen den Konzepten >Burnout` und `Depression` wird de facto an verschiedenen Stellen (kritisch) angemerkt, einerseits im fachlichen Diskurs (z. B. bei Reime/Steiner 2001: 304ff., PPmP; Dörr/Nater 2013: 75, PPmP, im QV unter 8.1.4; Korczak/Kister/Huber 2010: 24, im QV unter 8.1.5) und andererseits im fachexternen Diskursstrang (z. B. in den Artikelversionen auf Wikipedia zu Burnout ab dem Jahr 2010 oder in der ZEIT 49/2011, = Albrecht 2011a/b, im QV unter 8.1.6). Einige Texte konstatieren jedoch auch Unterschiede, bspw. in den Kernsymptomen, der Extension einzelner Symptome (z. B. in PH 5/2009, = Binkert 2009: 30 und PH compact 27/2011, = Benkert 2011: 45, im QV unter 8.1.7, vgl. auch Kap. 6.2.3.1 dieser Arbeit) oder in Intensitätsunterschieden, wie bei Reime/Steiner (2001: 304) in einer Originalarbeit der Fachzeitschrift „Psychotherapie, Psychosomatik, Medizinische Psychologie“:

Die Gemeinsamkeiten zwischen Burnout und einer Depression bzw. „dysthymen Störung“ ([3], S. 407) bestehen in „... Interessens-, Motivationsverlust und Apathie, Rückzug, negativem Selbstwertgefühl und dem Gefühl mangelnder Kompetenz“ ([4], S. 87). Die Differenzen betreffen z. B. die Intensität der Symptome. Unter einer Depression sollen die Betroffenen stärker leiden als unter Burnout, so dass erstere sich mehr auf den Alltag und das gesundheitliche Wohlbefinden auswirken soll [1] []ㅡ [6] [7]. Burnout soll sich im Gegensatz zur Depression vorwiegend im Berufsleben manifestieren und mit Arbeitsbelastungen wie z. B. hoher Fallzahl, Zeitdruck und fehlender Anerkennung durch Vorgesetzte zusammenhängen [1] [4] [5] [6] [7].

(Reime/Steiner 2001: 304, Fettmarkierung der Zahlen entfernt, Unterstreichungen, T.S.)

Ob diese Konzepte sich wirklich anhand einer unterschiedlichen Verwendung von sprachlichen Mitteln der Intensität bzw. Quantität voneinander abgrenzen lassen, wäre Gegenstand einer eigenen Studie, die Texte dezidiert zur `Depression` und zu >Burnout anhand qualitativer und quantitativer linguistischer Kriterien zur Konstituierung von Dauer, Frequenz und Intensität auswertet und vergleicht. 
Anker für diese ziemlich stabile Verortung der einzelnen Merkmale des Konzepts >Burnout` auf der Seite des 'ungesunden', 'nicht normalen oder erwünschten' Merkmale-Pols sind dabei verschiedene fachliche, aber auch Common-Sense-Normen - z. B. biologische und biopsychische Speziesnormen, sozialgesellschaftliche und kulturelle Idealnormen, berufliche und lebensweltliche Tauglichkeitsnormen, Individual- oder Gruppennormen -, die als implizite Vergleichsfolien ihre ein- und abgrenzende Wirkung entfalten. Sie formen die verschiedenen semantischen Dimensionen zwischen den Metakonzepten >Gesundheit</>Normalität` und >Krankheit</>Störung//>Abnormalität` aus. Es geht nicht nur um die Abwesenheit somatischer Auffälligkeiten im Vergleich zur Gesamtpopulation (Speziesnormen), sondern die Analyse der sprachlichen Oberfläche verweist auch auf berufliche und lebensweltliche Tauglichkeitsnormen (realistisches Engagement vs. unrealistischer Idealismus) und auf das Zusammenspiel individueller, gruppenspezifischer und gesellschaftlich-kultureller Erwartungsnormen in bestimmten Bereichen und Lebensphasen.

Im folgenden Kapitel 6.2.4.2 wird gefragt, welche in der Fläche der Diskursstränge wiederholt auftretenden Zeichenkombinationen Effekte stimulieren können, die eine Verfestigung der in diesem Kapitel erarbeiteten Ein- und Zuordnungspraktiken sowie die diskursive Durchsetzung bestimmter Definitionsvarianten bewirken und deren Geltung validieren.

\subsubsection{Sprachliche Stimuli definitorischer Praktiken der Verknappung, Gewichtung und Geltungsvalidierung im fachinternen und fachexternen Diskursstrang}

Analog zu Kapitel 6.2.4.1 wird in diesem Kapitel gefragt, welche wiederholt in der Fläche der Diskursstränge auftretenden Zeichenkombinationen Effekte stimulieren können, die eine Verfestigung und Geltungsvalidierung bestimmter Definitionsvarianten und Zuordnungspraktiken bewirken. Das folgende Kapitel widmet sich zunächst der Frage, welche sprachlichen sowie typo- und topografischen Stimuli auf Praktiken hindeuten, die dazu führen, dass einzelne Aspekte oder Definitionen hervorgehoben und dadurch besonders wichtig erscheinen (= Praktiken der Gewichtung). Daraufhin wird analysiert, durch welche intertextuellen Bezugnahme- sowie fachkulturellen und medialen Praktiken die Vielfalt von Definitionsvarianten in der Zusammenschau über einen gewissen Zeitraum hinweg reduziert wird und einzelne Definitionsvarianten eine dominante Diskursposition erhalten (= Praktiken der Verknappung). ${ }^{600}$ Zum Abschluss geht es darum, Marker der Intersubjektivität, Validität, und Neutralität herauszuarbeiten, mit

690 Schon Foucault hat auf Verknappungsprozeduren hingewiesen. Vgl. Kap. 4.1.3. 
denen die allgemeine Gültigkeit der (dominanten) Definitionen insbesondere im Bereich von Gesundheit und Krankheit weiter gestützt wird (= Praktiken der Geltungsvalidierung im medizinischen Bereich). ${ }^{61}$

Die Praktiken der Verknappung, Gewichtung und Geltungsvalidierung, die in diesem Kapitel beschrieben werden, sind Teil des in dieser Arbeit entwickelten Analyseprogramms der Unifizierung: Das heißt, es werden insbesondere sprachliche Mittel und Praktiken im Diskurs identifiziert, die statt einer agonalen eine vereinheitlichende, unifizierende Dimension transportieren und damit den Definitionsprozess unterstützen (vgl. dazu auch Kap. 6.2.1 dieser Arbeit). Im Zentrum des Analyseinteresses stehen Praktiken, die wie „Klassifikations-, Anordnungs-, Verteilungsprinzipien“ (Foucault [1970] 1993: 17 und Kap. 4.1.3) wirken und dazu führen, dass divergierende Konzepte im Diskurs weniger sichtbar werden und dadurch der Eindruck von Übereinstimmung entsteht. Damit sei nicht gesagt, dass dieser Konsens von den beteiligten Sprecherinnen und Sprechern immer intendiert erzeugt wird oder dass es keine sprachlichen Marker für Agonalität gäbe (vgl. dazu Kap. 7.3). Es wird gezeigt werden, dass sich fachlicher und gesellschaftlicher ,Konsens“ im Diskurs auch durch nicht (gänzlich) intendierte Verhaltensroutinen herauskristallisieren kann.

Die Praktiken der Verknappung, Gewichtung und Geltungsvalidierung werden direkt sowohl für die fachinternen als auch fachexternen Texte beschrieben. Denn auf diese Weise können die intertextuellen Bezugnahmepraktiken, die zur Verknappung des Diskurses beitragen, an einzelnen Beispielen über die Diskursstränge hinweg nachgezeichnet werden.

Im Rahmen der Analysen der definitorischen Passagen aus punktueller Perspektive wurde schon darauf hingewiesen, dass bestimmte Symptome durch Ausdrücke wie Kern- bzw. Warnsymptome, wichtigste Erkennungszeichen als besonders konstitutiv für >BURNOUT، herausgestellt werden und bestimmte Gruppen durch Ausdrücke wie insbesondere oder nicht nur ... auch als besonders 'betroffene' Personengruppen und -typen hervorgehoben werden (vgl. Kap. 6.2.2.1/6.2.2.2 und 6.2.3.1/6.2.3.2). Eine ähnlich gewichtende Funktion können typo- und topografische Strukturierungen innerhalb von „transkriptive[n] Bezugnahmeformen“ (Jäger 2012b: 16) bewirken: In Lehrbüchern, Abstracts sowie Magazin- und Zeitungsberichten wird diese Auszeichnungsart gerne verwendet:

\footnotetext{
691 Die Analyse der Praktiken der Geltungsvalidierung im medizinischen Bereich knüpft an linguistische Analysen zu sprachlichen Mitteln des Geltungsanspruchs an (Deppermann 1997; Vogel 2012, Schnedermann 2016). Auf einige dieser Sprachmittel, die die Geltung einer Definition stützen, wurde schon bei der Analyse der Definitionspassagen aus punktueller Perspektive eingegangen (vgl. Kap. 6.2.2.1 und Kap. 6.2.3.1 und die Überblickstabellen 1, 2.1, 2.2, 4, 5.1 und 5.2 in den Kap. 6.2.2.4 und 6.2.3.3).
} 
In folgendem Kapitel „Wirkungen der Arbeit“ und Unterkapitel „Spezifische Auswirkungen von Stress“ (Schaper ${ }^{3} 2014$; 531, im QV unter 8.1.2) im Lehrbuch zur Einführung in die Arbeits- und Organisationspsychologie von Nerdinger/Blickle/ Schaper ( ${ }^{3} 2014$ ) (siehe Abbildung 17) werden beispielsweise die Symptome „chronische Erschöpfung“ und „Zynismus-Aspekt“ sowie als Entstehungsbedingung die „Beziehung zwischen Klient und Betreuer“, was auf helfende oder Dienstleistungsberufe verweist, fett markiert. Farblich hervorgehoben und eingerahmt wird die Definition nach Maslach/Jackson (1984) präsentiert. Sie wird als eine „der bekanntesten Definitionen“ im Absatz zuvor beschrieben neben anderen Definitionen, die entweder stärker „das Individuum (Persönlichkeit des Helfers), die organisatorischen Bedingungen oder gesellschaftliche Prozesse fokussieren“ (Schaper ${ }^{3}$ 2014: 531, siehe Abb. 17 auf der folgenden Seite). ${ }^{692}$

Im Kapitel „Burnout“ von Berger/Berner/Schramm ( $\left.{ }^{5} 2015\right)$ im Lehrbuch für „Psychische Erkrankungen“ (siehe Abbildung 18 auf Seite 423) werden ebenfalls einzelne Namen und Merkmale hervorgehoben: einerseits „Herbert Freudenberger“ und andererseits „Christina Maslach“, deren Symptomtrias („emotionale[r] Erschöpfung, Depersonalisation und verringerte Leistungsfähigkeit bei beruflicher Überforderung“) ebenfalls im fetten Schriftsatz gedruckt wird. Als ,Betroffenen'-Gruppe werden „Menschen in sozialen Berufen“ schriftlich akzentuiert. Später im Text erfolgt eine weitere typografische Hervorhebung der Kritik an der weiten Extension des Begriffs 〉Burnout`, durch die dieser Begriff sich auf ,,jedwede Art von beruflicher Erschöpfung“ beziehe. Schließlich werden zwei weitere Distanzmarker in fetter Schrifttype gesetzt: Erstens wird das klassifizierende Rahmenkonzept „psychische Erkrankung bzw. eigenständige Diagnose“ mit Bezug auf die ICD-10 und das DSM-5 als nicht gültig beschrieben, und zweitens erscheint ein Hinweis auf „16 unterschiedliche Definitionen“. Doch trotz dieses Hinweises auf die Vielfalt der Definitionen wird direkt im Anschluss nur die Definition von Maslach/Jackson mit Bezug auf das MBI zitiert, wobei zwei Symptome wiederum typografisch hervorgehoben werden. In diesen Beispielen zeigt sich die Wirkmacht des Foucault'schen Prinzips des „Kommentars“, der zwar erlaube, „etwas anderes als den Text selbst zu sagen“ (Foucault [1970] 1993: 20), diesen aber dennoch reproduziere und zwar als „Spiel der Identität in der Form der Wiederholung und des Selben“ (Foucault [1970] 1993: 22, kursiv im Orig.; Kap. 4.1.3).

692 Weitere Beispiele aus dem Korpus, die zwar mehrere Definitionen bzw. Beschreibungskonzepte erwähnen und/oder Kritik an der zur „Diagnose“ gewordenen Definition >Burnout üben, dennoch aber die Definition von Maslach (und Jackson) deutlich hervorheben (z. B. typografisch oder topografisch zu Beginn des Kapitels oder durch eine vergleichsweise ausführliche Zitierweise), findet man auch bei Krapp/Weidemann ('2001: 326); Weber (32011: 426); Nowak (2010: 69); Groth (2013); Kunter/Pohlmann (2015: 274); Gerrig/Zimbardo $\left({ }^{20} 2015\right)$; alle Belege im QV unter 8.1.2. 
schlecht und dem Ausmaß privater Belastungen), dass die "objektiv“ ermittelte Arbeitsintensität mit dem Auftreten von depressiven Verstimmungen und Angststörungen in Zusammenhang stehen. Entsprechende Zusammenhänge konnten allerdings nicht für den objektiv ermittelten Handlungsspielraum mit den psychischen Beeinträchtigungen festgestellt werden.

Anhand einer Mediatoranalyse konnte außerdem ermittelt werden, dass die von den Arbeitskräften wahrgenommene Arbeitsintensität den Zusammenhang zwischen der objektiv ermittelten Arbeitsintensität und den psychischen Beeinträchtigungen teilweise vermittelt. Die psychischen Beeinträchtigungen treten schließlich umso häufiger auf, je höher die wahrgenommene Arbeitsintensität und je geringer der wahrgenommene Handlungsspielraum ist. Zumindest für den Zusammenhang von hoher Arbeitsintensität und psychischen Beeinträchtigungen lässt sich somit nachweisen, dass hierbei keine Urteilsverzerrungen der Betroffenen verantwortlich gemacht werden können. Anders liegt der Fall allerdings bei der Bewertung des Handlungsspielraumes, wozu unterschiedliche Erklärungen herangezogen werden können: Zum einen kann es sein, das psychisch beeinträchtigte Menschen aufgrund ihrer veränderten Wahrnehmung weniger Handlungsspielraum erleben. Zum anderen ist es aber auch möglich, dass der objektiv vorhandene Spielraum nicht genutzt werden kann (z. B. aufgrund eines zu hohen Arbeitsdrucks oder weil entsprechende Kompetenzen fehlen).

Unter den langfristigen Auswirkungen von Stress, die durch Arbeitsbelastungen entstehen, wurde außerdem die Entstehung von Herz-Kreislauf-Krankheiten (vgl. Siegrist, 1996) und das Burn-out-Syndrom in besonderem Maße untersucht. Im Folgenden soll das arbeitspsychologisch sehr gut untersuchte Burn-out-Syndrom genauer dargestellt werden.

\section{Burn-out-Syndrom}

Das Burn-out-Syndrom wurde ursprünglich vor allem in Sozial- und Pflegeberufen untersucht und bezeichnet einen besonderen Zustand berufsbezogener chronischer Erschöpfung. Es gibt unterschiedliche Definitionen von Burn-out, die danach unterteilt werden, ob sie das Individuum (Persönlichkeit des Helfers), die organisatorischen Bedingungen oder gesellschaftliche Prozesse fokussieren (vgl. Burisch, 2010). Eine der bekanntesten Definitionen nach Maslach und Jackson (1984) ist die folgende:

\begin{abstract}
Definition
Burn-out bezeichnet einen besonderen Zustand berufsbezogener chronischer Erschöpfung insbesondere in Sozial- und Pflegeberufen. Es wird meist als ein Syndrom aus emotionaler Erschöpfung, Depersonalisierung und reduzierter Leistungsfähigkeit beschrieben. Emotionale Erschöpfung ist durch hohe interpersonelle Anforderungen und die Beanspruchung emotionaler Ressourcen gekennzeichnet. Die Betroffenen fühlen sich durch den Kontakt mit anderen Menschen emotional überanstrengt und ausgelaugt. Depersonalisation beinhaltet negative, gefühlslose und zynische Einstellungen gegenüber Klienten, Kunden oder Patienten; ein Zustand, in dem die Betroffenen gefühlslose und abgestumpfte Reaktionen gegenüber ihren Klienten zeigen. Persönliche LeistungseinbuBen beschreibt die Tendenz, die eigene Arbeit negativ zu bewerten und ein Gefühl mangelnden bzw. schwachen beruflichen Selbstwertes zu entwickeln.
\end{abstract}

Der Aspekt der Depersonalisation, der am stärksten mit dem Kontext der helfenden Berufe verbunden ist, wurde in späteren, allgemeiner gefassten Definitionsversuchen auch als „Zynismus"-Aspekt bezeichnet, womit eine gleichgültige und distanzierte Haltung gegenüber der Arbeit sowie ihren Inhalten und Mitteln bezeichnet wird.

Als entscheidender Faktor für die Entstehung von Burn-out gilt die enge emotionale Beziehung zwischen Klient und Betreuer. Durch unrealistisch hohe Erwartungen des Betreuenden an seine Wirkungsmöglichkeiten im Rahmen solcher Beziehungen kann eine chronische emotionale Belastung entstehen, die nicht erfolgreich bewältigt wird und bis hin zu Zynismus gegenüber den zu betreuenden Klienten als Extremform der Depersonalisation führt. Die Entstehung von Burn-out ist ein komplexer Prozess und wird durch unterschiedliche Faktoren beeinflusst (Cordes \& Dougherty, 1993). Zum einen sind dies Anforderungen des Berufes und der Tätigkeit, zum anderen Persönlichkeitseigenschaften und Merkmale der persönlichen Lebenssituation. So wird insbesondere für die Tätigkeiten in helfenden Berufen ein hohes $\mathrm{Maß}$ an persönlicher $\mathrm{Zu}$ wendung und emotionaler Anteilnahme verlangt, die häufig nicht ausreichend gegeben werden kann, weshalb sich als Folge Gefühle des Versagens einstellen. Zudem sind die Tätigkeiten in diesem Bereich häufig körperlich belastend und es gibt nur wenige Möglichkeiten, auf die Arbeitsbedingungen Einfluss zu nehmen, was zu Unzufriedenheit, Hilflosigkeit und zu Resignation führen kann. Andere Faktoren können sich aber als Puffer gegen die Belastungen erweisen, so z. B. die wahrgenommene Autonomie oder Kontrolle bei der Arbeit, Merkmale der persönlichen Lebenssituation wie eine intakte Familie und intakte sozi-

\footnotetext{
Abb. 17: Typografische Hervorhebungen in Schaper ('2014: 531, im QV unter 8.1.2, hier in schwarz-weiß). Reprinted/adapted by permission from Springer Nature: Springer eBook, Wirkungen der Arbeit by Niclas Schaper, (C) 2014.
} 


\section{KAPITEL}

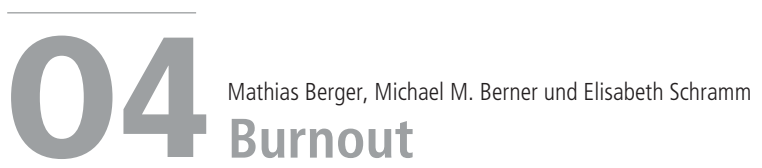

\subsection{Terminologie}

\subsubsection{Historische Entwicklung}

Der Begriff Burnout wurde erstmals von dem New Yorker Psychotherapeuten Herbert Freudenberger (1974) geprägt. Aufgrund selbst erlebter Erschöpfung durch eine Mehrfachbelastung als niedergelassener Psychotherapeut, als ehrenamtlicher Mitarbeiter in einer Ambulanz für drogenabhängige Jugendliche sowie als Dozent beschrieb er das Risiko, dass Menschen in sozialen Berufen durch idealistisches, übergroßes Engagement „ausbrennen“ können. Den aus der Raketentechnologie abgeleiteten Begriff übernahm er zur besonders bildlichen Darstellung eines Zustands, sich verausgabt und müde zu fühlen, verbunden mit Infektanfälligkeit, häufigen Kopfschmerzen, Magen-Darm-Problemen, Schlaflosigkeit, Kurzatmigkeit oder anderen vegetativen Symptomen. Im Kontakt mit Kollegen komme es zu emotionalen Ausbrüchen, leichter Reizbarkeit und rigidem unflexiblem Denken. Diese Symptomatik sei nur exemplarisch, da das Burnout sich interindividuell sehr vielfältig darstellen könne. Freudenberger betonte, dass es sich nicht um eine seelische Erkrankung, sondern lediglich um das Resultat einer beruflichen Überlastung handele.

Die Psychologin Christina Maslach bemühte sich ab 1981 um eine operationale Definition und die Entwicklung eines Messinstruments zu deren Erfassung und Quantifizierung (Maslach und Jackson 1981). Sie definierte Burnout als das gemeinsame Auftreten von emotionaler Erschöpfung, Depersonalisation und verringerter Leistungsfähigkeit bei beruflicher Überforderung. Das von ihr entwickelte Maslach Burnout Inventory (MBI) erfasst mithilfe von 22 Fragen das Vorliegen von Symptomen dieser drei Dimensionen. Das für die Berufsgruppe der Therapeuten entwickelte Instrument wird bei ca. $90 \%$ aller veröffentlichten Studien zu diesem Problem eingesetzt. Hingegen findet das von ihr im Nachgang für die Allgemeinbevölkerung entwickelte Instrument MBI-GS, von dem es keine validierte deutsche Version gibt, kaum Anwendung (Schutte et al. 2000). In den letzten Jahren hat sich der Begriff „Burnout“ insb. in Deutschland stark verbreitet und wird jenseits der sozialen Berufe mit zunehmender Unschärfe für jedwede Art von beruflicher Erschöpfung, aber auch häuslicher Überlastung, belastenden zwischenmenschlichen Beziehungen oder bei Überlastung im Leistungssport verwendet.

Rook (1998) stellte in einer Übersicht 16 unterschiedliche Definitionen des Begriffs „Burnout“ einander gegenüber. Weit verbreitet ist eine an den drei Dimensionen des MBI ausgerichtete Definition, die als wesentliche Elemente das Gefühl der emotionalen Er schöpfung und inneren Leere (emotional exhaustion), die zunehmende Depersonalisation i. S. einer Distanzierung $\mathrm{zu}$ den Inhalten der Arbeit und das fehlende Erleben von eigener Effizienz und Leistungszufriedenheit bei der Arbeit (personal accomplishment) ansieht.

\subsubsection{Klassifikation}

In der Internationalen Klassifikation Psychischer Störungen (ICD10) wird Burnout nicht als psychische Erkrankung bzw. eigenständige Diagnose im Kapitel V (F-Diagnosen) geführt. Lediglich im Anhang, d. h. im Kapitel XXI - Faktoren, die den Gesundheitszustand beeinflussen und zur Inanspruchnahme von Gesundheitsdiensten führen - wird der Begriff unter der Ziffer Z73 „Probleme verbunden mit Schwierigkeiten bei der Lebensbewältigung" angeführt. Hier wird unter Z73.0 „Ausgebranntsein“ mit den Unterpunkten „Burnout“ und „Zustand der totalen Erschöpfung“ aufgelistet. Die Burnout-Problematik könnte auch mit der Ziffer Z73.1 „Akzentuierung von Persönlichkeitszügen“, d. h. Typ-A-Verhalten (Verhaltensmuster, das durch zügellosen Ehrgeiz, starkes Erfolgsstreben, Ungeduld, Konkurrenzdenken und Druckgefühl charakterisiert ist), oder aber auch mit der Ziffer Z56 „Probleme in Verbindung mit Berufstätigkeit und Arbeitslosigkeit" erfasst werden. Auch in der anstehenden Revision ICD-11 ist nicht geplant, dem Burnout eine eigene diagnostische Kategorie zuzuordnen.

Im DSM-5 wird Burnout ebenfalls nicht als eigenständige Diagnose erwähnt. Burnout kann lediglich unter Ziffer 68.20 „Probleme im Beruf" eingeordnet werden. Entsprechend der initialen Definition von Freudenberger handelt es sich im DSM-IV nicht um eine Erkrankung, sondern um ein zu beobachtendes Phänomen. Da Burnout keine zu behandelnde Krankheitsdiagnose im ICD-10 darstellt, wurde es nicht in den Katalog der Deutschen Krankenkassen aufgenommen. Etwas anders wird das Problem des Ausgebranntseins von den Rentenversicherungsträgern behandelt. Die Rehabilitation zielt definitionsgemäß auf die Beseitigung oder Verminderung negativer Krankheitsfolgen auf den Ebenen der Funktionen, Aktivitäten und Partizipationen. Hierzu wird die Internationale Klassifikation der Funktionsfähigkeit, Behinderung und Gesundheit (ICF) mit einer entsprechenden Funktionsklassifikation genutzt. Da etwa die Hälfte der Rehabilitanden unter einer vitalen Erschöpfung leidet (auch Erschöpfung der Reserven bzw. BurnoutSyndrom genannt), gilt Burnout nach differenzialdiagnostischer Abgrenzung von Folgen körperlicher Erkrankung, Chronic-Fa-

Abb. 18: Typografische Hervorhebungen in Berger/Berner/Schramm (52015: e30, im QV unter 8.1.2, hier in schwarz-weiß). ( Elsevier GmbH, Urban \& Fischer, München, Berger, Psychische Erkrankungen. Klinik und Therapie. 5. Auflage 2015.

Ein ganz ähnliches Vorgehen sieht man beim Eintrag zu „Burnout“ im Überkapitel „Psychische Belastung und Beanspruchung“ im Lehrbuch für Arbeitsmedizin (= Groth 2013: 73). Sowohl die Symptomtrias nach Maslach/Jackson im grau hinter- 
legten Kasten oben als auch die „Symptomcluster[n]“ des Maslach Burnout Inventory weiter unten werden durch Fettdruck und Spiegelstriche hervorgehoben zitiert, und es wird zugleich darauf hingewiesen, dass >BURNOUT ^ nur ein „Erschöpfungssyndrom“ bzw. >Risikozustand ‘ und keine „Krankheit“ sei (siehe Abbildung 19):

\subsection{Burnout}

Burnout

- Risikozustand bei lāngerfristiger Arbeits überforderung

- Zustand von emotionaler Erschöpfung begleitet von Zynismus, Distanzierung und reduzierter Leistungszufriedenheit

- Keine Krankheit, aber bei fehlender Regeneration erhöhtes Risiko psychischer und psychosomatischer Folgeerkrankungen

Weder die Arbeitsmedizin noch andere Fachgebiete verfügen über eine verbindliche Definition des Burnouts. Burnout ist keine Krankheit und ist im ICD-10 als Erschöpfungssyndrom lediglich als ein mit Schwierigkeiten bei der Lebensbewältigung verbundenes Problem kodiert (ICD-10 Z73.0). Praktikabel ist, sich an dem Maslach-Burnout-Inventar (MBI) zu orientieren. Dabei handelt es sich um das international verbreitetste Inventar zur Testung einer Burnout-Gefahrdung. Die einzelnen Items des MBI lassen sich zu folgenden 3 Symptomclustern zusammenfassen:

- Emotionale Erschöpfung: Gefuhl der Oberforderung und des Ausgelaugtseins verbunden mit Energiemangel, Müdigkeit, Niedergeschlagenheit und Anspannungszuständen

- Zynismus und Distanzierung: Emotionaler Zustand, in dem aus dem ideal isierten mit positiven Erwartungen verbundenen Verhältnis zur Arbeit Frustration und eine Distanzierung von der Arbeit geworden sind. Die Betroffenen erleben Schuldzuweisungen und Verbitterung gegenüber den Arbeitsbedingungen, eine Abwertung der

[Seitenwechel von S. 73 zu S. 74, Groth (2013)]

Arbeit und einen Zynismus, der sich u. a. gegen Kollegen und auch ihre Klientel richtet. Dieser Zustand ist hāufig mit Schuldgefühlen oder auch einem vollständigen Gefuhlverlust (Depersonalisation) verbunden

- Verringerte Leistungszufriedenheit: Empfundene nachhaltige Minderung der Arbeitsleistung. Kompetenz und Kreativităt verbunden mit Konzentrationsstörungen und Arbeitsunzufriedenheit

[Nach diesen Spiegelstrichen erfolgt u. a. noch der Hinweis, dass es für ,die Diagnose eines BurnoutSyndroms" notwendig sei, ,dass die Betroffenen ihr Beschwerdebild als Folge ihrer Arbeitsbelastung erleben“" und dass ein „Burnout-Syndrom“ zu ,einer anderen psychischen/psychosomatischen Erkrankung führen" könne und ,wegen der symptomatischen Überschneidungen diagnostisch von diesen nur schwer abzugrenzen“" sei (Groth 2013: 74).]

Abb. 19: Typografische Hervorhebungen in Groth (2013: 73-74, Ausschnitte der Seiten, im QV unter 8.1.2). Reprinted/adapted by permission from Springer Nature: Springer eBook, Psychische Belastung und Beanspruchung by K. Groth, Copyright $\odot 2013$. 
Eine besonders verknappende Wirkung entfalten des Weiteren Schaubilder. In Kapitel 6.2.2.1 (Beleg 6) wurde schon ein Schaubild aus dem Lehrbuch von Nerdinger/Blickle/Schaper (32014: 532) beschrieben, das die drei Symptome nach Maslach/Jackson (1981; 1984) - im Schaubild per Pfeil aufeinander folgend - präsentiert und mit verschiedenen Stressorenquellen verbindet. In ähnlicher Weise verfährt auch das folgende Schaubild (siehe Abbildung 20), indem es die drei Hauptsymptome für >Burnout nach Maslach/Jackson (1981; 1984, siehe Beleg 2 in Kap. 6.2.2.1) in einen größeren Zusammenhang, in diesem Fall in das Klassifikationssystem nach der ICD-10, einordnet und 〉Burnout « damit als Folge von „Arbeitsüberforderung“ und als „Risiko-Zustand“ für „Folgekrankheiten“ platziert:

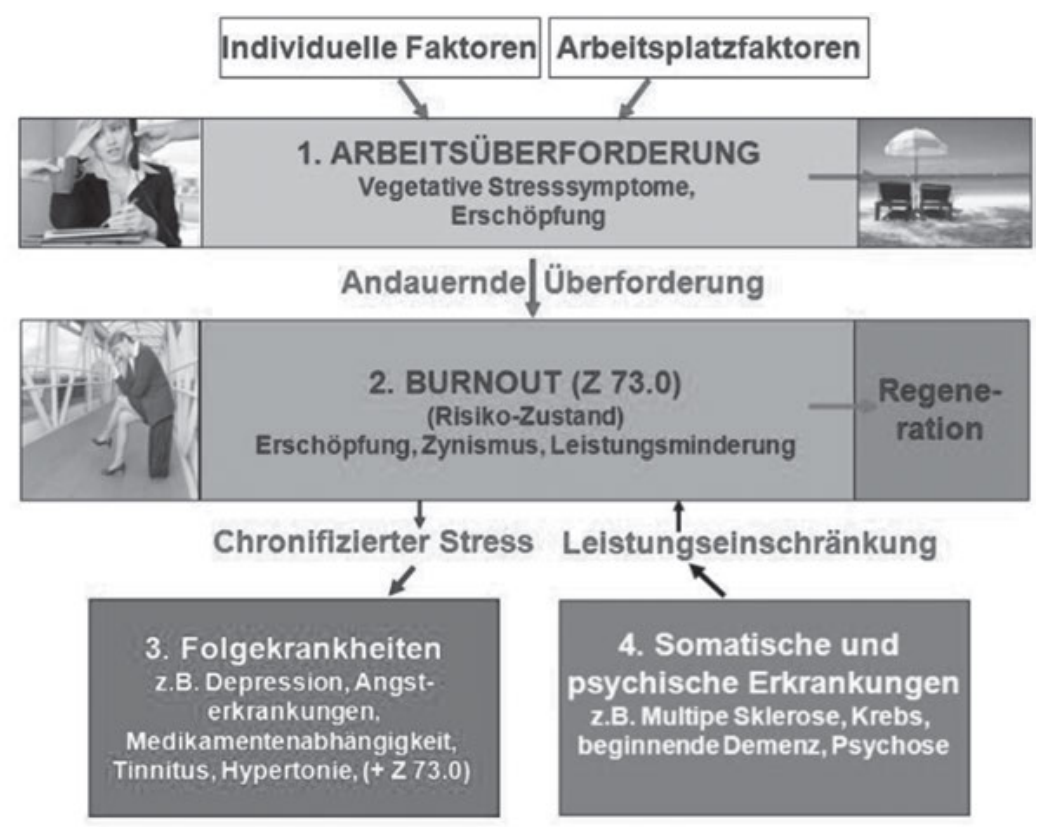

\section{Abb. 1 DGPPN-Konzept zum Öbergang von Arbeitsbelastung zur Krankheit}

Abb. 20: Einordnung von `Burnout mit den drei >Hauptsymptomen« nach Maslach/Jackson (1981/1984) im Kontext des Klassifikationssystems ICD-10 im Positionspapier der DGPPN (2012: 4, hier in schwarz-weiß), siehe im QV unter 8.1.9, @ DGPPN, M. Berger et al. 2012.

Es gibt allerdings auch Kapitel in Hand- und Lehrbüchern, in denen die Definition von Maslach/Jackson nicht in dieser dominanten Weise typografisch hervorgehoben erscheint oder sogar unerwähnt bleibt.

Im Lehrbuch von Sonntag/Frieling/Stegmaier (32012: 320) sind vier Definitionen in einer farblich hervorgehobenen Tabelle einander gegenübergestellt (siehe Abbil- 
dung 21), und im Fließtext erfolgt keine Gewichtung, welche Definition am meisten verbreitet sei, sondern die Definitionen werden danach unterschieden, ob sie sich eher auf individuelle Merkmale oder Faktoren der Arbeitsumgebung stützen. Das Thema „Burnout“ ist in das Großkapitel „Teil III: Personale Voraussetzungen und deren Förderung“ eingebettet, wie man durch den Seitenkopf (siehe Abb. 21) erkennen kann. In der mittleren Tabellenspalte werden Ausschnitte aus den Definitionen direkt bzw. in Übersetzung zitiert, und in der Spalte rechts daneben werden diese Definitionen über Erläuterungen im aufgelisteten Format beschrieben:

Teil III: Personale Voraussetzungen und deren Förderung

Tabelle III-23: Ausgewählte Definitionen und Konzepte von Burnout

\begin{tabular}{|c|c|c|}
\hline Autoren & Definition & Phänomenbeschreibung \\
\hline $\begin{array}{l}\text { Edelwich } \\
\text { \& Brodsky } \\
\text { [1984] }\end{array}$ & $\begin{array}{l}\text { «... zunehmender Verlust an } \\
\text { Idealismus und Energie .... (S. 12) } \\
\text { durch fortschreitende Desillusio- } \\
\text { nierung, hervorgerufen durch } \\
\text { Oberidentifikation mit Klienten } \\
\text { sowie durch Erfahrungen mit den } \\
\text { Arbeitsbedingungen. }\end{array}$ & $\begin{array}{l}\text { Vierstufiger Prozess der Desillusionierung: } \\
\text { 1. ideatistische Begeisterung (unrealistische } \\
\text { Erwartungen, übermäßiges Engagementl } \\
\text { 2. Stagnation: Unzufriedenheit mit organisatori- } \\
\text { schen Rahmenbedingungen der Arbeit, } \\
\text { weiterhin großes Engagement } \\
\text { 3. Frustration der idealistischen Erwartungen } \\
\text { 4. Apathie, Frustration, Zynismus, emotionaler } \\
\text { Rückzug. Vermeidung von Klientenkontakten }\end{array}$ \\
\hline $\begin{array}{l}\text { Pines, } \\
\text { Aronson \& } \\
\text { Kafry } \\
{[1983)}\end{array}$ & $\begin{array}{l}\text { «Ausbrennen ist das Resultat } \\
\text { andauernder oder wiederholter } \\
\text { emotionaler Belastung im } \\
\text { Zusammenhang mit langfristi- } \\
\text { gem, intensivem Einsatz für ande- } \\
\text { re Menschen* (S. 25). }\end{array}$ & $\begin{array}{l}\text { - körpertiche Erschöpfung (chronische Müdig- } \\
\text { keit, Kopfschmerzen, Schlafstörungen ...) } \\
\text { - emotionale Erschöpfung [Reizbarkeit, } \\
\text { Hitflosigkeit, Entmutigung. Leere ...) } \\
\text { - geistige Erschöpfung (negative Einstellung zu } \\
\text { sich selbst, zum Leben, zu anderen Menschen] } \\
\text { - emotionale und physische Distanzierung von } \\
\text { Klienten(-problemen) }\end{array}$ \\
\hline $\begin{array}{l}\text { Maslach \& } \\
\text { Jackson } \\
\text { [1984] }\end{array}$ & $\begin{array}{l}\text { «... Syndrom aus emotionaler } \\
\text { Erschöpfung. Depersonalisation } \\
\text { u. reduzierter Leistungsfăhigkeit } \\
\text { das bei Individuen, die in } \\
\text { irgendeiner Weise mit Menschen } \\
\text { arbeiten, auftreten kann» (S. 134). }\end{array}$ & $\begin{array}{l}\text { - Gefühl, durch den Kontakt mit anderen } \\
\text { Menschen emotional überanstrengt und } \\
\text { ausgelaugt zu sein } \\
\text { - gefühllose und abgestumpfte Reaktion auf die } \\
\text { zu betreuenden Personen/Klienten } \\
\text { - Gefühl des Kompetenz- und Erfolgsverlustes } \\
\text { bei der Arbeit }\end{array}$ \\
\hline $\begin{array}{l}\text { Cherniss } \\
\text { (1980) }\end{array}$ & $\begin{array}{l}\text { «... ein Proze日, in welchem ein } \\
\text { ursprünglich engagierter } \\
\text { Professioneller sich als Reaktion } \\
\text { auf in der Arbeit erfahrene (n) } \\
\text { Streß und Stre } 3-\text { Reaktion (stress } \\
\text { and strain) von seiner oder ihrer } \\
\text { Arbeit zurückziehts (Enzmann \& } \\
\text { Kleiber, 1989, S. 42). }\end{array}$ & $\begin{array}{l}\text { - Verlust von Kompetenzgefüht } \\
\text { - Apathie, Zynismus und Rigidität } \\
\text { - veränderte Einstellung zur Arbeit und zu den } \\
\text { Klienten } \\
\text { - Gefühle von Erschöpfung und Anspannung }\end{array}$ \\
\hline
\end{tabular}

Abb. 21: Gegenüberstellung von Burnout-Definitionen bei Sonntag/Frieling/Stegmaier ( 32012 : 320, im QV unter 8.1.2, hier in schwarz-weiß), @ by Verlag Hans Huber, Hogrefe AG, Bern, 2013 und Sonntag/Frieling/Stegmaier 2012. 
Nicht erwähnt wird die Definition von Maslach/Jackson vor allem in Handbuchtexten, wenn Burnout nur am Rande erwähnt wird (z. B. Dittmann-Kohli/ Sowarska/Timmer 1997; Ulich ${ }^{5}$ 2001; Dormann/Zapf 2007, im QV unter 8.1.2) oder wenn sich in einem Unterfach andere Konzepte verbreitet und durchgesetzt haben. So gibt es beispielsweise Handbuchkapitel aus dem Bereich der pädagogischen Psychologie, die eine andere Konzeptualisierung von >Burnout $\triangleleft$ bevorzugen oder zumindest gleichberechtigt verwenden (so z. B. Schaarschmidt 2008 und Krapp/ Weidemann ( ${ }^{4} 2001$ : 325, im QV unter 8.1.2). Denn in diesem Unterfach hat sich ein Diagnoseinstrument durchgesetzt, das entwickelt wurde, um im Kontext des Überthemas „Lehrerbelastung“ gesundheitsgefährdende und gesundheitsförderliche Muster der Belastungsbewältigung zu erheben: das Verfahren des Arbeitsbezogenen Verhaltens- und Erlebensmusters (AVEM), das ein „Risikomuster B“ ausweist (Schaarschmidt/Fischer ${ }^{2} 2003$ zitiert nach Schaarschmidt 2008: 202, im QV unter 8.1.2 und 8.1.5).

Betrachtet man aber insgesamt in den 53 im Korpus enthaltenen Fachlexikon-, Hand- und Lehrbuchtexten aus verschiedenen medizinischen und psychologischen Unterfächern, die das Thema „Burnout“ nicht nur erwähnen, sondern mindestens in einem Satz bzw. Abschnitt behandeln, ${ }^{693}$ wie häufig die Definition, die auf Maslach/Jackson zurückgeht, als einzige Referenz oder hervorgehoben zitiert wird, ${ }^{694}$ dann erhält man folgende Übersicht:

693 Manche dieser Lexikon-, Hand- und Lehrbuchartikel behandeln das Thema „Burnout“ ausführlich, einige Hand- und Lehrbuchartikel schneiden das Thema „Burnout“ im Rahmen anderer Kapitel an (diese Texte ergeben zusammen 54 im Korpus enthaltene Texte (darunter sind beim „Dorsch - Lexikon der Psychologie“ und „Pschyrembel - Klinisches Wörterbuch“ und teilweise auch bei anderen Lehrbüchern mehrere Auflagen enthalten, siehe das Quellenverzeichnis in Kap. 8.1.2)). Im Quellenverzeichnis dieses Unterkorpus sind aber auch Hand- und Lehrbücher enthalten, die das Stichwort „Burnout“ nicht enthalten (so z. B. Pawlik 2006; Kanning/Staufenbiel 2012; Bamberg/Mohr/Busch 2012, u. weitere) oder nur am Rande ohne weitere Bedeutungsangabe erwähnen (so z. B. Oerter/Bruhn 2005; Semmer/Grebner/Elfering 2010). Sie wurden als Quellen dennoch in das Unterkorpus aufgenommen, da dies zeigt, dass nicht jedes Lehrbuch einschlägiger Praxisbereiche, beispielsweise der Arbeitspsychologie, Organisationspsychologie oder pädagogischen Psychologie, Burnout zwingend enthält und dass in einigen Fachrichtungen wie z. B. der Allgemeinmedizin das Thema „Burnout“ nicht (z. B. Kochen ${ }^{5} 2017$; Jobst/Abholz ${ }^{4} 2013$ ) oder falls doch, nur am Rande genannt wird (so z. B. bei Gesenhues/Gesenhues/Weltermann et al. ${ }^{8} 2017:$ 1276).

694 Hervorgehoben zitiert heißt in diesem Zusammenhang, wenn die Definition oder Symptome der Definition von Maslach/Jackson (1981) und/oder das Maslach Burnout Inventory (MBI) reformuliert oder zitiert und dabei deutlich im Vergleich zu anderen Konzepten hervorgehoben werden, zum Beispiel durch fette Schrifttype, durch Einrahmung oder dadurch, dass diese Definition/diese Symptome zu Beginn des Hand- oder Lehrbucheintrags und am ausführlichsten zitiert werden (teilweise wird diese Definition auch mit einem Hinweis versehen, dass sie weit 
Tab. 10: Die Definition nach Maslach/Jackson im Korpus „Fachlexika-, Hand- und Lehrbücher“.

\begin{tabular}{|c|c|c|c|}
\hline $\begin{array}{l}\text { Fachlexikon-, Hand- u. } \\
\text { Lehrbuchtexte, die nur } \\
\text { die Symptomtrias, die } \\
\text { auf Maslach/Jackson } \\
\text { zurückgeht, zitieren/ } \\
\text { reformulieren (mit oder } \\
\text { ohne Quellenverweis, } \\
\text { teilw. auch } \\
\text { problematisieren) }\end{array}$ & $\begin{array}{l}\text { Fachlexikon-, Hand- u. } \\
\text { Lehrbuchtexte, welche } \\
\text { die Symptomtrias, } \\
\text { die auf Maslach/ } \\
\text { Jackson zurückgeht, } \\
\text { hervorgehoben neben } \\
\text { anderen Definitionen/ } \\
\text { Konzepten zitieren/ } \\
\text { reformulieren, teilw. } \\
\text { auch problematisieren }\end{array}$ & $\begin{array}{l}\text { Fachlexikon-, Hand- } \\
\text { u. Lehrbuchtexte, } \\
\text { die verschiedene } \\
\text { Definitionen } \\
\text { gleichberechtigt } \\
\text { nebeneinander } \\
\text { präsentieren, dabei } \\
\text { auch die Def. von } \\
\text { Maslach/Jackson, aber } \\
\text { nicht hervorgehoben }\end{array}$ & $\begin{array}{l}\text { Fachlexika, Hand- } \\
\text { und Lehrbücher, } \\
\text { die Burnout kurz } \\
\text { erwähnen, auf 1-2 } \\
\text { Quellen beziehen (die } \\
\text { nicht auf Maslach/ } \\
\text { Jackson zurückgehen) } \\
\text { oder ohne fremdes } \\
\text { (bzw. eindeutig } \\
\text { erkennbares } \\
\text { implizites) Zitat } \\
\text { definieren }\end{array}$ \\
\hline 11 & 24 & 6 & 12 \\
\hline
\end{tabular}

Die tabellarische Übersicht zeigt, dass die Definition, die auf das Maslach Burnout Inventory zurückgeht, in den Fachlexika und Hand- und Lehrbüchern in über der Hälfte der analysierten Texte als dominante Definitionsvariante konstituiert wird. Fachlexika, Hand- und Lehrbücher führen des Weiteren schon qua Publikations- und Textsorte diskursiv zu einer Verknappung der Quellen, weil sie für den geforderten komprimierten Überblick pro Thema eine Auswahl treffen müssen. Sie besetzen eine mächtige diskursive Position, da sie häufig als erste Anlaufstelle zum Einstieg in ein Thema und mit dem Anspruch, einen Überblick $\mathrm{zu}$ erhalten, konsultiert werden. Sie werden darüber hinaus von Personen verfasst, denen von Vertreterinnen/Vertretern einer Disziplin eine fachliche Reputation, bezogen auf dieses Thema, zugeschrieben wird. Die Einträge sind des Weiteren nach Regeln verfasst, die in dieser Disziplin gelten (vgl. Foucault [1970] 1993: 22ff.). ${ }^{695}$

verbreitet sei). Es werden in diese Zählung auch Texte aufgenommen, die zwar Distanzmarker in Bezug auf die Geltung der Definition setzen (z. B. dass es keine allgemeingültige Definition gebe), dann aber dennoch im direkten Anschluss ausschließlich die Definition bzw. die drei Haupt-Symptome nach Maslach/Jackson (1981) reformulieren/zitieren.

695 Ähnlich wie Fachlexika, Hand- und Lehrbücher können des Weiteren Abstracts im Rahmen von fachlichen Übersichtsartikeln Hervorhebungs- und Verknappungseffekte produzieren, wenn eine Definition als einzige im Abstract und damit topografisch im ersten wahrgenommenen Abschnitt des Fachartikels erscheint. In Kap. 6.2.2.2 wurde dies schon bei Beleg 7 (= Bauer/ Häfner/Kächele et al. (2003: 213) in PPmP 53 (5) im QV unter 8.1.4) beschrieben. Die Textsorte Abstract zeichnet sich meist dadurch aus, dass es strenge Vorgaben für die Zeichenanzahl gibt, und sie geht mit der Medialitätserwartung einher, dass der wichtigste Inhalt des Beitrags dort in kurzer Zusammenfassung präsentiert wird. Bauer et al. ziehen in ihrem Abstract den Namen 
Aus diesen Gründen dienen Lexika und Handbücher, teilweise vermittelt über Onlineplattformen wie Wikipedia, wahrscheinlich zudem häufig als Quellen für Vermittlungstexte, auch wenn diese Quellen medial selten eindeutig sichtbar gemacht werden. ${ }^{696}$ Studien legen nahe, dass Plattformen wie Wikipedia journalistisch genutzt werden, beispielsweise auch für die Suche nach weiteren Quellen. ${ }^{697}$ In Kapitel 6.2.3.2 wurde durch den Vergleich der Wikipedia-Artikelversionen $\mathrm{zu}$ „Burnout“ ausgewertet, dass der erste Definitions-Abschnitt in den ausgewerteten Artikelversionen der Jahre 2009 bis 2016 auf der Definition des Klinischen Wörterbuchs Pschyrembel ( ${ }^{261} 2007$, siehe Beleg 4 in Kap. 6.2.2.1) basiert (für einen genauen Vergleich der Pschyrembel- und Wikipedia-Versionen siehe Kap. 6.2.3.2). Ob die Medien sich an diesen Einträgen der Fachlexika, Hand- und Lehrbücher oder Wikipedia orientieren, ist nicht sicher herauszufinden, da selten die Quelle angegeben wird und einzelne Symptome nicht in derselben Strenge wie bei

Herbert Freudenberger und die drei Symptome, die auf Maslach/Jackson (1984) zurückgehen, so zusammen, dass diese Definition des „Burnout-Syndroms“ als Definition von Freudenberger interpretiert werden kann. Eine Seite später wird im Fließtext jedoch betont, „dass es keine allgemein gültige Definition von Burn-out“ gebe. Eine ähnliche nicht direkt im Kontext der Definition kenntlich gemachte Verschmelzung zweier Definitionsquellen, und zwar der klassifizierenden Einordnung von >Burnout in der ICD-10 und der Definition von >Burnout $`$ nach Maslach/Jackson (1984), findet man auch in einem Diskussionsbeitrag von Keller-Schneider (2016: 305) in der Fachzeitschrift PiEU: „Gemäß medizinischer Definition (ICD 10 der Weltgesundheitsorganisation [...]) ist Burnout ein depressives Syndrom, das sich in der Konstellation von emotionaler Erschöpfung, Depersonalisierung (Zynismus) und Leistungsmangel diagnostizieren lässt.“

Reviews bzw. Übersichtsbeiträgen kommt ähnlich wie den Fachlexika, Hand- und Lehrbücherm eine bedeutende Diskursposition zu, da sie von Forschenden und Fachleuten in der Praxis zur überblickshaften Informationsgewinnung über die wichtigsten Erkenntnisse und Studien und zum Einstieg in ein Thema genutzt werden. Auf einer übersichtlichen Seitenzahl sollen die wichtigsten Studien und Erkenntnisse zu einem Thema zusammengefasst werden.

696 Man findet im SPIEGEL-Korpus einen expliziten Beleg dafür, dass die Definition von „Burnout“ über Wikipedia mit Bezug auf den Pschyrembel zitiert wird (= Lobo 2013, DER SPIEGEL, im QV unter 8.1.5), wobei der Autor des SPIEGEL-Artikels nicht überprüft, inwiefern sich die Wikipedia-Version von der Version im Pschyrembel unterscheidet (siehe dazu Kap. 6.2.3.2). Einen Beleg, dass ein klinisches Wörterbuch wie der Pschyrembel von Journalistinnen und Journalisten verwendet wird, findet man zudem in einem Artikel der Zeitung DIE ZEIT aus dem Jahr 1999, in der in Bezug auf den Begriff 〉Burnout geschrieben wird: „In Nachschlagewerken wie dem Pschyrembel taucht der Begriff erst gar nicht auf“ (= Blech 1999, DIE ZEIT im QV unter 8.1.5). Burnout wurde erst in der 259. Auflage (2002: 251, im QV unter 8.1.2) in den Pschyrembel als Stichwort aufgenommen.

697 Dies legt ein Bericht der Bundeszentrale für politische Bildung aus dem Jahr 2012 nahe, der verschiedene Studien zur journalistischen Onlinerecherche und die Verwendung von Wikipedia zusammenfasst (Neubarth/Neuberger 2012, abrufbar unter: http://www.bpb.de/gesellschaft/ digitales/wikipedia/145822/die-wikipedisierung-des-journalismus? $\mathrm{p}=\mathrm{all}$, zuletzt eingesehen am 27.12.2019). 
Zitaten reproduziert werden. Daher ist eine genaue Auszählung, wie häufig die Symptome nach der Definition von Maslach/Jackson (1981/1984) oder anderen Definitionsformen benannt werden, nicht in der gleichen Weise möglich wie bei den Fachlexika, Hand- und Lehrbüchern. Die drei Symptome, die auf das Messinstrument des Maslach Burnout Inventory und teilweise auf Symptome nach Freudenberger (1974) zurückgehen oder diesen ähneln, ${ }^{698}$ werden jedoch über alle Dekaden wiederholt aufgerufen, wie die folgenden Belege aus dem Medienkorpus zeigen. Dabei wird das Symptom „Depersonalisierung“ sprachlich am stärksten variiert. Im ersten Beleg werden die Symptome zudem als „Phasen“ dargestellt, die aufeinander folgen (Unterstreichungen im Folgenden T.S.):

1) Süddeutsche Zeitung, 3.3.2001: V1/1 (= Brunner 2001, im QV unter 8.1.6)

Drei verschiedene Phasen gibt es im Burnout: a) Erschöpfung, b) Gereiztheit und Gleichgültigkeit, c) Leistungseinschränkungen.

2) Psychologie Heute, 10/2002: 24 (= Krumpholz-Reichel 2002, im QV unter 8.1.7)

Holländische Forscher untersuchen die psychische Ermüdung in der Arbeitswelt [als Zwischenüberschrift etwas größer gesetzt] [...]

In einem Fragebogen wurde der Grad der Erschöpfung, der Zweifel an der eigenen Kompetenz, aber auch das Maß an Zynismus als Kriterien für psychische Ermüdung evaluiert. [...]

Chronische Ermattung ist der Endzustand eines langen psychischen Prozesses: „Wer wegen Überforderung oder einem schlechten Betriebsklima aus der Balance gerät, zieht sich zunächst auf einen distanzierten Standpunkt zurück, um in der akuten Situation nicht das Gesicht zu verlieren“, erklärt Wilmar Schaufeli, Professor für Arbeits- und Organisationspsychologie an der Universität Utrecht und im Großprojekt Fatigue at work federführend für chronische Erschöpfung und Burnout zuständig. „Zu große Distanz im Job verschlechtert die sozialen Beziehungen und verhindert in einem nächsten Schritt gute Arbeitsergebnisse, was wiederum dazu führt, dass man sich psychisch der Arbeit nicht mehr gewachsen fühlt" - und ermattet kapituliert.

3) Frankfurter Allgemeine Zeitung, 3.4.2004: 55 (= Kals 2004, im QV unter 8.1.6)

Neben komplexen Wechselwirkungen läßt sich ein Burn-out-Syndrom durch drei Elemente charakterisieren: emotionale Erschöpfung, Dehumanisierung und Zynismus und schließ-

698 Das Symptom „Zynismus“, das häufig genannt wird und in den Aufzählungen teilweise das Symptom „Depersonalisation“ zu ersetzen scheint, nennt auch Freudenberger (1974: 161): "becomes the "house cynic". Freudenberger nennt auch einige somatische Symptome, die in Medientexten aufgegriffen werden: "exhaustion and fatigue, being unable to shake a lingering cold, suffering from frequent headaches and gastrointestinal disturbances, sleeplessness and shortness of breath". Emotionale Merkmale und Verhaltenssymptome sind bei Freudenberger z. B.: "Quickness to anger, [...] instantaneous irritation and frustration responses"; "cries too easily", "becomes excessively stubborn, and inflexible” (ebd.: 160f.). 


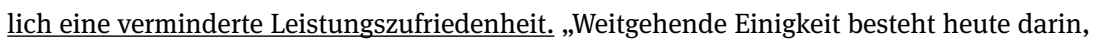
daß Distress, also negativer Streß, ein Schlüsselphänomen in der Entstehung des Burn-out darstellt“, sagt der Arbeitsmediziner Andreas Weber.

4) DIE ZEIT, 20.7.2006 Nr. 30 (= Kuhrt 2006, im QV unter 8.1.6)

ZEIT: Was unterscheidet denn Burn-out von Erschöpfung?

Siegrist: Burn-out ist durch drei Merkmale gekennzeichnet: emotionale Erschöpfung, den Abfall der Leistungsfähigkeit und das Auftreten von Entfremdungsgefühlen bei der Arbeit. Das heißt, dass man nicht mehr mit Freude an die Arbeit geht, sondern sich innerlich distanziert. Insofern ist Burn-out ein bisschen spezifischer als Erschöpfung.

5) SZ, 29.11.2008: V2-9 (SZ 2008a, im QV unter 8.1.6)

\section{Was ist Burnout?}

„Burnout“ bedeutet „Ausbrennen“. Der Psychoanalytiker Herbert J. Freudenberger prägte den Begriff erstmals 1974 in Deutschland. Bis heute gibt es allerdings weder eine allgemein akzeptierte Definition, noch gilt Burnout als klinische Diagnose. Der Begriff taucht in den ICD-10, der internationalen Klassifikation der Krankheiten, nur im Anhang auf. Zu den drei wichtigsten Symptomen von Burnout zählen den US-Forschern Maslach und Jackson zufolge: emotionale Erschöpfung, Depersonalisierung im Sinne einer nicht-mitfühlenden, ablehnenden oder gar aggressiv-zynischen Haltung gegenüber Kunden oder Klienten und das subjektive Gefühl mangelnder Leistungsfähigkeit. Hinzu kommen diverse seelische und körperliche Beschwerden wie Ängste, Abstumpfung, Reizbarkeit, ständige Müdigkeit, häufige Infektionen, Rücken-, Herzkreislauf- oder Magen-Darm-Probleme.

6) FAZ, 6.10.2010: N1 (= Kaulen 2010, im QV unter 8.1.6)

\section{"Burnout": Hat die Diagnose Methode, oder ist es Mode?}

Die inflationäre Verwendung des Begriffs Burnout suggeriert, dass eine ganze Gesellschaft psychisch erschöpft ist. [...]

Jeder hat ein intuitives Verständnis davon, aber weder die internationale Klassifikation der Krankheiten noch das „Diagnostische und Statistische Handbuch psychischer Störungen“ listen Burnout als eigenständige Erkrankung. Es fehlt auch ein allgemein anerkanntes Instrument, um die Störung von anderen Krankheiten, etwa der Depression und den Angsterkrankungen, abzugrenzen. Trotzdem wird sie laufend diagnostiziert. [...]

Der Begriff Burnout leitet sich aus der Arbeitswelt her. Die meisten Ärzte verstehen darunter einen Zustand aus arbeitsbedingter Erschöpfung, Selbstentfremdung, Zynismus und verminderter Leistungsfähigkeit [14]. Im praktischen Alltag zählen noch Dutzende weiterer Symptome dazu, etwa verstärkte Anspannung, Schlafstörungen, Unruhe, Konzentrationsschwäche, mangelnde Motivation und reduzierte Arbeitsleistung. Für Korczak sind diese Zuschreibungen ein beliebiger Katalog negativer Befindlichkeitsstörungen, die keine Rückschlüsse auf den Beginn der Symptome zulassen. In der wissenschaftlichen Literatur werde die Störung deshalb auch als „randunscharfe Menge“ bezeichnet, so der Medizinsoziologe im Gespräch. [...]. 
7) ZEIT ONLINE, 29. Juli 2010 (= Heyn 2010, im QV unter 8.1.6)

[...] ZEIT ONLINE: Wie kann ich feststellen, ob ich unter Burn-out leide?

Bergner: Erstes Symptom ist die emotionale Erschöpfung. Man kann sie selbst diagnostizieren. Sätze wie „Ich kann nicht mehr“ oder „Es macht alles keinen Sinn“ sind klassisch für emotional erschöpfte Menschen. Zweites Symptom ist die sogenannte Depersonalisation. Die Betroffenen ziehen sich zurück. Das dritte Symptom ist die Leistungsabnahme. Erst wenn alle drei Symptome erfüllt sind, lässt sich die Diagnose Burn-out stellen. [...]

8) DIE ZEIT, 8.7.2010, Nr. 28/2010 (= Rudzio 2010b) [Der folgende Ausschnitt, der als Screenshot dargestellt wird (siehe Abbildung 22), ist in dieser grauen Hinterlegung als „Lexikon-Abschnitt“ in den Fließtext des Zeitungsartikels integriert] ${ }^{699}$

\begin{tabular}{|c|c|}
\hline BURN-OUT & Was ist Burn-out? Von einem Burn-out wird heute gesprochen, wenn der Patient \\
\hline $\begin{array}{l}\text { WAS KANN ICH } \\
\text { DAGEGEN TUN? }\end{array}$ & $\begin{array}{l}\text { immer mehr von seiner Arbeit, zieht sich von Freunden zurück, da er keine Kraft } \\
\text { mehr für sie aufbringt, und hat das Vertrauen in seine Fähigkeiten verloren. }\end{array}$ \\
\hline \multirow[t]{3}{*}{$\begin{array}{l}\text { WIE KANN ICH } \\
\text { VORBEUGEN? }\end{array}$} & $\begin{array}{l}\text { Wie kommt es dazu? Gefährdet ist, wer dauerhaft an seine Grenzen geht, was Zeit } \\
\text { und Energie betrifft. Dazu kŏnnen Frustrationen kommen, wenn etwa ein Dozent } \\
\text { oder ein Chef die eigene Arbeit nicht anerkennt, wenn man nicht die Noten oder die } \\
\text { Bezahlung bekommt, die angemessen wăren. }\end{array}$ \\
\hline & $\begin{array}{l}\text { Bin ich gefährdet? Das können Sie mit diesem Test nachprüfen: www.burnout- } \\
\text { fachberatung.de/burnout-test.htm }\end{array}$ \\
\hline & $\begin{array}{l}\text { Auch wenn Sie noch nicht vor dem Zusammenbruch stehen, diese Zeilen Sie aber } \\
\text { zum Nachdenken gebracht haben, sollten Sie etwas gegen Ihre Öberlastung tun. }\end{array}$ \\
\hline
\end{tabular}

Abb. 22: Ausschnitt aus dem Artikel „Kostenstelle Mensch“, DIE ZEIT, 8.7.2010 (= Rudzio 2010b, im QV unter 8.1.6), () Zeitverlag Gerd Bucerius GmbH \& Co. KG 2010.

\section{9) Wikipedia, Artikelfassung zu „Burnout“ vom 26.12.2009}

Ein Burnout-Syndrom (engl. (to) burn out: „ausbrennen“) ist ein Zustand ausgesprochener emotionaler Erschöpfung mit reduzierter Leistungsfähigkeit, das als Endzustand einer Entwicklungslinie bezeichnet werden kann, die mit idealistischer Begeisterung beginnt und über frustrierende Erlebnisse zu Desillusionierung und Apathie, psychosomatischen Erkrankungen und Depression oder Aggressivität und einer erhöhten Suchtgefährdung führt.[1] $]^{700}$

699 In dem im dritten Abschnitt verlinkten Test werden diese Symptome und weitere in Form von Fragebogen-Items wiederaufgenommen, vgl. https://www.burnout-fachberatung.de/burnout-test.htm (zuletzt abgerufen am 22.11.2019). Eine Definition von Burnout, die in Form eines grau hinterlegten Kastens bzw. „Glossars“ in den Zeitungsartikel integriert ist, und eine Verlinkung auf einen Burnout-Test einer externen Seite (in diesem Fall auf folgende Seite: https://www. hilfe-bei-burnout.de/burnout-test-lang/), findet man beispielsweise auch bei Heinrich (2011, DIE ZEIT Nr. 40/2011, siehe im QV unter 8.1.6), vgl. dazu auch Abb. 12.

700 Unterstreichungen, die Links auf Wikipedia markieren, wurden hier entfernt, T.S. Diese Definition geht auf die Definition im Klinischen Wörterbuch Pschyrembel zurück. Das Symptom 
10) Wikipedia von 2011-2019: Unter Symptomen wird auf Wikipedia von 2011-2019 nur die Symptomtrias von Maslach/Jackson zugrunde gelegt und grafisch mit dem Copenhagen Burnout Inventory (CBI) verbunden (siehe die folgende Abbildung 23): ${ }^{701}$

\section{Symptome des Burnout-Syndroms}

(Nach dem Maslach- und Copenhagen Burnout-Inventory - MBI und CBI*)

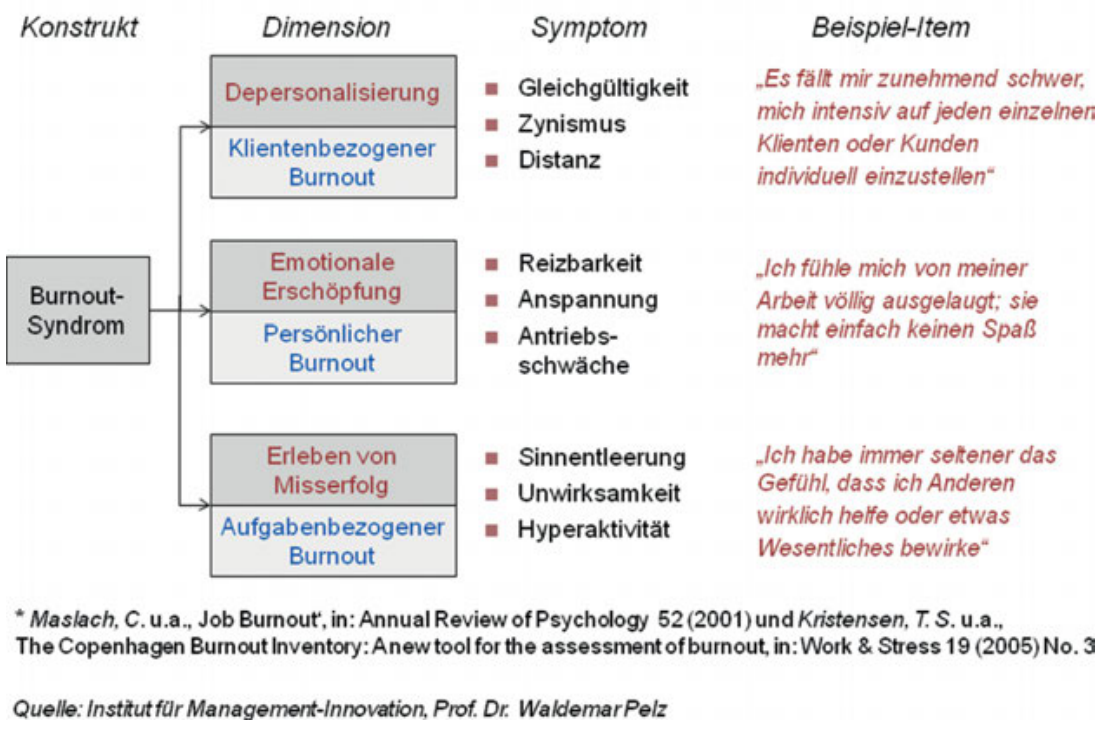

Abb. 23: Grafische Abbildung im Abschnitt „Symptome“ im Korpus der WikipediaArtikelfassungen von 2011-2019, CC BY-SA 3.0 DE (https://creativecommons.org/licenses/ by-sa/3.0/de/legalcode). ${ }^{702}$

\section{1) NetDoktor, 12.6.2014, (= Bannert 2014, im QV unter 8.1.8)}

In seiner Firma ist Schmidt nicht der einzige, der sich ausgebrannt fühlt. Ein mysteriöses Virus scheint sich unaufhaltsam in der Werbeagentur auszubreiten: Immer mehr Kollegen

der „Depersonalisierung“ wird in Wikipedia aber nicht übernommen. Diese Definition bleibt bis 2016 beinahe unverändert zu Beginn des Wikipedia-Artikels zu Burnout bestehen (siehe Kap. 6.2.3.2).

701 Die Zusammenführung des Copenhagen Burnout Inventory mit dem Maslach Burnout Inventory ist von der Forschung her nicht gedeckt. In Kap. 6.2.2.2 dieser Arbeit (siehe Beleg 6b) wurde gezeigt, dass die Autorinnen/Autoren des CBI ihren Fragebogen gerade aufgrund ihrer Kritik am MBI entwickelten (Kristensen/Borritz/Villadsen et al. 2005, im QV unter 8.1.5).

702 https://commons.wikimedia.org/wiki/File:Symptome_des_Burnout-Syndroms.png (Stand: 23.9.2021). Es wurde jährlich die letzte bearbeitete Version eines Jahres zum Vergleich verwendet: das heißt die Wikipedia-Artikel-Fassungen zu „Burn-out“ vom 27.12.2011; 30.12.2012; 31.12.2013; 16.12.2014; 16.12.2015; 28.12.2016; 21.12.2017; 2.12.2018; 6.12.2019. 
sind gestresst und überfordert. Sie infizieren andere mit ihrer emotionalen Erschöpfung und ihrer reduzierten Leistungsfähigkeit.

12) Gehirn\&Geist Dossier 1/2016: 25 (= Falkai 2016, im QV unter 8.1.7)

Betrachtet man den Symptomkatalog, so trifft einiges auch auf das Burnout-Syndrom zu. Dessen zentrales Merkmal, "anhaltende Erschöpfung und Leistungsminderung«, meint letztlich nichts anderes als das Hauptsymptom »Antriebsmangel und erhöhte Ermüdbarkeit« im Diagnosekatalog für Depressionen. Und hinter dem für Burnout typischen Zynismus verbergen sich möglicherweise »Gefühle von Schuld und Wertlosigkeit sowie negative und pessimistische Zukunftsperspektiven «, also Zusatzsymptome der Depression. ${ }^{703}$

Außerdem kann man die Symptome „emotionale Erschöpfung“, „Depersonalisation“ (medial-öffentlich häufig mit `Zynismus`, `Entfremdung`, oder `Distanz` gleichgesetzt) und „verminderte Leistungsfähigkeit“ in vielen Fallnarrationen als abstraktere Konzepte hinter den geschilderten Veränderungen im Verhalten und Erleben der Personen ausmachen:

\section{3) Der Spiegel, Nr. 52 (1988: 162, im QV unter 8.1.6)}

$\mathrm{Zu}$ Beginn seiner Karriere war der Berliner Professor für seine Studenten jederzeit zu sprechen. Neugierig und umtriebig engagierte sich der Wissenschaftler für Studienreform und Selbstverwaltung und war stets dabei, wenn ein ,autoritärer Zopf“ abzuschneiden war.

Heute beschränkt der inzwischen 53jährige Professor seine Begegnungen mit Studenten, „diesen narzißtischen Kretins“, auf ein kühles Minimum, sitzt Konferenzen seufzend ab und lebt müde und resigniert zwischen seinen Bücherwänden. Neuerdings ist der Akademiker regelmäßig beim Arzt: Er schläft miserabel, und sein Herz setzt zu unvermittelten Sprüngen an.

Die junge Lehrerin begann ihre Lehrzeit an einer nordrhein-westfälischen Gesamtschule voller Elan. Doch bereits nach einem Jahr im Schuldienst ist sie „ein Nervenbündel“, fällt nach dem Unterricht in einen Erschöpfungsschlaf und brütet nachts über ihren Stundenvorbereitungen: „Ich hätte nie gedacht, daß ich mal so werden könnte wie die säuerlichen alten Kollegen, die die Kinder bloß anschreien und Strafarbeiten aufgeben“, seufzt sie und erzählt, inzwischen haue sie auch dazwischen, ,anders kommt man einfach nicht durch“.

\section{4) Gehirn\&Geist Dossier, 1/2016: 20 (= Falkai 2016)}

Zunehmend wandelte sich die Freude am Job in Abscheu - insbesondere jüngeren Kollegen gegenüber, die vermeintlich leistungsfähiger waren. Hinze konnte sich immer schlechter konzentrieren und fühlte sich schon morgens erledigt. Dazu gesellten sich körperliche

703 Weitere Belege aus dem Korpus in der FAZ/FAZ.NET: mit explizitem Bezug auf Maslach (= Stock 2010); indirekter Bezug bei Nawrath (2007) „In der Wissenschaft wird Burn-out als Zustand emotionaler Erschöpfung, Distanziertheit und reduzierter persönlicher Leistungsfähigkeit bezeichnet“ und Dunsch (2016); im Karriere-SPIEGEL 31.10.2013 (= Burisch 2013); bis hier alle Belege im QV unter 8.1.6; GEOkompakt (08/2014, = Eberle 2014: 28, im QV unter 8.1.7). 
Beschwerden: Kopfweh, Magendrücken, Rückenschmerzen. Urlaub - sofern er sich denn welchen gönnte - vermochte die Lage nicht mehr zu verbessern.

In den Beispielen wird der zynischen Haltung der Personen (durch die Abwertung der Studierenden durch das Adjektiv „,äuerlich“ und „die Kinder bloß anschreien“ und „Abscheu“), der physischen und emotionalen Erschöpfung (,lebt müde und resigniert“, „Nervenbündel“, „Erschöpfungsschlaf“, ,schon morgens erledigt“) und der verminderten Leistungsfähigkeit („,brütet nachts über ihren Stundenvorbereitungen“, ,immer schlechter konzentrieren“) Ausdruck verliehen. ${ }^{704}$ Diese Textauszüge zeigen jedoch auch, dass die Symptome weniger eindeutig zugewiesen werden und dass noch weitere Symptome genannt werden (z. B. anfänglicher Elan sowie Schlafund Herzprobleme) bzw. die Symptome für >BURNOUT in den Medientexten stärker variieren. Die weitere Symptomatik, insbesondere der Wechsel der Merkmale sidealistische Begeisterung`, >Engagement‘, sverstärkter Einsatz` in ihr Gegenteil, wird im Diskurs über weitere intertextuelle Bezugnahmepraktiken verfestigt, die im Folgenden beschrieben werden.

Eine Verknappung der Symptome bewirkt sowohl in fachlichen Übersichtstexten als auch in fachexternen Problem- und Magazinberichten grafisch hervorgehoben Gedrucktes in Kästen und Abbildungen, die Phasen- oder Symptommodelle darstellen. Bei der Durchsicht der Quellen zeigte sich, dass sich in verschiedenen Textsorten des Burnout-Diskurses (implizite) intertextuelle Verweise zum einen auf die in Überkategorien untergliederte Symptom-Zusammenfassung von Matthias Burisch ( ${ }^{2} 1994 ;{ }^{3} 2006$; ${ }^{5} 2014$, im QV unter 8.1.5) und zum anderen auf ein Phasenmodell von Freudenberger/North (1985; ${ }^{9} 2002$, im QV unter 8.1.5) manifestieren.

Matthias Burisch, der eine in Deutschland mehrfach aufgelegte Monografie über das Burnout-Syndrom Ende der 1980er Jahre publizierte (1. Auflage 1989), hat nach einer eingehenden Literaturrecherche ,alle in der Literatur häufig genannten Symptome“ in einer „Übersicht [...] unter sieben Oberkategorien aufgelistet“ und „teils noch einmal in Unterkategorien aufgeteilt“ (hier zitiert nach Burisch $\left.{ }^{5} 2014: 25-29\right) .{ }^{705}$ Diese Oberkategorien werden in der Folge im fachlichen Diskursstrang vor allem in Übersichtsbeiträgen in Fachzeitschriften aufgenommen. Die folgende Übersicht zeigt eine Kurzfassung dieser Synopse beispielhaft (siehe Abbildung 24):

704 Ähnliche narrative Definitionsentfaltungen, in denen man die beschriebenen Symptome ausmachen kann, sind z. B. SZ, 31.12 .1994 (= Rheinz 1994, im QV unter 8.1.6); PH 4/2000 (= Smolka 2000: 39f., im QV unter 8.1.7); FAZ, 4.4.2004 (= Kals 2004: 55, im QV unter 8.1.6); SZ 30.10.2012 (Haas/Wolff 2012: 26, im QV unter 8.1.6).

705 Burisch spricht davon, dass das „Symptombild in den genannten Studien [...] sehr vielschichtig, andererseits aber von Studie zu Studie überraschend einheitlich“ sei (Burisch 52014: 25). 
Tabelle 2: Burnout Symptomatik nach Burisch ${ }^{21}$ (Kurzfassung)

\begin{tabular}{|l|l|}
\hline 1. Warnsymptome der Anfangsphase & $\begin{array}{l}\text { a) Vermehrtes Engagement für Ziele } \\
\text { b) Erschöpfung }\end{array}$ \\
\hline 2. Reduziertes Engagement & $\begin{array}{l}\text { a) Für Klienten, Patienten etc. } \\
\text { b) Für andere allgemein } \\
\text { c) Für die Arbeit } \\
\text { d) Erhöhte Ansprüche }\end{array}$ \\
\hline 3. Emotionale Reaktionen, Schuldzuweisung & $\begin{array}{l}\text { a) Depression, Schuldgefühle } \\
\text { b) Aggression }\end{array}$ \\
\hline 4. Abbau & $\begin{array}{l}\text { a) Der kognitiven Fähigkeit } \\
\text { b) Der Motivation } \\
\text { c) Der Kreativität } \\
\text { d) Entdifferenzierung }\end{array}$ \\
\hline 5. Verflachung & $\begin{array}{l}\text { a) Des emotionalen Lebens } \\
\text { b) Des sozialen Lebens } \\
\text { c) Des geistigen Lebens }\end{array}$ \\
\hline 6. Psychosomatische Reaktionen & \\
\hline 7. Verzweiflung & \\
\hline
\end{tabular}

Abb. 24: Kurzfassung der Synopse der Burnout-Symptomatik nach M. Burisch im HTA-Bericht Korczak/Kister/Huber (2010: 15, im QV unter 8.1.5), (c Korczak et al., DIMDI, Köln 2010.

Diese Oberkategorien von „1. Warnsymptome der Anfangsphase“ bis „7. Verzweiflung“ erscheinen nun auch in weiteren Übersichtbeiträgen und Monografien, z. B. bei Rook (1998: 125) ${ }^{706}$; Rösing (2003: 58-60); Beschoner/SchönfeldtLecuona/Braun et al. (2009: 216) ${ }^{707}$; von Känel (2008: 479) ${ }^{708}$; Nil/Jacobshagen/ Schächinger et al. (2010: 76) ${ }^{709}$, Kaschka/Korczak/Broich (2011: 782) ${ }^{710}$; Weimer/ Kraus (2011: 240) ${ }^{711}$; in der Übersichtsmonografie von Seibold/Schuh (52010: 156ff. ${ }^{712}$; bei Gumz/Brähler/Erices (2012: 34) ; $^{713}$ oder im Beitrag von Altfeld/Kellmann (2013: 48) $)^{714}$.

Im fachexternen Diskursstrang wurden diese sieben symptomatischen Oberkategorien von Burisch in den Artikelversionen zu „Burn-out“ des WikipediaPortals von 2005-2010 ${ }^{715}$ als Gliederungspunkte des Inhaltsverzeichnisses für das Kapitel „Die Burnout-Symptomatik“ ohne Verweis auf Burisch übernommen und

$706 \mathrm{Im}$ QV unter 8.1.5.

707 In der Fachzeitschrift PiD, im QV unter 8.1.4.

708 In der Fachzeitschrift Praxis, siehe im QV unter 8.1.5.

709 In der Fachzeitschrift Schweizer Archiv für Neurologie und Psychiatrie, siehe im QV unter 8.1.5. 710 Im Dt. Ärztebl., siehe im QV unter 8.1.4.

711 In der Fachzeitschrift Der Psychotherapeut, siehe im QV unter 8.1.5.

712 Im QV unter 8.1.5.

713 In der Fachzeitschrift PPmP, im QV unter 8.1.4.

714 In der Zeitschrift für Sportpsychologie, im QV unter 8.1.5.

715 Es wurde aus der Versionengeschichte des Wikipedia-Eintrags zu „Burn-out“ jährlich die letzte bearbeitete Version eines Jahres zum Vergleich verwendet (von 2005-2010). 
mit den Untersymptomen von Burisch und weiteren Merkmalsangaben kombiniert. Im Folgenden sei zur Veranschaulichung der erste Teil der Artikelgliederung des Wikipedia-Eintrags zu „Burn-out“ vom 21.12.2005 zitiert (graue Hinterlegung T.S.):

15) Wikipedia: Burn-out (21.12.2005)

\section{Inhaltsverzeichnis}

- 1 Kurzbeschreibung

- 2 Die Burnout-Symptomatik - Charakteristische Merkmale

- 2.1 Warnsymptome der Anfangsphase

- 2.2 Reduziertes Engagement

- 2.3 Schuldzuweisungen als emotionale Reaktion

- 2.4 Abbau

- 2.5 Verflachung

- 2.6 Psychosomatische Reaktionen

- 2.7 Verzweiflung

- 2.8 Zusammenfassung

- 3 Messung

- [...]

Auf der Plattform „Netdoktor.de“ wurden diese sieben Überkategorien nach Burisch zu einem Phasenmodell verbunden. Schrift und Umrandung der ersten Phasen sind optisch transparenter als die der letzten Phasen, wodurch die zunehmende Dringlichkeit des Burnout-Phänomens gestalterisch unterstrichen wird (siehe Abbildung 25 auf der nächsten Seite).

Nach Foucault greifen hier die Prinzipien des „Autors“ und des „Kommentars“: Sie verknappen die Ereignishaftigkeit und Zufälligkeit des Diskurses, indem sie im Fall des „Kommentars“ Textteile des zitierten Materials reproduzieren und im Fall des „Autors“ sprachlich-diskursiv gebundene Perspektiven auf das Phänomen/Konzept >BURNOUT` auf einen „Ursprung“ beziehen, damit gruppieren und in einem „Mittelpunkt ihres Zusammenhalts“ verdichten (vgl. Foucault [1970] 1993: 20ff. und Kap. 4.1.3). Die Verknappung durch die Stimme eines Autors wird im Burnoutdiskurs, hier durch den Autor Burisch, dadurch noch verstärkt, dass Burisch nicht nur in fachlichen Übersichtsbeiträgen (siehe oben) mit seiner Monografie und der symptomatischen Zusammenfassung, sondern auch in fachexternen Texten äußerst präsent ist. ${ }^{716}$ Burisch tritt außerdem im Medien-

716 So bezieht sich beispielsweise Gross (1992) in der FAZ vom 29.2.1992 in einem Problembericht auf die Monografie von Burisch (1989) und die dort erläuterten „Kategorien“ bzw. Stufen der Burnout-Symptomatik (im QV unter 8.1.6); Schwertfeger bezieht sich auf SPIEGEL ONLINE vom 22.7.2010 auf Burisch und seine Monografie, die sie als „Standardwerk“ bezeichnet; Bahnsen 
Die 7 Burnout-Phasen nach Prof. Burisch

\begin{tabular}{|c|}
\hline Phase VII \\
Verzweiflung und Bumout \\
\hline
\end{tabular}

Phase VI

Psychosomatische (körperliche) Erkrankungen

Phase V

Verflachung, Desinteresse und Gleichgültigkeit

\begin{tabular}{|c|}
\hline Phase IV \\
Abbau und schwindende Leistungsfähigkeit
\end{tabular}

\begin{tabular}{|c|}
\hline Phase III \\
Depression, Aggression, Schuldzuweisung
\end{tabular}

\section{Phase II}

Reduziertes Engagement und Ruckzug

Phase I

Extrem viel Energie und Ehrgeiz

Q rletDoktor.de

Das Phasenmodell nach Prof. Matthias Burisch: Nicht jeder Betroffene erlebt jede dieser Phasen.

Abb. 25: Transformation der Symptom-Gliederung nach Burisch (52014: 25-29) in ein Phasenmodell auf $($ C NetDoktor.de (= Dobmeier/Fux 2018, im QV unter 8.1.8, https://www. netdoktor.de/krankheiten/burnout/, zuletzt abgerufen am 6.4.2021, hier in schwarz-weiß).

und Vermittlungsdiskurs, teilweise auch in Fachmagazinen, als Interviewpartner und als Autor von Medienartikeln und Krankenkassenbroschüren auf. ${ }^{717}$

(2010) bezieht sich in einem Beitrag der ZEIT vom 8.7.2010 auf frühe Stadien des Burnout nach Burisch (im QV unter 8.1.6). Weitere Belege, die sich auf die Symptom-Oberkategorien bzw. Phasen nach Burisch beziehen sind z. B.: FAS, 13.9.2015 (= Oberhuber 2015: 35, im QV unter 8.1.6); PH compact, 27/2011 (= Schulze 2011: 41); PH 5/2009 (= Schulze 2009: 27, im QV unter 8.1.7); Geo kompakt, Nr. 40/2014 (= Eberle 2014: 26, im QV unter 8.1.7).

717 So z. B. in einem Interview in der Fachzeitschrift Psychotherapie im Dialog (= Burisch/Borcsa/ Wilms 2009, PiD, im QV unter 8.1.4); in einem Übersichtsbeitrag für PH 9/1994 (= Burisch 1994b: 22-26) oder in einer Rezension zum Buch von C. Cherniss ,Jenseits von Burnout und Praxisschock. Hilfen für Menschen in lehrenden, helfenden und beratenden Berufen“ in PH 4/2000 (= Burisch 2000: 72, im QV unter 8.1.7); oder in einem Problembericht zu „Burn-out“ im Lehrberuf in der Hamburger Lehrerzeitung 1/1985 (= Burisch 1985: 8-11, im QV unter 8.1.7); oder für die Präsentation eines Selbsttests auf SPIEGEL ONLINE vom 31.10.2013 (= Burisch 2013, im QV unter 8.1.6); oder als Inter- 
Fachextern wird des Weiteren das Zwei- und Zwölf-Phasenmodell von Freudenberger/Richelson (1980a: 41-83) und Freudenberger/North (1985; ${ }^{9} 2002$ ) in verschiedenen Textquellen zitiert. Das Zweiphasenmodell erscheint in den Artikel-Fassungen zu „Burnout“ auf Wikipedia in den Jahren 2005-2010 jeweils als erstes Phasenmodell neben anderen Phasenmodellen. ${ }^{718}$ Ab dem Jahr 2011 wird es durch das Zwölf-Phasenmodell getauscht, und die anderen Phasenmodelle werden 2012 aus dem Wikipedia-Artikel entfernt. Sowohl das Zweiphasenmodell (Freudenberger/Richelson 1980: 41ff.) als auch das Zwölfphasenmodell (Freudenberger/North 1985: 86ff.; ${ }^{9} 2002:$ 121ff.) transportieren eine Dichotomie zwischen einem Anfangsstadium, das durch einen hohen, scheinbar mühelosen Einsatz der eigenen Fähigkeiten und positiven Gefühlen geprägt ist, und einem Endstadium, das von chronischer Erschöpfung, einem Verlust von kognitiven, kreativen Fähigkeiten und negativen Gefühlen begleitet ist. Exemplarisch sei eine Abbildung aus der Apotheken Umschau zitiert, die das Zwölfphasen-Modell als absteigende Treppe ins Bild setzt und im Begleittext als „Abstieg“ betitelt und auf Herbert Freudenberger bezieht: ${ }^{719}$

Diese temporale und qualitative Dichotomie zwischen einem Anfangs- und Endzustand spiegelt sich auch im Titel des Beitrags aus der Apotheken Umschau „Erst Feuer und Flamme, dann ausgebrannt“ (AU 2005b: 40) und in vielen narrativen Definitionsentfaltungen wider, wie sie in Kapitel 6.2.3.1 analysiert wurden. Die Abbildung in der Apotheken Umschau (siehe Abbildung 26) zeigt des

viewpartner auf SPIEGEL ONLINE (= SPIEGEL ONLINE 2000; 2011d, im QV unter 8.1.6); oder im Interview mit der AU 10/2011-A (= Rotherbl: 34-37, im QV unter 8.1.7); oder als Autor der Broschüre „Burnout vorbeugen“ der Techniker Krankenkasse (= Burisch 2012, im QV unter 8.1.9).

718 In der Wikipedia-Artikel-Fassung zu „Burn-out“ vom 21.12.2005. Im Unterkapitel „Phasen des Burnout-Syndroms“ wird nach der folgenden Zwischenüberschrift „H. Freudenberger“ das Zwei-Phasenmodell wie folgt wiedergegeben: „Hier wird eine Entwicklung von einem empfindsamen zu einem empfindungslosen Stadium beschrieben.

- empfindsames Stadium: Negative Gefühle werden nicht beachtet, ein hoher Energieeinsatz zum Erreichen gewohnter Leistungen aufgebracht und chronische Müdigkeit verdrängt.

- empfindungsloses Stadium: es treten Symptome wie Gleichgültigkeit, Schuldzuschreibungen an die Umwelt, Angst nicht anerkannt zu sein und Desorientierung auf.“

719 Das Zwölf-Phasenmodell von Freudenberger/North (1985: 86ff.; ${ }^{9} 2002:$ 121ff.) wird u. a. in folgenden populärwissenschaftlichen Zeitschriftentexten abgebildet oder erwähnt: AU 11/2012A: 24 (= Schweiger 2012); AU 05/2014-A: 12-13 (= Wolfrum/Steinmüller 2014); GEO WISSEN, Nr. 48, 2011 (= Simon 2011: 56, im QV unter 8.1.7); Eine ähnliche Form der Treppendarstellung, jedoch mit Bezug auf „Erfahrungen von Patienten des Zentrums für seelische Gesundheit der Asklepios-Klinik Hamburg-Harburg“, findet man im Stern, Nr. 50/2015 (= Schmitz 2015: 53). In Fachtexten werden die zwölf Stadien in einem Kasten hervorgehoben gedruckt im HTA-Bericht von Korczak/Kister/Huber (2010: 15, im QV unter 8.1.5); im Dt. Ärztebl. bei Kaschka/Korczak/ Broich (2011, im QV unter 8.1.4) bei Burisch (52014: 41); Seibold/Schuh (52010: 169). 


\section{Rat\&Hilfe}

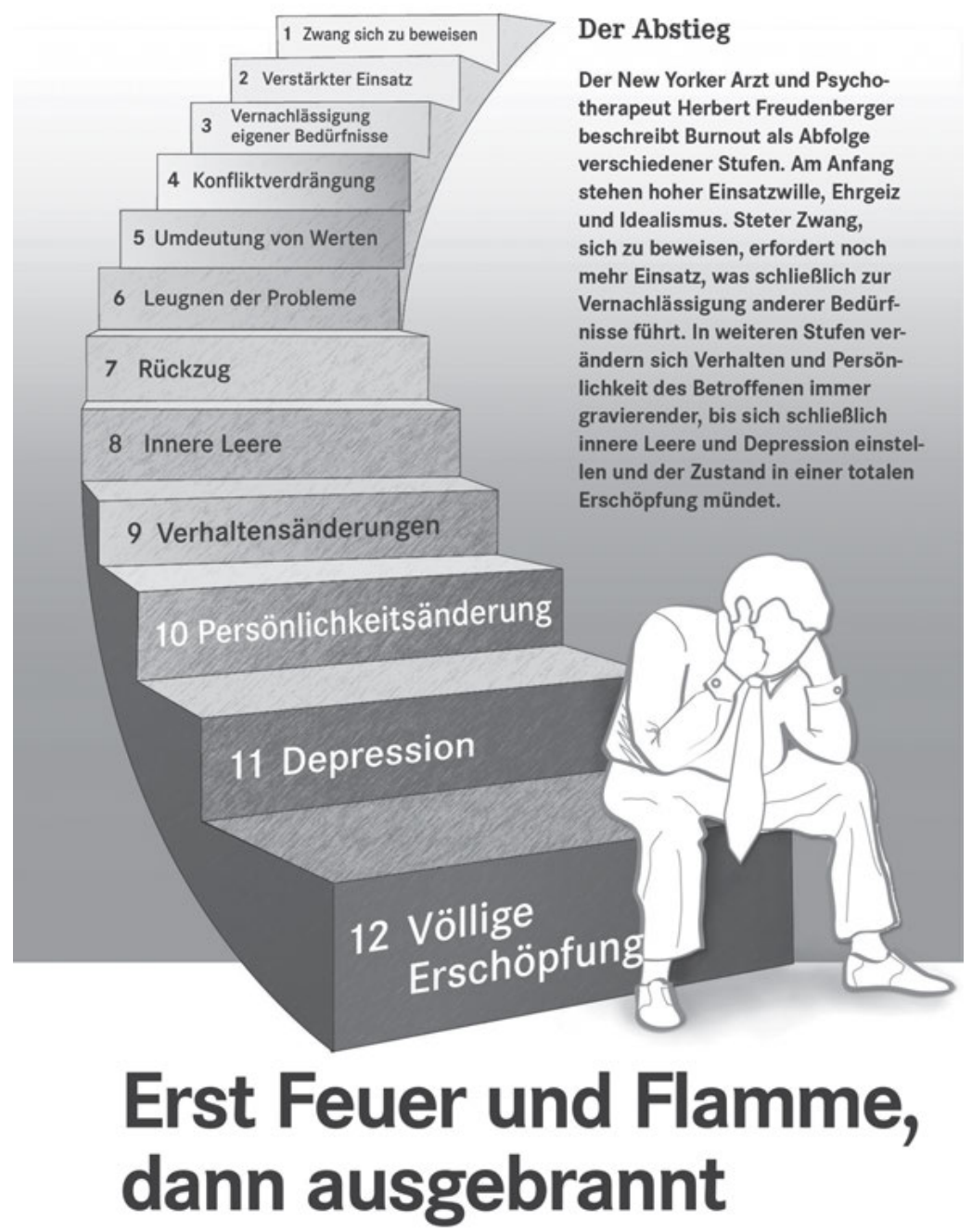

Arbeitswelt Ein „Burnout“ kommt nicht von heute auf morgen. Wer die Warnsignale rechtzeitig erkennt, kann gegensteuern

Abb. 26: Das Zwölf-Phasenmodell nach Freudenberger/North (1985: 86ff.; ${ }^{9}$ 2002: 121ff.) in Apotheken Umschau 5/2005-B: 40 (= AU (2005b) im QV unter 8.1.7, kopierter Ausschnitt in schwarz-weiß @ Wort \& Bild Verlag/Apotheken Umschau/Dr. Ulrike Möhle). 
Weiteren einige Attribute, die sich in fast allen Fotografien und Illustrationen, ${ }^{720}$ die in Medien- und Onlinetexten, aber teilweise auch in Lehrbüchern und Artikeln des Deutschen Ärzteblatts ${ }^{721}$ veröffentlicht werden, wiederholen: Die abgebildete Person wird als 'arbeitende' und 'erschöpfte' Person ikonisch-indexikalisch gekennzeichnet (durch Anzug, Krawatte, gesenkten Blick/Kopf, der auf die Hände gestützt ist, auf der untersten Treppenstufe nach einem mehrstufigen Weg abwärts statt aufwärts, siehe Abb. 26). Ähnliche Bildattribute werden auch in den folgenden Beispielen im Deutschen Ärzteblatt und im Magazin Gehirn\&Geist in Szene gesetzt. In den folgenden Fotografien (Abb. 27 und 28) werden zudem die 'übermäßige' >Belastung، durch einen ScHREIBTISCH VOLLER (KRANKEN-)AKTEN und das 'Arbeiten zu einer späten Stunde bzw. ohne Tageslicht' durch einen LAPTOP ALS LiCHTQUELle IM DUNKLEN RAUM dargestellt. Durch die Nahaufnahme sind Gestik und Mimik der dargestellten Personen zu erkennen, es wird eine gewisse Nähe suggeriert. Die Personen, um deren Befinden es geht, werden meist zentrumsnah im Vordergrund des Bildes scharf gestellt.

Die Fotografien plausibilisieren visuell den Kausalzusammenhang zwischen einem >übersteigerten Maß an Aufgaben` (sich türmende (Kranken-)Akten) und den sich in der Körperhaltung zeigenden `Erschöpfungs-Symptomen` (Kopf wird in die Hand gestützt etc.). Die Bilder wiederholen dadurch auf visueller Ebene die schon durch quantifizierende und graduierende Sprachmittel erreichte Ein-

720 Sylvia Bendel Larcher charakterisiert Fotografien im Kapitel „Bildanalyse“ im Rahmen ihrer Einführung in die Diskursanalyse als bedeutungsoffene ikonische Abbildungen, die auf ,real existierende konkrete Objekte verweisen (Bendel Larcher 2015: 128). „Illustrationen“ weisen nach Bendel Larcher ebenfalls Ähnlichkeiten mit dem Abgebildeten auf (= ikonischer Zeichenmodus), doch es müsse ihnen „kein realer Gegenstand in der Wirklichkeit“ vorausgehen, sondern sie könnten auf Personen, Gegenstände in einer fiktiven Welt verweisen (Bsp. Illustration einer konkreten Person in einer Märchenszene). Sie seien „bedeutungsfixiert“, da sie ausgehend vom Text jeweils „eine bestimmte Szene“ oder Situationen wiedergeben würden, weshalb sie auch „nicht [ohne Weiteres, T.S.] in andere Kontexte übertragbar“ seien (ebd.: 129). Bei den Fotografien im Burnout-Diskurs wird in der Regel jedoch auch „eine bestimmte Szene“ inszeniert. Dadurch bilden diese Fotografien zwar reale Personen und Gegenstände ab, aber mit der abgebildeten Szene und über Text-Bild-Bezüge werden Sachverhaltsfixierungsversuche unternommen.

$721 \mathrm{Im}$ Deutschen Ärzteblatt findet man Fotografien und Illustrationen in Texten, die unter den Rubriken „Themen der Zeit“, „Politik“ und „Varia“ aktuelle Status- oder Problemberichte zum Phänomenbereich Burnout im Ärztlichen, Sozialen Bereich/Bei Personengruppen mit hoher Belastung (z. B. ALLEInERZIEHENDE BERUfSTÄtige PeRsonen) sowie Nachrichten zu Fehlzeitenreports und Kurzzusammenfassungen von Studien zu diesem Thema präsentieren. In anderen Fachtexten (Übersichtsarbeiten und Originalarbeiten) des Dt. Ärzteblatts und anderer Fachzeitschriften findet man in der Regel keine Fotografien und Illustrationen, sondern Visualisierungen und Diagramme. Auf zwei Diagramme, eines in Kap. 6.2.2.1 (siehe die Beschreibung in Beleg 6) und eines in Kap. 6.2.2.2 (siehe Beleg 10, = Abb. 9) wurde im Rahmen der Analyse eingegangen. 


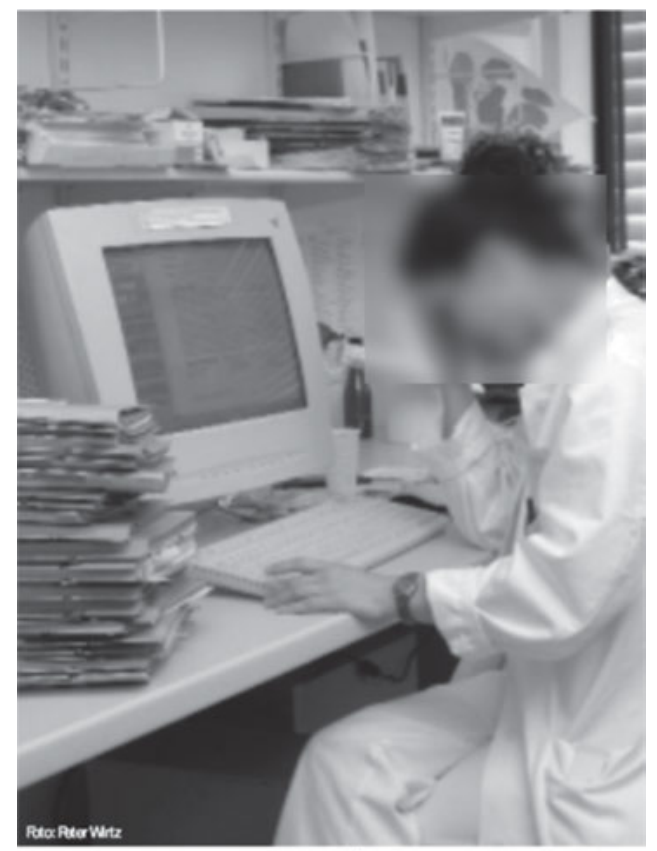

Burn-out ist die Krankheit des Oberengagements

Abb. 27: Fotografie (von () Peter Wirtz) im Beitrag von Bergner (2004: A 2234, im Deutschen Ärzteblatt in der Rubrik „Themen der Zeit“, im QV unter 8.1.4, hier in schwarz-weiß und anonymisiert).

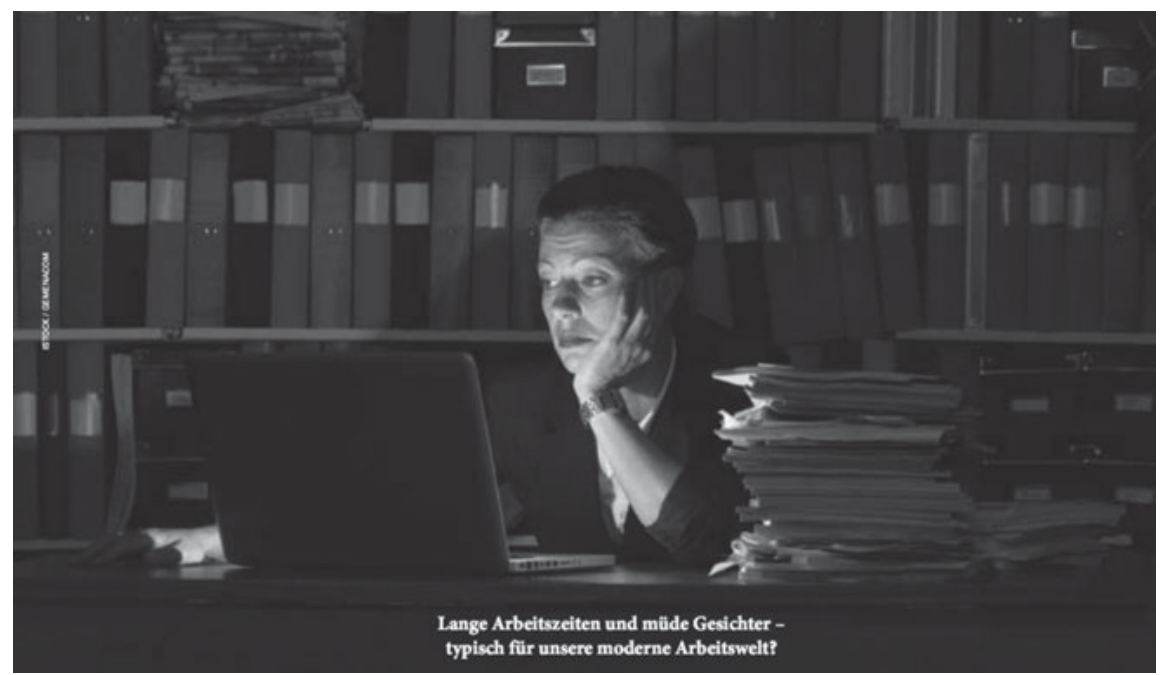

Abb. 28: Fotografie (Lizenz (C) iStock/Gemenacom) im Beitrag von Falkai (2016: 21, im Magazin Gehirn\&Geist, im QV unter 8.1.7, hier in schwarz-weiß) 
ordnung von >BURNOUT< oberhalb von 'normalen', d. h. 'nicht gesteigerten' und 'nicht-krankmachenden' Bedingungen Im ARBEITSLEBEn (vgl. dazu Kap. 6.2.2.1; 6.2.2.2 und 6.2.3.1).

Weitere intertextuelle Bezüge kann man zwischen der Metaphorik fachlicher Erklärungstheorien, die häufig im Zusammenhang mit >Burnout` erforscht werden (wie die Fachartikel und Keyword-Analyse zeigen, vgl. Kap. 6.2.4.1), und der in Medien- und Onlinetexten verwandten Metaphern erkennen. Diese Bezüge können am Beispiel der „Theorie der Ressourcenerhaltung“, des „ArbeitstätigkeitAnforderungen-Ressourcen-Modell[s]“ und des Modells der „Gratifikationskrise“ und Belegen aus dem Fach-, Medien- und Vermittlungstextkorpus aufgezeigt werden. Dazu wird zunächst eine Beschreibung der Modelle aus der Onlineversion des „Lexikons der Psychologie - Dorsch“ zitiert (Unterstreichungen (= Links) im Original, graue Hinterlegungen T.S.):

Nach der Theorie der Ressourcenerhaltung von Hobfoll (1989) sind Personen besser auf die Stressbewältigung vorbereitet, wenn sie über vielfältige Ressourcen zur Stressbewältigung verfügen und sie aufrechterhalten. Verluste machen die Personen sehr verletzlich. [.... ${ }^{722}$

(Greif 2021)

[engl.] «Arbeitstätigkeit-Anforderungen-Ressourcen-Modell», Abk. JD-R-Modell, [AO], wurde als arbeitspsychol. Modell i. R. der Burn-out-Forschung entwickelt. Es dient der Erklärung von pos. und neg. Befindensindikatoren im Arbeitskontext. Faktoren der Arbeit können demnach eingeteilt werden in Anforderungen (demands) und Ressourcen (resources). Durch eine Häufung von Anforderungen (z. B. Zeitdruck, ungünstige Umgebungsbedingungen, Schichtarbeit) kommt es über den sog. health impairment process zu neg. Folgen wie Erschöpfung oder Burn-out. Im Ggs. dazu führen Ressourcen (z. B. Autonomie, Unterstützung, Rückmeldungen) über den motivationalen Prozess des Modells zu höherem Engagement und besserer Leistung bei der Arbeit. Das Modell wurde zusätzlich um einen Interaktionseffekt zw. Anforderungen und Ressourcen erweitert. So können vorhandene Ressourcen die neg. Wirkung der Anforderungen abschwächen. AnforderungsRessourcen-Modell. ${ }^{723}$

(Faltermaier/Hübner 2021)

(= G.) [engl. gratification crisis; lat. gratificari sich gefällig erweisen], [AO, GES], das Modell der beruflichen G. [engl. Effort-Reward Imbalance Model; Effort-Reward-Imbalance Modell] wurde vom Medizinsoziologen Johannes Siegrist entwickelt. Es postuliert gesundheitliche Risiken bei einem Ungleichgewicht zw. Verausgabungen, die sich aus externalen Anforderungen und Verpflichtungen sowie einer internalen (übersteigerten) Verausgabungsneigung (auch als Overcommitment bezeichnet; Commitment) und Belohnung auf der anderen Seite (Lohn, Aufstiegsmöglichkeiten/Sicherheit, Wertschätzung) ergeben können.

722 Abrufbar unter: https://dorsch.hogrefe.com/stichwort/stress-am-arbeitsplatz, zuletzt eingesehen am 9.4.2021.

723 Abrufbar unter: https://dorsch.hogrefe.com/stichwort/anforderungs-ressourcen-modell, zuletzt eingesehen am 9.4.2021 (siehe im Literaturverzeichnis unter Faltermaier/Hübner 2019). 
Zahlreiche Studien belegen den Zusammenhang zw. G. und psych. Beanspruchungsfolgen (Burn-out) sowie Herz-Kreislauf-Erkrankungen. Gesundheit, berufliche Bedingungen. ${ }^{724}$

(Rigotti 2021)

Zusammenhänge zwischen diesen Modellen und Symptomen von >Burnout und mit beruflicher oder schulischer Belastung assoziierten psychosomatischen Beschwerden und/oder depressiven Symptomen wurden in verschiedenen Studien erforscht oder in Übersichtsbeiträgen dargelegt ${ }^{725}$ und zeigen sich im fachlichen Diskurs beispielsweise in den folgenden Formulierungen:

15) Buchwald/Hobfoll (2004: 247) in Psychologie in Erziehung und Unterricht (im QV unter 8.1.4, Unterstreichungen T.S.)

[...] Die ,Theorie der Ressourcenerhaltung، stellt Burnout als einen kontinuierlichen Prozess dar, dessen zentrale Ursache das andauernde, normalerweise schleichende Schwinden von Ressourcen ist. Die Entwicklung von Burnout entspricht einer Ressourcenverlustspirale, die ihre Dynamik durch das Zusammenwirken von Arbeitsstressoren und deren erfolgloser Bewältigung erlangt. Ausgebrannte Menschen erleben permanent drohende oder tatsächliche Ressourcenverluste oder erhalten nach andauernder Fehlinvestition von Ressourcen nur minimale Ressourcengewinne.

16) Klein/Grosse Frie/Blum et al. (2010: 375) in Psychotherapie, Psychosomatik und medizinische Psychologie (im QV unter 8.1.4, Unterstreichungen T.S.)

Beim Modell beruflicher Gratifikationskrisen von Siegrist [21] liegen psychosoziale Arbeitsbelastungen bei einer Verletzung der sozialen Reziprozität am Arbeitsplatz vor. Ein dauerhaftes Ungleichgewicht zwischen Verausgabung und Belohnung in Form von Gehalt bzw. beruflichem Aufstieg, Wertschätzung und Arbeitsplatzsicherheit führt dabei zu einer Stresserfahrung, welche das Krankheits- und Burnout-Risiko erhöht. Ergänzt wird dieses Modell durch die intrinsische Komponente der übersteigerten beruflichen Verausgabungsneigung. Dabei handelt es sich um ein psychisches Bewältigungsmuster, welches eine bestehende Gratifikationskrise zusätzlich verstärken kann. Assoziationen zwischen beruflichen Gratifikationskrisen und Burnout wurden für verschiedene Berufsgruppen ebenfalls bereits nachgewiesen [14, 22, 23].

724 Abrufbar unter: https://dorsch.hogrefe.com/stichwort/gratifikationskrise, zuletzt eingesehen am 9.4.2021 (siehe im Literaturverzeichnis unter Rigotti 2019).

725 Klein/Grosse Frie/Blum et al. (2010, in PPmP); Neubach/Schmidt (2004, in ZfAO); Freund/ Diestel/Schmidt (2012, in ZfAO); Wassermann/Hoppe/Reis et al. (2014, in ZfAO); Neuenschwander (2003, in PiEU); Buchwald/Hobfoll (2004, in PiEU); Gerber/Pühse (2007, in PiEU); Bodensteiner (2016, in PiEU); Keller-Schneider (2016, in PiEU); Berger/Falkai/Maier (2012, im Dt. Ärztebl.); Riedel-Heller/Luppa/Seidler (2013, in Der Nervenarzt); Abel/Schweitzer/Matthäus (2009, in PiD); Schulze (2009, in PiD); Albrecht/Giernalczyk (2016, in PiD); Siegrist (2016, in PiD); Koehler/ Koehler (2014, in DMW), alle Belege im QV unter 8.1.4; Ahola/Hakanen/Perhoniemi et al. (2014); Avanzi/Zaniboni/Balducci et al. (2014); Siebecke/Ciesinger/Klatt (2011), im QV unter 8.1.5. 
17) Schaufeli/Buunk (2003: 408) in The Handbook of Work and Health Psychology (im QV unter 8.1.2, Unterstreichungen T.S.)

Burnout as a Lack of Reciprocity [im Orig. als Überschrift etwas größer und kursiv gesetzt] By definition, the relationship between caregiver and recipient is complementary, which is semantically illustrated by the term caregiver and recipient; the former gives, the latter receives. However, according to equity theory, people pursue reciprocity in interpersonal relationships: what they invest and gain from a relationship should be proportional to the investments and gains of the other party in the relationship. Clearly, this is not the case in the human services: the caregiver-recipient relationship is unbalanced in terms of costs and benefits or investments and outcomes.

Diese Belege zeigen, dass diese Erklärungsmodelle mit der Metapher der „Ressourcenverlustspirale“, den ökonomischen Metaphern von `Investition`, ,Verbrauch`, `Gewinn`, `Belohnung`, >Erfolg bzw. erfolgreiche Investition` und ,Verlust und der Metapher des `Ungleichgewichts « zwischen diesen proportional aufeinander bezogenen Größen operieren. Die Metapher der „Ressource““726 zeigt des Weiteren, dass es eine Vorstellung davon gibt, dass bestimmte Größen zur Verfügung stehen, um Anforderungen zu bedienen bzw. negative Effekte oder die Investition und damit den Verbrauch dieser Ressourcen wieder auszugleichen. ${ }^{727}$

In Problem- und Magazinberichten aus Zeitungen und populärwissenschaftlichen Zeitschriften findet man teilweise direkte Bezüge auf die genannten Modelle oder indirekte Bezüge auf das `Ungleichgewicht` zwischen `Anforderungen`,

726 Die Gebrauchshäufigkeit des Wortes Ressource ist seit 1945 nach dem DWDSZeitungskorpus bis 2009 kontinuierlich angestiegen: https://www.dwds.de/r/plot?view=1\&corpus=zeitungen $\&$ norm $=$ date $\% 2$ Bclass $\&$ smooth $=$ spline $\&$ genres $=1 \&$ grand $=1 \&$ slice $=1 \&$ prune $=0 \&$ window $=3 \&$ wbase $=0 \& \operatorname{logavg}=0 \& \operatorname{logscale}=0 \&$ xrange $=1945 \% 3$ A2018\&q1 $=$ Ressource (zuletzt eingesehen am 31.12.2019). Dabei ist allerdings noch nicht erklärt, in welcher Häufigkeit es in übertragener Form für ’psychische Ressourcen « verwendet wird. Es ist jedoch interessant, dass sich in der belegreichen Monografie von Schuster zur psychiatrischen Schreibpraxis von 18001939 keine Belege für diese Verwendung des Ausdrucks Ressource finden. Es wäre daher möglich, dass die Verwendung der Bezeichnung Ressource als 'psychisches, menschliches Einsatzmittel' aus dem amerikanischen Englisch entlehnt wurde und auf die Human-Relations-Bewegung und das Human-Resource-Management zurückgeht. Diese These müsste jedoch an größeren Textkorpora überprüft werden.

727 Vgl. die Etymologie des Worts Ressource: ,entstanden aus dem Part. Perf. von afrz. mfrz. resourdre 'sich erheben, sich erholen', aus gleichbed. lat. resurgere; vgl. lat. surgere '(sich) erheben', kontrahiert aus subrigere, zu lat. regere 'geraderichten, lenken, leiten'“. (Pfeiffer/Braun 1993, Onlineversion im DWDS, siehe im Literaturverzeichnis unter den verwendeten Internetquellen unter 8.3). 


\section{>Investitionen bzw. Verbrauch von Ressourcen $`$ und `Belohnung winnung von Ressourcen $:^{728}$}

18) DIE ZEIT 21.10.1999 (= Reicherzer 1999, im QV unter 8.1.6, Unterstreichungen T.S.)

Fleißige Chefs sind stets im Einsatz, obwohl sie den Preis der Überstunden nur zu gut kennen. In der Studie von Cooper erklärten 72 Prozent der Befragten, die langen Arbeitszeiten würden ihrer Beziehung schaden, und 59 Prozent gaben zu, damit ihre Gesundheit zu ruinieren. Ihren deutschen Kollegen geht es nicht besser. Auch die Wissenschaftler aus Göttingen fanden bei ihrer Studie häufig Hinweise auf „drohende kulturelle Verarmung und persönliche wie soziale Entfremdung“. Und Hoff setzt hinzu: „Die Folgen sind immer wieder die gleichen. Produktivität und Motivation sinken, im sozialen Umfeld gibt es Probleme, und außerdem droht der Burnout.“

19) Psychologie Heute 4/2002: 39 (= Smolka 2000, im QV unter 8.1.7, Unterstreichungen T.S.)

Die Wirkung ist dann besonders tiefgreifend, wenn aufreibende Arbeit und dauernde Belastung von wenig Anerkennung und mitmenschlicher Unterstützung begleitet sind.

20) Gehirn\&Geist 11/2005: 17 (= Kraft 2005, im QV unter 8.1.7, Unterstreichungen T.S.)

Gratifikationskrise nennen es Experten, wenn das Gefühl, der hohe berufliche Einsatz würde nicht ausreichend gewürdigt, sich zum zusätzlichen Stressfaktor entwickelt.

21) Frankfurter Allgemeine Zeitung, 17.4.2013: N1 (= Sahm 2013, im QV unter 8.1.6, Unterstreichungen T.S.)

Das Profil der Anforderung in der Arbeitswelt hat sich geändert, im Medizinbetrieb in besonderer Weise, worauf Ulrich Egle, Psychosomatiker der Celenus-Klinik in Gängenbach, nachdrücklich hinwies. Mangelnder persönlicher Einfluss auf den Arbeitsablauf, administrative Zwänge, Verknappung der personellen Ressourcen und nicht selten auch der Mangel an Gratifikation, also Anerkennung der Tätigkeit, machen aus dem Berufsalltag eine Last.

22) Gehirn\&Geist Dossier 1/2016: 75 (= Altstötter-Gleich 2016, im QV unter 8.1.7, Unterstreichungen T.S.)

Auch Erschöpfung, vielleicht das zentrale Merkmal eines Burnout, entsteht aus einer übermäßigen Beanspruchung physischer und psychischer Ressourcen

728 Ähnliche Belege z. B.: „Ein Missverhältnis zwischen Verausgabung und Belohnung nannte Egle als allgemeine Ursache von Burn-out“ (Hetrodt 2013, FAZ, im QV unter 8.1.6); „Ein Betroffener zieht Bilanz: ,Gesundheit, Glück und Zufriedenheit lassen sich offensichtlich nicht mehr mit dieser Art von Arbeit in Einklang bringen.““ (PH 11/2000, = Kerber 2000: 34); „Erhalten Lehrer trotz hohen Engagements keine positive Rückmeldung von Schülern, Eltern oder Kollegen, dann entsteht eine sogenannte Gratifikationskrise, wodurch sich das Risiko für eine Burn-out-Entwicklung erhöht“ (PH 01/2004, = Krumpholz-Reichel 2004: 34); „Burnout entsteht ja dadurch, dass es eine Diskrepanz zwischen den Bewältigungsmöglichkeiten und den Anforderungen gibt“ (PH 9/2016, = Otto 2016), alle letztgenannten Belege im QV unter 8.1.7. 
23) Gehirn\&Geist Dossier 1/2016: 20 (= Falkai 2016, im QV unter 8.1.7, Unterstreichungen T.S.)

Zuletzt nahmen die Anforderungen in der Agentur jedoch ständig zu. Immer mehr Projekte wanderten auf seinen Schreibtisch. Die Tage füllten sich sukzessive mit Besprechungen, Kundengesprächen und Präsentationen. Anfangs gab ihm dieses Mehr an Aufgaben, das schnell getaktete Arbeitsleben einen gewissen Kick. Aber mit der Zeit nagte es zunehmend an seinen Ressourcen. Hinze opferte mehr und mehr Freizeit. [...] Der Kontakt zu Freunden schlief nach und nach ein, seine Frau zog sich zurück, und seinen kleinen Sohn sah er praktisch gar nicht.

Irgendwann ging gar nichts mehr. Hinze verließ die Agentur früher als gewohnt und ging direkt ins Bett. Trotzdem fühlte er sich morgens wie gerädert.

24) FAZ.NET, 29.10.2015 (= epd 2015, im QV unter 7.1.6, Unterstreichungen T.S.)

„Solange es mehr Ressourcen als Stressoren gibt oder das Verhältnis zumindest ausgeglichen wird, trägt das zur psychischen Gesundheit bei“, erläutert Lang. Kippe das Ver-

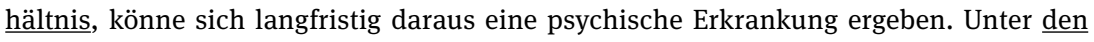
Ressourcen verbucht sie etwa die Vielfältigkeit der Aufgabe, den Handlungsspielraum, eine klare Zuständigkeit, gute Arbeitsmittel, die Möglichkeit zur Fortbildung sowie ein nettes Kollegium. Stressoren könnten dagegen eine Über- oder Unterforderung, eine ständige Erreichbarkeit auch zu Hause, Zeitdruck sowie ein schlechter Umgang mit den Kollegen sein.

Auch die Metaphern eines ıgestörten Gleichgewichts zwischen EnergieEntwertung und Energie-Aufladung ${ }^{729}$ und eines >Akkus, der nicht mehr aufgeladen werden kann ${ }^{730}$ weisen durch das konstatierte Missverhältnis zwischen Entwertung und Ladung Ähnlichkeiten zum metaphorischen Modell einer sunausgewogenen Ausgaben-Gewinn-Bilanzierung، auf. Diese Metaphern einer sozioökonomischen Sichtweise auf Krankheit (vgl. Kap. 3.2 und Eckart ${ }^{8} 2017$ : 323) erhalten dadurch eine definitorische Kraft im Burnout-Diskurs, dass sie variierend über verschiedene textuelle Erscheinungsformen hinweg auftreten.

Zum Abschluss dieses Kapitels geht es um Praktiken, die gleich impliziten Regeln im medizinisch-gesundheitlichen Bereich die Geltung einer Sachverhaltskonstituierung bzw. Definition stützen. Es geht dabei weniger um Faktizitäts- und

729 Vgl. dazu den Beleg auf FAZ.NET, 27.01.2012 (= Astheimer 2012, im QV unter 8.1.6, Unterstreichung T.S.): „Die Bundesanstalt für Arbeitsmedizin spricht von einem ,Endzustand emotionaler Erschöpfung', andere Erklärungen greifen auf die Physik zurück, indem sie Burnout beschreiben als System, dem dauerhaft mehr Energie entzogen als zugeführt wird.“

730 Spiegel-Online, 18.8.2011 (= Hucht 2011, Unterstreichung T.S.): „Letztlich habe [sic] in dem Jahr mit meinem zweiten Baby mehr gearbeitet als je zuvor. Meinem Mann ging es ähnlich. Im Nachhinein war das eine nicht so kluge Entscheidung. Es war wie bei einem Computer-Akku, der immer gut funktioniert, egal wie lange er geladen wird. Der dann aber ganz plötzlich nicht mehr zu laden ist.“ 
Autoritätsstimuli, die themen- und diskursübergreifend eingesetzt werden, ${ }^{731}$ sondern um fachkulturelle Praktiken, die insbesondere im medizinisch-semiotischen Prozess des Definierens geltungsverstärkend wirken und in den untersuchten Texten des fachlichen und fachexternen Diskursstrangs vermehrt zum Einsatz kommen. Dies wird zunächst an medizinischen Textsorten-Routinen und danach an Zeichenformationen in fachlichen und fachexternen Texten erläutert, die >Burnout` als typischen Sachverhalt des medizinisch-semantischen Funktionszusammenhangs verorten, dadurch dass sie bei der definitorischen Konstituierung von >BURNOUT < Bedeutungsaspekte evozieren, die sowohl in fachlichen als auch alltagssemantischen Kontexten häufig mit `Symptomen`, ১Syndromen und >Krankheit` verbunden werden (vgl. von Uexküll 1984b: 32f. und Kap. 3.1-3.3).

In Kapitel 5.3.3.2 wurden die gängigen medizinischen Textsorten beschrieben. Übersichtsarbeiten aus dem Bereich der Medizin bzw. Psychiatrie und Psychotherapie enthalten häufig, ähnlich zu Lehrbuchartikeln über Krankheiten, Abschnitte zu Definition, Ätiologie und Pathogenese, Prävalenz/Epidemiologie, Diagnostik und Differenzialdiagnostik, Prävention, Therapie (vgl. dazu Wiese 2000: 713f.). >BURNOUT< wird in den Übersichtsbeiträgen zumeist innerhalb dieses Textaufbau-Schemas behandelt und dadurch aus der Perspektive des Fachs Medizin dargestellt und diskutiert. Diese Arbeit argumentiert, dass dieser Textaufbau, der sich fachextern-öffentlich dann beispielsweise auch auf der Wikipedia-Plattform wiederfindet, die Geltung der Konstituierung des Phänomens als medizinischer Sachverhalt und damit indirekt auch seine Definition stützt, auch wenn das Ergebnis des Übersichtsbeitrags lautet: „Beim derzeitigen Kenntnis- und Diskussionsstand sollte der Begriff ,Burn-out“ nicht als Diagnose [...] verwendet werden“ (Kaschka/Korczak/Broich 2011: 786, im Dt. Ärztebl., im QV unter 8.1.4). Ein ähnlicher Effekt wird mit Titeln wie „Differentialdiagnostik des Burnout-Syndroms“ oder „Therapie des Burnout-Syndroms“ erzeugt. Das Phänomen/Konzept >BURNouT` wird damit auf Titelebene den praktischen Disziplinen der Differenzialdiagnostik und Therapie zugeordnet, auch wenn der HTA-Bericht zur „Differentialdiagnostik des Burnout-Syndroms“ zu dem Schluss gelangt, dass die bisher ,verwendeten Burnout-Messinstrumente [...] nicht differential-diagnostisch validiert" seien (HTA-Bericht von Korczak/Kister/ Huber 2010: 1). Hier greift das Foucault'sche Verknappungsprinzip der „Disziplin“ (Foucault [1970] 1993: 22f.), und es zeigt sich, dass Wissen im Rahmen von „Fachkulturen“ und deren Praktiken entsteht und dass innerhalb dieser Prakti-

731 Zum Beispiel Gebrauch des Indikativs in einem Kontext, in dem auf ,reale‘ Sachverhalte referiert wird, Verweis auf Diskursautoritäten oder diskursive Mehrheiten etc. Vgl. dazu Felder (2006b); Vogel (2012) im Kontext rechtlicher Normgenese; Deppermann (1997) für Glaubwürdigkeitsstrategien in Schlichtungsgesprächen. 
ken auch die semantischen Kämpfe stattfinden (Konerding 2015b: 72, siehe auch Kap. 4.1.3 und Kap. 7.3).

In den Kapiteln 3.1 bis 3.3 wurden die Grundkonzepte bzw. Termini medizinischer und psychologischer Wissensproduktion, z. B. das medizinischpsychologische Verständnis von `Symptomen`, `medizinischen Zeichen`, ১Störungen`, `Syndromen`, `Symptomclustern >Gesundheit und `Krankheit`sowie typische Zeichenaspekte und -Funktionen in diesen Praxisbereichen beschrieben. Dort wurden die Unterschiede, aber auch Gemeinsamkeiten dieser Konzepte herausgearbeitet. In diesem Kapitel sind im Zusammenhang mit Praktiken der Geltungsvalidierung für den medizinisch-psychologischen Bereich insbesondere die Attribute interessant, die über verschiedene Krankheits-Konzepte hinweg erscheinen. In Kapitel 3.3 wurden folgende Attribute herausgearbeitet (hier erfolgt eine komprimierte Auflistung, für eine ausführliche Übersicht und dazu passende Belege siehe die Kap. 3.1-3.3 dieser Arbeit):

Die Krankheitsursachen sind in der Regel nicht 'bewusst', 'vollständig kontrolliert', 'kompetent', 'motiviert' und 'freiwillig' von einer Person herbeigeführt. Die Attribute, die mit Symptomen, Syndromen und Krankheitszuständen verbunden werden, sind 'Anomalien vor dem Hintergrund von fachlichem und gesellschaftlichem Wissen' (im juristischen Krankheitskonzept 'regelwidriger Körper- und Geisteszustand'), 'Hilfs- bzw. Behandlungsbedürftigkeit', 'Mangel an selbstregulierenden Faktoren', ‘(drohende) eigendynamisch auftretende Funktions- und Möglichkeitseinschränkungen', 'Unwohlsein', 'Leiden' und 'Unbewusstheit'; besonders in juristischen und versicherungstechnischen Kontexten ist das Attribut der 'Arbeitsunfähigkeit' zudem wichtig.

Betrachtet man nun verschiedene Belege des fachlichen und fachexternen Diskursstrangs, in denen Burnout definiert wird, so stößt man in diesen Kotexten auf eine Fülle grammatischer und autosemantischer Zeichen, die ähnliche semantische Dimensionen transportieren.

So findet man sowohl in Lexikon-, Hand- und Lehrbuchtexten als auch in vielen fachexternen Texten Formen, die den geschilderten Zustand/Prozess als ein Geschehen perspektivieren, das 'eigendynamisch', ohne gänzlich kontrolliertes oder bewusstes Zutun der beteiligten Menschen abläuft. Folgende Bezeichnungen und Formulierungen können eine solche Wirkung, dass das Sүмртом wie ein Wetterzeichen auftritt, ohne dass es bewusst produziert würde (vgl. Peirce CP $8.185^{732}$ ), unterstützen:

$732 \mathrm{Vgl}$. folgendes Zitat von Peirce: „But it appears to me that all symptoms of disease, signs of weather, etc., have no utterer“ (CP 8.185) und Kap. 2.2.1 dieser Arbeit. 
Neben der Nennung verschiedener (beruflicher) Gruppenbezeichnungen ist häufig die Rede von Betroffenen/ ... sind betroffen ${ }^{733}$ oder auch von Patienten ${ }^{734}$, Opfern $^{735}$ (vermehrt in fachexternen Texten) und gefährdeten Arbeitnehmern/ ... sind gefährdet ${ }^{736}$. Sie sind durch diese Formulierungen „Patiens-Partizipanten“

733 Weitere Beispiele: „Der Begriff Burnout [...] bezeichnet ein Syndrom, von dem vor allem Angehörige von Sozialberufen betroffen sind“ (Schüpbach/Kause: 2009: 505, im QV unter 8.1.2); „ein Zustand, in dem die Betroffenen gefühlslose und abgestumpfte Reaktionen gegenüber ihren Klienten zeigen“ (Schaper ${ }^{3} 2014$ : 531, im QV unter 8.1.2); Barth 22001/42010, (im QV unter 8.1.2); Bauer (2009: 251, PiD); Bergner (2004: A-2232) und Wittmund (2004: A-2679) im Dt. Ärztebl., alle zuletzt genannten Beiträge im QV unter 8.1.4; siehe auch im fachexternen Diskursstrang AU 05/2005-B: 40 (= AU 2005b); AU 2011d, 18.10.2011 und PH 01/2004 (= Krumpholz-Reichel 2004), im QV unter 8.1.7; ZEIT ONLINE 29.7. 2010 (= Heyn 2010, im QV unter 8.1.6); „Sarah Günther findet es deshalb richtig, dass Betroffene den Weg an die Öffentlichkeit wählen“ (Wenzel 2012, FAZ. NET, im QV unter 8.1.6); „Burnout und arbeitsbedingter Stress träfen immer mehr Menschen“ (= dpa/dapd/end 2013, SPIEGEL ONLINE, im QV unter 8.1.6).

734 Z. B. „Programm für stationäre Burn-out-Patienten“ (Stier-Jarmer/Frisch/Oberhauser et al. (2016: S. III im Anhang des Beitrags), im Dt. Ärzteblatt, im QV unter 8.1.4; Bauer (2009: 253, PiD); Kissling/Mendel/Förstl (2014: 2588, DMW, im QV unter 8.1.4); Reime/Steiner (2001: 304, PPmP) und Söllner/Behringer/Böhme (2016: 227, PPmP), im QV unter 8.1.4; in fachexternen Texten: DIE ZEIT, Rudzio (2010b); SPIEGEL (Nr. 52, 1988); FAZ, Weiguny/Nienhaus (2014a); GEO WISSEN, Frömel/Recht (2011); .

735 SPIEGEL ONLINE/manager magazin: „Gerade die Leistungsfähigen sind die Opfer. (= Klepsch 2011); „Wachsende Opferzahl“ (= Werle 2012b) im QV unter 8.1.6); „,Heute‘, schrieb Byung-Chul Han, ,beutet man sich selbst aus, wobei man sich in Freiheit wähnt. Das Leistungsobjekt ist Täter und Opfer zugleich“ (Freidel 2013, FAZ); Interessant in diesem Zusammenhang ist auch die Mehrworteinheit Kampf gegen Burnout und welche Personengruppen hierbei im Kotext als Akteure eingesetzt werden, um Opfer zu vermeiden/Personen vor `Burnout zu schützen: z. B. „Arbeitgeber sollen den Kampf gegen ,Burn-out“ aufnehmen“ (FAZ, 30.1.2013, = SteinauSteinrück (2013); vgl. auch FAZ 2013c); weitere Belege mit der Verwendung des Ausdrucks Opfer: G\&G Dossier 1/2016: 20 (= Falkai 2016, im QV unter 8.1.7); DIE ZEIT, 28.10.2010, Nr. 44 (= Spork 2010, im QV unter 8.1.6); kritisch zum Begriff `Opfer in DIE ZEIT, 1.12.2011, Nr. 49 (= Pawelzik 2011, im QV unter 8.1.6); SZ 6.5.1997 (= Weinfurtner 1997, im QV unter 8.1.6); Dpa/svs/tine (2015, FAZ.NET, im QV unter 8.1.6); PH 8/2014 (= Ernst (2014b: 3, im QV unter 8.1.7); Kleinschmidt beschreibt, dass die Erkenntnis des eigenen Anteils in der Therapie aus der „Opfersituation“ herausführe (PH 4/2017: 72, = Kleinschmidt 2017); in fachlichen Texten seltener, aber es gibt auch Belege: Freudenberger (1974); Kleiber/Enzmann (1990: 11, im QV unter 8.1.5); Seibold/Schuh (52010: 64ff.) überschreiben ein Kapitel mit dem Titel „Opferrolle ablegen“ (im QV unter 8.1.5); Bergknapp (2009: 241, PiD, im QV unter 8.1.4).

736 „Gefährdet sind Menschen in sozialen und pflegerischen Berufen“ (= Kals 2004, in FAZ); vgl. auch Meck (2010, FAZ.NET); Weiguny (2015, FAZ); Heimann (2008, manager magazin); alle Belege im QV unter 8.1.6; in Fachtexten: „Es besteht die Gefahr, an einem Burn-out-Syndrom zu erkranken“ (Meldung im Dt. Ärztebl., siehe Bühring 2005: A-1705) und Madel (2003), ebenfalls im Dt. Ärztebl.; „Pastoren mit dem burnoutgefährdeten Muster“ (Voltmer/Büssing/Thomas et al. 2010: 431, in PPmP); Albrecht/Giernalczyk (2016, in PiD); beide Belege im QV unter 8.1.4. 
eines (selbstinduzierten) Vorgangs oder einer „Zustandsveränderung““737, auch wenn sie innerhalb dieses Zustands teilweise als Handelnde und damit in einer agentivischen oder agensähnlichen Rolle auftreten. Denn die absichtsvollenintendierten Handlungen, die den Personen zugeschrieben werden, sind häufig negiert oder mit Handlungseinschränkungen verbunden oder im Kontext als inadäquat etikettiert (z. B. Aufgaben nicht mehr bewältigen können, sich immer schlechter konzentrieren, reduziertes Engagement, die Kinder bloß anschreien). Sonst erscheinen sie meist in „Proto-Agens-Rollen: ${ }^{738}$

1. als Experiencer/Zustandsträger ${ }^{739}$ von nur bedingt kontrollierbaren Gefühlen und Einschätzungen oder von Empfindungen, für deren Deutung die BurnoutLiteratur sensibilisieren möchte ${ }^{740}$ (fühlte sich schon morgens erledigt; Zwang, sich zu beweisen; sind gestresst und überfordert; sich immer weniger kompetent und erfolgreich fühlt, Wie kann ich feststellen, ob ich unter Burn-out leide?);

2. als „Vorgangsträger“ selbstinduzierter Bewegungen/telischer Vorgänge (schläft miserabel; Entwicklung dysfunktionaler Einstellungen; entfremdet sich immer mehr von der Arbeit; hat seine seelischen Kräfte völlig verbraucht; dass man sich emotional überfordert ${ }^{741}$ );

737 Beatrice Primus bestimmt die Patiens-Rolle wie folgt: „Als Patiens wird die semantische Rolle eines Partizipanten bezeichnet, der in dem vom Prädikat bezeichneten Geschehen physisch manifest betroffen ist und dessen Zustand sich physisch verändert“ (Primus 2012: 31f.). Unter einer „Zustandsveränderung“, die Primus auch unter die Patiens-Rollen einordnet, versteht sie Folgendes: „Eine Zustandsveränderung ist an die Aktionsart des Satzes gebunden und setzt keinen weiteren Verursacher-Partizipanten voraus“ (ebd.: 32).

738 Siehe dazu Primus (2012: 25ff.), die sich auf den Proto-Agens-Begriff von David Dowty bezieht.

739 „Sentience (Experiencer/Zustandsträger)“ hat nach Primus „mehrere Ausprägungen, u.a. Wahrnehmung (sehen, hören, auffallen), Empfindung (frieren), Emotion (mögen, hassen, fürchten), Bewertung (schätzen, bevorzugen) oder epistemische (d.h. wissensbezogene) Zustände (wissen, glauben, kennen, einfallen). Jeder psychische Zustand setzt einen Experiencer (auch Zustandsträger) voraus“ (Primus 2012: 25).

740 Die ,Betroffenen` sollen lernen, ihre Empfindungen ,richtig‘ zu deuten, um dann wieder zu kontrollfähigen Akteuren zu werden: „Kenne ich die Signale meines Körpers, kann ich darauf reagieren“ (Gottschalck 2012, manager magazin, im QV unter 8.1.6); Stimmen, die die Zuschreibung >Burnout kritisieren, setzen auch bei der ,richtigen' Wahrnehmung und Bewertung an: „,Die Mitarbeiter wollen auch selbst zu viel' [...]. Es gelte, mehr auf sich selbst aufzupassen“ (Knust 2012, SPIEGEL ONLINE, im QV unter 8.1.6).

741 Die Formulierungen entfremdet sich immer mehr von der Arbeit und dass man sich überfordert werden in diesem Kontext nicht als Handlung mit einem gänzlich kontrollfähigen, absichtlich handelnden Agens interpretiert. Denn wenn man diese Konstruktionen im Hinblick auf die semantischen Merkmale 'Kontrolle' und 'Absicht' durch Hinzunahme der Handlungsverben unterlassen, tun oder des Adverbs absichtlich sowie durch Anwendung des Imperativs testet, 
3. als vormalige „Besitzer“ von Fähigkeiten/Ressourcen/Idealen (verlieren auch die Energie für ihr Privatleben; Betroffenen geht die psychische und physische Leistungsfähigkeit sowie die Kraft zum Regenerieren verloren; konnte ich nicht mehr abschalten $)^{742}$.

BuRnouT und dessen Symptome stehen regelmäßig in Subjektposition (Burnout ist ein schleichender Prozess) und erscheinen durch Behältermetaphorik (Burnout, Depersonalisation beinhaltet $^{743}$ ) oder Personifizierung (Syndrom, das ... auftritt ${ }^{744}$, hinzu kommen diverse seelische und körperliche Beschwerden, sein Herz setzt zu

so erscheinen die Konstruktionen „hinsichtlich der Absichtlichkeit“ zumindest vage zu sein (vgl. Primus 2012: 18ff.):

"Der Mitarbeiter entfremdet sich absichtlich von der Arbeit / "Was der Mitarbeiter unterließ, war es, sich von der Arbeit zu entfremden / "Entfremden Sie sich bitte von der Arbeit!; "Was man tut, ist sich bei der Arbeit zu überfordern (Die Konstruktion Was man unterlässt ist, sich bei der Arbeit zu überfordern ist aber durchaus plausibel / "Man überfordert sich absichtlich bei der Arbeit / "Überfordern Sie sich bitte bei der Arbeit! Weitere Beispiele, die im Hinblick auf die Merkmale 'Kontrolle' und 'Absicht' nicht eindeutig sind, wären Beispiele mit dem Verb regenerieren („,nehme die Fähigkeit [...] ab, nach Belastungen zu regenerieren“ (Bühring 2000: A602, Dt. Ärztebl., im QV unter 8.1.4) oder den Verben entspannen und aus- und abschalten, die zwar als Handlungsanweisung häufig in Burnout-Ratgebertexten formuliert werden, aber nicht (mehr) gänzlich kontrolliert werden (,wie sie sich in ihrer Freizeit optimal entspannen“ (Lesti 2008, FAZ) versus „Unfähigkeit, sich zu entspannen“ (Belz 2012, FAZ; „,wenn es nicht mehr gelingt, die Stressreaktion des Körpers ,auszuschalten““ (PH 2/2002, = Nuber 2002: 21).

742 Die „Betroffenen“ werden in diesen Beispielen in der semantischen Rolle von vormaligen `Besitzern` präsentiert. Die verlorenen Besitzgegenstände sind die abstrakten Größen `Energie`, >Leistung`, `Kraft‘. In der Theorie der semantischen Rollen wird bei Besitzrelationen jeweils unterschieden, ob der >Besitzı 'veräußerbar' oder 'unveräußerbar' ist, also ob der >Besitzer ` das Erwerben oder Veräußern kontrollieren kann (Primus 2012: 18-19). Der Umgang mit diesen ,Ressourcen' wird durchaus als kontrollierbar bis zu einem bestimmten Grad angesehen, was durch Formulierungen wie Haushalten mit den eigenen Kräften oder die Energie gut einteilen etc. deutlich wird. Korpusbelege in Auswahl: „Kollegen, die [...] ihre Ideale verloren haben“ (Drees 2003 im Dt. Ärztebl., im QV unter 8.1.4); „hat das Vertrauen in seine Fähigkeiten verloren“ (Rudzio 2010b, DIE ZEIT, im QV unter 8.1.6.); , ,[D]och Linde verliert das Gefühl für sich, er ist wie besoffen von seinen Erfolgen“ (Briseño 2012, SPIEGEL ONLINE, im QV unter 8.1.6).

743 Ein weiteres Beispiel dazu ist: „,Erstens beinhaltet Burn-Out die Symptome einer Depression, wie etwa Überforderung, Erschöpfung oder Leistungsabfall', sagt Ruhwandl“ (= Steinkohl/ Hölper 2003: 35 in der SZ, im QV unter 8.1.6); siehe z. B. auch Schaper (32014: 531f., im QV unter 8.1.2);

744 Dies ist eine kookkurrente Wortverbindung - Nebenwirkung(en)/Krankheit(en)/Störung(en) ... treten/tritt auf - wie eine Recherche des Kookkurrenzprofils des Verbs auftreten in der Kookkurrenzdatenbank CCDB des Leibniz-Instituts für Deutsche Sprache zeigt (zuletzt abgerufen am 29.12.2019). Die Datenbank ist unter http://corpora.ids-mannheim.de/ccdb/ erreichbar (= Belica 2001). Vgl. dazu auch Schnedermann (2016: 239). 
unvermittelten Sprüngen an .... $)^{745}$ als aktives Zentrum des Satzes. Dies bewirkt, dass die Blickrichtung auf das Phänomen von den Symptomen ausgeht.

In den Fachlexika, Hand- und Lehrbüchern und in Textstellen, in denen der Nominalstil dieser Textsorten übernommen wird, herrscht eine komprimierte, ${ }^{746}$ unpersönliche Ausdrucksweise vor, bei der die Vorgänge über nominalisierte Nebenprädikate (Erschöpfung, Depersonalisierung) versprachlicht werden, wodurch die beteiligten Personen nur implizit als involvierte Größen erscheinen. Denn bei einer Formulierung wie Syndrom, das bei professionellen Helfern als Folge von Überlastung auftritt lässt sich nicht genau bestimmen, ob dem Nomen Überlastung eine transitive oder reflexive Lesart zugrunde liegt und von welcher Instanz die Handlung bei einer transitiven Lesart ausgeht. Ähnlich verhält es sich bei Formulierungen wie hohe interpersonelle Anforderungen, Maße beeinträchtigten Befindens oder den gestellten Ansprüchen nicht mehr zu genügen.

Dadurch, dass Burnout und die einzelnen Symptome als Elemente perspektiviert werden, die ohne menschliches Zutun auftreten, wird eine Unabhängigkeit von subjektiven Standpunkten konstituiert, die den definitorischen Anspruch auf objektorientierte Sachverhaltskonstitution/Begriffsbildung unterstützt. In Sprachgebrauchsformen, die antonyme und komplementäre Gegensätze ausdrücken und dadurch sozial-kulturelle Bewertungsnormen evozieren (vgl. Kap. 6.2.4.1), manifestiert sich zudem eine stabile sozial-kulturelle Vergleichsfolie, vor deren Hintergrund einzelne Merkmale des zu definierenden Sachverhalts erst zu erklärungsbedürftigen Signalen, sprich Symptomen, werden. Diese medizin-semiotische Praxis, die in Kapitel 3.1 beschrieben wurde, verstärkt die realsemantische intersubjektive Wirkung dieser Definitionen. Denn die damit implizit transportierte Aufforderung, für diese „Signale“ eine Beschreibung und Erklärung zu finden, entsteht aus der Diskrepanz zwischen erwarteten ,normalen' und den deskriptiv-narrativ dargelegten Zuständen und Handlungs- und Verhaltensweisen. Dadurch scheint der Impuls

745 Weitere Beispiele für Personifizierung: „Die subjektive Anstrengung steigt, aber die Qualität der Leistung sinkt“" (Schmidbauer (1991: 41); Zum Prinzip der Personalisierung als sprachliches Mittel des Geltungsanspruchs im medizinischen Bereich siehe auch Schnedermann (2016). Dieses Mittel wird auch in anderen Bereichen zur Verstärkung der Geltung der Aussage eingesetzt, für den juristischen Bereich findet man bei Vogel (2012) eine ausführliche Analyse.

Eine weitere personifizierte Größe, die gegen das Wohl der Person ,handelt‘ und die mehrfach im Kontext von >Burnout genannt wird, sind die sogenannten dysfunktionalen „inneren Antreiber“: „Wir haben in Burn Out-Gruppen (sic) häufig festgestellt, dass Lehrer unter ,inneren Antreibern“ leiden“ (PH 12/1997, = Smolka 1997: 43).

746 Die Textstellen sind im Sinne von P. von Polenz’ Satzsemantik „komprimiert“, da die Zahl der zu verstehenden Inhaltsteile größer als die Zahl der Ausdruckseinheiten ist (vgl. von Polenz ${ }^{3} 2008:$ 25ff.). In Kap. 6.2.2.1 wurde einer dieser Textstellen in explizite Formulierungen aufgelöst. 
zum Definieren ,von den Dingen selbst“ auszugehen. Dies bewirkt eine ,empirische‘ Validierung, die auf die gesamte Definition ausstrahlt.

\subsubsection{Zusammenfassung}

In Kapitel 6.2.4.1 wurden sprachliche Stimuli definitorischer Praktiken der Zuweisung, Einordnung sowie Ein- und Abgrenzung herausgearbeitet, die >Burnout` dadurch ein- und abgrenzen,

- dass sie das Konzept stabil einem Bereich zuweisen (z. B. über fachliche Rubriken in Hand- und Lehrbüchern, Keywords in Fachzeitschriften, Ressorts und Rubriken in Zeitungen oder über Berufsgruppen, die besonders beforscht werden/über die am meisten medial berichtet wird). >Burnout wird dadurch über die Einzeltexte hinweg besonders in Verbindung gebracht mit den Themen „Stress“, „Belastung“, „Leistungsprobleme“ und „psychische Gesundheit“ und folgenden beruflichen Personengruppen: Arbeitnehmer/innen im sozialen und schulischen Bereich, mit hoher Verantwortung/ Leitungsfunktion, mit hoher Motivation/hohem Engagement und der Gruppe Leistungssportler/innen;

- dass sie das Konzept im Kontext fachkultureller Praktiken konstituieren (in klinischen Fächern durch Bezug auf die ICD-10 und Zurückweisung der Gleichsetzung des Begriffs >Burnout`mit den Begriffen `Syndrom〈/>klinische Diagnose und in anderen psychologischen Anwendungsfächern z. B. durch Bezug auf etablierte arbeitspsychologische und -soziologische Modelle). Diese fachlichen Zuordnungspraktiken werden in fachexternen Texten aufgegriffen und vermittlungstechnisch weiterverarbeitet. Ein metaphorisches Modell, das sowohl fachintern als auch fachextern Bedeutung besitzt, ist das Konzept einer >unausgeglichenen Bilanz` (vgl. dazu Kap. 6.2.4.2);

- dass sie das Konzept wiederholt an verschiedenen Stellen auf ähnliche implizit mitgemeinte kollektive Wissensbestände (Bezugsnormen wie durchschnittliche Arbeitszeiten, Belastungen, biologische Durchschnittswerte oder sozialkulturelle Idealnormen und deren Gegenpole) beziehen. Die Attribute von >Burnout` werden dadurch über die fachlichen und fachexternen Einzeltexte hinweg in ähnlicher Weise (in fachexternen Texten jedoch deutlich expliziter und teilweise im Vergleich zu den fachinternen Texten auf einer höheren Steigerungsstufe) von 'erwarteten', 'normalen' somatischen und psychischen Funktions- und Leistungsprofilen und Arbeitsbedingungen abgegrenzt. Das ‘übersteigerte Maß an Aufgaben’ wird zudem in Fotografien und Illustrationen in Presse- und Magazintexten und in fachlichen Texten, die sich an eine breitere Öffentlichkeit wenden, musterhaft ins Bild gesetzt (siehe Kap. 6.2.4.2). 
In Kapitel 6.2.4.2 wurden außerdem sprachliche Stimuli definitorischer Praktiken der Gewichtung, Verknappung und Geltungsvalidierung untersucht, die durch typo- und topografischen Hervorhebungsmittel und intertextuell sichtbar gemachte Zitations- und Reformulierungsnetze zur Durchsetzung bestimmter >Kernsymptome` (z. B. chronische emotionale und physische Erschöpfung, Zynismus/Depersonalisation, reduzierte Leistungsfähigkeit, berufliche Überforderung, fehlende Regenerationsfähigkeit) und Phasen-Symptom-Modelle (z. B. von Burisch ${ }^{5} 2014$ und Freudenberger/North 1985/ ${ }^{9} 2002$ ) beitragen. Durch die diskursiv verbreiteten Phasenmodelle wird der Kontrast zwischen einem umtriebigen >Anfangsstadium` (vermehrtes Engagement für Ziele, hoher Energieeinsatz, Zwang sich zu beweisen) und einem quantitativ und qualitativ entgegengesetzten $>$ Endstadium` (Abbau der kognitiven Fähigkeit, Rückzug, völlige Erschöpfung) konstituiert. Dieser Kontrast wird in verschiedenen Ausprägungen auch in fachlichen deskriptiven Definitionsentfaltungen (teilweise nur implizit) und in den meisten fachexternen narrativen Verfahren des Definierens durch sprachliche Mittel der Gradierung und Polarisierung diskursiv ausgestaltet.

Schließlich wurden in Kap. 6.2.4.2 mit Rückgriff auf Kategorien der medizinischen Semiotik und Krankheitskonzepte (siehe Kap. 3) Praktiken der Geltungsvalidierung für den medizinisch-psychologischen Bereich herausgearbeitet. Burnout und einzelne Merkmale erscheinen durch diese Praktiken als Sachverhalte, die unabhängig von subjektiven Standpunkten realsemantisch definiert werden. 